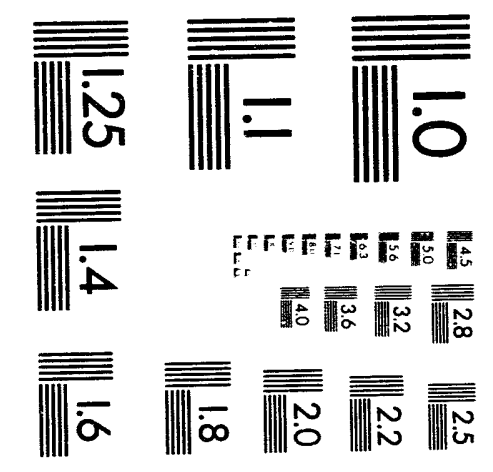



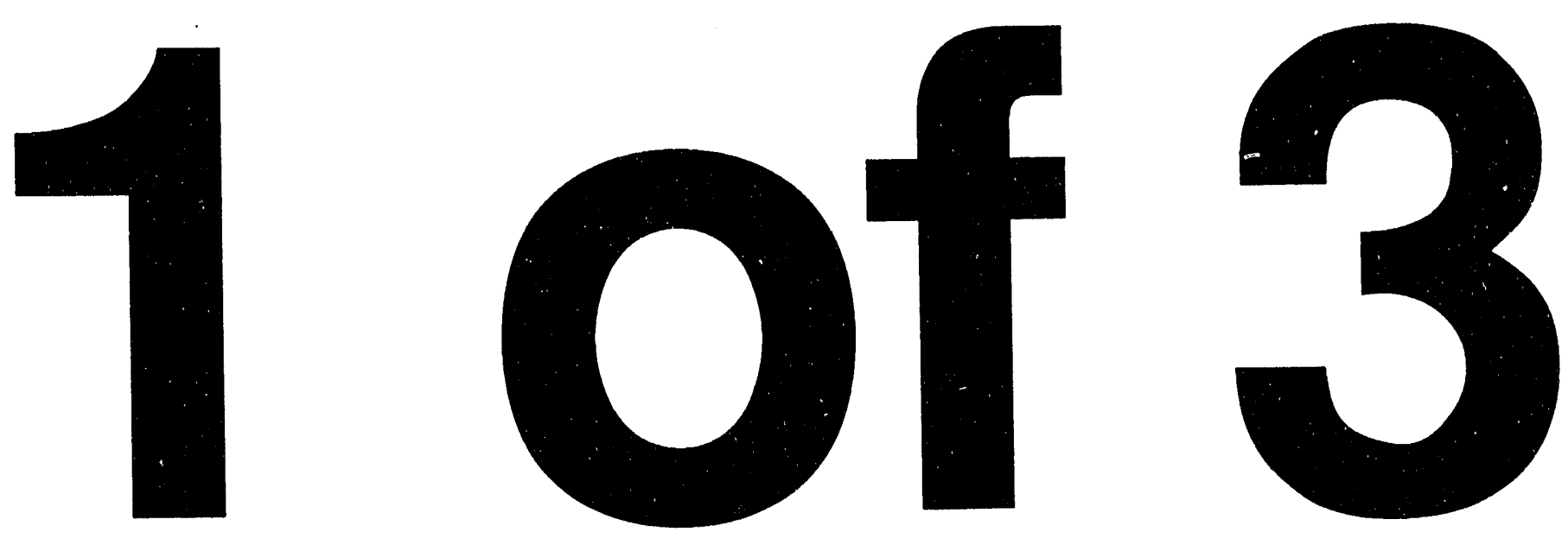


\section{IDECLASSIFIED ]}

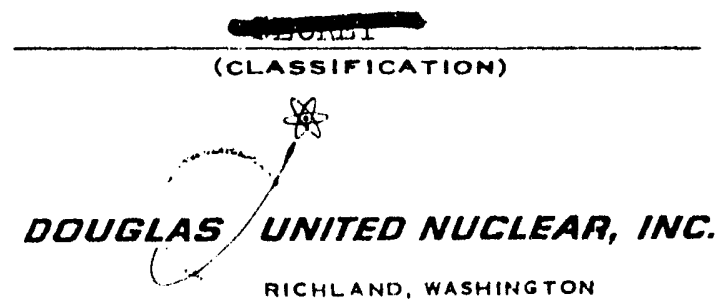

DOCUMENT NO.

DUNN $-5929 \cdot 4$

Hanford Codes C57-C65

SERIES AND COPY NO.

$$
\text { A } 1
$$

DATE July 1, 1969
$X$ RESTRLCTED DATA

THIS DOCUMENT CONTAINS RESTRICTED DATAAS DEFINED IN THE ATOMIC ENERGY ACT OF 1954. ITS TRANSMITTAL OR THE DISCLOSURE OF ITS CONTENTS IN ANY MANNER TO AN UNAUTHORIZED PERSON IS PROHIBITED.

[ OTHER OFFICIAL CLASSIFIED INFORMATION THIS MATERIAL CONTAINS INFORMATION AFFECTING THE NATIONAL DEFENSE OF THE UNITED STATES WITHIN THE MEANING OF THE ESPIONAGE LAWS, TITLE 18, U.S.C., SECS. 793 AND 794, THE TRANSMISSION OR REVELATION OF WHICH IN ANY MANNER TO AN UNAUTHORIZED PERSON IS PROHIBITED BY LAW.

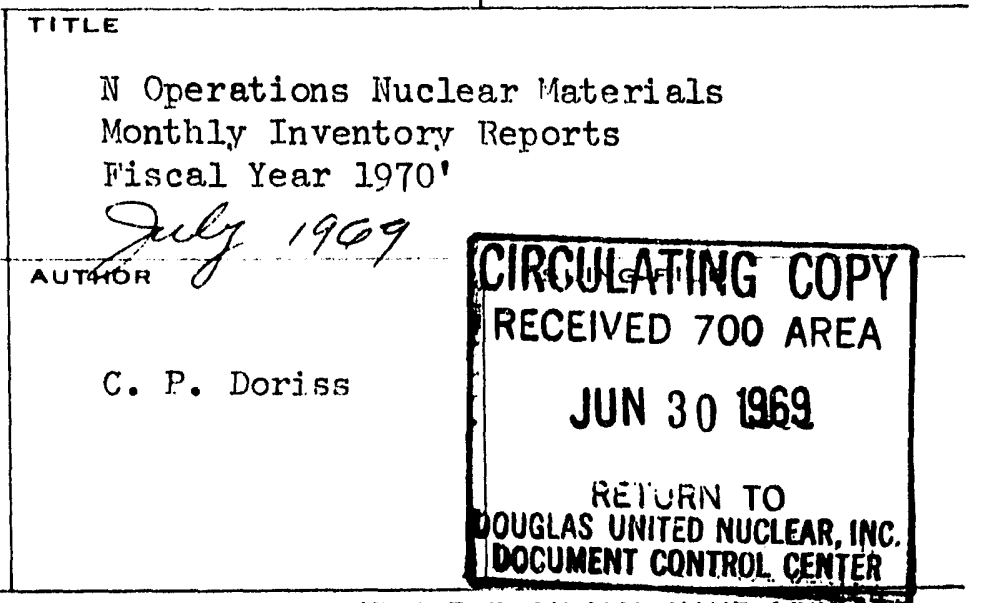

THIS DOCUMENT MUST NOT BE LEFT UNATTENDED OR WHERE AN UNAUTHORIZED PEIR SON MAY HAVE ACCESSS TO IT. WHEN NOT IN USE, IT MUST BE: STORED IN AN APPROVED LOCKED REPOSITORY WITHIN AN APPROVED GUARDED AREA. WHILE IT IS YOUR POSSESSION AND UNTIL YOU HAVE OBTAINED A SIGNED RECEIPT FROM CLASSIFIED FILES, IT IS YOUR RESPONSIBILITY TO KEEP IT AMD ITS CONTENTS WITHIN THE LIMITS OF THIS PROJECT AND FROM ANY UNAUTHORIZED PERSON. ITS TRANSMITTAL TO, AND STORAGE AT YOUR PLACE OF RESIDENCE IS PROHIBITED. SECRET OR CONTROLLED CONFIDENTIAL DOCUMENTS CANNDT BE DUPLICATED. IF ADOITIONAL COPIES ARE REQUIRED, OBTAIN THEM FROM THE RELATED ISSUING FILE. ALL. PEFSONS READING THIS DOCUMENT ARE REOUESTED TO SIGN IN THE SPACE PROVIDED BELOW.

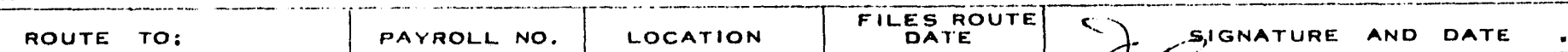

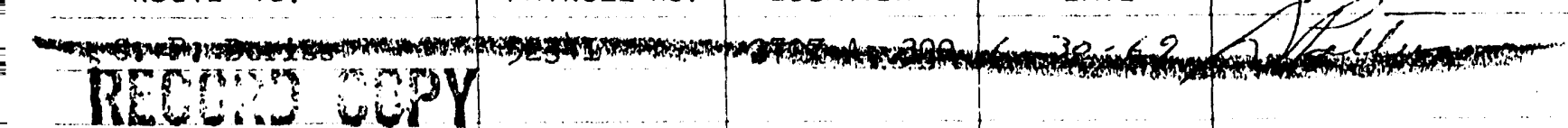

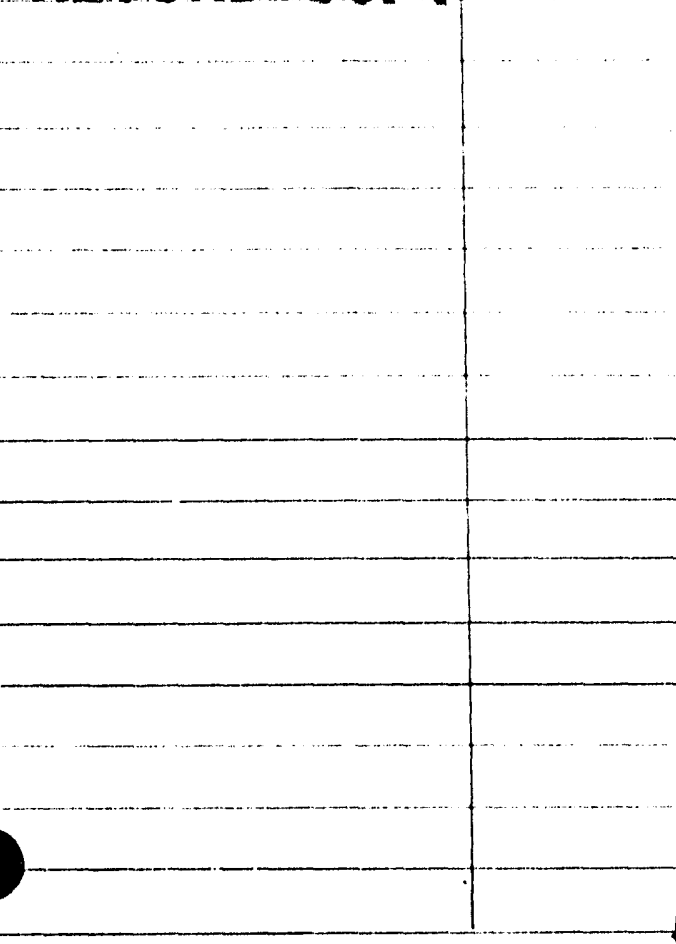

$54-5100-165 \quad(5-68)$ AEC.RL RICHLAND, WASH

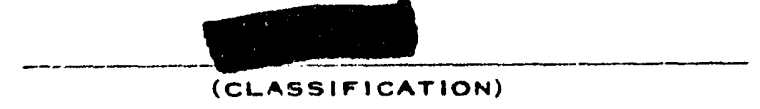
$8 \%$ r 


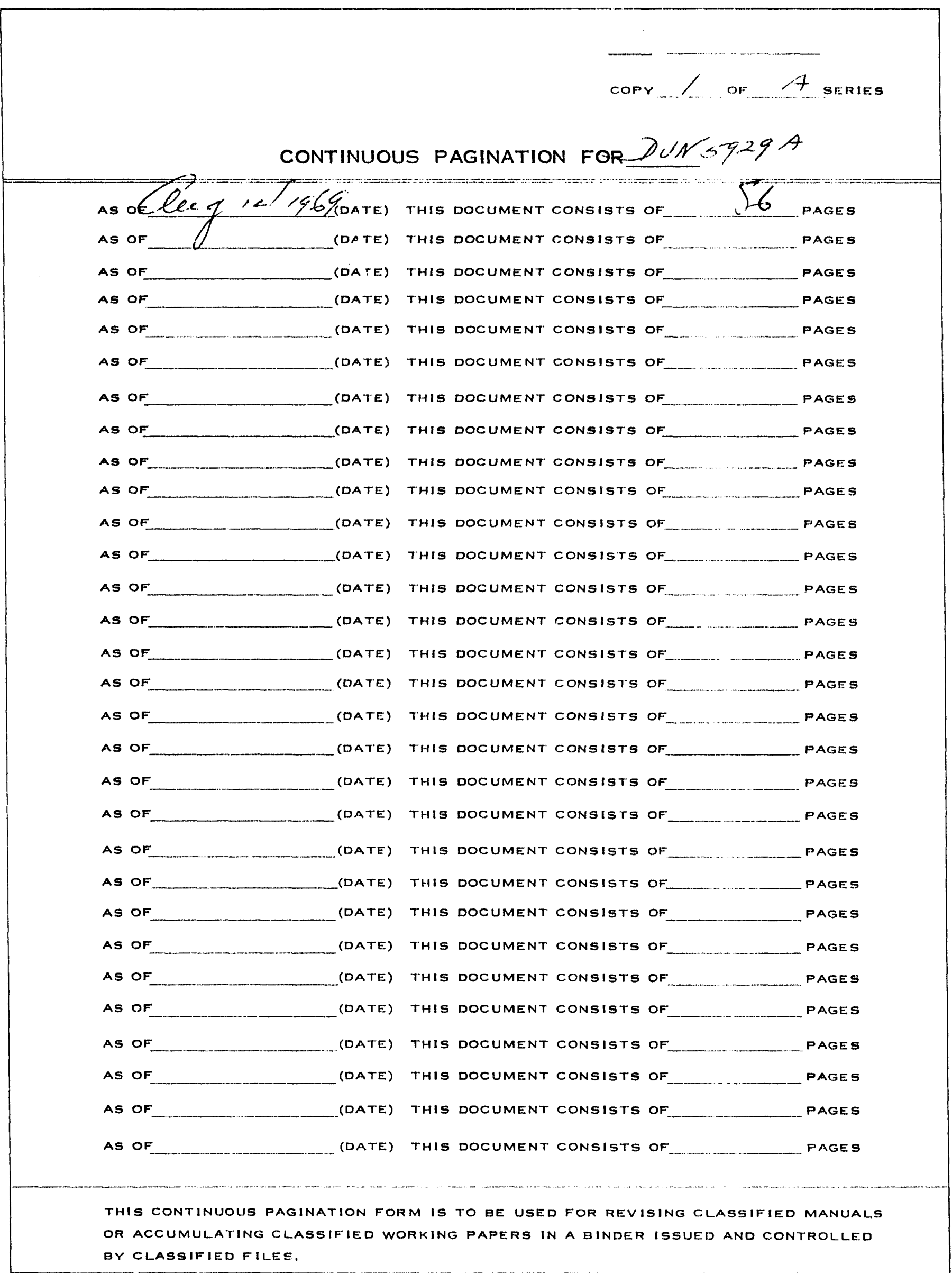


| DECLRUIFIED |

$$
\begin{aligned}
& \text { DUN-5929A } \\
& \text { Copy A } 1
\end{aligned}
$$

Hanford Code C-65, C-57

July 1,1969

This document classified

by 'I' Prudich

DISTRIBUTION

C. P. Doriss

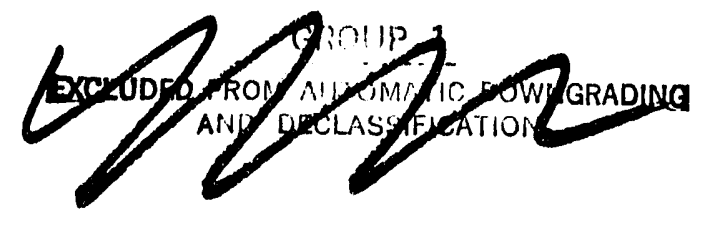

N OPERATIONS NUCLEAR MATERIALS

MONTHLY INVENTORY REPOR'SS

FISCAL YEAR 1970

$$
\begin{gathered}
T 04469 \\
\text { Author } P . \text { Doriss }
\end{gathered}
$$

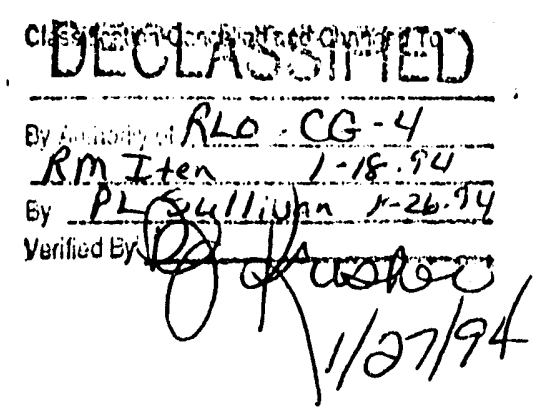

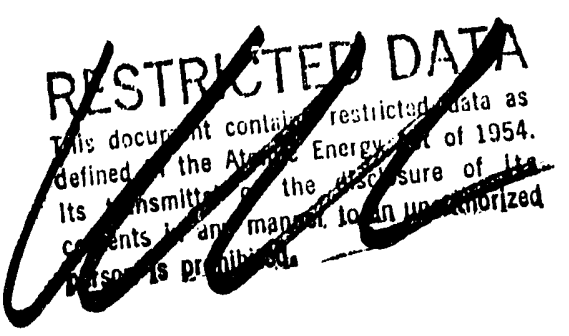

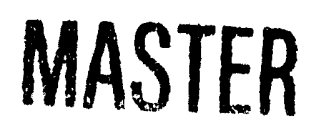

-DECLASGIFIEL , fe

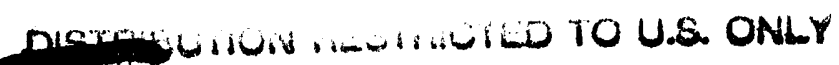




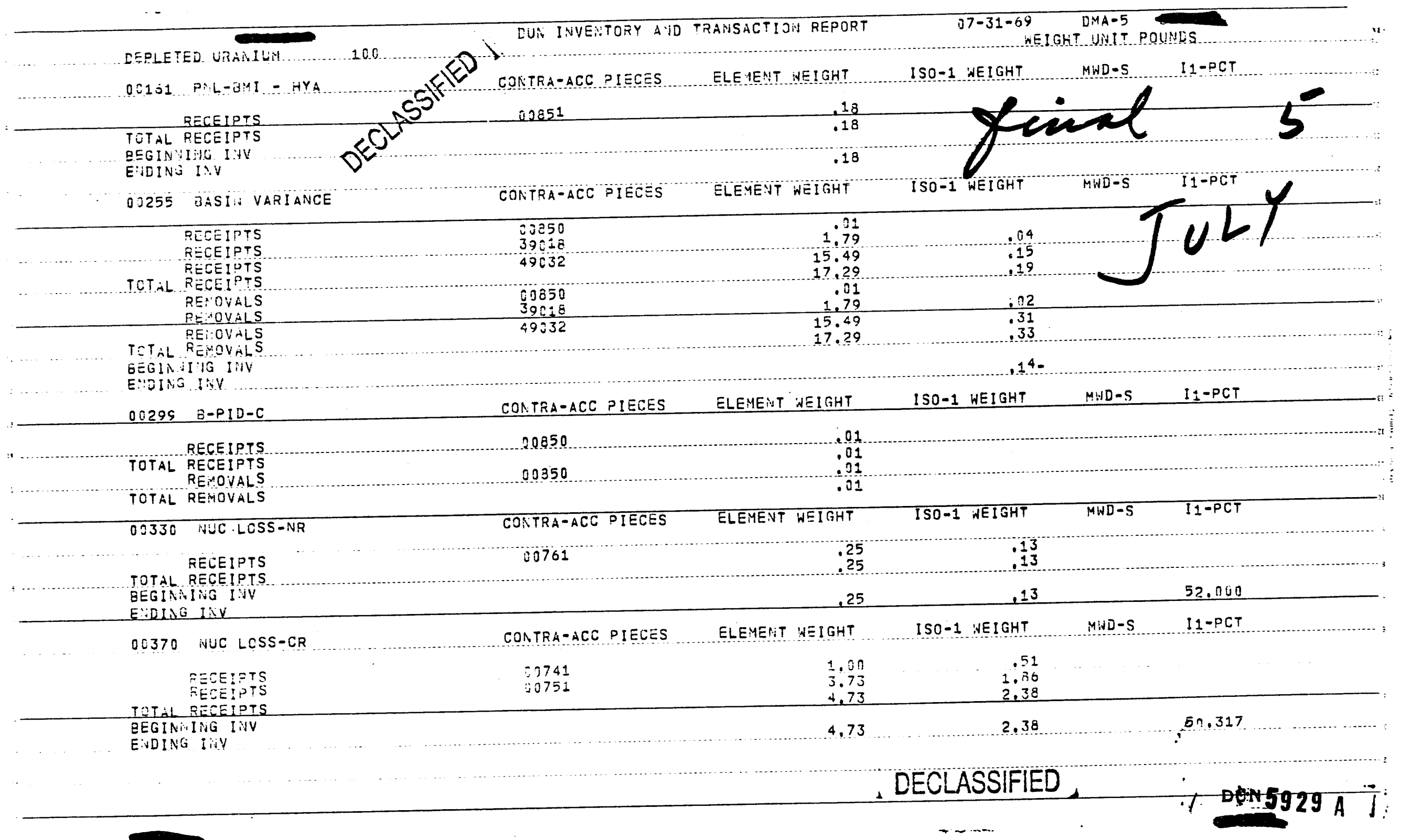




\section{-}

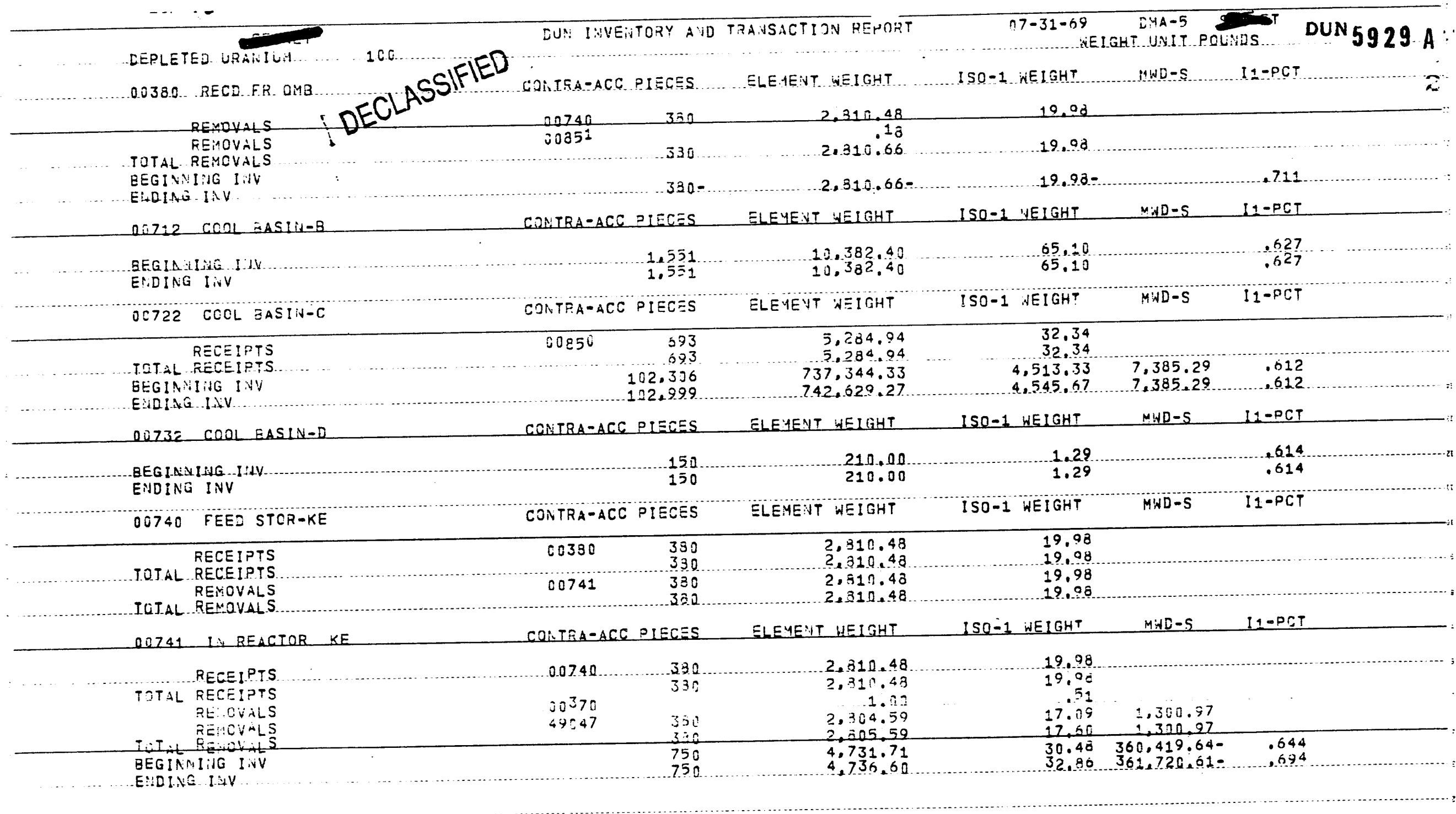




\section{-}

$07-31-59$ DYA-5

CJ: INVEITORY AIID TRAISACTION REPORT NE!GHT UNIT FOUNDS

DEPLETED URASILY

00742 COCL SASIN-KE 100

CONTFA-ACC PIECES ELEYEVT WEIGHT

ISO-1 NEIGHT

MWD-S I1-PCT

TOTAL RECEIPTS

EEGINEISG IIU

$49: 32$

595,78

131.364 .17

$131,979.95$

76
76
18.326
18.432

ELETENT

3.65
3.65
303.85
807.50

3.65
303.85

$807.50 \quad 72.035 .30 \quad .612$

ENDING IVIV

CONTRA-ACC PIECES

ELEMENT NEIGHT

ISO-1 WEIGHT

$M W D-S$ II-PCT

$O O 751$ IV REACTOR KW

00370

REOOVALS
TOTAL RENOVALS

BEGINIING IIVV

ENDISG I:V

$0[752$ COOL BASIN-Kii

$12357 \ldots \ldots \ldots$ 9.2736.59

3.73
3.73
.740 .32
.736 .59

1.36

1.36

$60.71259,631.13-\quad .685$

$\begin{array}{lll}66.71 & 259,631.13- & .655 \\ 64.84 & 256,031.13-\ldots . .566\end{array}$

$39218 \ldots \ldots 942.24$

ISO-1 WEIGHT

MWD-S

$11-P C T$

TOTAL RECEIPTS

EEGIN THO IOY

29.455

29,559

994.24
694.24
$223.643,80$

$223,643.80$
$224,538.04$

ENDING INV

COMTRA-ACC PIECES

ELEMEYT WEIGHT

.25

REMOVALS

JOTAL REMOVALS

ENDING IVY

00330

.25
732.25
732.08

EDIRG LiV

00752 COOL EASIN-NR

CONTPA-ACC PIECES

ELEHENT NEIGHT

730.28
730.28

ISO-1 WEIGHT

5.47

$\begin{array}{rrr}1.369 .61 & 93.288 .70 & .612 \\ 1,375.79 & 93,288.76 & .612\end{array}$

$1,375.83$

BEGINUIIUG IIVV

EIJDING . $V \mathrm{~V}$

OS820 CHEM-METAL-NE

COATRA-ACC PIECES

NEIGHT

4.1

$\frac{4.18}{4.18}$

MWD-S

$11-P C T$

QEGIINIOG IN

E.D? $\mathrm{I} v$

QCgSE PLAI AS TES-GE

COR TEA-ACE PI $\because C:=S$

$\begin{array}{ll}26 & 361.30 \\ 23 & 261.33\end{array}$

ISO-1 WEIGH'

\begin{tabular}{cc}
1.79 & \\
1.79 & .655 \\
ISO-1 HEIGHT & .655 \\
\hline
\end{tabular}

$\begin{array}{lll}.13 & & \\ .02 & 712.210 & .685 \\ 90 & 712.21- & .669\end{array}$

RECEIPTS
RECEIPTS
RECEIPTS
TUTAL RELEIPTS GEIOVALS

ELENE $T T$ VEIGHT

\begin{tabular}{cc}
1.79 & \\
1.79 & .655 \\
ISO-1 HEIGHT & .655 \\
\hline
\end{tabular}

MWD-S

$\square I-P C T$

\begin{tabular}{l} 
RECEIPTS \\
RECEIPTS \\
RECEIPTS \\
TUTAL TELEIPTS \\
REIOVALS \\
\hline
\end{tabular}

60255
00299
00850
00255

.01
1
3.60
3.52
.01

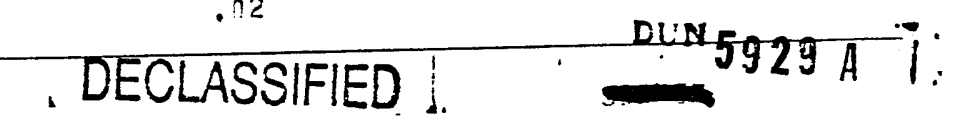




\section{-}

$-\cdots$

LUR INVENTORY AVD TRANSACTIJU REPORT

07-31-69

DMA-5

NEIGHT UNIT FOUALS

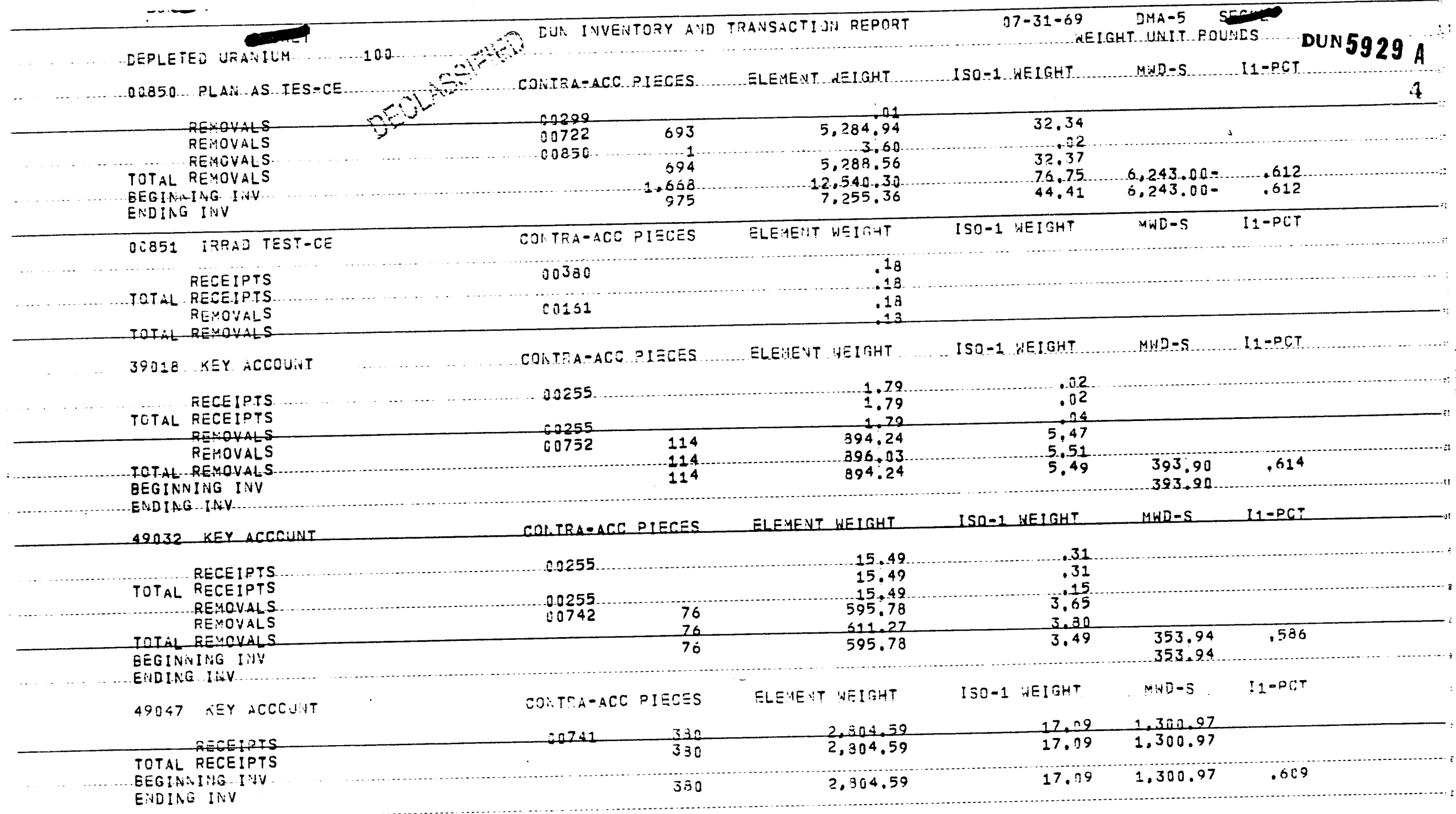


712.21 .529 EyDIns IinV 


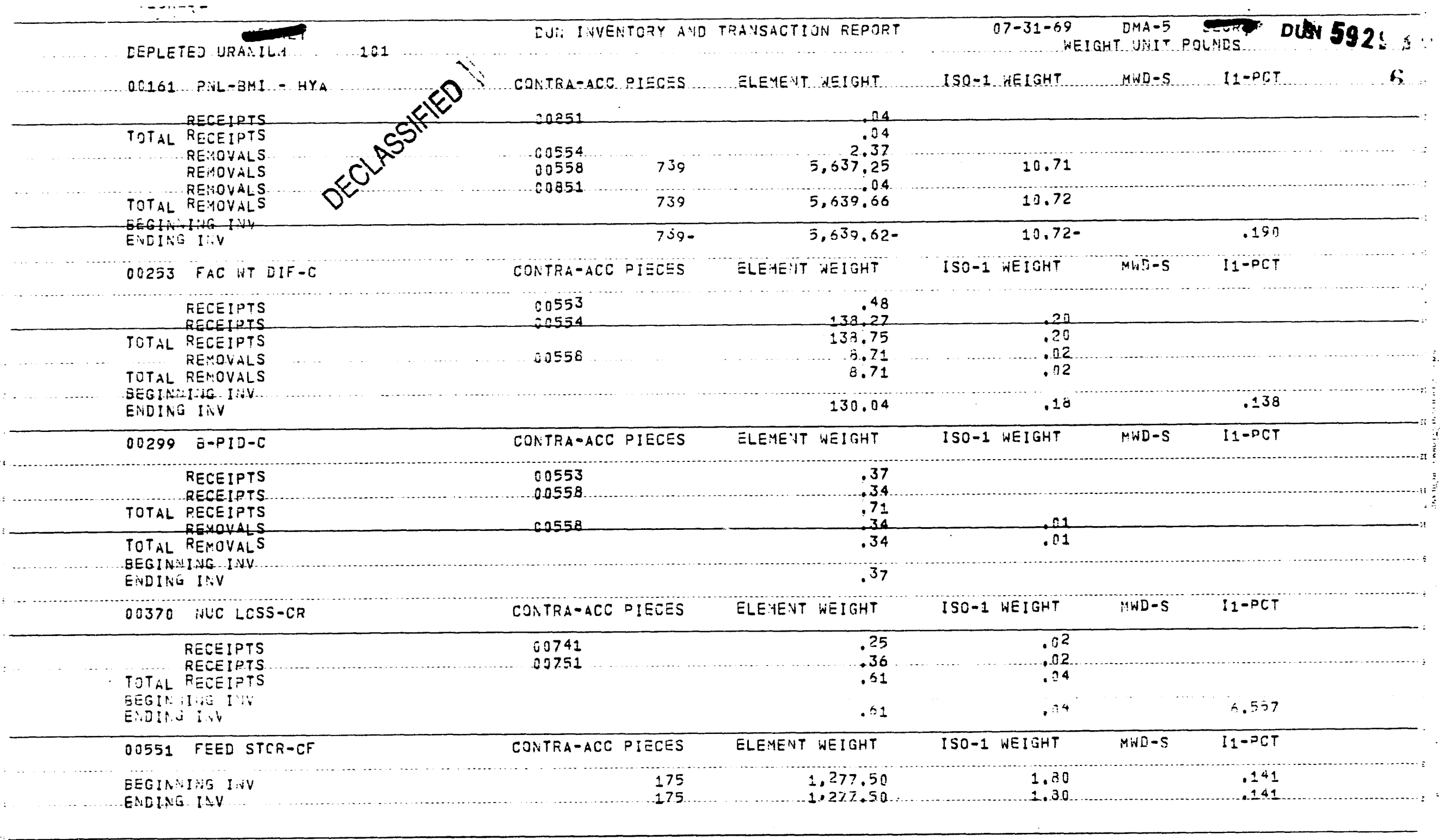


$07-31-6$ nEIGHT UNIT POULES

IEPLETED URALILI

00553 DEF SCRAP-CF

101

DU:. INVENTORY AID TZANSACTION REPOR

CORTRA-ACC PIECES EL... ELEMENT WEIGHT

TOTAL RECEIPTS

REUCVALS.

REMOVALS

TOTAL REMOYALS

EEGINIIING INV

ENDING IN:V

$0 C 554$ UI SCPAF-CF

RECEIPTS
RECEIPTS

TOTAL RECEIPTS

REMOVALS

RE OOVALS

REYOVALS

TOTAL RENOYAL

EEGINUING I

ENDING IV

0055E IN PROCESS-CF

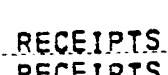

RECE IPTS

RECEIPTS

TOTAL RECEIPTS

REMOVALS

TOTAL FEMOVALS

EEGINALIIG INV

EAdDIfa linv

00616 313 PFO $\equiv \mathrm{VG}-\mathrm{CF}$

EEGINAIA IN

E Divi TSY

$\begin{array}{rr}0.0554 & 2.37 \\ 2.37\end{array}$

30253

00299

$421 \quad 3.473 .48$

3.475 .00

4.89

a 9

141

COITRA-ACC PIECES ELEYEIUT NEIGHT

ISO-1 WEIGHT

$12,296.06 \quad 17.34$

\begin{tabular}{|c|c|c|c|}
\hline 00151. & $\begin{array}{l}1, j 53 \\
1,558\end{array}$ & & $\begin{array}{l}17.34 \\
17.34\end{array}$ \\
\hline 0253 & 1.350 & $\begin{array}{r}130.40 \\
130.27 \\
2.37\end{array}$ & .20 \\
\hline
\end{tabular}

00553

$0055 \mathrm{a}$

1.003

1,003

1,327

$7,700.80$

$7,0.01 .44$

$10,758,83$
$15,215.8$

10.30

11.06

21,91

.145

CONTFA-ACC PIECES ELEMENT NEIGHT

ISO-1 WE!GHT

$M W D-S$

$I 1-P C T$

$\begin{array}{rrr}0.0161 & 739 & 5.637 .25 \\ 00253 & & 8.71 \\ 00299 & 1,003 & 7,700.8 \\ 00554 & 1.742 & 13,347: 1 \\ 00299 & & 12.296 .0 \\ 0.0554 & 1.601 & 12.296 .4 \\ & 1.001 & 5.523 .51 \\ & 711 & 6,574.21\end{array}$

COUTRA-ACC PIECES

46.23
46.23

10.71

1.01

10.86
21.59

17.34

17.34

$\frac{7.76}{12.0^{4}}$

. .141

.20

$M W D-S$ 11-PCT

173
.173
ELEUE TT VEIGHT

ISO-1 NEIGHT 


\section{$\bullet$}

$-$

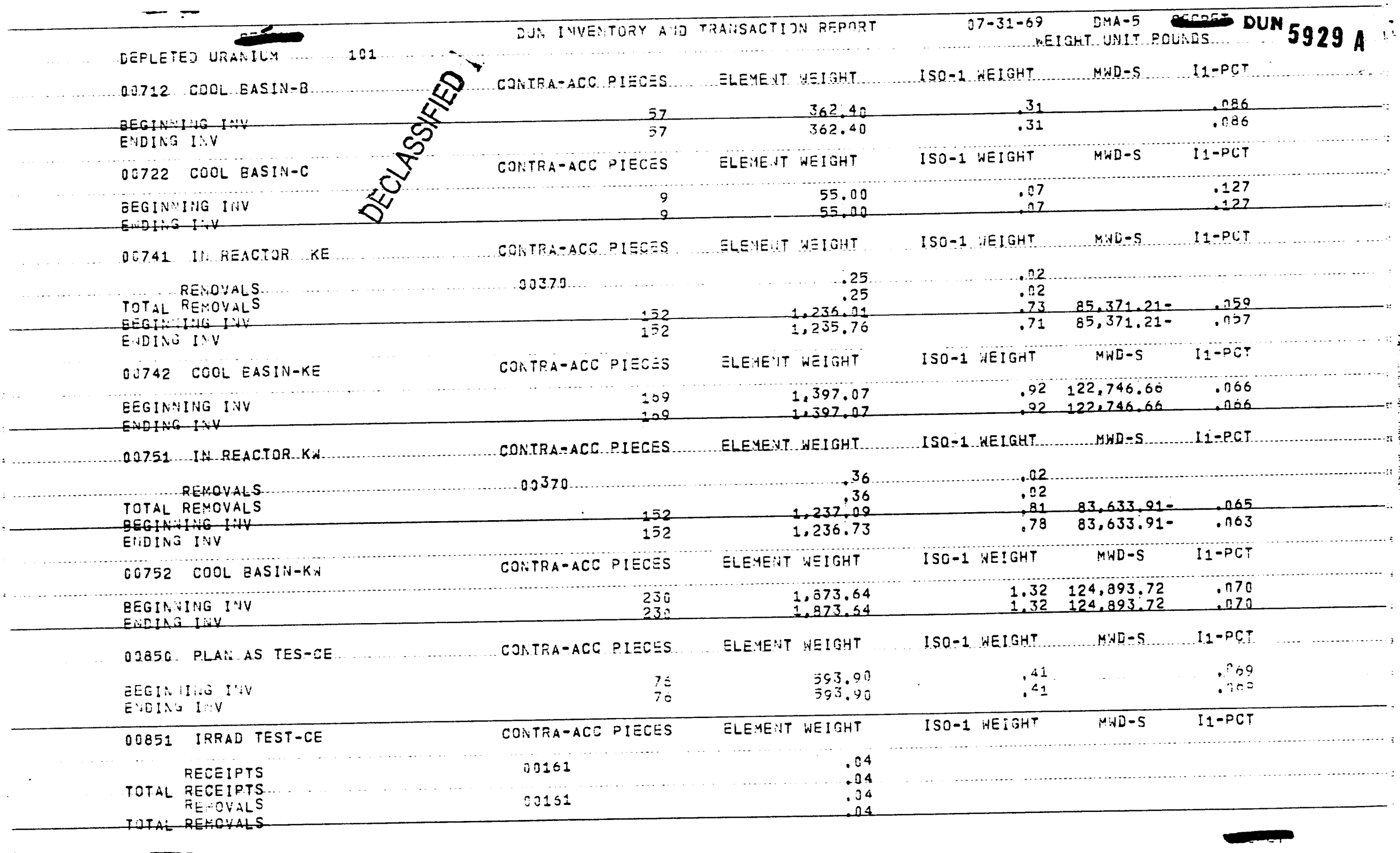




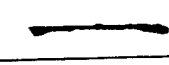
DEPLETED URADILM 00855 INST EL DEV-CE EEGINAING LiV ENDING liV
DUA IYVENTORY A'ID TRANSACTI JN REPOPT CORTRA-ACC PIECES ELEMENT NEIGHT

$3-\frac{13.05}{13.05}$

\section{$07-31-6$}

DMA-5 SETrL

.101

$\frac{3}{3}$
DEIGHT UNIT POLPIES ISO-1 WEIGHT $M W D-S \quad I 1-P C T$ $\begin{array}{ll}.02 & .153 \\ .02 & .153\end{array}$

\section{DECLASSIFIED}




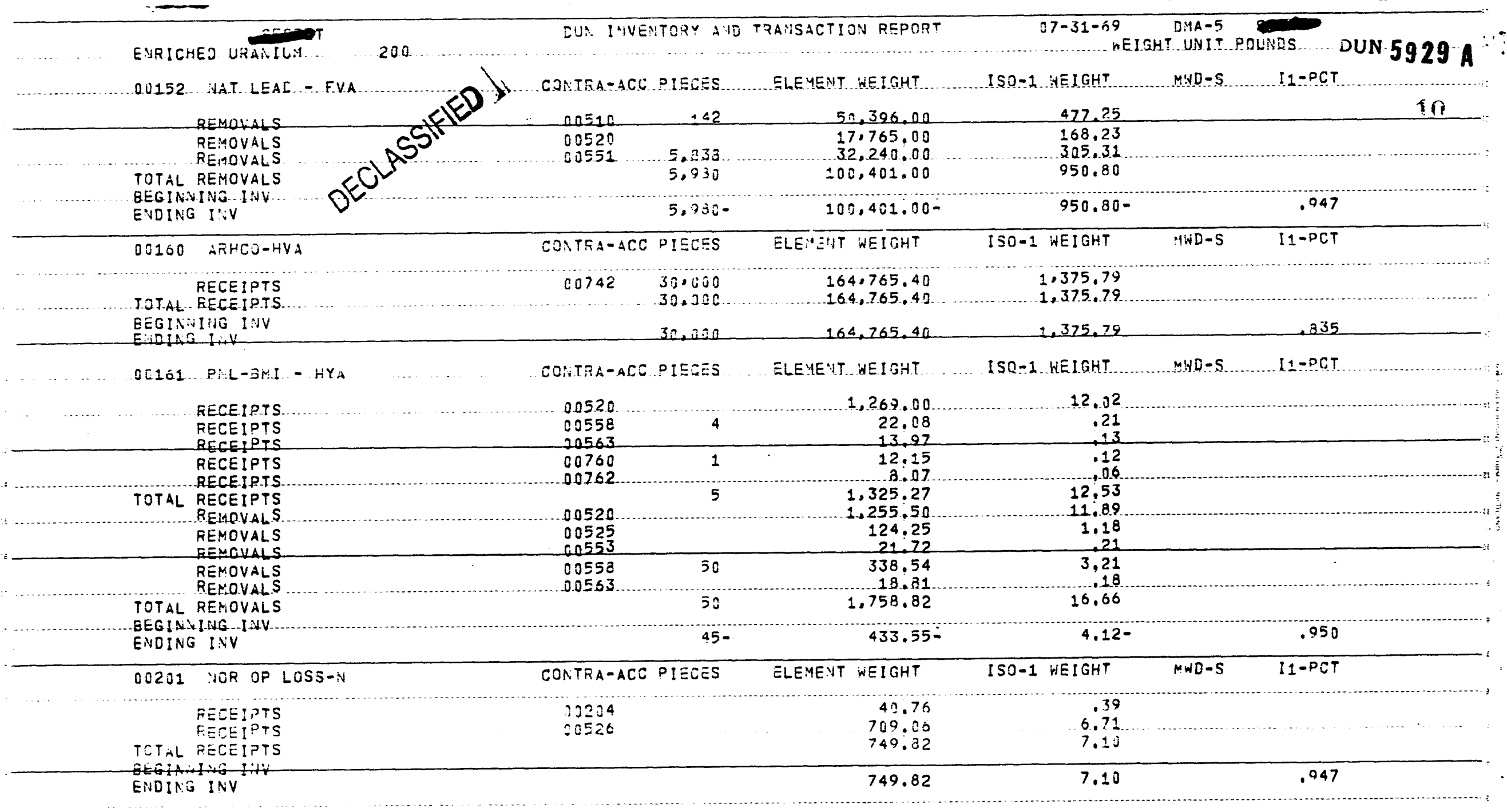




\section{-}

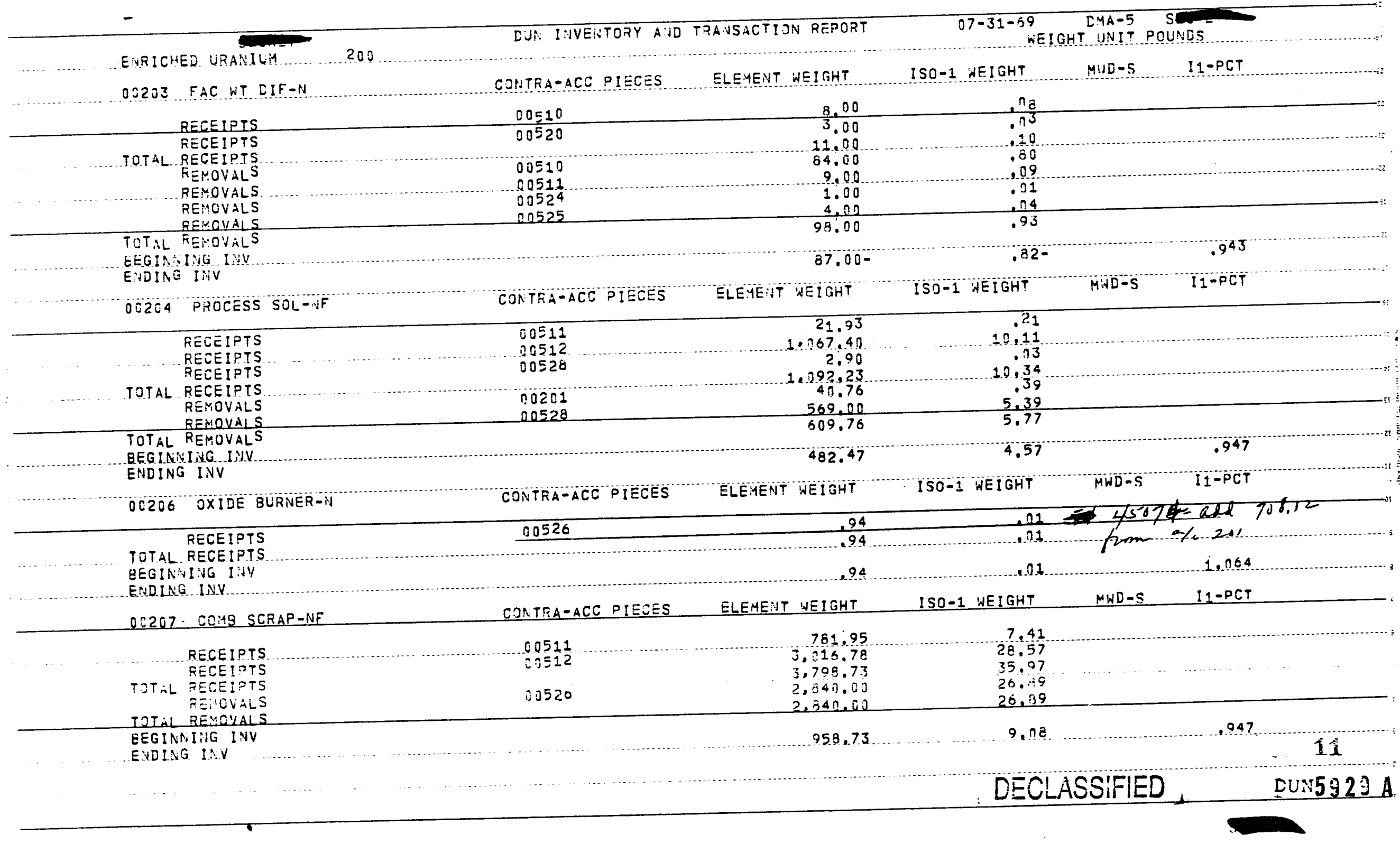




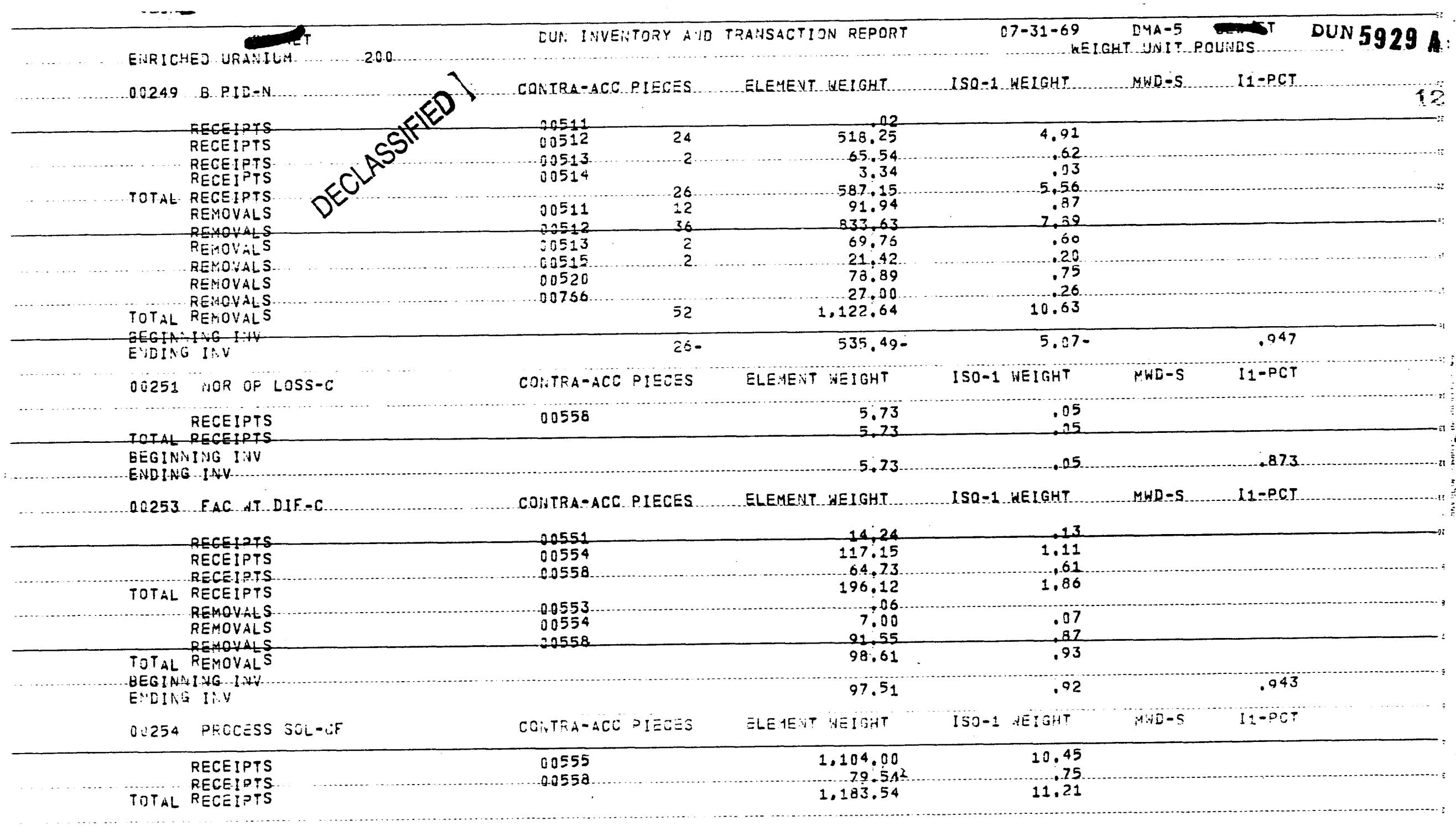

TIAL RECEIPTS 


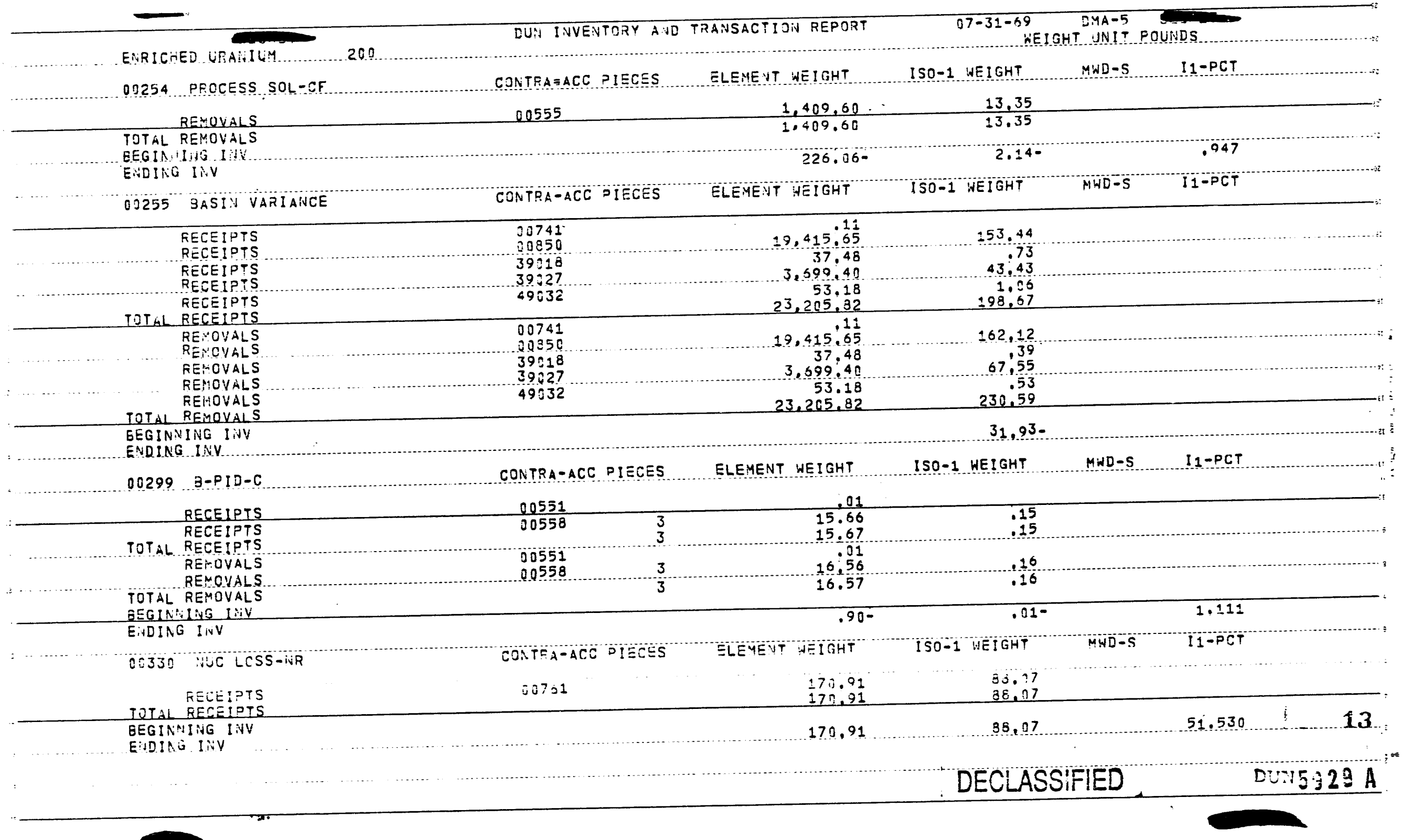


$-\cdot \quad-$

DUN INVENTOZY AND TPAISACTION REPORT

$97-31-69$ WEIGHT UNIT. POUNDS

DUN5929 A

E:RICHED URAAIUM 202

8

CONIPA-ACC PIECES ELI...ELEMENT WEIOHT ISO-1 HEIGHT MWD-S I1-PCT

.29370 ULLC.LOSS-CR. 00741 214.52

383.60 116.37

\section{REEETRTS}

TOTAL RECEIPTS

TOTAL RECEI TS

EHDING INV

OO510 EEEO STCR-UF

$598.12 \quad 326.19$

54.536

CONTFA-ACC PIECES ELEMEUT WELOHT

ISO-1 WEIGHT

$M+D-S$

$11-P C T$

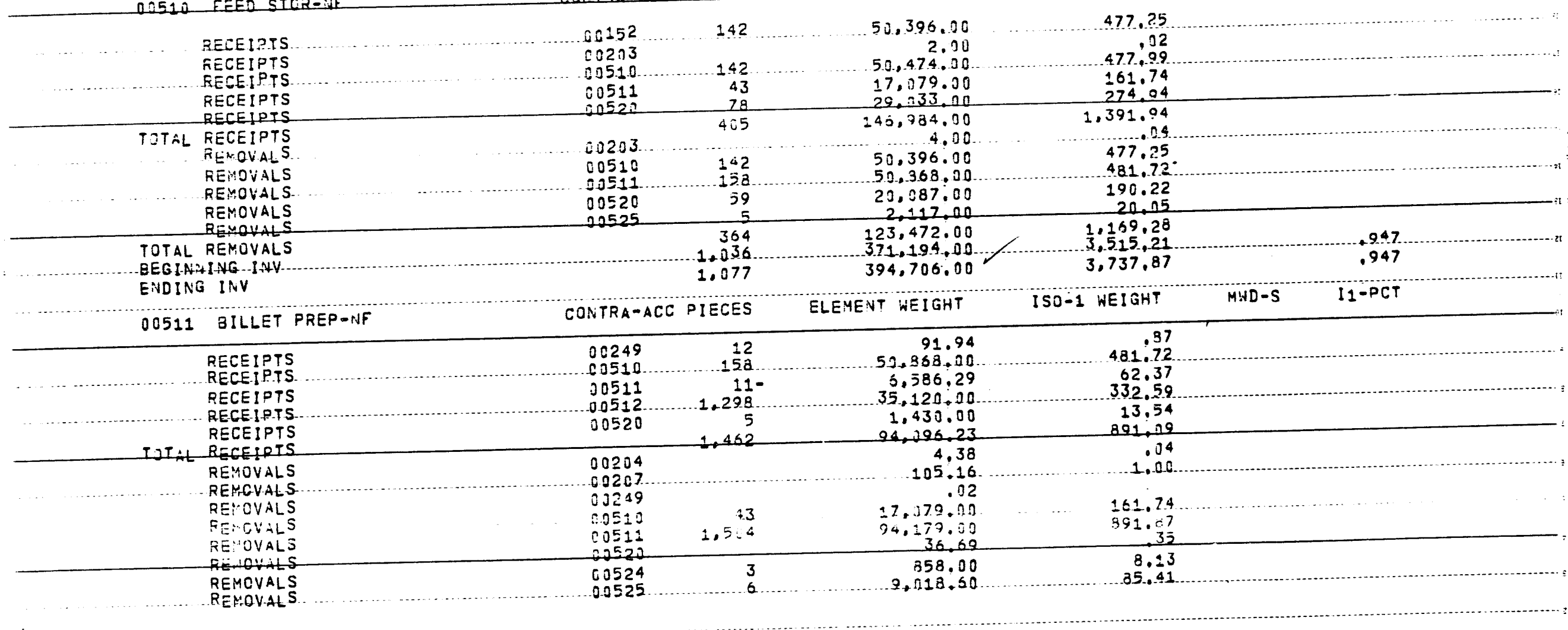




\section{0}

$-$

CUN IVVENTORY AWJ TRANSACTIDN REPORT

$07-31-69$

HEIGHT UNIT POUNES

ENRICHED LFANILM.

200

00511 BILLET PREP-NF

RENOVALS

TOTAL REMOVALS

EEGIN:IIVG IIIN

ENDING IAV

$005: 2$ TUEE PREP-IJF

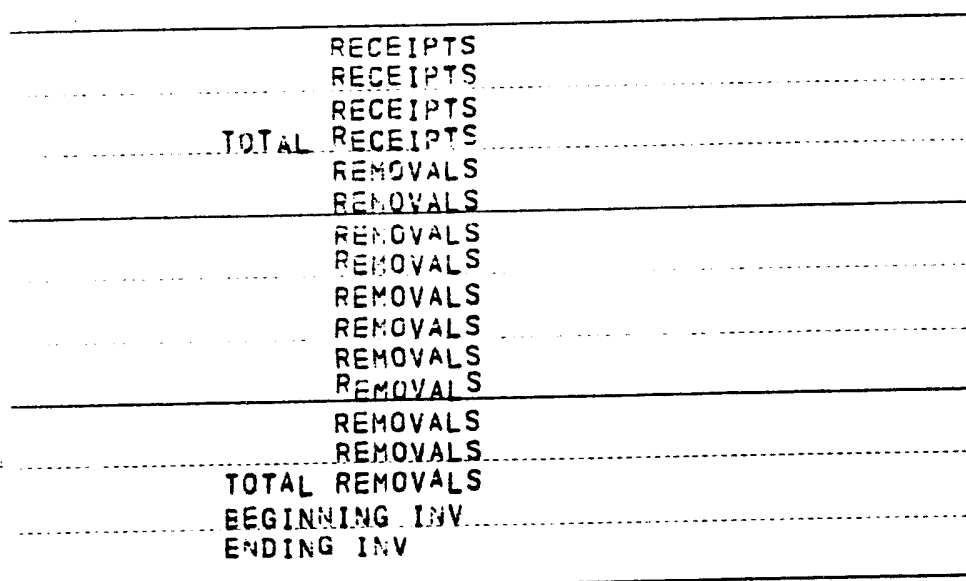

80563

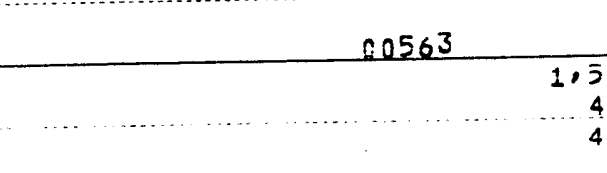

CONTRA-ACC PIECES
CONTFA-ACC PIECES ELEMEUT WEIGHT

ISO-1 WEIGHT

MD-S I1-PCT

\begin{tabular}{|c|c|c|c|}
\hline $\begin{array}{l}00249 \\
00511\end{array}$ & $\begin{array}{r}36 \\
4.034\end{array}$ & $\begin{array}{r}33.3 .63 \\
86,007.37\end{array}$ & $\begin{array}{r}7.39 \\
823.01\end{array}$ \\
\hline 00512 & 4,070 & $\begin{array}{r}17.10 \\
87.753 .10\end{array}$ & 831.87 \\
\hline $\begin{array}{l}00204 \\
00207 \\
\end{array}$ & & $\begin{array}{r}1.067 .40 \\
3,016.78 \\
\end{array}$ & $\begin{array}{l}10.11 \\
28.57 \\
\end{array}$ \\
\hline $\begin{array}{l}00249 \\
60511\end{array}$ & $\begin{array}{r}24 \\
1,293\end{array}$ & $\begin{array}{r}513.25 \\
35.120 .00\end{array}$ & $\begin{array}{r}4.91 \\
332.59\end{array}$ \\
\hline & 2,565 & $64,760,50$ & $613^{.12}$ \\
\hline & $\begin{array}{r}7 \\
249 \\
\end{array}$ & $\begin{array}{r}188.42 \\
5.272 .13 \\
\end{array}$ & $\begin{array}{r}1.78 \\
49.93 \\
\end{array}$ \\
\hline $\begin{array}{l}00520 \\
0.0525\end{array}$ & $\begin{array}{r}16 \\
493\end{array}$ & $\begin{array}{r}300.82 \\
11.779 .87\end{array}$ & $\begin{array}{r}2.85 \\
111.56\end{array}$ \\
\hline & $\begin{array}{l}4.652 \\
5,983 \\
6.001\end{array}$ & $\begin{array}{r}122,041,27 \\
166,293,49 \\
132,010.32\end{array}$ & $\begin{array}{l}1,155,73 \\
1,574,80 \\
1,250,14\end{array}$ \\
\hline
\end{tabular}

ISO-1 WEIGHT

$613: \frac{.66}{28}$

$\begin{array}{cc}2 & 69,76 \\ 5 & 64,760,50 \\ 7 & 1,930.29 \\ 4 & 66,761,55 \\ 2 & 65,54 \\ 9 & 662.72 \\ 6 & 57,027.17 \\ 2 & 59.19 \\ 9 & 59,500.51 \\ 6 & 11,732.52 \\ 41 & 13,386.46\end{array}$

.04
1.148 .57
503,68

5.03 .68
246.21

047

$121,284.69$

$25,998.89$

PIECES

$\begin{array}{rr}00249 & 2 \\ 00512 & 2.555 \\ 00750 & 77 \\ & 2.044 \\ 00249 & 2 \\ 00520 & 19 \\ 00522 & 2.406 \\ 00558 & 2 \\ & 2.429 \\ & 020 \\ & 841\end{array}$

13.386 .46

ISO

TOTML REETOVALS

ENDING IIV 


\section{-}

$\sim$

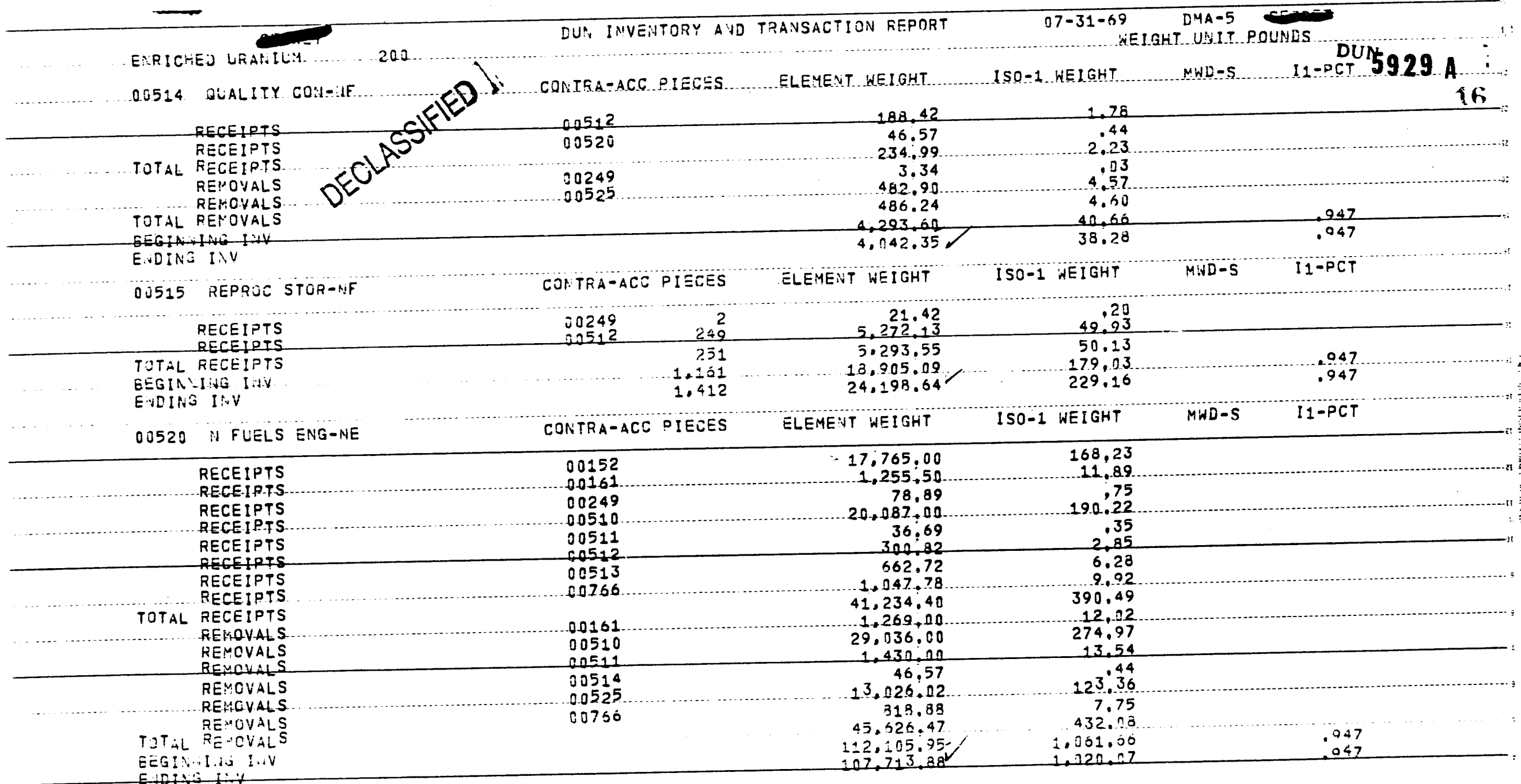

ECG1...1.A I.iV 


\section{$\bullet$}

...

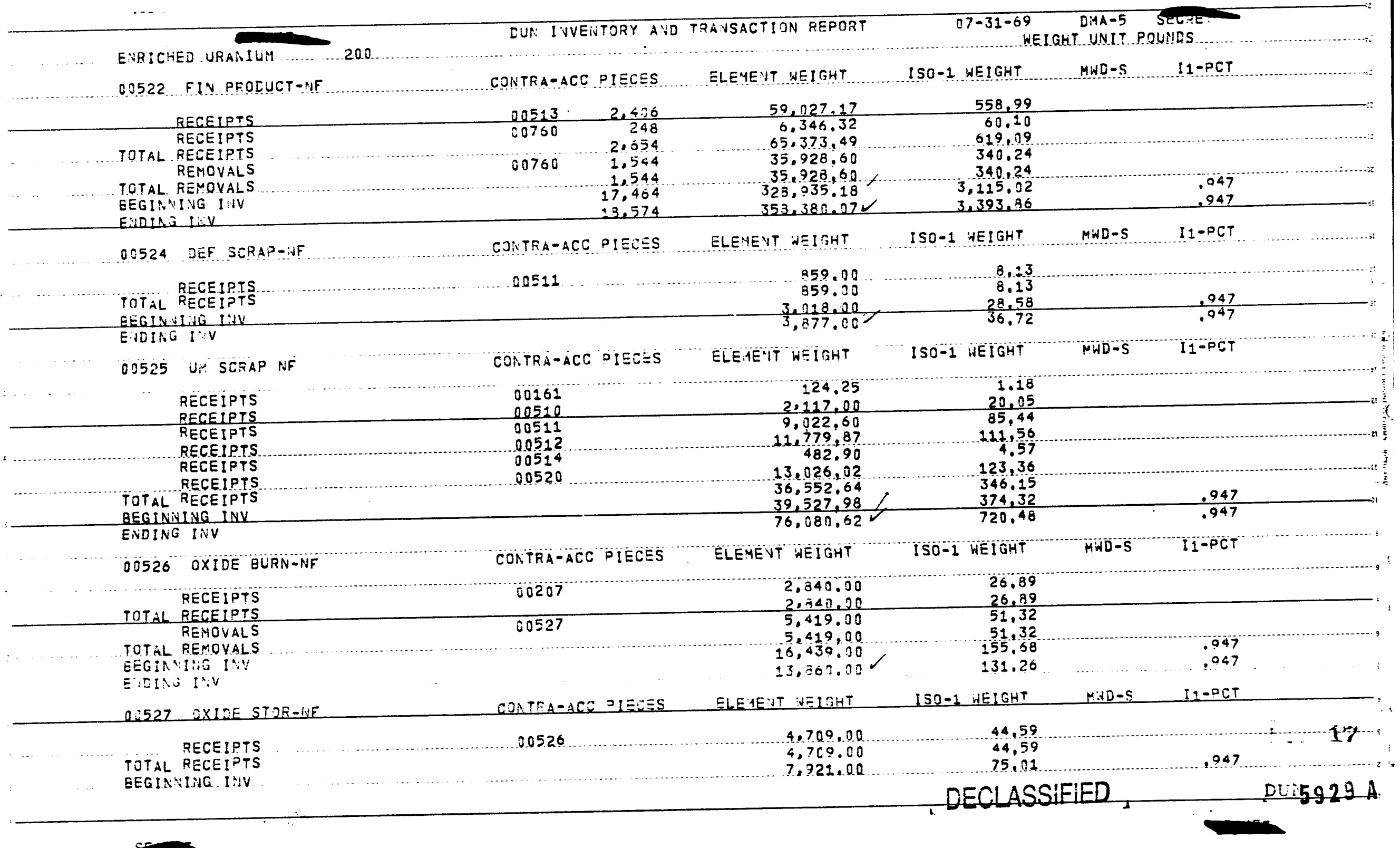




\section{-}

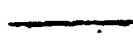

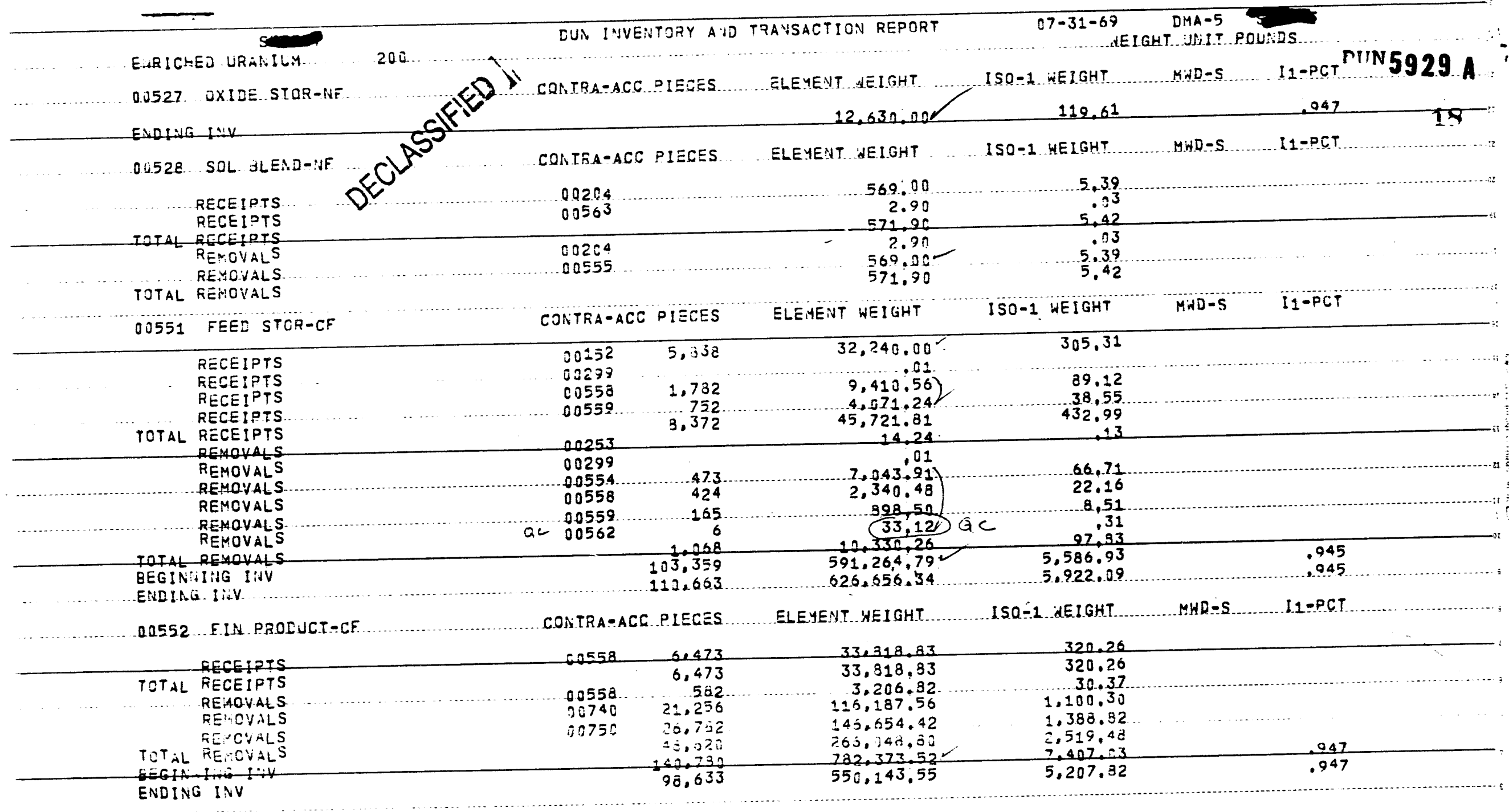


$\infty$

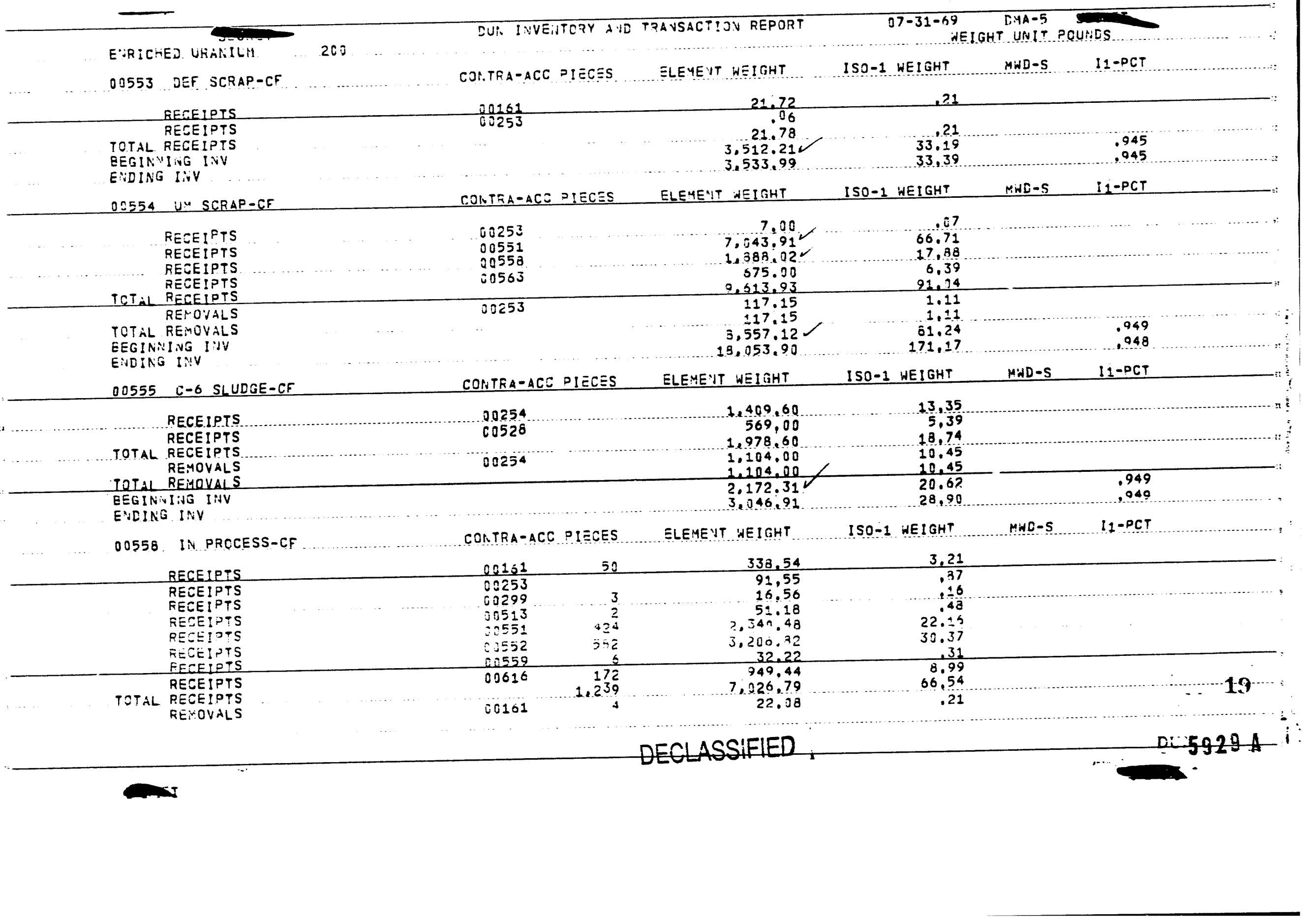




\section{-}

ESRICHED LRARIIL:A DUI. IMVENTOR: AVD TRAVSACTIOV REPORT

$07-31-69$ D:14 - 5

$-\cdots$

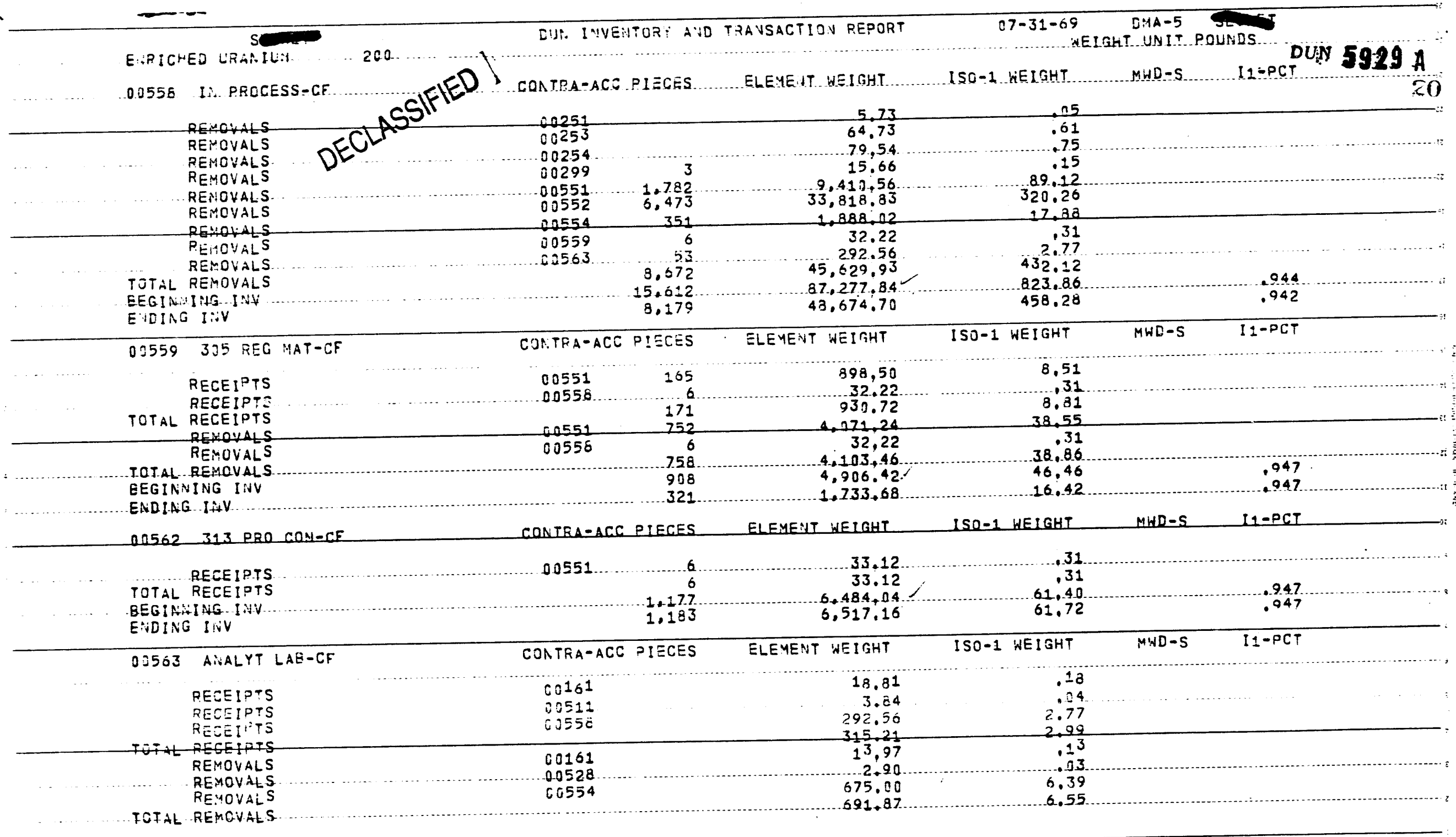

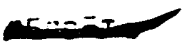




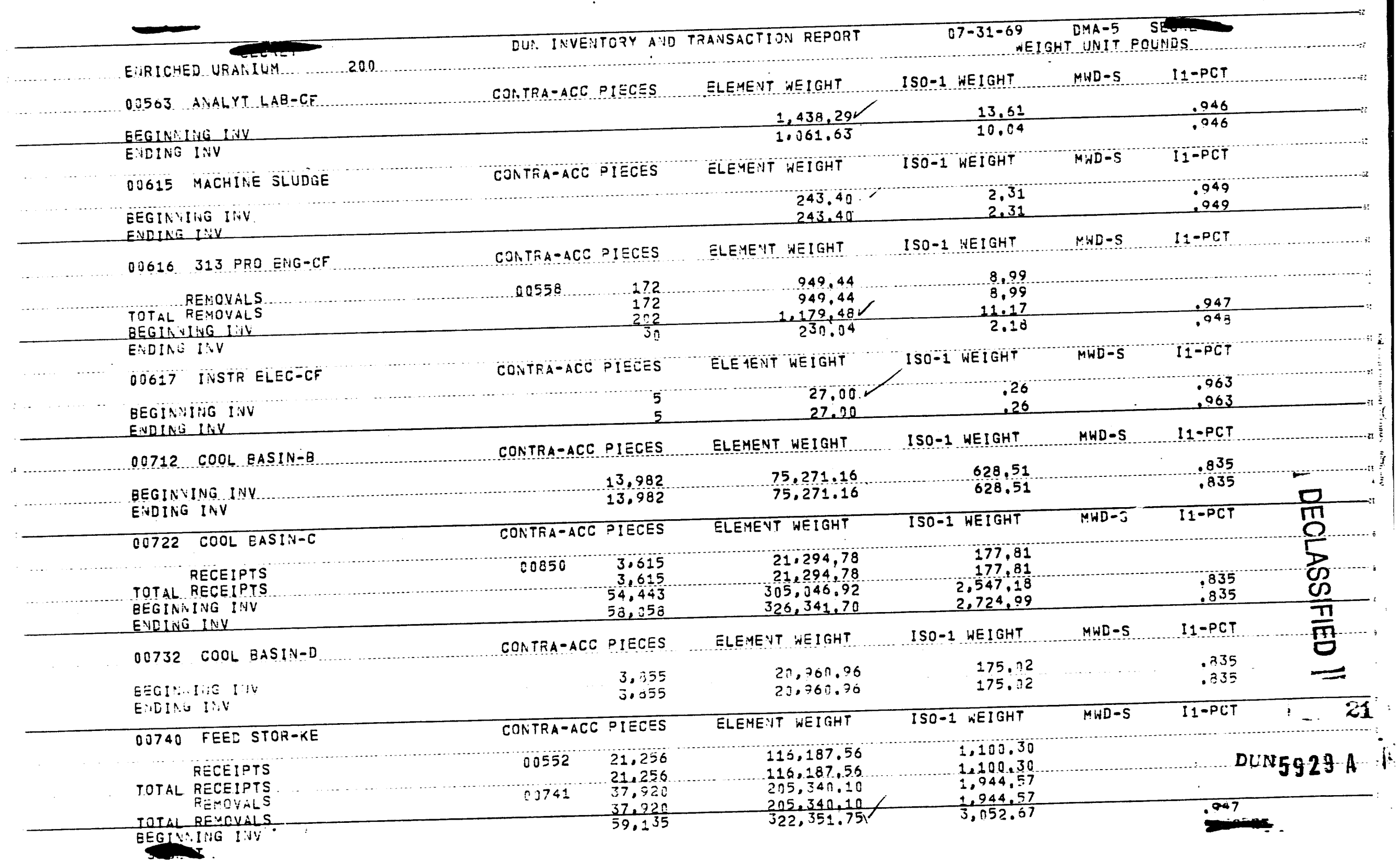


- -

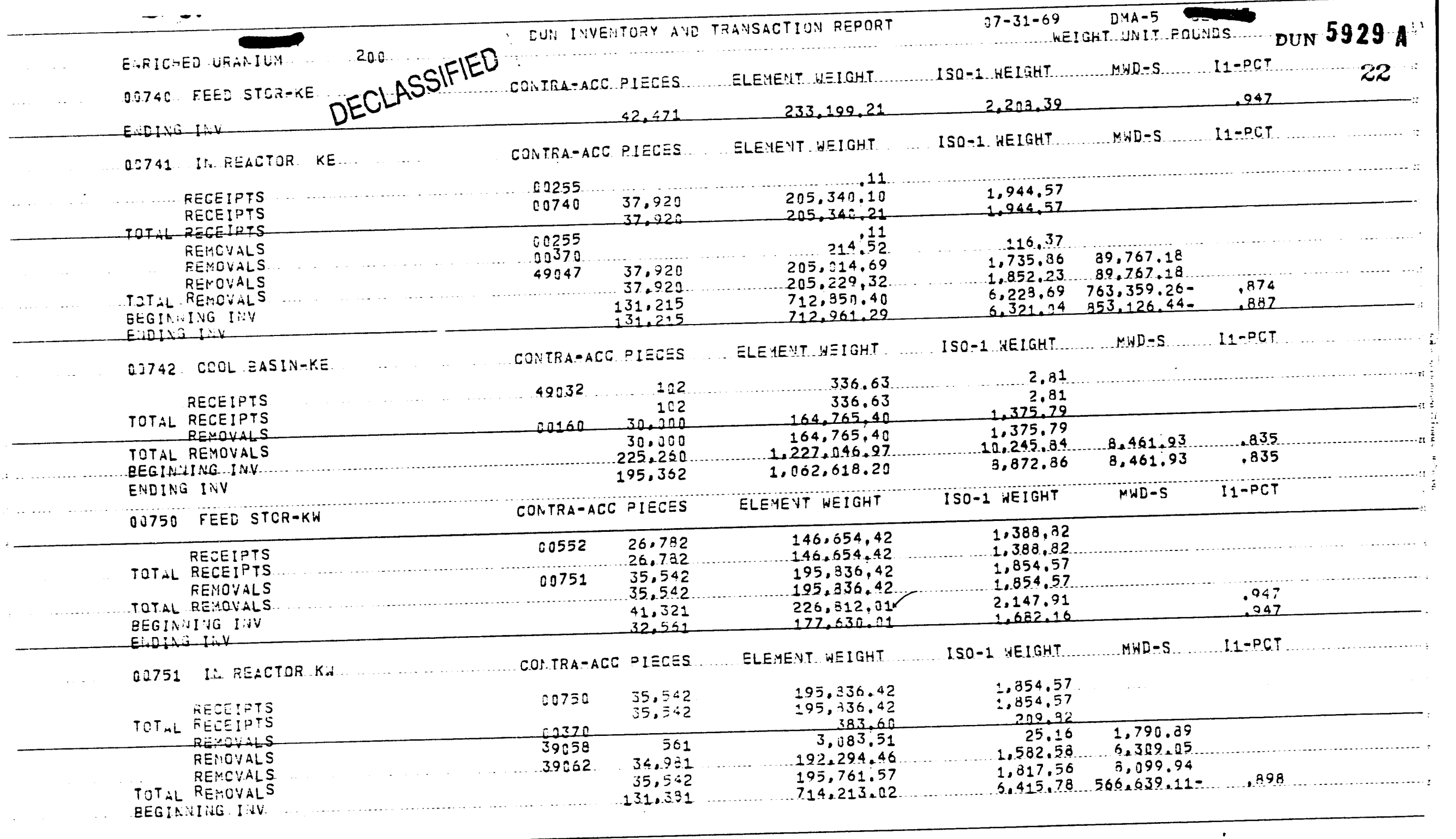


ENFICHED URAIILIA

00751 I: REACTOR KH

CONTRA-ACC PIECES

ELEMENT WEIGHT

ISO-1 WEIGHT

MWD-S I1-PCT

ENDIAG Lik

$$
131,381
$$

$714,287,87$

$6,452,78 \quad 574,739.05$

.003

00752 CCOL EASIN-KW

CONTRA-ACC PIECES

ELEMENT WEIGHT

ISO-1 WEIGHT

RWD-S

$11-P C T$

\section{RECEIPTS}

EEGINIIIUL INV

39218
39027

$\begin{array}{rr}446 & 2.350 .01 \\ 51.683 & 281.658 .24 \\ 52.229 & 284.208 .25 \\ 165.426 & 896.247 .67\end{array}$

896.247 .67
1.180 .255 .92

$2,371,47$

217,555

ELEYENT WEIGHT

7.483 .66

$190.920 .74 \quad .835$

CONTRA-ACC PIELES

$35,928,60$

00522
00760
00161
00513
00522
00750
00761

REMOVALS

TOTAL REMOVALS
OEGINNING INV

ENDING INV

00761 IN REACTOR-NR

CONTRA-ACC PIECES

\begin{tabular}{|c|c|c|}
\hline 772 & $35,928,60$ & 340 \\
\hline & 48.78 & \\
\hline 773 & $35.977,38$ & 340 \\
\hline 37 & $\begin{array}{r}12,15 \\
930,29\end{array}$ & \\
\hline 124 & $6,346,32$ & \\
\hline$\frac{1}{06}$ & 12,15 & \\
\hline $\begin{array}{l}.006 \\
.109 \\
.241\end{array}$ & $\begin{array}{r}43 \\
51 \\
151 \\
135\end{array}$ & $\begin{array}{r}41 \\
49 \\
43 \\
28\end{array}$ \\
\hline
\end{tabular}

ELEMENT WEIGHT

ISO-1 WEIGHT

MWD-S

.947

MWD-S I1-PCT

TOTAL RECEIPTS

REMOVALS

REMOVALS

RENOVALS

REYOVALS

REMOVALS

TOTAL REMOVALS

EEgIN II:A ITUV

E'vilag Iivy

$\begin{array}{rr}00760 & 1.006 \\ 00330 & 1,006 \\ 59044 & 16 \\ 59051 & 1.08 \\ 59064 & 1.002 \\ 59065 & 43 \\ & 1.079 \\ & 14,091 \\ 14,516\end{array}$

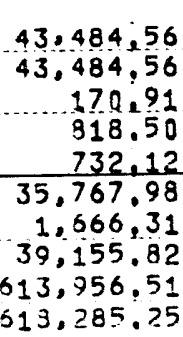

411.80

411.80

88,07

. $.54 \quad 86.78$

$\begin{array}{rr}5,74 & 559,94\end{array}$

$272,07 \quad 33,131,32$

$386,57 \quad 35,023,44$

4,591

$5,124,55 \quad 408,328.23-$

$5,149,78 \quad 443,356.67-\quad .833$

00762 COOL BASIN-NR CONTEA-ACC PIECES

00161

$$
\text { RENOVALS }
$$

TOTAL REYCVALS

ELEMENT WEIGHT

8.07

8,07
MWD-S

I1-PCT 


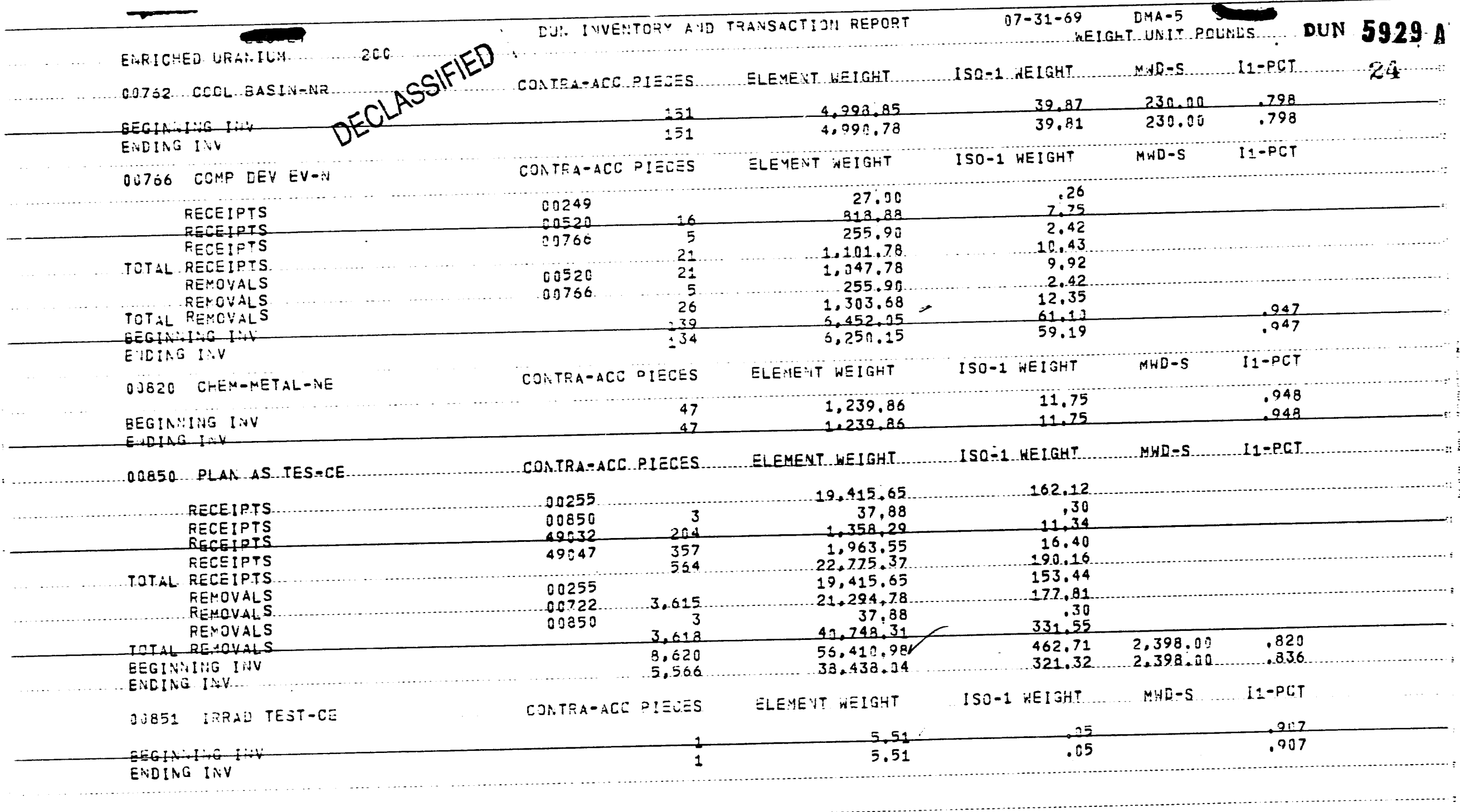




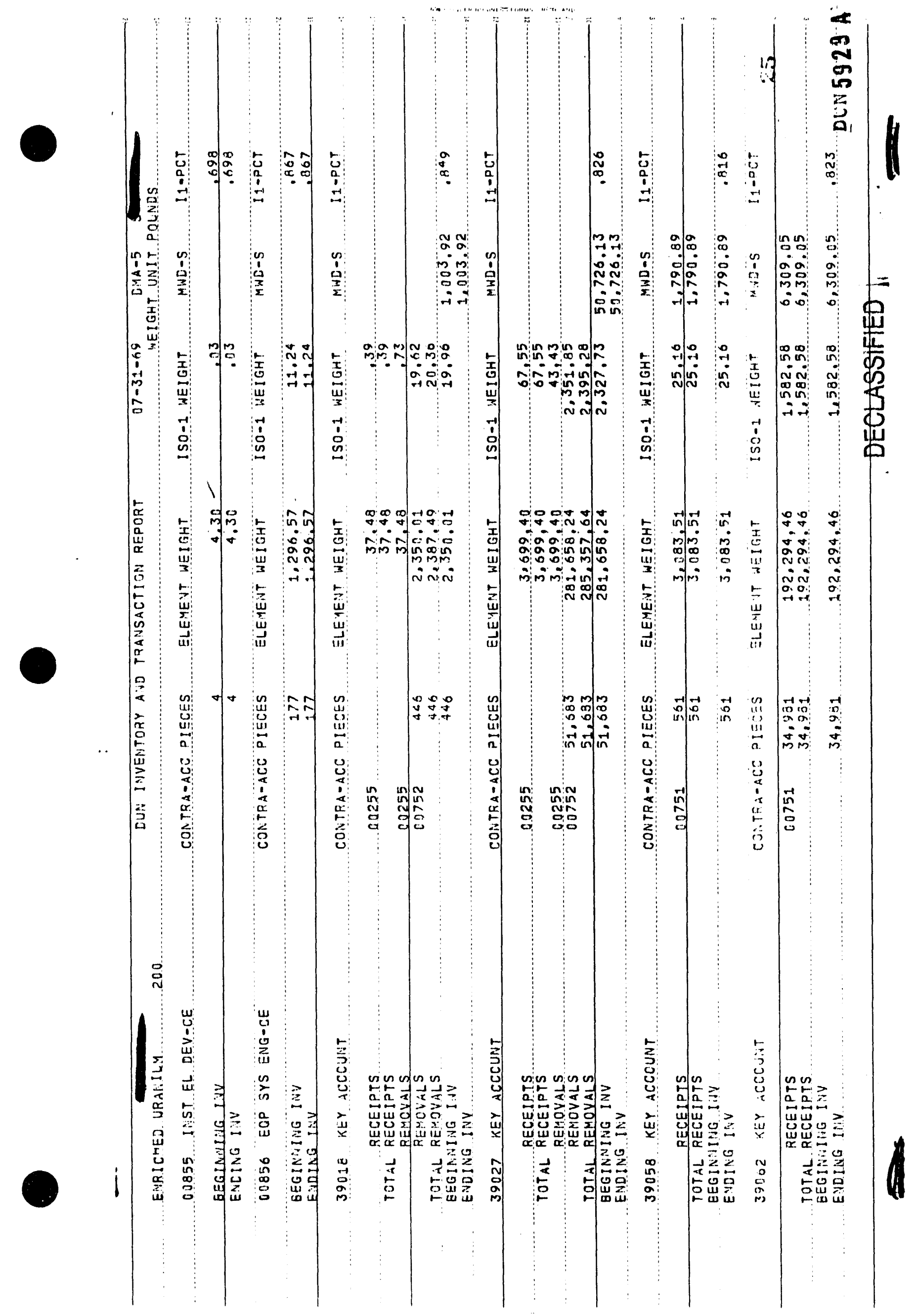




\section{$\longrightarrow$}

EIFICHED URAALIL

4.9032 KEY ACCCUNT

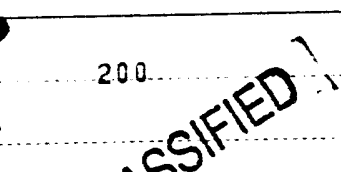

TOT RECETPTS RECEIPTS REIHOVALS REMOVALS REYOVYALS

TOTAL FEMOVALS

EEGIANIITG TH

49047 KEY ACCOUNT

RECEIPTS

TOIAL RECEIPTS

TOTAL REIOVALS

EEGINIIING I:Y

ENDING ISV

$58922 \mathrm{KEY}$ ACCCUNT

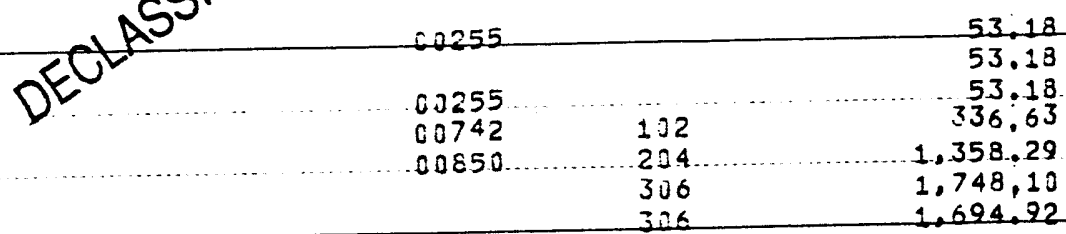

REGINIING INV

EINDING INV

58993 KEY ACCOUNT

CONTFA-ACC PIECES

00741

c) 850

37.920
37.920
357
357

ELEMENT WEIGHT

37.563

CONIRA-ACC PIECES

2.363
2,503

CONTRA-ACC PIECES

402

BEGINNING INV

ENDIAG .ILV

59033 KEY ACCCUNT

CONTRA-ACG PIECES

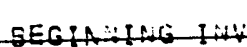

EYDING INV

59044 KEY ACCOUNT

RECEITTS

TOTIL RECETRTS

EEGIN.UING INV

ENDING INV

$1,963.55$

1.763 .55

ELEYENT WEIGHT

$98 \cdot 273,21$
$98,273,21$

ELEYENT WEIGHT

364,48

CONTFA-ACC PIECES ELEMENT WEIGHT
37-31-69 DMA-5 WEIGHT UHIT PCUIIQS

DUN 5929 A

26

.53
.53
1.06
2.31
11.34
15.22
14.68

.53
.53
.26
.31
.34
.22
.68

MWD-S

$11-P C$

6

.......

585.80

585.80

MWD-S II-PCT

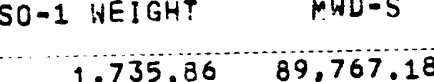

$1.735 .86 \quad 80.767 .18$

16,40

16.40

$1,719.46 \ldots 89,767.18 \ldots \ldots .847$

-1 WEIGHT MWD-S U1-PCT

$\begin{array}{rrr}712.26 & 109,322.86 & .725 \\ 712.26 & 109.322 .86 & .725\end{array}$

ISO-1 WEIGHT MHD-S II-PCT

$\begin{array}{llll}12,205,29 & 102.77 & 5,630.08 & .842 \\ 102.77 & 5,630.08 & .842\end{array}$

CONTRA

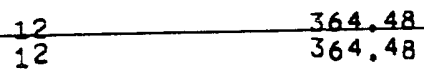

ISO-1 WEIGHT MWD-S I1-PCT

$\frac{3.45}{3.15}$

$131.82 \quad .864$

ISO-1 WEIGHT

MWD-S

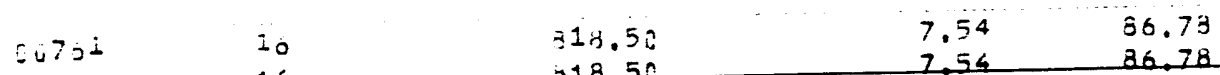

\begin{tabular}{l}
$18.50 \quad 7.54 \quad 86.78$ \\
\hline
\end{tabular}

818.50

7.54

86.78 



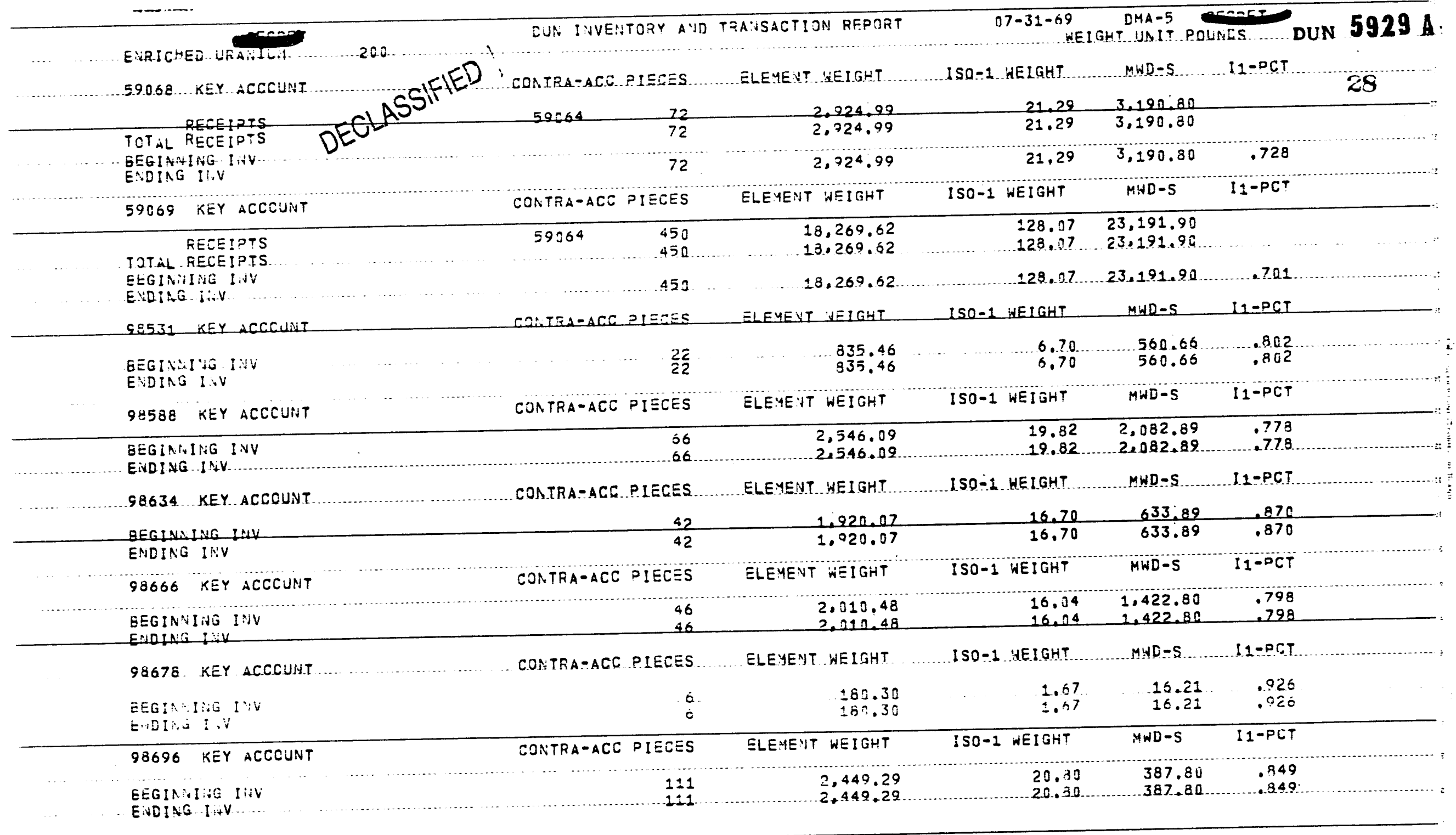




\section{0}

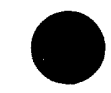

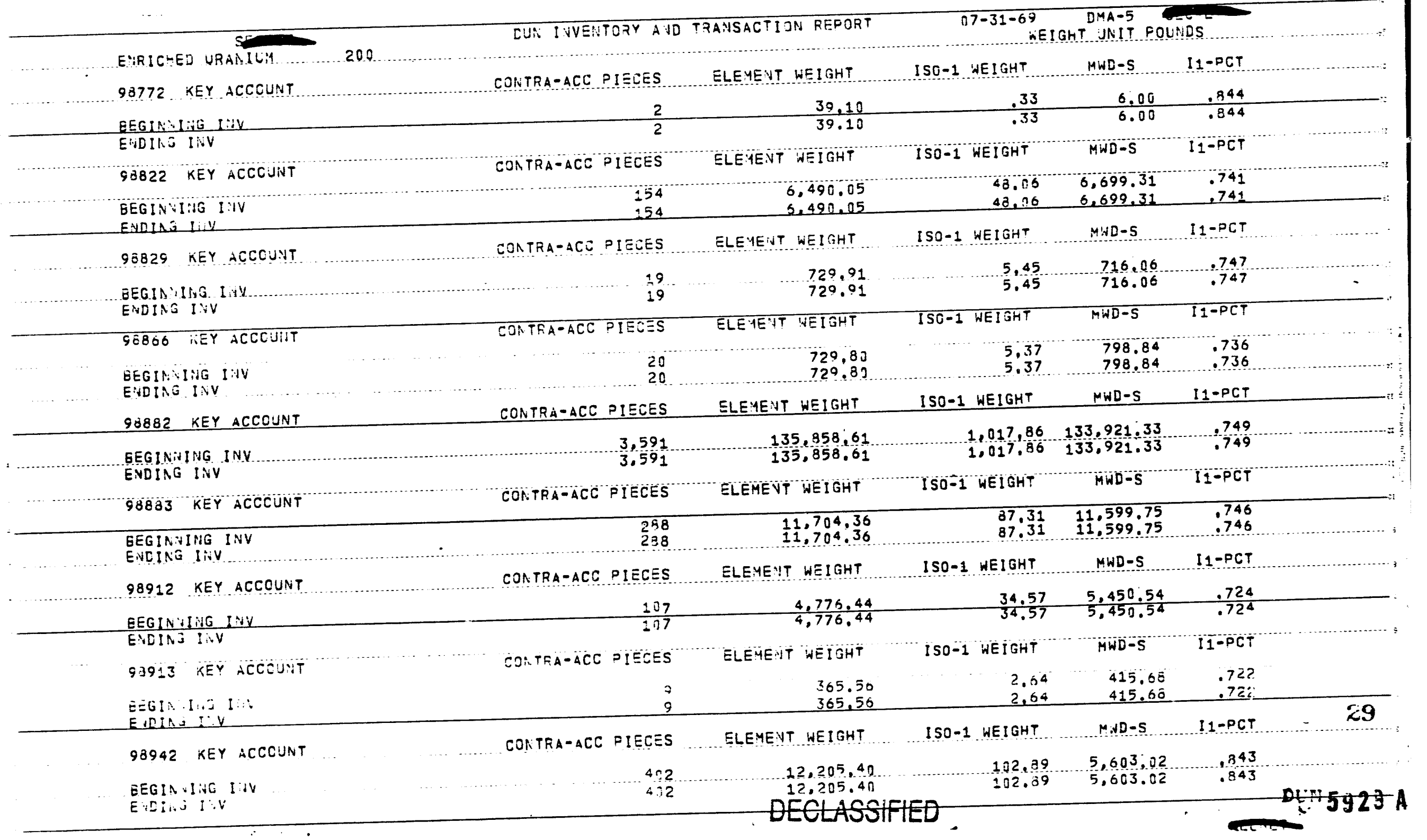




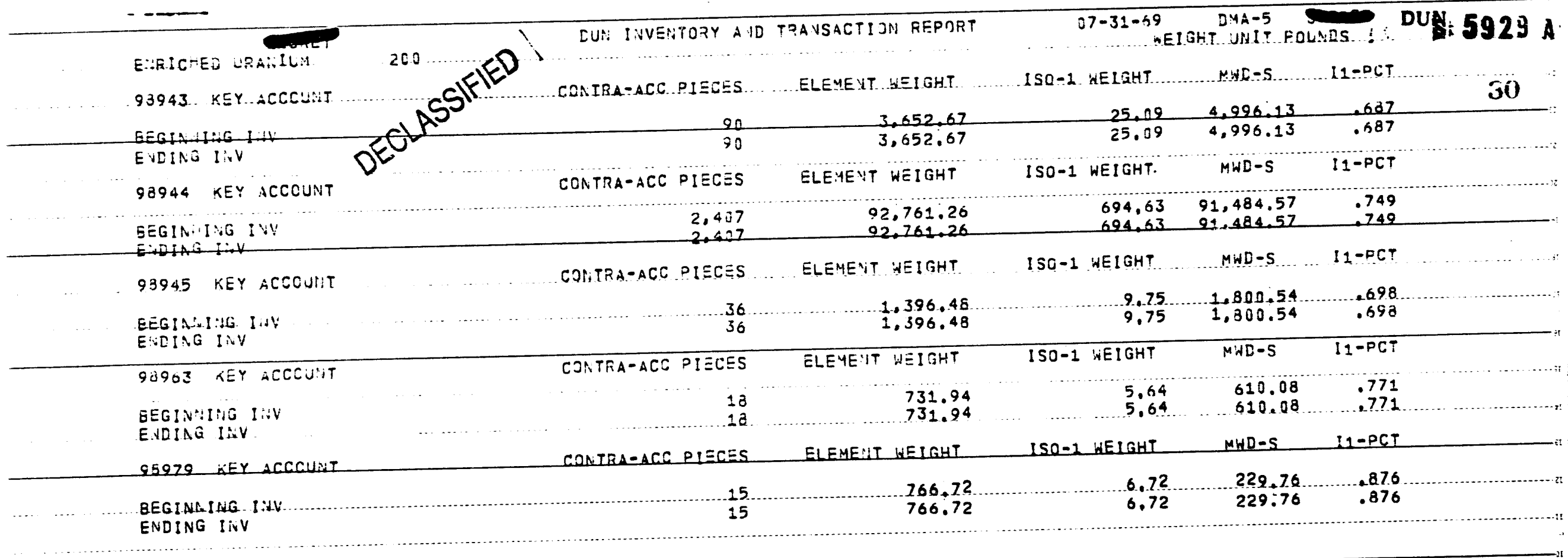




\section{-}

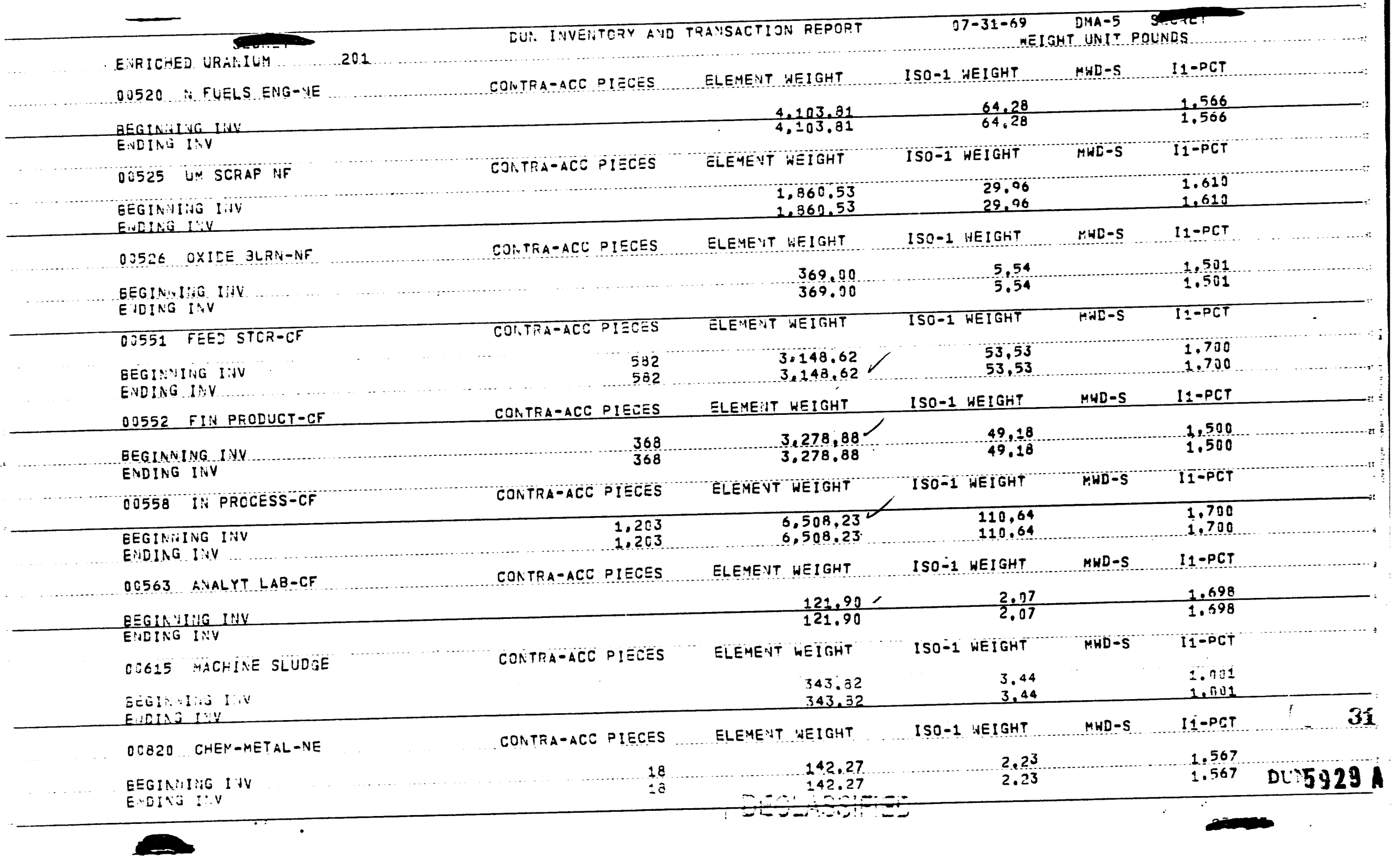




\section{○}

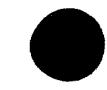

-.....

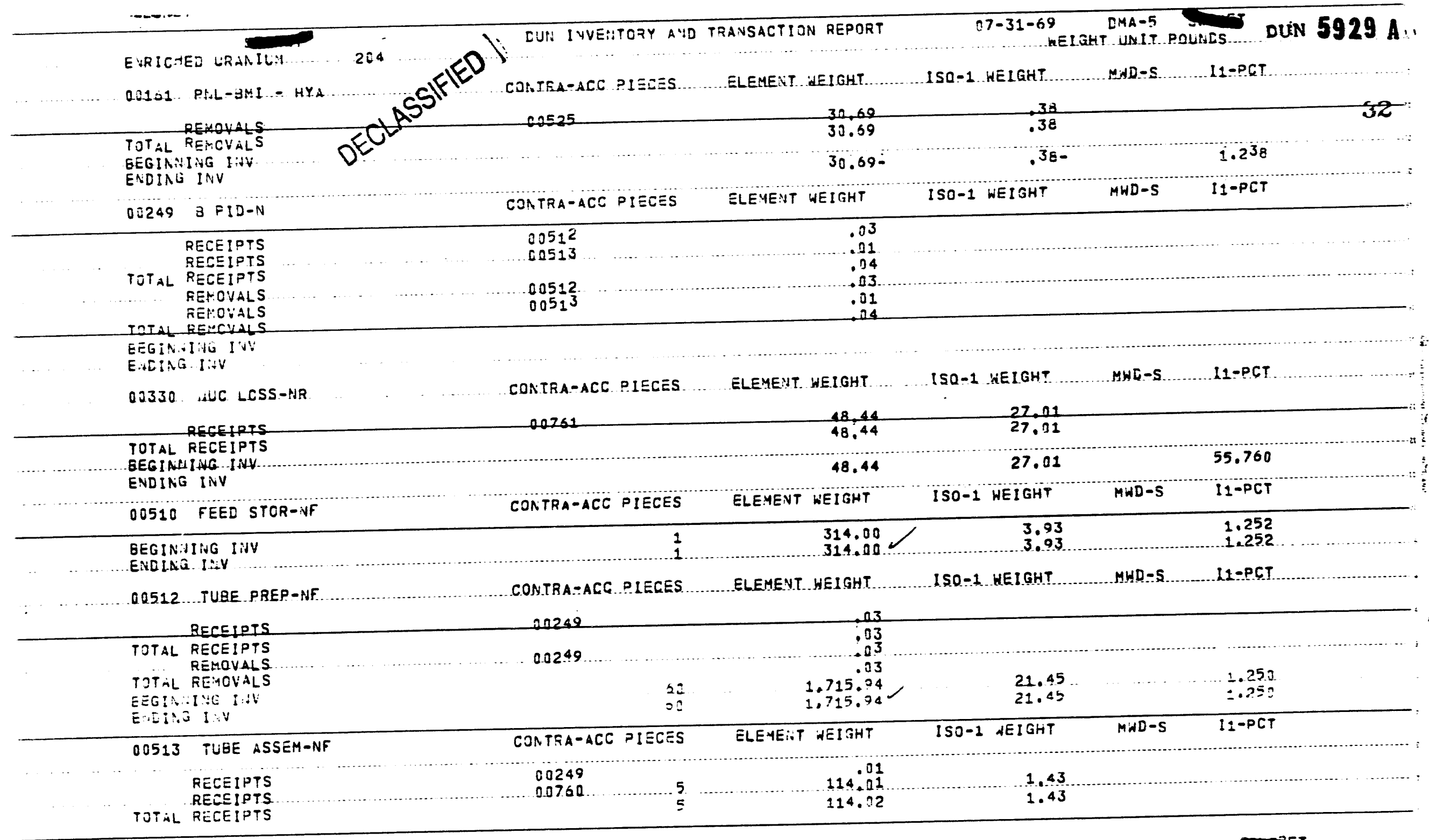




\section{5} ENRICMED UFAAILY Q0513 TUBE ASSEM-NF

$\begin{array}{ll}\text { REHAVVALS } & 00249 \\ \text { FEMOVALS } & 00522\end{array}$

... REMOVALS

TOTAL REMOVALS

BEGIA:IING INV

ENDINï INV

00514 GUALITY CON-inF

EEGIMING IHA

ENEINo IVV

00515 REPROC STOR-IF

EEGIN:IUG IUN

EiNDING IRV

00520 N FUELS ENG-VE

EEGINIING INV

ENDING INY

03522 FIN PROLUCT-NF

$$
\text { RECEIPTS }
$$

TOTAL RECEIPTS

GEGINUING INY

ENDING INV

00524 DEF SCRAP-NF

EEGIN J IVÉ IIV

ENDINS LIVV

DO525 U: SCEAAF H:F RECEIPTS RECEIPTS

TOTAL RECEIPTS

GEGINIING IIV

ENDING IN:V

00251
CUI. INVENTORY AND TRANSACTION REPORT

ELEYENT WEIGHT

$\begin{array}{ccc} & .01 & \\ 50 & 986.88 & 12.34 \\ 77 & 148.63 & 1.86 \\ 57 & 1.135 .52 & 14.19 \\ 174 & 3.225 .22 & 40.31 \\ 122 & 2.203 .52 & 27.54\end{array}$

12.34
1.86
14.19
40.31
27.54

ISO-1 WEIGHT

1.25

1.25

100,34

ELEMENT NEIGHT

1SO-1 WEIGHT

133.00
133.00

$10.64 \pi .16$
10.640 .16

ELEMENT WEIGHT

55.64

CONTRA-ACC PIECES $55.6432 .64 ?$

ISO-1 WEIGHT

B,

ISO-1 WEIGHT

ELEMENT WEIGHT

$\begin{array}{rrrr}00513 & 50 & 986.88 & 12.34 \\ 50 & 986.88 & 12,34 \\ 3,447 & 81.296,63 & 1.016,21 \\ & 3,497 & 82.283 .51 & 1.028 .54\end{array}$

12.34

12.34

$1.016 .21 \quad 1.250$

ELEMENT WEIGHT

$2,002.00$
$2,002.00$

CDR.TIAA-ACO PIECES

ELEITENT NEISHT

39.69

$14 \hat{2} .63$

179.32

30.955 .22
40.134 .54

30.955 .22
$40,134,54$

ISO-I WEIGHT

25.13
25.03

ISO-1 WEIGHT

$.3 \mathrm{a}$

1.80
2.24
499.44

$\begin{array}{ll}499.44 & 1.250 \\ 501.60 & 1.250\end{array}$

1.250
1.250

Din $5929 A$ 


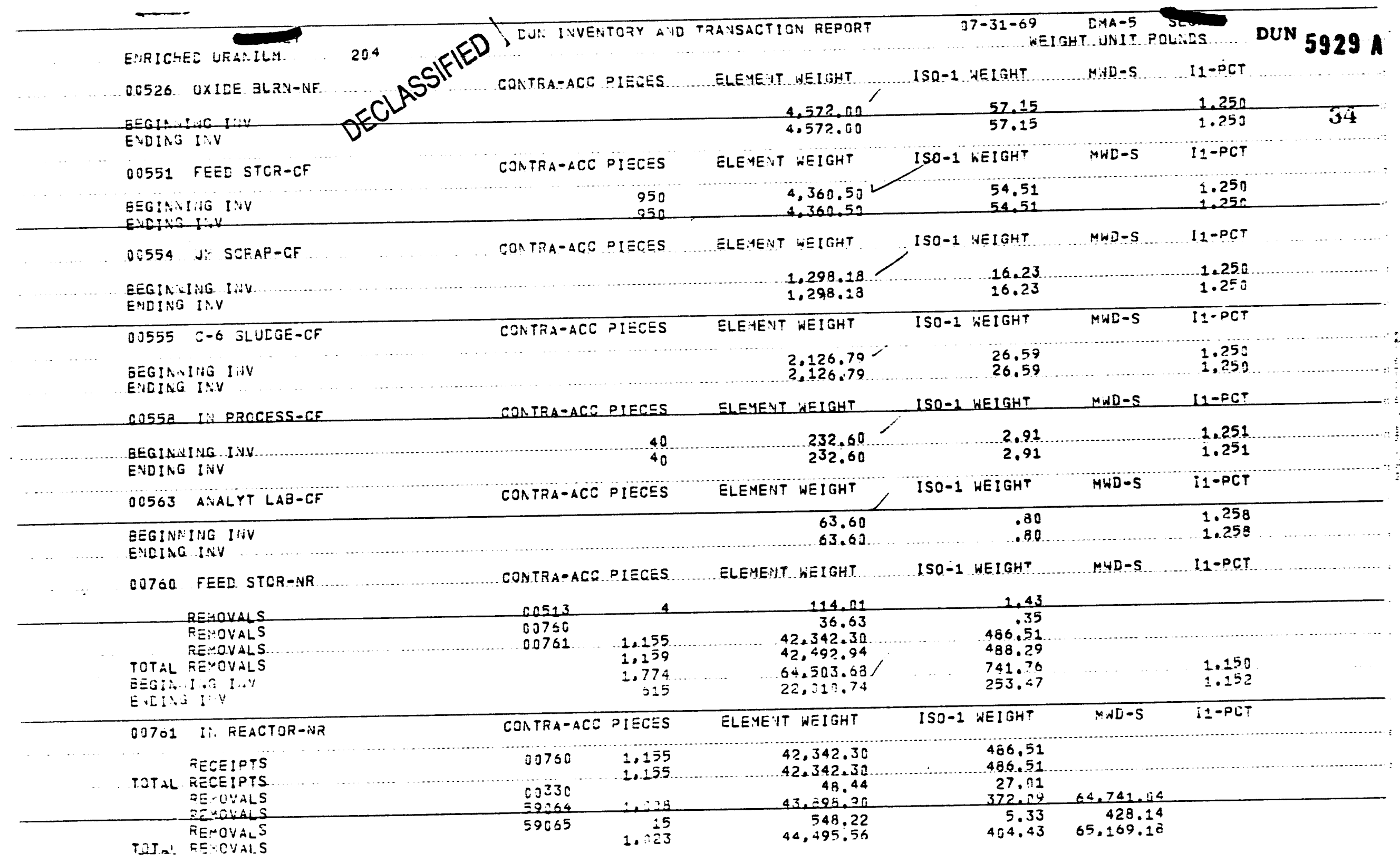




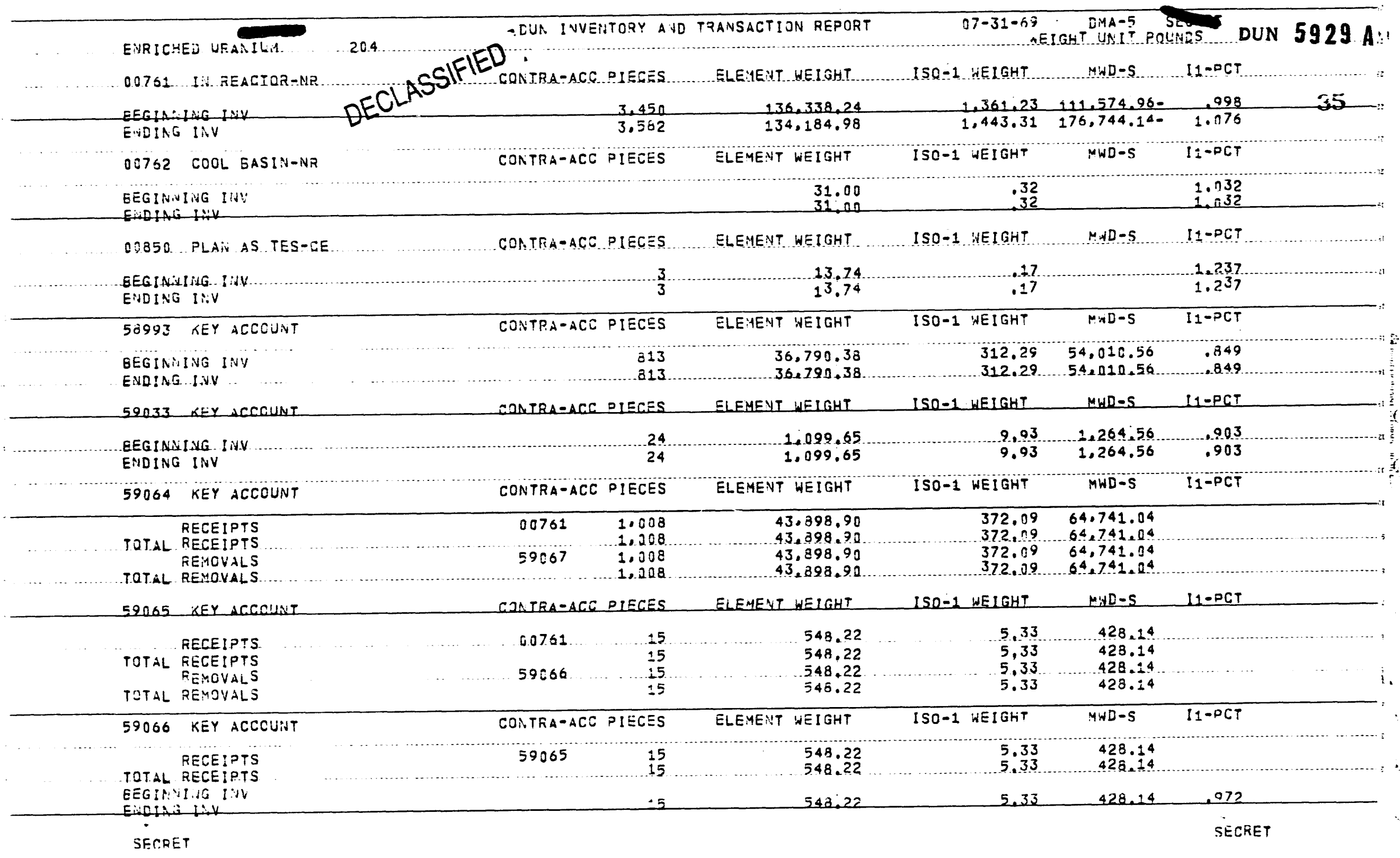





\section{punass}

3

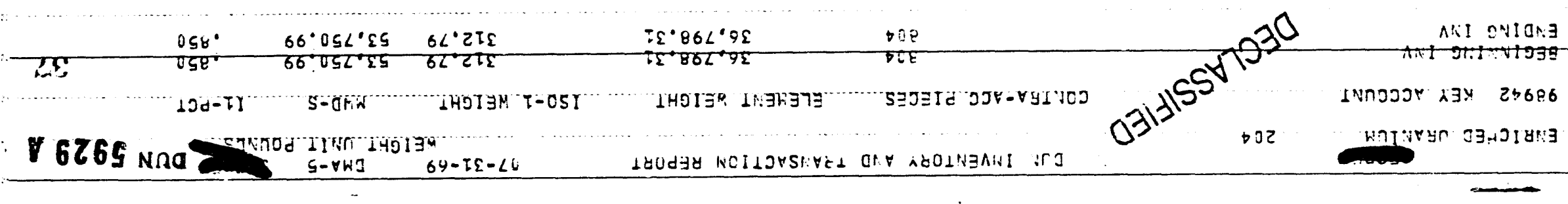




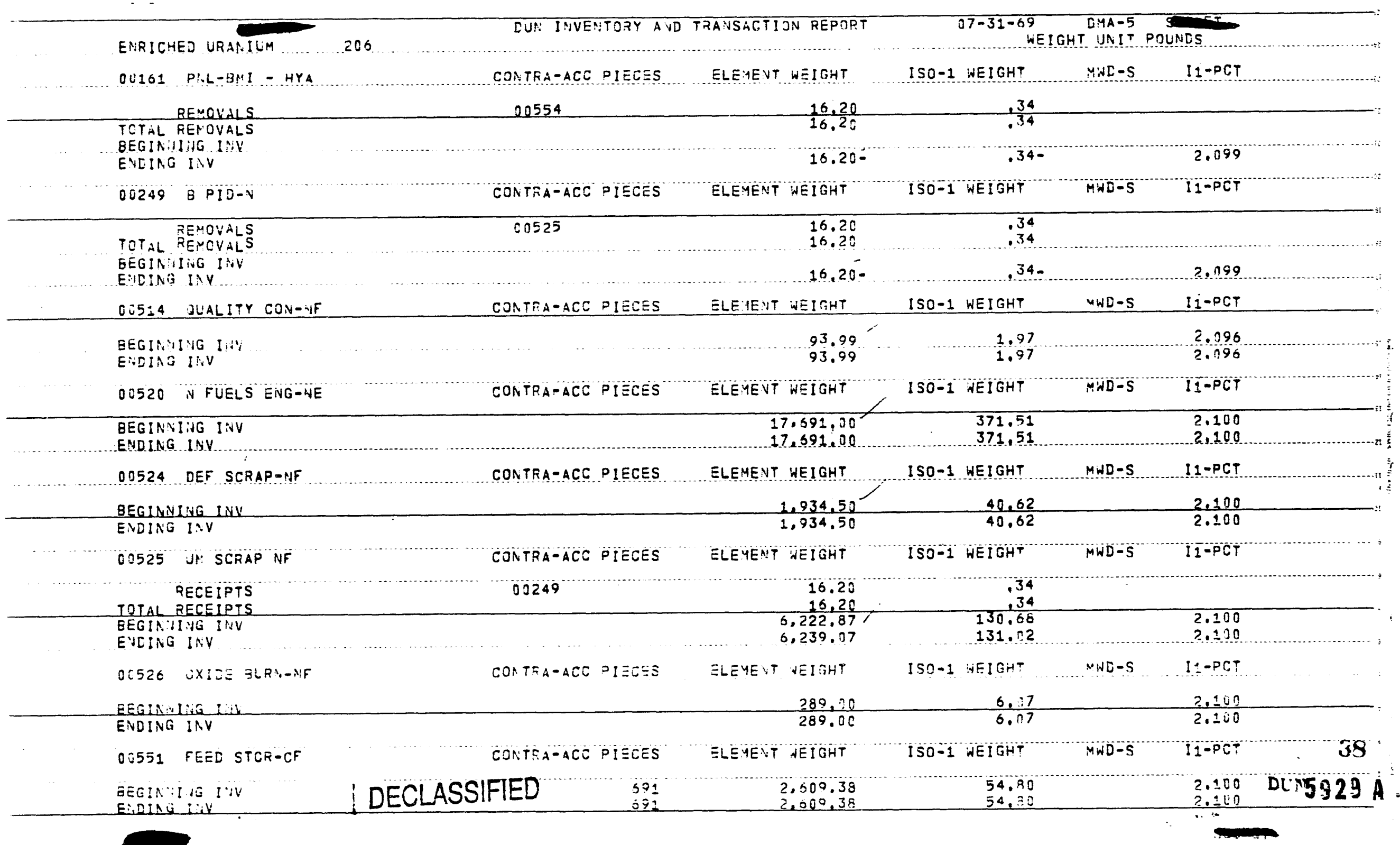




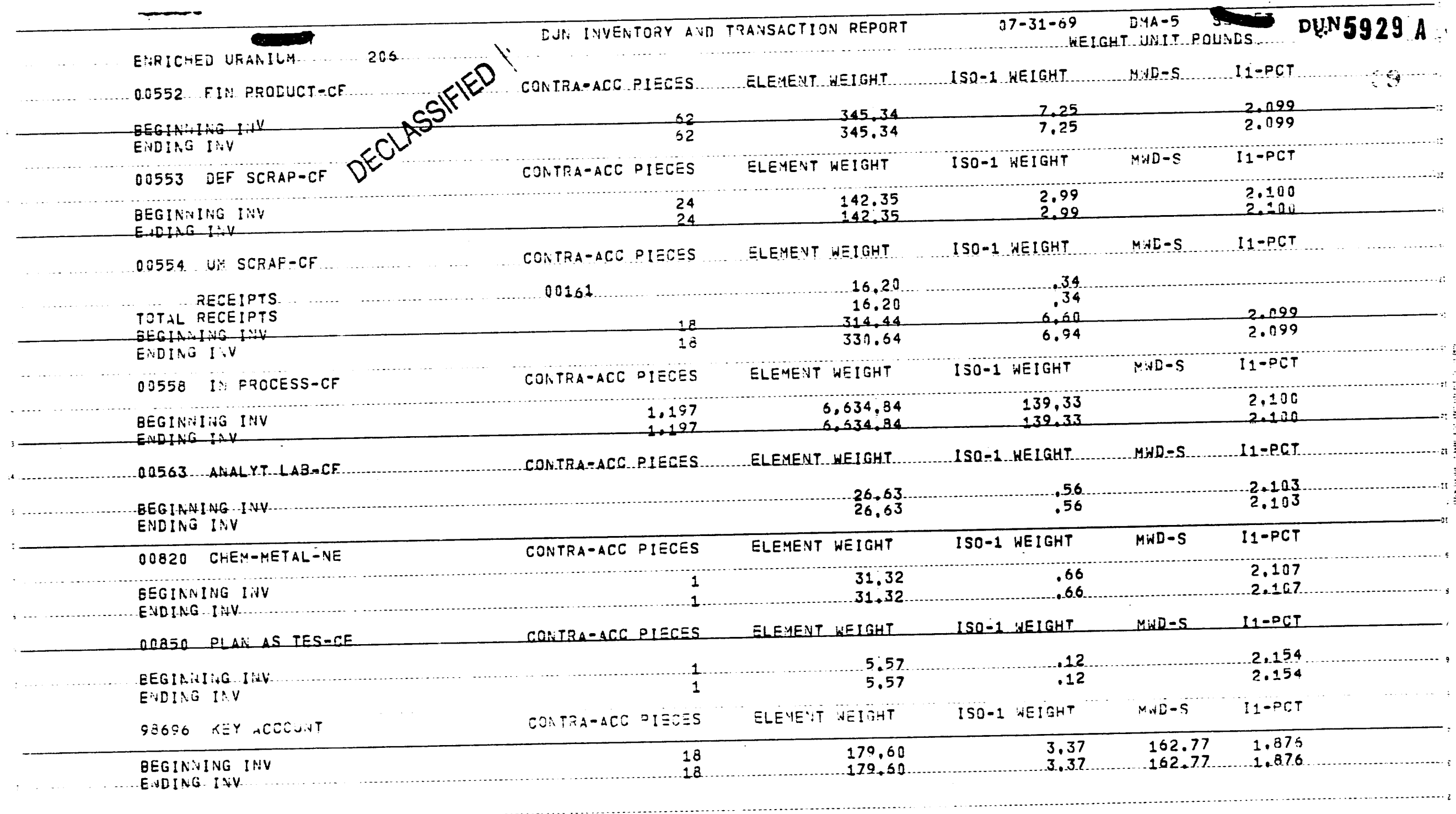




\section{$\bullet$}

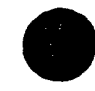

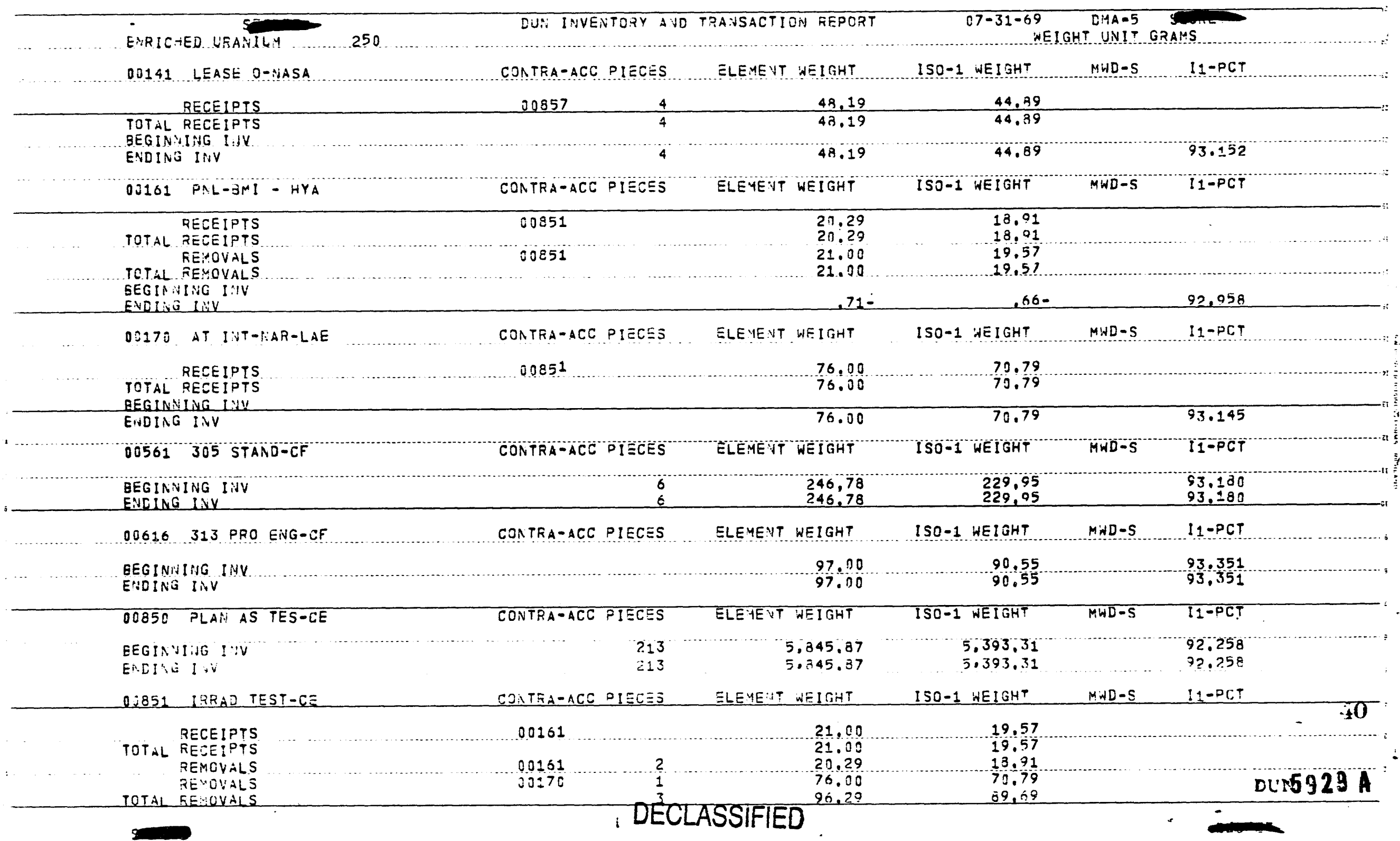




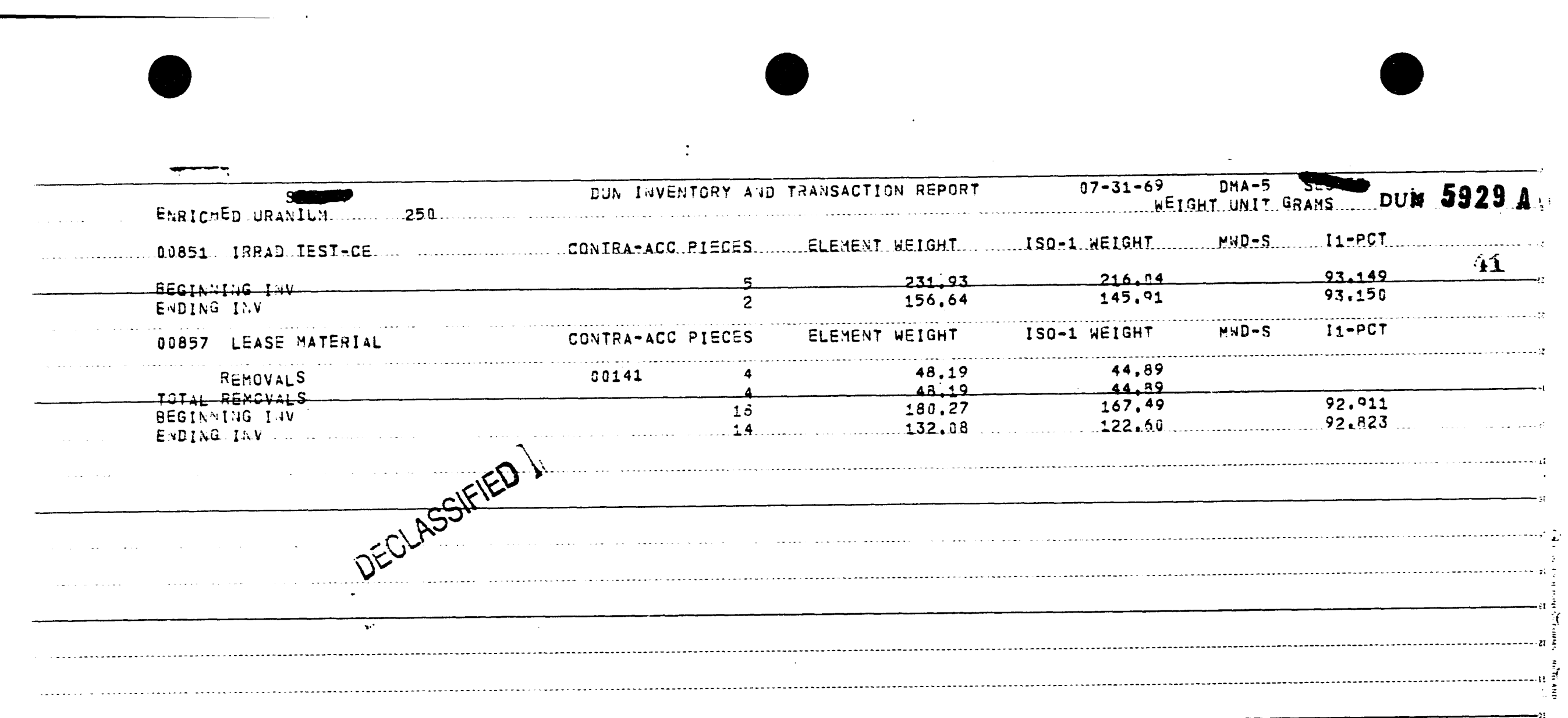




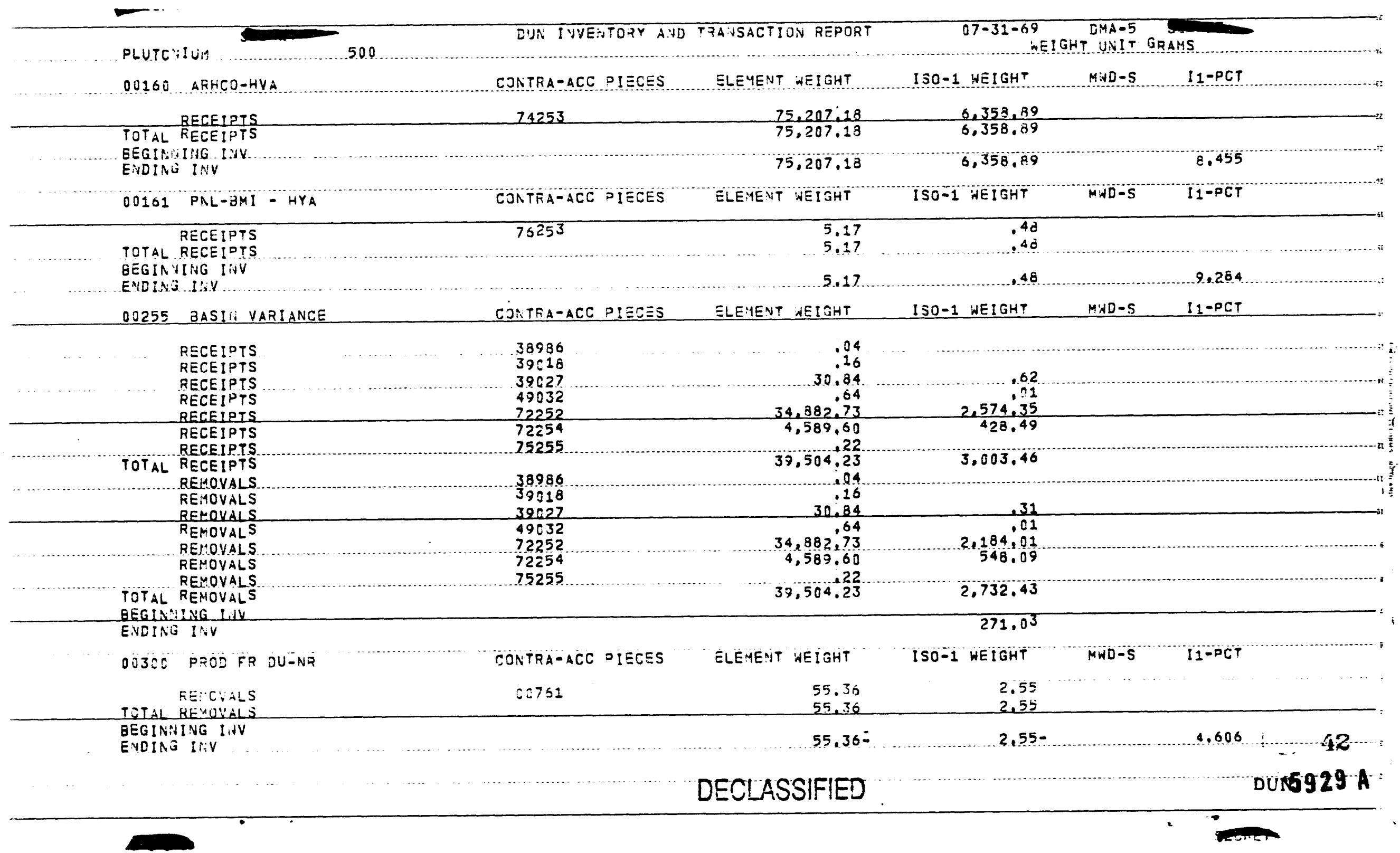




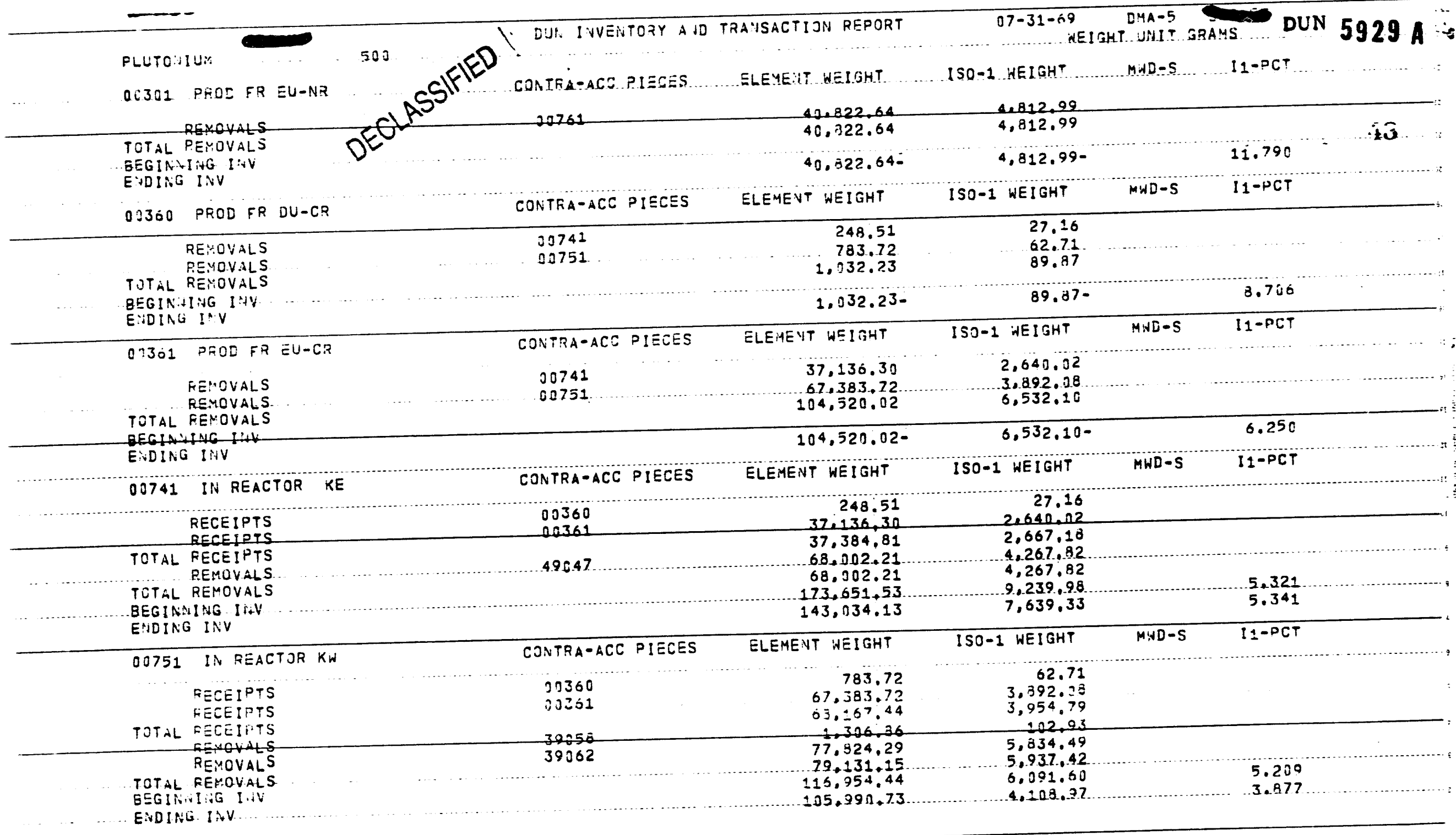



PLUTC:IUM

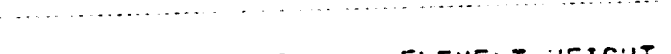
ISO-1 NEIGHT . . MND-S .... I1-PCT

39018. KEY ACCCUINT $1,289.42$ 20.05 6.513 $+5$

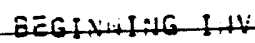
ENDINOS INV

39027 KEY ACCCUNT sक

II-PCT

CONTRA-ACC PIECES ELEYENT WEIGHT

ISO-1 WEIGHT

MWD-S

30.84 30.84

RECEIPTS REMOVALS

TOTAL REMCVALS TOTAL REMOVALS EEGIANING INV INV

$\begin{array}{lr}00255 & 30.84 \\ 00255 & 30.84 \\ 75253 & 30.34 \\ & 112.258 .64 \\ & 110.289 .48 \\ & 110.258 .64\end{array}$
.31 $110,289.48$ 110.258 .64 .62 3.092 .920 $3,093.60$ 7.340 39058 KEY ACCCUNT CONTRA-ACC FIECES ELEMENT NEIGHT $1,306.86$ 00751 1306.86 TCTAL RECEIPTS gegINUI ING INV ENDIAS SiN

1.306 .86

39062 KEY ACCOUN.T CONTRA-ACC PIECES .......ELEMENT.NEIGHT RECEIPTS TOTAL RECEIPTS OEGTAVING IHI

00751

$77 \cdot 324 \cdot 29$ ENDING INV

49032 KEY ACCCUNT $77,824.29$ RECEIPTS

TOTAL RECEIPTS REMOVALS REFOVALS REMOVALS REYCVALS

TOTAL RE:CVALS

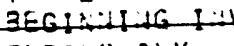

ENDING IiN

CONTRA-ACC PIECES ELEMENT WEIGHT

$\begin{array}{ccc}\text { ISO-1 NEIGHT } & \text { MWD-S } & \text { I1-PCT } \\ 102.93 & & \\ 102.93 & \\ 102.93 & & \\ & & 7.376\end{array}$

00255 .

ISO-1 HEIGHT

MHD -S N.....11-PGT

$5,834, .49$

5.834 .49

ISO-1 HEIGHT

7.497

00255

00255

00850

74252

74253

.64
.64
.64
358.11
92.14
295.74
746.53
745.79

.01
.01
.01
19.10
5.93
29.22
53.30
53.25

.01

19.01

7.92

D稌 5929! 


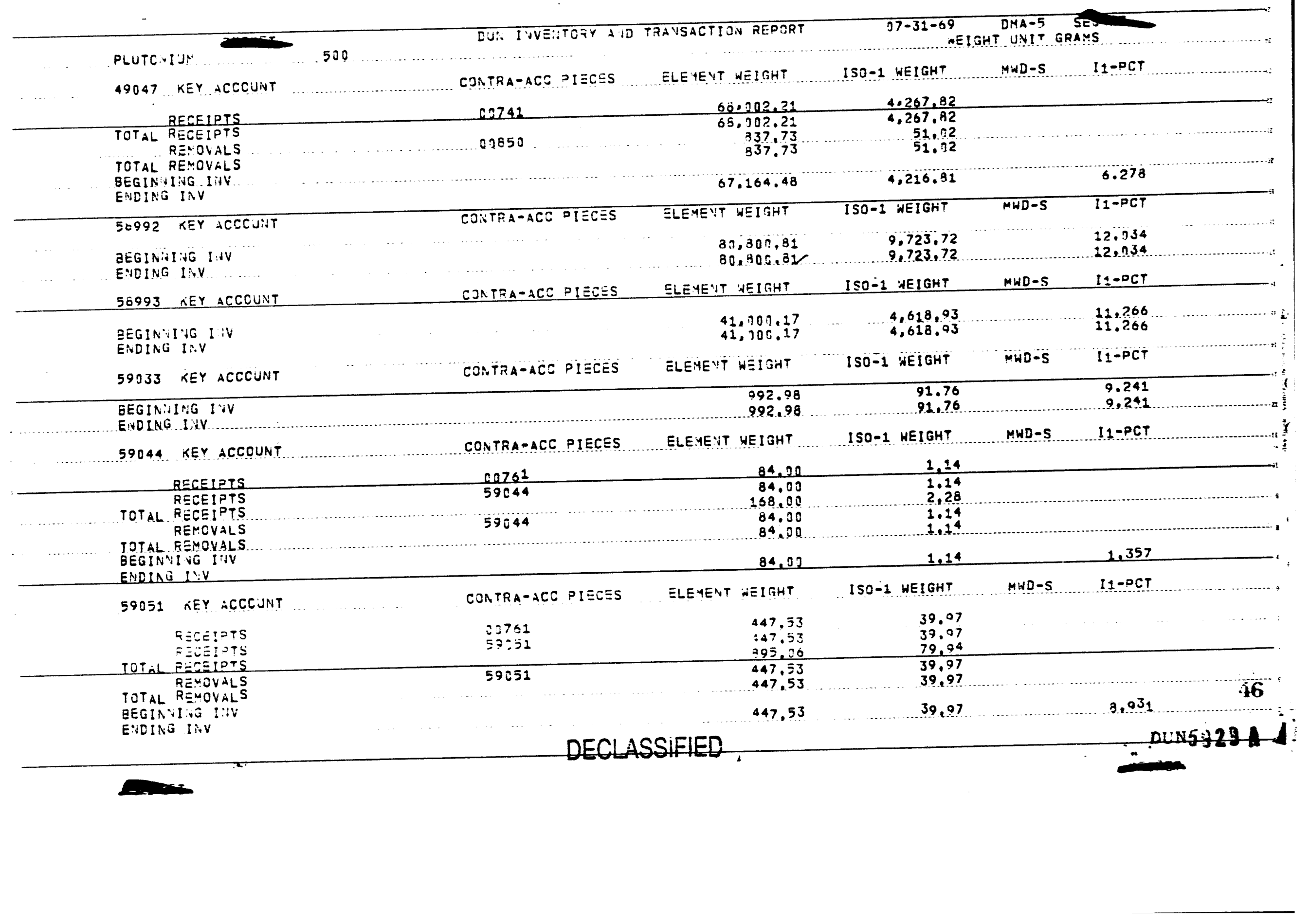




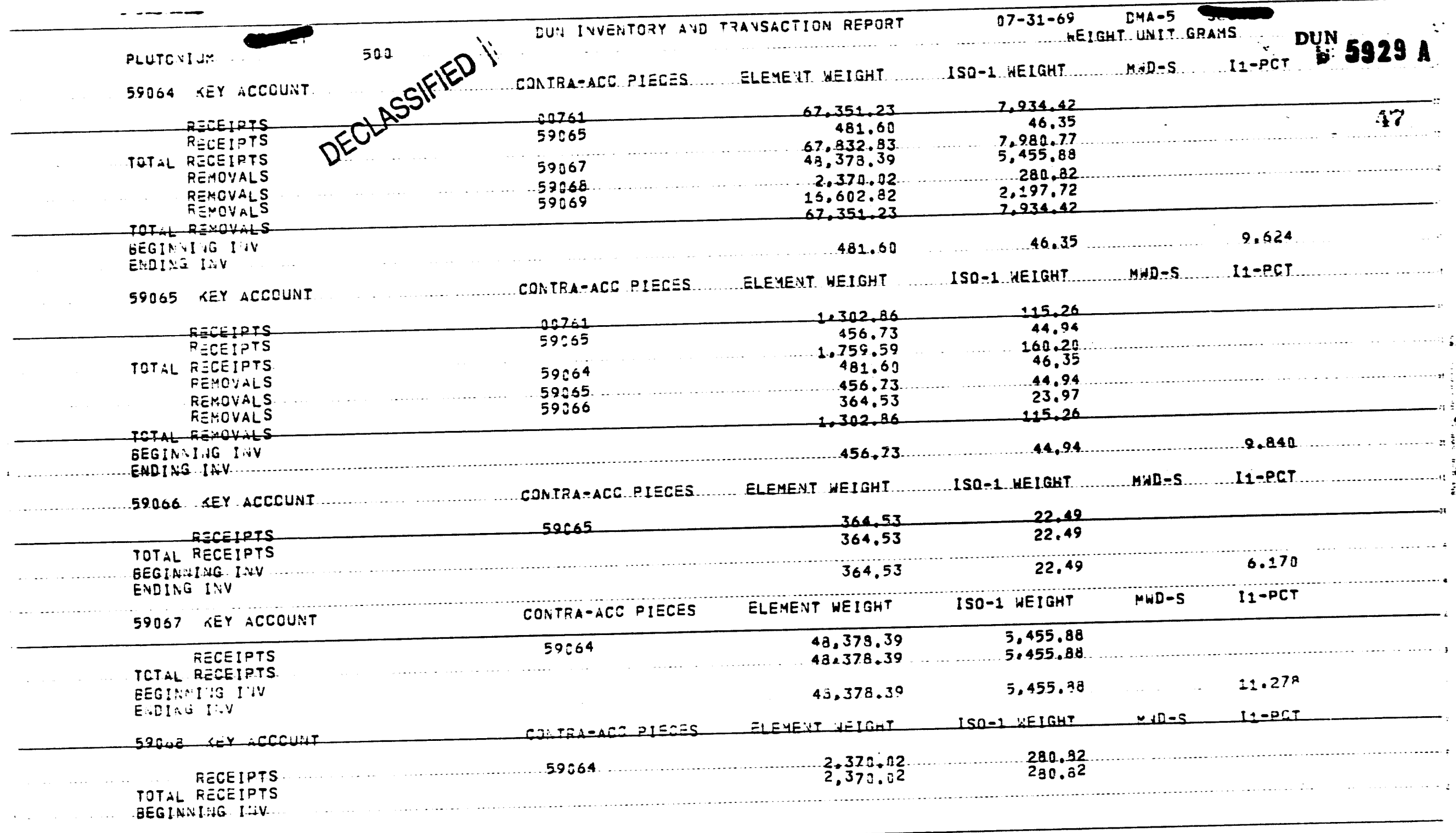


PLUTC:IJT

59060 KEY ACCCUNT

CONTFA-ACO PIECES ELEMEVT WEIGHT

15O-1 WEIGHT

MWD-S 11-PCT

ENDISO livi

$$
2.379 .02
$$

280.82

11.349

59069 KEY ACCOUNT

CONTFA-ACC PIECES .... ELEYENT WEIGHT

ISO-1 WEIGHT.

MHD-S

$11-P C T$

TOTAL RECEIPTS

TETAL RECEIPTS

ENDING IINV

59264

15.502 .82

2.197 .72
2.197 .72

ENDING ISN

$16,602,32$

16.502 .32

2.197 .72

71251 I BAS PL T51

CONTRA-ACO DIECES

ELEMEYT WEIGHT

ISO-1 WEIGHT

EEGINAIIVG IVV ENDING Liv

71252 S EAS PL T51 19.98 10.98

gEgINivINo lid

ENDING INV

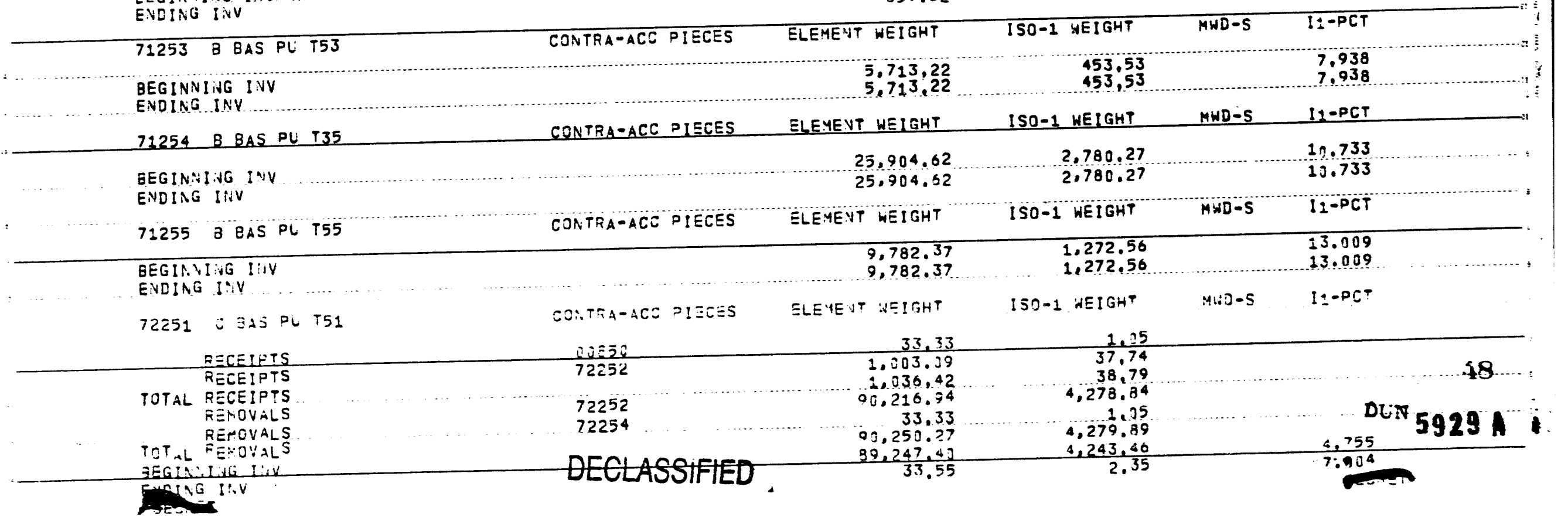


- -

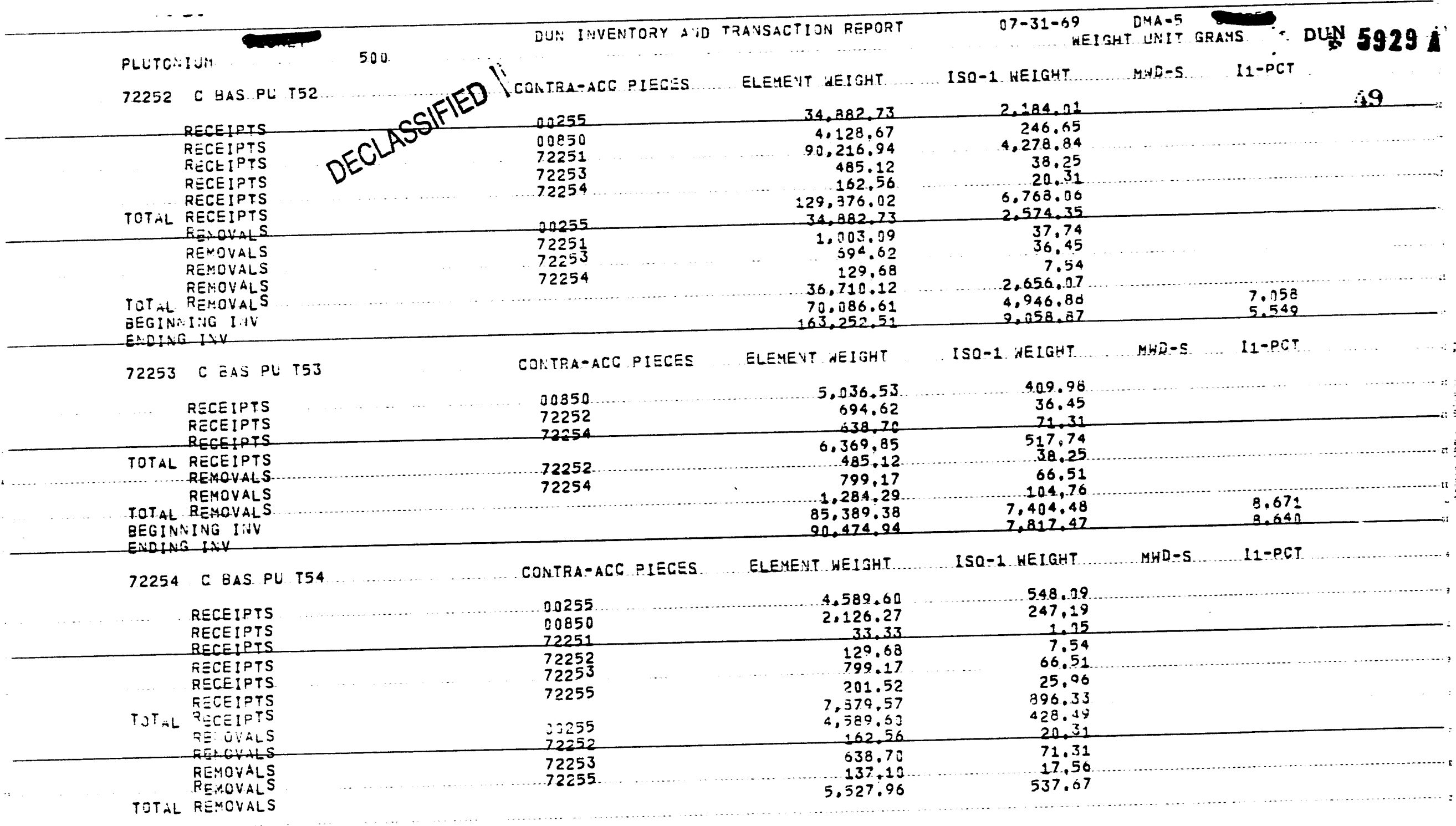

TOTAL REMCVALS 


\section{-}

-

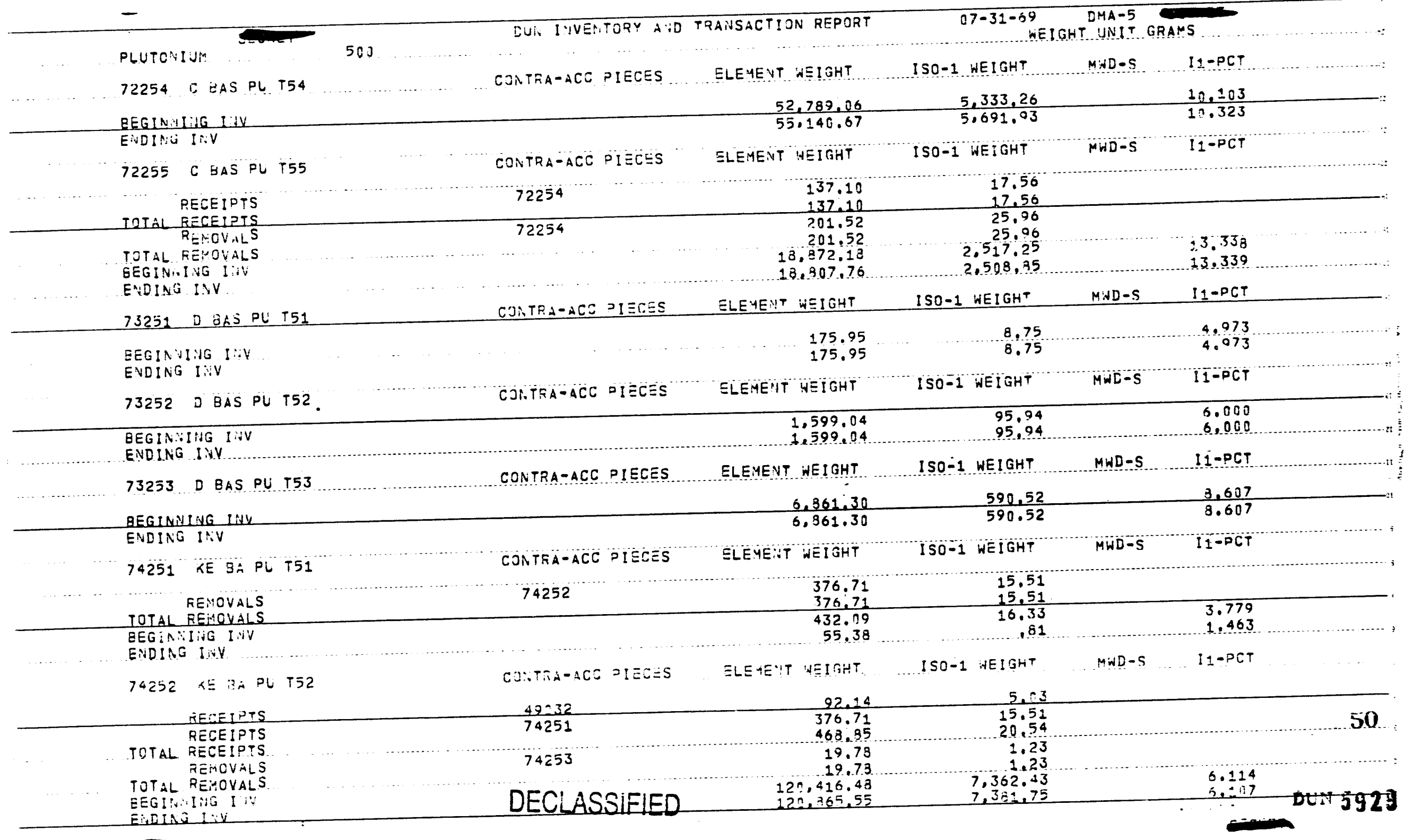


DUi, IUVE TORY A ID TRAISACTION REPJRT

$07-31-69 \quad 044-5$ NEIGHT UNSIT GRAMS

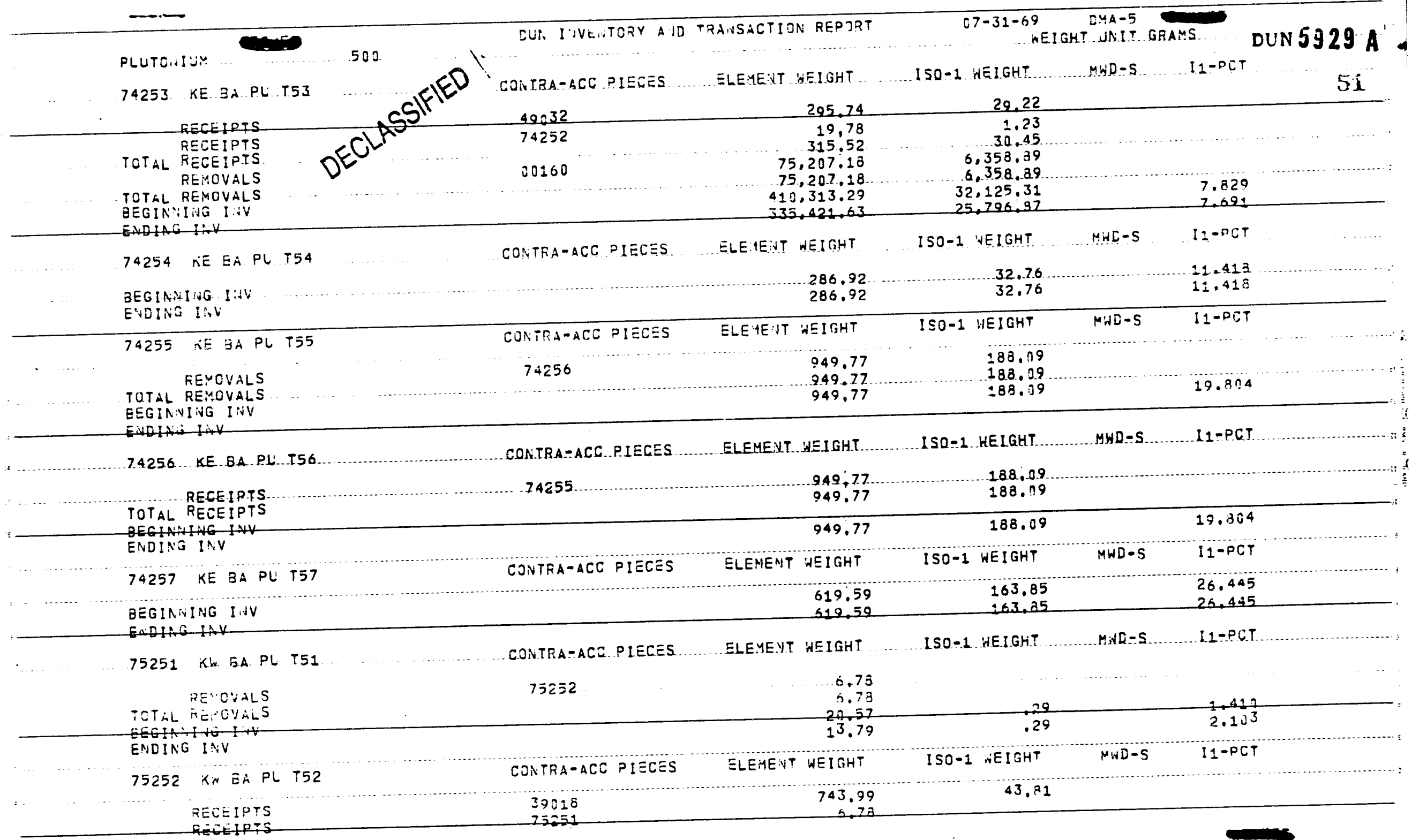




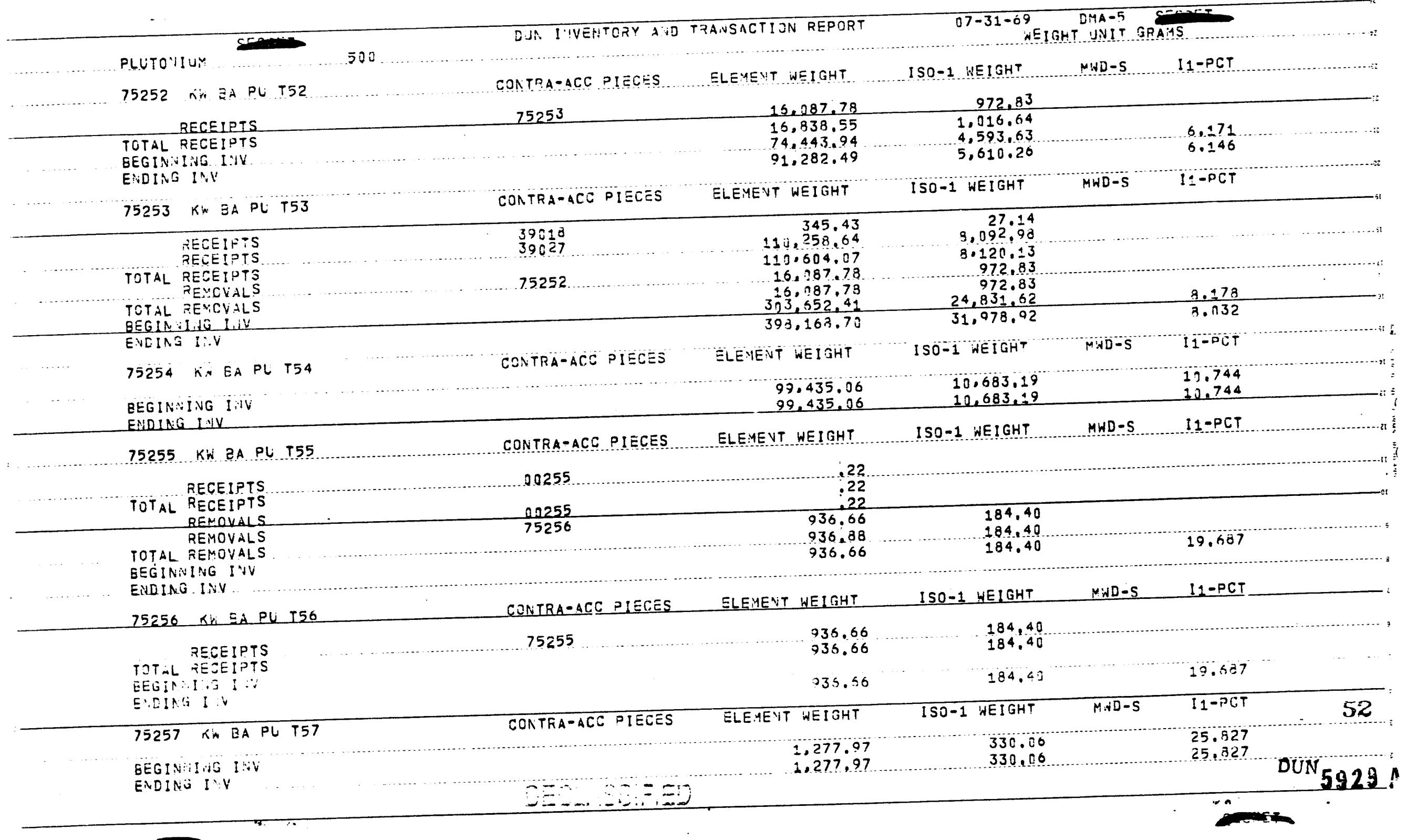




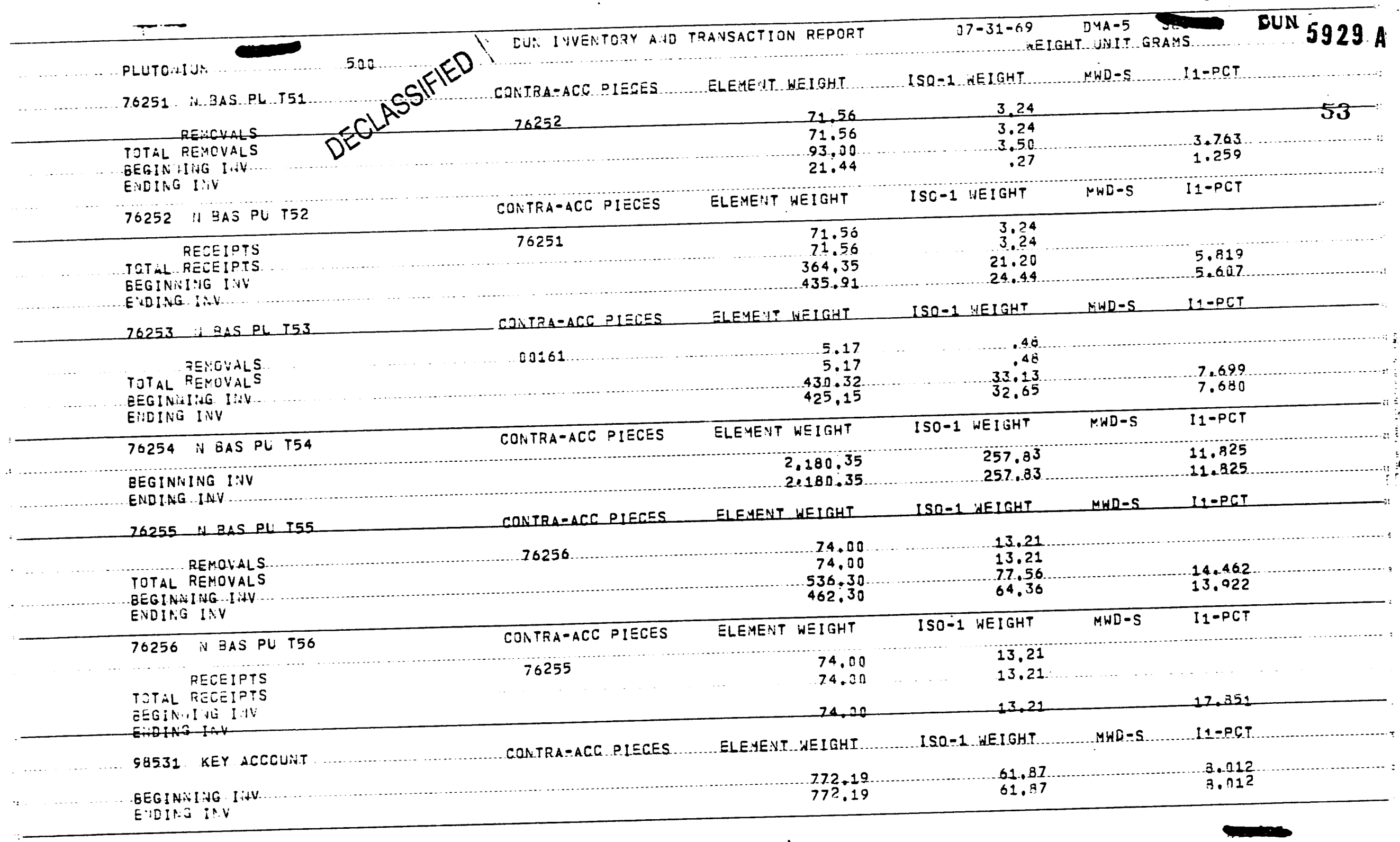




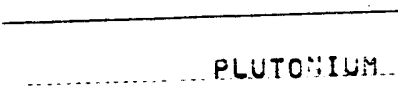

\$8560 KEY ACCCUNT

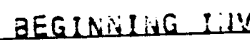
ENDING INV

98588 KEY ACCOUNT

BEGINIING IIJV EIDISG LiV

98634 KEY ACCCUNT

EEGINUIYG IIV EVDIAS ISV

98606 KEY ACCOURT

BEGINIVING INV EVDING INV 98678 KEY ACCOUNT

EEGINNING IIYY ENDING INV

$98696 \mathrm{KEY}$ ACCOUNT

EEGINNING IIIV

ENDING INV

\$8772 KEY ACCOUNT

GEGINNIIVE LiV

ENDING INV

$98822 \mathrm{KEY}$ ACCCUNT

EEGIN.IIIG I I ENDIVi ISV

98829 KEY ACCOUNT

EEGIR:t IHG bis E:DING ?:V
LUN: INVENTORY AYD TRANSACTION REPORT CONTRA-ACC PIECES ELEMENT WEIGHT

511.81

511.81

CONTRA-ACC PIECES

ELEMEVT WEIGHT

1.098 .30

1.998 .30

CONTRA-ACC PIECES ELEMEIT WEIGHT

991.45

991.45

CONTRA-ACC PIECES

CONTRA-ACC PIECES ELEMEYT WEIGHT

15.33
15.33

CONTRA-ACC PIECES ELEMENT WEIGHT

2.392 .08

2.392 .08
$2,392.08$

CONTRA-ACC PIECES ELEYEVT WEIGHT

10.05

CONTRA-ACU PIECES

ELEMENT WEIGHT

5.060 .24 5.960 .24

CONTAA-ACC PIECES ... ELEMENT WEITHT

544.93

544.93

(20)

07-31-69 DMA-5 NEIGHT UNIT GRAMS

ISO-1 WEIGHT MWD-S II-PCT

$\begin{array}{ll}30.95 & 6.047 \\ 30.95 & 6.047\end{array}$

ISO-1 WEIGHT MWD-S I1-PCT

$183.21 \quad 9.108$

ISO-1 WEIGHT MWD-S I1-PCT

4,346

$\begin{array}{rr}43.11 & 4.349 \\ \text { ISO-1 WEIGHT MWD-S II-PCT }\end{array}$

$\begin{array}{ll}118.39 & 8.529\end{array}$

SO-1 WEIGHT MHD-S II-PCT

.18

WHAT II-PCT

ISO-1 WEIGHT MWD-S I1-PCT

$57 \quad 5.672$

$\begin{array}{rr}.57 & 5.672 \\ .57 & 5.672\end{array}$

$\begin{array}{lll}1.20 .16 & \text { MWD-S } & 11.563 \\ & & \end{array}$

$\begin{array}{rr}689.16 & 11.503 \\ \text { ISC-1 HEIGHT } & 11-P C T\end{array}$

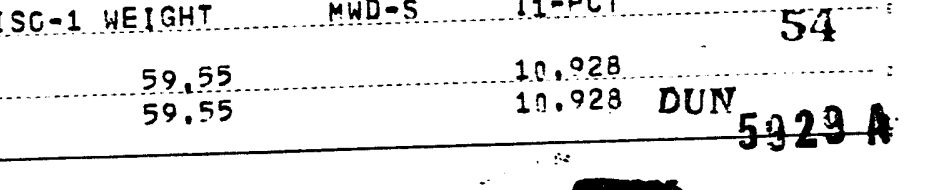




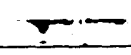

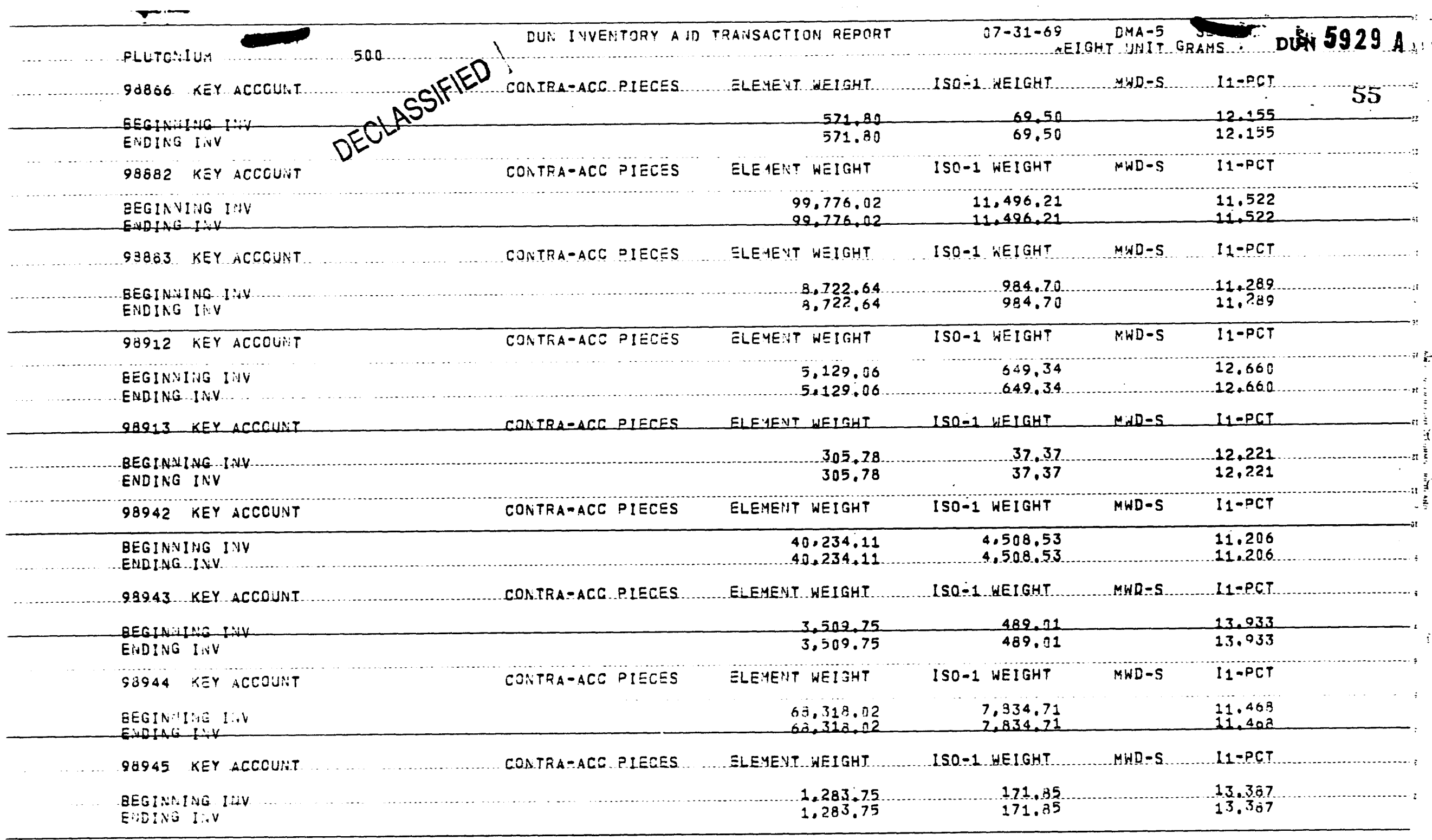

REGINMING IHV

1.283 .75 


\section{$\bullet$}

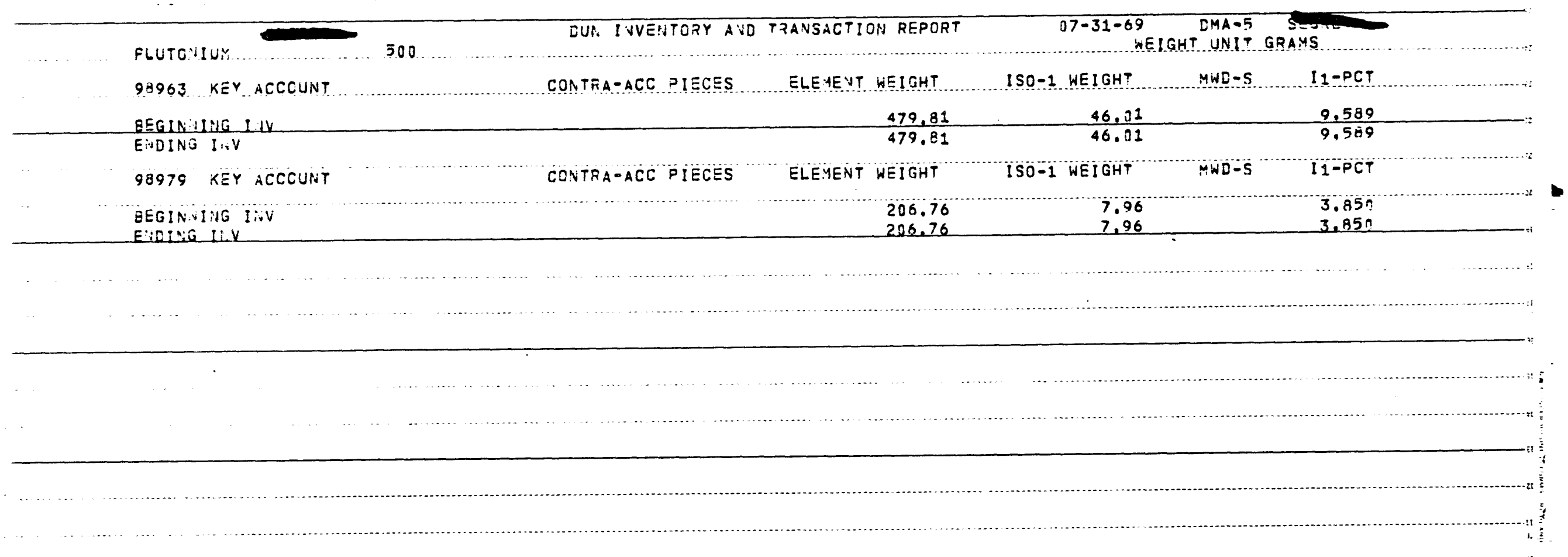

FLUTO'IU:

98963 KEY ACCCUNT

98979 KEY ACCCUNT

EGINVIIV IIV

Earive liv 
$+-\cdots$

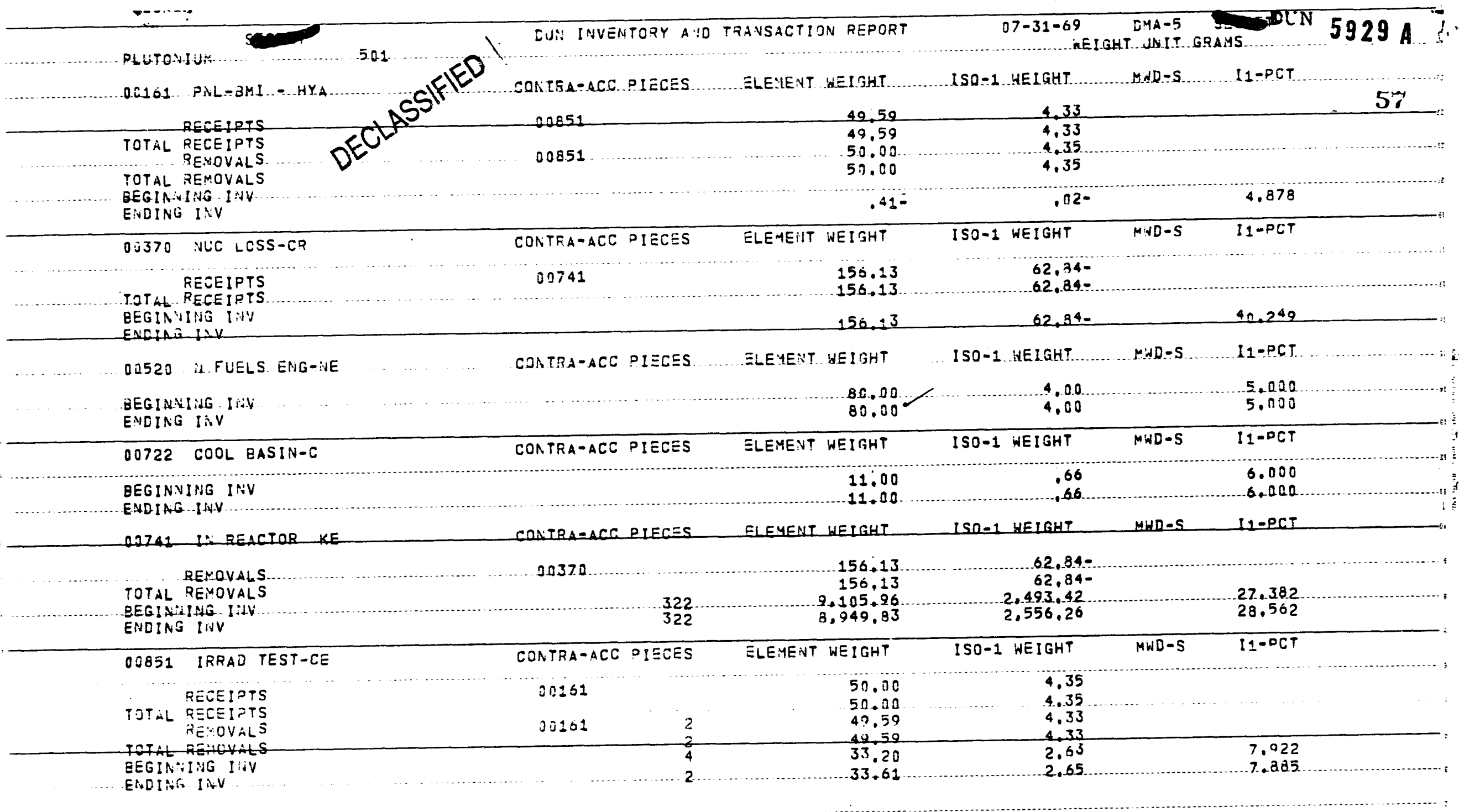




\section{○}

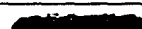

$71252 \quad 3$ SAS PU T51

BEGINVLIVG LiUV

CONTRA-ACC PIECES

ELEMENT WEIGHT

90.40

90.40

ISO-1 WEIGHT

EIGHT DNIT GRAMS

ENDING IiV

$\frac{4.52}{4.56}$

MND-S I1-2CT

5.000

5.000 


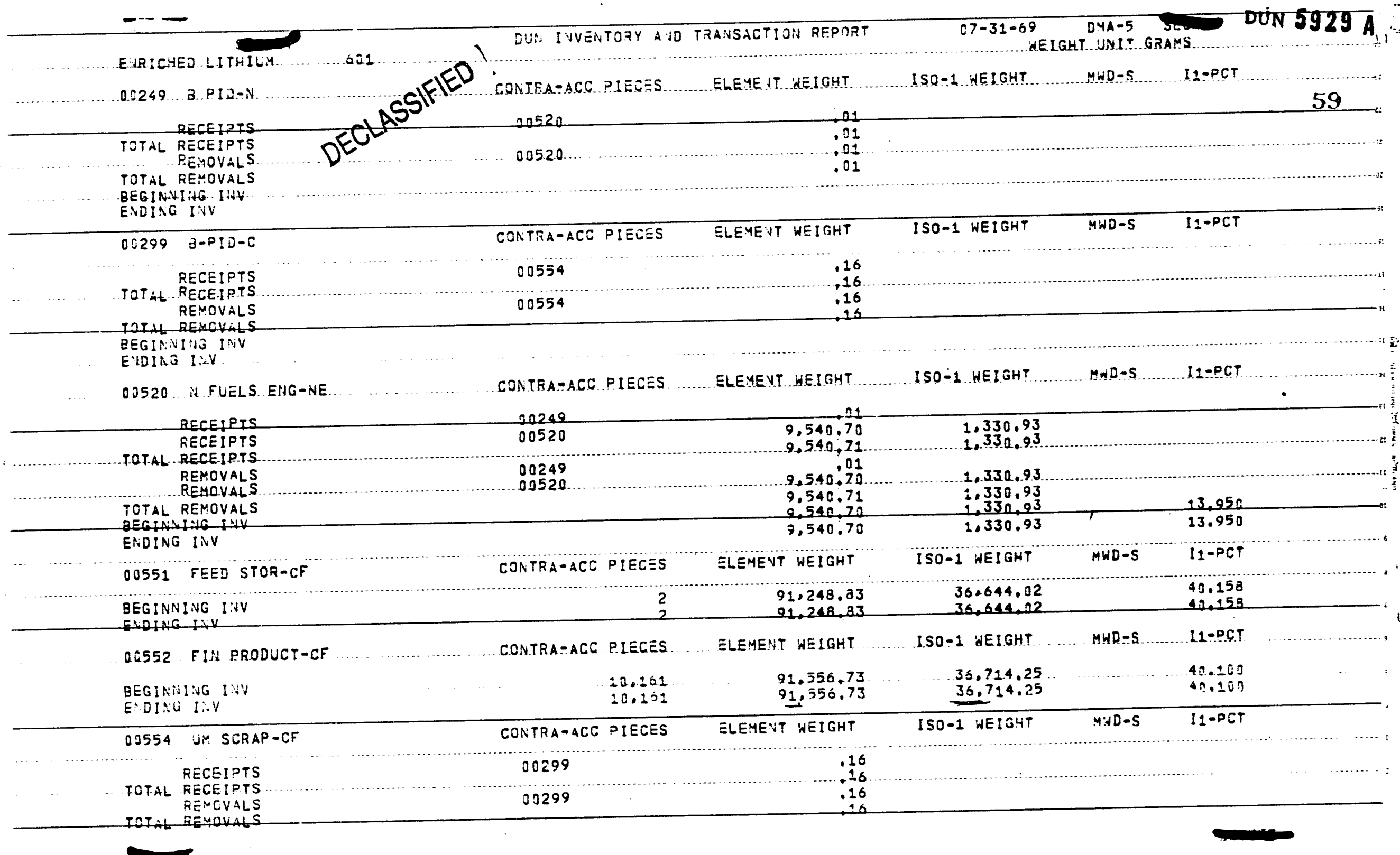


:

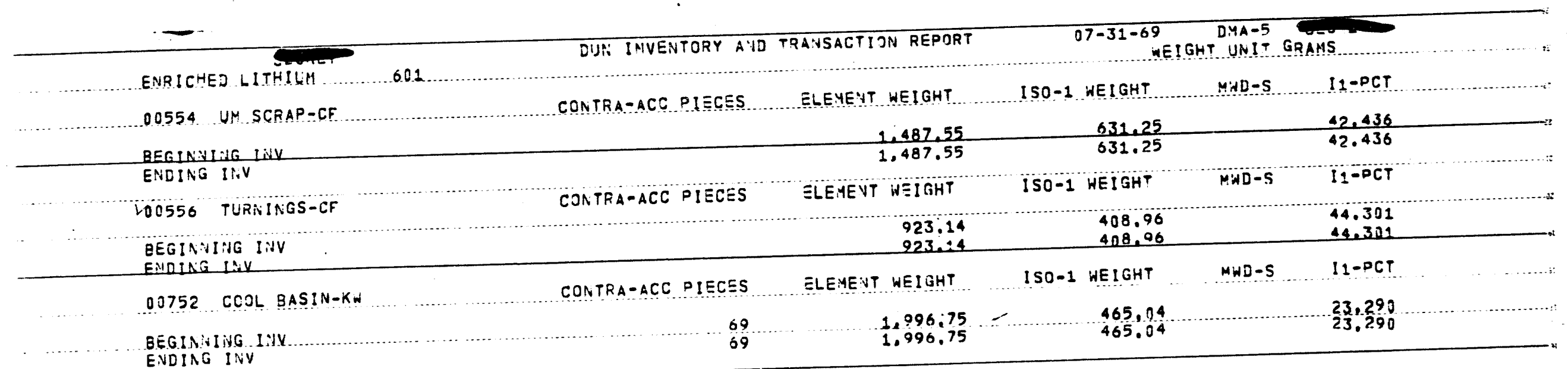




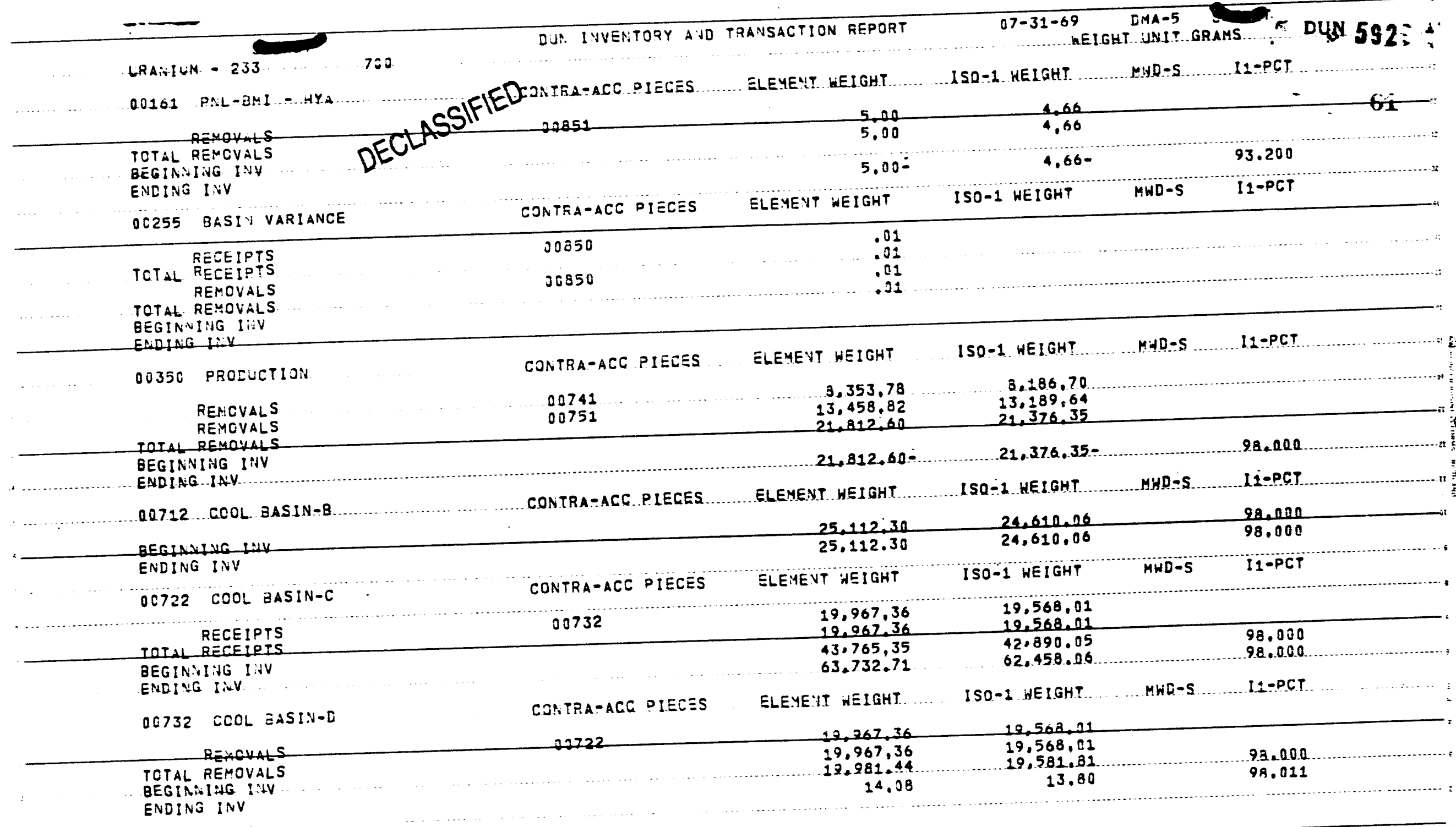





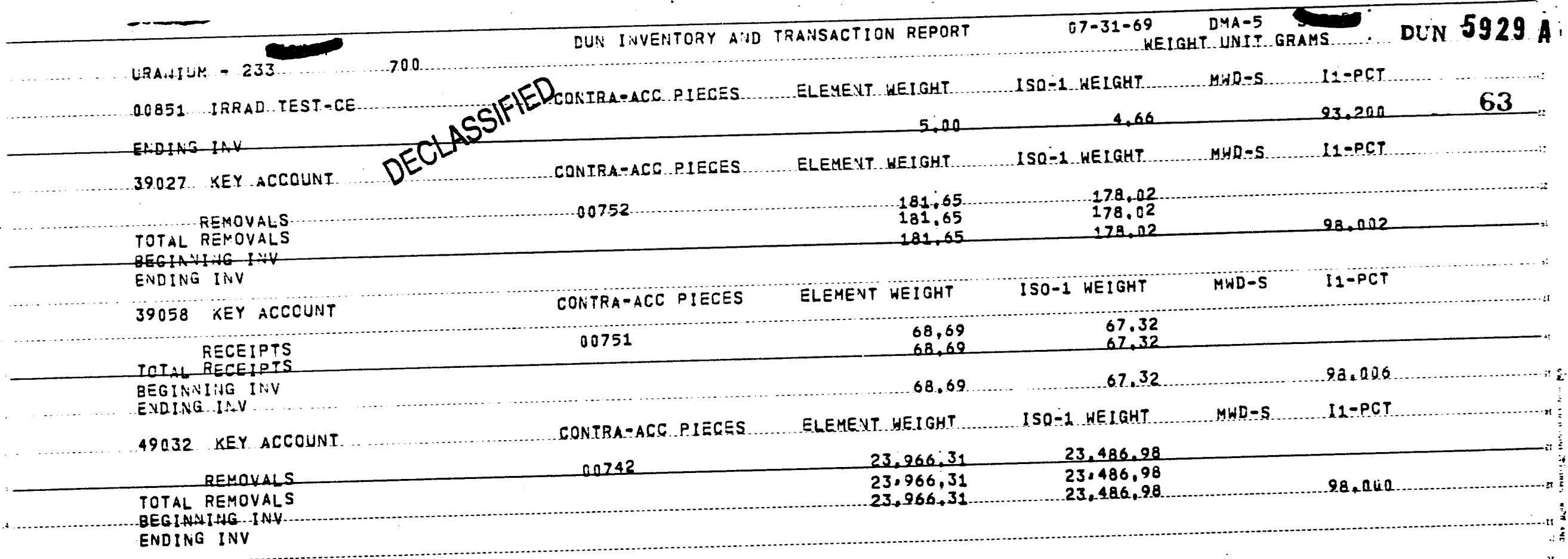




\section{-}

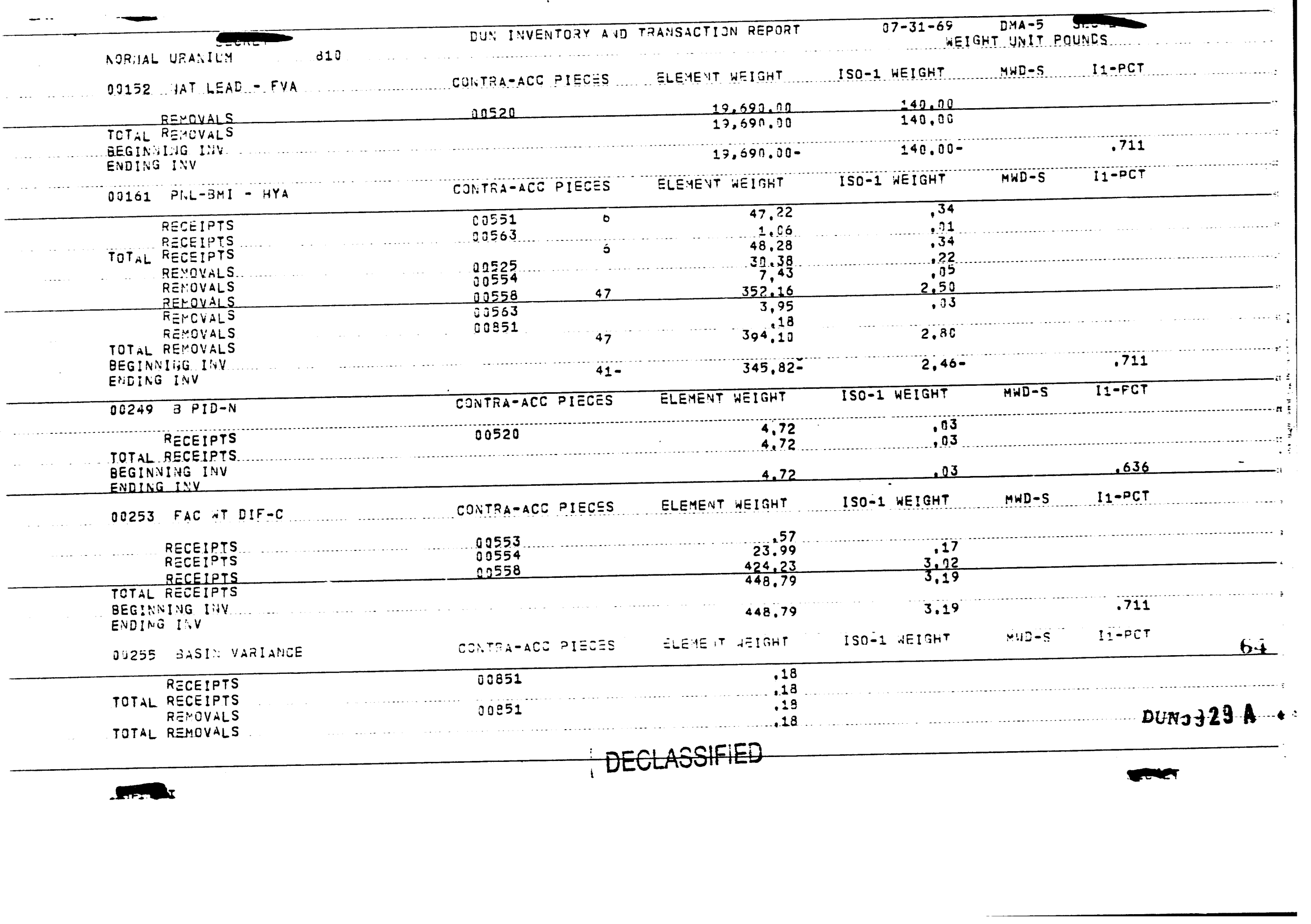


○

$\cdots$

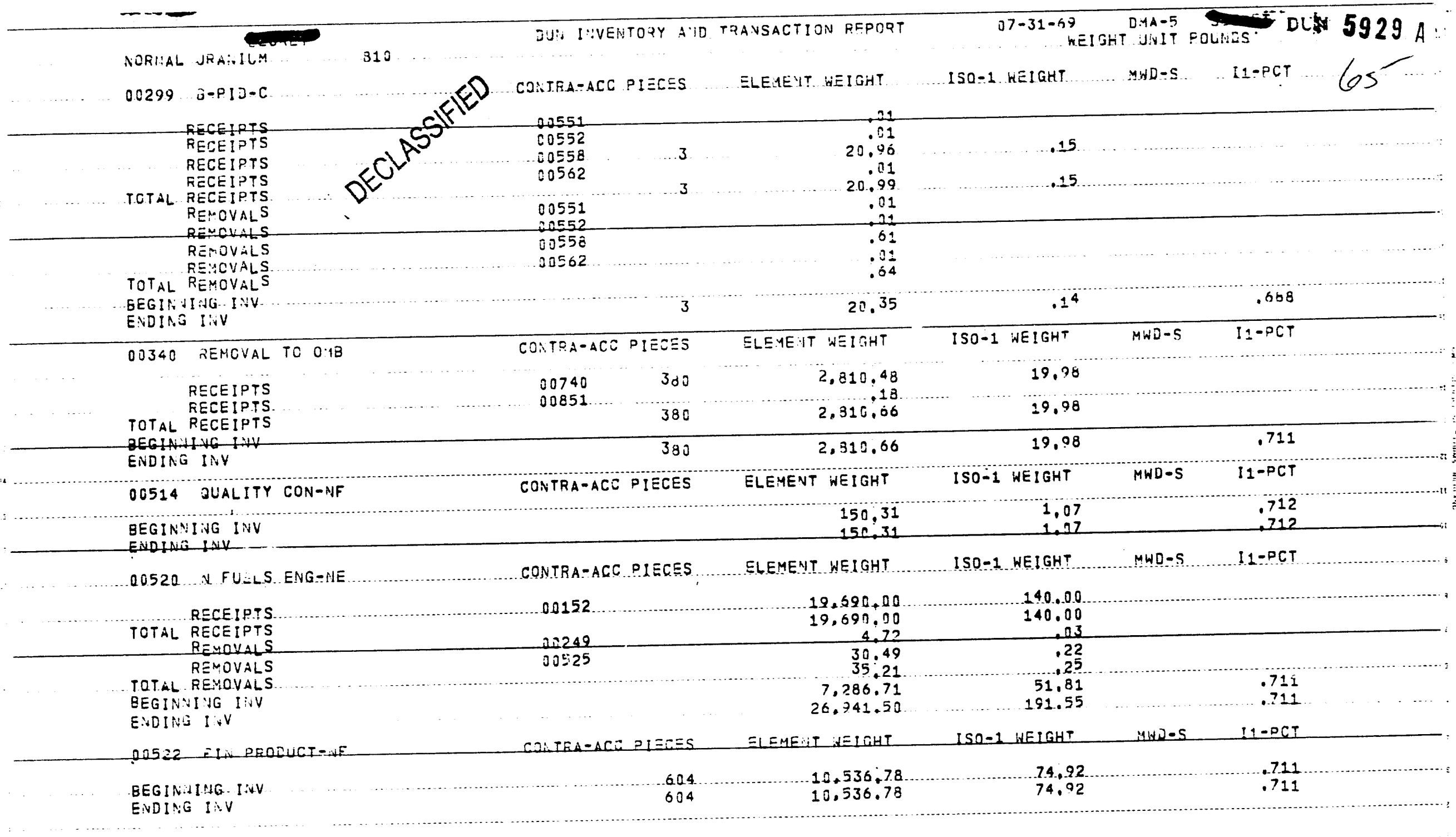


-

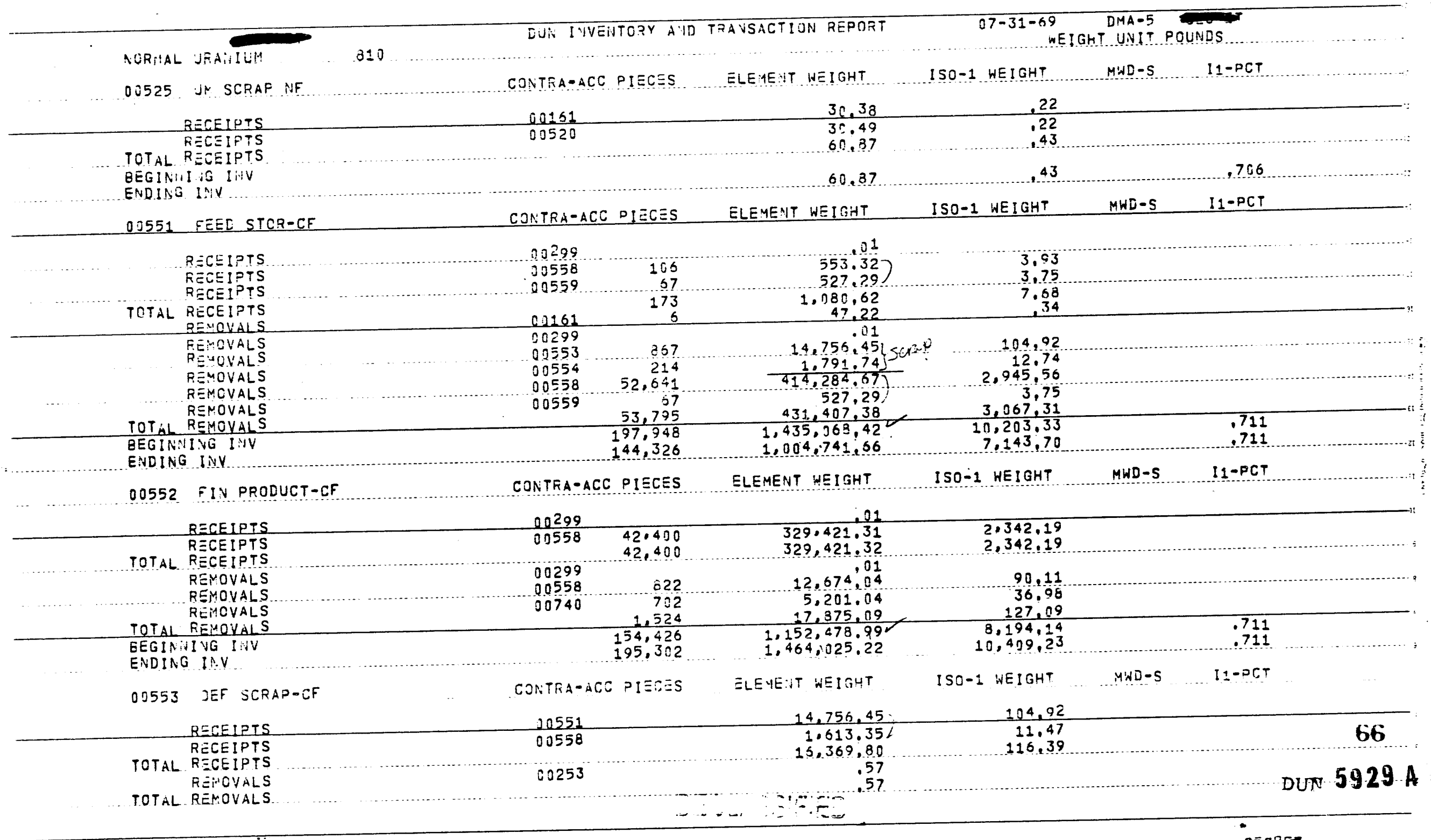




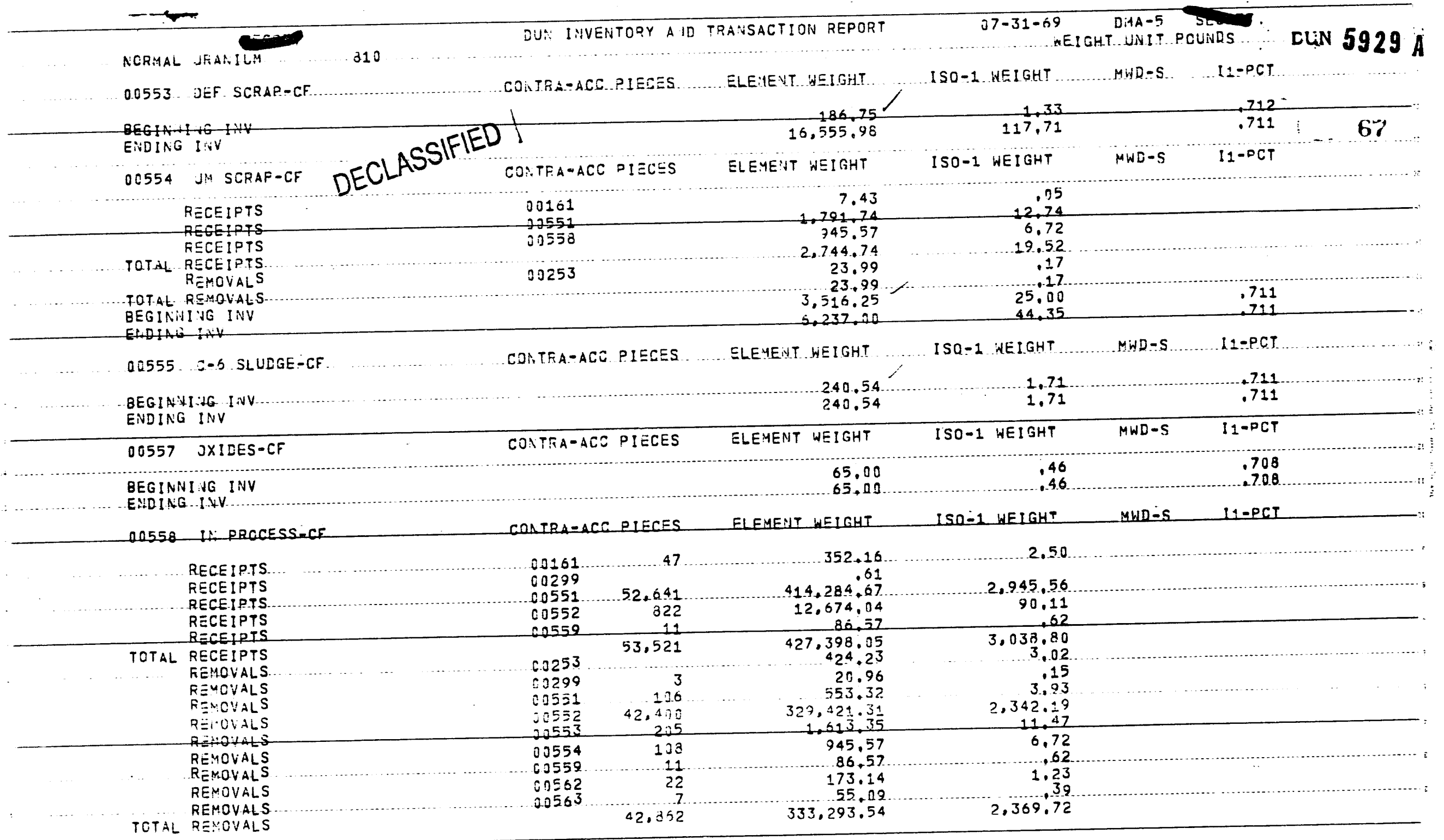




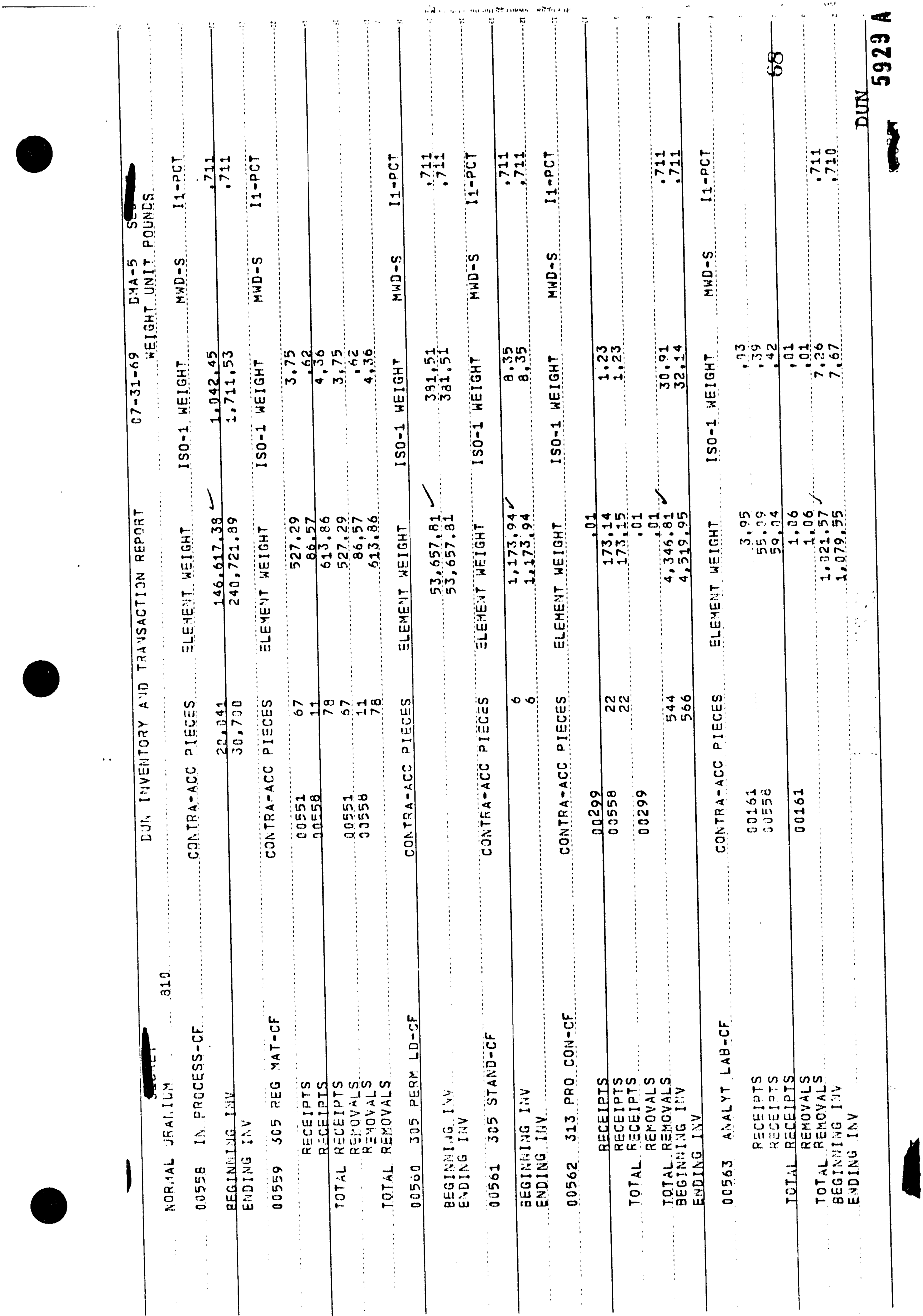




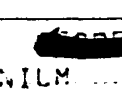

\& EUL IYVEI!TCRY AVD TRANSACTION REPORT EUV IVVELTORY AVD TRANSACTION REPORT

$77-31-69$ WEIGHT UMAIT POUNCS.

NCR:IAL JRAIUILM CONTEA-ACE PIECES ........ ELEMENT WeIGHT Q0615 MACHLI:E SLUDSE CDNTSA-ACC PIECES ......... ELEMENT WEIGHT EEGIAUIIIG IHUV
ENCING IIVV

00616313 PRO ENG-CF GEGINIIIIG INIV 932.40 $1 \$ Q-1$ WEIGHT...

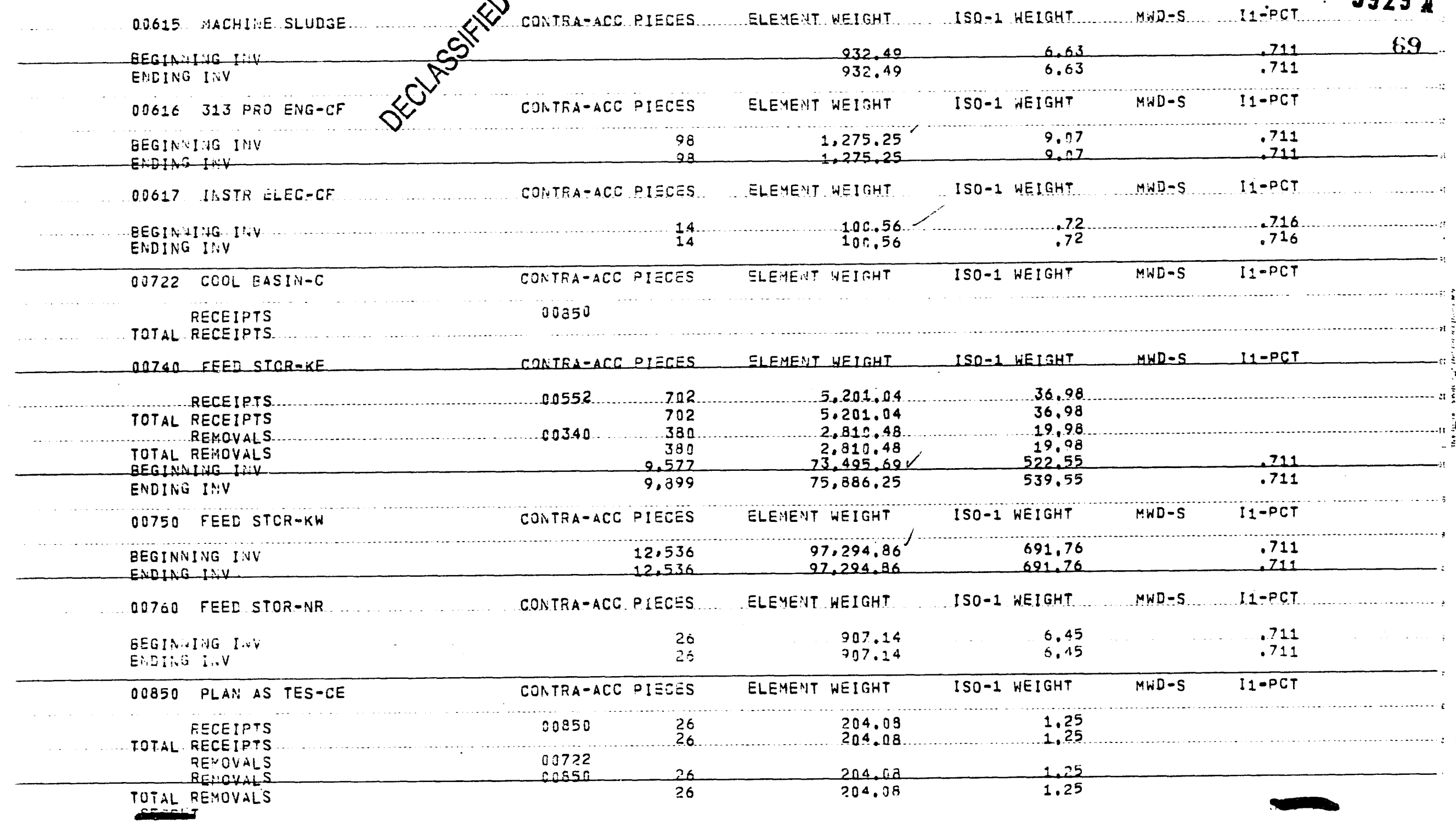




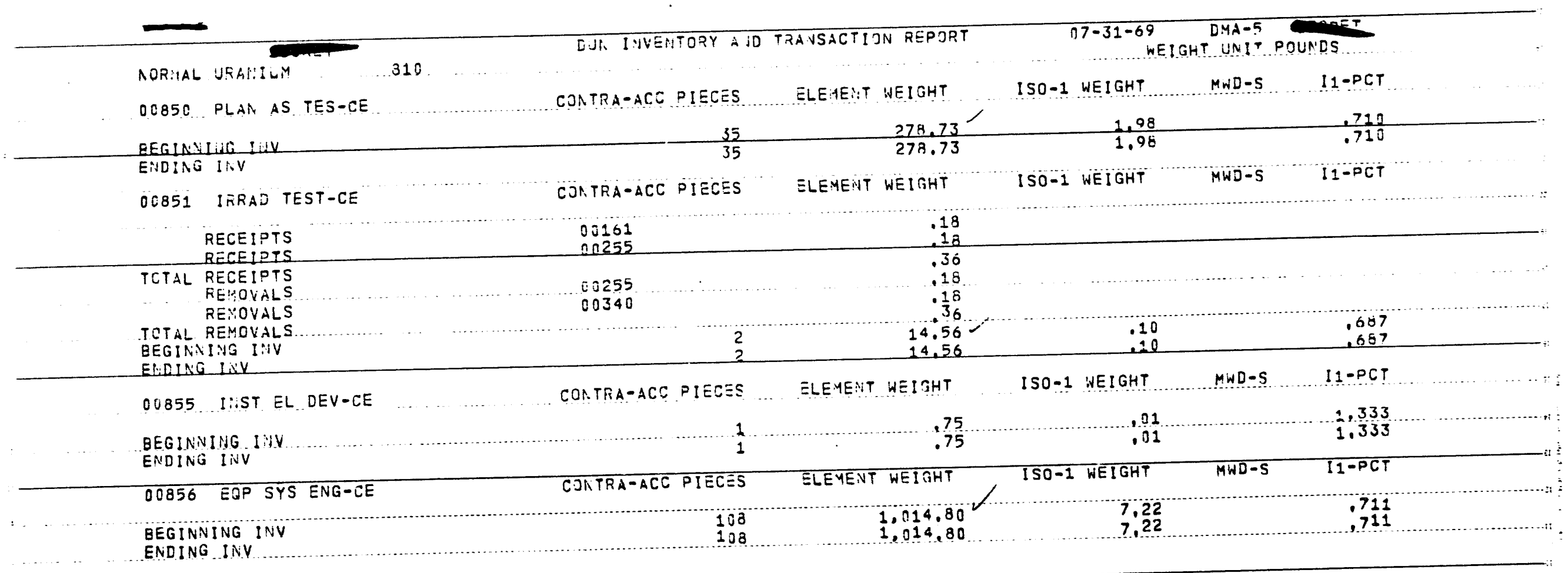




\section{-}

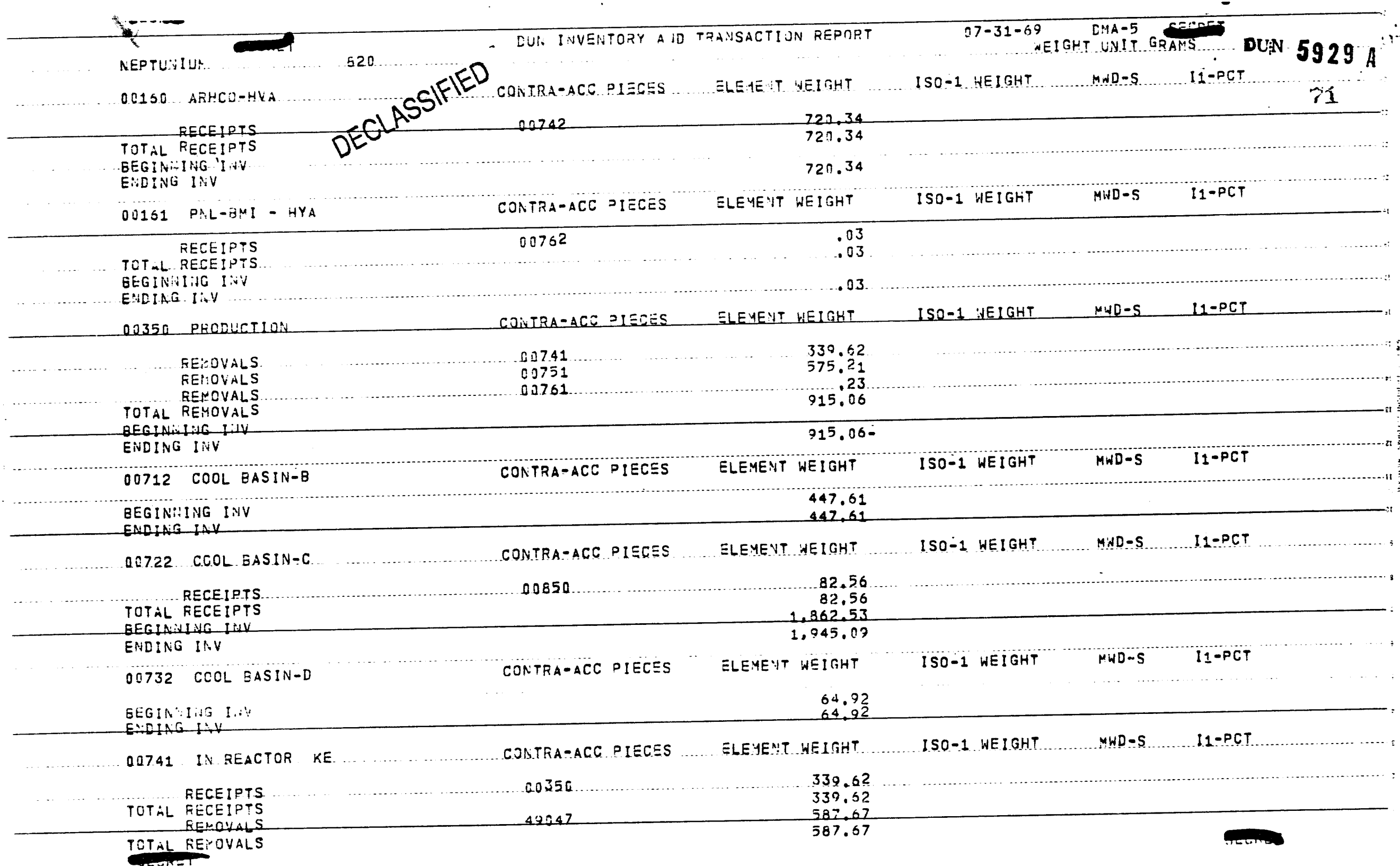


NEPTUNIUY

00741 IN FEACTOR KE

EEGINilitite LII

Eiving INV

00742 CCOL BASIN-KE

FECEIPTS

TOTAL RECEIPTS

FENOVALS

TOTAL FEMOVALS

GEGINIIING INV

ENDING ISV

00751 IS REACTOR KN

TOTAL FEECEIPTS

REMOYALS

TOTAL EFMOVALS

BzGINidING I

ENDING IIIV

00752 COOL BASINZKA

$$
\text { RECEIPTS }
$$

TOTAL RECEIPTS

GEGINDING INV

ENDING INY

00701 IN REACTOR-NR

FECEIPTS

TOTAL RECEIPTS

REMOYALS

REY:OVIL

RE:AJVALS

TOTAL REMOVALS

GEGINNING IN

EWDING I:UV
320

CONTRA-ACC PIECES ELE. ELEYT NEIGHT

$\frac{1,393,38}{1,145,33}$

CONTFA-ACC PIJCES ELEMEYT WEIGHT

\begin{tabular}{rr}
49032 & 2.34 \\
& 2.34 \\
\hline
\end{tabular}

$00160 \quad 720.34$

$4,724.50$

724.50
4.006 .50

COATRA-ACC PIECES ELEMENT WEIGHT

ISO-1 NEIGHT

MWD-S

$11-P C T$

$00350 \ldots \ldots \ldots \ldots \ldots \ldots .21$

$39058 \quad 575.21$

$39062 \quad 720.44$

732.71

934,96

777.46

CONTRA-ACC PIECES ELEMENT WEITHT

ISO-1 WEIGHT MWD-S I1-PCT

$\begin{array}{rr}39018 & 8.01 \\ 39027 & 1,017.38\end{array}$

$1,025: 39$

$4,135.95$

$5,161.34$

CONTRA-ACC PIECES ELEMENT WEIGHT

ISO-1 WEIGHT

MWD-S

$11-P C T$

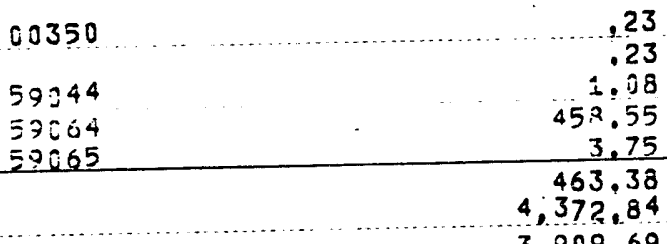

3.909 .69

DUN $5929 A$ 
.

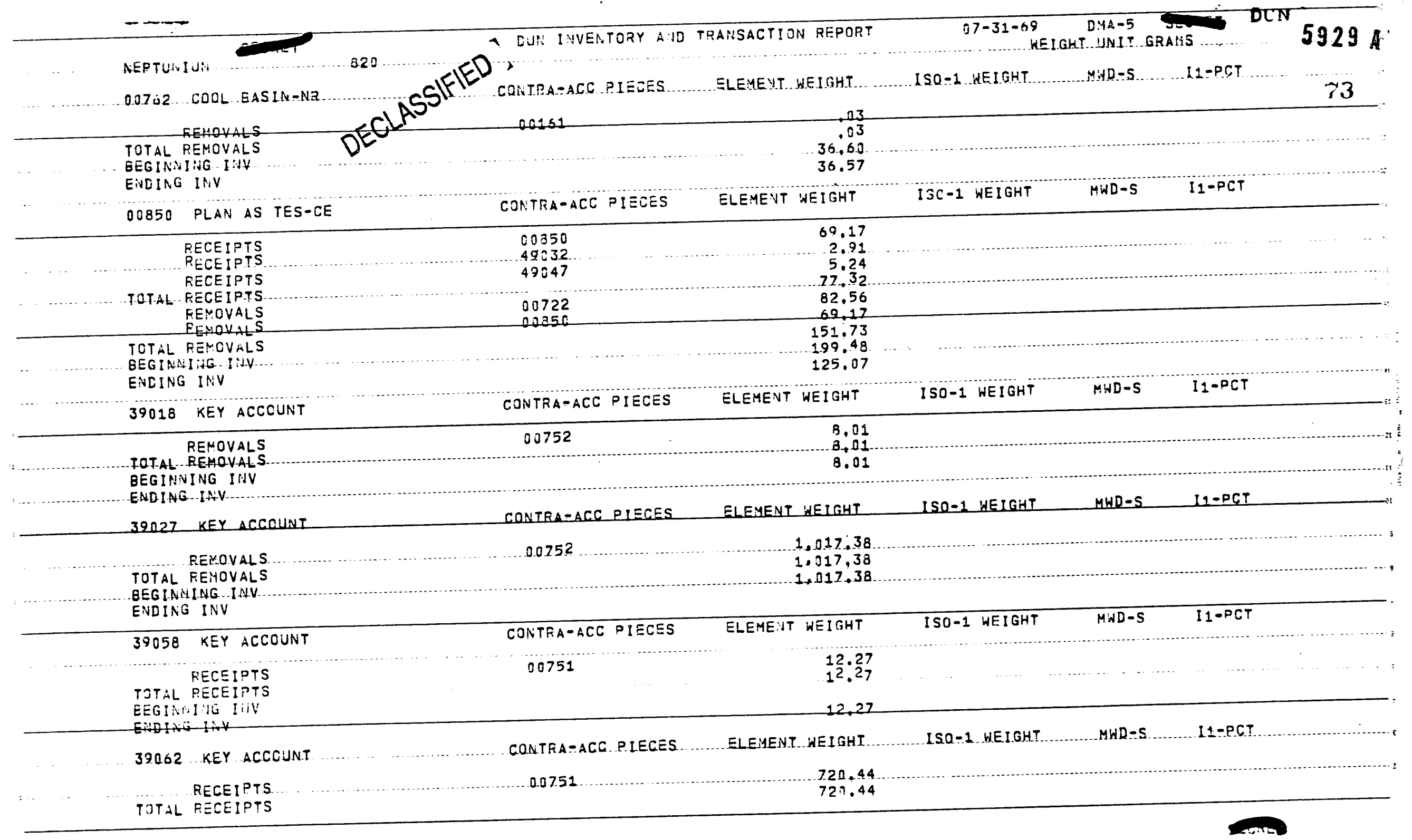




\section{○}

NEPTL:IU:A

MWD-S I1-PCT

39062 KEY ACCCUNT

$72 . .44$

EEGINIIUTG LHY
ENDING IIIV

49032 KEY ACCOUNT

CONTRA-ACC PIECES

ELEMEVT WEIGHT

ISO-1 WEIGHT

MWO-S $\quad$ STCT

$$
\text { REMOVALS }
$$

TOTAL:OVALS

00742

00550

EEGINTIING IIV

ENDING INV

49047 KEY ACCOUNT

CONTRA-ACC PIECES

ELEYENT WEIGHT

2.34

2.21

5.25

\section{RECEIPTS}

REHCYALS

TOTAL RENOVALS

BEGINIV ?ING INV

ENDING LVY

\begin{tabular}{|c|c|}
\hline 30741 & $\begin{array}{l}587.67 \\
587.67\end{array}$ \\
\hline 00050 & $\begin{array}{l}5,24 \\
5,24\end{array}$ \\
\hline
\end{tabular}

53992 KEY ACCCUNT

BEGINNING INV

ENDING INV

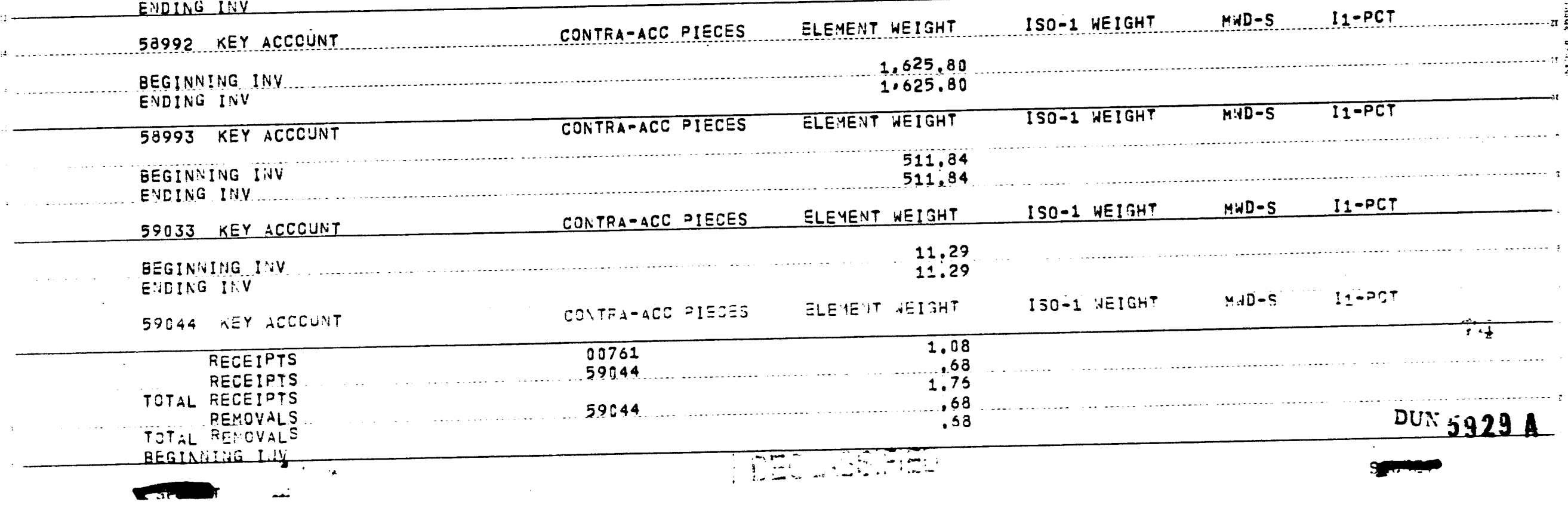




\section{$\bullet$}

$-\cdots$

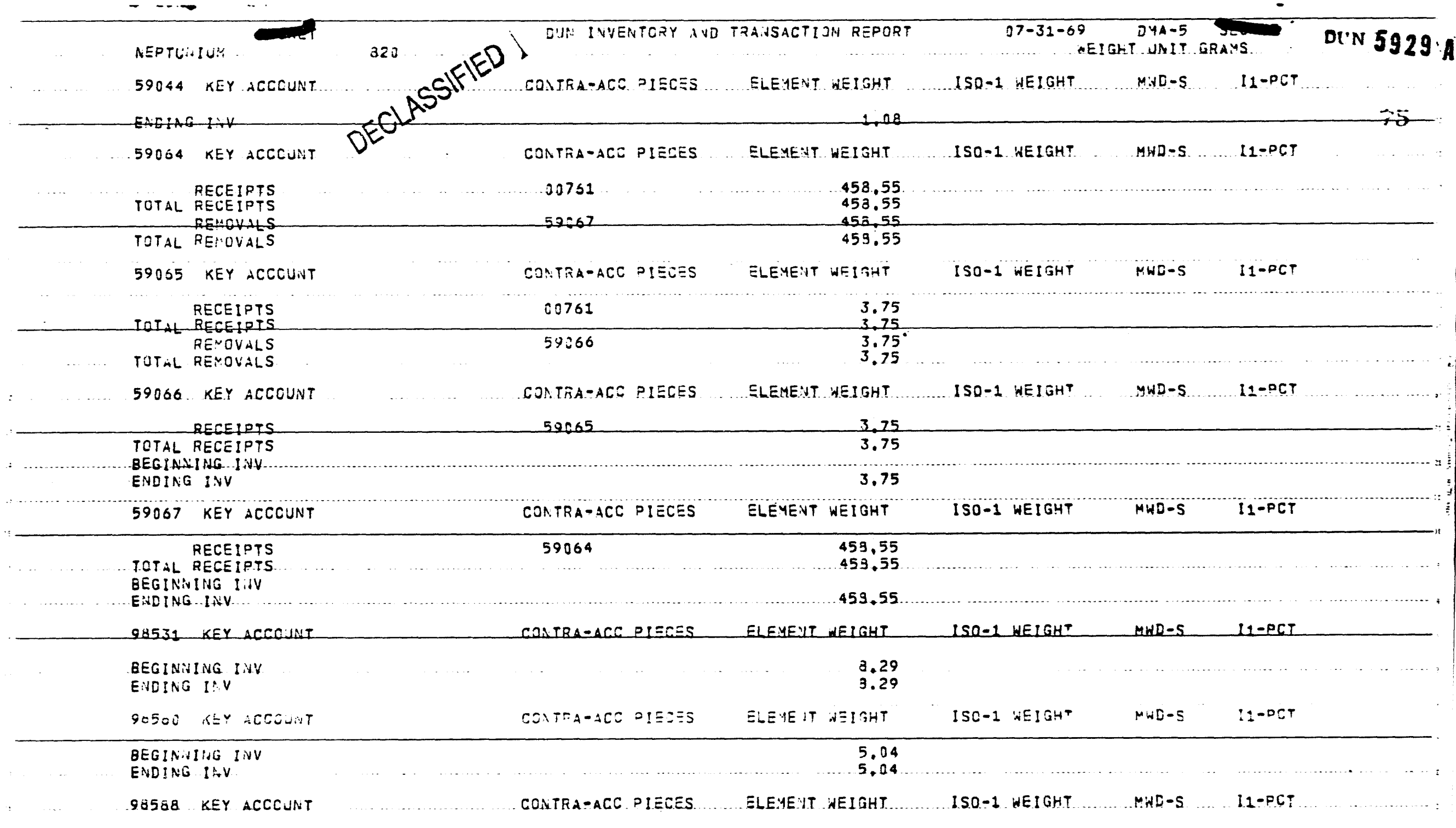

Etoinulito In

ENDING IS.V

$\frac{22.37}{22.97}$ 


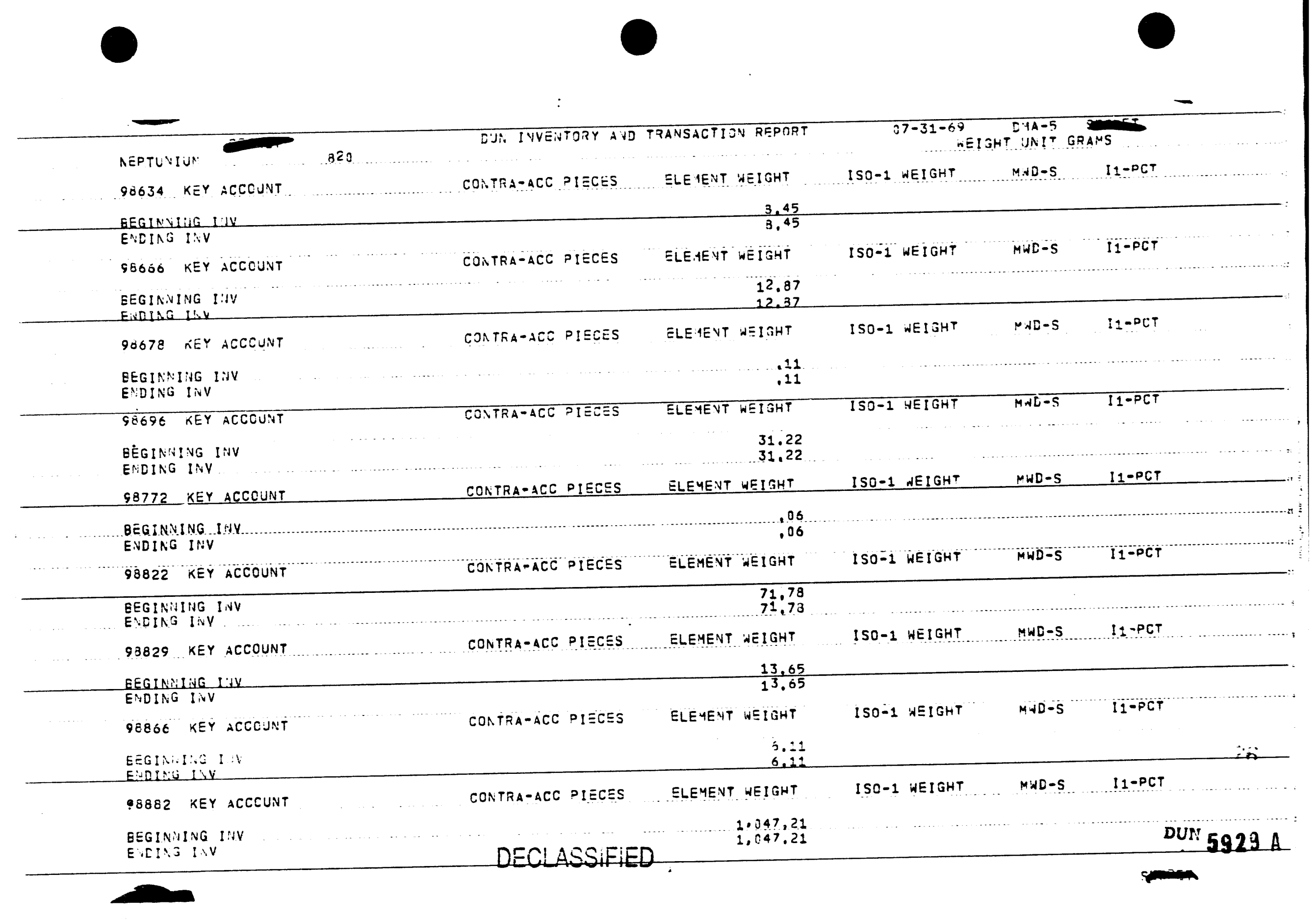




\section{-}

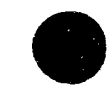

$---$

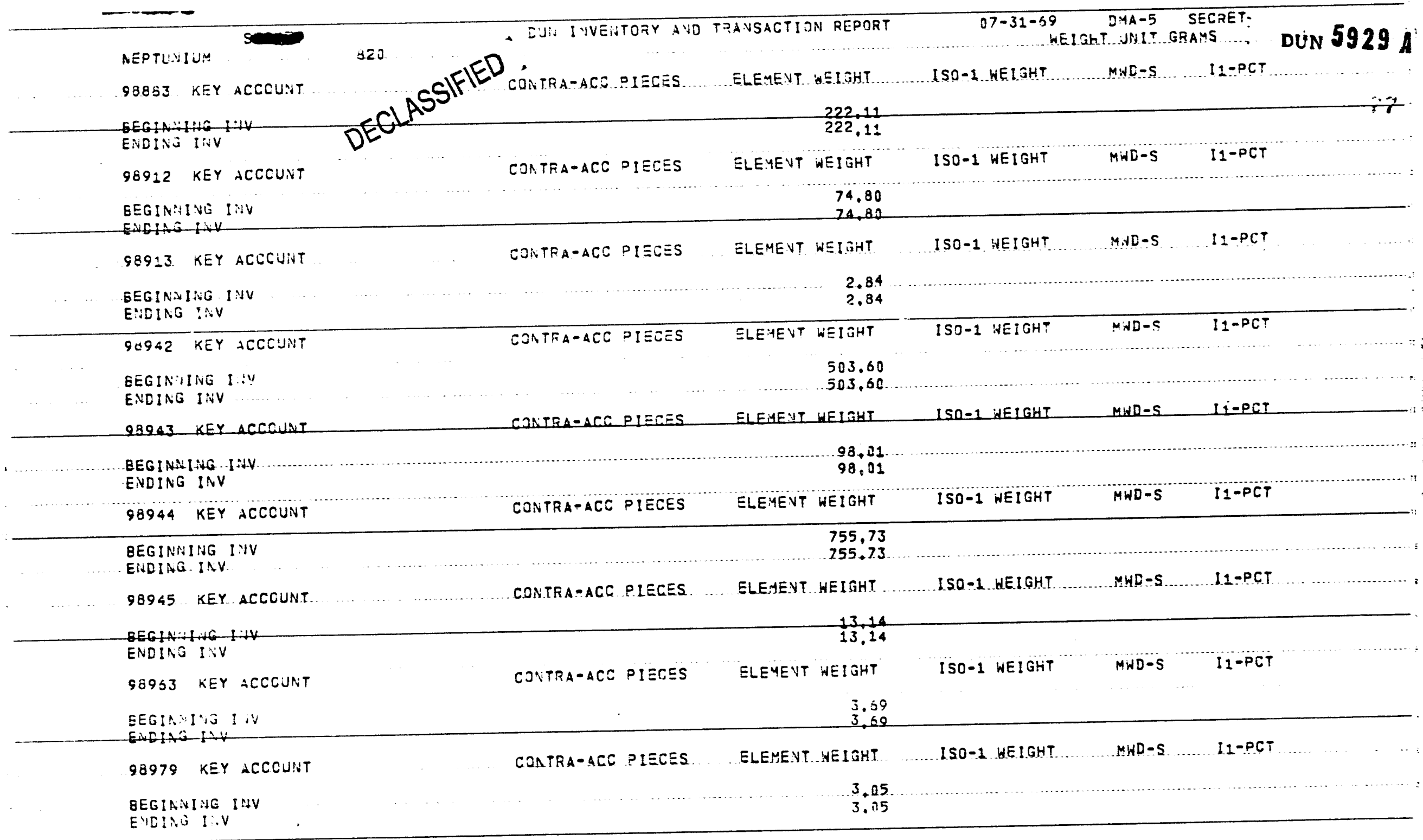




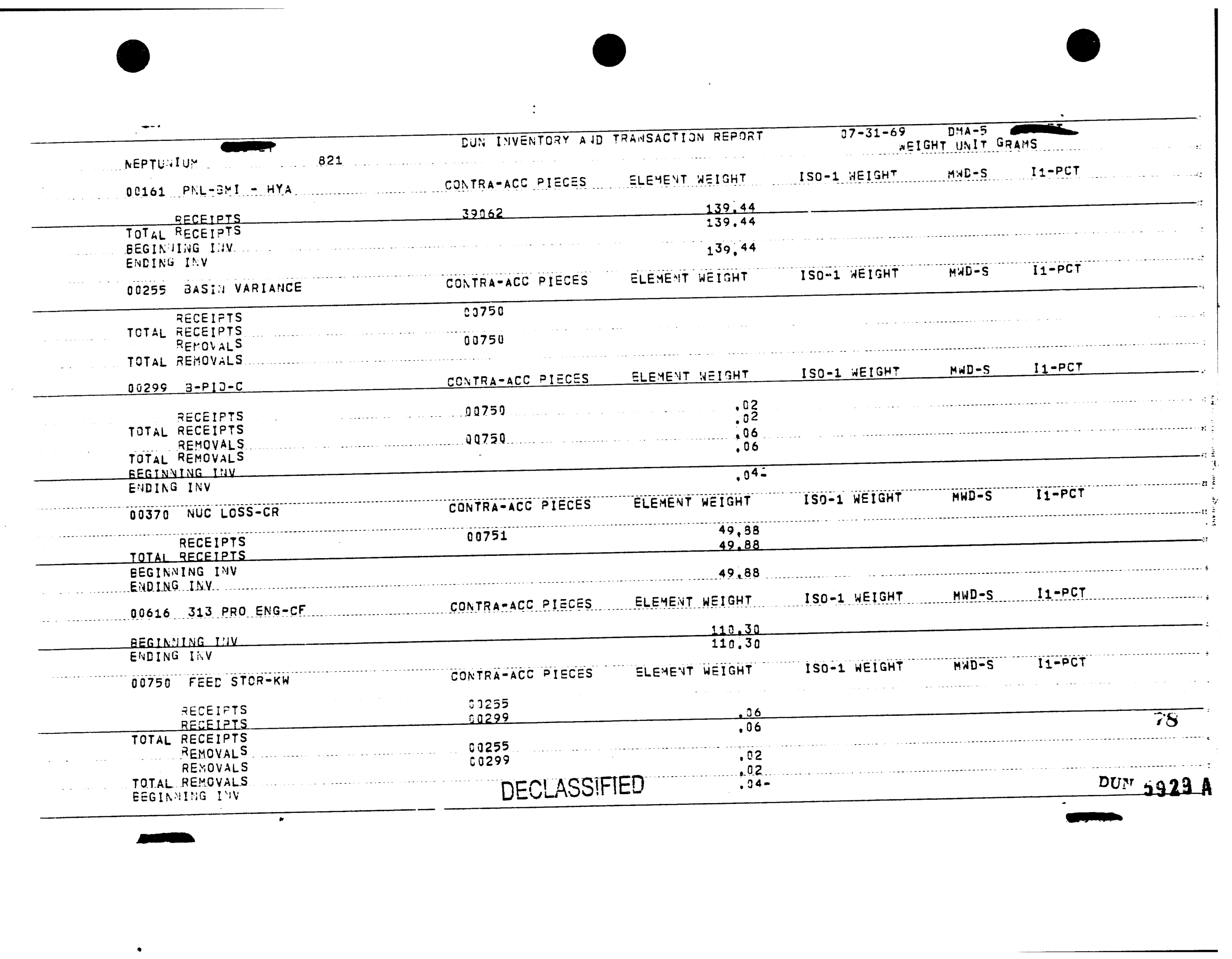


NEPTUNLU
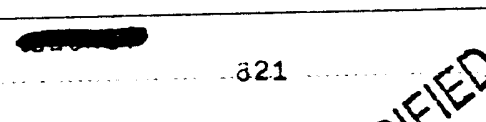
DUM INVENTORY AND TPANSACTION REPORT

OC750 FEEL STCR-KW CONTEA-ACC PIECES ........ELEMEVI WEIGHT

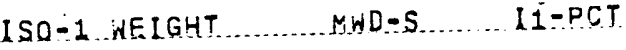

ENDTAE-INU 00751 . Ji REACTOR. Kiv $p^{5}$ ig

REFO:ALS TOT It FEMO:ALS EEGINAI:UG I ENDIA' ISV

39062 KEY ACCCUNT RECEIPTS GECEIPTS

TOTAL RECEIPTS REHOVALS RE, OVALS

TOTAL REMOVALS

EEGIANHUL I IN CONTRA-ACC PIECES .......ELEMEXT HEIGHT

ISO-1, HEIGHT.

MWD $-S \ldots \ldots \ldots 11-P C T$

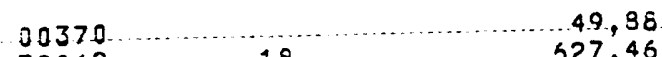

$\begin{array}{lll}00370 & 49,98 \\ 39062 & 18 & 527,46\end{array}$

677.34

$1,054,66$

CONTFA-ACC PIECES ....... ELEMENT WEIGHT

ISQ-1 NEIGHT _...MWD-S _...LI-PCT

$\begin{array}{lcr}0,751 & 13 & 627.46 \\ 39.062 & & 139,44 \\ 03161 & 13 & 765.90 \\ 39062 & 4 & 139.44 \\ & 1 & 139.44 \\ & 2 & 278,88 \\ & 4 & 488,02\end{array}$




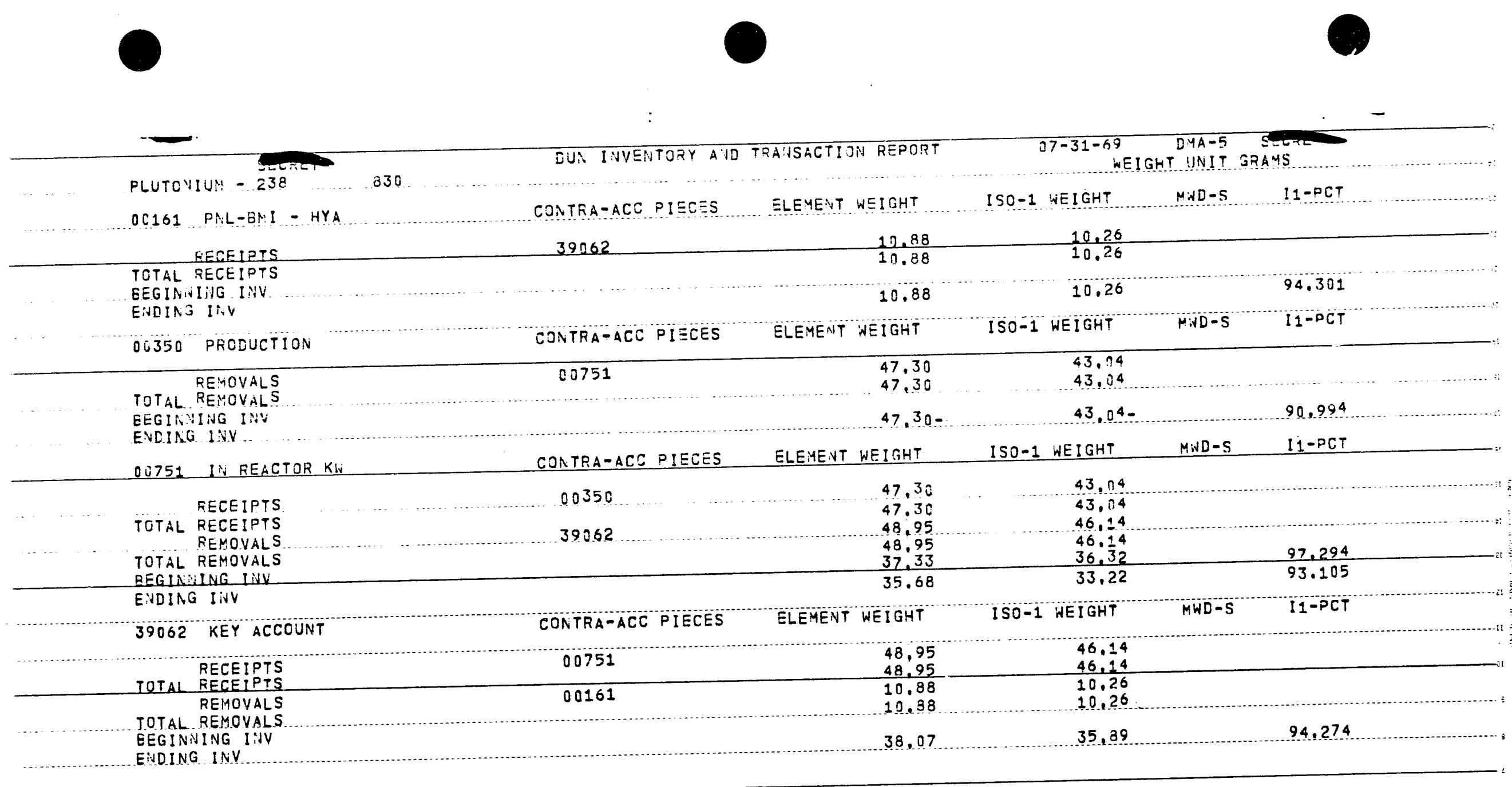




\section{$\bullet$}

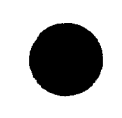

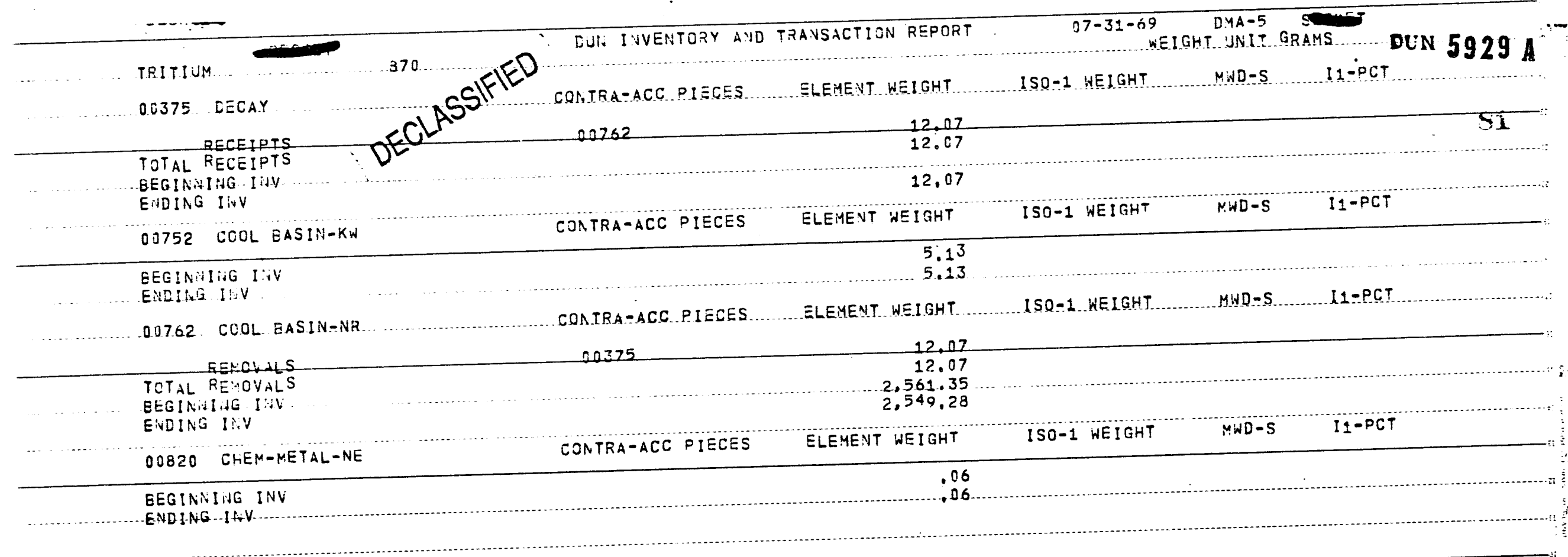


THORIUM

DUN INVEIITORY AVD TRAIISACTION REPORT

07-31-69 DMA-5 WEIGHT UNIT POUNDS

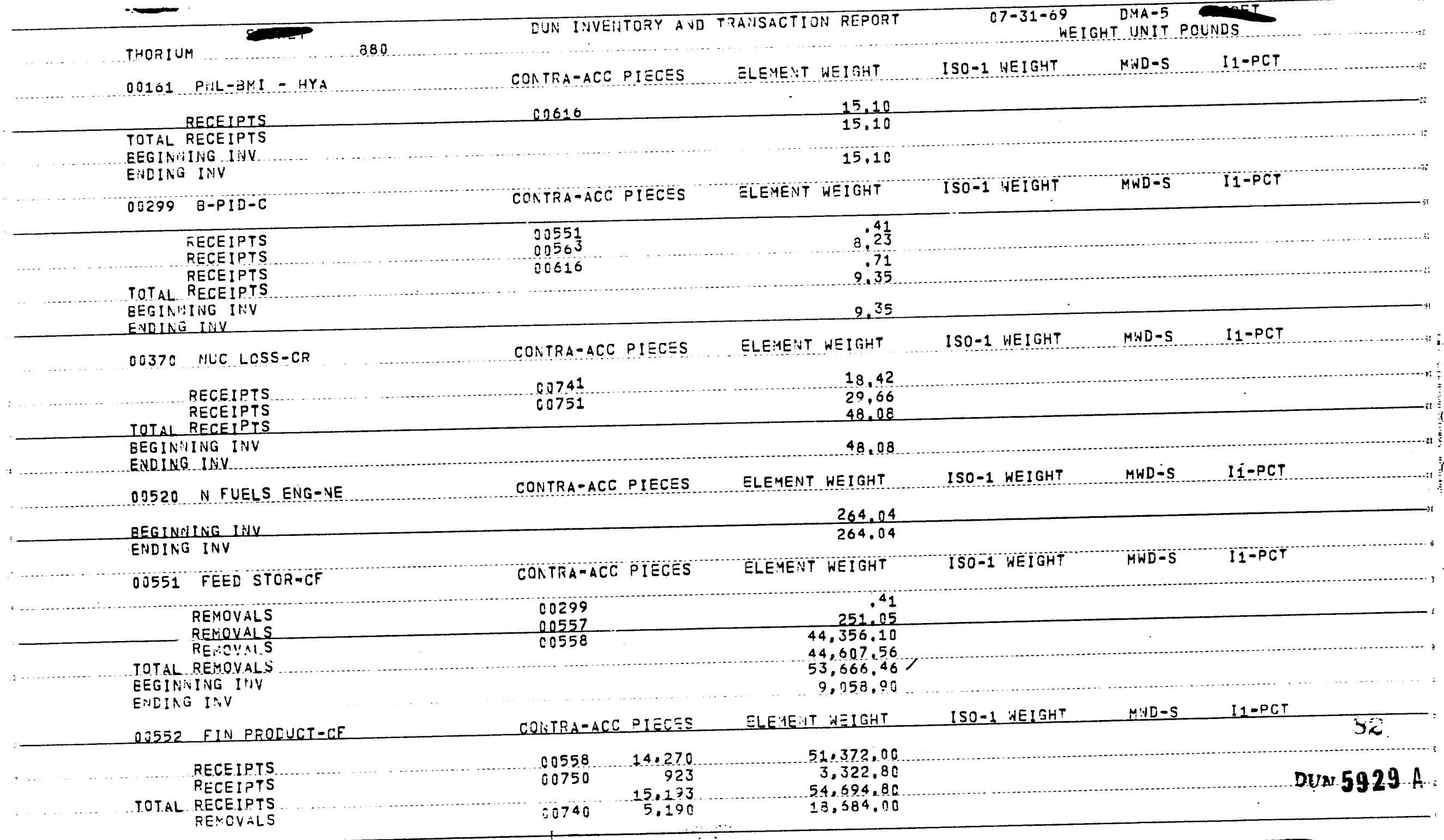




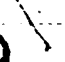

0.0552 FIN PRODUCT-CF कासे CONTRA-ACG PIECES ..... ELEMENT WEIGHT 1SO-1 RELGHT WEIGHT UNIT POLNES

\section{Din $5929 i$}

REYOVALS

TOTIL REIOVALS

EEGIKIAIHG INV

ENDING INV

$\begin{array}{rrr}00750 & 10,381 & 37.371 .60 \\ 15.571 & 56.055 .60 \\ 4.747 & 16.267 .38\end{array}$

$4,747 \ldots 16,267.38$

14.906 .58

00557 OXIDES-CF

CONTRA-ACC PIECES

ELEMENT WEIGHT

ISO-1 WEIGHT

MWD-S II-PCT

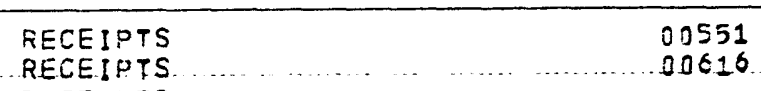

TOTAL RECEIPTS

BEGINII I W I I I V

EVDING IIUY

251.05
00551

293,54

$5 ., 298.42$

$5,591.96$

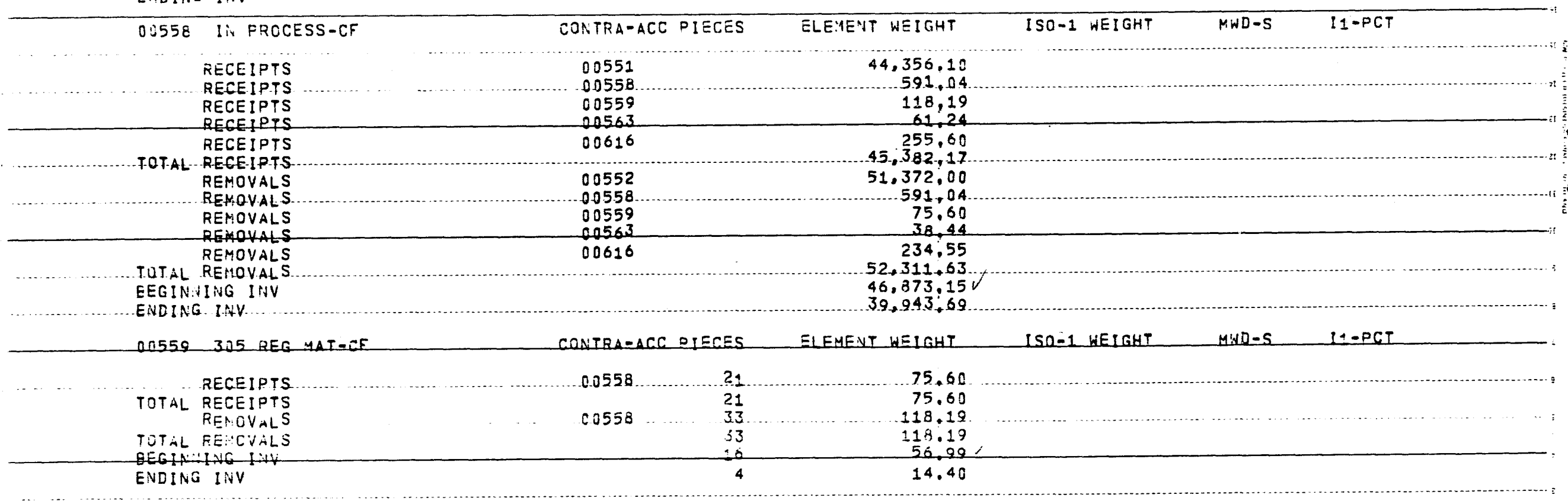


DUi INYENTORY AUD TZANSACTION REPORT

CONTRA-ACC PIECES ELEHEVT WEIGHT 128,94 123.94

EEEINANIUG IUV ENDING INV

130563 ANALYT LAB-CF RECEIPTS

TOTAI RECEIPTS REMOVALS REMCVALS

TOTAL REMOVALS EEGIN!ING IYY. EVDING INV

00616313 PRO ENG-CF RECEIPTS

TOTAL RECEIPTS REYCVALS

REMOVALS REMOVALS

TOTAL REMOVALS

EEGINNING INY

ENDING IMV

00712 COOL BASIN-B

EEGINNIING INV

ENDING INIV

00722 COOL EASIN-C

\section{RECEIPTS}

TOTAL RECEIPTS

EEGIN!INO INN

E LI:U I\%V

00732 COOL BASIN-D

REMOVALS

TOTAL RETOVALS

EEGINYING !'IV

\section{0}

$\frac{\text { CONTPA-ACC PIECES }}{00550}$

ONTPA-ACC PIECES

ELEIIENT WEIGHT

38.44

$\frac{38.44}{6.23}$

61.24

120.15

89.12

CONTRA-ACC DIECES ELEHEVT WEIGHT

234.55

00558
0.5151 $\quad \begin{array}{r}234.55 \\ 234.55 \\ 15.10\end{array}$

00151

00299

00557

42.49

42.49
255.60

313,90

$71 \ldots .038 .81$
959.46

$71 \ldots .038 .81$
959.46

CONTRA-ACC PIECES ELEMENT NEIGHT

$14,492 \quad 35,732,55$

$14,492 \quad 35.732 .55$

CONTRA-ACC PIECES ELEMENT WEIGHT

8.0732

9,364
9,304
16,100
$75, \div 64$

$25,377,87$

25,377

45.314 .27
75.592 .14

CON TRA-ACC PIECES

$25,377.87$

00722

9.364
9.354

9.354
9,376

$25,377.87$
$25,405.25$
ISO-1 NEIGHT

ELE.MENT WHEIGHT
MWD-S

$11-P C T$

WEIGHT UNIT POUNDS

ISO-1 NEIGHT MWD-S I1-PCT

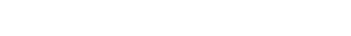

O-1 WEIGHT MWD-S II-PCT

DECLASSIFIED MUD-S I1-PCT




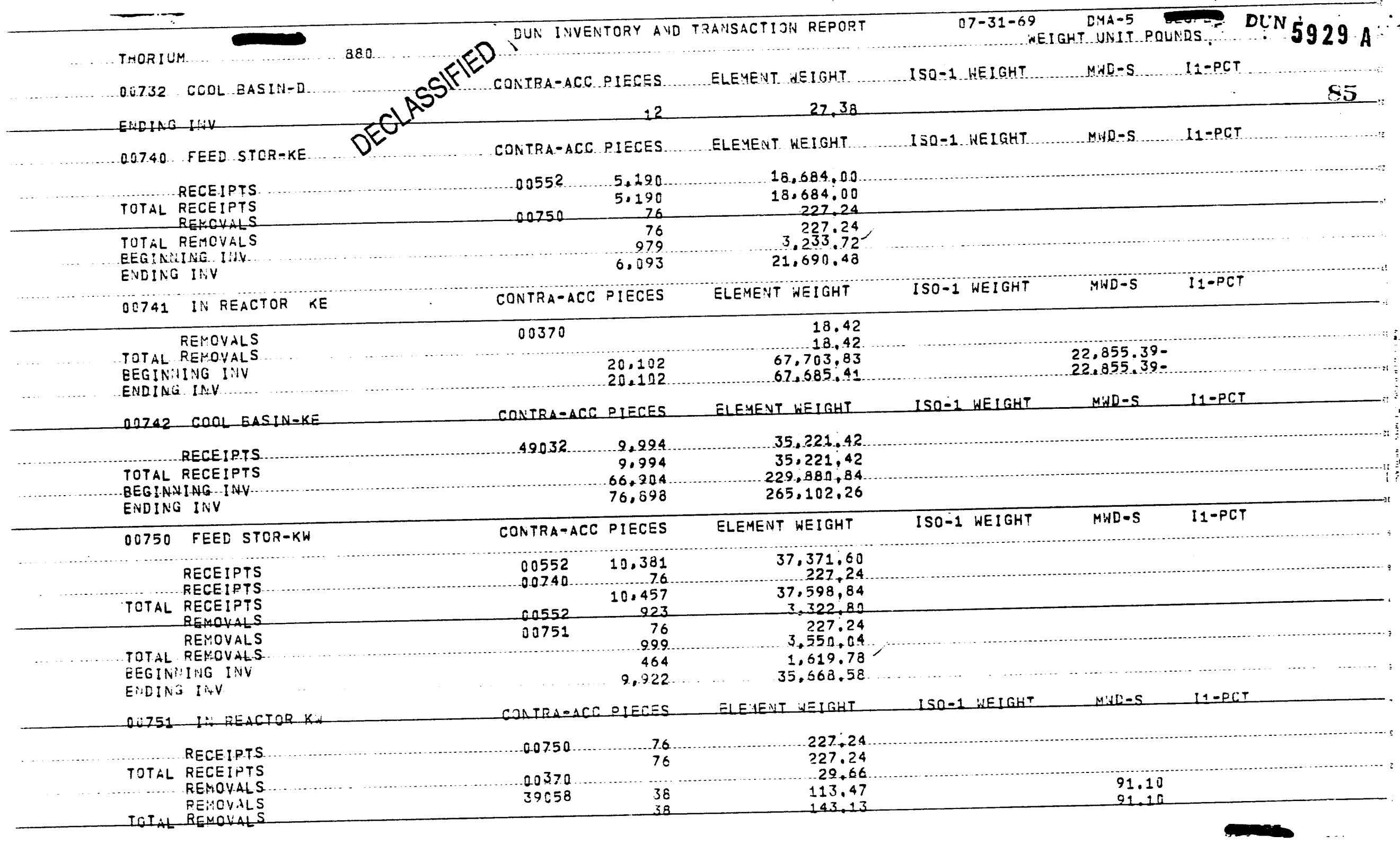




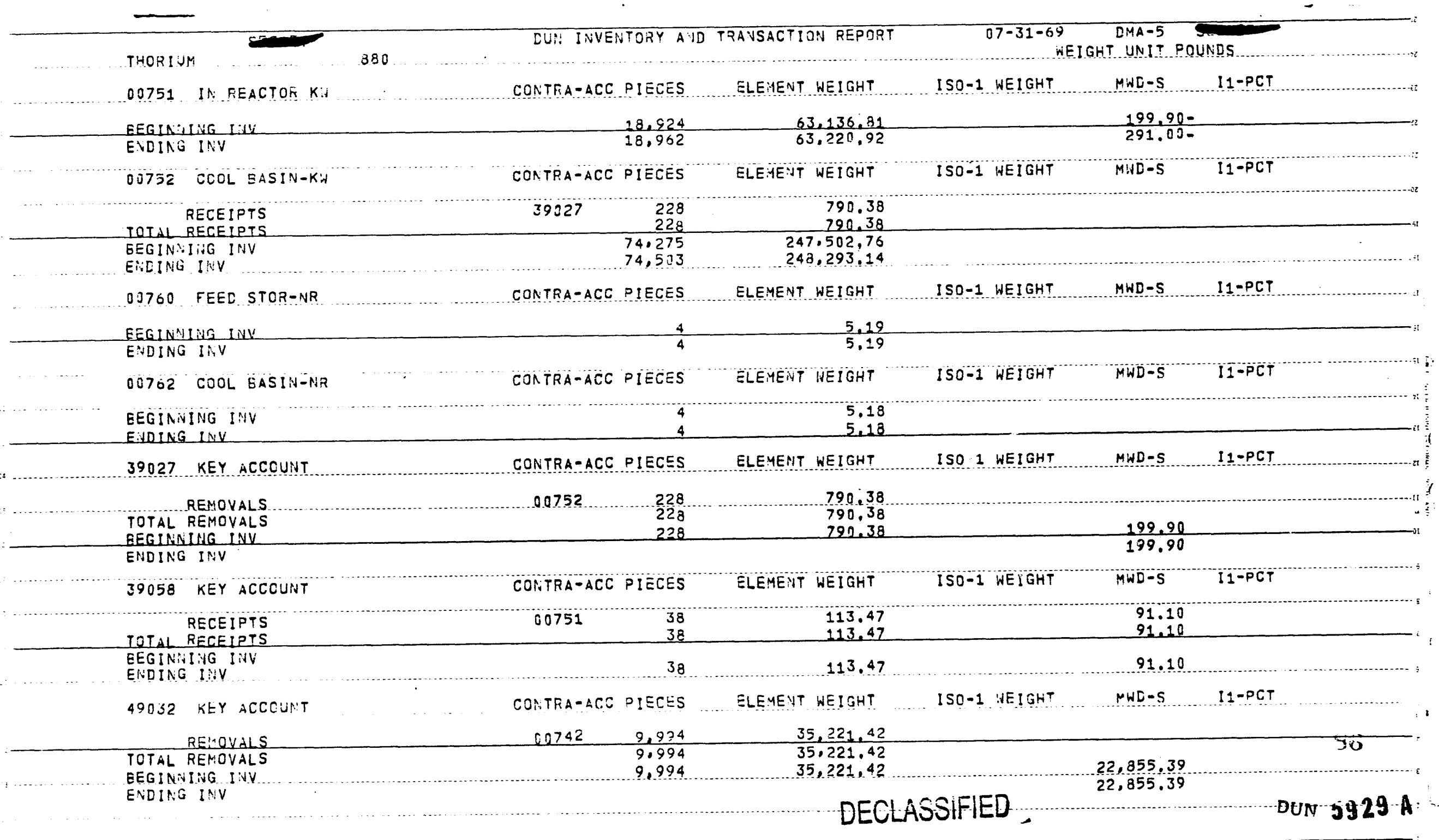




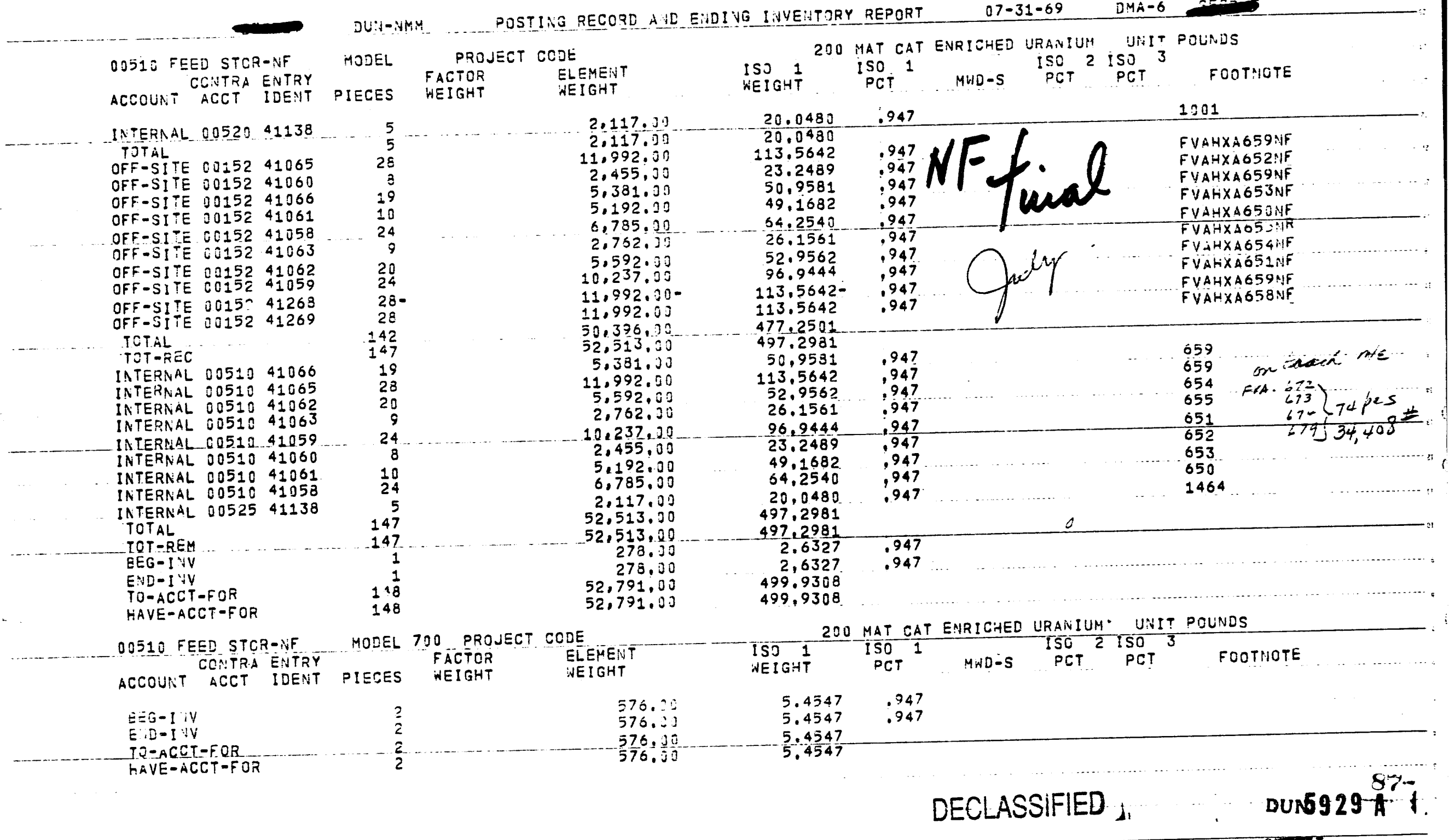




\section{…-}

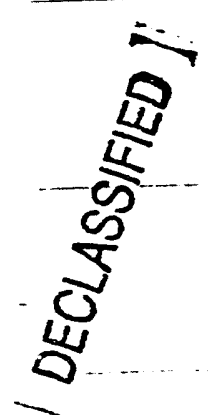

00510 FEED STCR-IAF CONTTA E:VTRY

EEG $=1: \mathrm{iV}$

E:ID-IJIV

TO-ACCT-FOR

HAVE-ACCT-FOR

00510 FEEI STOR-VF

ACCOUIT CONTSA ENTRY

$B E G-j I V$

EAD - I:V

TO-ACCT-FCR

HAVE-ACCT-FOR

O0510 FEED STOR-INF CONTRA ENTRY

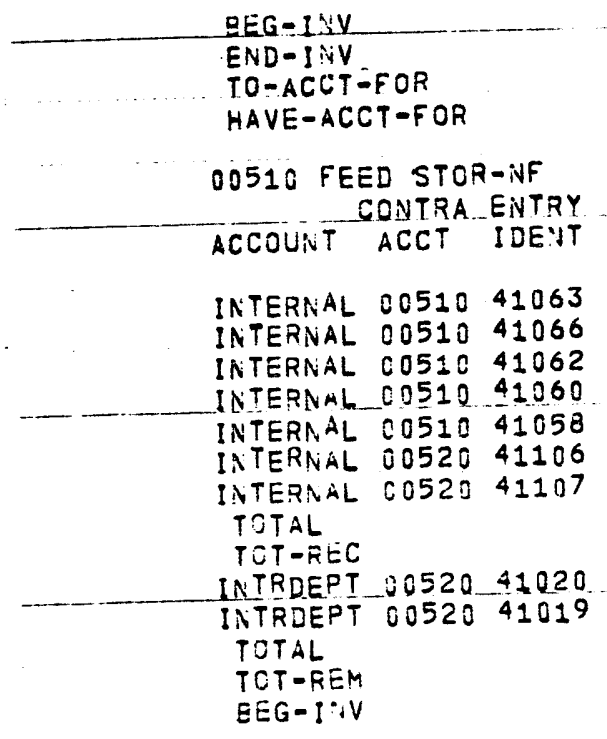

UWV-UMY POST LIVG RECCRD AVE EVDIVG INVENTTORY REPORT

$07-31-69$

DMA-6

200 MAT CAT ENRICHED URANIUM UNIT POUNDS

YODEL 701 PROJECT CODE FACTOR ELEMENT

PIECES WEIGHT WEIGHT

ISO 1

ISO 2 ISO

FOOTHOTE

$\begin{array}{lll}250.02 & 2.4622 & .947 \\ 260.00 & 2.4622 & .947 \\ 260.00 & 2.4622 & \\ 260.07 & 2.4622\end{array}$

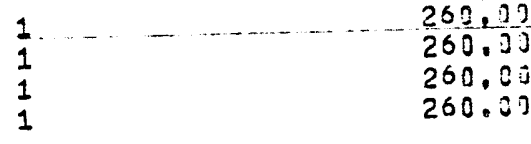

HODEL 702 PROJECT COJE

200 MAT CAT ENRICHED URARIUM UNIT POUNES

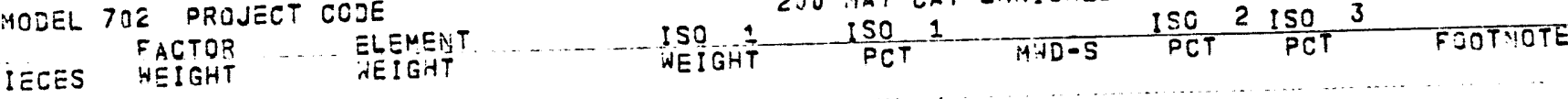

43
43
43
43

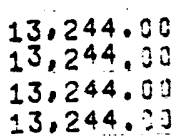

MODEL $7: 7$ PROJECT CODE

FACTOR
PIECES WEIGHT NEMENT

\subsection{1}

$94749 \div \div-2$

125.4191

125.4191

$3.244 .23 \ldots \ldots \ldots 125.419$

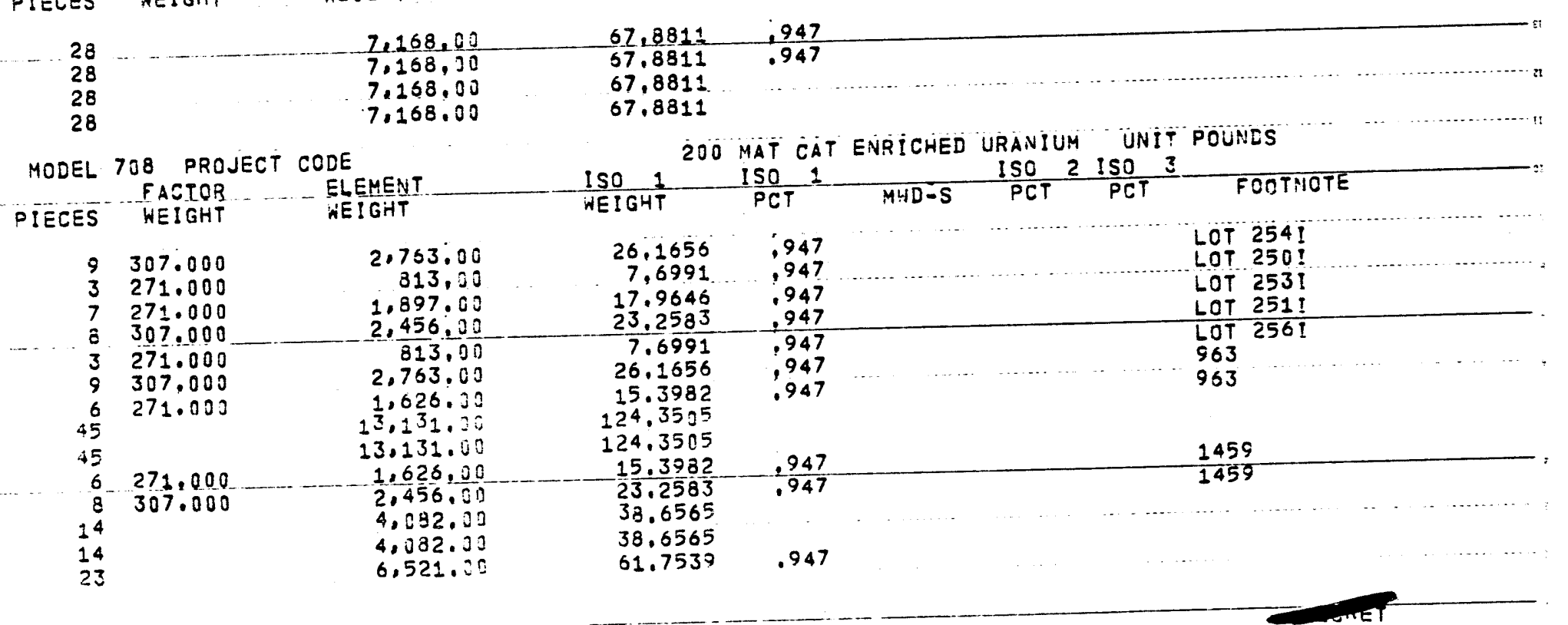


$07-31-69$

DMA-6

OOS10 FEED STCR-NF

HODEL 708 PROJECT CCDE

OOSIO FEEL STCR-NFT CCNTRA ENTRY

ACCOUNT ACCT IDENT

$\begin{array}{ll} & \text { FACTOR } \\ \text { PIECES WEIGHT } & \text { NEIGHENT }\end{array}$

230 MAT CAT ENRICHED URAIVIUH UNIT POUNDS

- TOD-ACCT-FOF

TO-ACCT - FOF
HAVE-ACCT $F O R$

OO510 FEED STOR-NF

CONTRA EVTRY

ACCOUAT ACCT IDENT

54
$6 \mathrm{a}$

INTERNAL 0020341174

1041066

INTERNAL 0051241058

INTERNAL OO510 41062

INTERAAL 00511.41145

INTERNAL OO520 41106

TOTAL

TOT-REC

INTERNAL 0020311123

INTERNAL 0020341123

INTERNAL O0511 41077

INTERNAL O0511 41018

INTERNAL OO51I 41040

INTERNAL

TOTAL 0052041019

INTRDEPT OO520 41021

TOTAL

TOT-REM

EEG-INV

EIID-INV

TO-ACCT -FOR

HAVE-ACCT-FOR

O0510 FEED STOR-NF

CCNTIAA EITRY

58

$15.570,00$
19.652 .00

$19,652.00$

MODEL 799 PROJECT CODE

$\begin{array}{ll} & \text { FACTOR } \\ \text { PIECES WEIGHT ELEENT }\end{array}$

ACCOUNT ACCT IDEVT

INTERNAL C0511 41009

INTERNAL 0051141010

TOTAL

INTERNAL DO511 $41 \pm 46$

TUTAL

$\begin{array}{rr}16 & 286.000 \\ 21 & 286.000 \\ 13 & 286.000 \\ 11 & 286.000 \\ 24 & 286.000 \\ 1 & \\ 86 & \\ 86 & \\ & \\ 25 & 286.000 \\ 52 & 286.000 \\ 41 & 286.000 \\ 118 & \\ 24 & 286.000 \\ 6 & 286.000 \\ 30 & \\ 148 & \\ 402 & \\ 346 & \\ 494 & \\ 494 & \end{array}$

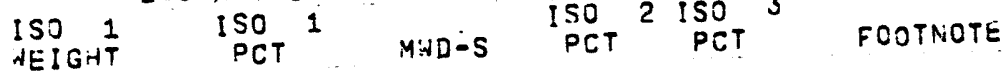

$\frac{147.4479}{136,1044}$

136,1044

.947

200 MAT CAT EVRICHED URANIUM UNIT POUNDS

NEIGHT PCI 1 MND-S PCT PCT FOOTMOTE

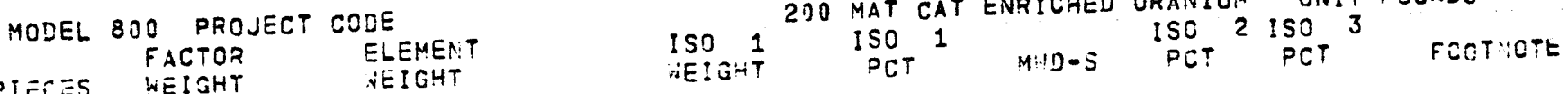

2.00
4.576 .00

6.036 .00

$3,718.00$

3.146 .03

238.0

$24,600.00$

$24.600,00$

2.00

$7,150.00$

$14,372.00$

14.372 .00
$11,726.00$

$11,726.00$

$33,752,00$
$6,864,00$

$1,716,0$

8.530 .00

$42,332.00$

$116,688,00$

98.956 .00

141.238 .03

141.288 .00

EIG

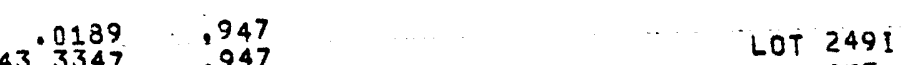

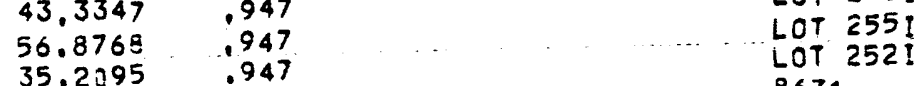

$29.7926 \ldots .947$

$9.0021-947$

8631

2,7274

232,9620

232.9620

947

1000

.0189

67.7105

140.8378

310.6313

65,0021

65.0021
16,2505

31.2526

400.8839

1.105 .0354

937,1135

1.337 .9974

ISO 1 ISO 1 MUT

$\begin{array}{ll}947 & \text { NT DIF } \\ 947 & \text { NFE } 1000 \\ 947 & 1462\end{array}$

.947
947
947

.947

, 947

.947

$30.4734 \quad 947 \quad 947859$

$\$ 9$

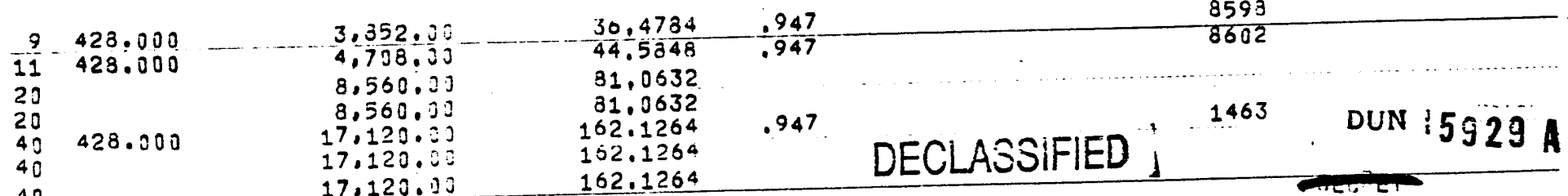


$17-31-69$

2.4A-6

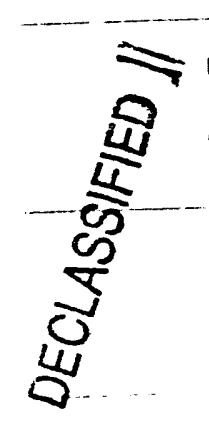
MODEL 300 PROJECT CODE

0510 FEEC STCR-AHF PIECES HEIGHT WEIGHT ISO 1 ISO

JNIT POLNSS $E E G-I D Y$

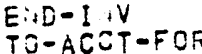

HAYE-ACCT-FOR

DOS10 FEEE STOR-INF

COUTRA ENTRY ACCOUNT ACCT IJE:T

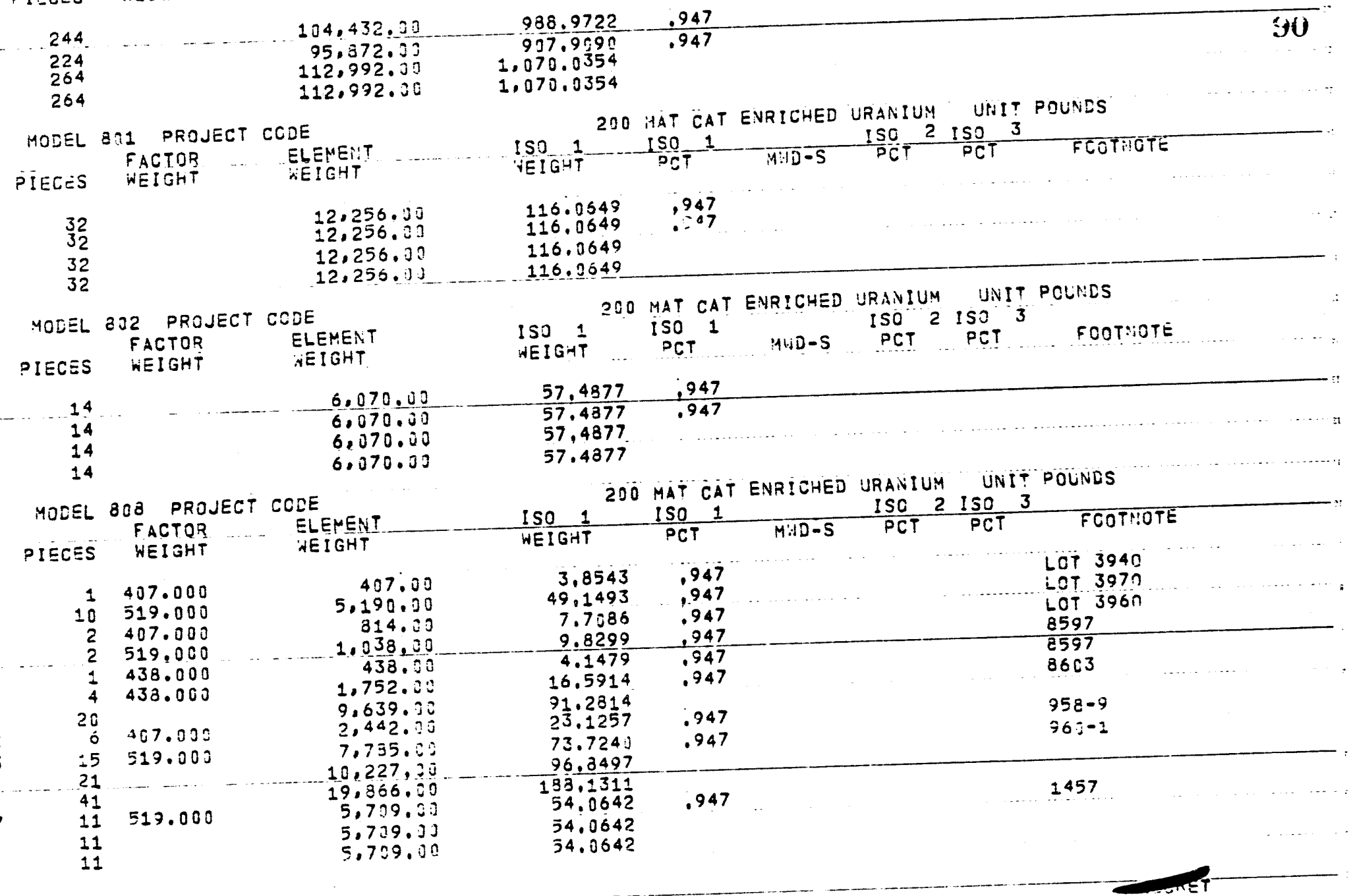


7-31-69 D44-6

DUY

POSTIIG RECORD AID ENDIVIG INVENTORY TEPORT

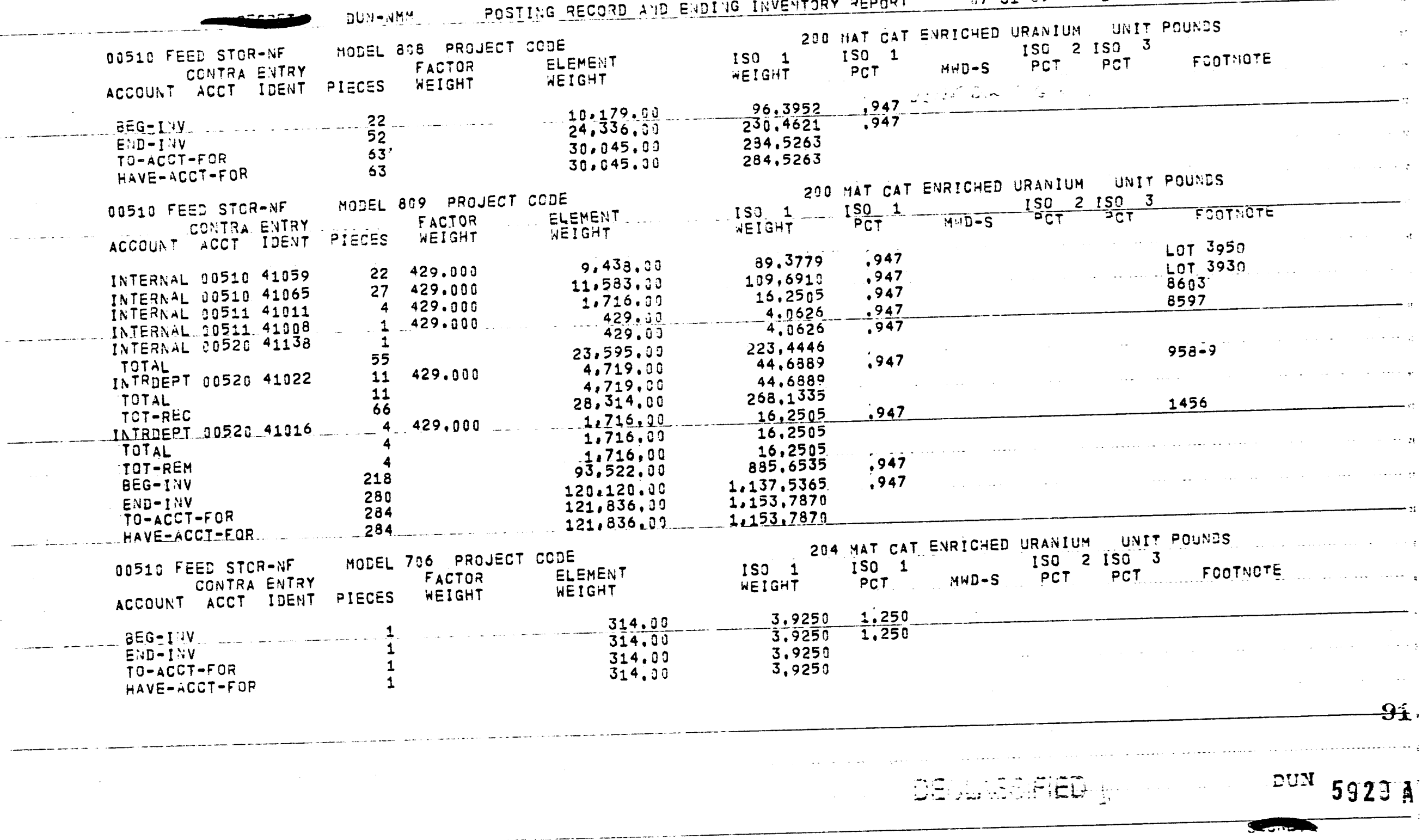




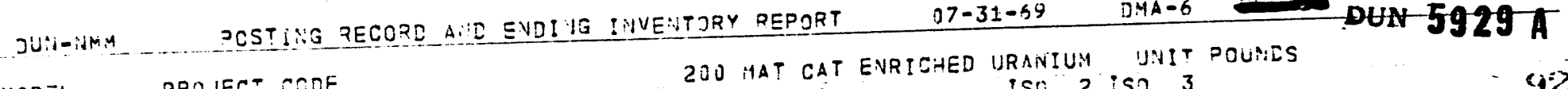

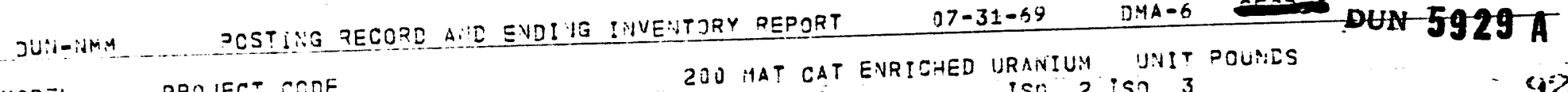

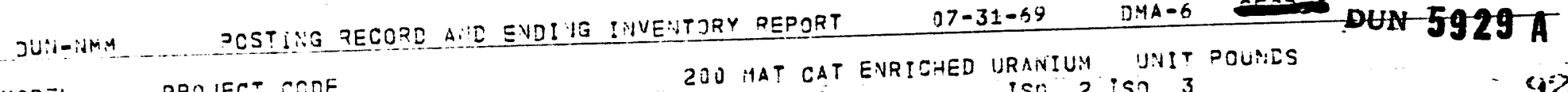

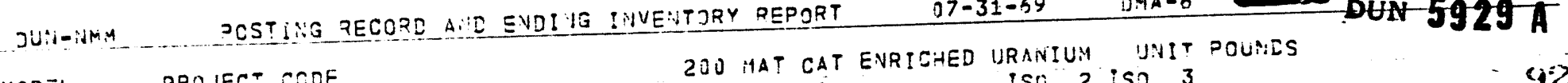

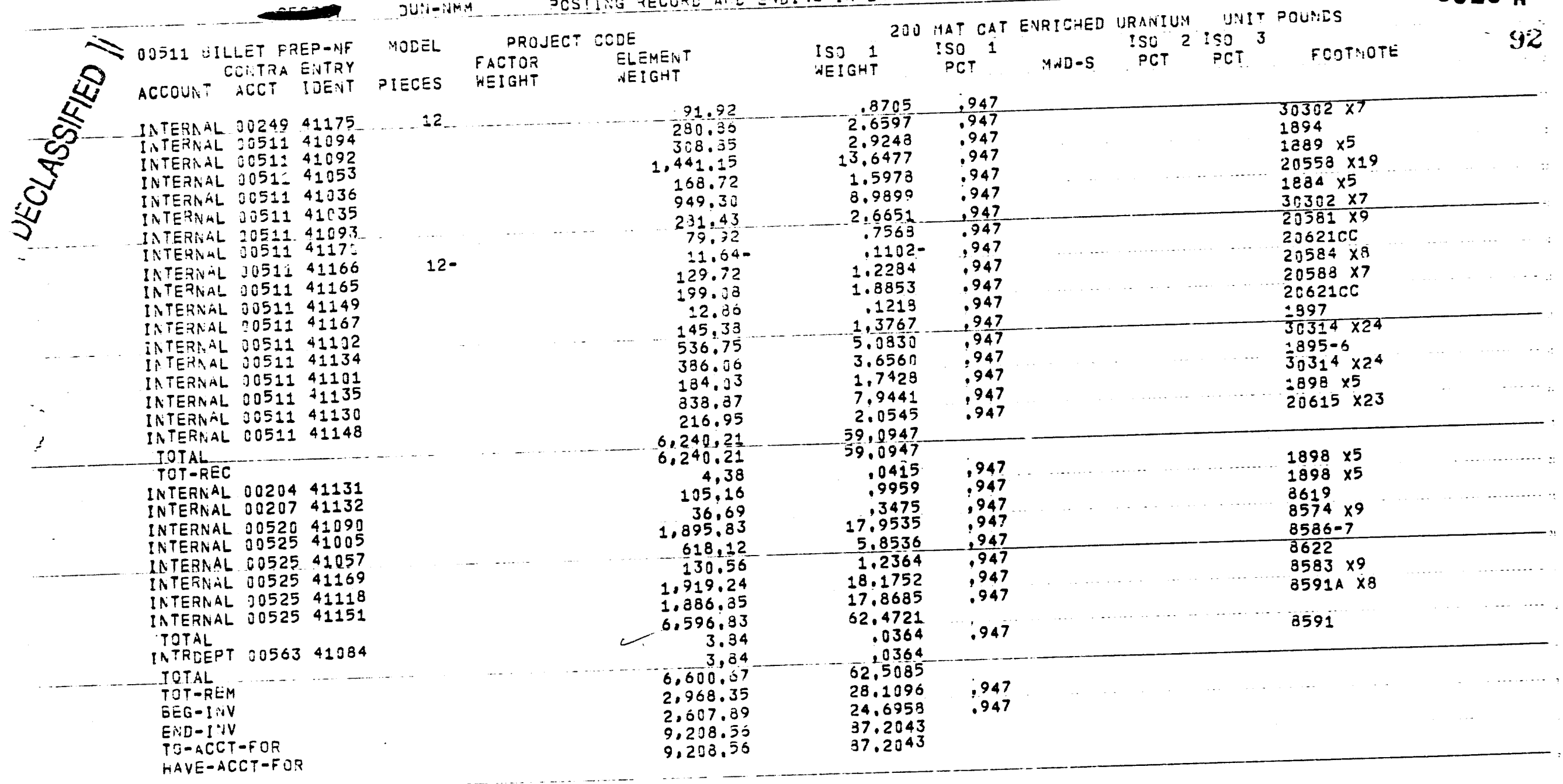




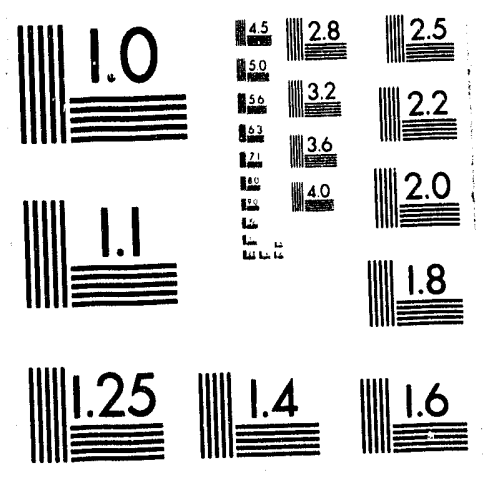



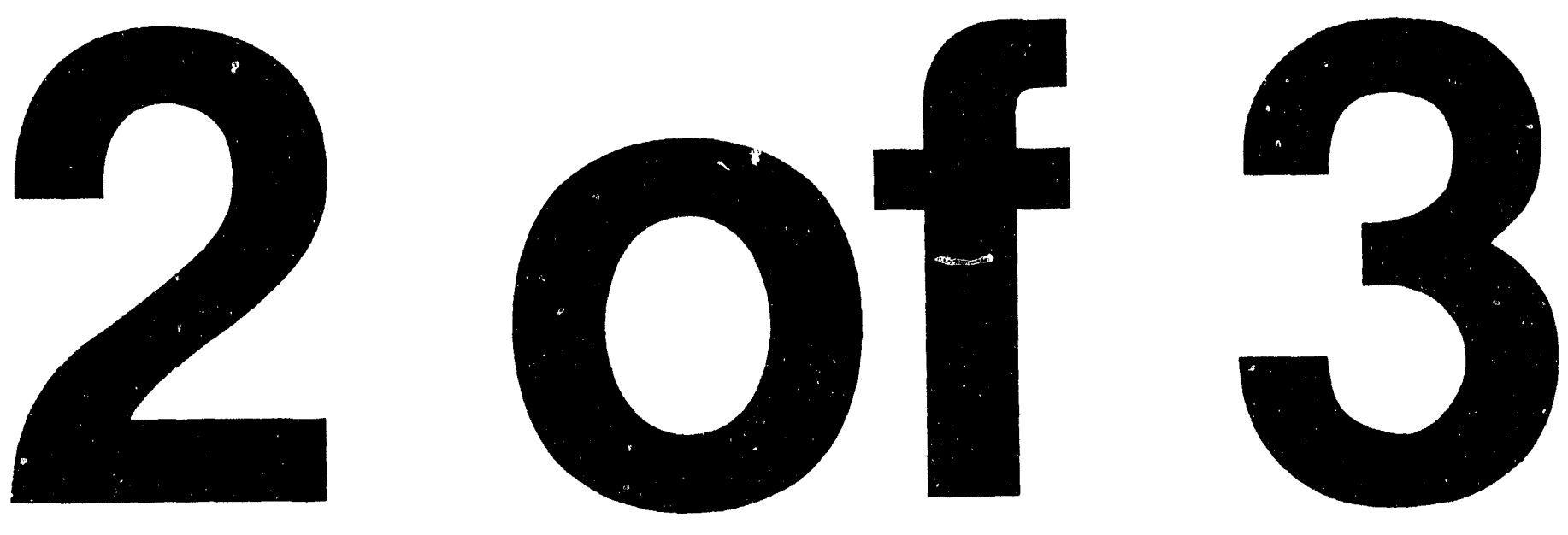
DUR-iVMA POSTING RECORD AND ENDIVG INVENTORY REPORT 07-31-59 DMA-6

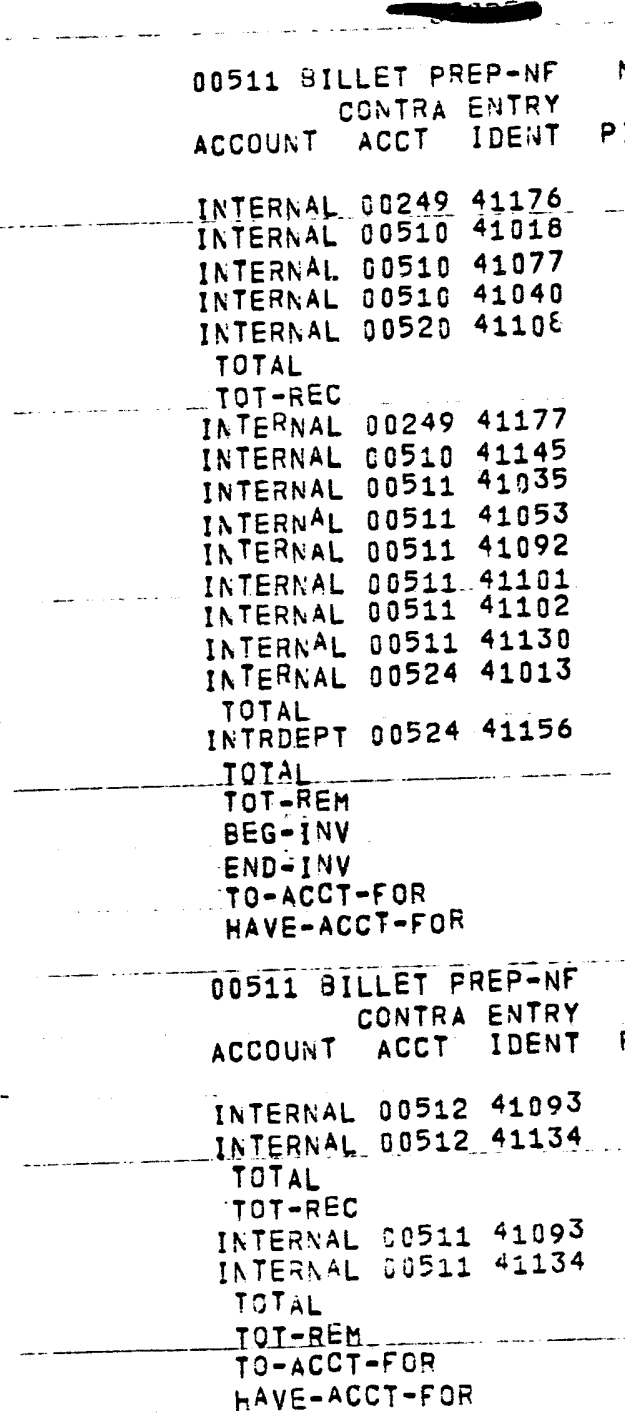

MODEL 7 IO PROJECT CODE $\begin{array}{ll} & \text { FACTOR } \\ \text { PIECES HEIGHT } & \text { WEIGHTH }\end{array}$ 200 MAT CAT ENRICHED URANIUM UNIT POUHDS

ISO 1 ISO 1 MIND-S $\begin{aligned} & \text { ISO } 2 \text { ISO } 3 \\ & \text { PCT }\end{aligned}$ FOOTNOTE DEHT NEIGHT

$\begin{array}{rrr}52 & 286.000 & 1 \\ 25 & 286.000 & \\ 41 & 286.000 & 1 \\ 5 & 286.000 & \\ 123 & & 35\end{array}$

$14,872,02$ $11,726,00$ 1.430 .00 $35,178,02$ $35,178,02$ 123

TOT-REC

INTERNAL 0024941177 INTERNAL C0510 41145 INTERNAL OO511 41335 051141092 NTERNAL 00511.41101 INTERNAL 00524 41013 INTRDEPT O0524 41156 TOT-REM

TOT-REM

O051I BILLET FREP-NF OOS11 BILLET PREPANT ENTRY ACCOUNT ACCT IDENT

$\begin{array}{ll}11 & 286.000 \\ 48 & 286.000\end{array}$

$72 \quad 286.000$

$16 \quad 286,000$

$20 \quad 286.000$

286,000

41286.000

$21 \frac{1}{2} 286,000$

219
96

219
219

3.178 .02
3.146 .02

$13,728,00$

$20,592,00$

$4,576.00$

$5,720,00$

2.238 .00

$11,726,00$
286,00

$62,062,02$

572,00

572,00

$62,634,02$

150

$140.0002 \quad 1,000+1947-1458$

$\begin{array}{lll}67.7105 & 947 & 1462\end{array}$

$\begin{array}{lll}111.0452 & , 947 & 9461\end{array}$

MODEL 720 PROJEC̄T CODE

$62,634,02$

111,0452

1461
962

333.1358

$\begin{array}{rrr}333,1358 & & 150 \\ 29,7004 & 2,000 & 863\end{array}$

130.0042

$\begin{array}{rr}195,0062 & .947 \\ 43,3347 & 947\end{array}$

$.947 \ldots . . .1894 \times$

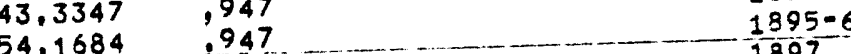

$21,6674,947 \ldots 97$

$\begin{array}{llll}111.0452 & 947 & 94 \ldots \ldots \ldots & 1398 \times \\ 1607-8\end{array}$

2,7084

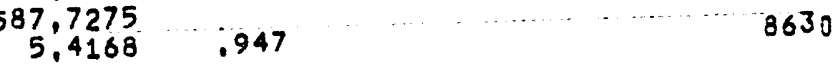

MODEL 720 PROJECT CODE
FACTOR
HELEMENT

5,4168

593,1443

260.0085

IECES WEIGHT

733,50
$4,564,00$

4516.300

16.300

$4,564,00$
$5,297,50$

$5,297,50$

5.297 .50
733,50

4.554 .00

5.297 .50

$5.297,50$
5.297 .50

$5,297,50$

593,1443
593,1443

593.1443

325
45

280
325

325
325

325

200 MAT CAT ENRICHED URANIUM UNTT POUNDS

ISO 1
WEIGHT PCT $^{1}$ MHD:S ${ }_{\text {PCT }}{ }_{\text {PCT }}$ FOOTNOTE

$6.9462 \quad 047 \quad 30302 \times 7$

$93.947 \quad 30314 \times 24$

$53.2211 \ldots .947$

$50.1673 \quad 303$ ?2 $\times 7$

$43.2211 \quad .947$

$30314 \times 24$

50.1673

50.1673

50.1673 


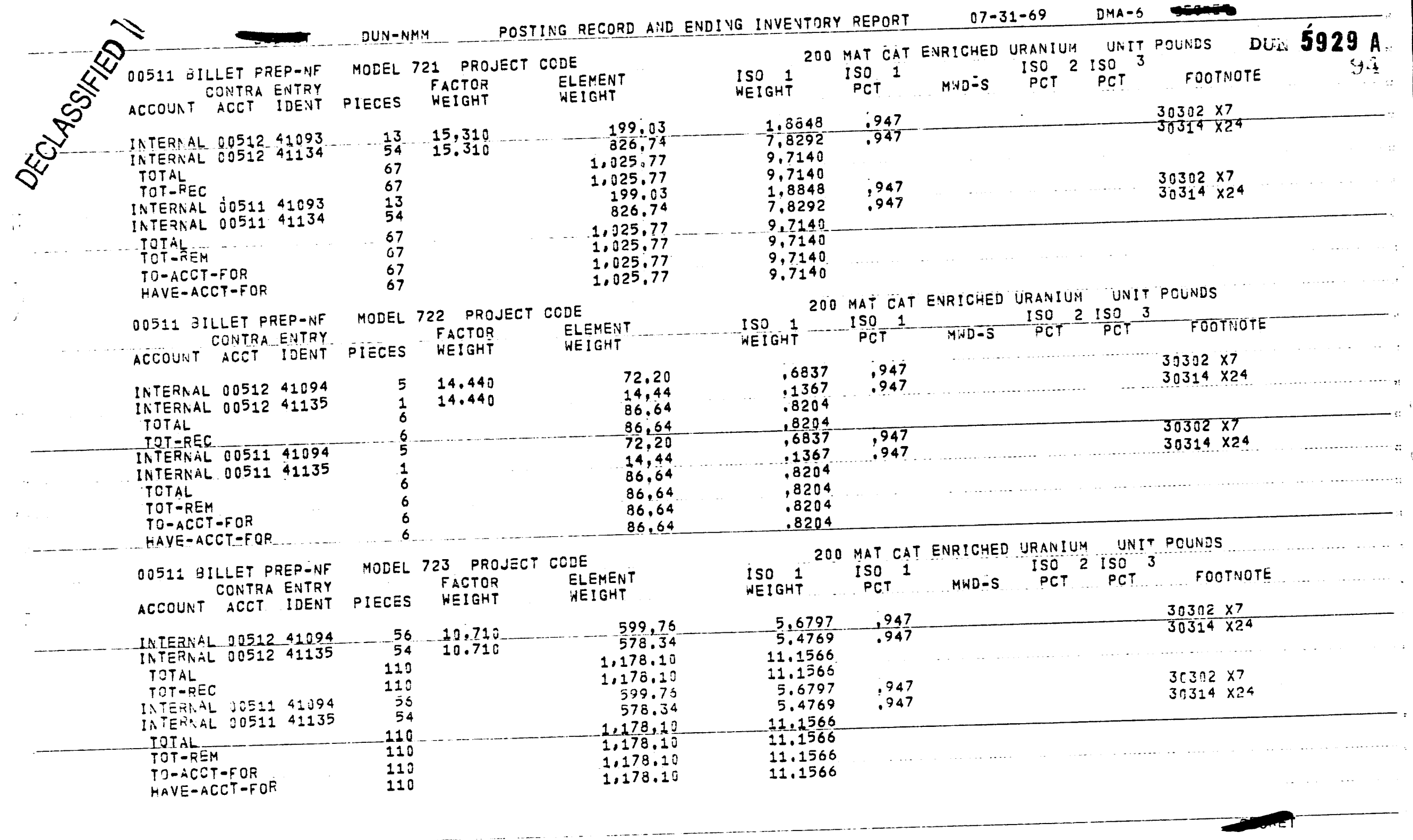




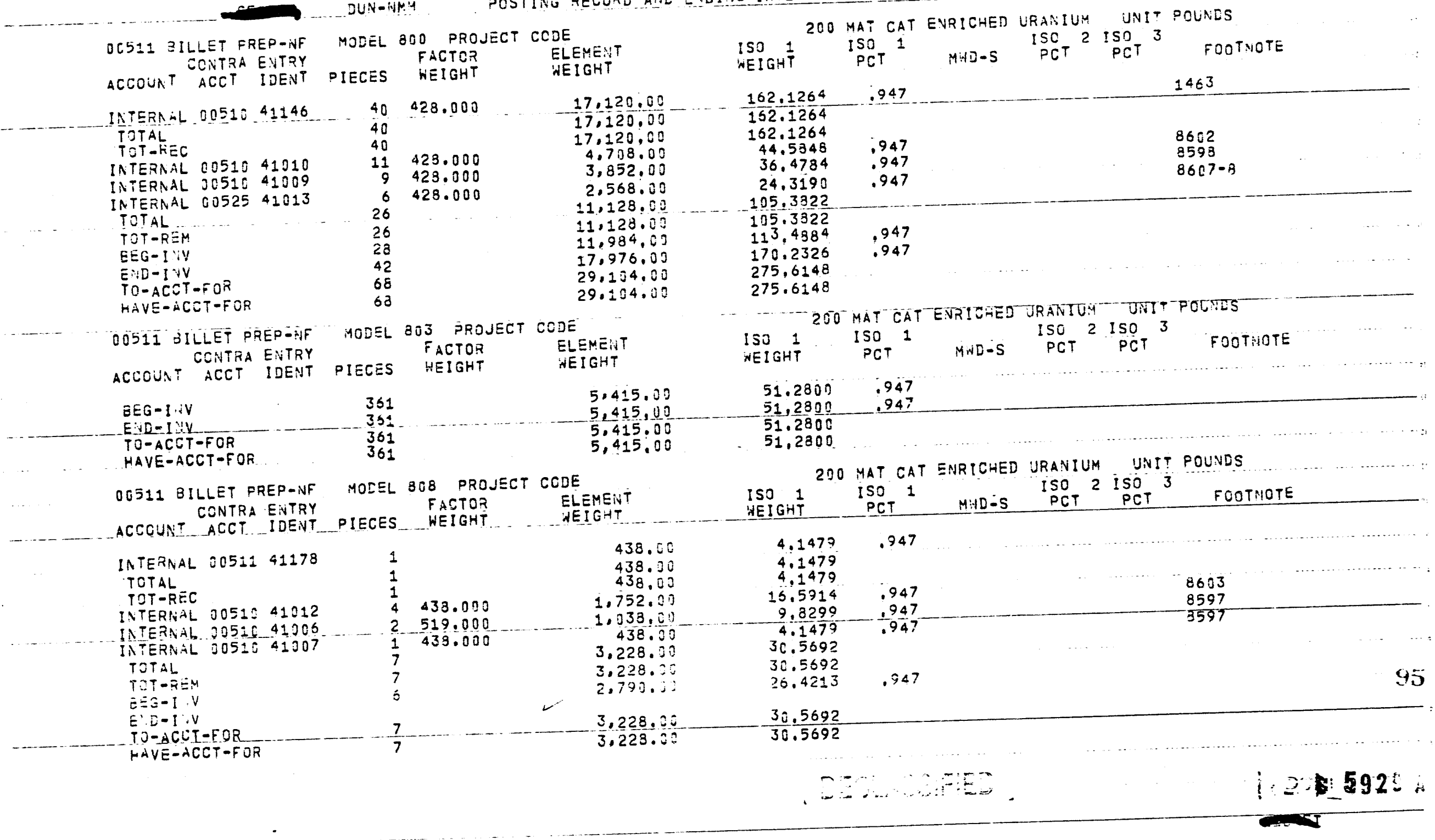




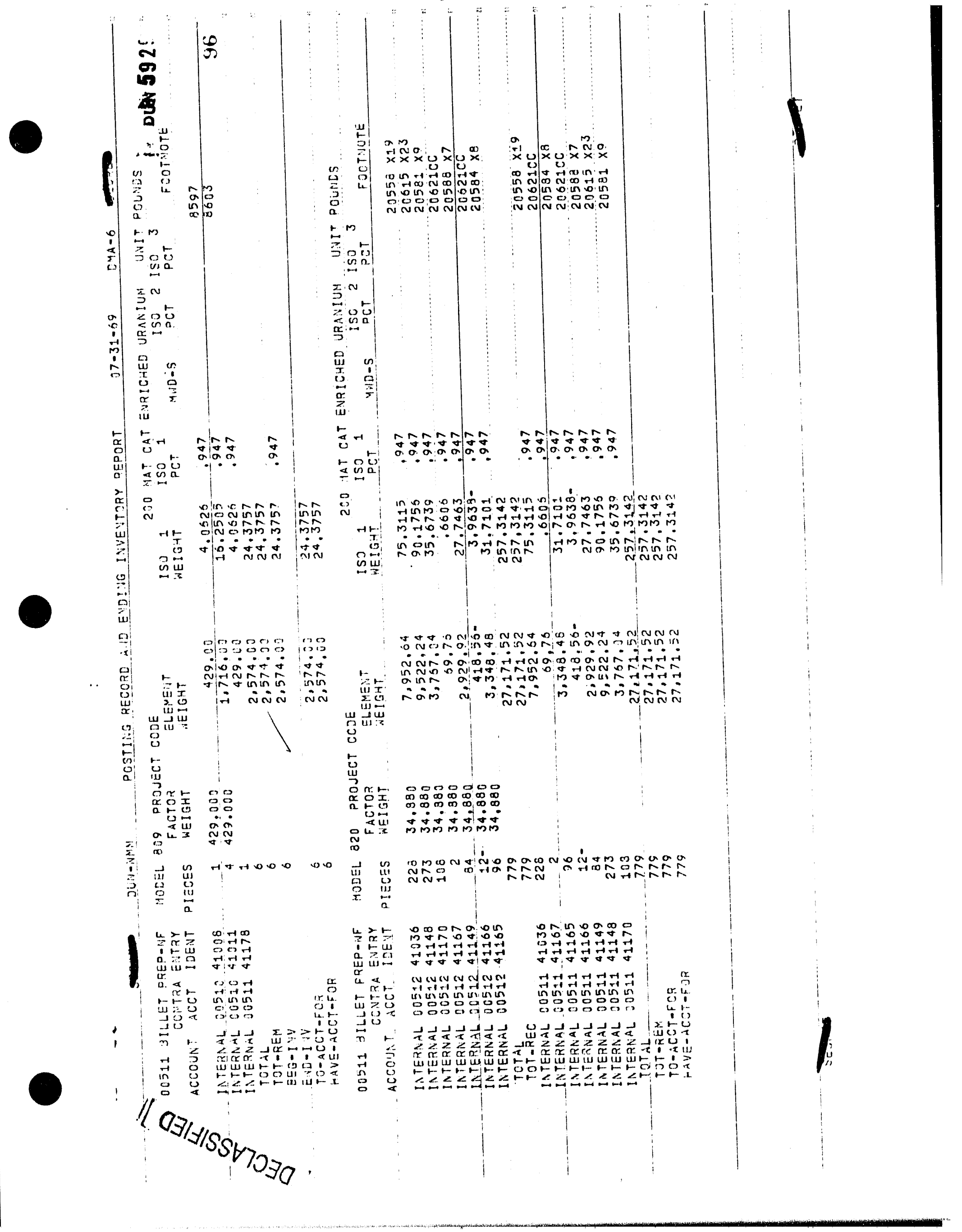


:

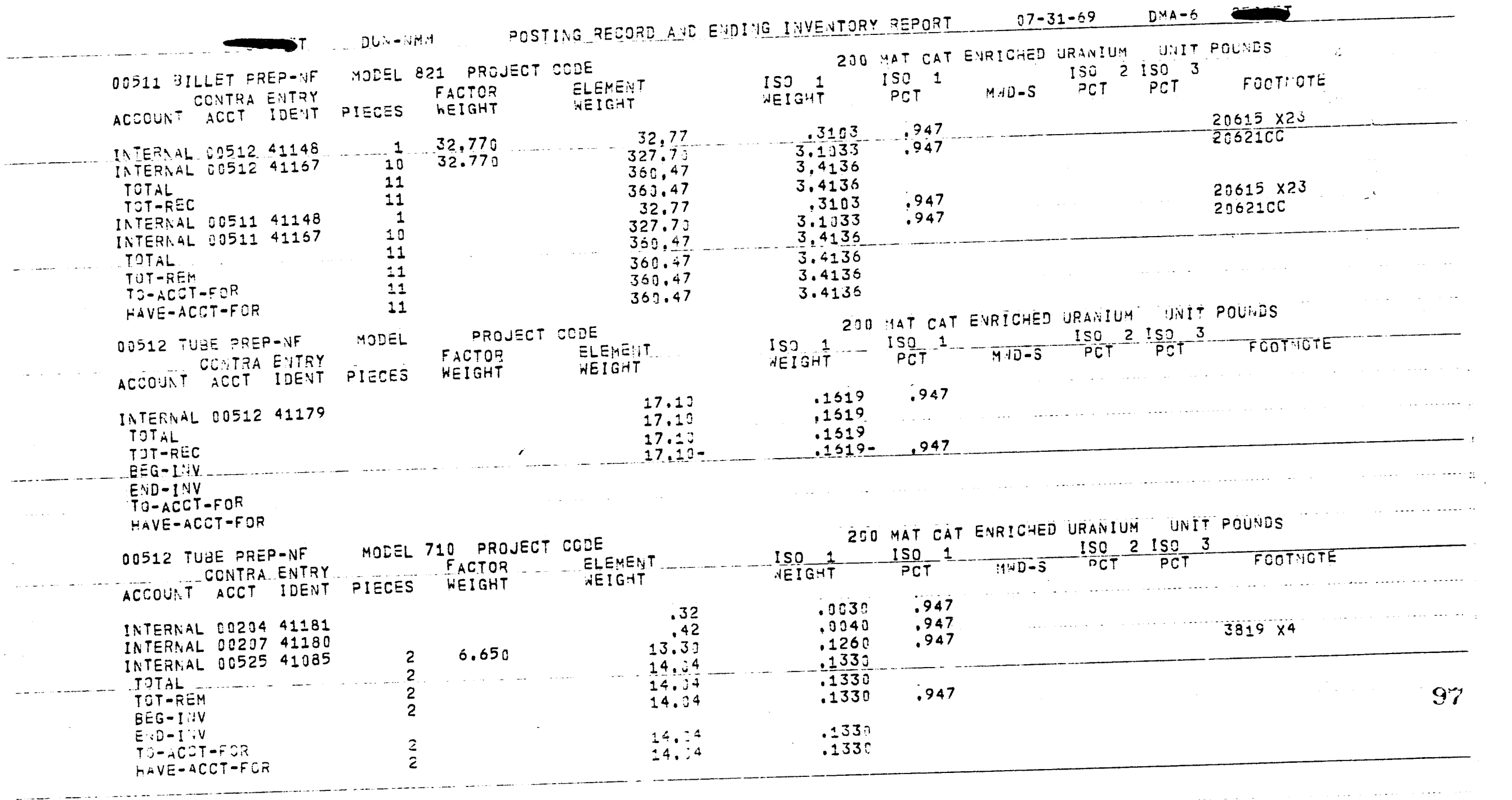


OOJ12 TUUE PREP-NF CUNTPA ENTRY HJEEL 7I3 PROJECT CODE FACTOR ELEMENT ISO 1 ISO ISO 2 ISO
POD-S PCT FEOTHOTE ACCOUNT ACCT IDENT PIEC 5 WEIGHT WEIGHT

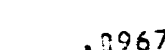

$$
\text { PCT }
$$

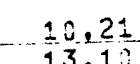

INTERAAL OC20741182 INTERNAL CO515 41075 ITERARL O0515 41015 IITENAL O0525 41036 INTERAML O0525 41112 TOIAL.

TCT-REN

aEG-INV

TO-ACCT-FOR
END-INV

HAVE-ACCT-FO

00512 TUEE PREP-AF

OCUTRA ENTRY

ACCOUNT ACCT IDENT

INTERNAL OD511 41035 INTERNAL O0511 41292 INTERNAL O0511 41053 INIERNAL O0511 41101 INTERNAL O0511 41130 TOTAL.

0020441186 INTERAAL 0020741185 INTERAAL O0249 41184 IiTERNAL O0249 41109 INTERNAL O051141093 INIERNAL OO511 41134 INTERAAL O0513 41097 INTERAAL Q0513 41249 INTERNAL 00513 41121 INTERNAL CO514 41142 INTEPAAL OOSE4 41147 INTEFiNL O0S2Z 41030 INTERNAL =0520 41100 INTERIAL C0520 41113 INTERIVAL D0525 41152

12.210
12.210
12.210
12.210
$\frac{10.21}{13.10}$ 30.22 390.72 30.05 61.35 792.54 792.54 792.54
792.54 63

.0967 .947

95

YOEEL 720 PROJECT CODE PIECES WEIGHT WEIGHT

$720 \quad 15.860$

$\begin{array}{r}240 \\ 1.079\end{array}-16.860$

30016.860 12.139 .20 4.046 .40
18.191 .94 5.053 .00 1.254 .50 $\begin{array}{ll}2.414 & 40.700 .24 \\ 2.414 & 40.700 .34\end{array}$ 2,414 214,90 $\begin{array}{lll}214.90 & 2.0351 & .947 \\ 644.77 & 6.1053 & .947 \\ 134.83 & 1.2773 & .947\end{array}$

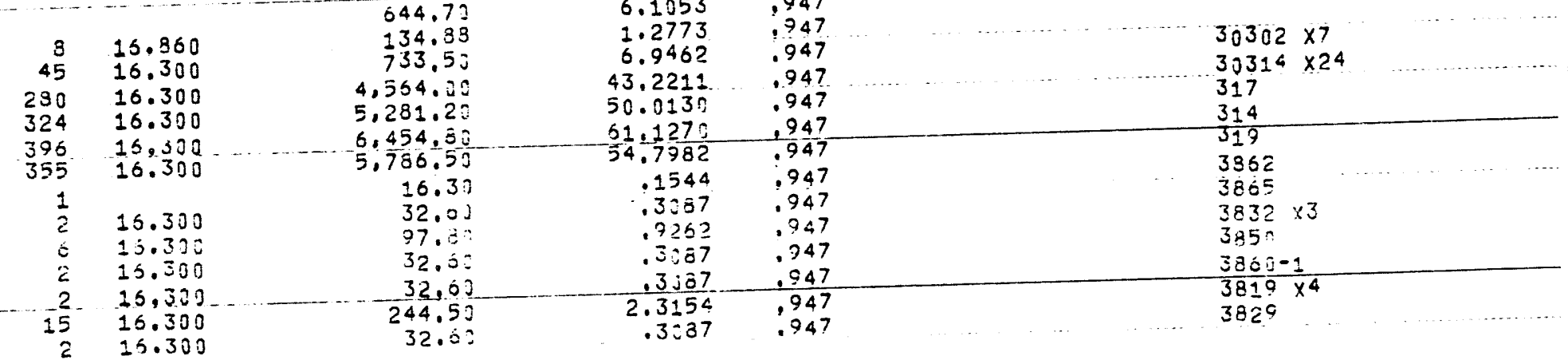

44.50

$3: 37$ 
DUiv-ivM?

00512 TIE FREP-NF ACCOUAT ACCT IDENT

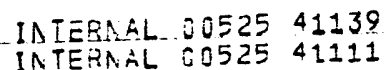
TOTAL

TOT-REM

$E E G-I j J$

TO-ACCT -FOR
ETID-ITV

A AVE-ACCT-FO

00512 TUAE FREP-NF CONTRA ENTR ACCOUAT ACCT IDENT

INTERNAL COE11 41102 INTEENAL D0511 41133 INTERNAL TOTAL

INTEREC 0020441189 INIERNAL $00204 \quad 41189$ INTERNAL 0024941187 INTERNAL 0051141093 INTERNAL O0511 41134 INTERNAL 0051341050 INIERN
TOTAL

TOT-REM

EEG-IIV

END-INV

TO-ACCT-FOF

HAYE-ACCI -EQE

00512 TUBE PREP-NF COITRA BITTYY

ACCOUNT ACCT IJEUT

IA TERNAL 6024941190

INTERAIL 0051141130

INTERAML DO511 41130

TOTAL

TOTALEC

WEI GHT

7
356
31
344
344

15.000
MOEEL 720 PRIJECT CCDE
FACTOP. ELEME:H

PIECES WEIGHT WEIGHT

75
30
1,543
1,543
1,312
2,183
3,726
3,726

$1,222,5$

16.300

10DEL 721 PROJECT CODE

FACTOR PLEMENT
PIECES WEIGHT HEIGHT

128

$288 \quad 15.970$

230

646

$\begin{array}{rr}0 & 15.870 \\ 13 & 15.310\end{array}$

15.310

15.310
15.310

15.310

$10442.46 \quad 10,443.46$

$13,534.72$

$13,534.7 ?$

489.30

$26,014.98$

$22,120.32$

$36,305.38$

$62,320,35$ $62,02.00$

$3,650.20$

$10,252,12$

$\frac{26.60}{78.83}$

126.96

199.03

326.74

1.038 .17

244,96

856

IC ENDISG
50
30
78
98
32
38
$35-$
36

DG INEVTSPY REPORT

$07-31-69$

D4A-6

ENRICHED URANIUM 2 ISO 3 TOUNLS

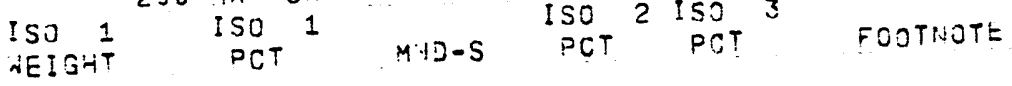

$\begin{aligned} & 11.5771-.947 \\ & 4.6303\end{aligned}-\frac{3854 \times 5}{3843-945}$

246,3519

246.3619

$209.4794 \quad .947$

$348.5469 \quad .947$

$594.978 \overline{8}$

594.9033

200 HAT CAT ENRICHED URANIUM UNIT PULANS

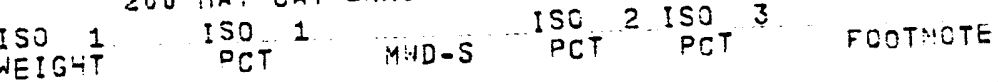

$\begin{array}{lll}19.2370 & .947 & 1897 \\ 43.2332 & .947 & 1898 \times 5 \\ 34.5564 & .947 & 3.314 \times 24\end{array}$

34.5564

97.0866

$.2519-947$

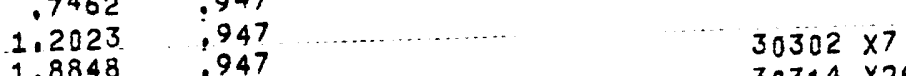

$1.8848 \quad 9947 \ldots \ldots \ldots \ldots 314 \times 24$

15.5135

.947

30314

2.3199

29,7477
29,7477

29.7477
31.5506

91.5606

98.8995

128,6472

MODEL 722 PROJECT CODE

AEIOAT

ISO

200 MAT CAT ENRICHED URANIUM UNIT POUIVDS

947

$12003 \operatorname{lin} 100$ t 207

Sugut ever

105.00
4.590 .20
465.32
5.160 .30
5.150 .20

.9944

SU 1 ISO 2 ISO

$4: 10-5$

ISOT
ISOT

FOOT:STE

99

43.4673

.947

4.4035

48.8553

.947
.947

$1898 \times 5$

$-\cdots+2=$

DIN 5223 
Doos12 TUSE FREP-VF CONTRA EVTRY
ACCOUN ACCT IDEINT

INTERAAL 20204 41192 INTERNIL CO207 41191 INTERNAL O0511 41135 INTERAAL

TDT-REM

TDT - FEM

ED- $-1,4$

TO-ACCT-FOR

KAVE-ACCT-FOR

00512 TUBE PREP-NF CCIUTRA ENTRY

ACCOUNT ACCT IDENT

POSTINA RECORD AIS ENDI LG IVIVETTORY REPOPT ?7-31-69

$D 4 A-6$

HOVEL 722 PREJECT CODE

FACTOR HOSEL 722 PLEE:TT

200 MAT CAT ENIRICHED URANIUHA JIIT POUNLS

ISO 1 ISO 1 MOTT MOS ISO 2 ISJ 3 POT ROOTICTE

DUN $5929 \mathrm{~A}$

INTERAAL 0024941193

INTERNAL O0249 41193

ii)

INTERNLL Q0511 41035

WEIGHT WEIGHT

5
1
6
6
13
351
357
357

2.54
72.20
14.440
14.440
90.44
90.22
90.20
195.20
$5,255.20$
$5,355.30$
$5,355.30$

HOEEL 723 PROJECT CODE

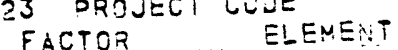

PIECES WEIGHT W WEIGHT

ATERA C0511-41004

INTERAAL O0511 41094

INTERNAL O0511 41101

INTERNAL O0511 41135

INTERNAL 0051141130

TQTAL

TOT-REC 0020441195

INTERNAL 0020441195

INTERNAL O0207 41194

INTERNAL OO511 41094

INTESAAL D0511 41135

INTERNAL 0052541111

INTERNAL O0525 41139

TOTAL

$T$ TO

$3=G-I$ i

$E V D=\operatorname{LiV}$

TO-ACCT F OR
HAVE-ACCT-FOR

10
69
16
46
34
8
20
35
41
279
279

56
54
3
5
6
124
124
273
428
552
552

11.27

$11.27 \mathrm{~J}$

11.270

11.270

11.270

11,270

11.270

11.270

112.70
777.63

180.32

513.42

333.19

394.45

462.77

$3,144,33$

3.144 .33

$44.33 \quad-29.7767$
17.7767

$.048 ? \quad .947$

$.0230 \quad .947$

$\begin{array}{lll}.0837 & .947 & 30302 \times 7\end{array}$

$30302 \times 7$
$30314 \times 24$

ivo

.1367
.8523

$1.8466 \quad 947$

49.8596

30.7119

200 MAT CAT ENRICHED URAIIUM INNIT POUNUS

10.710

52.58

599.75

578.34

10.710

10,710

10.710

32,13

$53.5 \overline{5}$

10.710

1.397 .40

$1,397.40$

$3,076.74$

4.223 .50

$\begin{array}{ll}4.221 .04 & 58.9131 \\ 6.221 .34 & 58.9131\end{array}$

ISO $\frac{1}{\text { ISIGHT }} \frac{150}{\text { PCT }}-\frac{\text { ISO } 2 \text { ISO }}{\text { MCT }}$

$1.0673 \quad .947 \quad 1839 \times 5$

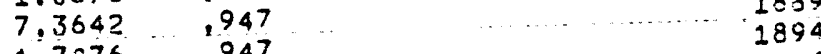

$\begin{array}{ll}1.7075 & .947 \\ 4.9094 & 947\end{array}$

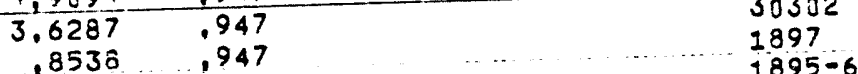

$1894 \times$

$1895-6$

$30314 \times 24$

$1898 \times 5$

\begin{tabular}{lll}
3.076 .71 & 13.2341 & \\
1.947 & 29.1354 & .947 \\
\hline & 45.6790 & .947
\end{tabular}


JUV-AMM MOLEL 724 PROJECT CODE

DOJ12 TUBE PREP-NF CONTZA ENTAY
ACCOUNT ACET IDENT INTERIAL O05:1 41093 Ii TEOAAL O0511 41134 TOTAL

TOT-REC 020441197 INTERNAL 0020741196 INTERNAL 2051341164
INTERAAL DO525 41139

TUTAL

TOT-REM

IEG - IIV

E.TD-I

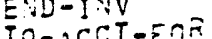

HAVE-ACCT-FOH

00512 TUZE PREP-NF CONTRA ENTRY

ACCONAT ACCT IDENT

INTERNAL 0024941198

TOTAL

TOT-REC

INTERNAL C0204 41200

INTERAL 0020741199

NTERNAL D0525 41085

INTERNAL $=052541112$

TOTAL

TOT-REM

GEG-IIV

END-ITIV

TO-ACCT -FOR

HAVE-ACCT-FOR

00512 TUEE FREP-.NF

OOS12 TUOTTAA EUTYY

ACCOUNT LCET IDENT

INTERNAL OO204 41202

INTERIAL $T 020741201$
MOLEL 724 PROJECT COJE
FACTOR
PIECES WEIGHT NEMEITT

$\begin{array}{rrr}35 & 7.550 & 541.75 \\ 93 & & 732.13 \\ 176 & & 1.343 .70 \\ 178 & & 1.343 .90 \\ & & 7.70 \\ 49 & 5.990 & 23.10 \\ 6 & 5.990 & 41.51 \\ 55 & & 415.25 \\ 55 & & 415.25 \\ 67 & & 50.35 \\ 190 & & 1.434 .50 \\ 245 & & 1.349 .75 \\ 245 & & 1.849 .75\end{array}$

HODEL 730 PROJECT CODE

HODEL 730 PROJECT CODE FATOR
PIECES WEIGHT NEMT

…............

$19.010 \quad 9.01$

1

$20 \quad 8,640$

8.640

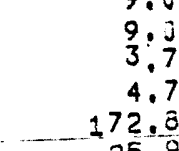

$\frac{9}{3}: \frac{31}{3}$

4.79

172.00
25.92

207.25

207.23

198.22

207.23

207.23

23

MODFL 733 PROJECT OCDE
FACTOZ WEIGHT

PIECES NEISHT

$07-31-69$

DMA-6 SESRET

EVRICHED URANIUM IJNIT FOUNIES

ISO 1 ISO 1 MCT MOS ISO 2 ISO 3 PCT FOOTHOTE

$\frac{6.0774}{6.6494} \quad .947$

$6.6494 \quad .947$

12.7263

12.7263

$.0729 \quad .947$

$.2188 \quad .947$

$3.2436 \quad .947$

321

.3772

3.9325

3.9325
4.7904

$4.7904 \quad .947$

$13.5847 \quad .947$

17,5172

200 HAT CAT ENRICHED URANIUM UNIT PCUNES

ISO 1 ISO 1 MWD-S PCT POT PCT ROTHOTE

$3854 \times 5$

$\begin{array}{lll}2.27 & .0215 & .947 \\ 2.91 & .0275 & .947\end{array}$



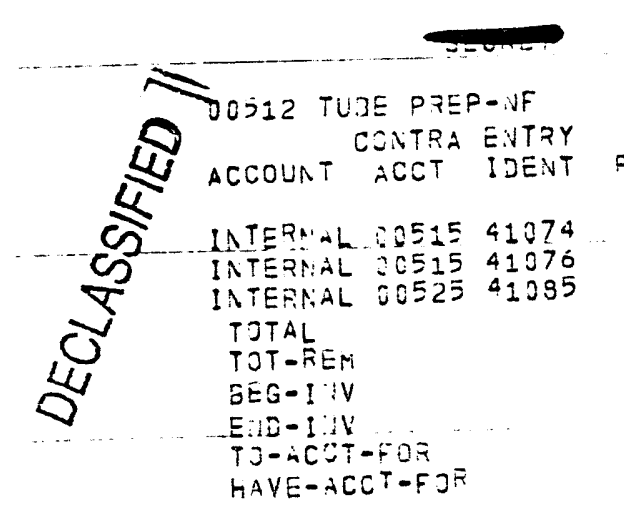

OOS12 TUSE PREP-NF CCNTRA ENTRY
ACCOUNT ACCT IJEUT

INTERNAL 0020441204 INTEPNAL 0020741203 INTERAAL OO2525 41112 INTERNAL
TOTAL

TOTALEM TUI-FEM
SEG $=I Y V$

END-I IV

TO-ACCT-FCR

HAVE-ACCT - FOR

O0512 TUBE PREP-NF

DO512 TUBE PREPENFA EVTYY

ACEOUNT ACCT IDEVT

INTERNAL 0024941206 TOTAL

IIT-REC

INTERNAL J0249 41205

TOTAL

$T O T-R E M$

$3 E G-I: V$

E

HAVE-ACCT-FOR

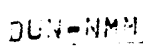

POST:IVG RECORE \& ID ENDIUG IVVEITTRY REPORT

$07-31-69$

$D M A-6$

MODEL 733 PROJECT COJE

MOEEL 733 PACTOR NEREMENT
PIECES WEIGHT NEIGHT

PIECES WEIGHT WEIGHT IST $1 \frac{1}{1}$ ISO 1 ISO 2 ISO 3

Jivit POLIIES

DUN $323 \mathrm{~A}$

$6 \ldots 11.430 \ldots 58.53$

$211.430 \quad 22.35$

11.430

$$
58.23
$$

155.20

155.20

105.23

155.2

$\pm 05.22$

$\frac{14}{14}$

WEIST

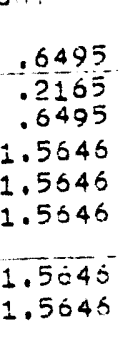

MODEL Q1O PROJECT CCJE

FACTOR ELEMENTT

P!ECES. WE!GHT

HAT CAT ENRTCHED URANIUY UNIT POUNES

ISO 1 ISO 2 ISO 3

FEOTNOTE

$\begin{array}{rrrr}1.97 & .0187 & .947 & \\ 6.17 & .0584 & .947 & 3837-9 \\ 146.35 & 1.3907 & .947 & \end{array}$

1113.350

$6 \cdot 17$

146.35
154.39

154.39

154.99

11

154.99
154.99

10DEL 814 PROJECT CODE

FACTOR ELEMETI

ELEMENT

200 MAT CAT ENRICHED URANIUM UNIT POUNDS

PIECES WEIGHT

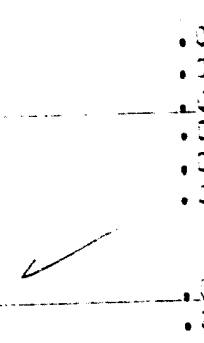

1.3907

1.4575

$\frac{1}{1.4673}$

.947

$42007-8$ IS0

$\begin{array}{lll} & & \\ 31 & .0002 & 2,000 \\ 11 & .0002 & \\ 01 & .0002 & \\ 21 & .0001 & 1.000 \\ 21 & .0001 & \\ & .0201 & \\ & .02010 & \end{array}$


:

$07-31-n 9$

D.4A-6

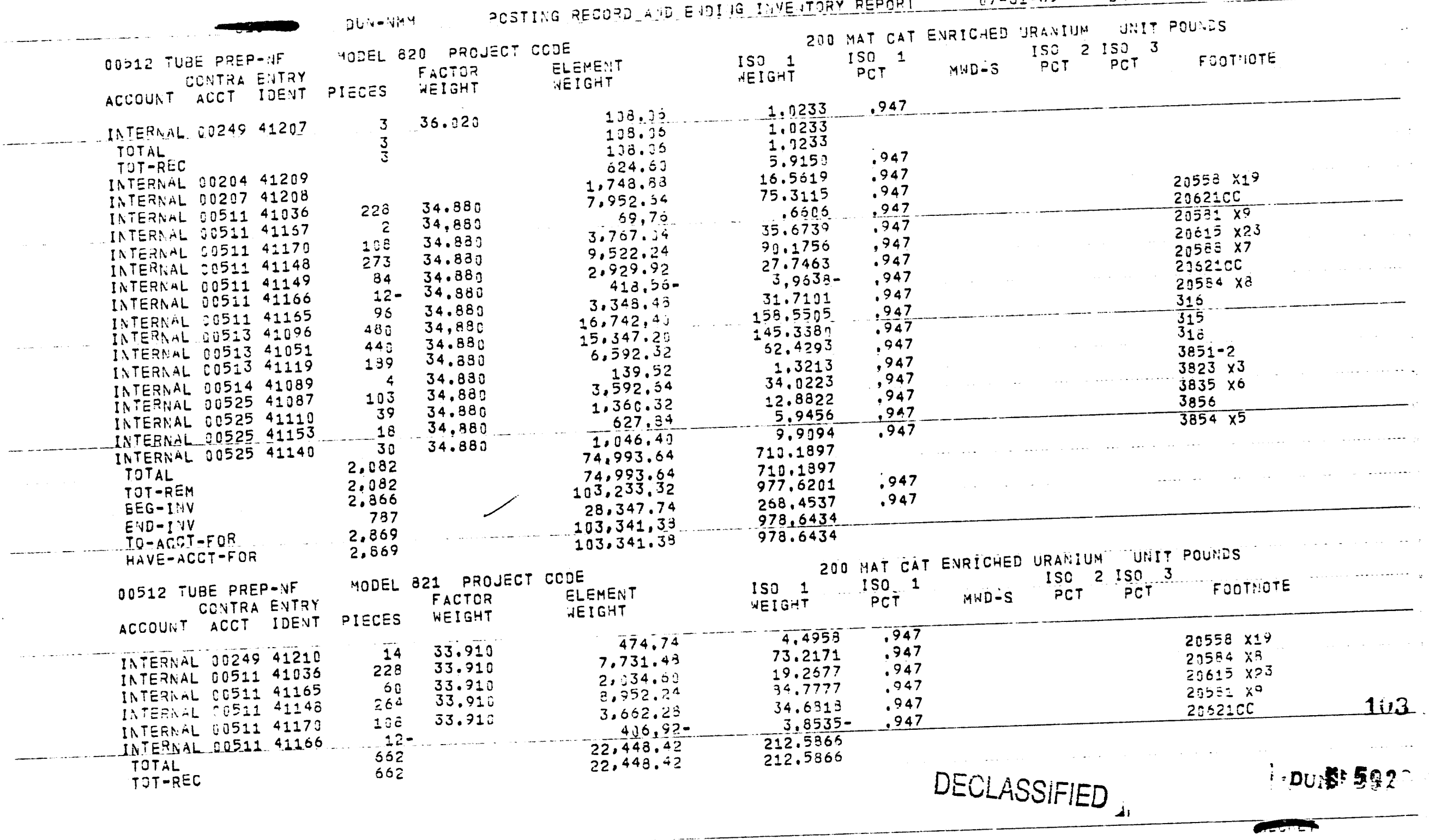




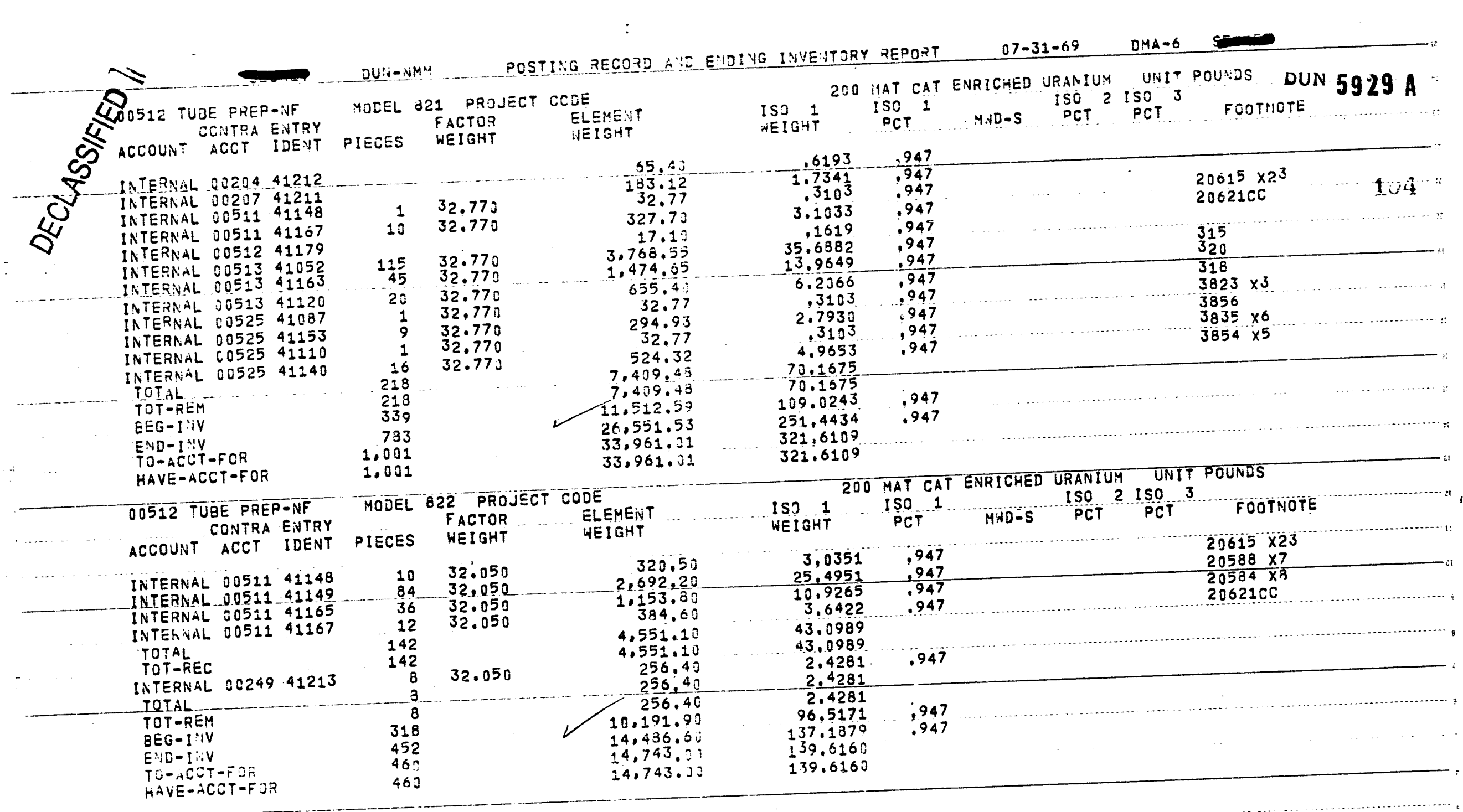


Duit-ivit!

OOSTIIG RECORD AIE EVDIYG IVVEYTORY PEPORT

$07-31-6$

DMA- 6

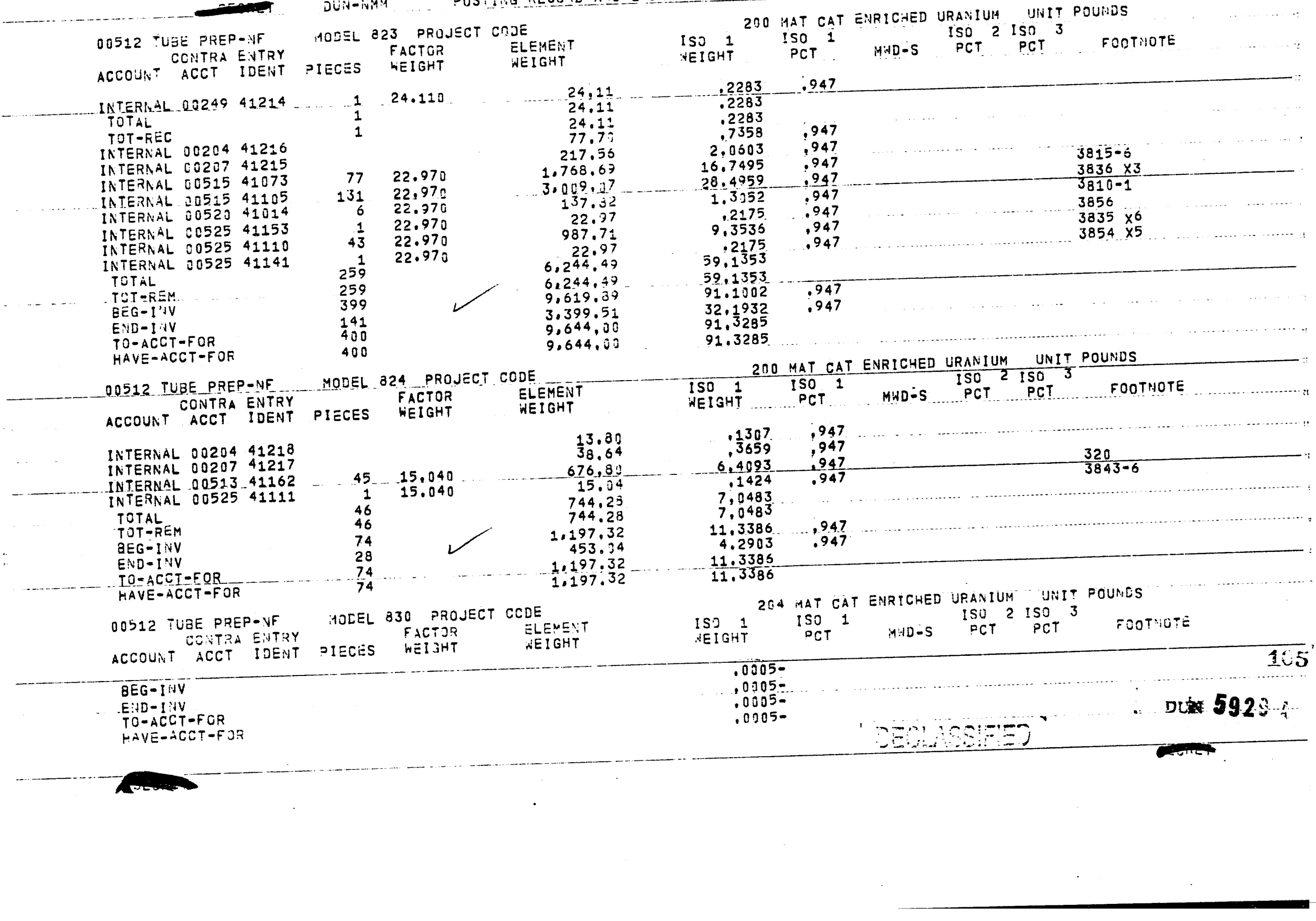




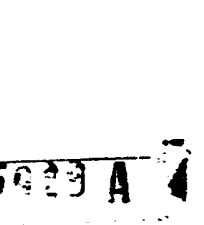

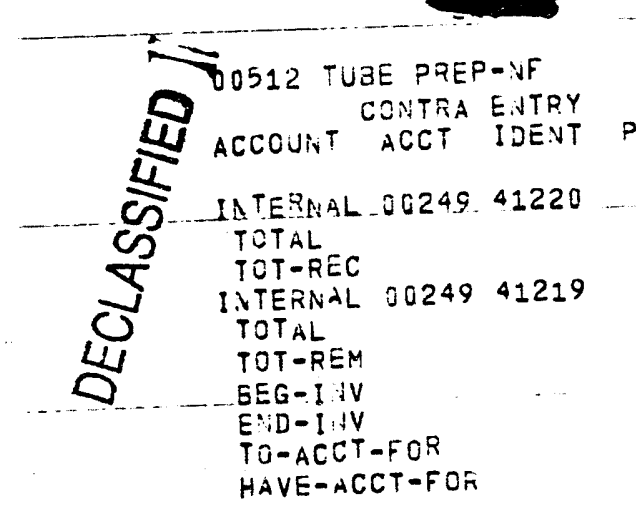

$D L Y-1, M M M$

POSTING RECOZD A D ENDIVG INUENTORY REPORT

$07-31-69$

DMA-6

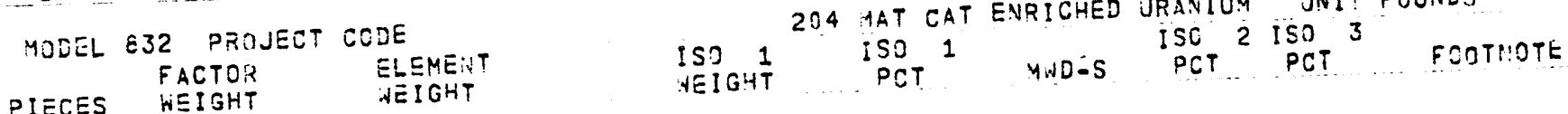

$\ldots .0006 \quad 2.000$

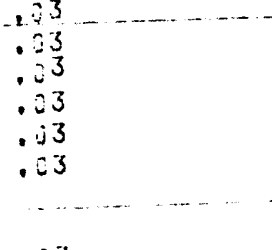

$\begin{array}{ll}.23 & .0003 \\ .03 & .0003\end{array}$

00512 TULE PREP-YF CCNTPA E.YTRY

ACCOUNT ACCT IDENT

WODEL $\$ 33$ PROJECT CCDE

1.0006

.0006

106

.0303

.0003

003

FACTOR ELEME::T WEIGHT

.0003

EEG-IiIV

END-IiNV

TO-ACCT-FOS

HAVE-ACCI-EOR

O0512 TUBE PREP-NF

CONTRA ENTRY

ACCOUNT ACCT IDENT

MODEL 840 PROJECT CODE
FACTOR ELEMENT

FACTOR ELEMEN

IS

234 MAT CAT ENRICHED URANIUM UNIT POUNES

PIECES WEIGH

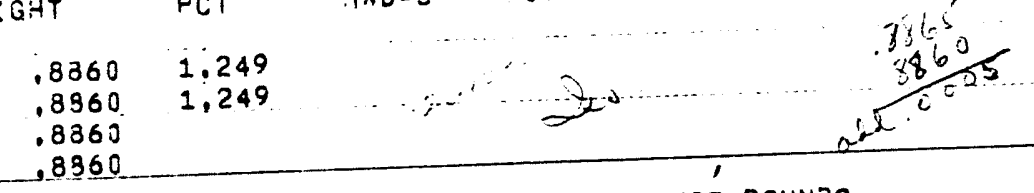

204 MAT CAT ENRICHED JRANIUM UNIT POUNDS

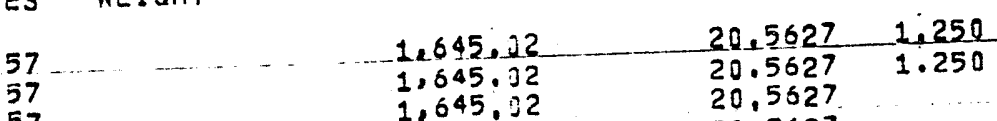

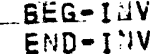

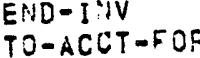

HAVE-ACCT-FOR

OOS13 TUBE ASSEIA-NF

COCOUNT COUTRA ENTRY

2ON HAT CAT ENRICHED URANIUM UNIT POUNDS

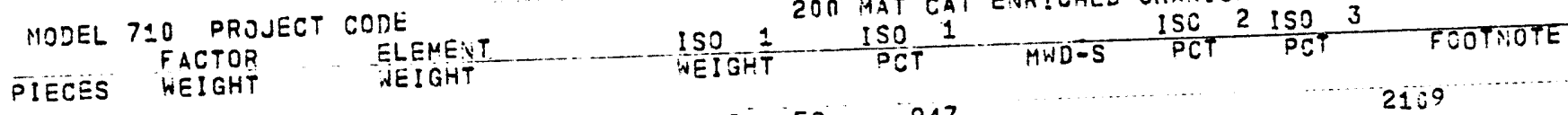

INTERNAL OOS22 41027

TETAL

TOTAREM

ENDD-IIV

TO-ACCT-FOR

HAVE-ACCT-FOR

6.650

212. 万]

212.

212.50

266.0 .03

53.23

256.30

$2.0152 \quad .947$

2.0152

2.0152

$2.5190-\frac{.947}{.947}$

2.5190

2.5190

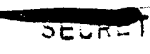


DYA $=6$

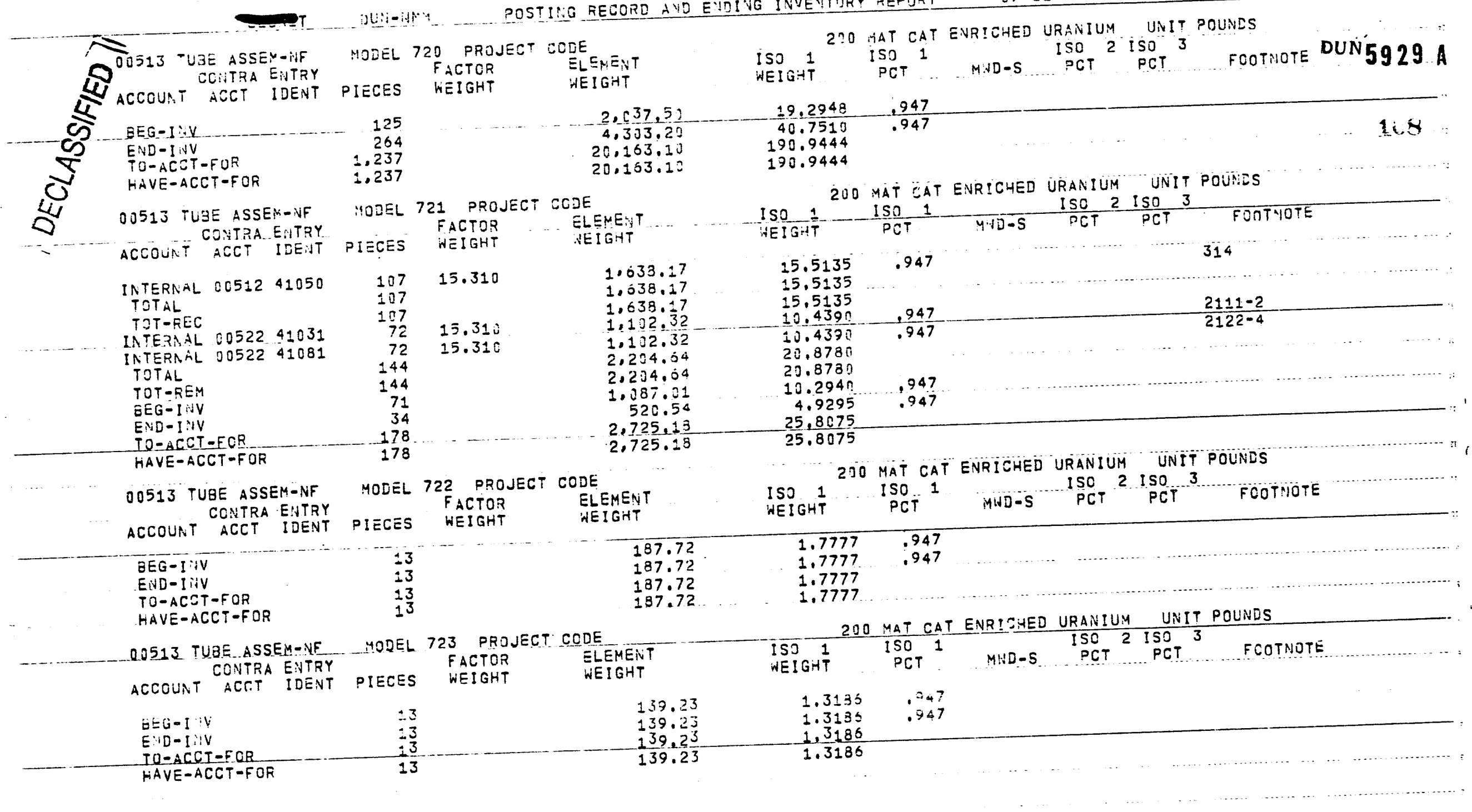


Di:T-VMM

POSTING RECOPD AUE EYDIVG IUVENTORY REPORT

$97-31-69$

D.1A-6

OOS1J TUEE ASSEM-INF EONTRA ENTRY

ACCOUIT ACCT IDENT PIECES

MOEEL 724 PROJECT CODE
FACTOR TLEMENT

200 AAT CAT ENRICHED URANIUM UNIT POLNES

WEIGHT 'AEICHT

INTERLAL S0512 $41164 \ldots \ldots$. 49

TOTAL

TOT-KEC

BEG-INV

TOM-ACST-FOR
EID-ITV

HAYE-ACEI-FO?

6.990

… 342.51

342.51

342.51

342.31

342.51

49
49

O0513 TUAE ASSEY-IJF

CONTRA ENTRY
ACCOUNT

49

IS

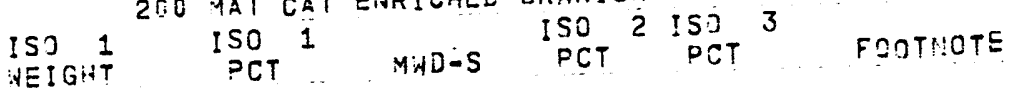

MODEL 730 PQOJECT CODE

ACCOUNT ACCT IDENT

IATEOAAL 5052241025

FACTOR T ELEMENT

$342.52 \ldots . .$.

\begin{tabular}{l}
3.2436 \\
$\begin{array}{l}3.2436 \\
3.2436 \\
3.2436 \\
3.2436 \\
3.2436\end{array}$ \\
\hline
\end{tabular}

TOTAL

TUT-REM

EEG-I:IV

END-I'IV

TO-ACCT-FOR

00513 TUBE ASSEM-NF MODEL 733 PROJECT CODE

IGHT

CONTRA ENTRY ACCOUNT ACCT IDENT

\begin{tabular}{|c|c|c|}
\hline 33 & $8.64 \mathrm{~J}$ & 255.12 \\
\hline 33 & & 285.12 \\
\hline 33 & & $235 \cdot 12$ \\
\hline $\begin{array}{r}39 \\
6 \\
-0\end{array}$ & & $\begin{array}{r}336.96 \\
51.84\end{array}$ \\
\hline $\begin{array}{l}39 \\
39\end{array}$ & & $\begin{array}{l}336.96 \\
336.95\end{array}$ \\
\hline
\end{tabular}

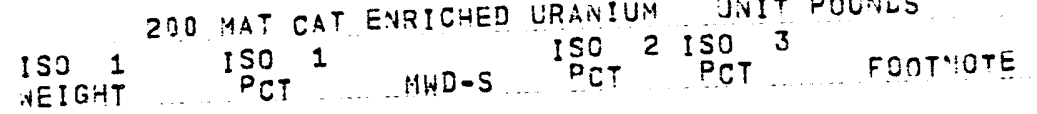

210

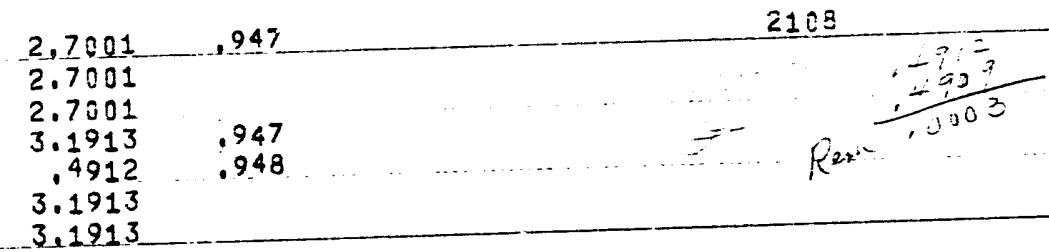

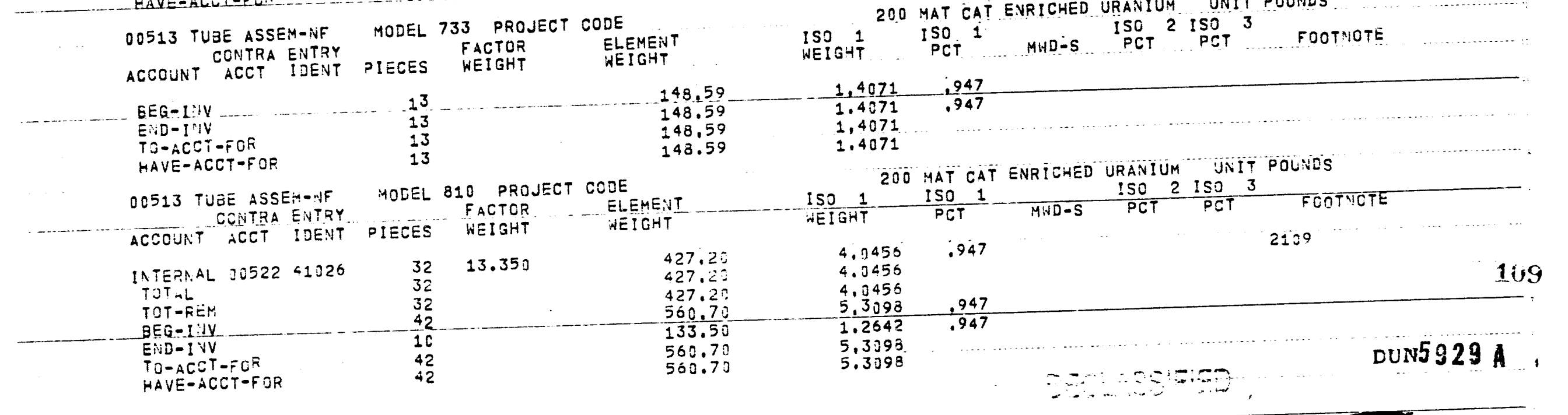




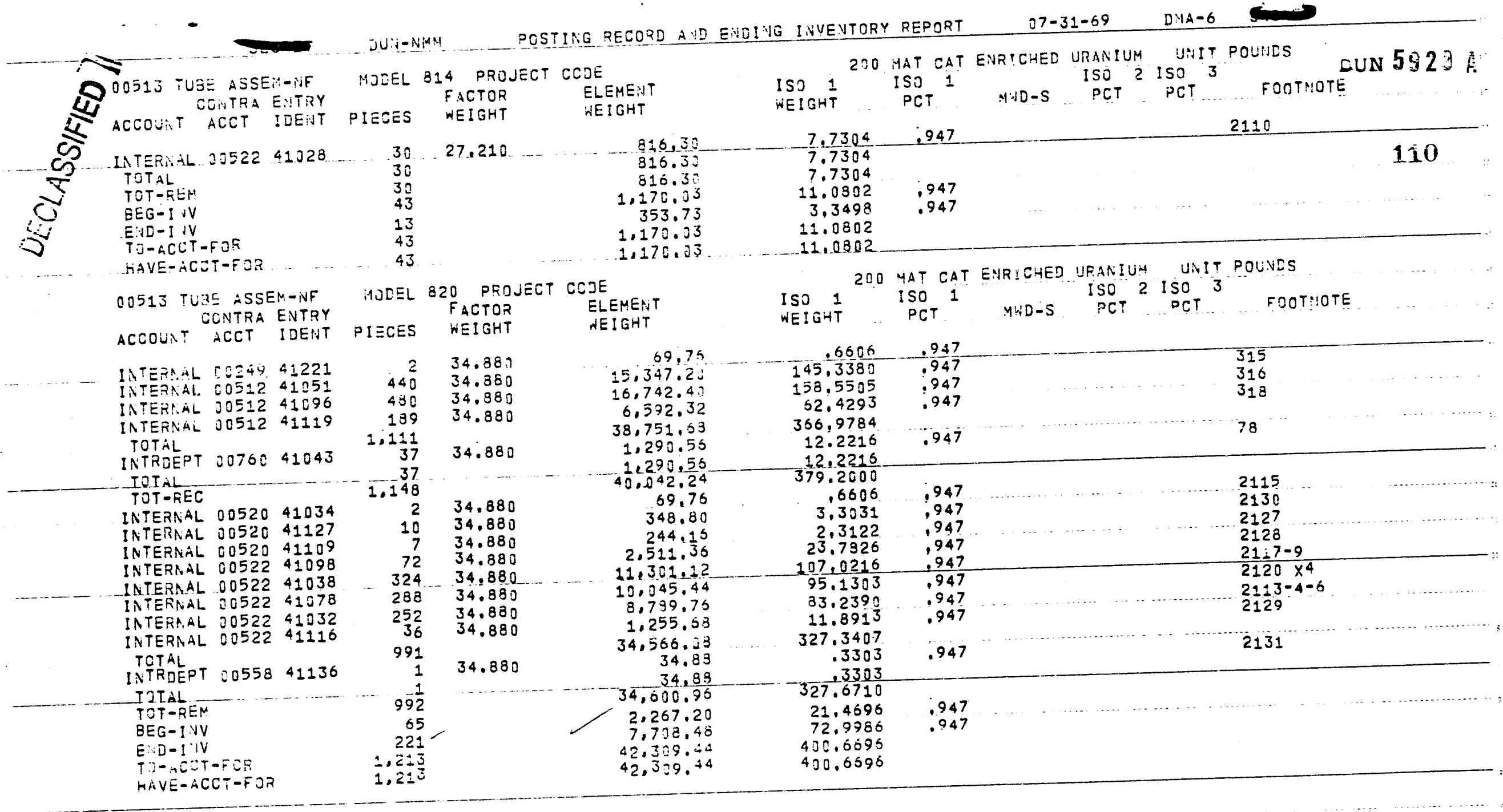


OМ513 TU3E ASSEK-NF CCNTRA ENTRY ACCOUNT ACCT IDENT

INTERNAL 0051241052 INTERIVAL O0512 41120 INTERAAL CO512 41163 TOTAL

TOT-FEC

INTERNAL 3224941222

INTERATL OIS22 41030

INTERINAL 00522 41030

TOTAL

TOT-REM

EEG-I:IV

END-1 1 TO

TO-ACST-FOR

AVE-ACCT-FOR

00513 TUEE ASSEM-NF CONTRA EVTRY
ACCOUNT ACCT

\section{$B E G-I N V$}

END-INT

TCT-FOR

00513 TUBE. ASSEM-NF

CONTRA ENTPY

ACCOUNT ACCT IDENT

EEG-IVHV

EID- I IV

TO-ACET-FQR

HAVE-ACCT-FOR

00513 TUAE ASSEH- UF

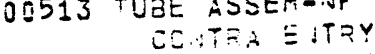

ACCOUNT ACCT IJENT

INTERNAL 00512 41162

TOTAL

TOT-REC

$E E G-I$ IV 07-3i-09 DHA-6

2U:i-UMH

DCSTIIUG RECORE A YDEEUDIIG IYVEUTORY REPORT

UNIT FOUADS

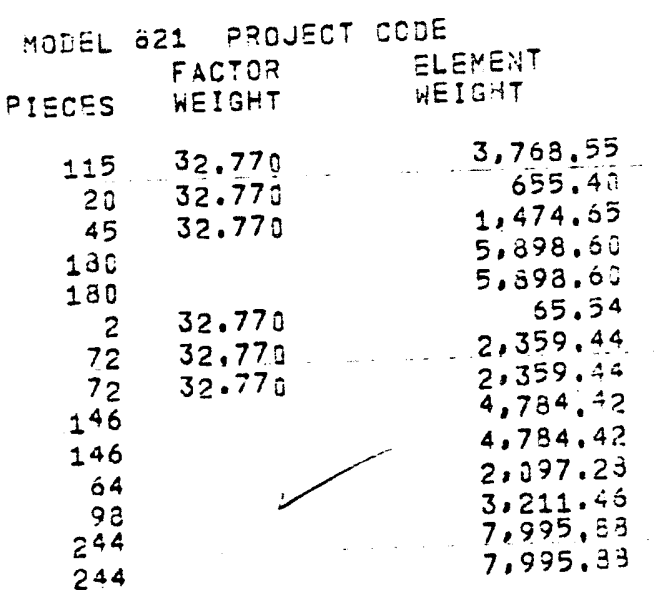

MOEEL 822 PROJECT CODE

FACTOR ELEYENT

PIECES WATGHT NEIGHT

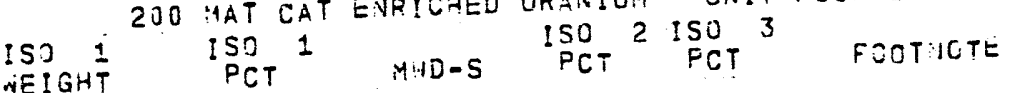

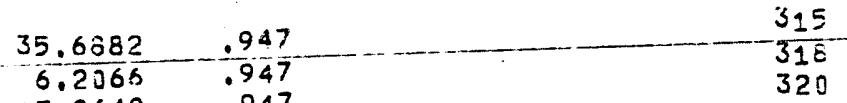

$6.2065 \quad .947$

55.8597

55.8597

\begin{tabular}{rr}
.6297 & .947 \\
$22.3439-.947$ & $2111-2$ \\
\hline $2122-4$
\end{tabular}

$22.3439 \quad .947$

45.3085

45.3085

$19.8613 \quad .947$

$30.4125 \quad .947$

$75.721 ?$

75.7210

200 MAT CAT ENRICHED URATIUM UNIT POLNES ISO 1 ISO 1 MET MUTE PCT PCT POTNCTE

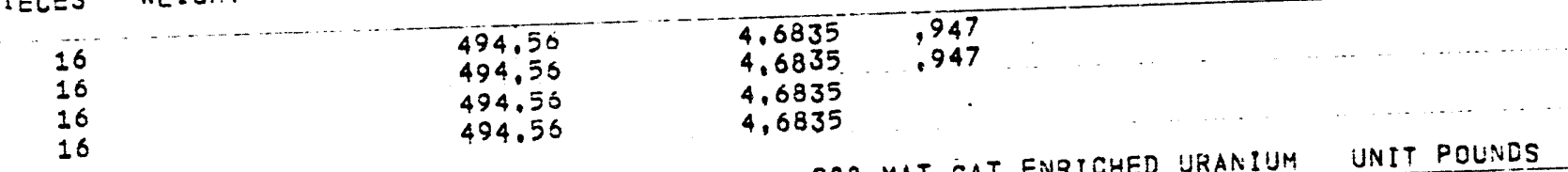

MOUEL 823 PROJECT CODE
FACTOR
PIECES WEIGHT

$\begin{array}{lll}133.75 & 1.7401 & 947 \\ 133.75 & 1.7401 & .947 \\ 133.75 & 1.7401 & 1.7401 \\ 133.76 & \end{array}$

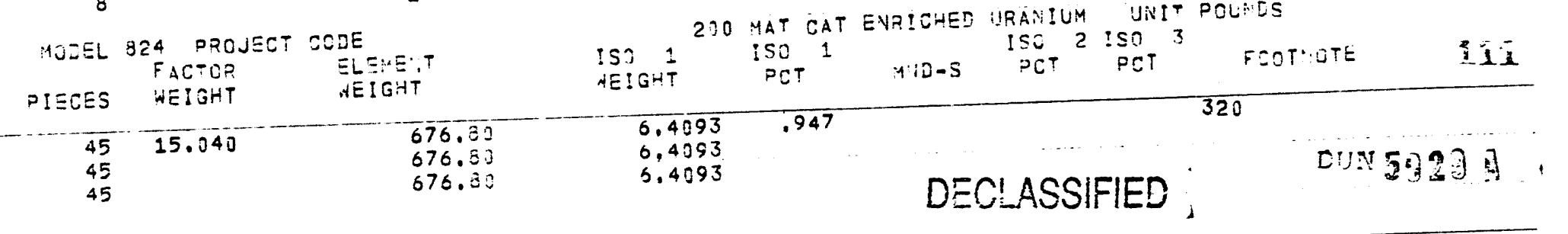




\section{-}
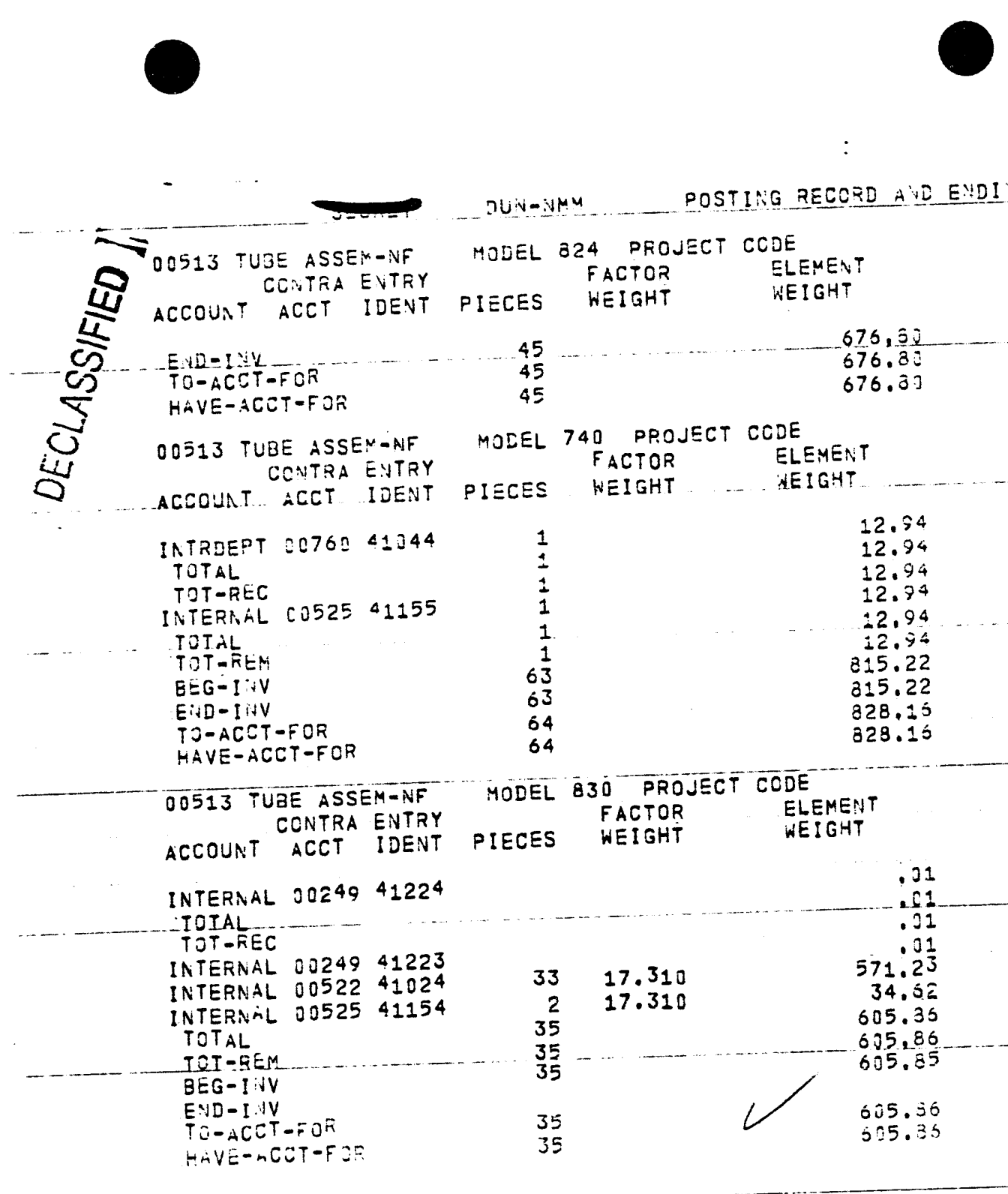

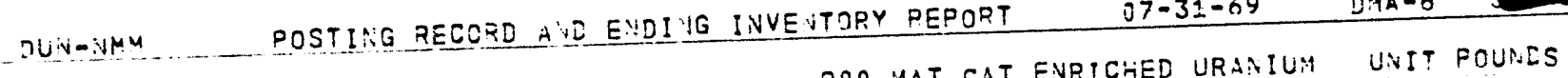

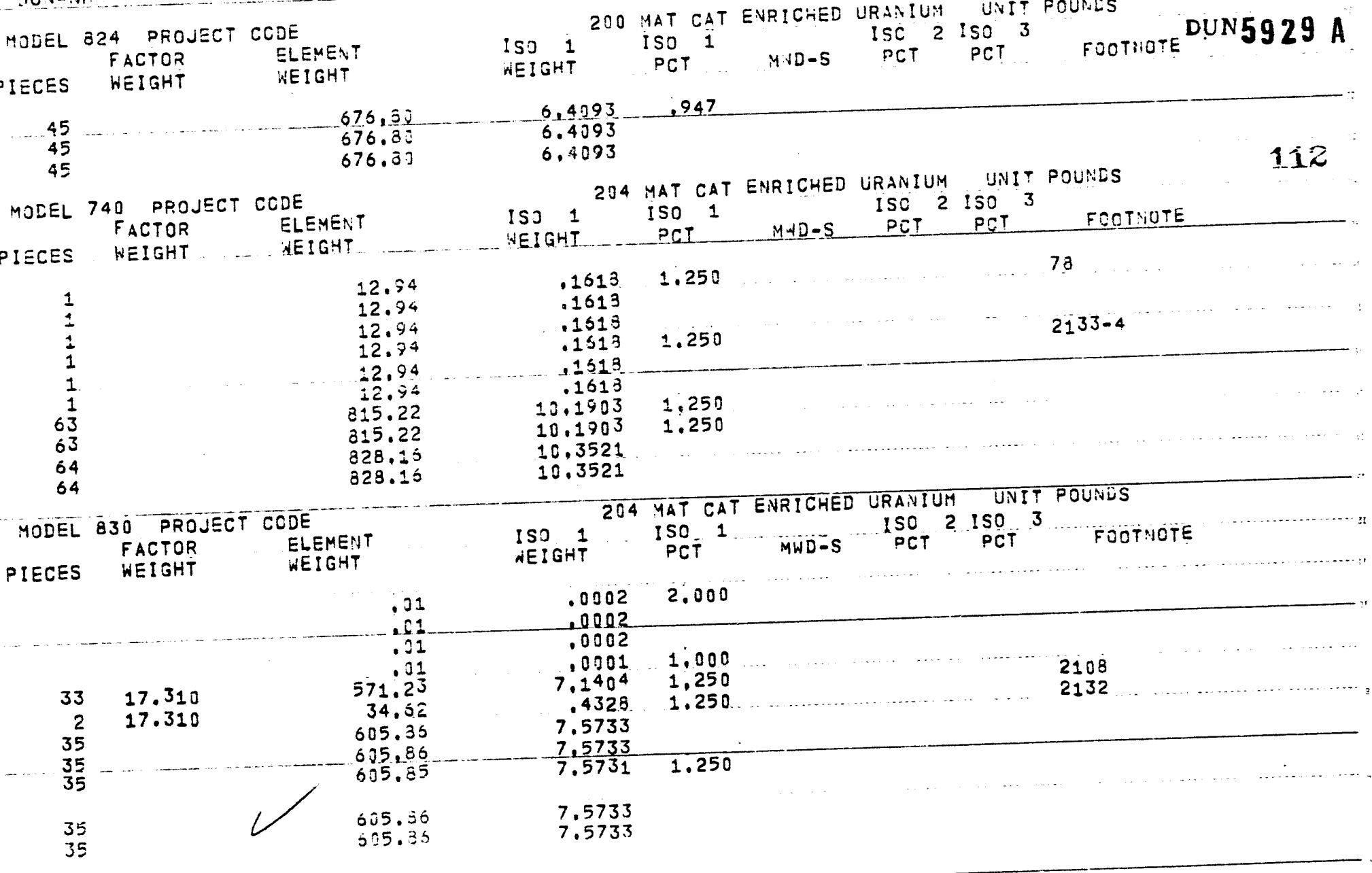




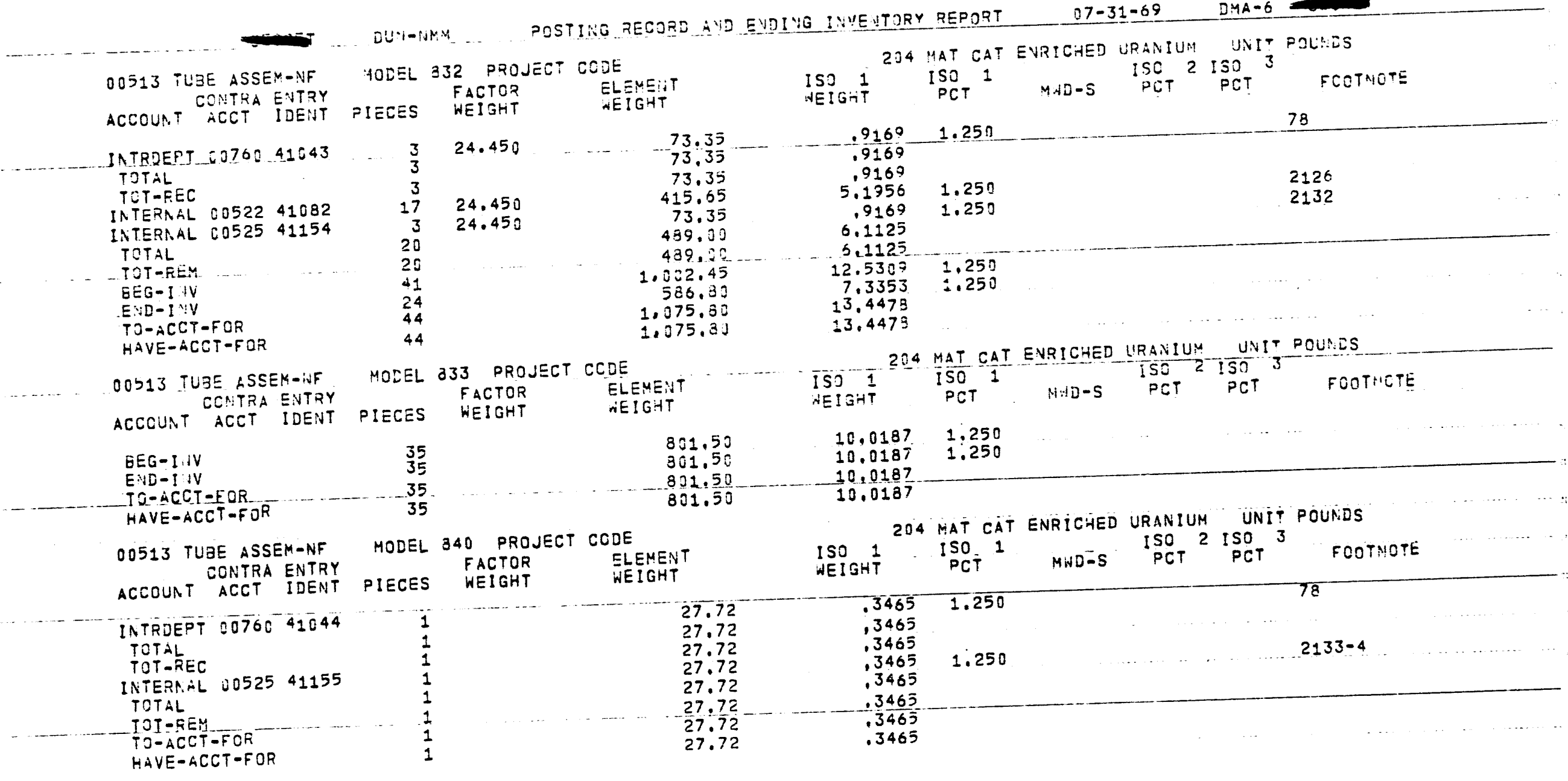

113

$$
7=\text { SOFE }
$$




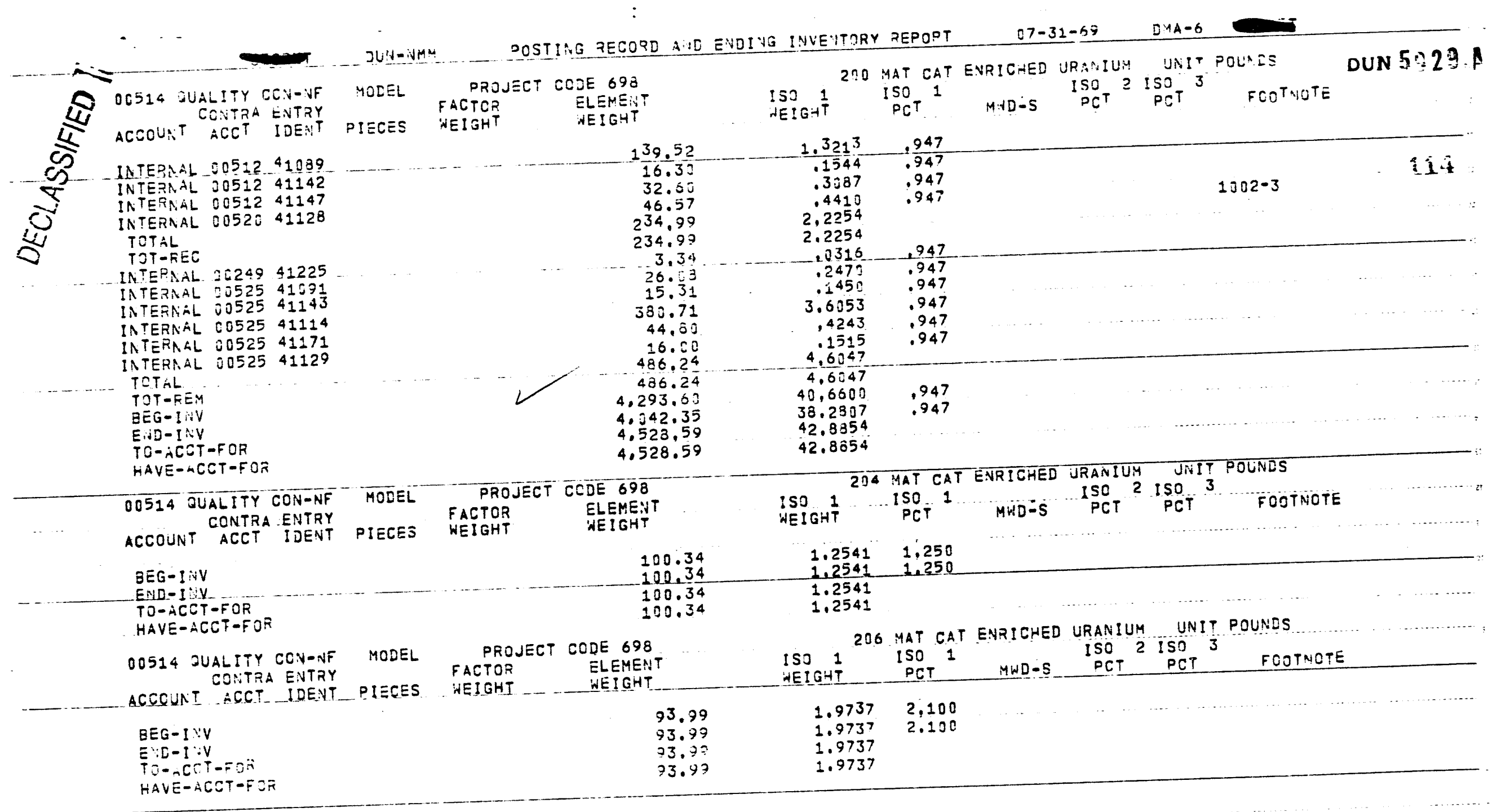


-

:

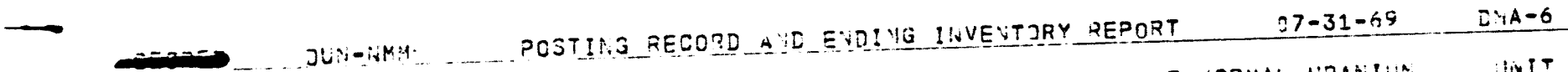

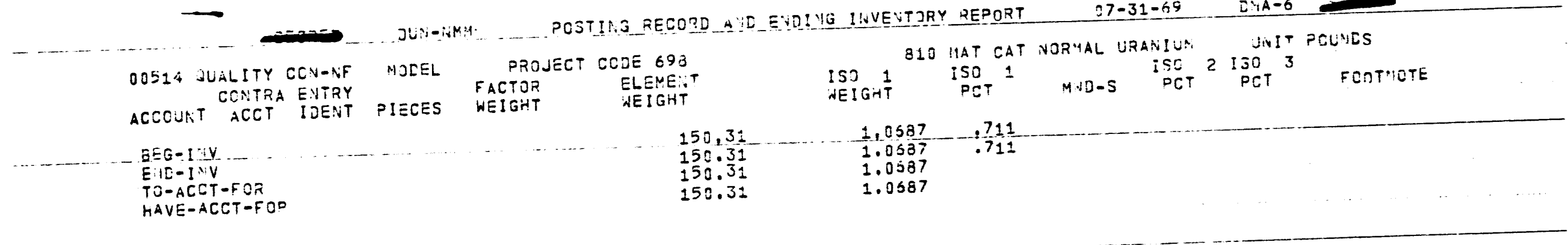


DLiv-ivMM POSTING RECOQD AVE EIDIVG IAIVENTORY PEPORT

$17-31-69$

[., $4 A-6$

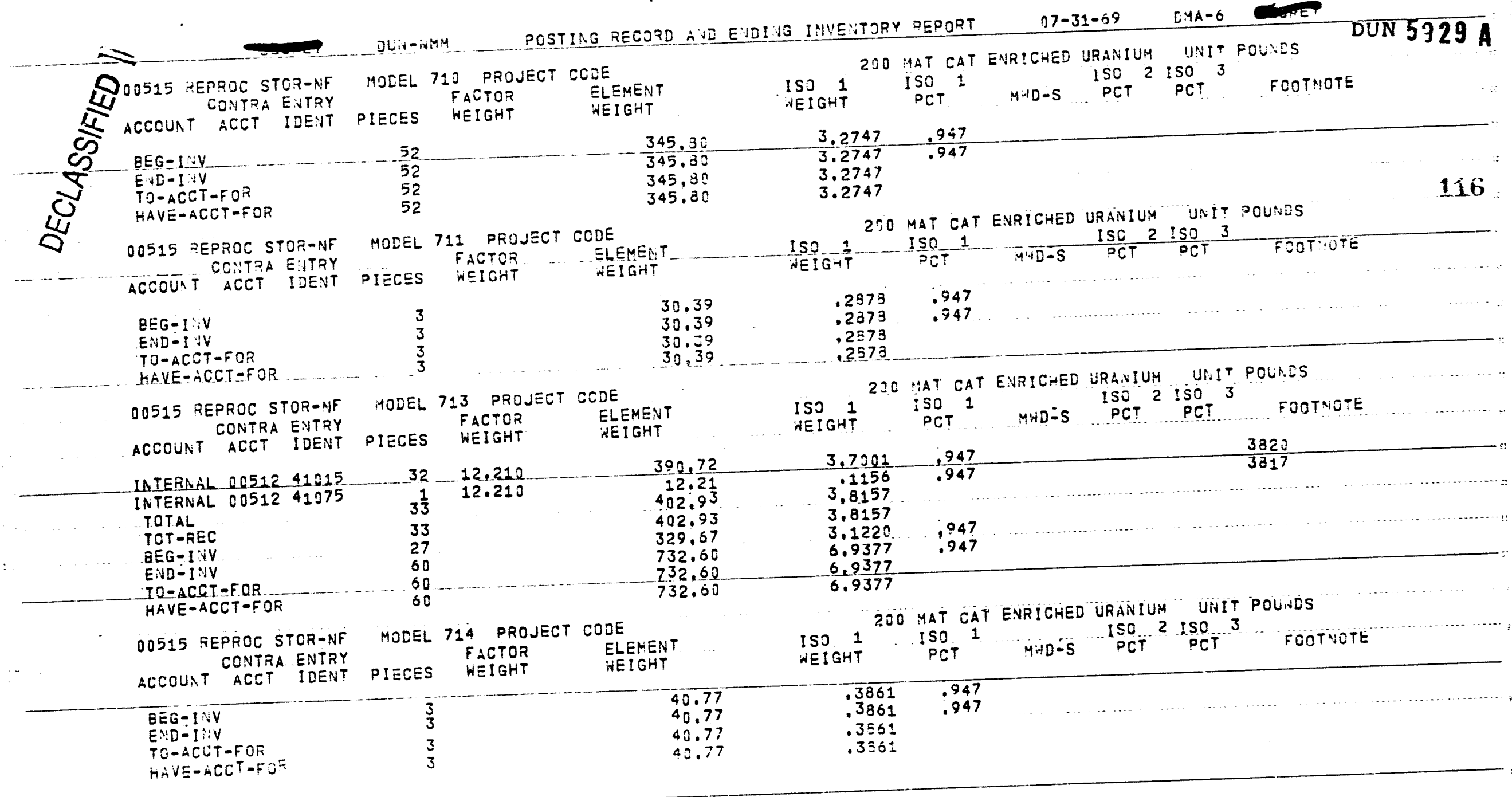


JUN-NMM

POSTI:U RECORD AVD E!DIIG IVYE TTORY REPORT

$07-31-69$

DMA-6

HOEEL 720 PROJECT COD

DOS15 REPRDC STCR-NF CCNTRA ENTRY
ACCOUNT ACCT IDENT $a E G-I M Y$
$E: D-I: V$ END - I V V V TS-ACCT-FOR

OO515 REPPOC STCR-HF ACCOUNT ACCT IDENT

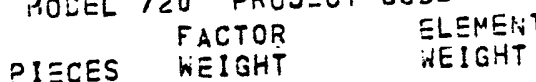

230 HAT CAT EVRICHED UPANIUM UNIT POLSUS ISJ $1 \frac{1}{T}$ $\begin{array}{lll}\text { ISO } 1 & \text { ISO } 2 \text { ISO } \\ \text { PCT } & \text { MHD-S } & \text { PCT }\end{array}$ FUOTNOTE

795
795
795
95

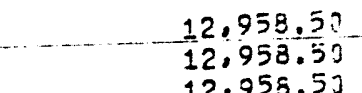

MODEL 722 PROJECT CODE

FACTOR MODEL ELEMENT

PIECES WEIGHT W.... NEIGHT

200 MAT CAT ENR?CHED URANIUY UNIT PQUNES

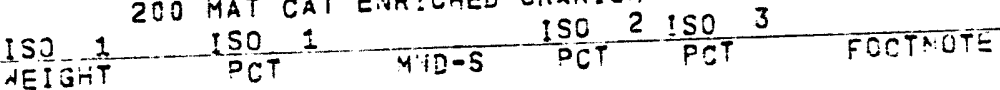

$\begin{array}{ll}.2735 & .947 \\ .2735 & .947\end{array}$

.2735

28.83

28.8

23.39

TO-ACCT-FOR

KAVE-ACCT-FOF

DO515 REPFOC STCR-NF CCYTRA ENTRY ACCOUAT ACCT IDENT

MODEL 723 PROJECT CODE FACTOR ELEMENT PIECES WEIGHT UEIGHT

200 MAT CAT ENRICHED URANIUY UHIT POLNES

UTERNAL C0249 41226

TOTAL

TOT-KEC

BEG-INV

TO-ACCT-FOR

TOVE-ACCT-EOR
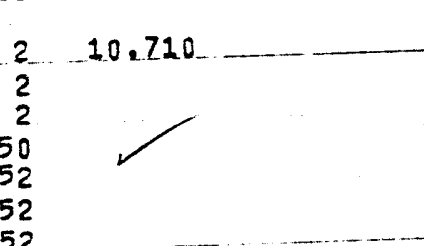

OOS15 REPROC STOR-NF CONTRA ENTRY ACCOUNT ACCT IDENT

MODEL 730 PROJECT CODE<smiles>[Z16][14CH]CCC</smiles>
ISO 1 ISO 1 MHDES ISO 2 ISO 3 PCT .... FOOTYOTE

$202^{\circ}$ .947 2029 5.0711 5,2739 5.2739

9947

$\begin{array}{rr}21.42 & 5.0711 \\ 535.50 & 5947\end{array}$

556,7

556.92 FACTOR ELEMENT

200 MAT CAT ENRICHED URANIUM UNIT POUNDS

ISO 1 ISO ${ }^{2}$ ISO 1 MHD-S ISO 2 ISO 3

FOOT:OTE

$\frac{.5727}{.5727}-\frac{.947}{.947}$

$\begin{array}{ll}50.48 & .5727 \\ 60.45 & .5727 \\ 50.48 & .947 \\ 69.40 & .5727\end{array}$

EMD $-1: V$

TO-ACCT-FOR
OAN

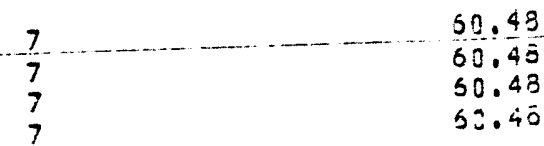

HAVE-ACCT-FOF 
07-31-69 DMA-6

DLIII-UMM

PCSTING RECORD AYD EMDING INVEVTJRY REPORT

200 MAT CAT ENR!CHED URANIUM UNIT POUSDS DUYN 5929 A

Q 00515 REPROC STCR-NF MODEL 733 PROJECT CODE

CONTRA EINTRY
ACCOUNT ACCT IDENT PIECES WEIGHT

INTERNAL 3051241074

INTERNAL 0051241076

TOTAL

BEG-INV
END-INV

TO-ÄCCT-FQR
HAVE-ACCT $-F O R$

$6 \quad 11.43306 .53$

6
2
8
8
31
39
39
39

0515 REPROC STOR-NF MODEL 822 PROJECT CODE

CONTRA ENTRY

ACCOUNT ACCT IDENT

22.66

91,44

91.44

354.33

445.77

$445,7 \frac{7}{445}$

$E E G-I N V$

TOA ACCT-FOR
END I IV

TO-ACCT-FOR
HAVE-ACCT $-F O R$

HEISHT

NEIGHT

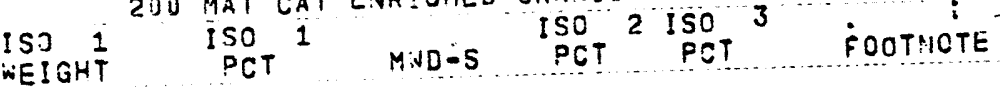

$\begin{array}{rr}.6495 & .947 \\ .2165 & .947\end{array}$

.8660

$3.3555 \quad .947$

$\begin{array}{ll}4.2255 & .947 \\ 4.2215 & .947\end{array}$

IIAT CAT ENRICHED URANIUM UNIT FOUNES

Q0515 REPROC STOR-NF YODEL 823 PROJECT CODE

Q0515 REPROC STCR-NF
CONTRA ENTRY
ACCOUNT ACCT IDENT PIECES WEITER

INTERNAL D0512 41073 INTERNAL 0051241105

IOIAL

$\begin{array}{rr}77 & 22.970 \\ 131 & 22.970 \\ 208 & \end{array}$

TOT-REC

BOT
BND-INV

TO-ACCT-FOR

HAVE-ACCT-FOR

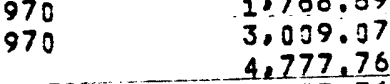

$4,777.75$
3.767 .03

3.767 .08

8.544 .64

$8,544.54$

ISO 1 ISO 1 MCT 1 ISD-S PCT POT 2 FOOTNOTE

00515 REPROC STOR-NF MODEL 824 PROJECT CODE CONTRA ENTRY

ACCOUNT ACCT IDENT PIECES WEIGHT

24 PROJECT CODE
FACTOR
WEIGHT ELEMENT

$$
\begin{array}{ll}
.8781 & .947 \\
.8781 & .947 \\
.8781 & \\
.8781 &
\end{array}
$$

200 MAT CAT ENRICHED URANIUM UNIT POUNDS

EEG $-I: V$

TUD-I:IY

HAVE-ACCT -FOR

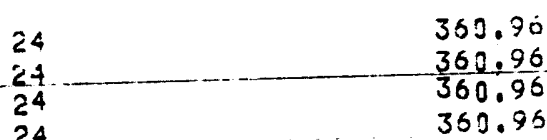

350.90
360.96
360.96

360.95

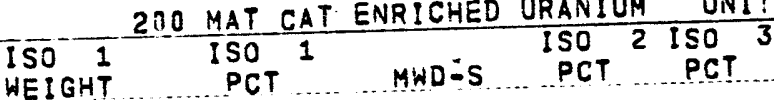

$16.7495 \ldots 94$

$28.4959 \quad .947$

45,2454

45.2454

$35.6742 \ldots .947$

$80.9196 \quad .947$

80.9196

80.9196
30.9196

200 MAT CAT ENRICHED URANIUM UNIT POUNDS

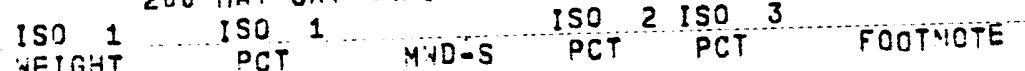

$3.4183 \quad .947$

$3.4183 \quad .947$

3.4183

47 


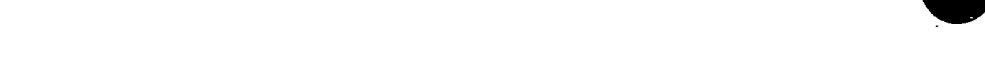

DUiv-ivM POSTIYG PECOPD AUD EIDIVG ISIVENTOPY REPORT $07-31-69$ DMA-6

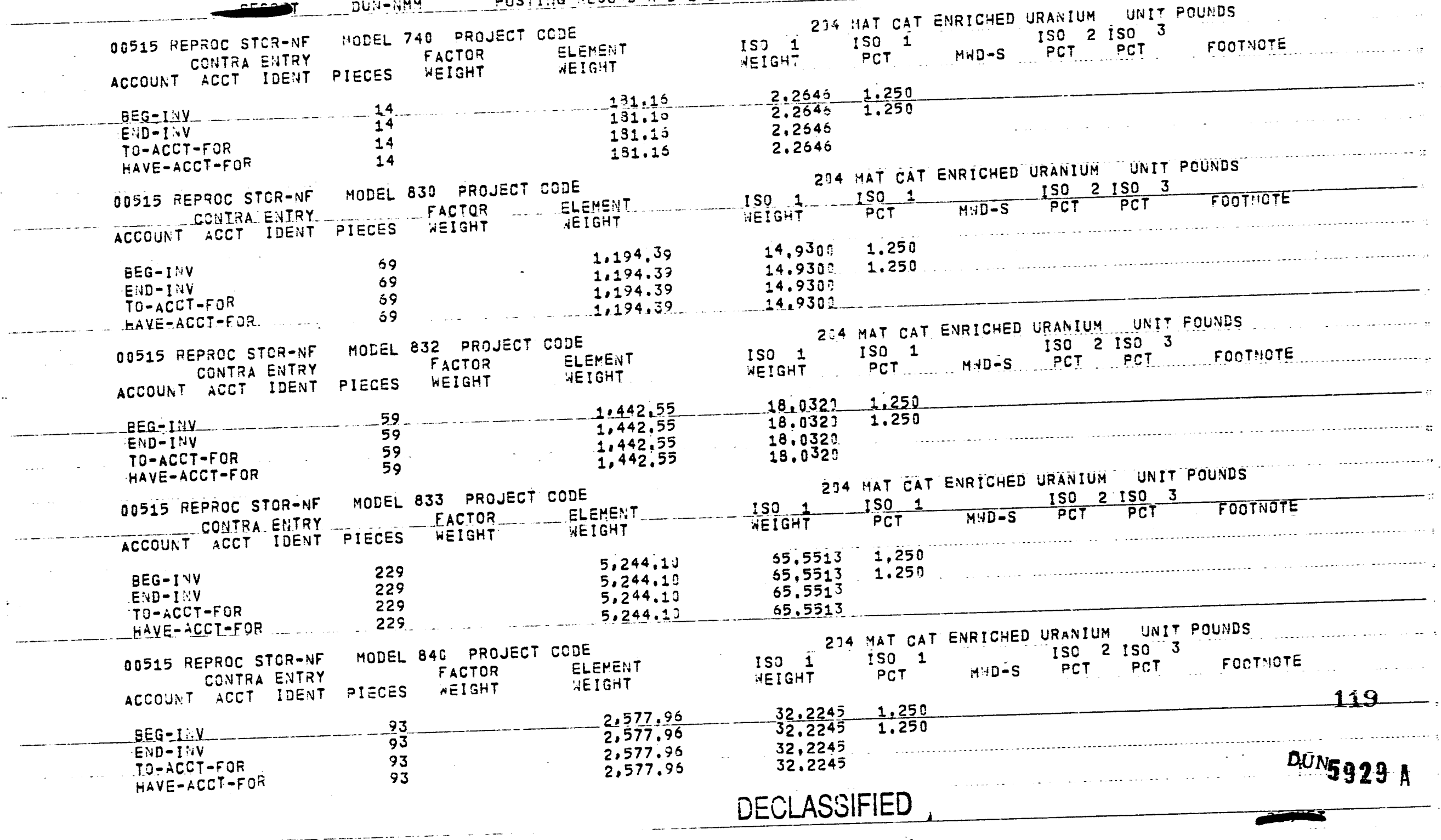


:

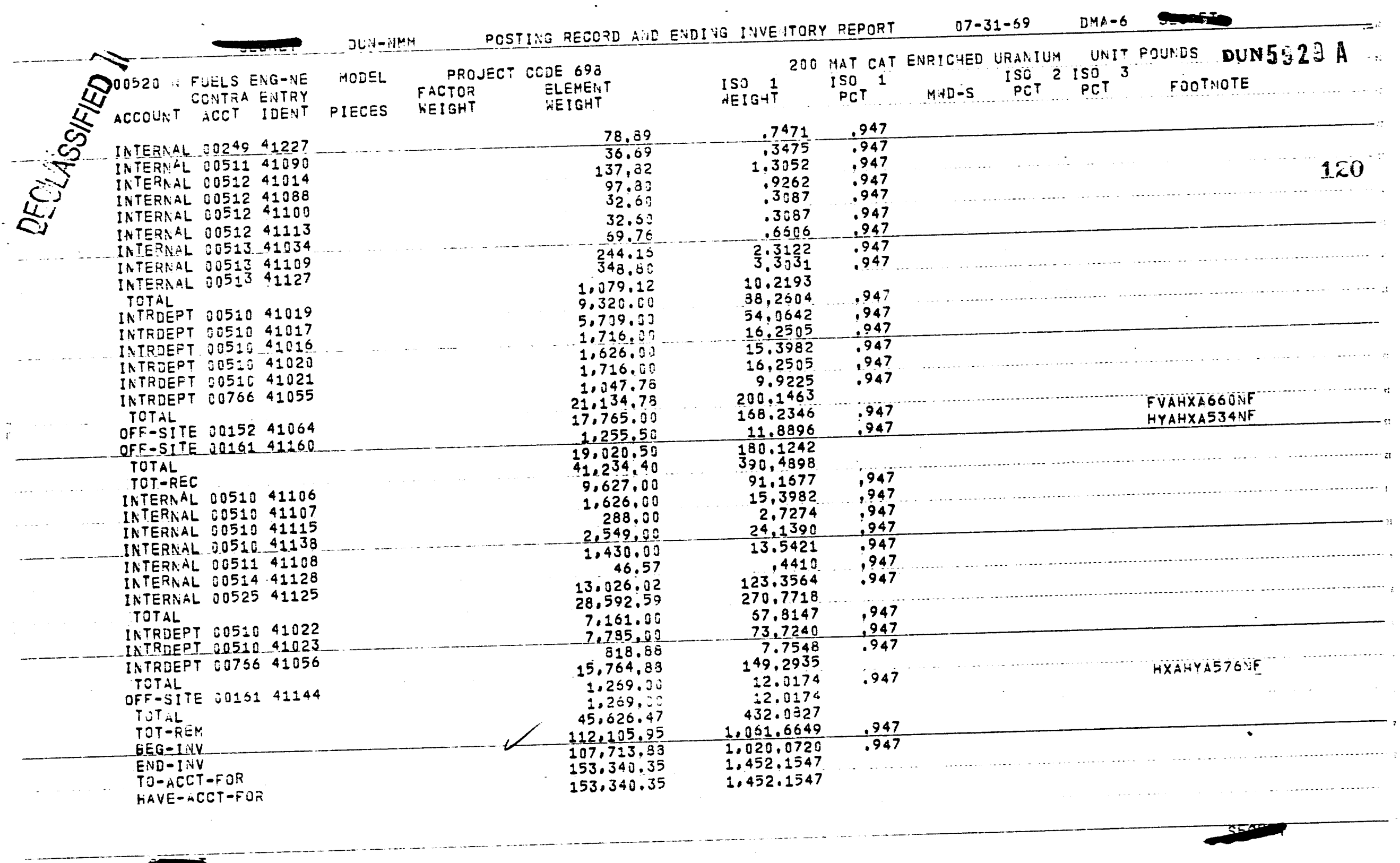


$: \quad 077-31-69$ DMA-6

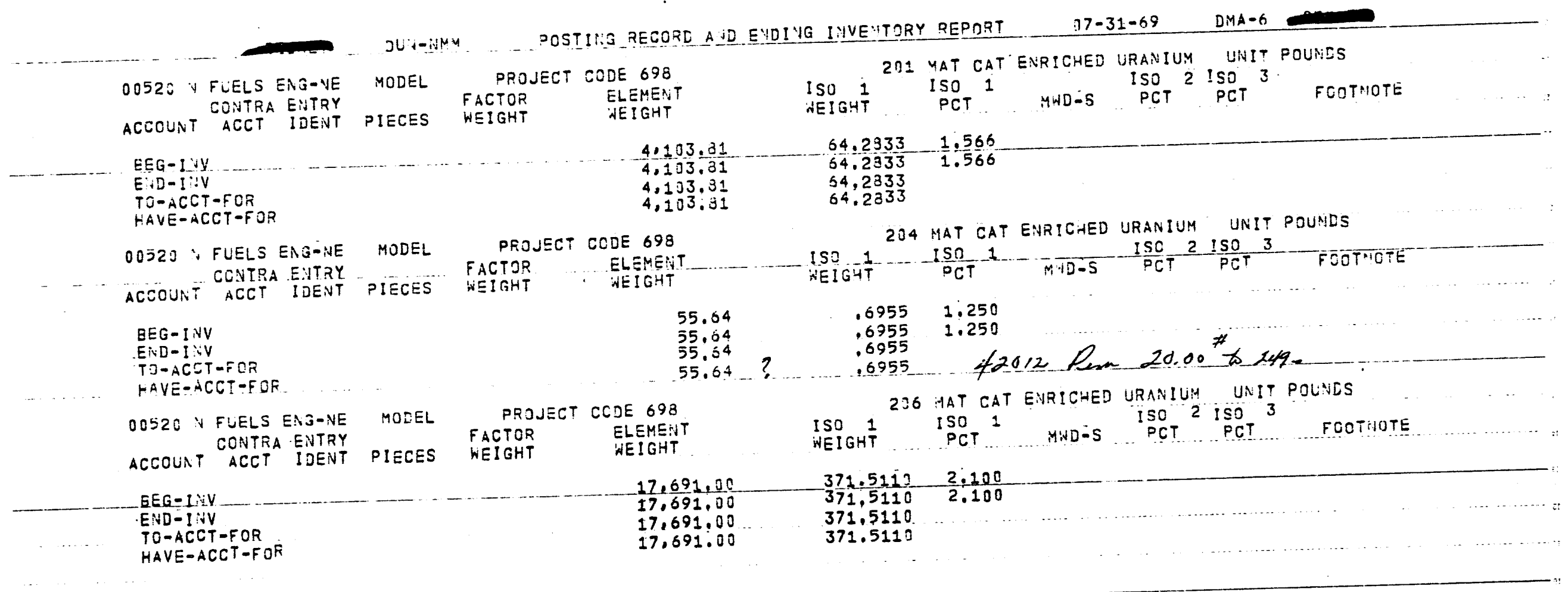




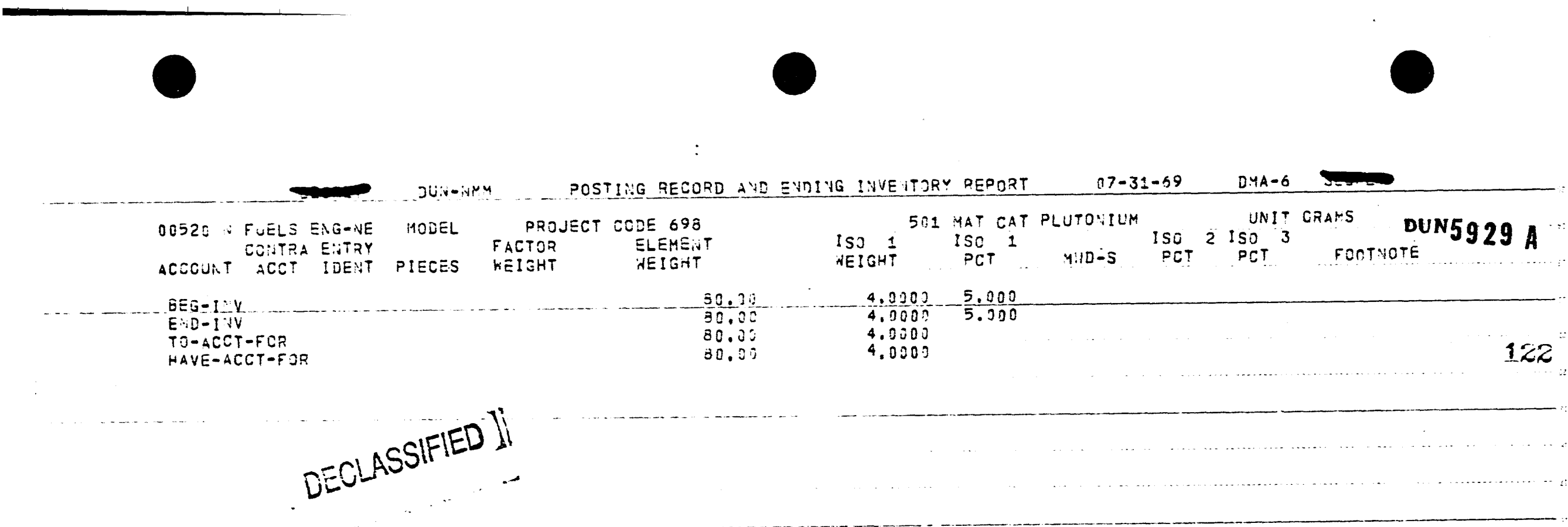




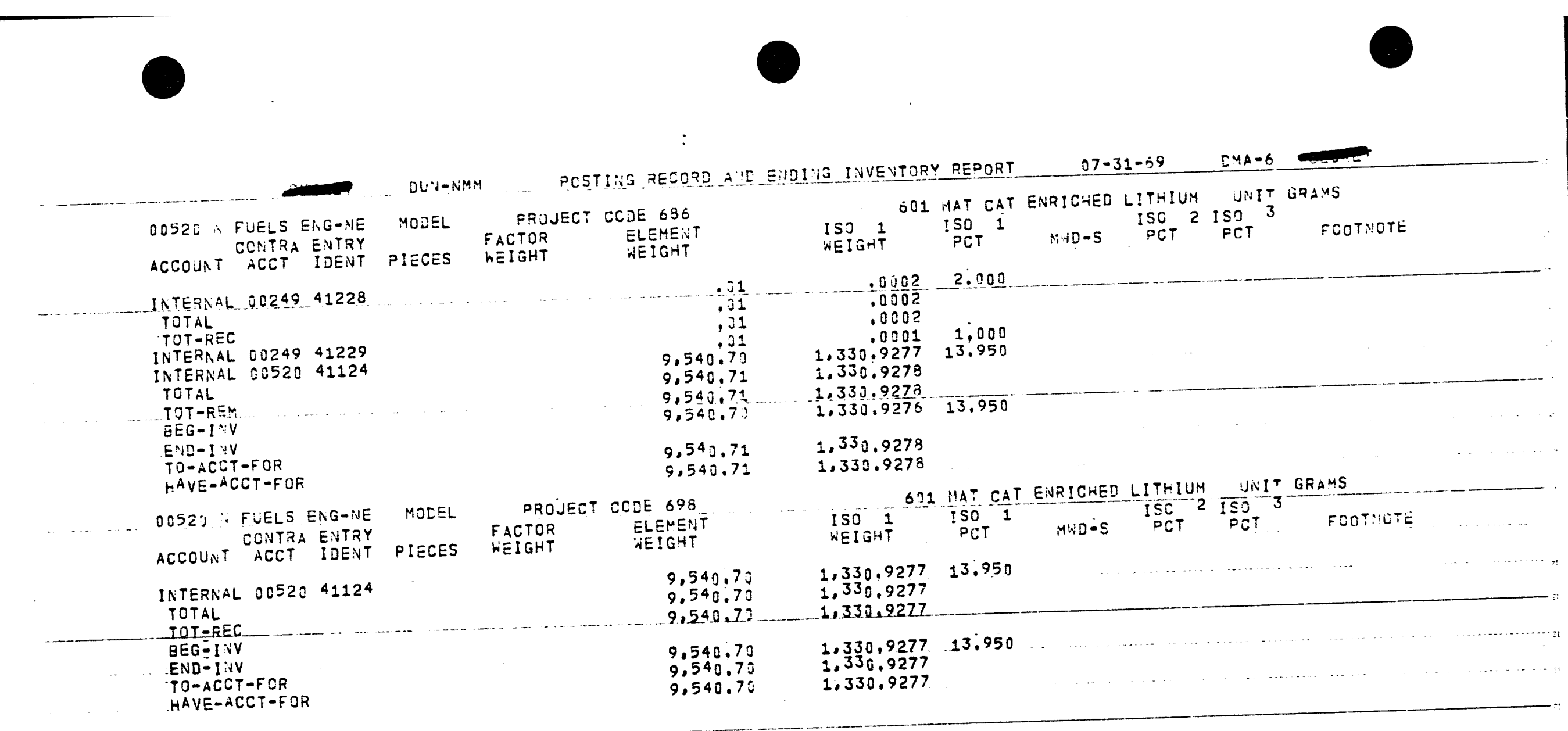




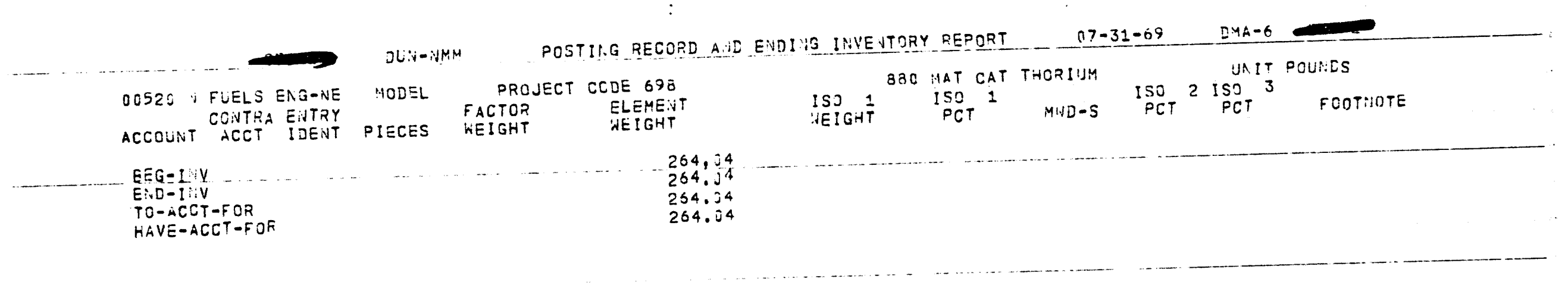


$07-31-69$ DMA-6

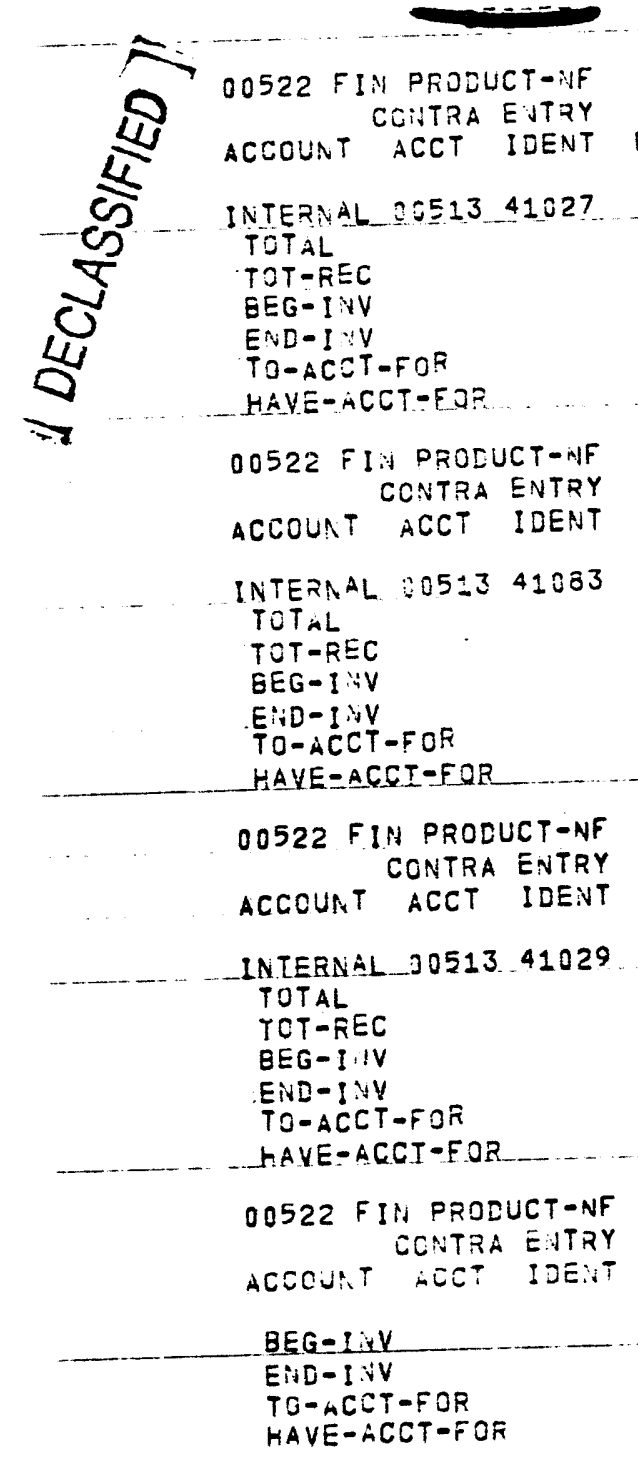

DLiA-iNM

POSTING PECOPD A IE ENDI IG INVEVTORY REPORT 200 MAT CAT ENRICHED URANIUM UNIT POUIDS

MOLEL 710 PROJECT CCOE

FACTOR ELEMENT WACTORT WEIGHT

ISO 1 ISO 1 MOT 1 ISO 2 ISO 3 PCT FCOTIOTE PIECES

EIGHT

\begin{tabular}{ll}
2.0152 & .947 \\
\hline 2.0152 & \\
2.0152 & \\
56.6282 & .947 \\
68.6434 & .947 \\
58.6434 & \\
53.6434 &
\end{tabular}

$\begin{array}{rrrr}32 & 6.650 & & 212.38 \\ 32 & & & 212.30 \\ 32 & & & 212.30 \\ 1.058 & & & 7.035 .70 \\ 1.090 & & 7.248 .50 \\ 1.090 & & 7.248 .50 \\ 1.090 & & & 7.248 .50\end{array}$

MOLEL $7 \pm 3$ PROJECT CODE

FACTOR PROJECT CODE

PIECES WEIGHT IVEIGHT

$\begin{array}{rrr}17 & 12.210 & 237.57 \\ 17 & & 237.57 \\ 17 & & 297.57 \\ 2.412 & & 29.450 .52 \\ 2.429 & & 29.658 .09 \\ 2.429 & & 29.653 .09 \\ 2.429 & & 29.653 .29\end{array}$

ISO

20 MAT CAT ENRICHED URANIUN UNIT POLNLS

PCT MUD-S DCT PCT

$\pm .0657 \quad .947$

1.9657

$278.8931 \quad: 947$

$\begin{array}{rr}278.8931 & .947 \\ 230,8583 & .947\end{array}$

230,8588

280.8583

714 PROJECT CODE

MOEEL 714 PROJECT CODE
FACTOR ELEMENT

PIECES WEIGHT WEIGHT

ISO 200 MAT CAT ENRICHED URANIUM IS ISO 1 ISO 2 ISO 3

WEIGHT PCT MWDES PCT PCT FOOTNOTE

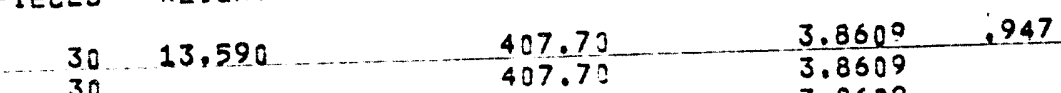

$\begin{array}{rrrr}30 & 407.75 & 3.8609 & \\ 30 & 48.964 .77 & 463.6759 & : 947 \\ 3.603 & 49.372 .47 & 467.5368 & .947 \\ 3.633 & 49.372 .47 & 467.5568 & \end{array}$

$\begin{array}{lll}3.633 & 49.372 .47 & 467.5568 \\ 3.633 & 49.372 .47 & 467.5569\end{array}$

3.633 -

MODEL 715 PROJECT CCDE

FACTOR PLEMEIT
DIECES WEIGHT NEIGHT

200 MAT CAT ENRICHED URANIUM UNIT POLNDS

\begin{tabular}{rrrr}
197 & 3.235 .95 & 31.1195 & .947 \\
\hline 197 & 3.285 .96 & 31.1198 & .947 \\
197 & 3.285 .95 & 31.1193 & \\
197 & 3.235 .96 & 31.1199 &
\end{tabular} 
DUiviam

DOSTII.S RECORD AUE ENDI IG INVE'TTPY FEPORT

77-31-59

$3: 4 A-6$

00522 FIIV PRODUCT-NF ACCOUNT CONTAA ENTRY $E E G=I: V$

END - IIV

TO-ACET-FOA

HAVE-ACCT-FOR

OO522 FIN PROEUCT-NF COTZA ENTRY ACCOUNT ACCT IDE'IT

INTERNAL O0513 41079 INTERIIAL ODJ13 41033 INTERMAL O0513 41099 INTERIAL BO513 41J30 HTEPNAL $=05: 3+1117$

TOTAL

INTRDE?

TOT-FEC

TOTOREC

TOTAL

-TOF-REM

EEG-IVV

END-I I

TO-ACCT-FOR

HAVE-ACCT-FOR

OO522 FIN PROEUCT-NF

CCHTRA ENTRY

ACCOUAT ACCT IDENT

NTERAAL 0051341081

INTER:AL 0051341031

TOTAL

TOT-ZEC

TOT iL

EEG-IVV

E.iD-I V

TO-ACCT-FOR

HAVE-ACCT-FOC
MODEL 716 PROJECT CCDE

FACTOR
PIECES WEIGHT NEMENT

18
18
12
18

MODEL 720 PROJECT CODE

FACTOR
PIECES WEIGHT NEME:TT

$\begin{array}{rrr}238 & 16.300 & 4.694 .40 \\ 252 & 16.300 & 4.107 .50 \\ 72 & 15.300 & 1.173 .50 \\ 324 & 15.300 & 5.251 .20 \\ 36 & 15.300 & 536.50 \\ 972 & & 15.043 .00 \\ 124 & & 2.321 .20 \\ 124 & & 2.021 .20 \\ 1.096 & 16,300 & 17.354 .80 \\ 340 & 5.542 .00 \\ 340 & & 5.542 .00 \\ 340 & 5.542 .00 \\ 1.480 & & 24.124 .00 \\ 2.236 & & 36.446 .80 \\ 2.576 & & 41.983 .00 \\ 2.576 & & \end{array}$

MOEEL 721 PROJECT CODE

FACTOR ELEMENT

WEIGHT WEIGHT

15.310

15.310

15.312

258.12

258.12
ISP

230 MAT CAT EVRICHED URANIUH UNIT POULDS

GHT PCT MND-S PCT PET FCOTHOTE

$2.4444 \ldots .947$

$2.44 \overline{44}$

2.4444

2.4444

11T CAT ENRICHED URANIUA UM:T POLNES

230 AAT CAT ENR!CHED URANIUIA UNIT POLNDS

ISO 1 ISIGT

EIGHT

$\begin{array}{rr}44.4560 & .947 \\ 38.8790 & .947 \\ 11.1140 & .947 \\ 50.0130 & .947 \\ 5.5570 & .947\end{array}$

150.0390

120.0300

19.1403

169,1798

52.4327

52,4327

52,4327

228.4548

345.1519

397.6346

.947

.947

$2120 \times 4$
$2113-4-6$

2128

$2117-9$

2129

79

.947

200 MAT CAT ENRICHED URANIUM JHIT POLNDS

ISO 1 ISO 1 PCT MNDE ISO PCT ISO 3 PCT FOTMOTE

$10.4390 \quad 947 \quad 2122-4$

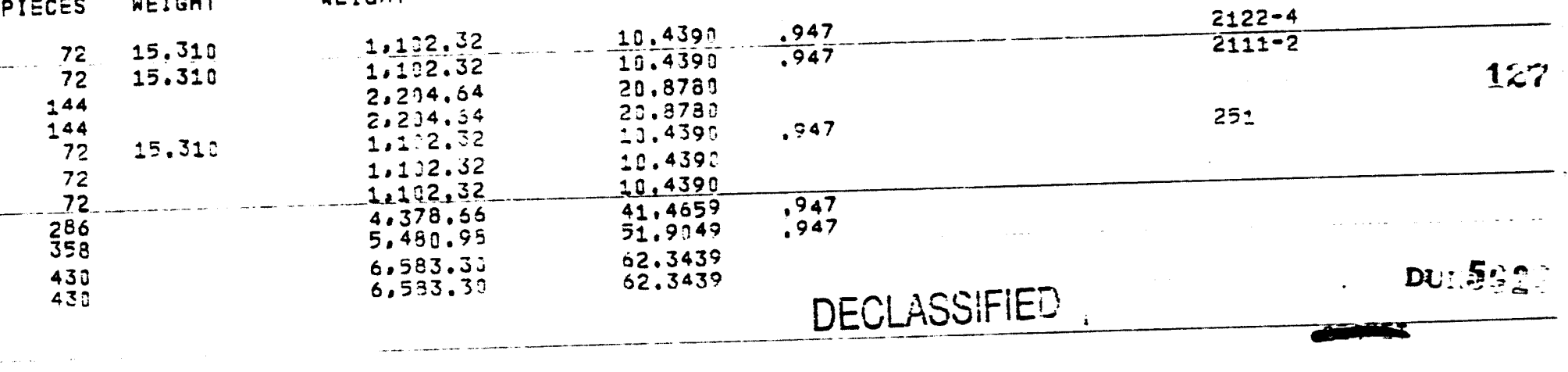


DYA-6

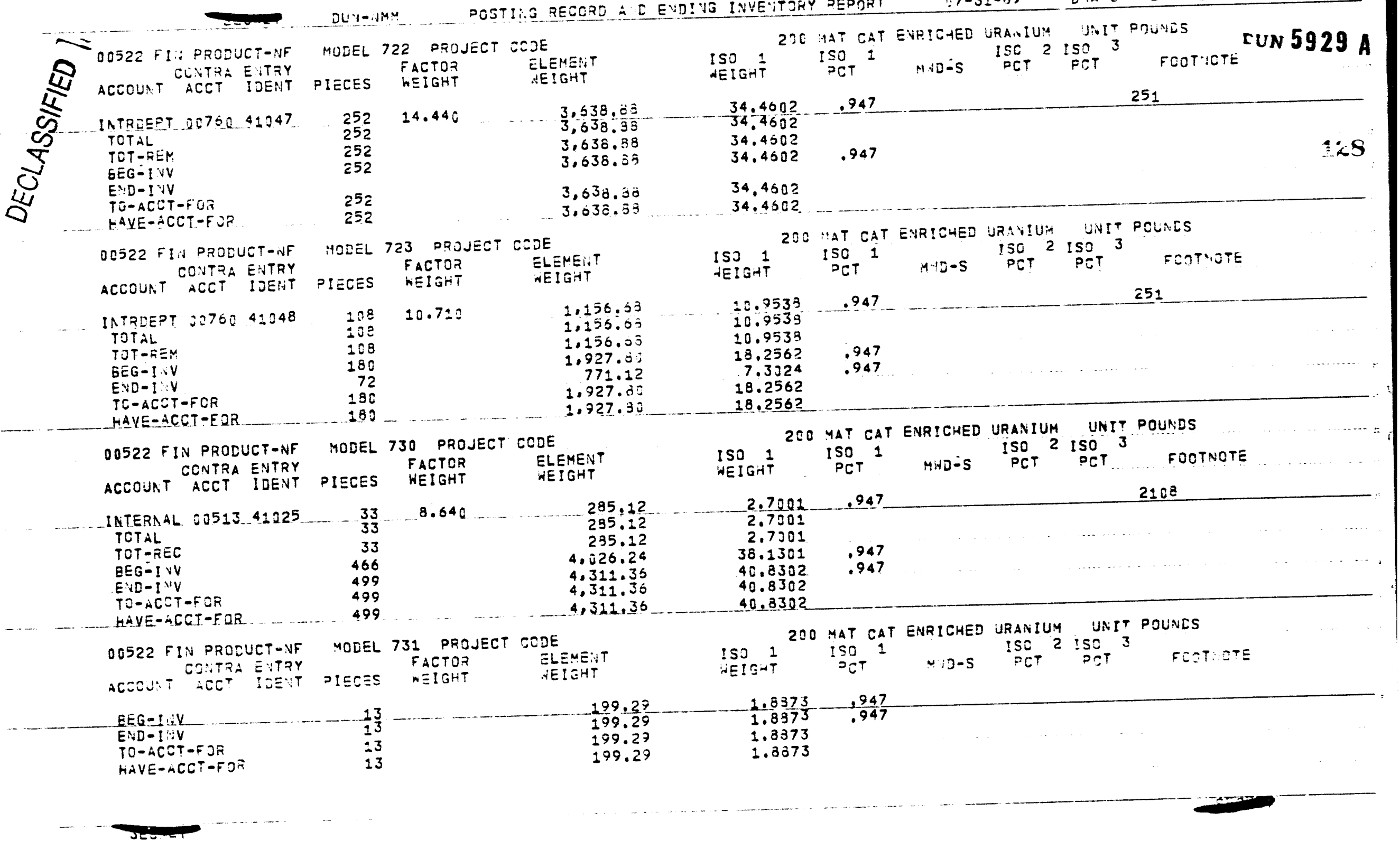




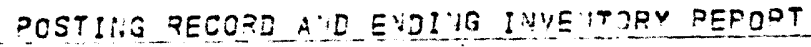

$07-31-69 \quad 2 \times 4-6$

00522 FI: PRODUCT-NF COCOUAT COTSTA EITTRY

DLVIVIM $\begin{array}{lll} & \text { FACTOR } & \text { ELEME:TT } \\ \text { PIECES WEIGHT } & \text { HEIGHT }\end{array}$

$2,468 . \overline{8} \quad 23,3303$ $2,458.83 \ldots 23.3503$ $\begin{array}{ll}2.458 .35 & 23.3303 \\ 2.468 .35 & 23.3303\end{array}$

$E G-1:$

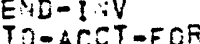

HAVE-ACCT-FOR

00522 FIN PRODUCT-NF

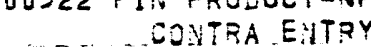
ACCOURT ACCT IDENT

INTERNAL C0513 41026 TOTAL

TOT-FEC

EEG-I:V

EAD-IN

HAVE-ACCTAFOA

OO522 FIN PROLUCT-NF

OOS22 FIN POROLUCTENF EUTRY

ACCOUNT ACCT IDENT

INTERNAL O0513 41228

TOTAL

TOT-REC

$B E G-I Y V$

END- I Y Y

TO-AC:T-FOR

HAVE-ACCT-FOP

00522 FIN PROELCT-INF

OOS22 FIN PRODUCTONF

- ACCOUAT ACCT IDENT

EEG-I:V

E ID-I IV

TO-ACST-FOC

HAVE-ACCI-FO

216
216
216
216

MOEEL 810 PROJECT LCDE

$\begin{array}{rrr}32 & 13.350 & 427.23 \\ 32 & & 427.20 \\ 32 & & 427.23 \\ 1.058 & & 14.124 .32 \\ 1.090 & 14.551 .3 ? \\ 1.070 & 14.551 .52 \\ 1.090 & 14.551 .50\end{array}$

MOLEL 814 PROJECT CODE

$$
\text { FACTOP }
$$

PIECES

$\begin{array}{r}30 \\ 30 \\ 30 \\ 3,603 \\ \hline\end{array}$

27.210

816,30
816,30
816,30
98.037 .63
98.353 .93
$98.853,93$
98.353 .93

3.633

3,633

98.353 .93

HODEL 815 PROJECT CCDE

FACTOR ALEMEINT

$$
\begin{aligned}
& \text { FACTOR } \\
& \text { WEIGHT ELEMEINT }
\end{aligned}
$$$$
3.3303
$$
MOEEL 733 PROJECT CCOE

FACTOR ELEMEATT

230 HAT CAT ENRICHED URANIU:A UNIT POL:DS

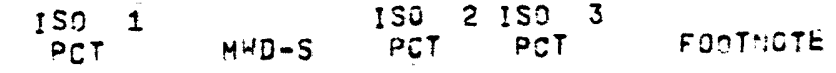

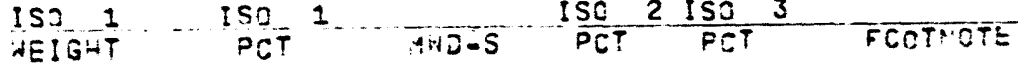

200 MAT CAT ENR!CHED URANIUM UNIT POLNCS

4.0456
4.0456
4.0456
$133.757 ?$
137.8225
137.8025
137.8026

9472109

.947

$.94 \overline{7}$

200 MAT CAT ENRICHED URANIUM UNIT POLNLS ISO 1 ISEIGHT $\frac{\text { ISO } 1 \text { PCT }}{\text { MHD-S }}$ PCT 2 ISO 3 FOT

$\begin{array}{rr}7.7304 & .947 \\ 7.7304 & \\ 7.7304 & \\ 928.4164 & .947 \\ 936.1460 & .947 \\ 936.1465 & \\ 936.1469 & \end{array}$

230 MAT CAT ENRICHED URANIUM UNIT POUNOS

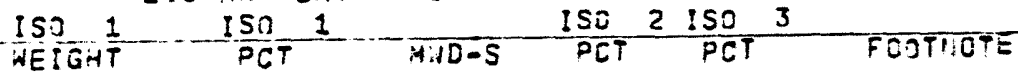

$\begin{array}{llll}197 & 5.732 .75 & 54.2347 & .947 \\ 197 & 5.732 .7 & 54.2947 & .947 \\ 137 & 5.732 .77 & 54.2947 & \\ 197 & 5.732 .73 & 54.2647 & \end{array}$


7-31-69 DYA-6

PUT-NMY POSTING RECORD AIL ENDIIG IIVEYTORY REPORT

2 TO MAT CAT ENRICHED JRANIUM UNIT POUNDS

MODEL B16 PROJECT CODE

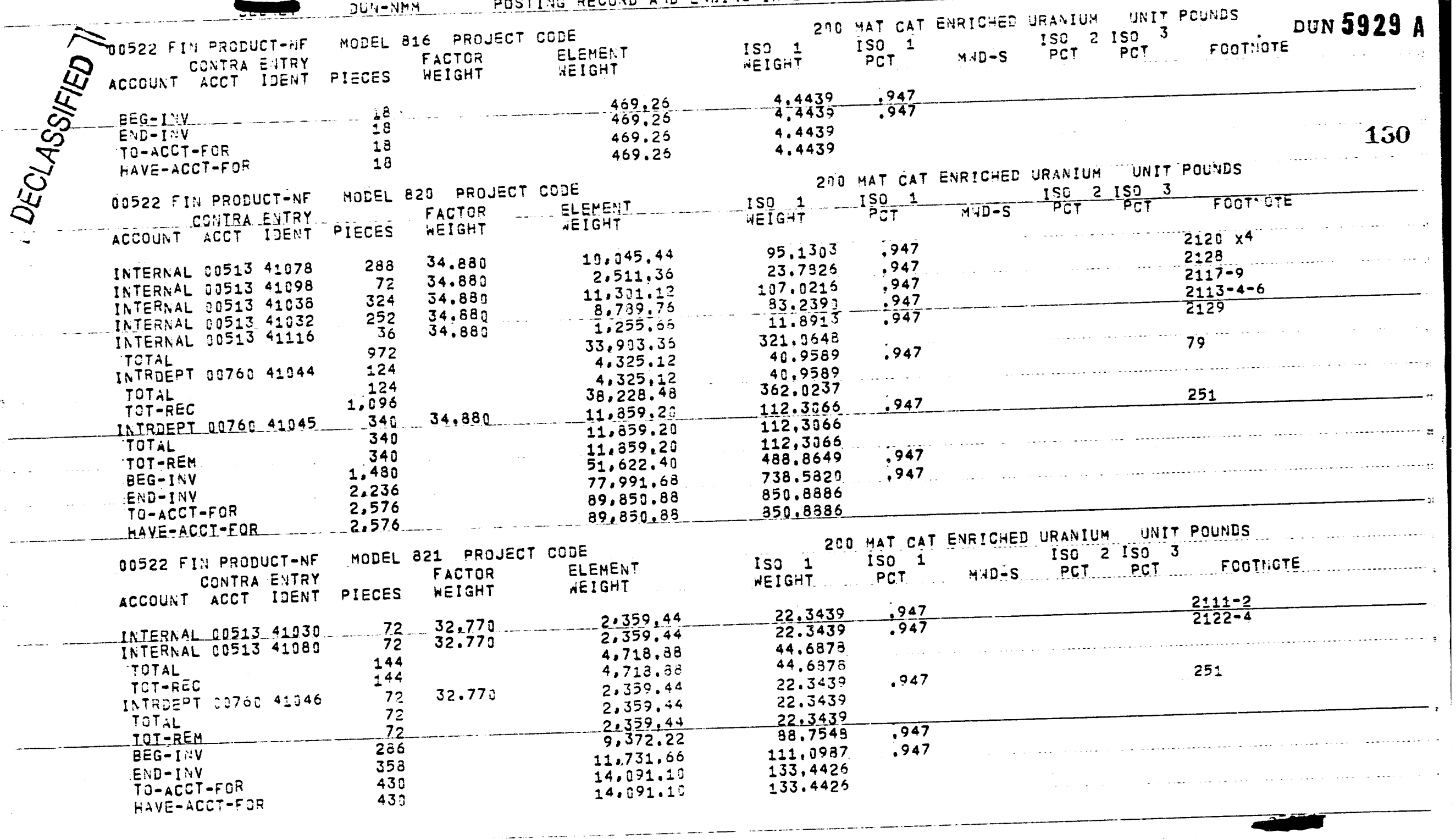




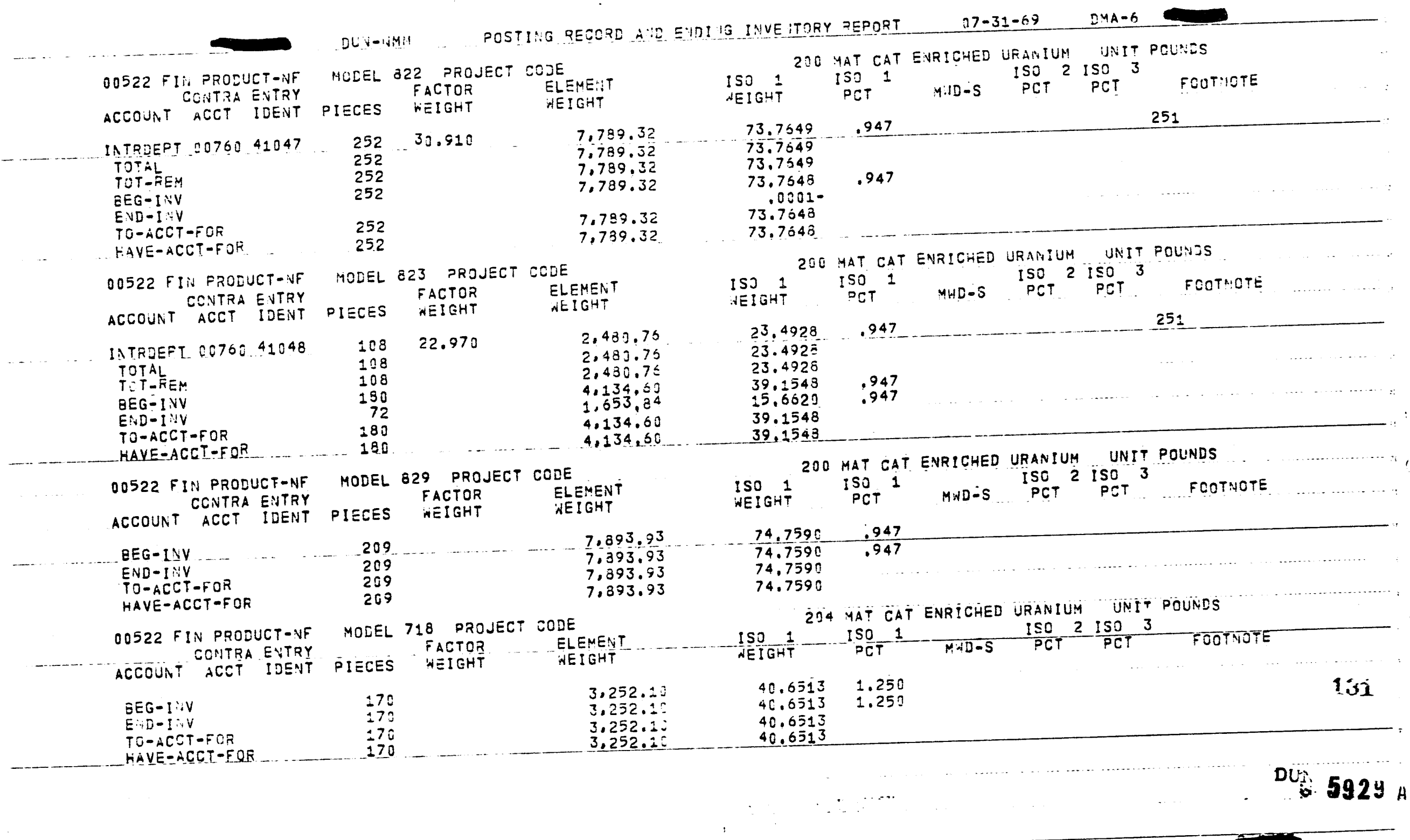


DUL-IIMM

POSTING RECORD A ID EODI IG INVENTORY REPORT

$07-31-69$

D.MA-6

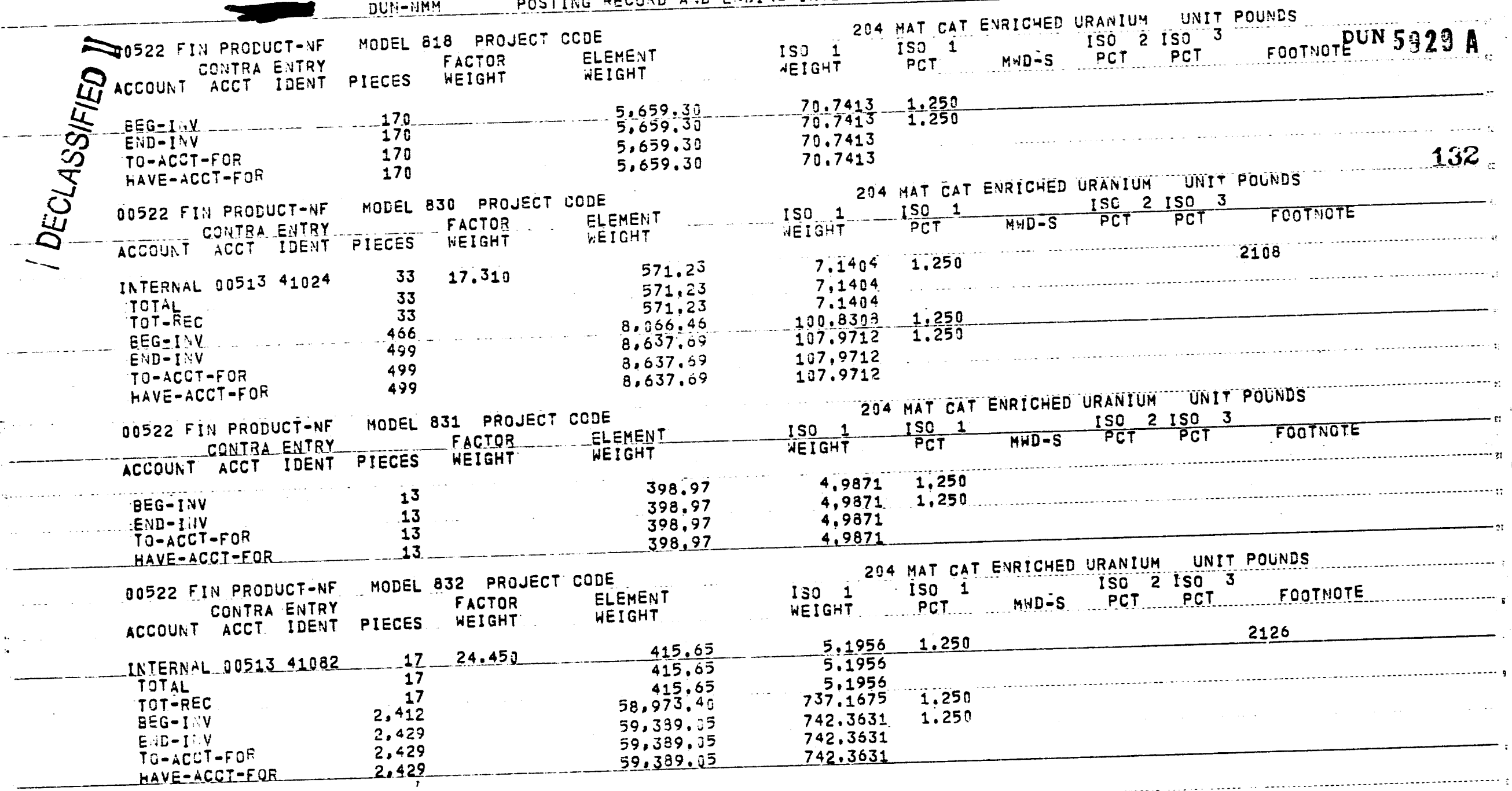




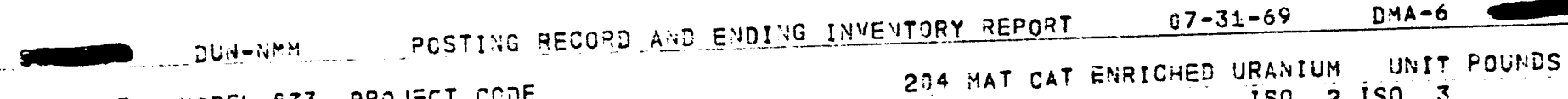

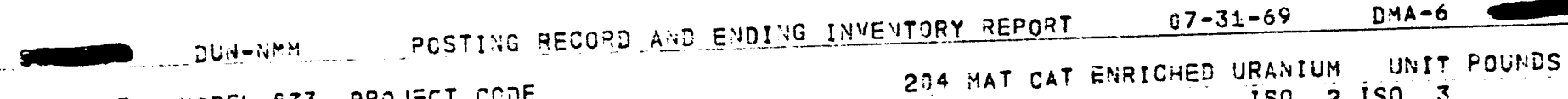

00522 FIN PFCEUCT-NIF MOLEL 833 PROJECT CCDE

CONTRA EUTRY PITE PIECES WAETORT
ACCOUNT ACCT IDENT

EEG-1:U

END-I.VV

TO-ACCT-FOA

HAVE-ACCT-FOR
216

216

216
ISJ 1

ISO 1 ISO 2 ISO 3

FOOTNOTE EIGHT

$61.8500 \quad 1,250$

$1.2500 \quad 1.250$

$4.946 .40-1.6 \frac{61.830}{51.830 n}$

4.946 .40
4.946 .40 $\quad 51.8300$

4.946 .40

61.8300

DECLASSIFIED 

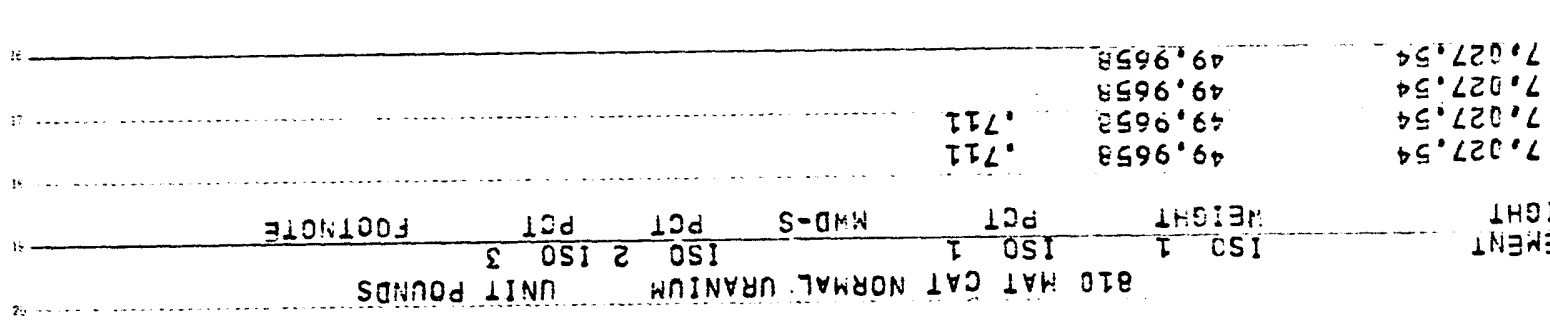

ESI $\begin{array}{r}8056^{\circ} b 2 \\ 8056^{\circ}+2 \\ \text { IIL: } \quad 5056^{\circ}+2 \\ \hline \text { ITL: }\end{array}$ เNヨฝヨา rolot

$20 \Omega$
$20 \Omega$
208

208

टण

SรวId $7 \exists 30 \mathrm{w}$

उ०ू

502
502
505
505

उอร

$+2^{\circ} 605 \cdot \varepsilon$

$\frac{32}{2} \cdot 605 \cdot \frac{2}{2}$

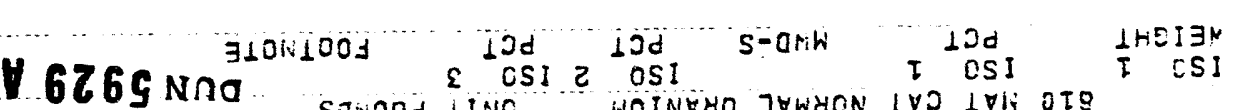

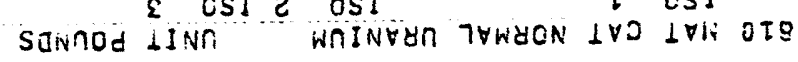
1H9I
$1 H E I \exists M$

YODJOH

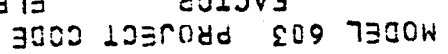

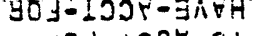

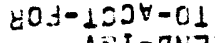

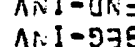

$11: 301 \quad 1202 \quad 1$ VnONO2

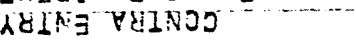
INA-100008d 11 \& 22500

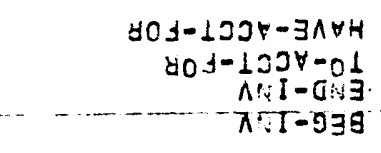

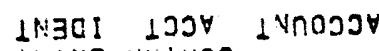

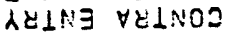

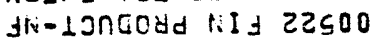


$07-31-69$

Di1A-6

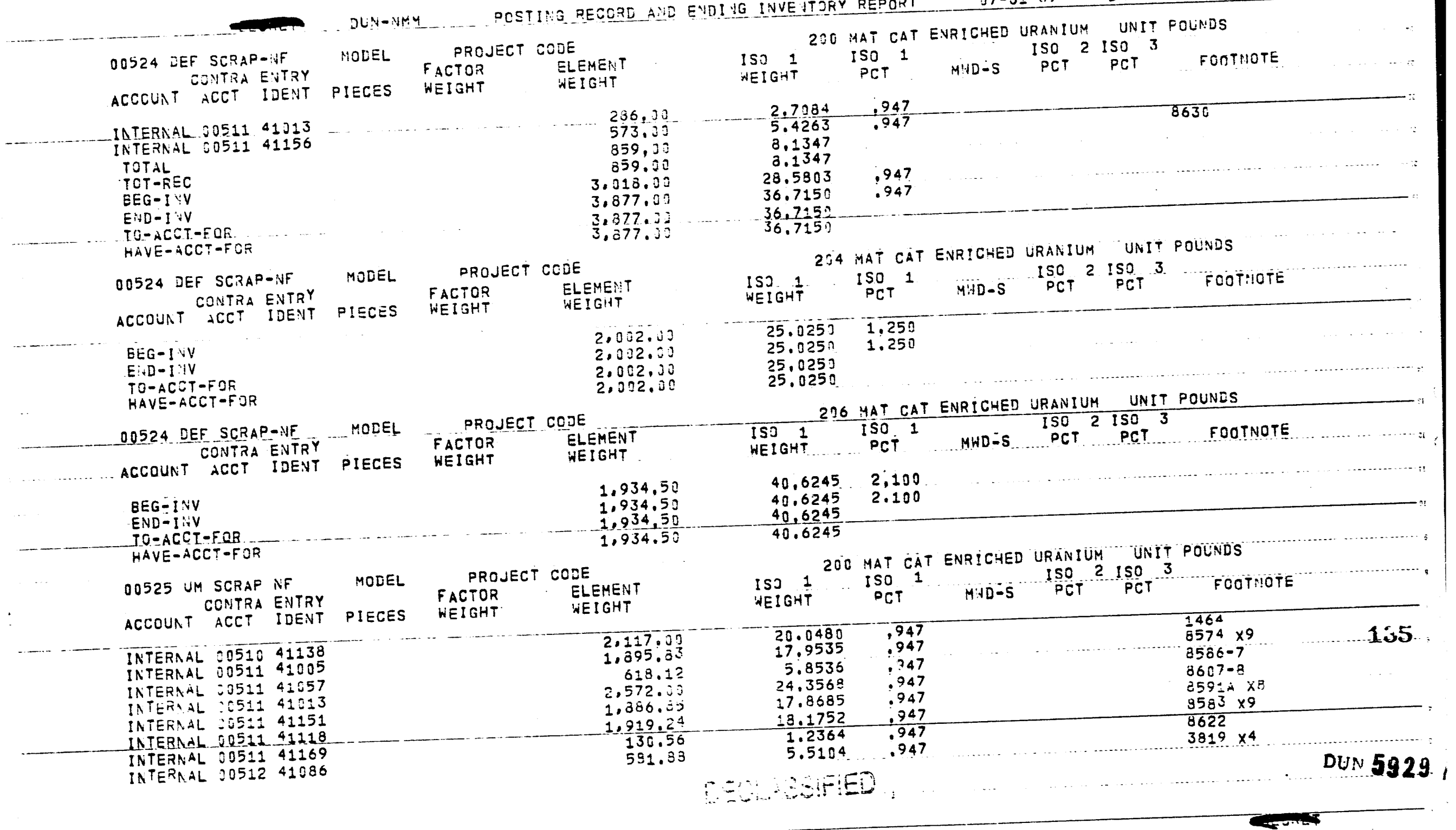


D.1A-6

DUit-yry

POSTILO RECOPD AUD EVDIVG INVE TTORY REPORT

$07-31-69$ UNIT FOUNDS

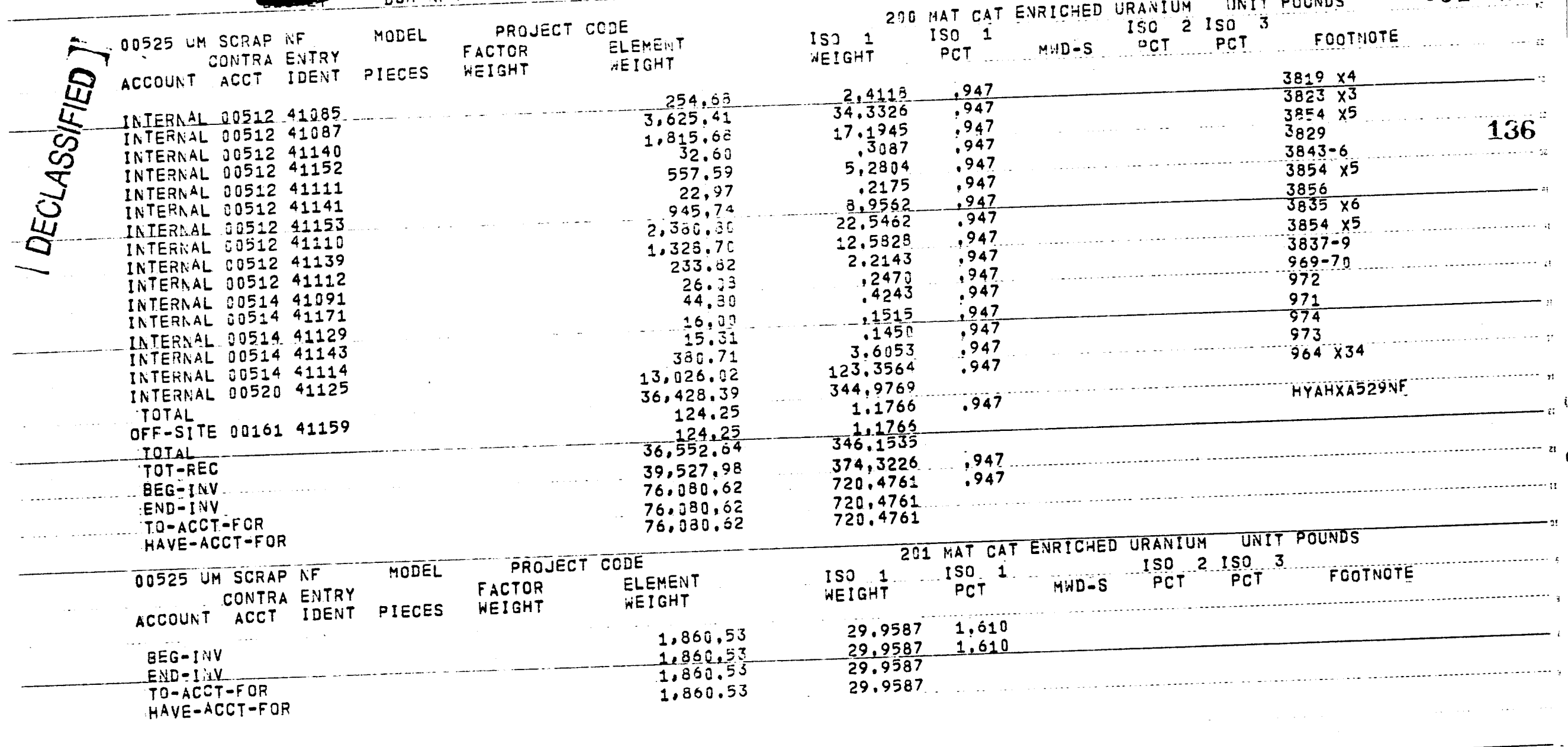


:

INVEMTOEY REPORT $07-31-69$ D:14-6

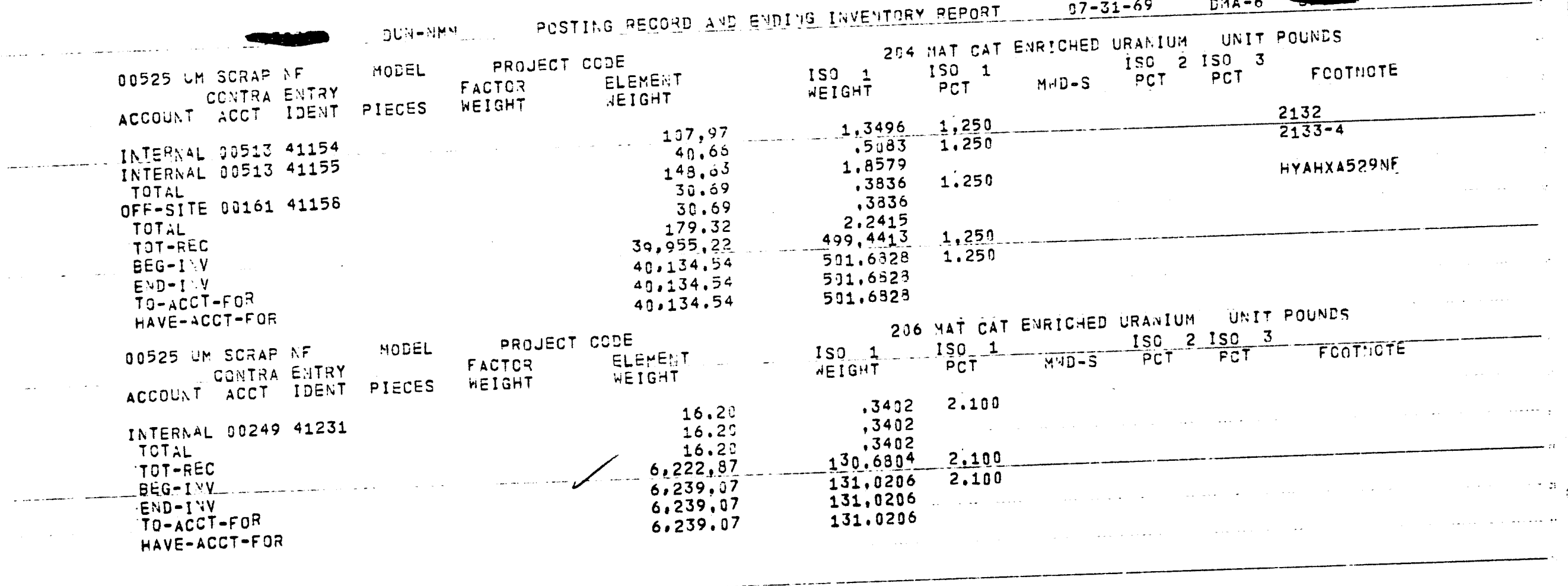

10 ' 


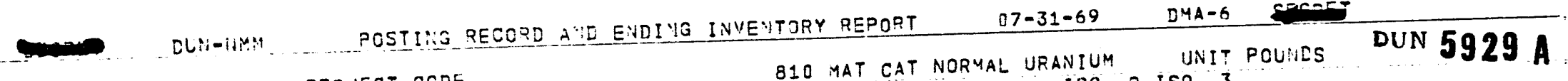

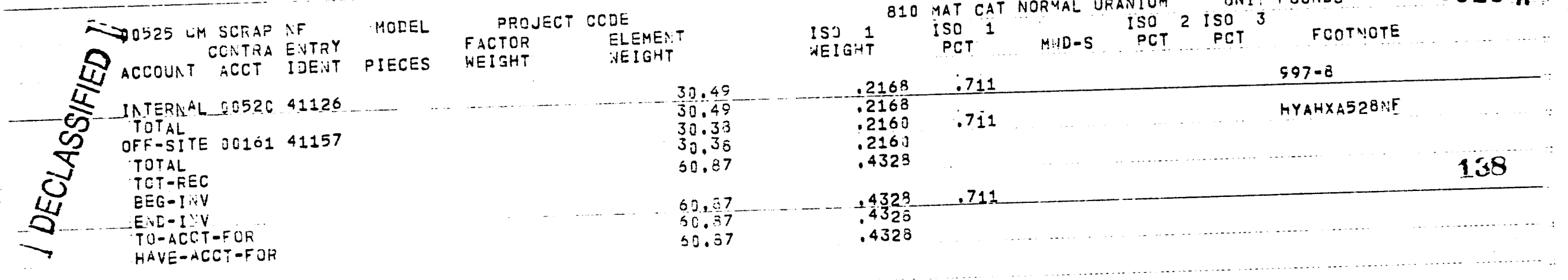


$\cdots-$

D0520 OXIEE ALRI-NF ACCOUNT CONTRA EITRY

INTERNAL CC207.41071

INTERKAL 0020741072

INTERNAL 00237 41095

INTERNAL O020741103

INTERAAL OQ207 41103

INTERNAL j0207 41161

INTERNAL J02J7 020741168

TOTAL

TOT-REC

INTERNAL O0527 41122

TOTAL

TOT $=\bar{R} E M$

EEG-I

TO-ACCT-FOR

HOAVE-ACCT TOF

00526 OXIDE BURM-NF

ACCOUNT $\frac{\text { CCNIRA ENTRY }}{\text { ACCT IDENT }}$

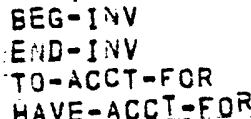

BEG-INV

END-INV

TO-ACCT-FOR

HAVE-ACCT-FOA

DO526 OXIDE OURN-NF MODEL

00526 AXIDE BURN ONF

ACCOUNT ACCT IDENT

$E E G=1$ iV $Y$

END-I IN

TO-ACCT-FOF

HAVE-ACLT-FOA

MODEL

PIECES

PIECES
DUid-idMM

POSTILG RECOSD AUD ENOIUG IUVEYTORY REPORT

$27-31-69$

DMA-6

UNIT POLNDS

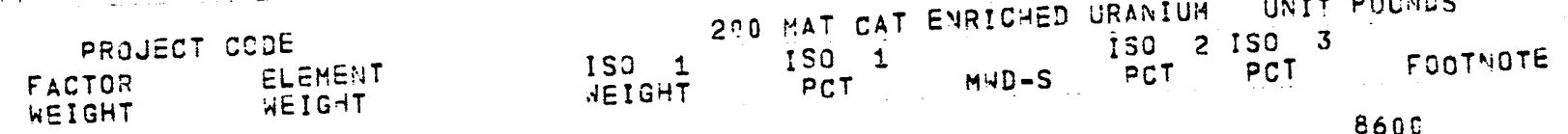

$1,061,0$

893.32

$117 .:$

174.00

137.00

322.20

$2,340$.

2.840 .5

5.419 .30

$5,419.00$

$5,419.35$

16.439 .0

13.360 .0

19.279 .00

19.279 .00

PROJECT CODE

FACTOR

WLEMENT

10.0477

$10.0477 \quad .947$ 8600

$8.4567-.947 \quad 3812 \times$

1.1080

1,6475

1,2974

3.0493

1.2379

26.8945

51.3179

51,3179

51.3179

155.6015

131.2585

132,5764

WEIGHT

201 HAT CAT ENRICHED URAMIUM UNIT FOUNDS

[S] 1

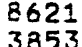

3853

$3863 \times 3$

$.947 \quad 3867$

.947

$1073-97$

.947

.947

ISO 1 MWT $\frac{\text { ISO }}{\text { PCT }}$ ISO

FOOTNOTE EIGHT

$\begin{array}{ll}5,5350 & 1,500 \\ 5,5350 & 1.500 \\ 5,5350 & \end{array}$

204 MAT CAT ENRICHED URANIUM UNIT POUNES

PROJECT CCDE

369.00

369.00

369.0

FACTOR
WEIGHT NLEMENT

ISO 1 ISO 1 MHDES ISO 2 ISOT

ISO 2 ISOT $^{3}$ FCT FCOTYOTE

PIECES WEIGHT

$\begin{array}{ll}4.572 .00 & 57.1500 \\ 4.572 .30 & 57.1507\end{array}-\frac{1.250}{1.250}$

$4,572.00 \quad 37.1500$

109 
07-31-69 DMA-6

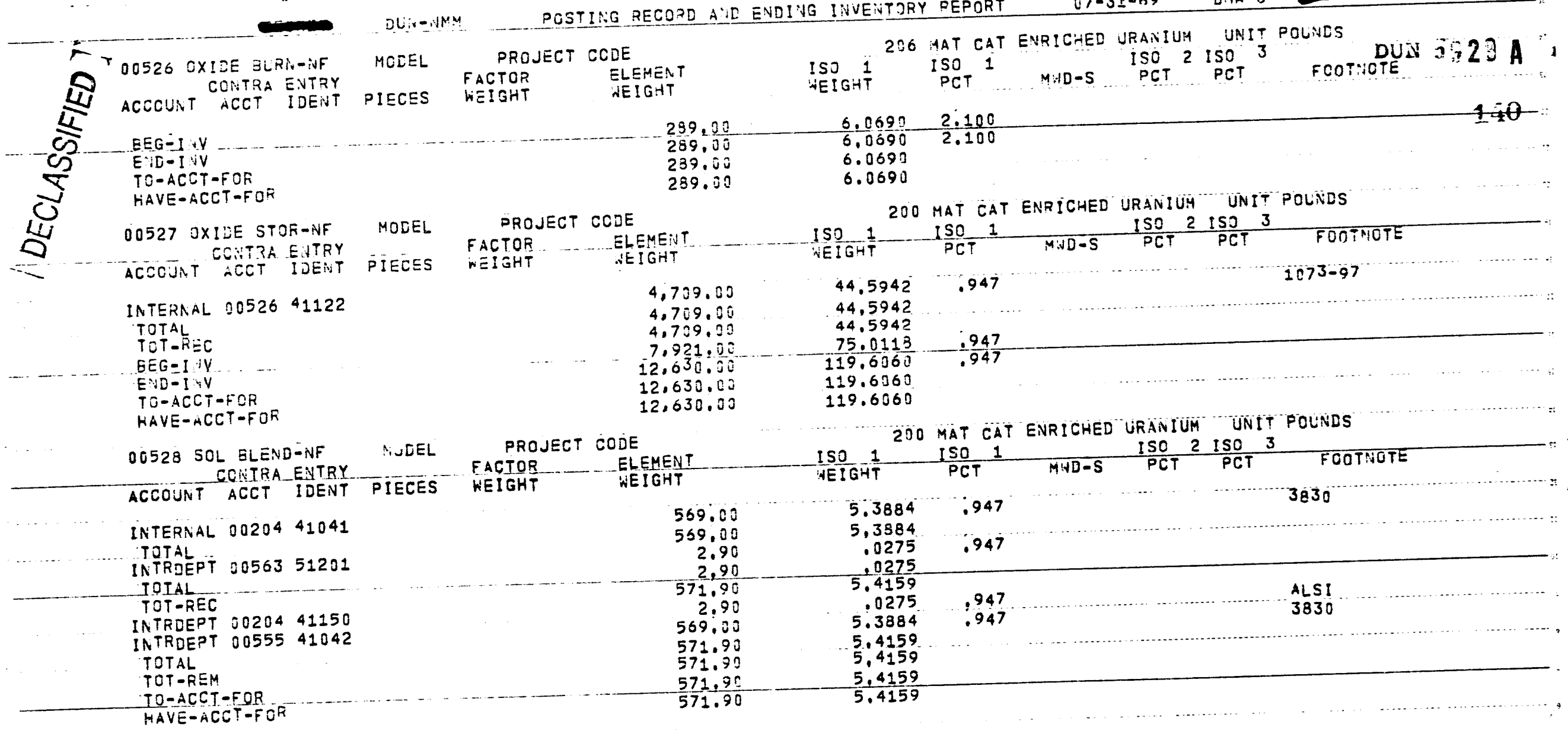




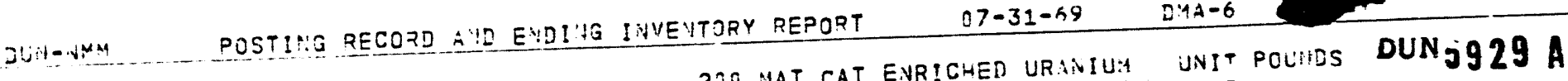

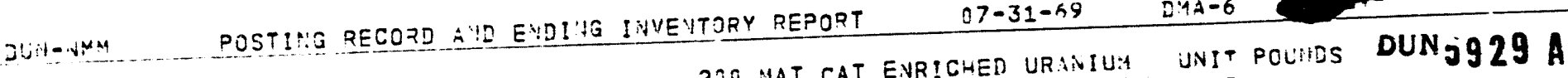

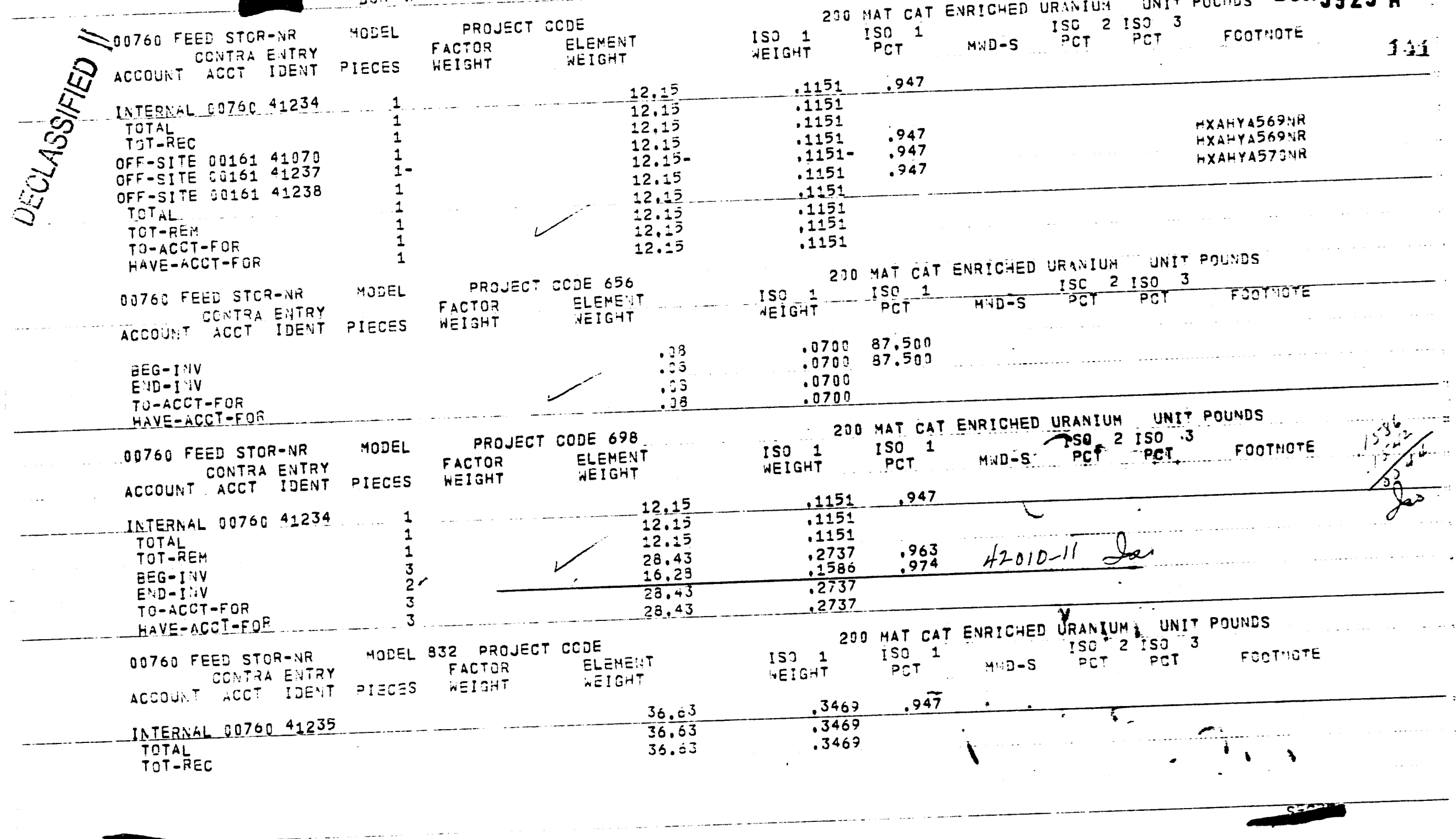


POSTING AECORD AID EIDIVG IIVEYTTRY REPORT

$87-31-69$

DMA-s

00760 FEED STCR-NR

YODEL Z32 DROJECT CODE

200 MAT CAT

ISO 1

IOT CAT EVRICHED URANIUHA UNIT PCUNES

CCNTEA ENTRY
ACCONR: ACET IDENT

PIECES HEIGHT

ELEMENT

HEIGHT

ISO 1

ISC 5 ISO

FOOTMOTE

$E E G=I: Y$

ETO-ACUT-FOR

HAVE-ACCT-LOR

00760 FEEE STOR-NR

00760 FEEE STOR-NR
COITTFA EUTRY

ACCOUHT MOLTA IDEYT

MODEL 910 PROJECT COCE

$\begin{array}{lll}36.65 & .3469 & .947 \\ 35.63 & .3469 & \\ 36.63 & .3469\end{array}$

$36.53 \quad .3465$

IATEFIIAL O0751 11122

TOTAL

TCT-REM

EET-I.IV

ETD-IVIV

HAVE-ACCT-FJR

00760 FEED STCR-NR

DO760 FEEL STCRENR ACCOUNT CONIRA ENTRYY

$68 \quad 20.000$

200 MAT CAT ENRICHED URANIUH UNIT POUNES

ELEMENT

$B E G-I N V$

EYD-IUV

TO-ACCT-FOR

HAYE-ACCT-FOR

00760 FEED STCR-NR

CONTRA ENTRY

58
1,07
1,00

1.350 .00

$1,360.02$

$21,522,39$

20.160 .63

21.520 .00

1,076

MOLEL 911 PROJECT CCDE MIECES WEIGHT W WELMENT
WEIGT

$\begin{array}{ll}12.8792 & .947 \\ 12.8792 & \\ 12.8792 & \\ 203.7944 & .947 \\ 190.9152 & .947 \\ 203.7944 & \\ 203.7944 & \end{array}$

200 HAT CAT ENRICHED URAMIUM UNIT POLNDS WEIGHT 1 POT 1 ISO 21503 PUD-S PCT

CONTRA ENTRY
ACCOUNT ACCT IDENT PIECES WEIGHT WET WEIGHT

\begin{tabular}{lll}
334.62 & 3.1687 & .947 \\
334.52 & 3.1687 & .947 \\
334.52 & 3.1687 & \\
334.62 & 3.1687 & \\
\hline
\end{tabular}

$\begin{array}{rr}11 & 334.62 \\ 11 & 334.52 \\ 11 & 334.52 \\ 11 & 334.62\end{array}$

MODEL 912 PROJECT CODE

290 MAT CAT ENRICHED URANIUM UNIT POLNDS

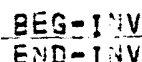

TOMACCT-FOR
END-IVIV

HAVE-ACET TOF

7
7
7
7

232.75
232.75

232.75

232.75

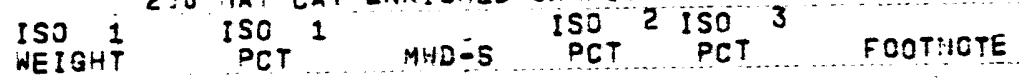

$2.2041 \quad .947$
2.2041
2.2041
2.2041




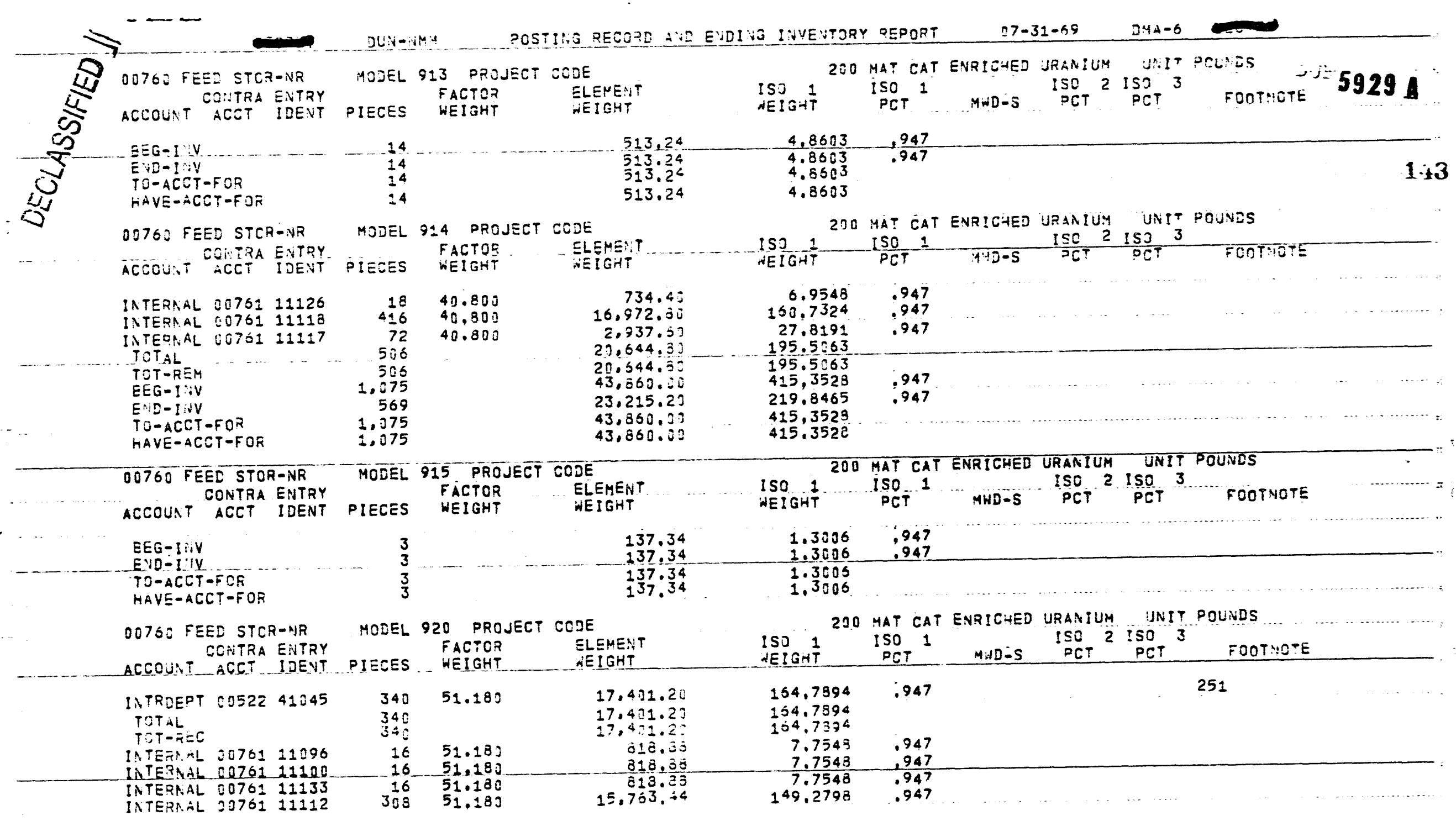


$-$

DO760 FEED STCR-NR CONTRA ENTRY ACCOUNT ACCT IDENT INTERNAL 00761.11137 INTERNAL 0076111111 TOTAL

TOTAL 0051341043

INTRDEPT 00513404040

TCTAL

TOT-REM

EEG-I::V

TO-ACET-FCR

HAVE-ACCT-FOR

O076U FEES STOR-AR

CCNTRA ENTRY

ACCOUNT ACCT IDENT

ATRDEPT 0052241046

TOTAL.

TOT-REC

BEG-INV

TOM-ACCT-FOR

HAVE-ACCT-FOR

00760 FEED STCR=NR 00760 FEED STOR ERTRY CONTRA ENTRY

ACCOUNT ACCT IDENT

INTRDEPT D0522 41047

TOTAL

TOT-REC

8EG-IIV

END-I $Y V$

TO-ACOT-FOR

HAVE-ACCT-FJR

POSTING RECORD AIS ENDI IG INVEYTTORY REPORT

$07-31-69$

DYA-6

MODEL 920 PROJECT CCDE

$\begin{array}{ll} & \text { FACTOR } \\ \text { PIECES HEIGHT } & \text { HEIGHE: }\end{array}$

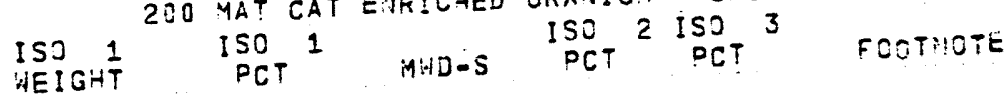

16 - $51.130 \ldots$

51.180

$1,226.3$
$20,267.2$

20.267 .23

1.893 .06

6.346 .32

8.239 .93

$28,507.25$

$84,549.35$

$73,443,3 \mathrm{~J}$

101.950 .56

$101,950.56$

1.435

1,992

DODEL 921 PRJJECT CCDE

FACTOR

FACTOR

PIECES WEIGHT

$3,461.76$

48.080

3.461 .76

$3,461.76$

3.461 .76

$3,461,76$

$3,461: 76$

72

MODEL 922 PROJECT CODE

22 PROJECT CODE

PIECES

WEIGHT WEIGHT

45.350

11.428 .20

11.428 .20

$11,428.23$

11.428 .23

$11,428.23$

$11,428.23$
$11,420.23$

$7.7543 \quad .947$

11.632 ? .947

191.9312

$\$ 7.9330 \quad, 947$

$60.0997 \quad .947$

78

78.0327

250.9530

$800.6825 \quad .947$

695.5080

965.4719

965,4719

200 MAT CAT ENRICHED URANIUM UNIT POUNOS

ISO 1 ISO 1 POD-S PCT PCT FOTNOTE

251

32.7329

$-\underline{2} .7829$

32.7829

32.7829
32.7329

200 MAT CAT ENRICHED URANIUM UNIT POUNDS

ISO 1 ISO 1 MWD-S ISO 2 ISO 3 PCT ${ }_{\text {PCT }}$ FOOTNOTE

$108.2251 \quad .947$

108,2251

108.2251

108.2251

138.2251

1วล. 2:51

DECLASSIFIED 
$97-31-69$

$5+4-6$

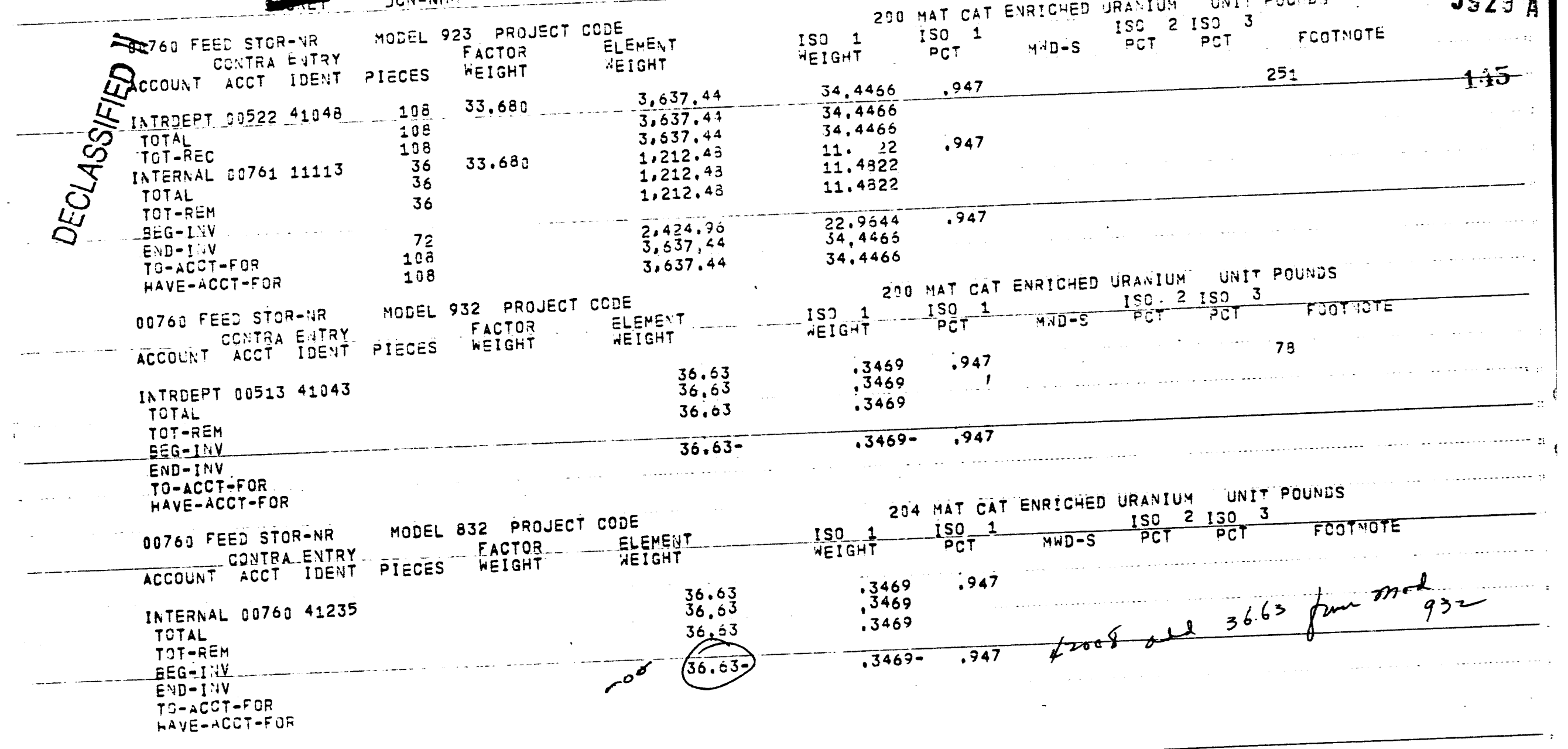


7-31-59 D4A-6

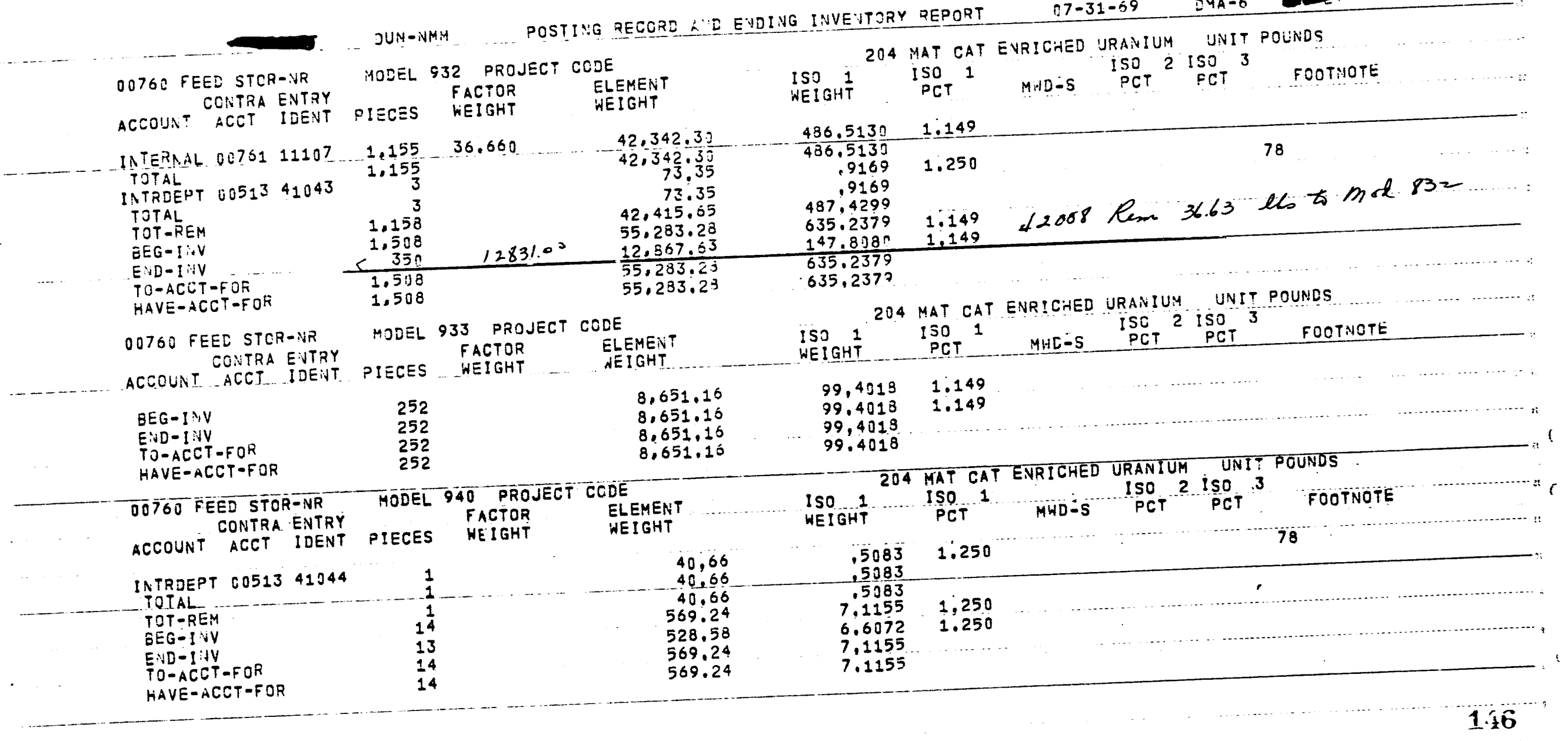




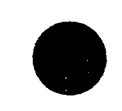

\section{-}

$\ldots$

DUN-NMM

POSTING RECORD AND EVDIYG INVENTORY REPORT

$07-31-69$

DMA-6

MODEL 605 PROJECT CODE

810 HAT CAT NORMAL URANIUM UNIT POUNDS ISO 1 ISO

ISO 2 ISO

PCT 3 FOCTNOTE

DUะ5929 A

O00760 FEED STCR-INR FACTOR ELFMENT ACCOUAT ACCT IDENT

PIECES HEIGHT

WEIGHT

$\begin{array}{rr}6.4498 \\ 6.4498 & .711\end{array}$

EEG $=I I V$

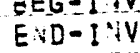

TO-ACET-FOR

$26 \ldots$

$\begin{array}{ll}26 & 907.14 \\ 26 & 907.14\end{array}$

6.4498

6,449

147

4

HAvE 

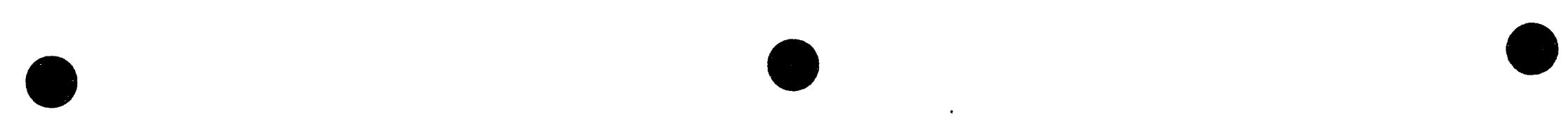

:

DUN-NMM POSTING RECORD AND ENDIVG INVENTORY REPORT

$07-31-69$

DMA-6

DUN-NMMM _. POSTING RECORD AND ENDINO MAT CAT THORIUH

00760 FEED STCR-ANR MODEL

CCNTRA ENTRY

FACTOROAECT CODE 656

FACTOR ELEMENT

ISO 1

WEIGHT

UNIT POUNDS

ACCOUNT ACCT IDENT PIECES

$\frac{5.19}{5.19}$

BEG-IIIY

TO-ACCT-FOR
EIVD-INV

HAVE-ACCT-FOR

\begin{tabular}{r}
4 \\
\hline 4 \\
4 \\
4
\end{tabular}

5.19
5.19 

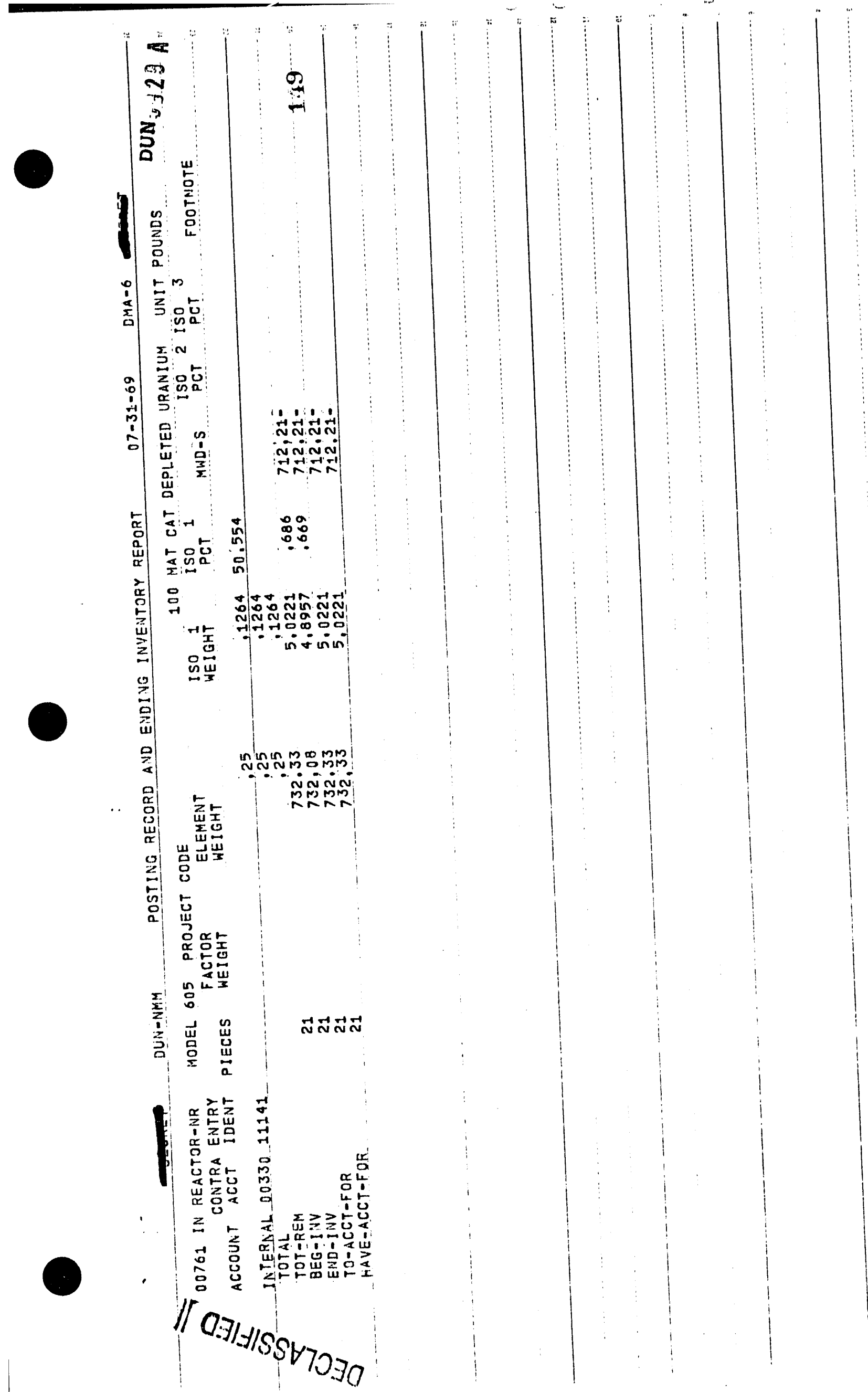
:

DUN-NMM .... POSTING RECORD AND ENDIVG INVENTORY REPORT

07-31-69 DMA-6

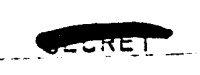

00761 IN REACTOK-NR CCNTRA ENTRY ACCOUNT ACCT IDENT INTERNAL O0330.11150 TOTAL TOT-REM BEG-INV TO-ACCT-FOR HAVE-ACCT FOR.

DO761 IN REACTOR-NR CONTRA ENTRY
IDENT IATERAAL 0076011122 TOTAL TOTAL GEG-INV EEG-INV
END-INV TO-ACCT-FOF HAVE-ACCT-FOR

00761 IN REACTOR-NR CONTRA ENTRY ACCOUNT ACCT IDENT INTERNAL 00330.11153 INIERNAL 00330.11153 INTERNAL 0033011146 INTERNAL 59064 11101 INTERNAL
TOTAL

TOTAL

EEG-INV

END-INV

TO-ACCT-FOR

HAVE-ACCT-FOR

\section{MODEL \\ PIECES \\ PROJECT CODE \\ PROJECT CODE
FACTOR
WEIGHT}

200 MAT CAT ENRICHED URANIUM UNIT POUNDS

$\begin{array}{lll}\text { ISO } 1 & 1 \text { ISO } 1 \text { ISO } 3 & \\ \text { WEIGHT } & \text { PCT } & \text { MWOTNOTE }\end{array}$

$\begin{array}{rrrr}.03 & .0124 & 41.192 & \\ .03 & .0124 & & \\ .03 & .0124 & .679 & 317,94= \\ 97,16 & .6596 & .660 & 317,94= \\ 97,13 & .6472 & .660 & 317,94= \\ 97.16 & .6596 & & 317,94-\end{array}$

200 MAT CAT ENRICHED URANIUM UNIT POUNDS

MODEL 910 PROJECT CODE

FACTOR ELEMENT

ISO 1 ISO 1 MUT ${ }^{1}$ ISO 2 ISO 3 PCT FOTNOTE

PIECES WEIGHT WEIGHT

$\begin{array}{llrlr}63 & 20.000 & 1,360.00 & 12.8792 & .947 \\ 68 & & 1,360.00 & 12.8792 & \\ 68 & 1,360.00 & 12.8792 & \\ 68 & 1,360.00 & 12.8792 & , 947 \\ 68 & 1,360.00 & 12.8792 & \\ 68 & & 1,360.00 & 12.8792 & \end{array}$

\section{MODEL 911 PROJECT CODE}

FACTOR ELEMENT

200 MAT CAT ENRICHED URANIUH UNIT POUNDS

ISO ${ }_{\text {WEIGHT ISO } 1} 1$ MWD-8 PCT 2 PCT 3 FOOTNOTE

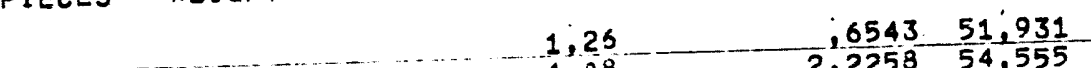

$2,2258 \quad 54,555$

$122.7079 \quad .342 \quad 6.748 .62$

$3.0158 \quad .028 \quad 194,18$

$\begin{array}{rrr}3,0158 & .828 \quad 6,942,80 \\ 128,6037 & 6,942,80\end{array}$

128.6037
339.5368

364,23
$14,942,94$

14.942 .94

$38.825,59$

23.832 .65

38.825 .59

$\frac{339}{210} \cdot .5368$

$.87511 .364 .92-$

$210.9831-.88318 .307,72-$

$339.5368 \quad 11.364 .92-$

339.5363

$11,364,92-$

150 
$-\quad-\cdot$

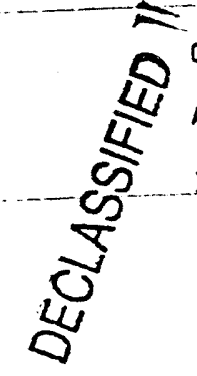
CUITRA ENTRY ACCOUNT ACCT IDENT

INTERNAL 00330.11152 TOTAL

TOT - REM

EEG-IIV

ENIT-INV

TS-ACCT-FOR HAYE-ACCT -F DR

O0761 IA TEACTOR-HR CONTRA ENTRY ACCOUNT ACCT IDENT

INTERAAL C033011147 INTERNAL DO330 11151 TOTHL

TOT-HEM

BEG-IJV

ESD-I IN

TO-ACCT-EOR

00761 IN REACTOR-NR

CONTRA ENTRY

ACCOUNT ACCT IDENT

ITERNAL 0076011117

TERAAL 0076011126

INTERNAL 00760 11118

TOTAL

INTERNAL 2033011144

INTERALL C0330 11145

INTERANL O0330 11154

IITERIAL 5033r 11156

IN TER 11 59051 11097

IN TER 5930411114

AN Eñ 5905411119

IN ERAML 5905411119

INTERNAL 590305 11130

TJTAL

TOTAREM
DUN-NMM

PCSTING RECORD AND EVDIVG INVEVTORY REPORT

$7-31-69 \quad D M A-6$

200 MAT CAT ENRICHED URANIUH UNIT POUNDS

TiN 5929

MODEL 912 PROJECT CODE

$\begin{array}{ll} & \text { FACTOR } \\ \text { PIECES HEIGHT } & \text { WLEMEITT }\end{array}$

ISO 1 ISO 1 MCT

FOOTNOTE

$\begin{array}{rrr}1.14 & .5969 & 52.358 \\ 1.14 & .5969 & \\ 1.14 & .5969 & \\ 5.111 .10 & 43.3642 & .84821 .134,05= \\ 5.109 .95 & 42.7673 & .83721 .134 .05= \\ 5.111 .10 & 43.3642 & 21.134,05= \\ 5.111 .10 & 43.364 ? & 21.134 .05=\end{array}$

$15 i$

154
154
154

154

200 MAT CAT ENRICHED URANIUM UNIT POUNDS

MOCEL 913 PROJECT CCDE

FACTOR ELEMENT

PIECES WEIGHT

ELEMEN

ISO

ISO 1 CAT ENRICHED URANIUM ISO 2 ISO

FDOTMOTE

Iont

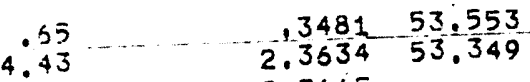

$5.28 \quad 2,7115$

2,7115

$22,329,03 \quad 193,2992$

$22,323,95 \quad 190.5877$

$.866 \quad 61.657,87$

$.85461,657.87$ $\begin{array}{lll}22.329 .03 & 193.2992 & 61.657,87- \\ 22.329 .03 & 193.2992 & 61.657 .87=\end{array}$

610

610

MODEL 914 PROJECT CODE

FACTOR ELEMENT

ISO 1 ISO 1 PCT 1 MHDES ISO 2 PCT PCT

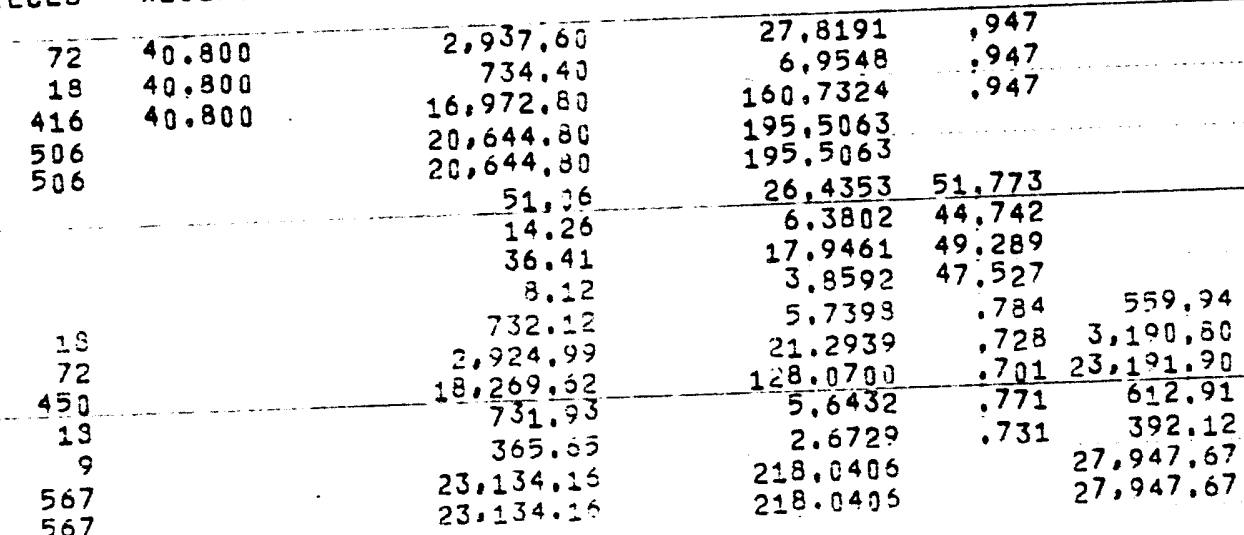


(1)

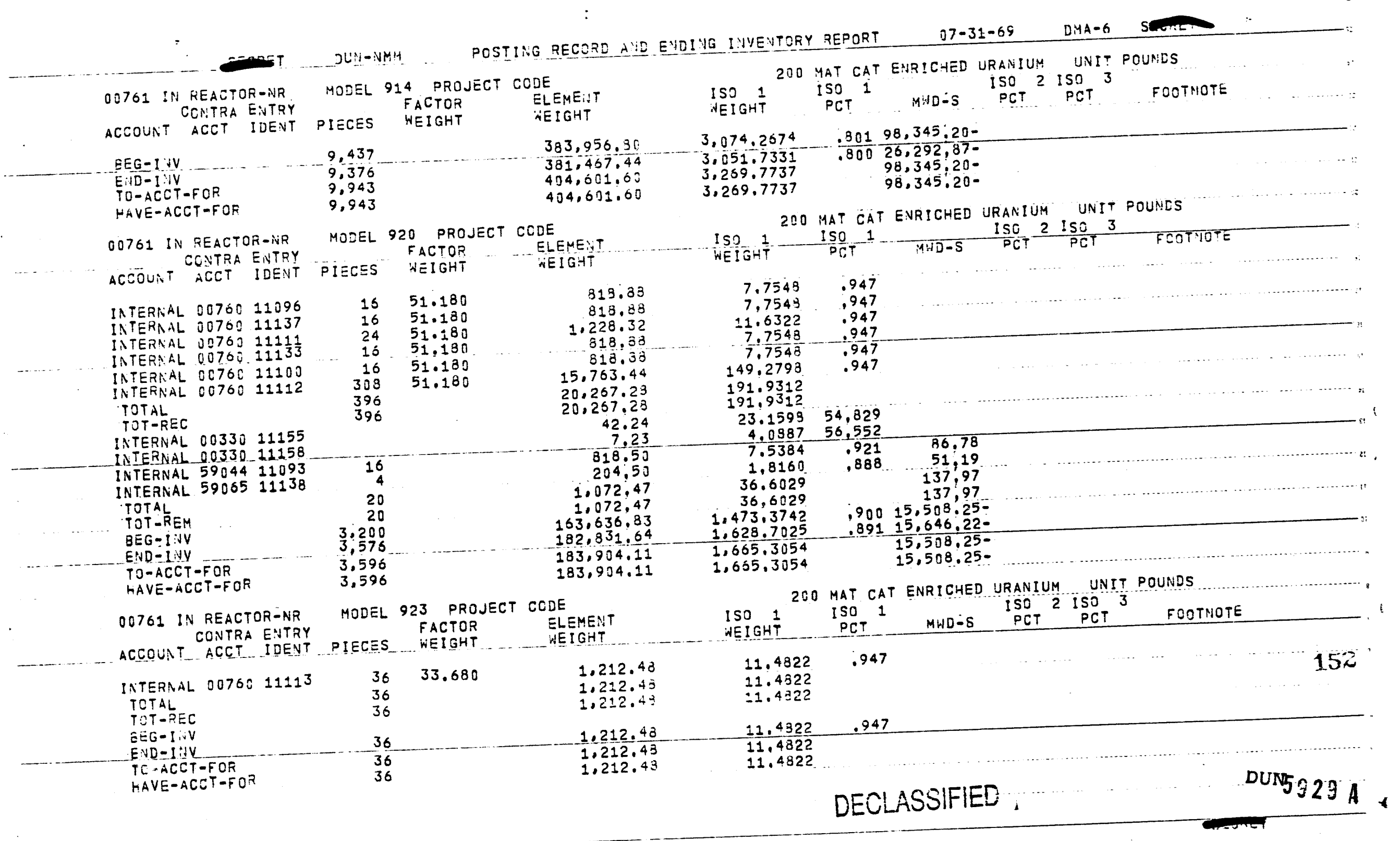


- -

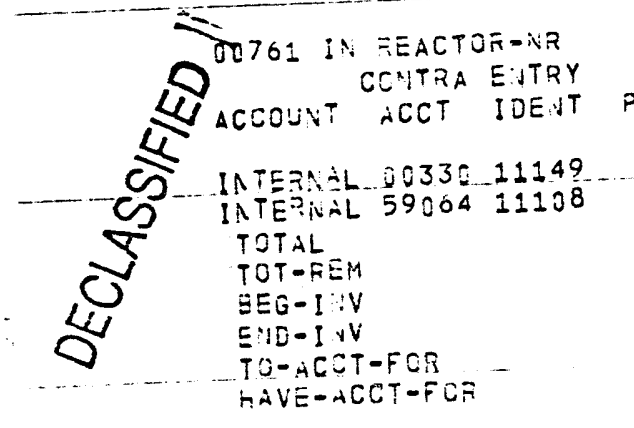
OOT61 IN IEACTOA-NR
CCNTRA EUTRY

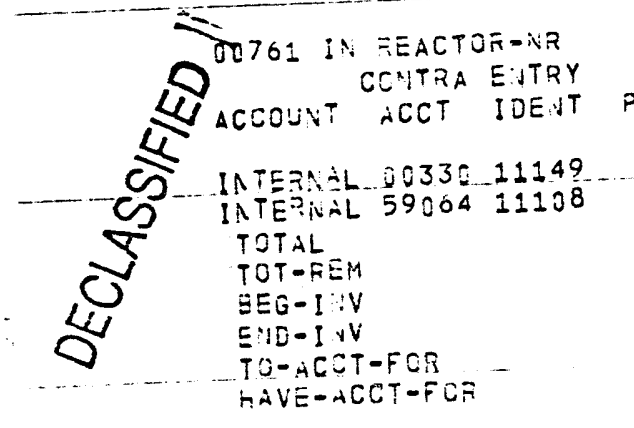

00761 IN FEACTOR-NR OONTRA ENTRY IATEDAL 8076011107 INTE T:

TOTAL

TITERAL 0033011148 INTERNAL O0330 11148 INTERAL 59205 - 11134 TOTAL TOT-REM

BEG-INV

END-INV

TO-ACCT-FOR

HAVE-ACCT -FOR.

0076 I IN REACTOF-NR 00761 IN REACTOE ENTRY ACCOUNT ACCT IDENT

WIERXIL 2033011157

TOTAL

TOT-REM

EEG-I

TJ-ACOT-FCO

HAYE-ACET-EOR

DOSTING REEORE AUD EVDIVG IUVEUTTRY REPORT

$07-31-59$ C:1A-6 31 PROJECT OOJE
FACTOR
WEIGHT

ISO 1 ISOT 1

UNIT POUHDS

MHDOS ISO 2 DCT PET FCOTMOTE

\section{IECES WEIGHT}

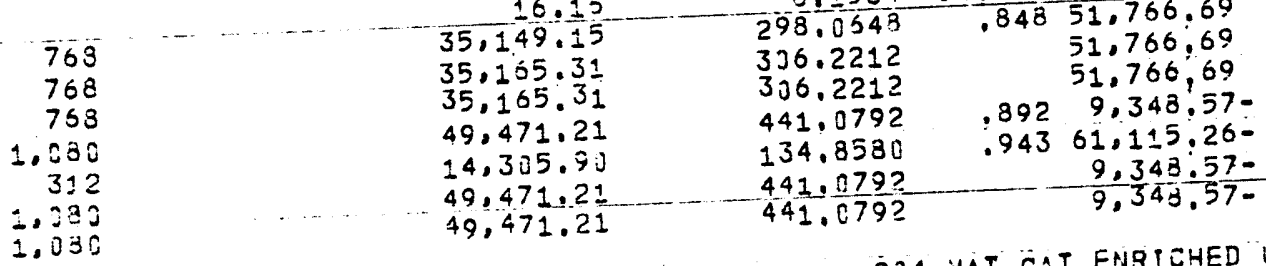

1.030

204 MAT CAT ENRICHED URANIUM UNIT POUNES

MODEL 932 PROJECT CCDE

FACTOR BLEMENT

SO 1 ISO 5 ISO

FCOTIOTE

IECES

$1.155 \quad 36.600$

1.155

1,155

ISO 1 ISO 1 MCT MODS

240
+15
255

255

255

2,340

3,240

3,495

$42.342 .30 \quad 485.5130 \quad 1.149$

$\begin{array}{ll}42.342 .30 & 436.5130 \\ 42.342 .30 & 486.5130\end{array}$

$42.342 .30 \quad 486.5130058 .320$

$\begin{array}{rrr}31.91 & 18.6099 & 58.320 \\ 8.749 .75 & 74.0229 & .34612,974,35\end{array}$

8.749 .75

548.22

9.329 .88

$9,329,88$

$85,647,44$

$118.659,86$

127.999 .74

MOEEL 940 PROJECT CCDE

MOLEL
FACTOR

5.3287

97.9515

$.346 \quad 12,974,35$

$13,402,49$
$13,402,49$

$905,0414 \quad 1,057 \quad 2,226,39-$

$293,5929 \quad 1.090 \quad 15,628,88-$
$2,226,39-$

.391 .5544

2.226 .39 -

204 MAT CAT ENRICHED URANIUM UNIT POUNDS

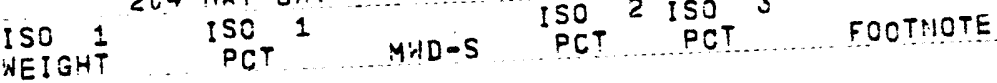

$.2445 \quad 66.069$

$\begin{array}{rrr}.37 & .2445 & 66.069 \\ .37 & .2445 & \\ .37 & .2445 & \\ 1.219 .59 & 15.1053 & 1.230 \\ 1.219 .22 & 1.8613 & 1.219 \\ 1.229 .59 & 15.1053 & \end{array}$


$97-31-69 \quad 0 M A-6$

$J i-i n M M$

0076: IN REACTOF-INR CONTRA EINTRY ACCOULT ACET IDENT

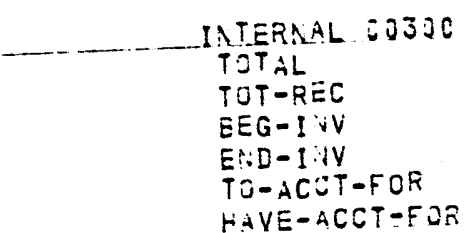

00761 IN REACTOF-NR

CONTRA ENTRY

ACCOUNT ACCT IJENT

INTERALL 80331.11159

TOTAL

TOT-FEC

NTERAAL 5904411094

INTERNAL 5905111098

INTERNAL 5906411105

INTERAAL $59064-11120$

INTERNAL 5906411109

INTERNAL 5906411102

INTERNAL 5906411115

INTERNAL 59065-11128

INTERNAL 5906511139

INTERNAL 59065.11131

INTERAAL 590651113

INTERNAL 590651112

TOTAL

TOT-REM

EEG-1YV

EOD-IT

HAVE-ACCT-FOA
DOSTING RECORD AVE ENDIVG IIUENTTRY REPORT

500 MAT CAT PLUTOYIUM

UNIT GRAMS

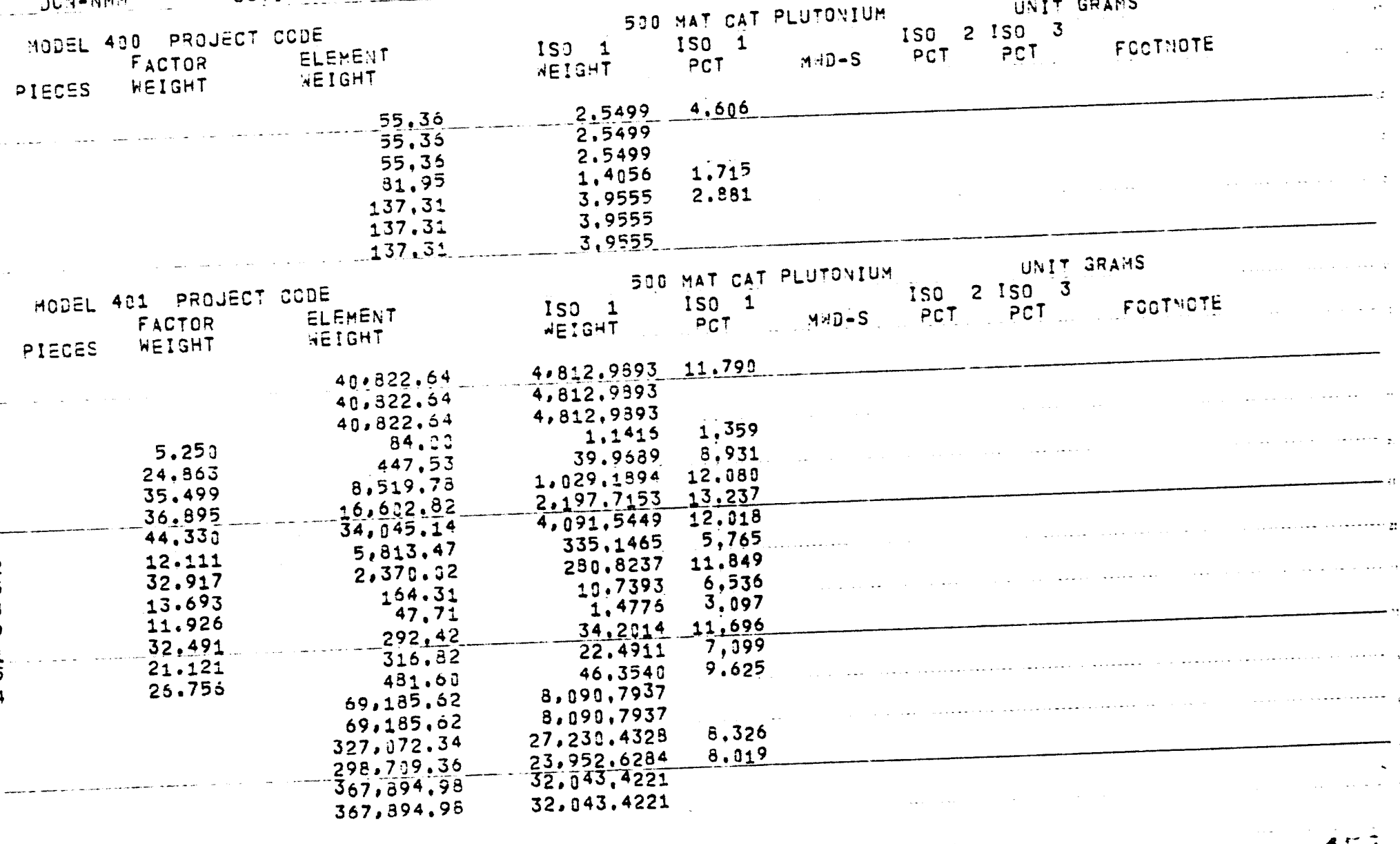




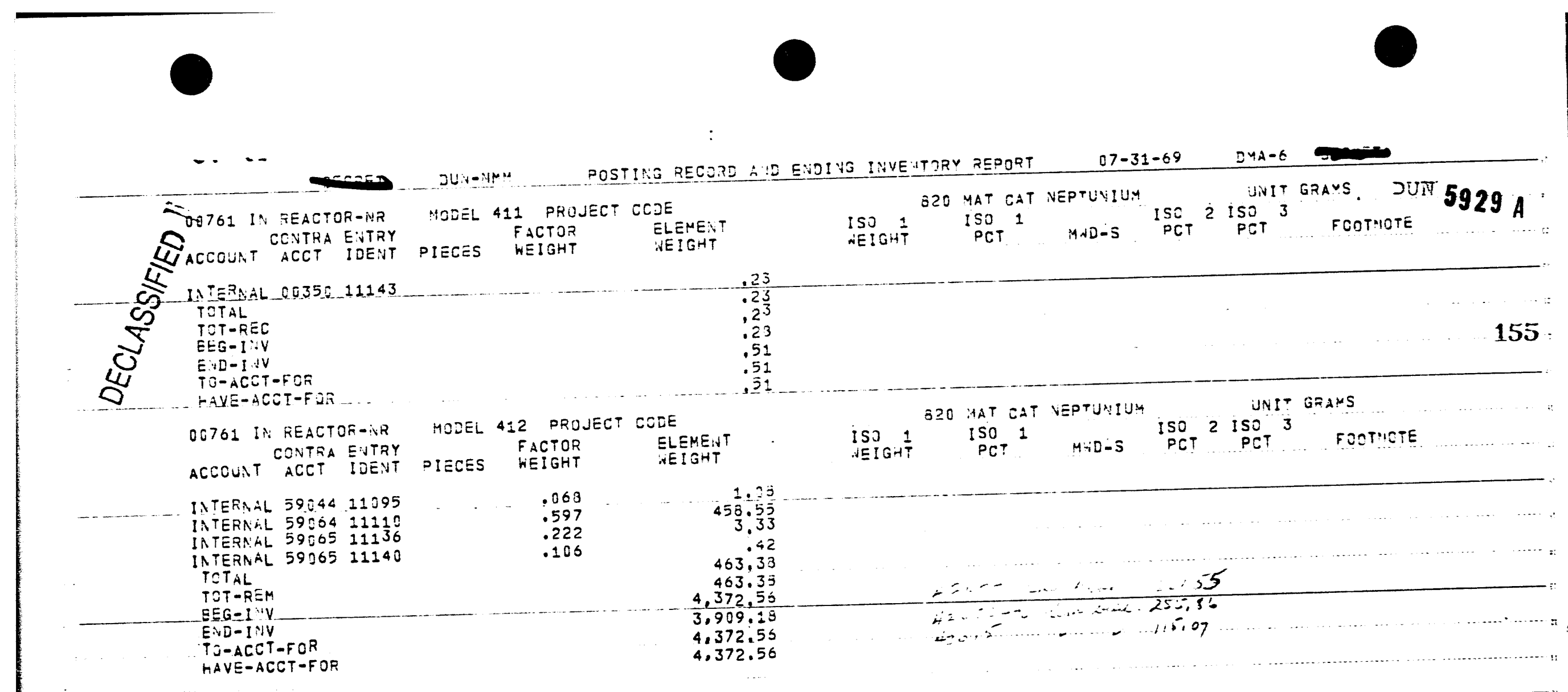




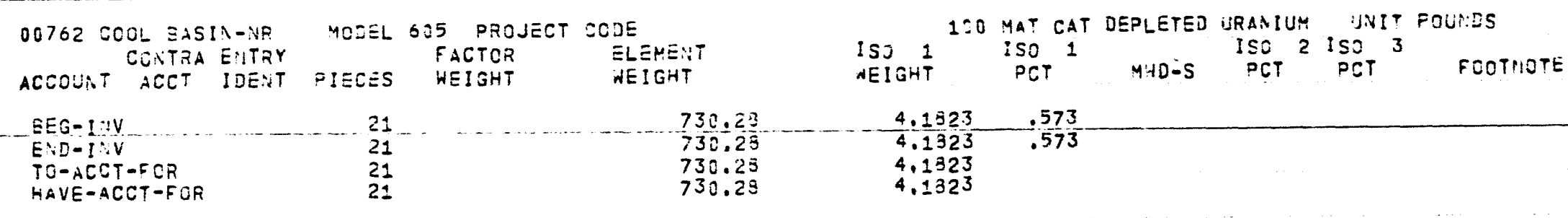

150 


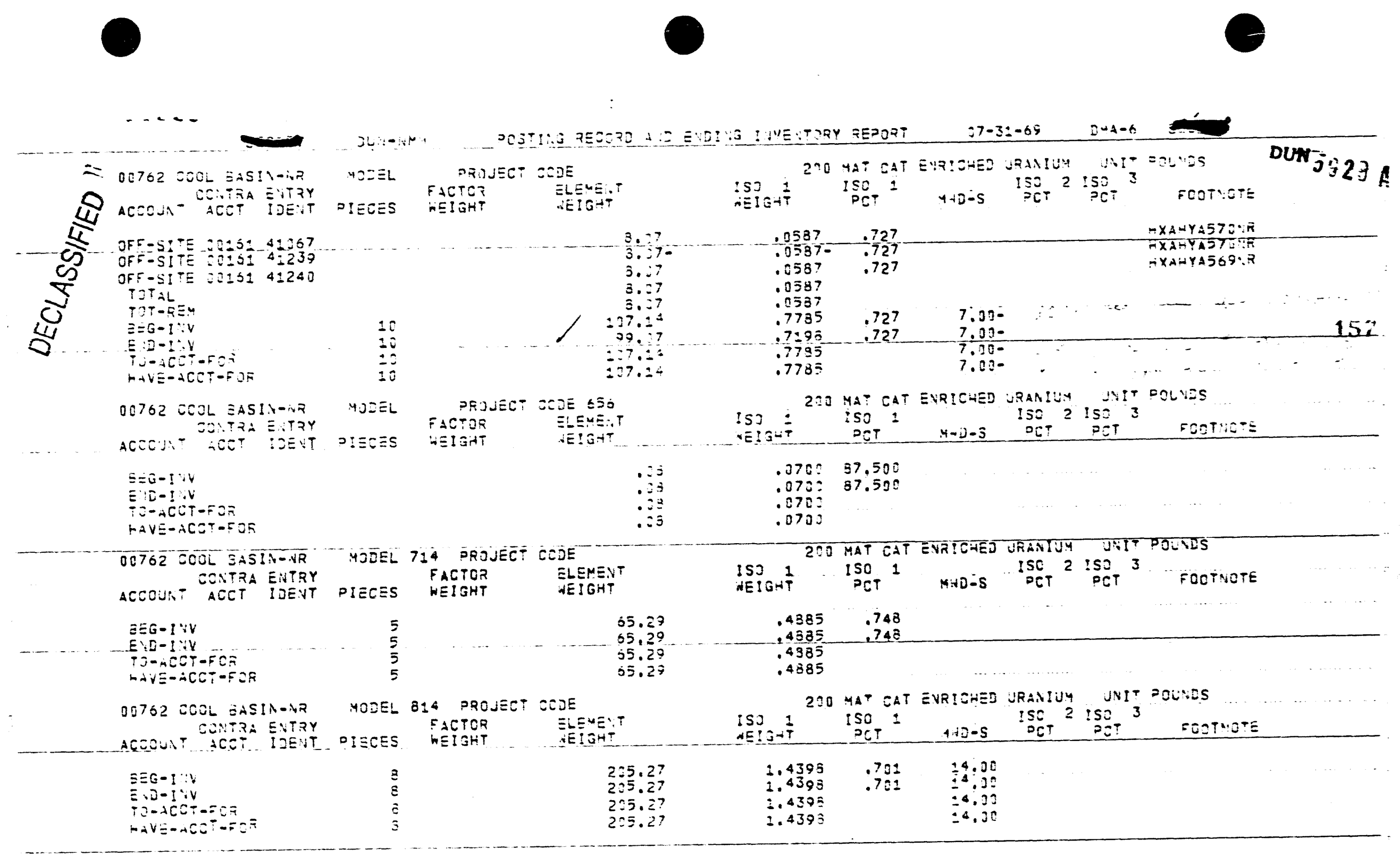


OL762 COOL EASII.-AR OCOTHEA EUTRY MOLEL B2O DRIJECT COEE
FACTOR OLEMENT ACCOUET ACCT TDEAT 200 HAT CAT ENRICHED URAVIUY JUIT FOUIES $E E G=I I Y$ TDOACCT-FOE TOAVE-ACCT - FOR
TAVE

OU762 LOOL EASINA-IVR ACCOUAT CEUTHA EITTRY IECES WEIGHT MEIGHT

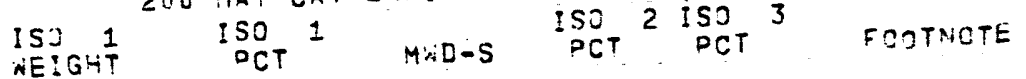
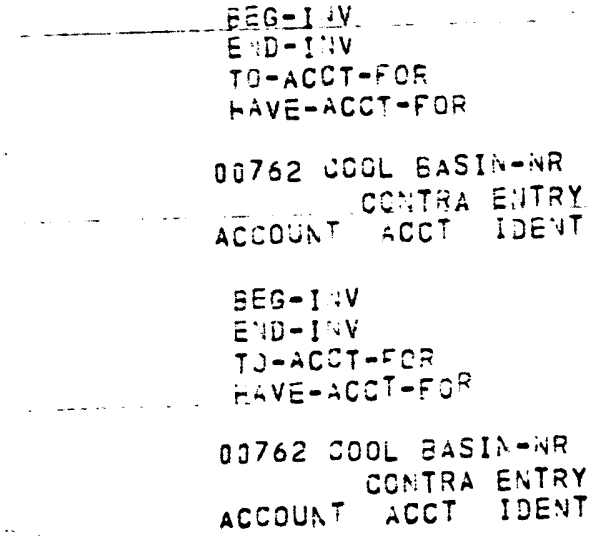

MODEL B29 PROJECT CCDE

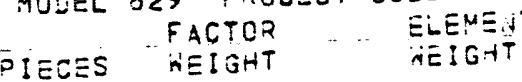

200 MAT CAT ENRICHED URAMIUM UNIT PCUMIS

$\begin{array}{ll}15 & 565.61 \\ 15 & 565.51 \\ 15 & 565.51 \\ 15 & 555.31\end{array}$

MJEEL 910 PROJECT CODE

FACTOR ELEMENT

4.62
4.8529

PIECES WEIGHT

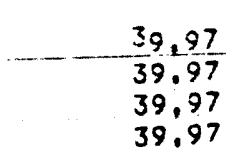

$\begin{array}{lll}39.97 & .3585 & .897 \\ 39.97 & .3385 & .897 \\ 39.97 & .3585 & \\ 39.97 & .3585\end{array}$

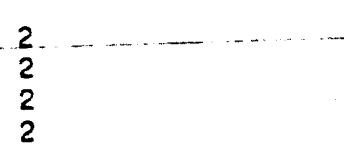

HODEL 911 PROJECT CCDE

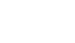

0

200 HAT CAT ENRICHED URANIUM UNIT POUNDS

PIECES WEIGHT - W WEIGHT ISO 1

200 IAAT CAT ENRICHED URANIUM ISN 2 ISO 3

230 MAT CAT ENRTCHED URANIUHA JI:IT POUNES

ISO 1 ISO 1 WOTOS ISOT 2 ISO ${ }^{3}$ PCT FOTTMOTE

$\begin{array}{ll}48 & 1.453 .49 \\ 48 & 1.453 .49 \\ 42 & 1.453 .49 \\ 49 & 1.453 .49\end{array}$

\section{$\begin{array}{ll}10.4831 & .721 \\ 10.4831 & .721\end{array}$}

10.4831

MODEL 914 PROJECT CCDE

200 MAT CAT ENRICHED URANIUM UNIT POUNDS

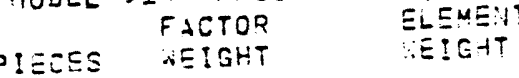

IS 3

200 MAT CAT ENR!CHED URANIUM UNIT

PCT 3 FOOTNOTE

IEHT

$\begin{array}{rrr}22.5061 & .043 & 58.00 \\ 20.5681 & .843 & 58.00 \\ 20.5681 & & 53.00\end{array}$

$20.5681 \quad 53.00$

$\begin{array}{lll}2.440 .84 & 20.5681 & 53.00 \\ 2.449 .84 & 20.5681 & 58.00\end{array}$

PCT PCT

158

TO-ACLT-FCE

2.440 .04

$$
(-\infty-\cdots)
$$

Dun 522 
PCSTI::O RECORE A E EVDIVG INUE ITJRY REPORT

ก:7-31-59 D4A-6

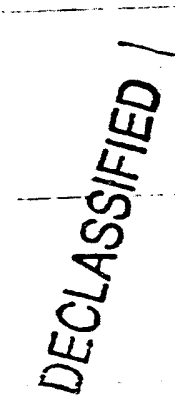

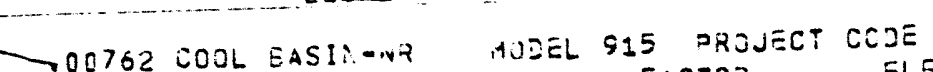

O CONTIAa ENTRY PIECES WEIGHT W NEIGHT

200 HAT CAT EVRICHED URANIUH JNIT POUIES

JUN $5929 A$

ACCOUIT ACCT IDENT

IST 1 IGHT PCT
ISD $\quad$ ISOT 2 ISOT

FOOT'IOTE

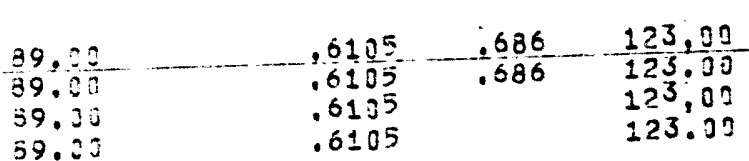

$E: A D-1: T$ V

TO-ACOT-FCR

HAVE-ACCT-FOR

08762 COOL $32 S I M-V R$

ACCOUAT AOCTRA IOVTRY

59.23

$.6 \pm 05$

MOLEL PROJECT CCDE

FACTOR BLEMENT

234 MAT CAT ENR!CHED URANIUM UNIT POUNDS

PIEここS

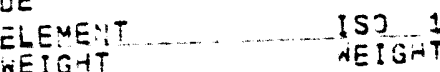

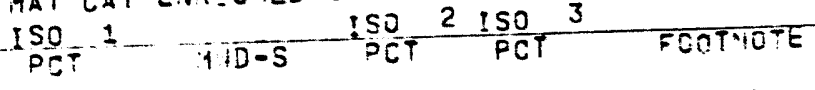

EEG-IYV

$E: D-I 11 \mathrm{~T}$

TO-ACET-FOR

31.53

31.

$\begin{array}{rr}31.02 & .3193 \\ 31.0 & .3193\end{array}$

.3193
.3193
.3193
.31933
.3193


:

DUN-HiMY 00702 LOOL EASIKGINR CCNTRA ENTRY ACCOUNT ACCT IDENT

EEG-IJy

ETID-IiiV

HAVE-ACCT-FOR

00762 COOL 3ASIN-NR 00702 COOL BASIN-NR CONTRA EVTRY
ACCOUNT ACCT DDENT

MODEL 412 PROJECT CCDE

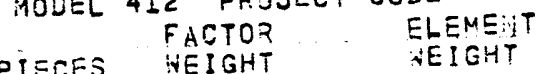

OFF-SITE 0016141068

OFF-SITE 0016141233

OFF-SITE J0161 41232

TSTAL

TUT-REN

EEG $-I: i V$

END-I INV

TO-ACCT-FOR

HAVE-ACCT-FOR

PIECES WEIGHT NEIGHT
MOLEL 411 PROJECT CODE

07-31-69 DMA-6

UNIT GRAMS

320 MAT CAT NEPTUNIUH

ISO 'I

ISO 1

$M ! I D \div S$

150
PCT

FCOTIOTE

1.95

1.95

1.95
1.95

1.90 PIECES WEIGHT .35

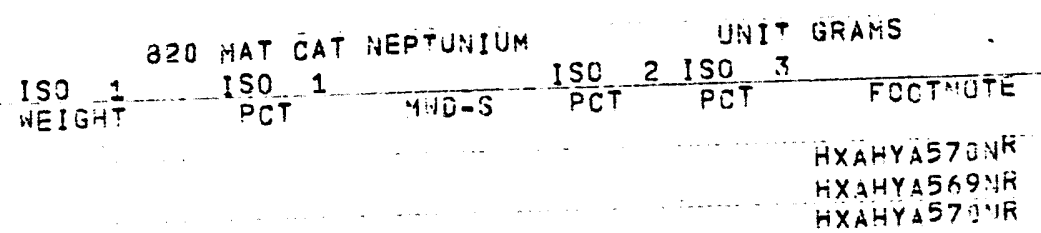

1 
Dut-idiny POSTING PECORD AIL EVDI NG INYEUTORY REPORT $07-31-69$ DMA-6

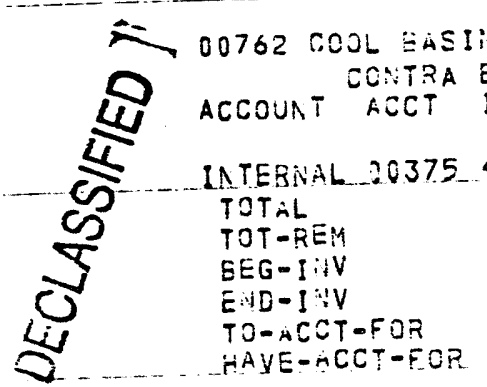
MODEL PROJECT CCDE PROJECT CCDE
FACTOK ELEMENT FACTOR TET WLEMENT 1S. 1 ISO 1 UHIT GRAMS DUN 32 . $A$

F 00762 COOL EASII-IVR NEIGH WEIGH tritiot ISD 2 iso POT 3 FOTIOTE DECAY INTEBNAL 20375
TOTAL
TOT-REM
GEG-IIV
EAD-IUV
TO-ACCT-FOR
HAYE-ACCT-FOR 00762 COOL BASIN-IHR ACCOUIT CONTRA ENTRY PIECES FACTOR HEIGT ELEMENT
MODEL 420 PROJECT CCDE

$\therefore \therefore \quad \therefore \quad \therefore$ INTETAL $20375 \quad 41172$ TOTAL TOTALEM

EEG-IIV

END-INV

TO-ACCT-FOR WAYE-ACC.T-EOE

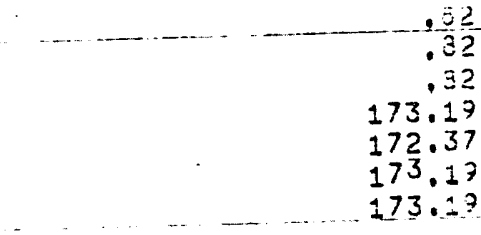
173.19 172.37 173.17 173.19

870 MAT CAT TPITIJM ISO 1 ISO 1 MCT MO-S ISO ${ }^{2}$ ISO 3 PCT FOOTOTE
$16 \hat{1}$

EECAY 
POSTING RECORD AID EVDIUG IVVEVTORY REPORT 37-31-69

$D M A-6$

DU:T-NMM PQOJECT CCDE 656 00762 COOL BASIA-INR CCNTRA ENTRY
ACCOUTT ACCT IDENT FACTOR PROJECT CCJE 6SO ACCOUST ACCT IDENT PIECES 3SO MAT CAT THORIUM ISO 1 ISO 1 UNIT POUNDS $\begin{array}{lcc}\text { EEQ-1 } & 4 & 5.13 \\ \text { EVD-I:VV } & 4 & 5.13 \\ \text { TO-ACCT-FOR } & 4 & 5.13 \\ \text { HAVE-ACCT-FOR } & 4 & 5.13\end{array}$

$16 \bar{a}$ 
:

and

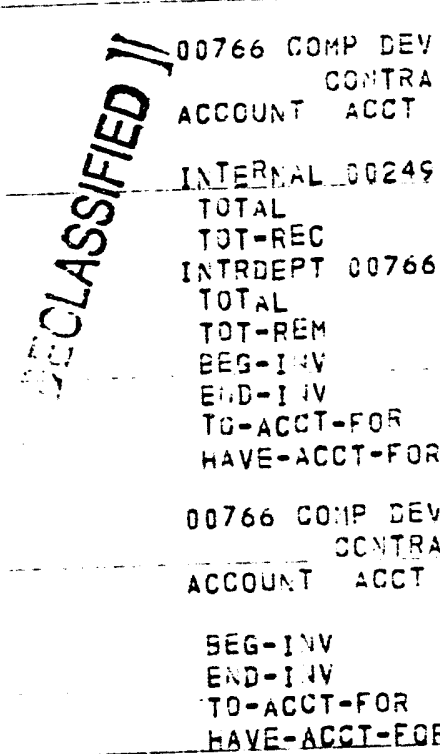

00766 COMP DEV EV-N CONTRA ENTRY ACCOUNT ACCT IDENT

INTRDEPT DO520 41055

INTRDEPT 0076641054

TOTAL

TOT-REM

EEG-IIIV

EVID-Iit

TO-ACET-EAR

HAVE-ACCT-FOF

00766 COMP DEV EV-4 ODITRA E ETTEY ACCOUNT ACCT IDENT IATRDEPT J0520 41056 INTRUEPT DOT6́6́ 41054 TOTAL

13

13

13

MODEL 9:4 PROJECT OCDE 690 FACTOR ELEMENT WEIGHT KEIGHT
CSTIMG RECOAE EUE ENDIVG INYENTORY REPORT
200 MAT CAT ENRICHED URANIUM UNIT POUNLS PROJECT CODE 693
FACTOR
WEISHT IST IGHT ISO 5 ISO

FOOTIOTE

5929 A

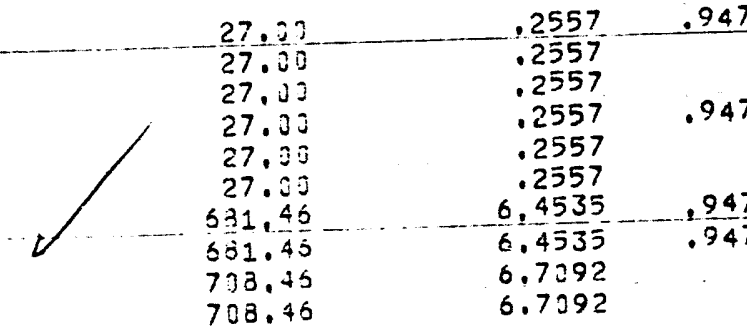

163

200 MAT CAT ENRICHED URANIUM UNATT POLUNDS

15011 ISO 1 ISO 2 ISO 3 FCT

$6.9548 \quad 947$

64

6.9549

$\begin{array}{ll}734.40 & 6.9549 \\ 734.40 & 6.9548 \\ 734.40 & 6.9549\end{array}$

MODEL 915 PROJECT CODE 698

MODEL 915 PAOTECT CODE 698 ELEMEN FACTOR ELEMENT

200 MAT CAT ENRICHED URANIUM UNIT POUNDS

PIECES WEIGHT

5
5
10
10
10
10
10

223,95

ISO 1 ISO 1 MND-S ISO 2 ISO 3

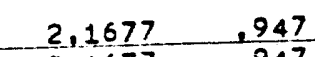

$\begin{array}{lll}2.1677 & 947\end{array}$

$457.30 \quad 4,3354$

$457.03 \quad 4.3354$

$457.33 \quad 4.3354 \quad .947$

$457.00 \quad 4, \frac{3354}{43354}$

MOEFL 920 PSOJECT CODE 693

FACTOT OLENEIT
HEIGHT HEIGHT

200 MAT CAT EVRIOHED URABIUM UIIT POUNES

PIECES WEIGHT

15011 ISO 1 HOT ISO 2 ISP

FODT.MTE

$\begin{array}{rrrrr}16 & 51.180 & 313.08 & 7.7548 & .947 \\ \equiv & 51.180 & 255.93 & 2.4234 & .947 \\ 21 & & 1.074 .73 & 10.1782 & \\ 21 & 1.574 .73 & 10.1782 & \end{array}$

450 
00760 OCAP DEV EV-A CONTRA ENTRY
ACCOUNT ACCT IDENT

DUII- INMM

MOEEL 920 PROJECT CODE 690

$\begin{array}{ll} & \text { FACTOR } \\ \text { PIECES HEIGHT NEMENT }\end{array}$

INTRDEPT 00520.41055

TOTAL

TOT-FEM

ESID-IVIV
GEG-ITV

TO-ACCT-FOR

HAVE-ACCT-FO.

00766 ZOHP EEV EV-N

CONTRA ENTRY ACCOUNT ACCT IDENT

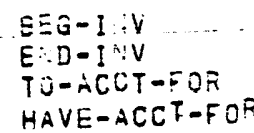

PIECES WEIGHT

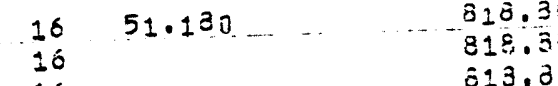

134

MOEEL 924 PROJECT CCDE 695 FACTOF BLEMENT WEIGHT

NEI GHT

DMA-6

013,03

4.247 .74

4.5 .73 .04
5.322 .72

$5,322,72$

$5,322.72$

332.45

333.45

330.45

230 MAT CAT EVRTCHED URANIUM UNIT POUNDS

ISJ 1 ISO 1 HCT

$7.7543 \quad 947 \quad 88-9$

7.7543

40.2279

42.6513

50.4061

50.4961

947

.947

.947

200 MAT CAT ENRICHED URANIUM UNIT POUNES

ISO 1 ISO 1 MUT ${ }^{1}$ ISC 2 ISO 3 PCT POT POTOTE

$.1204 \quad . .947$

$3,1294 \quad .947$

3.1294

3.1294 
:

DOSTIIUG RECORD \& ED ENDIIG INVENTJRY PEPORT 07-31-69 DMA-6

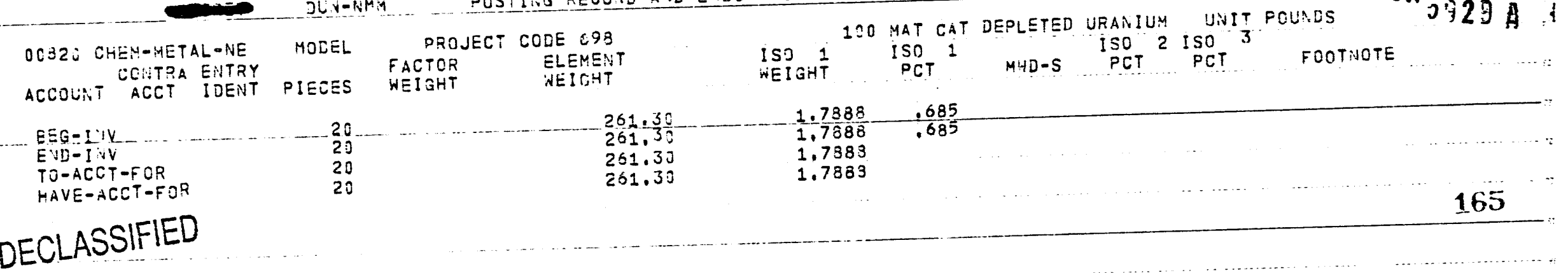


Juit-jun

00322 IHEY-METAL-NE CCNTRA E:UTRY
ACCOUNT ACCT IDENT

EEG-IVV

EOD-ACT-EOR

TOS-ACOT-FOR

OOS2E VHEY-NETAL-NE 00050 GHEMENETAL-NE ACCONAT ACCT IDENT

BES-I:V

tr-aCCt-Foh

have-ACCT-tose

DOE20 CHEM-METAL-NE CONTAA ENTRY

ACCOUNT ACCT IDEVT

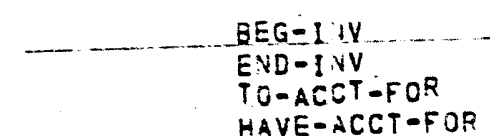

-ACCT $-F O R$

OOO20 CHEM-METAL-NE

COMTOA ENTRY

ACCOUNT ACCT IDENT

\section{HODEL}

PIECES

MOEEL 714 PROJECT CCDE G尹B

MOEEL 714 PACTOR BLEMEMT

HOLEL E14 BROJECT CODE 695

FACTOP OLEMENT
PIECES WEIGHT WEIGHT

ACCOJNT ACCT IDENT PIECES

BEG-IIV

EVID-INV

TO-ACOT-FOF

HAVE-ACCT-FCE

OOZ2J GHEN-METAL-IVE

OONTAA EVTRY

ACOOLO ACET IDEVT

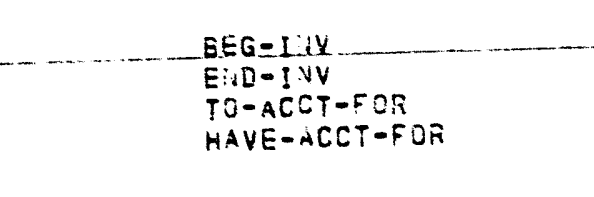

20
20
20
20

MODEL

$21=0 ;: 2$

F

PROJECT CODE 695
FACTOR N ELENENT
WEIGHT

$\begin{array}{ll}2 & 40.43 \\ 2 & 40.45 \\ 2 & 40.41 \\ 2 & 40.41\end{array}$

$\begin{array}{ll}10 & 135.93 \\ 10 & 135.93 \\ 10 & 135.75\end{array}$

10 135.9

408.15
15
15
15
15

MODEL 821 PROJEET CODE 698

MODEL BACTOR

$37-31-69$

DYA-6

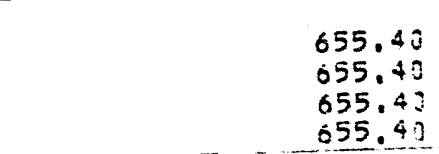

PROJECT CCLE 630 FACTOF AEISHT OLTERT

AIC EVDI IG INYEVTORY REPORT

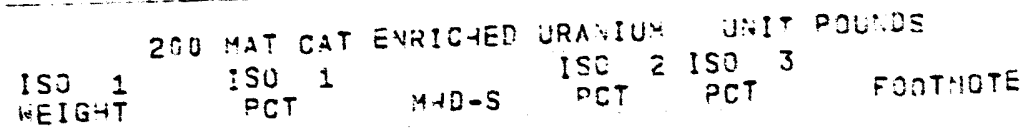

$.3826 \quad .947$

$.3526 \quad .947$

.3825

.3325

200 MAT CAT ENRICHED URAVIUM UNIT POUNES

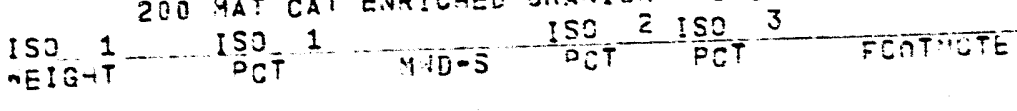

$$
\begin{array}{ll}
1.2900 & .949 \\
1.2900 & .940 \\
1.2700 & \\
1.2900 &
\end{array}
$$

2:0 AAT CAT ENRICHED URANIUY UITT POLSES.

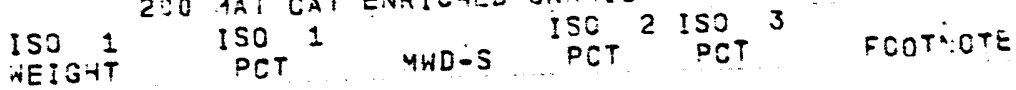

$$
\begin{aligned}
& 3.8700 \\
& 3.3700 \\
& 3,8700 \\
& 3.8700
\end{aligned}
$$

200 HAT CAT ENRICHED URANIUM UNIT POUNOS

200 MAT CAT ENRICHED URANIUM
ISO ISTET ISO 1 ISO 2 ISO 3
MCT

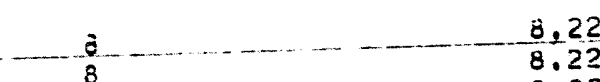

8.22
8.22
8.22
8.22

.22
.22
.22
.22

$\begin{array}{ll}6.2067 & .947 \\ 6.2067 & 947\end{array}$

6.2067

6.2067

201 MAT CAT ENRICHED URANIUM UNIT POUNLS

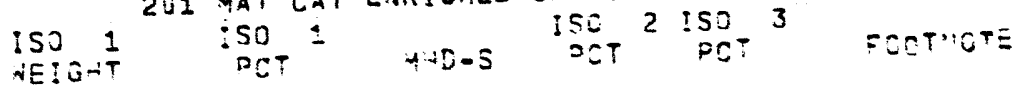

$.3297-4 \cdot \frac{.011}{3.011}$

$.3297 \quad 4.011$

3297
UECLASSIFITE

166

SUN $5 \div 2 \div$ 
- $\cdots$

:

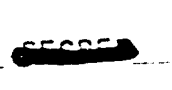

DO820 CHEIM-METAL-ME ACCOUNT ACITTA ENTRY

DLii-ivMm

POSTING RECORD ADD EVDIVG INYEUTORY REPORT $07-31-69$

DMA-6

MUEEL PROJECT CODE 693

PIECES RAIGHT ELEMENT

$2 J 1$ MAT CAT ENR!CHED URANIUA UNIT PCLMDS

IS. 1

ISO \& ISO

DUN

5929 A

EEG $=3$ iV

$E=I N-I V$

TO-ACST-FU

HAVE-ACCT-FOF

OCSZS CHEH-HETAL-NE MODEL 863 PROJECT CODE 698

$\frac{13}{12}$

$\frac{134.5}{134.05}-\frac{1.9015}{1.9015}-\frac{1.419}{1.419}$

$134.5 \quad 1,9015$

$134.05 \quad 1.9315$

ACONA TONTAA EYTRY

HODEL 863 PRIJECT CODE 698

206

206 MAT CAT ENRICHED URANIUM UNIT POUNLS

\section{EEG-IYV}

E :D-I:QV

TO-ACOT-FOR

HAVE-ACCT-FOA

PIECES WEIGHT

IEIGi

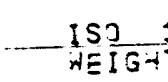

ISO 1 ISO 2 ISO 3 FOT FOTIOTE

$31 \cdot 32$

$31 \cdot 32$

.65772 .100

2.100

.6577

DECLASSIFIED 
OOE20 CHEVHEMTAL-HE MODEL 400 PROJECT CODE 695 CONTRA ENTRY
ACCOUNT ACCT IDENT PIECES WEIGHT W WEIGHT

500 HAT CAT PLUTONIUH

UHIT GRAMS

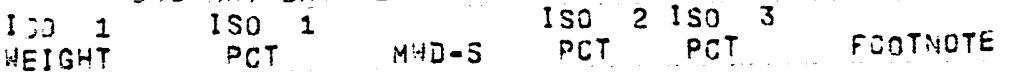
EEG $=1 \mathrm{VIV}$ EMD-IIV TO-ACCT -FCR HAVE-ACCT-FJE

45.31 00320 CHEY-METAL-NE MODEL 401 PROJECT CODE 633 ACCOUNT ACCT IDENT PIECES HEIGHT

ELEVEN EEG-IVN

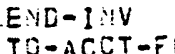
HAVE-ACCT-FOF

00920 CHEK-METAL-NE MODEL 401 PRJJECT CCDE 698

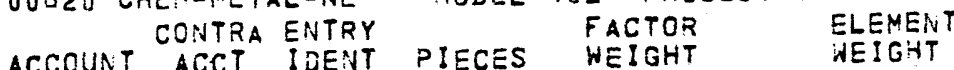
45.31 $45.81 \quad .4123$ $45.31 \quad .4123$ EEG-I:IV END-IINV

TO-ACCT-FOR HAVE-ACCT $F O R$

$\begin{array}{rrr}20.57 & 1.0181 & 4.949 \\ 20.57 & 1.0181 & 4.949 \\ 20.57 & 1.0181 & 1.0181 \\ 20.57 & 1.01\end{array}$

500 MAT CAT PLUTONIUM

UNIT GRAMS

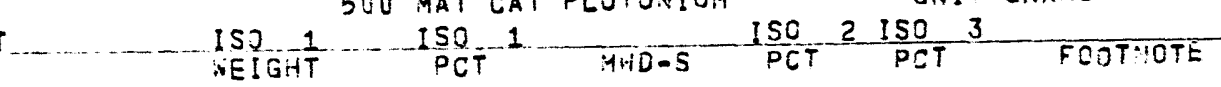

$\begin{array}{lll}13.94 & 2.0910 & 15.000 \\ 13.94 & 2.0910 & 15.000\end{array}$

2.091?

13.94 $2.091 ?$

\section{MAT CAT PLUTOHIUM UNIT GRANS}

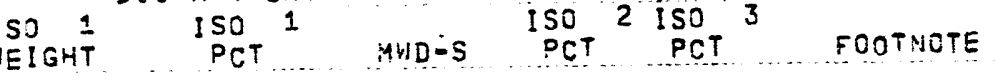

DECLASSIFIED ! 
POSTING RECORD AVE EVDITG JIVEETTORY REPORT

$07-31-69$

DMA-6

UNIT GRAYS DUN 00020 GHEHOHETAL-WE MOLEL PROJECT CODE 693 CONTRA ENTRY FACTOR P ELEMEIT
ACCOUAT ACCT IDENT DIECES WEIGHT 370 YAT CAT TZITIJIA 370 MAT CAT

$150^{3} 3$ WEIGHT ISO

MND-S

ISO 2 ISO

FCOTHOTE BEG-IIV

END-INV

HAVE-ACCT-FOF

.25
.85
.05
.05

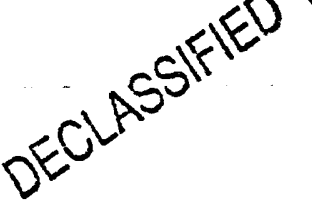


$:$

DUN-NHM

POSTINO RECOAD ALLEVIDIVG IIVE TTORY REPORT

$07-31-69$

EMA-6

58992 KEY ACCOUNT

CCATRA ENTRY
ACCOUNT ACCT IDENT

EEG $=1$ IIV

ENL-IVIV

TO-ACCT-FOR

HAVE-ACCT-FOF.

58992 KEY ACCOUNT

CONTRA ENTRY
ACCOUTT ACCT IJENT

MODEL 914 PROJECT ICDE

PIECES

FACTOR

ELEMENT

WEIGHT

$\begin{array}{ll}2.142 & 97.012 .49 \\ 2.142 & 97.012 .40 \\ 2.142 & 37.012 .40 \\ 2.142 & 87.012 .41\end{array}$

MOLEL 920 PROJECT CODE

PIECES FACTOR

WEIGHT AEIGHT

221
221
221
221

END-INV

TO-ACCT-FOR

HAVE-ACCT-FOP

11.260 .01

11.250 .31

11.260 .31

11.260 .31
200 MAT CAT ENRICHED URAVIUIA UNIT POUNES

ISO 1 ISO 1 MUT ISC 2 ISO 3 PCOT PCT PCT FOTE

$630.8399 \quad .725 \quad 96,640: 22$

$630.8399-.72596 .640 .22$

$630.8399 \quad 96,640.22$

$630.8399 \quad 96,640.22$

200 MAT CAT ENR!CHED URANIUM UAIT PQUNES

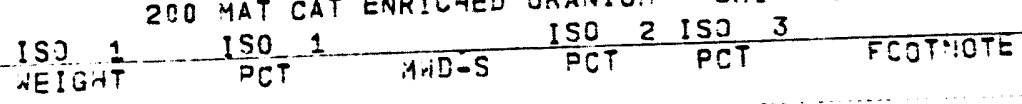

\section{$31.4157 \quad, 723 \quad 12,632,64$}

$91,4157 \quad .723 \quad 12,682.64$

31.4157

$31.4157 \quad 12.682 .64$ 
-

58992 REY ACCCUNT COITRA ENTRY ACCOUAT ACCT IDENT

EEG-LLV

$E N D-I T V$

TO-ACCT-FCR

HAVE-ACCT-FOR

58992 KEY ACCCUAT

CCNIRA EUTRY
ACCEUT ACCT IDENT

EEG- I:UV

END-IIVV

TO-ACCT-FOR

... HAVE-ACCT-FOS

JUI-NMH

MODEL 914 PROJECT SCDE

PIECES

FACTOZ

WEIGHT

POSTING RECORE AIE EVDI HE INVEYTIRRY TEPORT

$07-31-69$

DMA-6

UNIT GRAMS

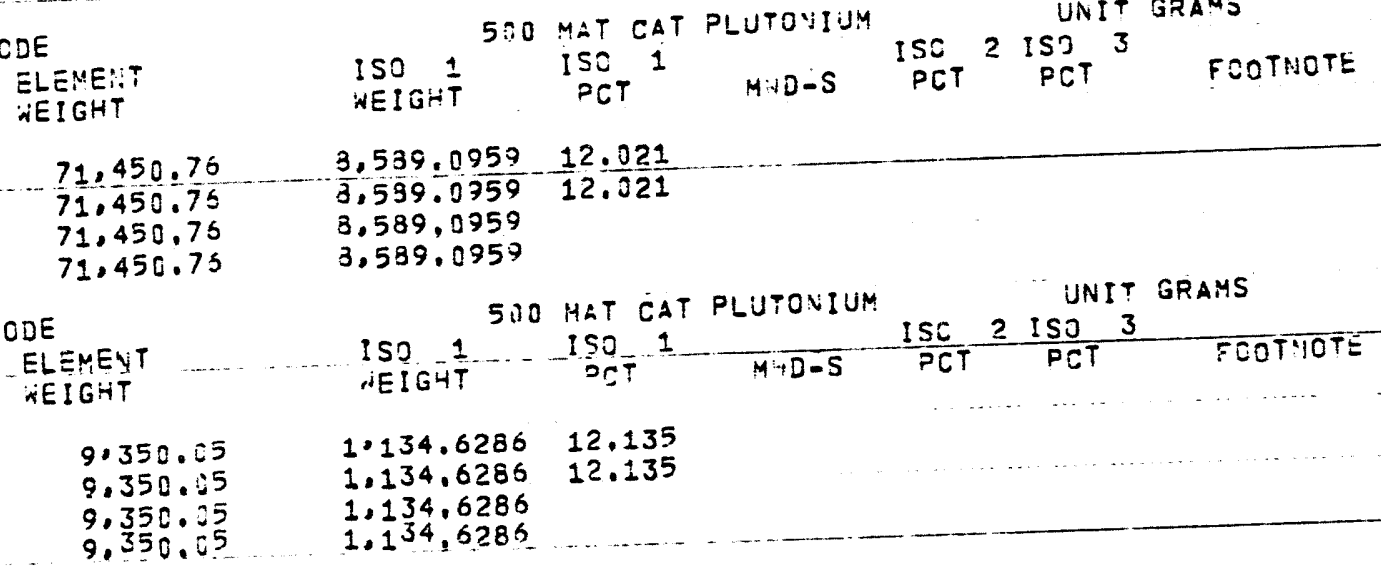

MODEL 920 PROJECT CODE FACTOR … ELEMEYT PIECES WEIGHT

9.350 .05

$1,134.6286$ 

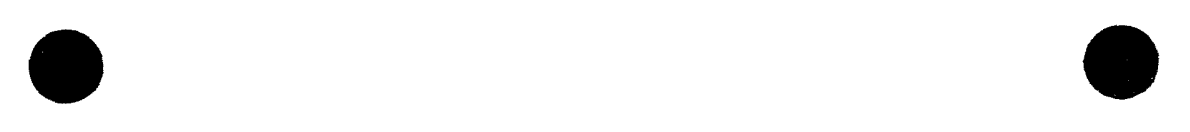

$-$

geczes . DLit-ivMA

POSTIHG RECOPE A OE EYDIYG IHVEYTORY DEPORT

$07-31-69$

DMA-6

58992 KEY ACCCUNTT MJEEL 914 DROJECT SODE

ACCOUNT CCNTRA ENTRY MCET IDENT PIECES WESTGRT BLEMENT

IST TSTEAT ISE 1

B20 HAT CAT NEPTINIIUM

UNIT QRAYS

Thing 29

ACcour aCCT I0,436,92

Eving

$1,435,92$

$1,435,92$

TO-ACET-FOA

$1,436,72$

HAVE-AECT-FOR

MODEL 920 PROJECT SCDE

58992 KEY ACSOUNT COITZA EITZY

ACCOUNT ACCT IJE:TT

PIECES

FACTCE

GLEMENT

NEIEHT

820 MAT CAT NEPTUMIUM UNIT GRAAS

$\operatorname{isc} 2$ IST

FEOTEOTE

…:

EEG-IVV

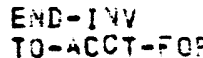

RAVE-ACOT-Fó

130.05

130.30

138.53

125.55

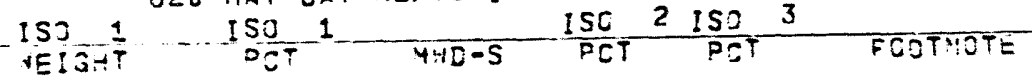

DECLASSIFIED] 
:

DUiv-ivis HJLEL 605 PRJJECT LCDE 58993 KEY ACCOUNT CONTTA ENTRY
ACCOURT ACCT IDENT EEG-I:Y

ENO-I IVT-FOR

HAVE-ACCT-FOF
HOLEL 605 PRDJECT ECEE
FACTOR PIECES WEIGHT

21
21
21
21

$07-31-69$

$04 A-6$

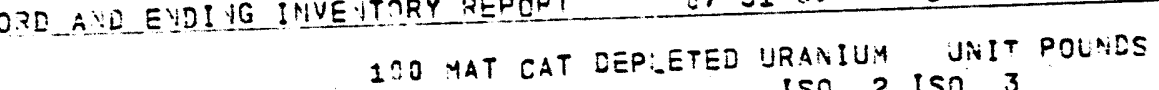

ISO 1 WEIGHT 100 MAT CAT DEP:-ETED URANIUM ISNIT
ISO 2 ISO PCT PCT FOOTNOTE ISO M.ง -5

3.8501 742.21

729,09

727.67

729.67

3.8601 $.529 \quad 712.21$

3.8501

729.57

712.21 
JUN-AMM POSTINO RECORD AIOD EVDIVG INYEITTORY PEPORT 07-31-69 DMA-6

58993 KEY ACCCUNT HODEL 91: PROJECT CCDE 58993 KEY ACSCUNT ACCOUNT COETRA EINTRY

$\begin{array}{ll} & \text { FACTOR } \\ \text { PIECES WEIGHT } & \text { ELEMEIHT }\end{array}$ 200 MAT CAT ENRICHED URANIUM JNIT POUNDS,

DUN $5929 A$

\section{EEG $-I: Y Y$
END-IIIV}

TO-ACCT-FCR

HAVE-ACCT-FOA

402

12.205 .29 ISO 1 ISO 1 ISO 2 ISOT 3 FOT FOTHOTE

58993 KEY ACCOUNT ACCOURT COVIRA ENTRY

402

\subsection{5 .29} 12.205 .29 102.7585 $M+N=S$ MODEL 931 PROJECT CODE
FACTOR

$-\frac{842}{.842}-5,630.08$

102.7685

102.7685

204 MAT CAT ENRICHED UKANIUM UNIT POUNDS

$298.0543 \quad .84851,734,17$

$\begin{array}{lll}35.149 .09 & 298.0643 & .848 \\ 35.149 .19 & 298.0643 & .048 .51 .784 .17\end{array}$

$\begin{array}{rrr}35.149 .89 & 298.0643 & .848 .51,794.17 \\ 35.149 .89 & 298.0643 & 51.784 .17 \\ 35.149 .99 & 298.0543 & 51.734 .17\end{array}$

END-IIV $\quad 760$

$35.149 .7 ?$

TO-ACCT -FOF
HAVE-ACCT $F O F$

768

$51,734,1$

58993 KEY ACCOUNT MODEL 932 PROJECT CCDE

58993 KEY ACCOUNT
CONTRA ENTRY MACTOR

204 MAT CAT ENRICHED URANIUM UNIT POUNES

ISO 1 ISO 1

ISO 2 ISO

ACCOUNT ACCT IDENT

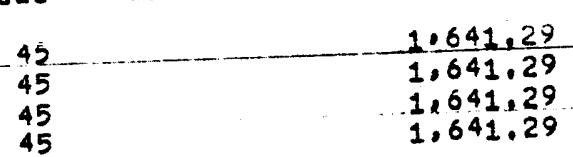

$14.2300 \quad 867 \quad 2.226,39$

$14.2300-.867 \quad 2.226,39$

$B E G=L I Y$
END - JNV

END - JNV

HAVE-ACCT-FOR 
·

JUN-IHM POSTING RECQRD A:D ENDIYG INVEYTORY REPORT

$07-31-69$

DA-6

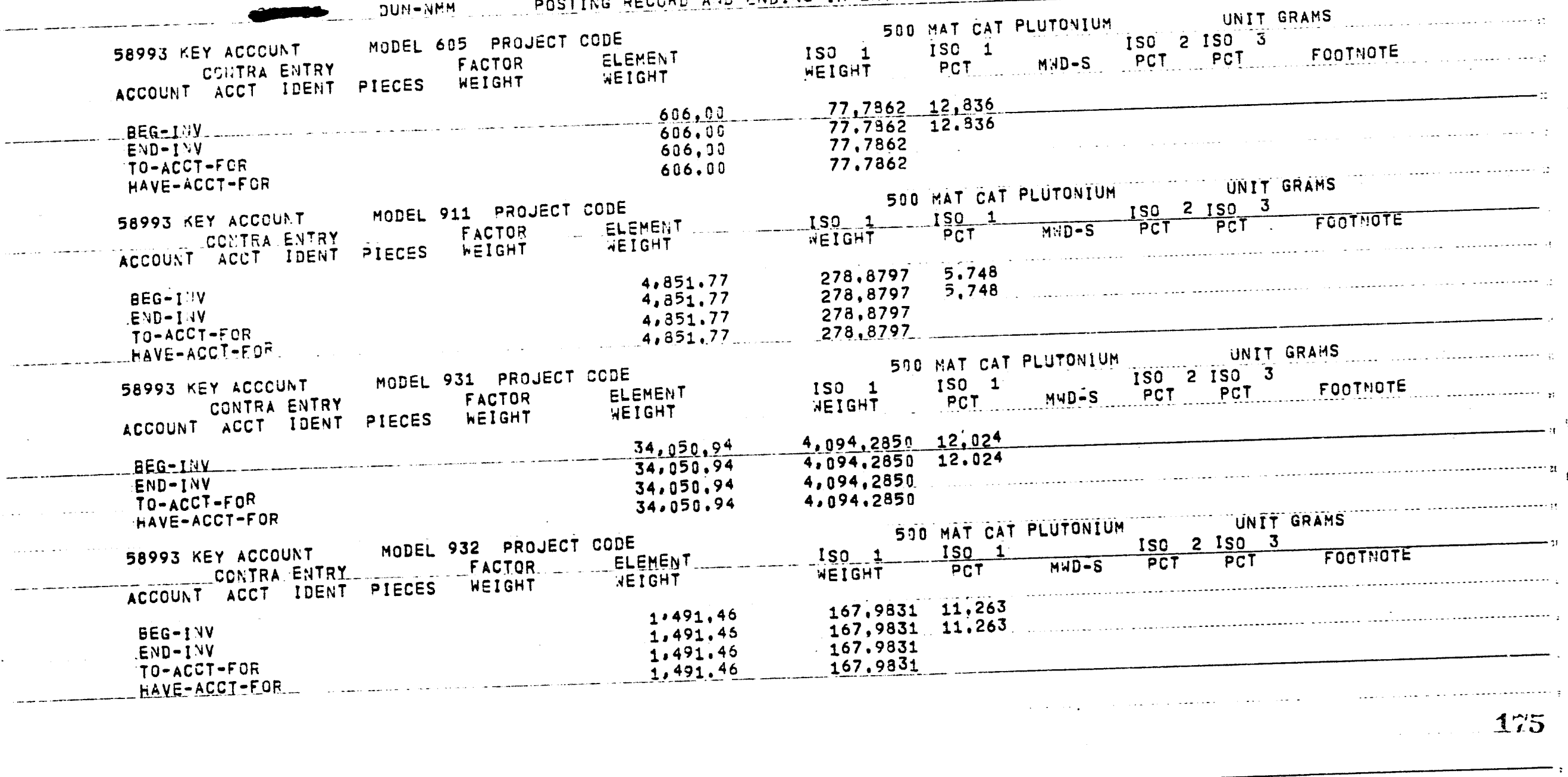




\section{-}

....

POSTING RECORD AID ENDIVG INVEYT JRY REPORT
820 MAT CAT NEPTUVIUM

POSTING RECORD AUD ENDIVG INVEYTJRY REPORT DUIV-UMM O7-31-6
820 MAT CAT NEPTUVIUM

$07-31-69$ C.MA-6

58993 KEY ACCCUNT YODEL PROJECT CODE

$\begin{array}{llll}58993 \text { KEY ACCCUNT } & \text { YODEL } & \text { PROJECT CODE } \\ \text { CONTRA EVTRY } & & \text { FACTOR } & \text { ELEHENT } \\ \text { ACCOUKT ACCT IDENT PIECES WEIGHT } & \text { NEIGHT }\end{array}$ ISO I I ISO 1

UNIT GRAMS

ISO 1 MWDES ISO 2 ISO

FONTHOTE

EUN5929 A

ACCOUNT ACCT IDENT PIECES

EEG-IJV

END - I VV

TO-ACCT-FOK

HAVE-ACCT - F OR

58993 KEY ACCOUNT

CONTRA.EMTRY

ACCOUIT ACCT IDENT

911 PROJECT CODE
FACTOR N D. ELEMENI

$\frac{5.34}{5.34}$

5.34
5.34

BEG-INV

END- I IV

TO-ACCT-ECA
END I T

HAVE-ACCT-FOR

58993 KEY ACCCUNT

CONTRA ENTRY
ACCOUAT ACCT IDENT

MODEL 931 PROJECT CODE

MODEL GACTOR ELEMENT

FACTOR ELEMENT

820 MAT CAT NEPTUNIUM

\section{GEG-IVV}

END-INV

TO-ACCT-FOR

HAVE-ACCT-FOF

58993 KEY ACCOUNT MODEL 932 PROJECT CCDE
CONTRA ENTRY FACTOR

458,74

458,74

458.74

458.74

ACCOUNT ACCT IDENT

IECES WEIGHT

WEIGHT

ISQ 220 MAT CAT NEPTUNIUM
ISIGHT
ISO I IST

$$
\begin{aligned}
& 28.33 \\
& 28.33 \\
& 28.33 \\
& 28.33
\end{aligned}
$$

$8 E G-I N V$

END-INV

TO-ACCT-FOR

HAVE-ACCI-EOR

HAVE-ACCI-EOR

19.43

19.43 
59033 KEY ACCCUNT

CONTRA ENTRY ACCOUNT ACCT IDENT

EEG-IYV

END-I:T

TO-ACCT-FOR

HAVE-ACCT-FOR

59033 KEY ACCOUNT

ACCOUNTT COATEA ENTRY IDENT

EEG-INY

EVD-TNV

TO-ACCT-FOR

$\begin{array}{rr}\text { TO-ACCT }-F O R & 24 \\ \text { HAVE-ACCT-FOR } & 24\end{array}$

\section{MODEL 911 PROJECT CCDE} FACTOR

12
12
12
12

ELEMENT

354,48

364.4

364.4

364.48

12

MODEL 931 PROJECT CCDE

FACTOB $\ldots$ ELEMENT

\section{$1,099.65$}

$1,099,0$

1.099 .65

$1,099.65$

24
24
24
24

200 MAT CAT ENRICHED URANIUM UNIT POUNDS

ISJ 1 ISO 1 MUD-S ISO PCT PCT ${ }^{2}$ FOOTNOTE

$3.1455 \quad, 363-131,82$

$\begin{array}{rrr}3.1455 & 863 & 131,82 \\ 3.1455 & 131,82\end{array}$

$3.1455 \quad 131.32$

204 MAT CAT EHRTCHED URANIUM UNIT POUNADS

ISO 1EIGHT $\frac{1 S O}{\text { PCT }}$ MUD-S $\frac{\text { ISO } 2 \text { ISO } 3}{\text { PCT }}$ POT FOOTIOTE

$\begin{array}{lll}9,9298 & .903 & 1.264,56 \\ 9.9298 & 903 & 1.264,56 \\ 9.9298 & & 1.264 .56 \\ 9.9293 & & 1.254 .56\end{array}$




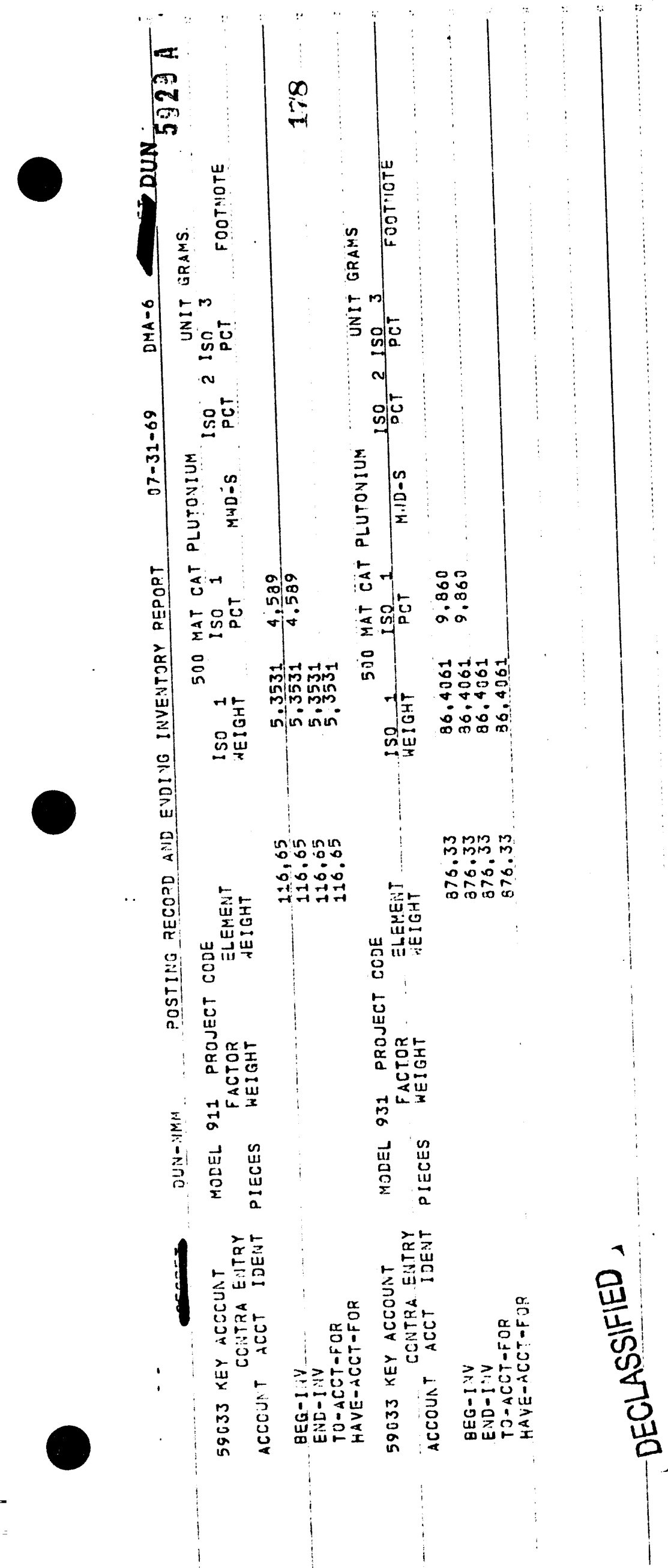


07-31-69 DMA-6

OLA-UMM PCSTING RECORO AVO EVDIVG INVEUTORY REPORT

number.

59033 KEY ACCCUN.T CONTRA ENTRY ACCONITT ACCT IDENT

\section{EEG-IIIV}

TO-ACCT-FOR

HAVE-ACCT-FOR

59033 KEY ACCOUNT

ACCOUNT CONTRA ENTRY

MODEL 911 PROJECT CODE

PIECES NEIGHT

ACTOR NEIGHT

ISO 1 ISO 1

UNIT GRAMS

EIGHT

.52

.02

.52

MODEL 931 PROJECT CODE

MODEL 931 FACTOR

FACTOR

NEIGAT

BEG-I, IV

END-IIAV

TOACST-FOR

HAVE-ACCT-FOR

$$
\begin{aligned}
& 62 \\
& \\
& 67 \\
& 67
\end{aligned}
$$

10.67

19.57
10.57

10.67 


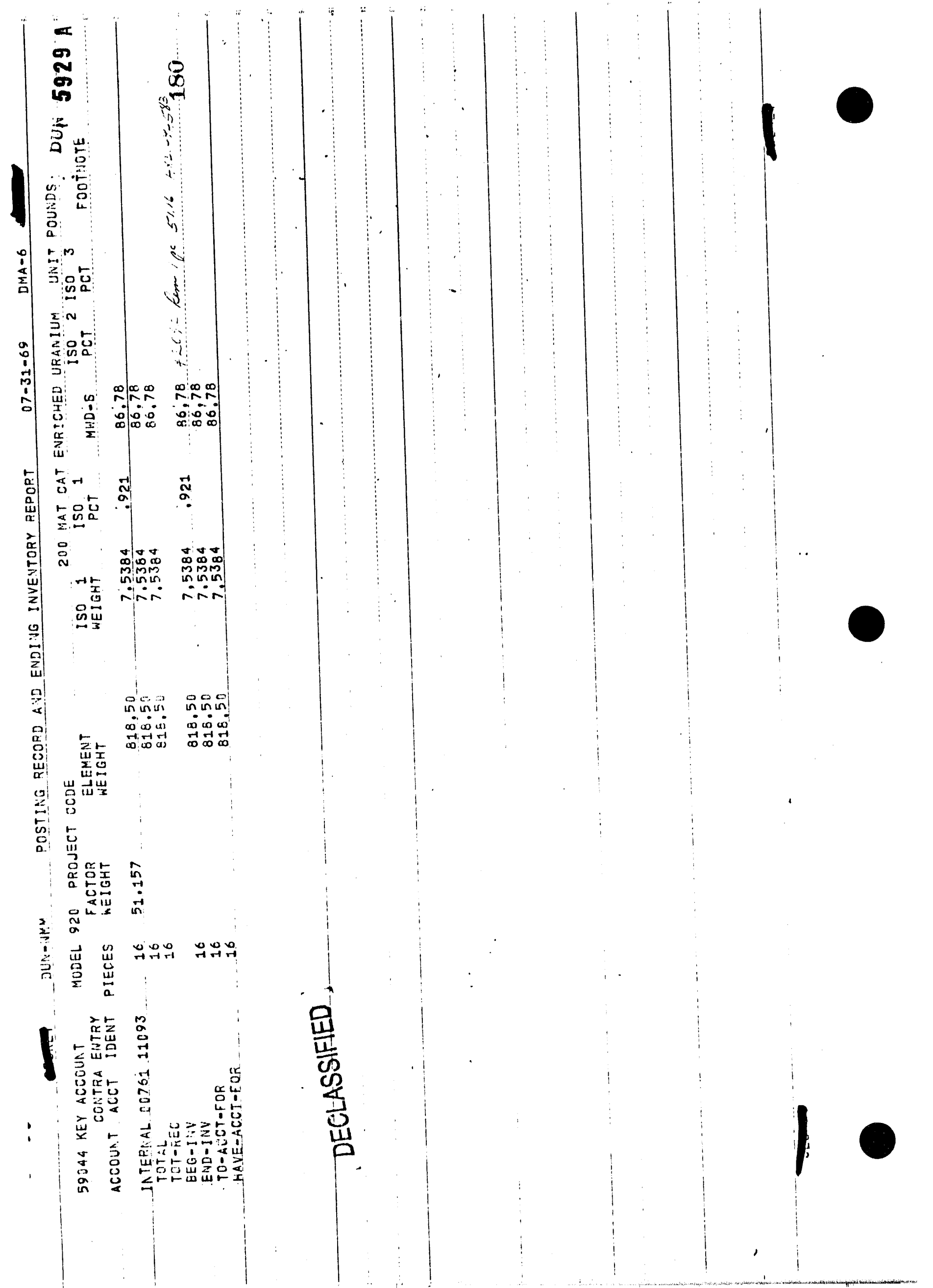




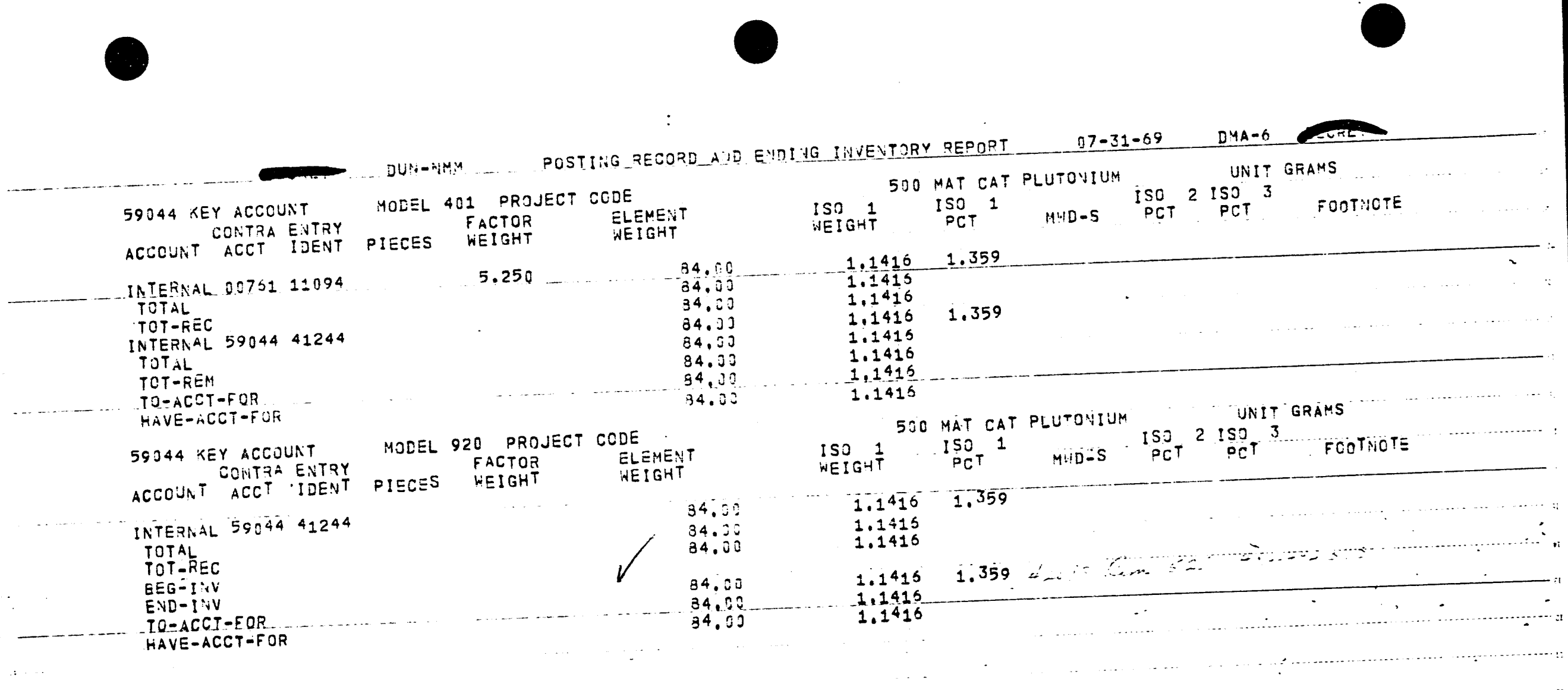

$15 i$ 
. $07-31-69 \quad 0,14-6$

POSTILG PECORD A UD ENDIIG INUENTARY PEPORT $07-31-69$

UNIT GRAHS

DUN 323 月

59044 KEY ACCCUIT MODEL 412 PROJECT CODE

59044 KEY ACCCUIT
CONTRA ENTRY MOLEL 4 FACTOR
ACCOUNT ACCT IDENT PIECES WEIGHT

O20 MAT CAT NEPTUNIUM NEIGHT ISO 1 PCT MHDES ISOT PCT FOOTHOTE INTEENAL 075111095 TOTAL TOT-REC

NTERNAL $59044 \quad 4124$

TOTAL

TOT-REM

EEG-IIV

TO-ACCT-FO

HAVE-ACCT-FOR

59044 KEY ACCCUR.T CONTSA ENTPY

ACCCUNT ACCT IDENT

MODEL 920 PROJECT CCDE $.068 \ldots$

INTERNAL 5904441245

TOTAL

TOT-RE

BEG
END-IVIV

TO-ACCT -FOR

HAVE-ACCT-FOR

1.03

$\frac{1.28}{1.03}$

1.08
.68

.63

$.63 \quad 42009$ oft 6 Mol 920

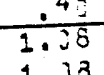

820 MAT CAT NEPTUNIUM UNIT GRAMS

PIECES IXEIGHT NLEMEIT IST 1 ISHT 1 ICT 1 MWD-S $\frac{15022 \text { ISO } 3}{\text { PCT }}$

DECLASSIFIED 
OUN-IJMM POSTING PECQRD AID EVDIJG IAVEITORY REPORT

$07-31-69$ DMA-6

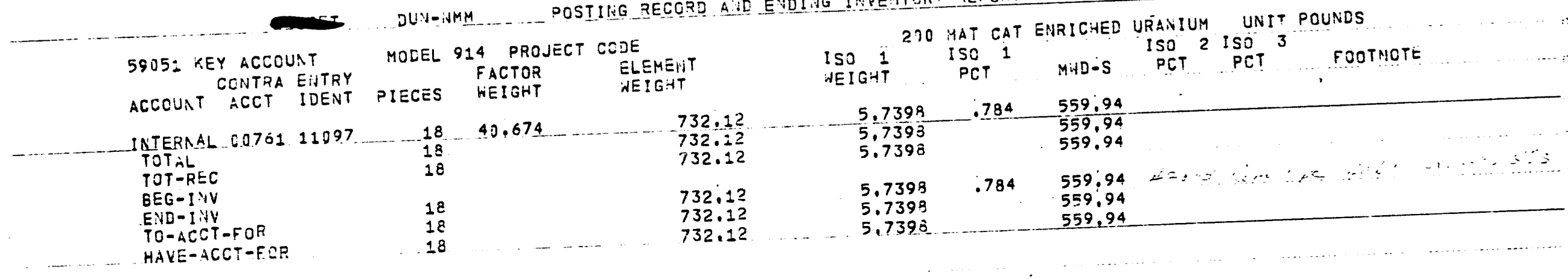


:

JUYi-nMiM

POSTING FECOOD A UE EIDI IG INVEUITORY PEPORT

500 HAT CAT PLUTOMIUH

MOEEL 401 DROJECT CCDE

59051 KEY ACCCUNT CONTRA ENTRY ACCOUNT ACCT IDEINT WEIGHT NEIGHT ISJ 1 ISC 1 MCT 150,2 iso JNIT GRAMS INTEGinAL OC751 11092 TOTAL

-REC 5905141246

INTERN.AL

TOTALL

TO-ACCT -FOR

HAVE-ACCT-F $\triangle A$

59051 KEY ACCOUNT CONTÁA ENTRY ACCOUAT ACCT IDENT

MOLEL 914 PROJECT CODE PIECES WACTOR

IATERAAL 5905141246

TOTAL

TOT-REC

BEG-1:V

END-IIV

TO-ACCT $=F C S$

24.863

447.53 $-39.9389-6.931$

39.9689

$447.53 \quad 39.9689 \quad 3.931$

40.53098

$447.53 \quad 39.9650$

$\begin{array}{ll}447.53 & 39.9659\end{array}$

447.53
447.53

HAVE-ACCT-F CR

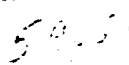
$i=1$.
ELEMENT

\subsection{9}

$\begin{array}{ll}447.53 & 39.9639 \\ 447.53 & 39.9689\end{array}$

$447.53 \quad 39.9689$
4.931

$\begin{array}{r}447.53 \\ 447.53\end{array}-39.9689$

gO MAT CAT PLUTONIUM UNIT GRAMS

${ }_{\text {ISOT }} 1$ MUDES ISO 2 ISOT $_{\text {PCT }}^{3}$ FOCTNCTE

0.931

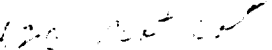

$447.53 \quad 39.9689$ 


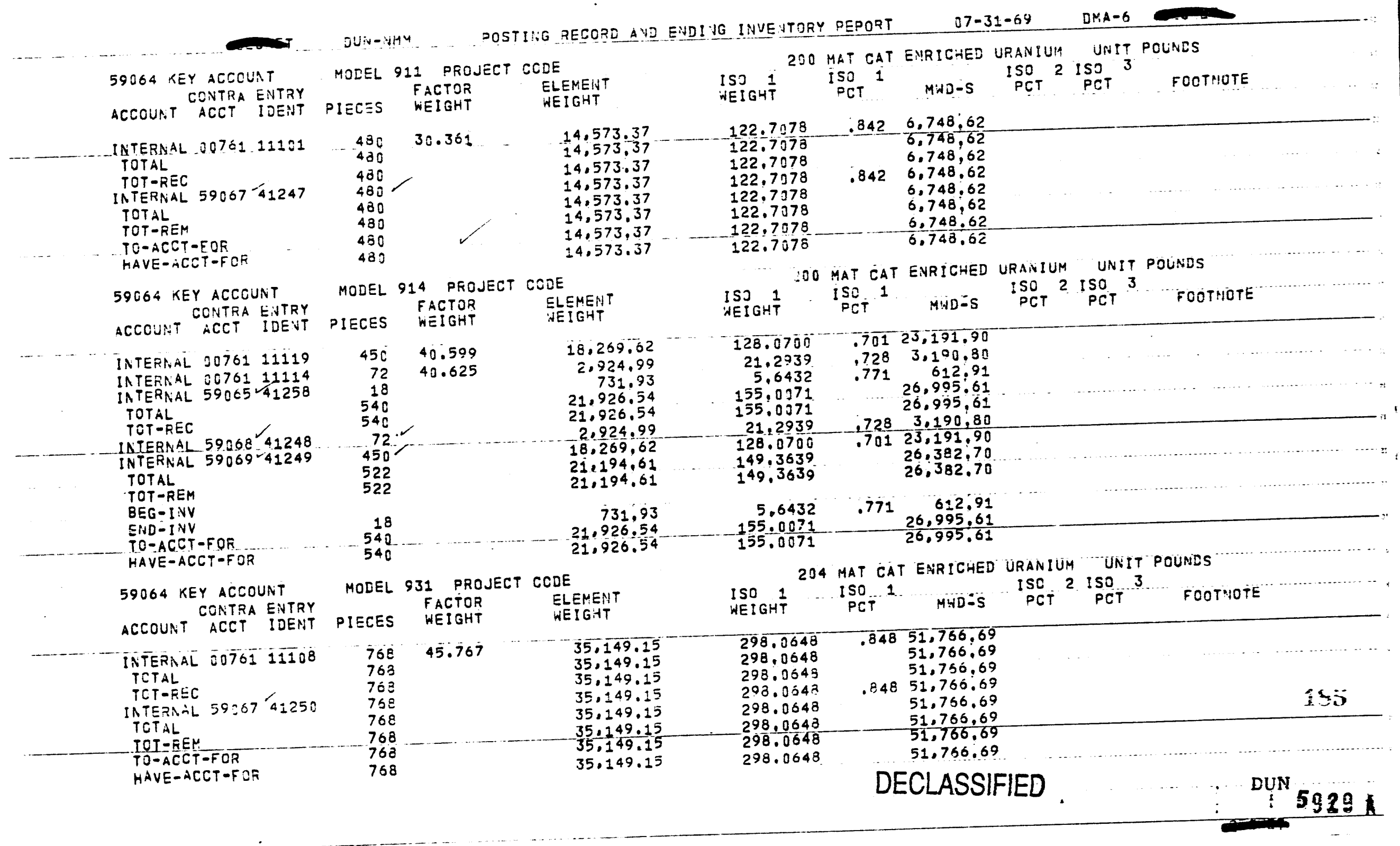


-
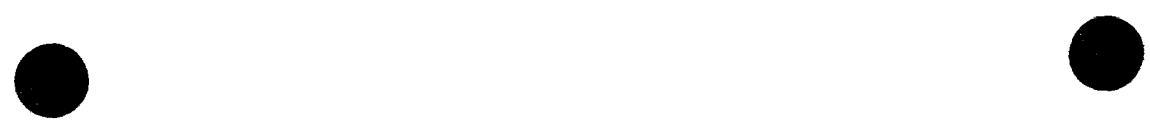

$\ldots$

POSTIING RECORD AID ENDITG INVEITORY REPORT

$07-31-69$

$D M A-6$

DUN

DUVIV-NMY

BOTET CODE

MOEEL 932 PROJECT CODE

204 MAT CAT ENRICHED URMINIUN UNIT POUNES

59064 KEY ACCOUNT CONTRA ENTRY FACTOA

ACCOUAT ACCT IDENT

ECES NEIGHT

NEIGHT

INTERIAL 0.75111104 TOTAL

TOT-REC

INTERAAL 5906741251

TOTAL

TO-ACCT-

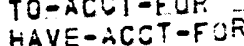

$240 \quad 36.457$

ISJ 1

240

240

240

240

240
240

8.749 .75

8.749 .75

$8,749,75$

$8,749.75$

8.749 .75

8.749 .75

8.749 .75

$74.02590 .346,12.974^{\circ} 35$

PCT PCT FCOTHOTE

$74.0229-.34612 .974 .35$

$74.0229 \quad 12.974: 35$

$74,0229 \quad 346 \quad 12,974,35$

74.0229

$74.0229 \quad 12.974 .35$

$74.0229-12.974 .35$

DECLASSIFIED! 
:

07-31-09 DMA-6

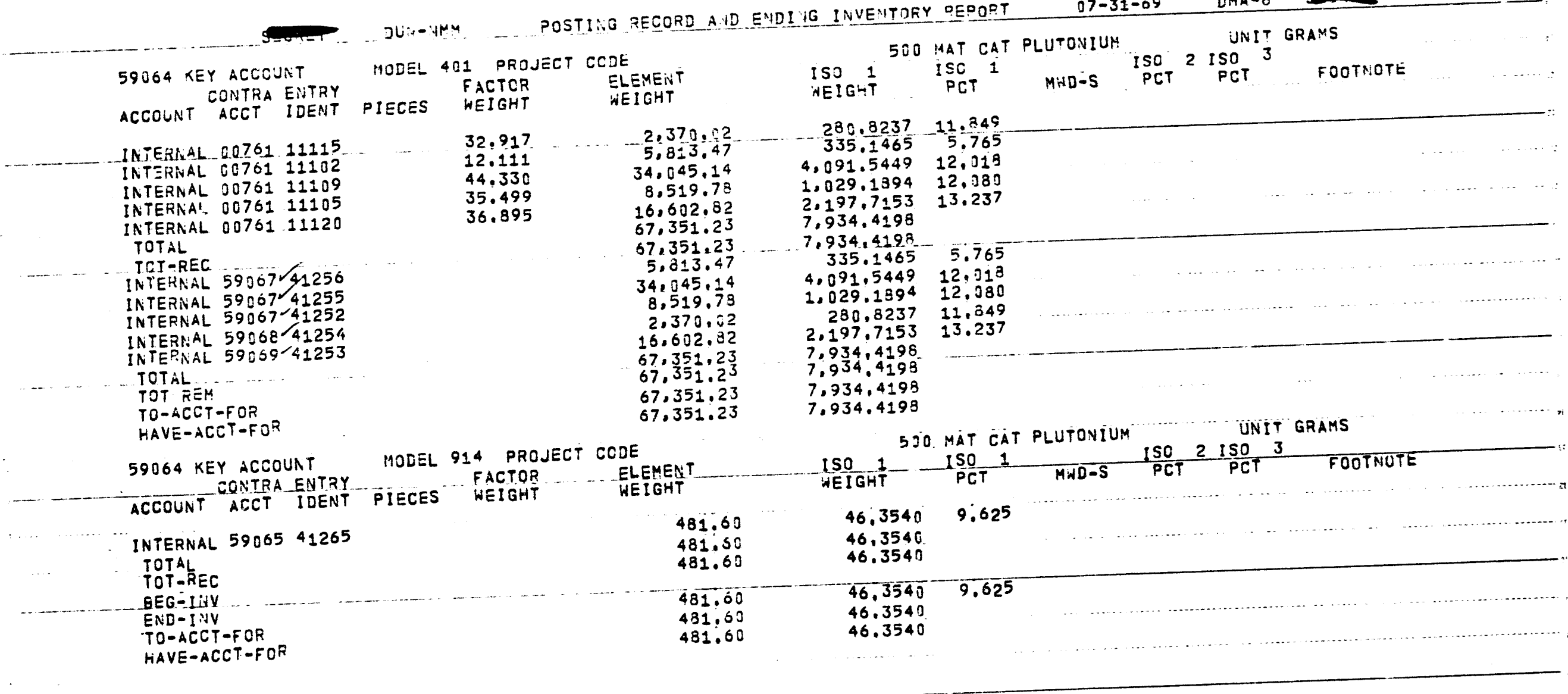




$$
\begin{aligned}
& \text { 震 }
\end{aligned}
$$

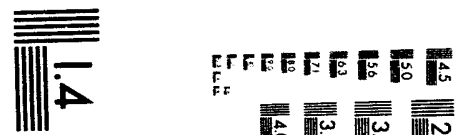

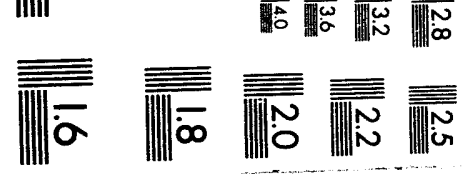




$$
\begin{aligned}
& \omega \\
& \stackrel{o}{\rho} \\
& \omega
\end{aligned}
$$


-

59065 KEY ACCOUNT

CONAT EDNTFA ENTRY

I. IEPNAL D0761.11127.

TOTAL

TOT - FEC

EEG-I:V

END-I:IV

TO-ACCT-FOR

HAYE-ACOT-FOS

59065 KEY ACCCUNT

CONITRA ENTRY

ACCOUNT ACCT IDENT

INTERNAL. $35761-11130$

INTER:AL 0076111123

TOTAL

TOT-REC

INTERNAL $59064^{/ / 41258}$

TOTAL

TOT-BEM

EEG-INV

END-INV

TO-ACCT -FOR

HAVE-ACET-FOR

59065 KEY ACCOUNT

CONTRA E.VTRY

ACCOUNT ACCT IDENT

INTERNAL O0761 11138

TOTAL

TOT-REC

INTERNAL

TOTAL

TOT-REM

TO- $A C O T-F O A$

HAVE-ACOT-FOR
DLin-vms

POSTIIG RECORD A ID EUDIIG INUENTORY REPORT

$07-31-59$

DPA A - 6

MODEL 911 PRJJECT CODE

$\begin{array}{ll} & \text { FACTOR } \\ \text { PIECES WEIGHT } & \text { ELEMENT }\end{array}$

200 MAT CAT ENRICHED URANIUM UNIT POUNDS

$12 \ldots 30.353$
12
12
12
12
12

MODEL 914 PROJECT SEDE

$\begin{array}{ll} & \text { FACTOR } \\ \text { ELEMENT }\end{array}$

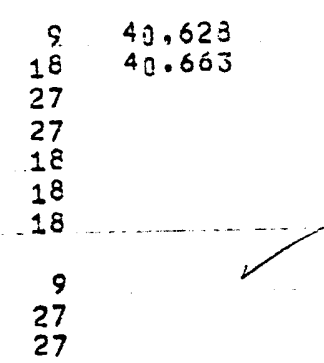

365.65
731.93
1.097 .58
1.097 .55
731.93
731.93
731.93
365.65
1.097 .58
1.097 .58

MOLEL 920 PROJECT CODE

FACTOR ELEMENT

364.23

364.23

354.23

354.23

354.23

PIECES WEIGHT

NEIGH

204.50

204.50
204.50

204.50

204.50

204.39

2.4 .5

234.50
ISO $1 \frac{1}{\text { IEIGHT ISO } 1}$ PCT MHD-S ISO 2 ISOT 3 PCT FOOTNOTE

$\begin{array}{lll}3.0158 & .828 & \frac{194.18}{194.18} \\ 3.0158 & & 194.18 \\ 3.0158 & & \\ 3.0158 & .828 & 194.18 \\ 3.0158 & & 194.18 \\ 3.0158 & & 194.18\end{array}$

200 MAT CAT ENR!CHED URANIUM UNIT POUNES

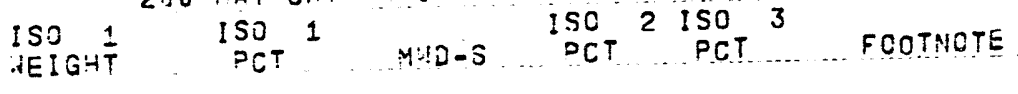

$2,6729 \ldots, 731 \ldots 392,12$

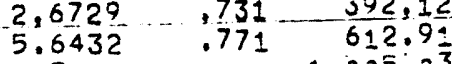

$8.3161 \quad 1.005 .03$

$8.3161 \quad 7, \quad 1,0.05,03$

$5.6432 \quad 612.91$

$5.6432 \quad 612,91$

$2.6729 \quad: 731 \quad 392 ; 12$

$8.3161 \quad 1.005 .03$

8,3161

200 MAT CAT ENRICHED URANIUM UNIT POUNDS

ISO $\frac{1}{1}$ ISO 1 MWDES ISO 2 ISO 3 PCT FOOTHOTE

$1,8160 \quad 51,19$

$\begin{array}{lll}1,8160 & 888 & 51,19 \\ 1,8160 & 51,19\end{array}$

1.8160
1.8160

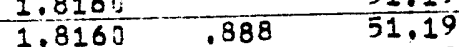

$\begin{array}{lll}1.8160 & .888 & 51.19 \\ 1.8160 & & 51.19\end{array}$

$1.8160 \quad 51: 19$

1.8160
$1.816 ?$

$1.816 ?$

$1.8160 \quad 51.19$ 
- -

POSTING PECORD A UE EVDIVG INVEUTORY REPORT

$07-31-69$

DMA-6

sones

DUNE:23A

59005 KEY ACCOUNT MODEL 932 DRDJECT CCDE

59005 KEY ACCOUNT
CONTFA ENTRY MOCEL
ACCOUAT ACCT IDENT PIECES WACTOR WIGHT

ISO 1 ISO 1

MAT CAT ENRICHED URANIUM UNIT
ISO 1 ISO 2 ISO 3

PCT

ISO
PCT ISO PCT

FOOTNOTE

INTERNAL OOT61 11134

TOTAL

TOT -REC

INTERNAL $59066^{41260}$

TOTAL

TO- ACOT-FO

HAVE-ACCT-FOF

15
15
15
15
15
15
15
15

543.22

546.22

548.22

548.22

548.22

548.22

548.22

548,22
548.22

$5.3287 \quad .972 \quad 428: 14$

$5.3287 \quad 428,14$

$5,3287 \quad 423,14$

$5.3287 \quad .972 \quad 428.14$

$5,3287 \quad 428,14$

$5.3287 \quad 428.14$

5.3287
5.3287

DECLASSIFIED, 



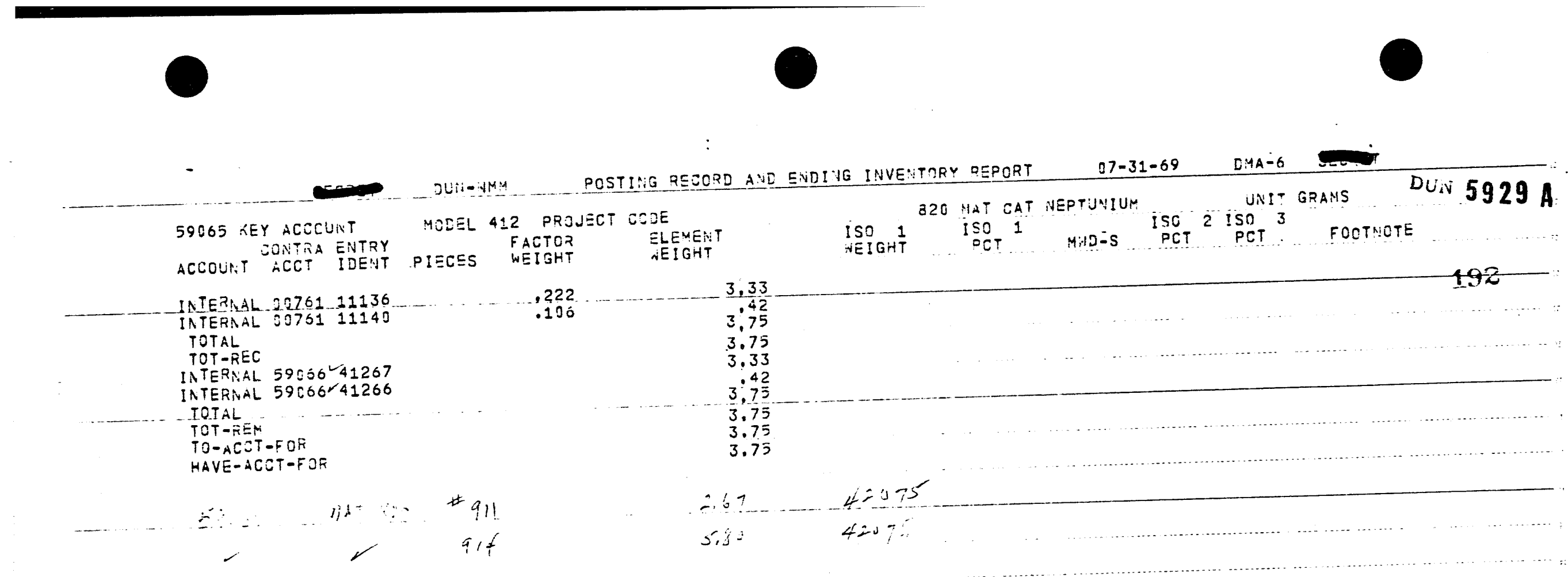

DECLASSIFIED) 
$:$

POSTING RESORD AUE EUDIVG INVESTORY REPORT

$07-31-69$

DMA- 6

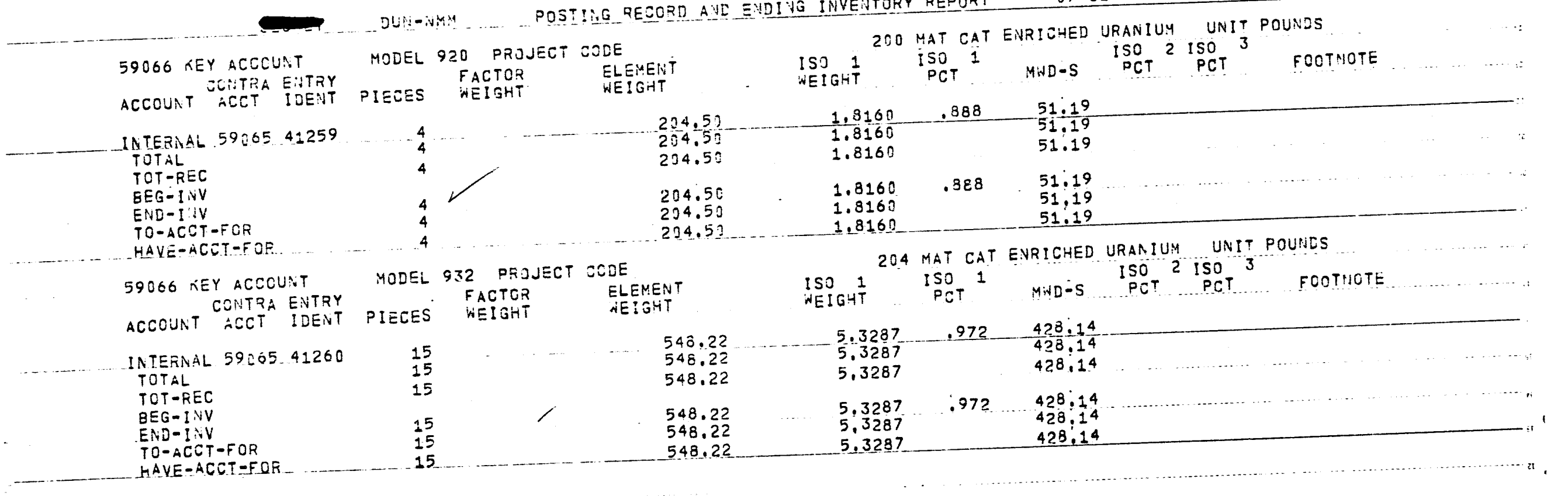



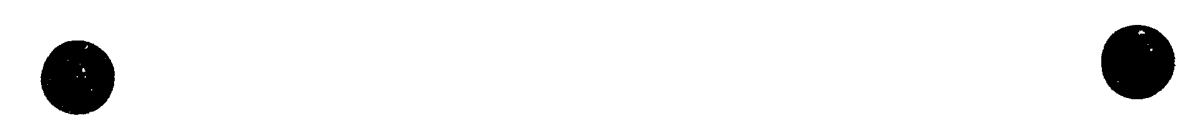

$\cdots$

:

DUV-SMY _ POSTHEO PECORD AND EVDIVG INVEYTORY REPORT

[i1A-6

59000 KEY ACCOUNT MUDEL 920 PROJECT OCDE

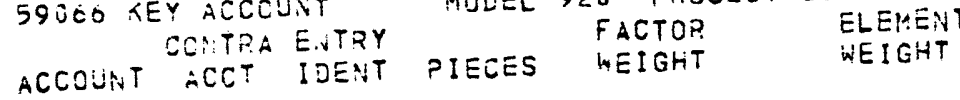
500 MAT CAT PLUTONIUM IN TERIAL 5925541263 TOTAL

TOT-REC

BEG-INV

EUD-IIV

TO- $\angle C E T$-FOR

HAYE-ACOT-FSE

59560 KEY ACECUNT

COATRA ENTRY

ACCOLNT ACCT IDEIAT

ISO 1 ISO

ISO 1 MUDE-S

150
$P C$

UNIT GRAMS

DUN $\div$

IATEEAAL $59: 554: 262$

TOTAL

TUT-REC

EEG-IN

THO-ACCT-FOR
TOAD I

47,71 IEIGHT

PCT FOOTHOTE

HAVE-ACST-ENS

47.71

47.7

MOLEL 932 PROJECT CCOE

MOLEL 932 FACTOR PRSJCT CCOE ELEMENT

47.71 500 HAT CAT PLUTOVIUM

ISO $\mathrm{S}$ ISO 1

POT 1 HWOS

ISO 2 ISO 3

UAIT GRAMS

PIECES WEIGHT

NEIGHT

$22.4711 \quad 7.099$

316,32

316.32

22.4911

$3 \pm 0 . \equiv 2$

22.4911

316.82

316.82

$22.4711 \ldots 7.099$

$316: 82$

22.4711

22.4911

DECLASSIFIED

- 


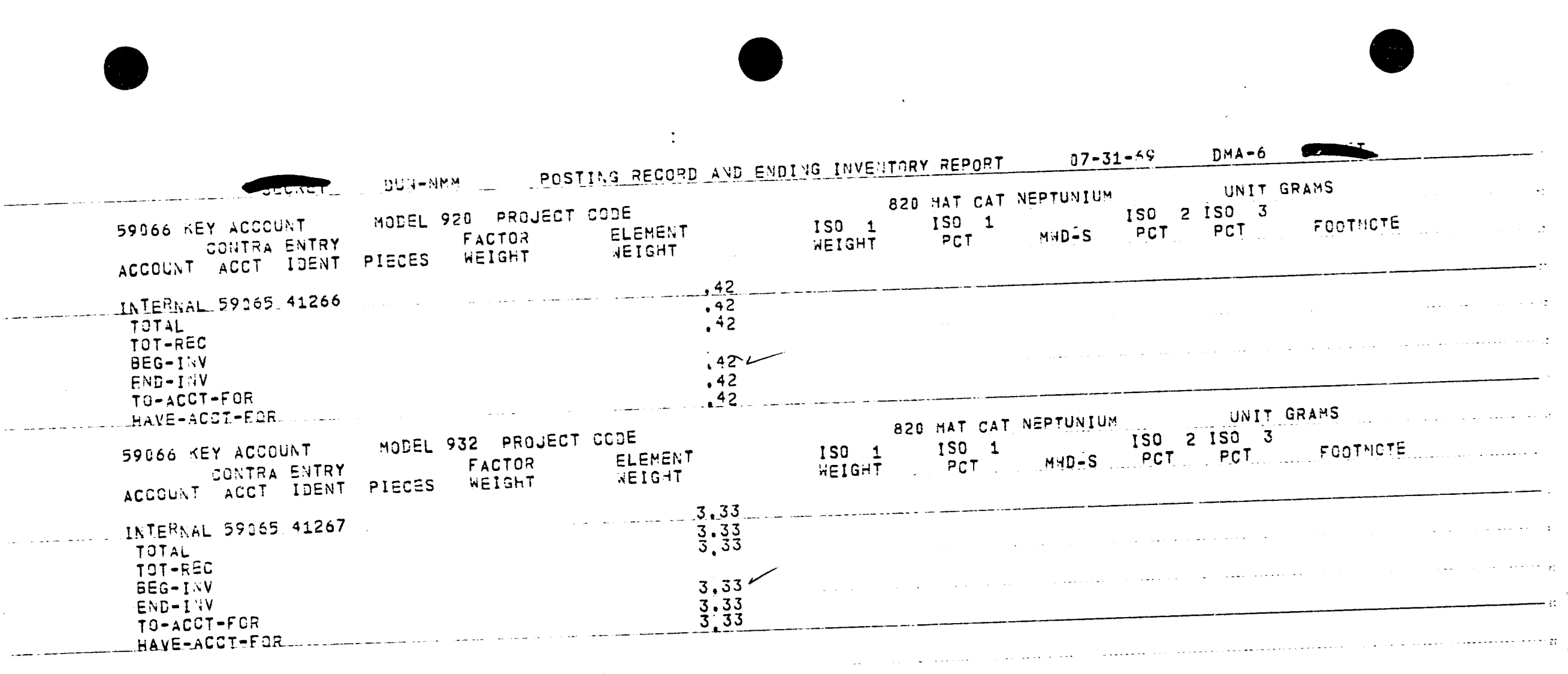

155

\section{DECLASSIFIED}




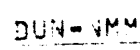

PCSTING RECORD AND EUDI IG INVEUTORY REPORT

$07-31-69$ Di:A-6

59067 KEY ACCCLNT MOLEL 911 DROJECT CODE ACCOUNT ACITSA EITTRY FACTCP OLEMEIIT PIECES HEIGHT UEIGHT

$\begin{array}{ll}\text { ISO } 1 & \text { ISO } 1 \\ \text { WE!SHT } & \text { PCT }\end{array}$

CHED URANIUA

UNIT POUNES

$5929 \mathrm{~A}$

ACCOUNT TOTAL
TOT-REC

EEG-I:IV

END-I:IV

TS-ACCT-FOR

HAVE-ACOT-FOT

59067 KEY ACSOUNT

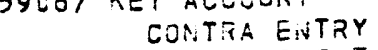

ACCOUNT ACET IDENT

$430 \quad 14,573.37$

$\frac{14,573,37}{14.573 .37}$

ISE

$M: A D=S$

ISO 2 iso 3

PCT FOOTNOTE

196

INTEENAL $59004 \quad 4 \$ 250$

TOTAL

TCT-FEC

$B E G-I i, V$

EVID-IDV

TO-ACCT-FCR

HAVE-ACCI-FOR

59067 KEY ACCOUNT

CENTRA ENTRY
ACCOUNT ACCT IDENT

430

14.573 .37

122.7073

$.842 \quad 6,748: 62$

$\begin{array}{ll}122.7073 & 6.748 .62 \\ 122.7078 & 0.748 .62\end{array}$

$\checkmark \quad 14.573 .37$

14.573 .37

122.7078

$\begin{array}{ll}480 & 14,573.37 \\ 430 & 14,573.37\end{array}$

$\begin{array}{ll}122.7073 & 6.748 .62 \\ 122.7379 & 6.748 .62\end{array}$

$.842 \quad 6,740,62$

OLEL 931 PROJECT CCDE

MOCEL 931 PACTOR ELEMENT 6.748 .62

PIECES WEIGHT WEIGHT

MAT CAT ENRICHED URANIUY JNIT POLIOS
ISO 1 ISO 2 ISO 3
PCT MIVD-S PCT PCT FOTIOTE

INTERAAL $59064 \quad 41251$

TOTAL

TOT-REEC

EEG-IYV

TO-ACCT-EOR

HAVE-ACET-FOR

\begin{tabular}{|c|c|}
\hline $\begin{array}{l}760 \\
76 s \\
768\end{array}$ & $\begin{array}{l}35.149 .15 \\
35.149 .15 \\
35.149 .13\end{array}$ \\
\hline $\begin{array}{l}768 \\
768 \\
768\end{array}$ & $\begin{array}{l}35.149 .15 \\
35.149 .15 \\
35.149 .15\end{array}$ \\
\hline
\end{tabular}

$298.0643 \quad \frac{.848 \quad 51.766 .69}{51.756 .69}$

$298.0643 \quad 51.756 .6$

298.0543

51.766 .69

$\begin{array}{ll}298.0648 & 51.766 .69 \\ 298.0648 & 51.756 .69\end{array}$

MOCEL 932 PROJECT CODE $51,756,69$

$\begin{array}{ll} & \text { FACTOR } \\ \text { PIECES HEIGHT } & \text { ELEMENT }\end{array}$

204 MAT CAT ENRICHED URANIUM JNIT POLNDS

PECES REIGHT

8.749 .75

ISO 1

ISO 1 MUDOS SOT 2 ISO

FOOTNOTE

$\begin{array}{lllr}240 & 8.749 .75 & 74.0229 & .846 \\ 240 & 8.749 .75 & 74.974 .35 \\ 240 & 8.749 .75 & 74.0229 & 12.974 .35 \\ & & 12.974 .35 \\ 240 & 8.749 .75 & 74.0229 & .846 \\ 240 & 8.749 .75 & 74.0229 & 12.974 .35 \\ 240 & 8.749 .75 & 74.0229 & 12.974 .35\end{array}$

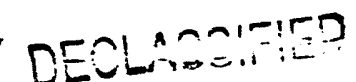


: 37-31-69 [MA-6

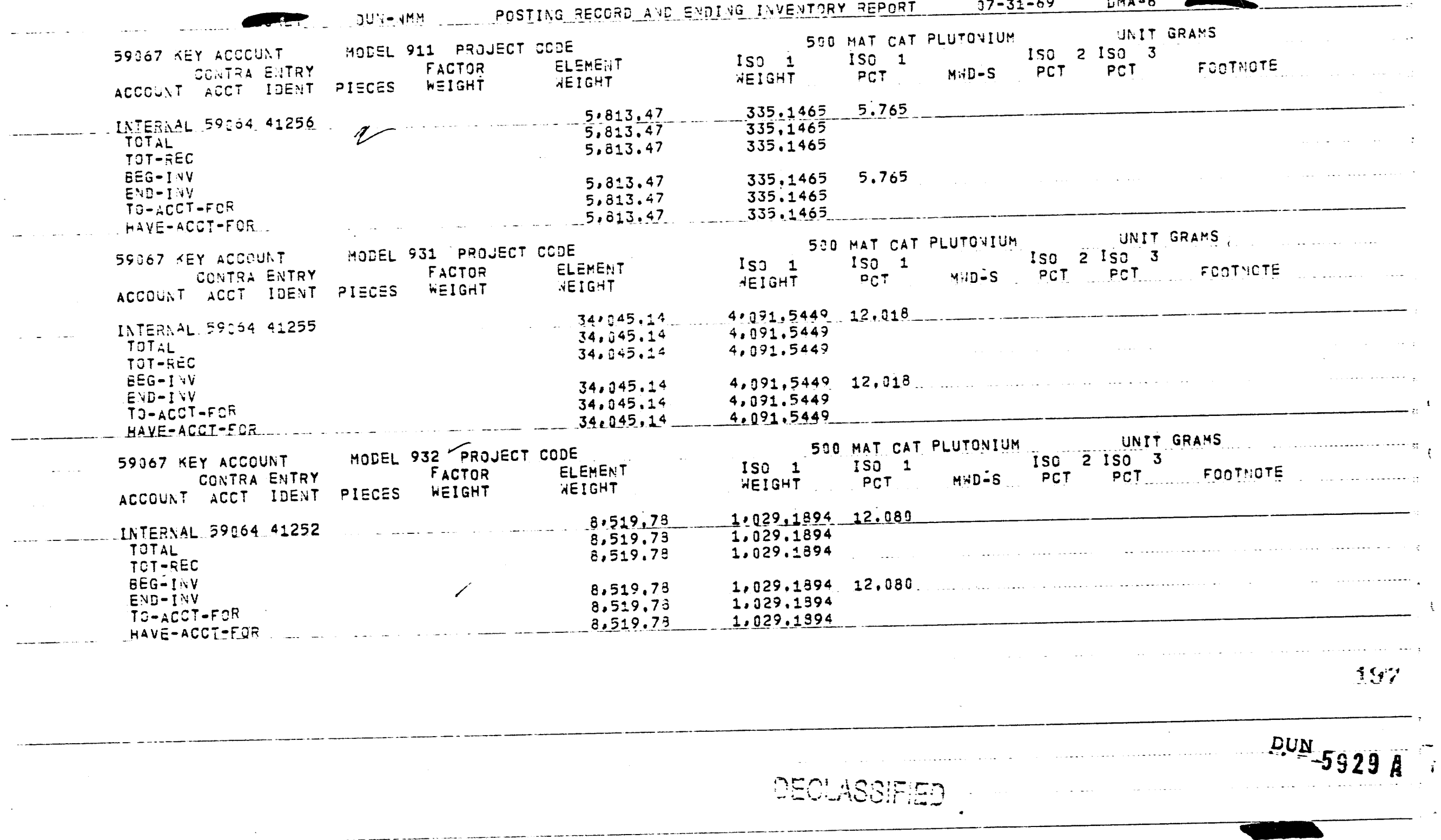



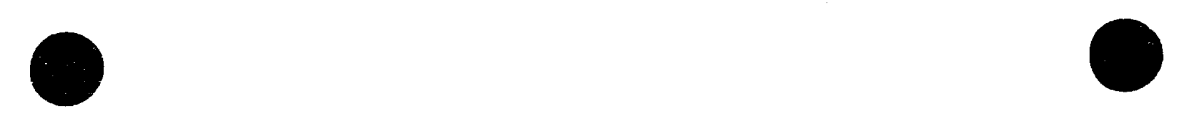

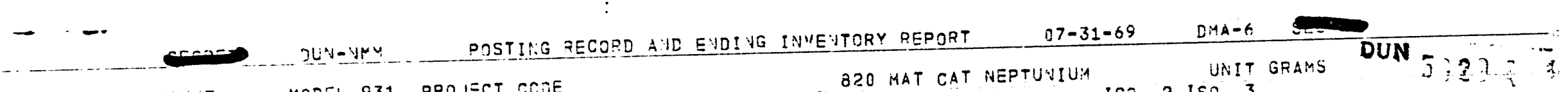

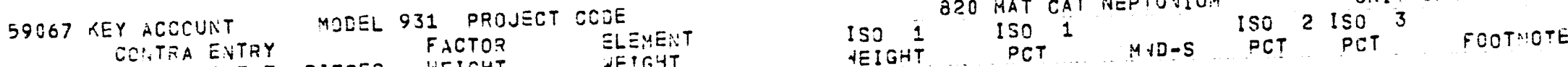
ACCOURT ACCT IDENT PIECES WEIGHT AEIGHT

TOTAL

453.55 458.55
458.55

TOT-REC

BEG-INV

END-INV
TO-ACCT -FOP

458.55

458.55

HAVE-ACCI-EDR

$459.5=$

$$
\begin{aligned}
& 59067 \text { NOD9s2 } \\
& 115,07 \quad 42015 \\
& \text { mard } 91 T^{3} 3.99 \\
& 42011
\end{aligned}
$$

DECLASSIFIED 


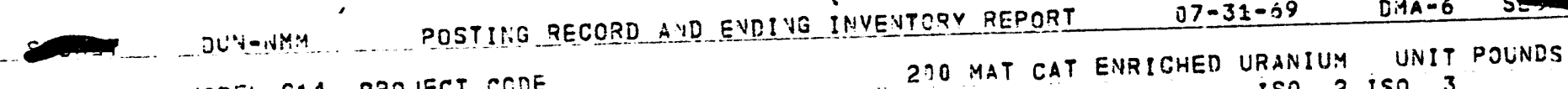

$07-31-59$

DiAA-6

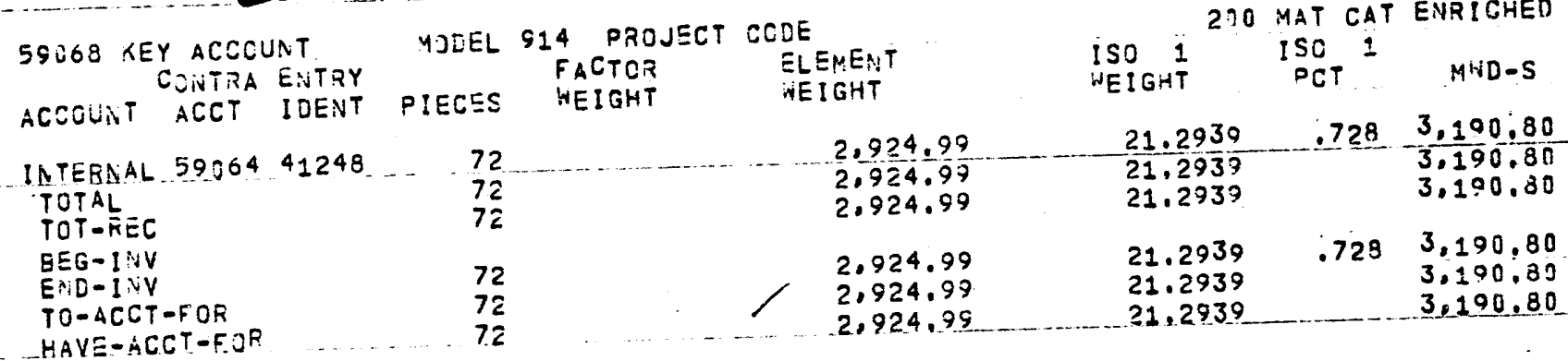




\section{•}

\section{-}

$\ldots$

:

POSTING RECORD AID ENDIVG INUEVTORY QEPORT

DYA-6

UNIT GRANS

39068 KEY ACCOUNT MODEL 914 PAOJECT CODE ISO 500 HAT CAT PLUTOVIUM ACCOUNT CDIUTRA ENTRY PIECES WEIGHT WEIGHT

INTEENAL $59064 \quad 41254$

TOTAL

EEG-INV

ETID-IVY

TO-ACCT-FCS

HAVE-ACET-FO?

-

2.370 .02 NEIGHT

ISO 1

UNIT

\section{$5929 \mathrm{~A}$ \\ 0}

$230.8237 \quad 11: 849$

$\frac{2.379 .102}{2.373 .22}-\frac{290.8237}{230.8237}$

$2,373.02 \quad 280.8237$

MUD-S PCT ${ }^{2}$ ISOT FOOTHOTE

$2.370 .02-280.823711 .849$

2,370

2.370 .32

280.8237

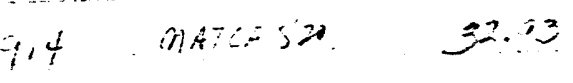

$4237=$

ASSIFIED, 

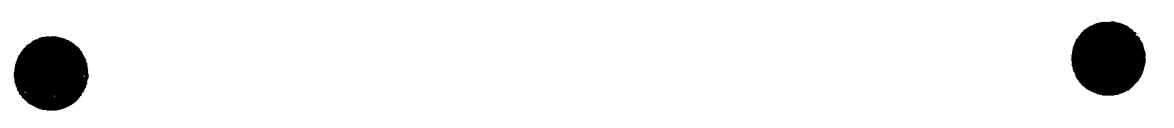

POSTISO RECORD AVD ENDI IG INVEUTORY REPORT

$07-31-59$

DMA-6

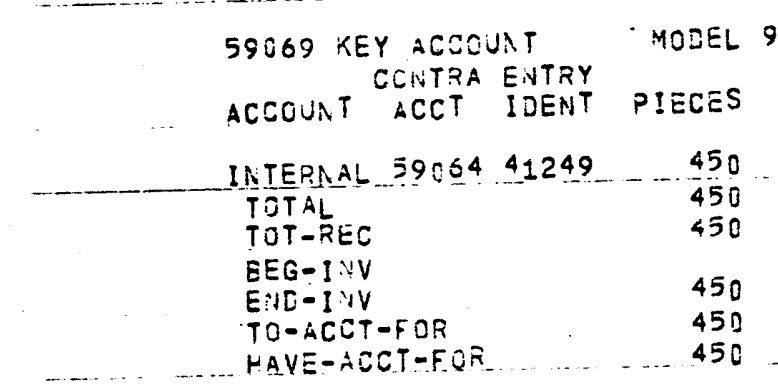

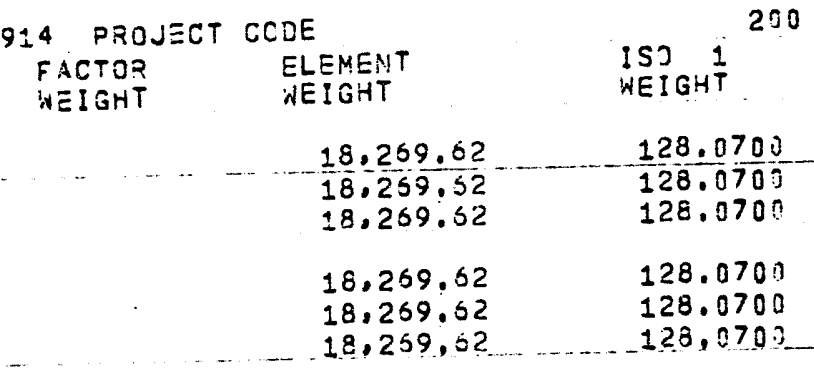

IST 1 ISTH ISO 1

CHED URANI

UNIT FOLAES

450

701230191

ISO 5 ISO 3

SOT 3 FEOTNOTE

$z \mathfrak{x}$ 


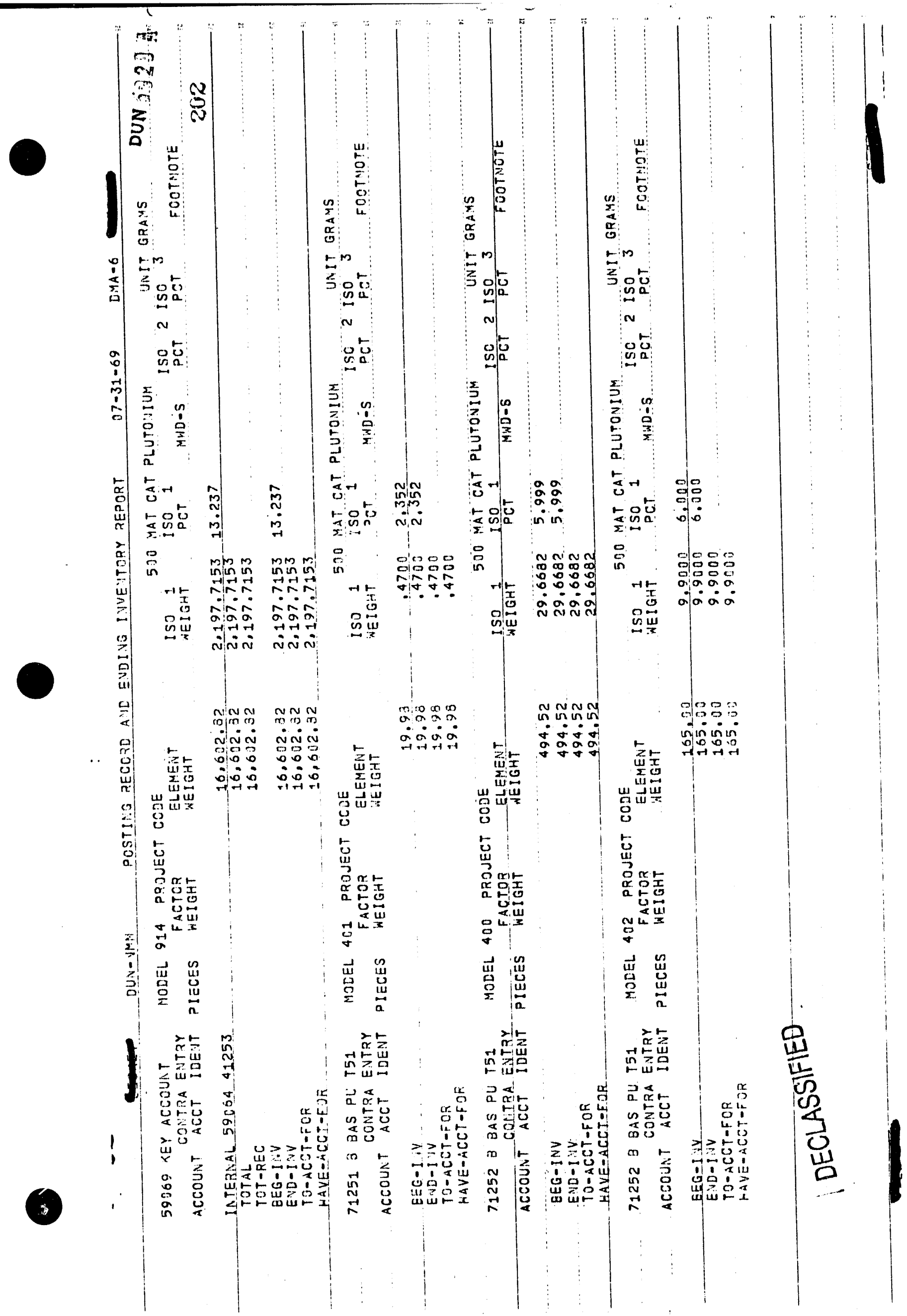


$27-31-69$

DMA-6

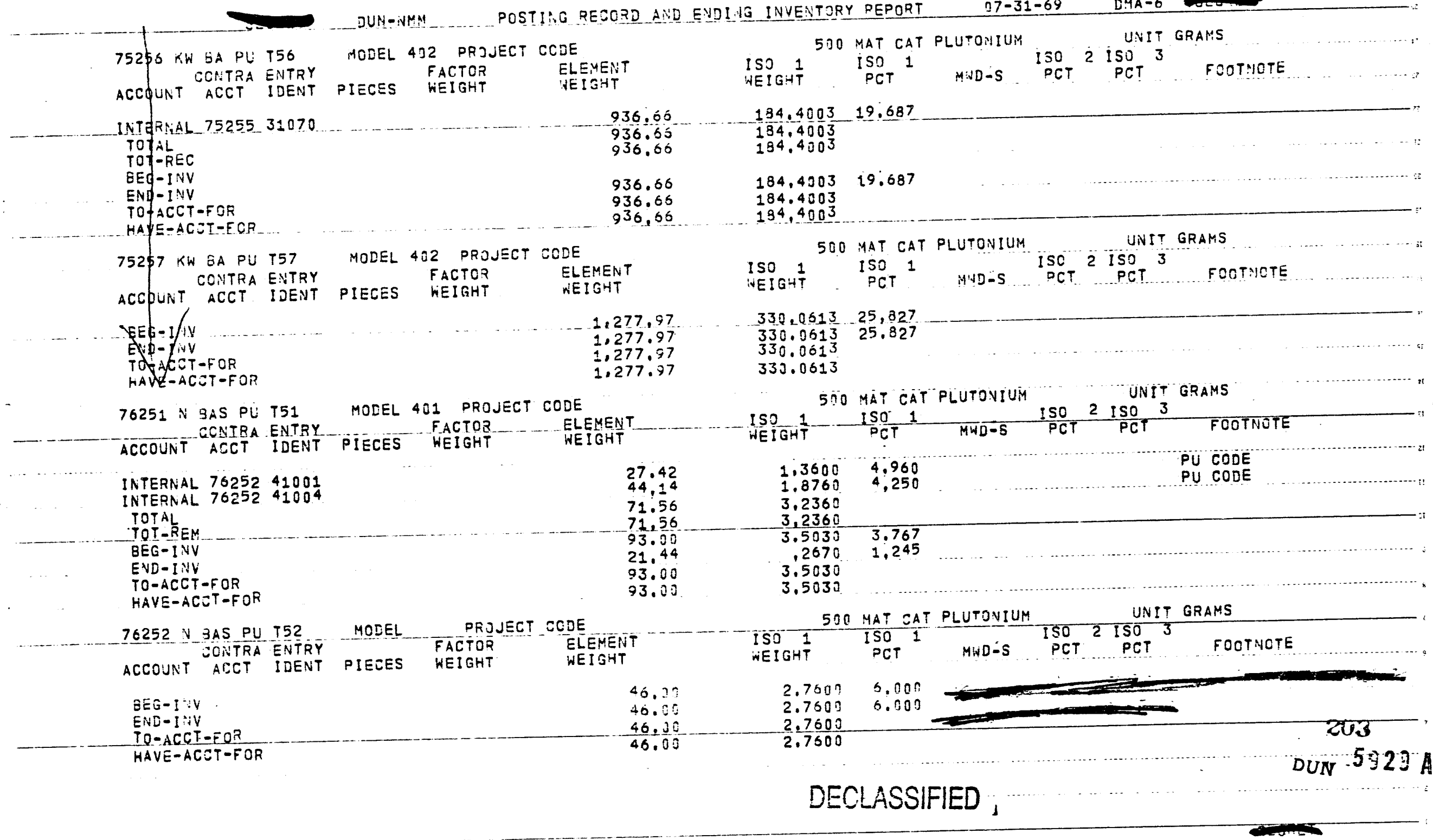


DMA-6

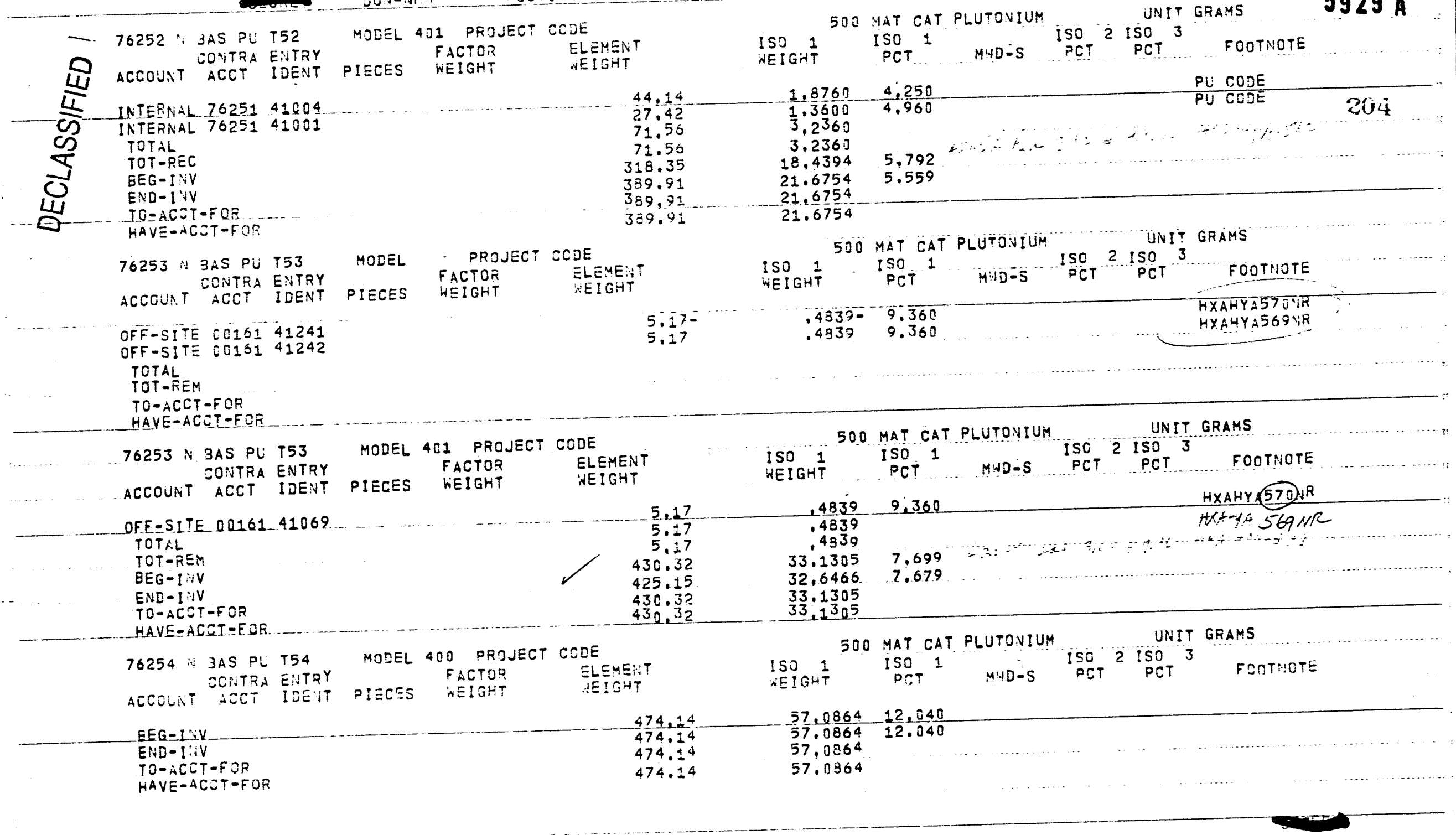


:

7-31-69 DMA-6

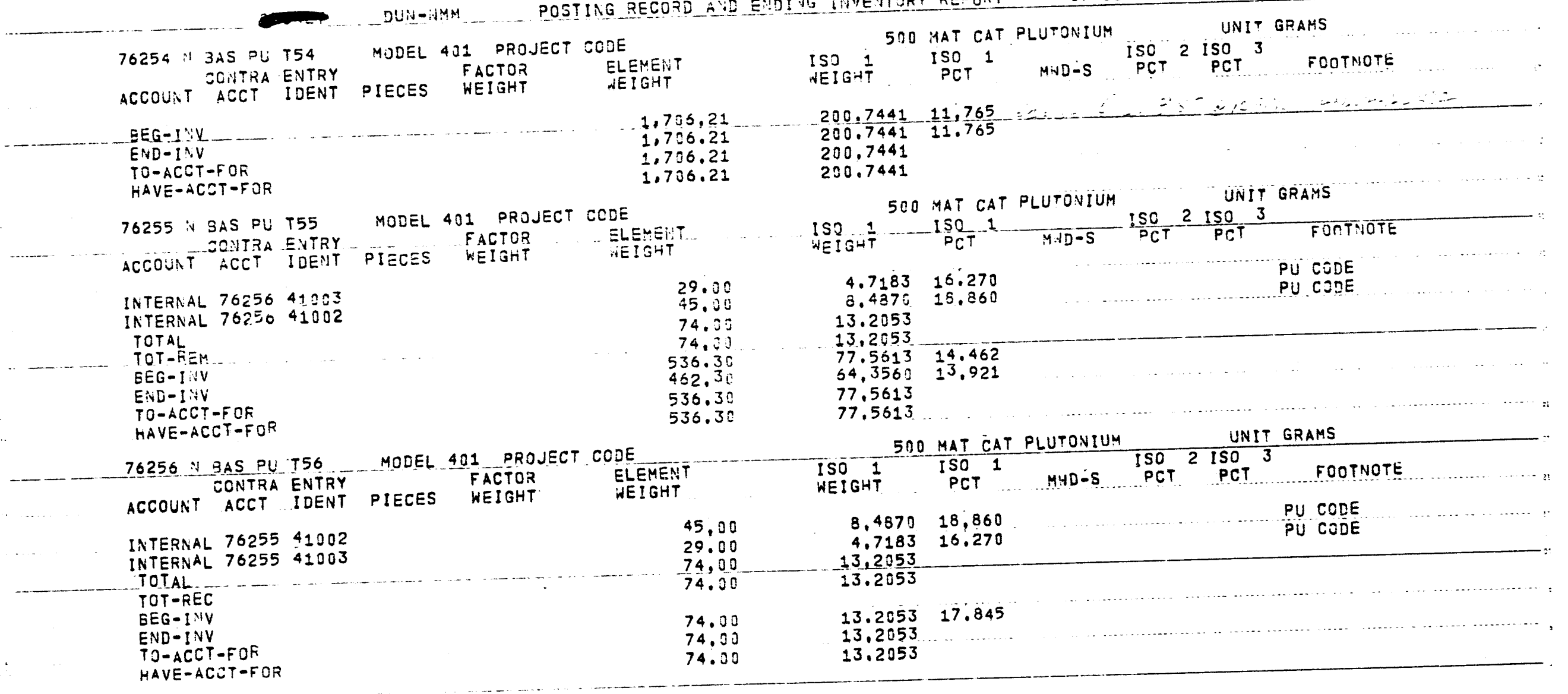


POSTII.G RECORD A ID EVDIYG IUVEUTORY PEPORT

$07-31-69$

D.AA-6

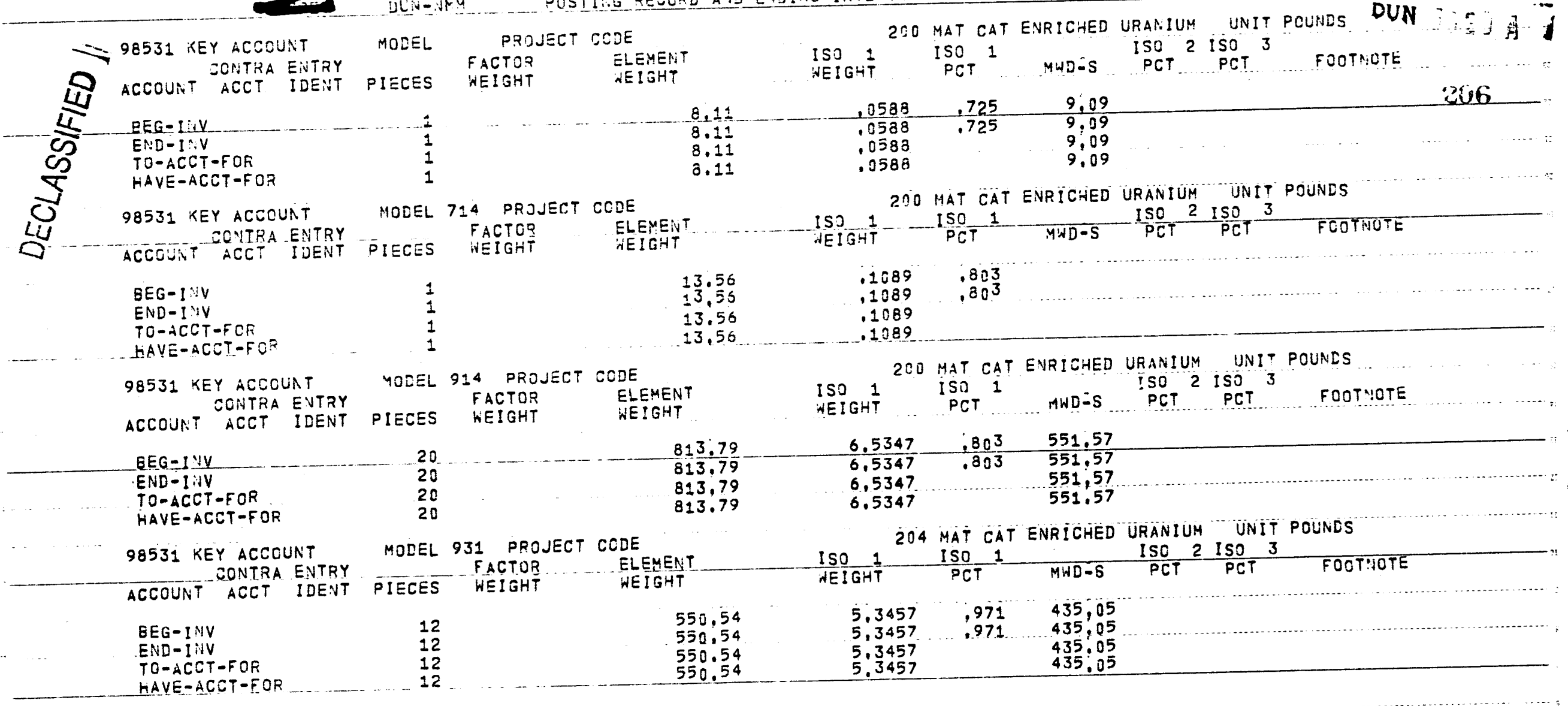



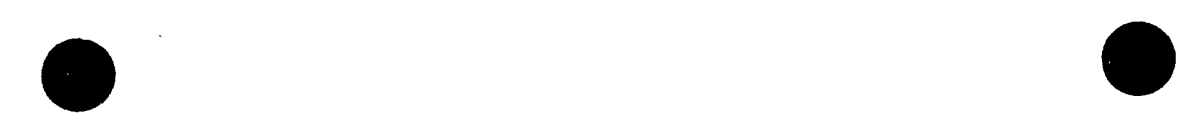

SUN-IMM

POSTII:G RECORD AIL ENDIIG INVEVTORY REPORT

07-31-69 D.4A-6

\section{KEY ACCCURT MODEL 401 PRJJECT CCDE}

DOHTRA ENTRY
ACCOUNT ACCT IDENT

PIECES FACTOR
WEIGHT

ELEMETT

500 MAT CAT PLUTOYIUM

IST 1 ISO

UNIT GRAMS

\section{BEG-IYY}

EIND-IIV

TO-ACCT-FOR

HAVE-AC $T$ T-FOF

772.19

772.19

772.19

772.19

$61.8738 \quad 8,013$

$\frac{1}{1.8738} \quad 9.013$

51.8738

61.8733

8

กUN 329A

DECLASSIFIED 
$07-31-69$

98531 KEY ACCOUNT COINTRA ENTPY ACCOUNT ACCT IDENT

MODEL 412 PROJECT COJE
FACTOR
PIECES WEIGHT NEMENT
$\begin{array}{ll}820 \text { MAT CAT } \\ \text { ISO } 1 \\ \text { MEIGHT } & \text { ISO } 1 \\ \text { PCT }\end{array}$ NE! GHT EPTUYIUY ACTO BEG-IYY

TO-ACCT-FOR

HO- ACCT $-F O R$
HAVE-ACCT FOR

8.29
8.29

8.29

8.29

ISO 2 ISO
DMA-6 UNIT GRAMS

SO 3

$929 A$

PCT FONTNOTE

\section{DECLASSIFIED}


DLiv-NMM

PCSTI::G RECOPD AII ENDIVG INVEYTORY REPORT

$07-31-69$

DMA-6

98560 KEY ACCCUNT

SCNTRA ENTRY

ACCOUNT ACCT IDENT

MODEL 718 PROJECT CODE

204 MAT CAT ENRICHED URANIUM INNIT POUNDS

$B E G=I: Z V$

TO-ACCT-FOF

HAVE-ACIT -FOF

9856 J KEY ACCOUAT

ACCOUNT ZONTRA ENTRY IDENT

EEG-I:S

EIrD-ITH

TO-ACCT-FOR

HAVE-ACET - FOF

98560 KEY ACCOUNT

98560 KEY ACCOUNT

ACCOUITT ACCT IDENT

EEG-I:UY

END-INV

TO-ACCT-FOR

HAVE-ACCF-FOR

98560 KEY ACCOUNT OONTRA ENTRY
ACCOUAT

\section{$B E G-I N V$}

END-INV

TO-ACCT - FOR

HAVE-ACET-FOR
FACTOR ELENENT

2
2
2
2

ISO 1 ISO 1 MCT ${ }_{\text {PCT }}{ }_{\text {PCT }}$

FCOTMOTE

-.. 33,52

$33,52 \ldots-.3955-1,180$

$33.52 \quad .3955$

MOEEL 818 PROJECT CODE

MOEEL 818 PRUJECT CODE
PIECES WACTOP........ ELEMENT

204 MAT CAT ENRICHED URANIUM UNIT POUNDS

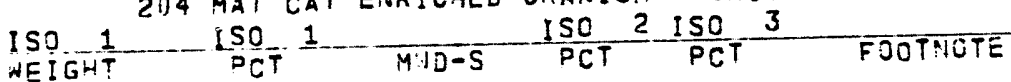

$\begin{array}{ll}2 & 67.01 \\ 2 & 67.01 \\ 2 & 67.01 \\ 2 & 67.21\end{array}$

MDDEL 918 PROJECT CODE

MODEL 918 FACTOR ELEMENT

NEIGHT

523,57

523.57

523.57

523.57

$.7907 \quad 1.180$

.7907

.7907

67.01

10
10
10
10

MODEL 931 PROJECT CODE

MACTOR - ELEMEN
PIECES WEIGHT

$1 . .7907$

204 MAT CAT ENRICHED URANIUM UNIT POUHDS

ISO 1 ISEIGHT ${ }_{\text {PCT }} 1$ MHD-S ISO 2 ISO 3 PCT FOOTNOTE

$\frac{6.1729}{6.1729} \frac{1.179}{1.179}-\frac{181,00}{181,00}$

$0.1729 \quad 1.179 \quad 181,00$

6.1729
6.1729

204 MAT CAIT ENRICHED URANIUM UNIT POUNDS

$\begin{array}{llll}1 \text { SO } 1 & \text { ISO } 1 & \text { ISO } 2 \text { ISO } 3 & \\ \text { NEIGHT } & \text { PCT } & \text { MID-S } & \text { PCT }\end{array}$

GH

$\begin{array}{lll}5,2001 & 945 & 506,08 \\ 5,2001 & 945 & 506,08 \\ 5.2001 & & 506,08 \\ 5,2001 & & 506.08\end{array}$

$\begin{array}{ll}33.52 & .3955 \\ 33.52 & .3955\end{array}$ 


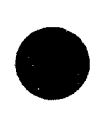

$07-31-69$ D.MA-6

98560 KEY ACCOUNT DOUTRA ENTRY MODEL 401 PROJECT CODE MODEL 401 PROJECT CODE ACCOUNT ACCT IDENT

NEIGHT

500 MAT CAT PLUTONIUM

EEG-I:VY

TO-ACCT TOR WEIGHT

ISO 1 ISO

UNIT GRAMS

DUN5:2]

$511.81 \quad 30.9539-6.048$

$30.9539-6.048$ MHDOS

ISO 2 ISO

30.9539

$\begin{array}{ll}511.81 & 30.9539 \\ 511.81 & 30.9539\end{array}$

210 DECLASSIFIED 
-
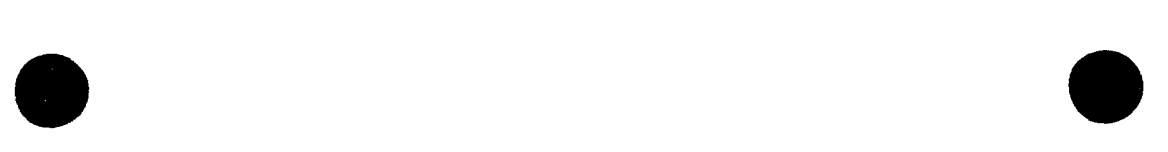

:

DO... DUN-IMMY POSTING RECORQ AVE EULIVG INVEUTORY REPORT

$07-31-69$

DMA-6

98560 KEY ACCOUNT CONTRA ENTRY MOEEL 412 PRJJECT CCDE

ELEMENT

820 YAT CAT NEPTUNIUM ISJ 1 ISIGHT POT 1 WEIGHT

UNIT GRAIS ACCOUNT ACCT IDENT FACTC? WEIGHT MHD-S SC $2150^{\circ} 3$ QEG-1:Y TND - I V V HAVE-ACCT-FOR

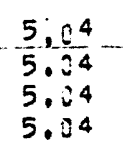

818

nUN 
$\cdots+$

POSTING RECCRD AVD EVDI'VG INVEVTCRY REPORT

$07-31-69$

DMA -6

DUYY

DULA-NMM DEOJECT CCDE

98588 KEY ACCOLNT CONTRA EVTRY ACCOUNT ACCT IDENT

PIECES

FACTOR
WEIGHT

200 MAT CAT ENRICAED URANIUM UNI POUNES

$\begin{array}{ll}\text { ISO } 1 & \text { ISO } \\ \text { WEIGHT } & \text { PCT }\end{array}$

$M \because 1 D-5$

ISO 2 ISO 3

FOOTMOTE

212

EEG-1YY

TO-ACCT-FOA

HAVE-ACCT TOR

$\frac{3}{3}$
3
3

$24 \cdot 30$
$24 \cdot 30$
$24 \cdot 30$
$24 \cdot 30$

98588 KEY ACCOUNT

EOUTSA ENTRY

ACCOLIT ACCT IDEVT PIECES

MODEL 913 PROJECT CODE

$\begin{array}{lrr}.1696 & .698 & 31,33 \\ .1696 & .698 & 31: 33 \\ .1696 & & 31,33\end{array}$

$.1696 \quad 31,33$

FACTOR … ELEMENT

200 MAT CAT ENRICHED URANIUM UNIT POUNDS

WEIGHT

WEIGAT

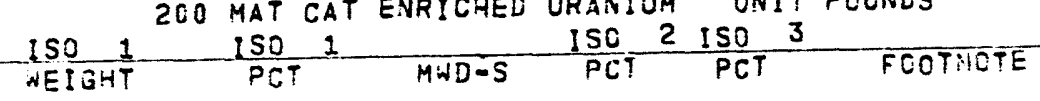

END-I:V

TO-ACCT -FOR

HAVE-ACCT -FOR

$\begin{array}{ll}10 & 366.5 \\ 10 & 366.50\end{array}$

$10 \quad 356.50$

366.50

98588 KEY ACCOUNT

CONTRA ENTRY
ACCOUNT ACCT IDENT PIECES HEIGHT PLTOR WEIGHT

MOEEL 914 PROJECT CODE

BEG-IIV

END-Iidy

HAVE-ACCT-FOR

53

2.155 .29

2.155 .29

2.155 .29

2.155 .29

98588 KEY ACCCUNT CONTRA ENTRY ACCOUNT ACCT IDENT

53$$
2.155 .2
$$

MODEL 918 PROJECT CODE PIECES WEIGHT NACTOR NEIGHT

NEIGHT

BEG-INV

END-1.V

TO-ACCT-FOR
HAVE-ACIT-FO

DECLACCIFIED $\begin{array}{lll}3,4121 & 931 & 22,98 \\ 3,4121 & 931 & 22,96\end{array}$

$3.4121 \ldots 22,78$

3.4121 22,98

ISO I ISO ISHT MCT 1 MHDES ISOT 2 ISO PCT 3 FOCTNOTE

$\begin{array}{lll}16.2342 & .753 & 2.028 .58 \\ 16.2342 & .753 & 2.028 .58\end{array}$

$\begin{array}{lll}16,2342 & .753 \quad 2,028,58 \\ 16,2342 & 2.028,58\end{array}$

$16,2342 \quad 2,028.58$

204 MAT CAT ENRICHED URANIUM UNIT POUNOS

$14,4464 \quad 1,154 \quad 540,96$

$14,4464 \quad 1,154 \quad 540,96$
200 MAT CAT ENRICHED URANIUM UNIT POUNES

$\begin{array}{lllll}1 S O & 1 & \text { ISO } 1 & \text { ISO } 2 \text { ISO } 3 & \\ \text { PCT } & \text { MWD-S PCT PCT } & \text { FOOTNCTE }\end{array}$

\begin{tabular}{lrrrr}
24 & 1.251 .85 & 14,4464 & 1,154 & 540,96 \\
24 & $1,251.85$ & 14,4464 & 1.154 & 540,96 \\
24 & 1.251 .85 & 14.4464 & 540,96 \\
24 & 1.251 .85 & 14.4464 & 540,96 \\
\hline
\end{tabular}


$07-31-69$

DUN-ANM MOEEL 401 PROJECT CODE 98580 KEY ACCOUNT CONTRA ENTRY ACCOUNT ACCT IDENT

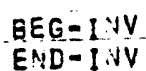

END- INV

TC-ACCT-FOR

HAVE-ACCT-FOR
500 YAT CAT PLUTONIUM ISO 1 ISO 1 MHD-S Iso

$D M A-6$

UNIT GRAMS

ISO 3 FOOTHOTE

213

Dư $5929 . A$ 
$07-31-69$

DMA-6

98588 KEY ACCOUNT MODEL 412 PROJECT CCDE

S2O MAT CAT NEPTUMIUM

FACTOR
CONTRA ENTRY ELEMENT
ACCT IDENT PIECES WEIGHT ISO 1 ISO 1

UNIT GRAMS

ACCOUNT ACCT IDEN

22.97

BEG-I IV

TO-ACCT-FOR
END I INV

HAVE-ACCT-FOR

22.97

22.97

22.97

DUN 59294

DECLASSIFIED 
DUP-HMM

POSTING RECORD AYD ENDI IG INVEATORY REPORT

$07-31-69$

DMA-6

98034 KEY ACCOUNT MODEL 915 PROJECT CODE
CONTRA ENTRY MACTCR P ELM CONTRA EITTRY
ACCOUNT ACCT IDENT PIECES HEIGHT

$B E G=1: I V$
END $-1, I V$

TO-ACCT-FGR

$42+1,920,07$

$1,920.07$
1.920 .07

$1,920.07$

200 MAT CAT ENRICHED URANIUY UNIT POUNES

HAVE-ACCT-FOR

ISO 1 ISO 1

MWD-S

ISO 2 ISO
PCT

FCOTMOTE

98634 KEY ACCOUNT MODEL 918 PROJECT CODE

98634 KEY ACCOUNT MODEL 918 PROJECT CODE

$\begin{array}{lll}16.6982 & 870 & 633 \\ 16.6982 & 89\end{array}$

633,89

$\begin{array}{ll}16.6982 & 633.89 \\ 16.6982 & 633.89\end{array}$

204 MAT CAT ENRICHED URANIUM UNIT POUNDS

ACCOUNT ACCT IDENT PIECES WEIGHT

ELEMENT

15011204 MAT 1 MWD-S 150 PCT 150 PCT

GEG-INV

TO-ACCT - FOR

22
22
22

HAVE-ACCT-FOR.

$\begin{array}{ll}22 & 1.151 .05 \\ 22 & 1.151 .05\end{array}$

WEIGHT PCT

MWD-S

3 FOOTMOTE 


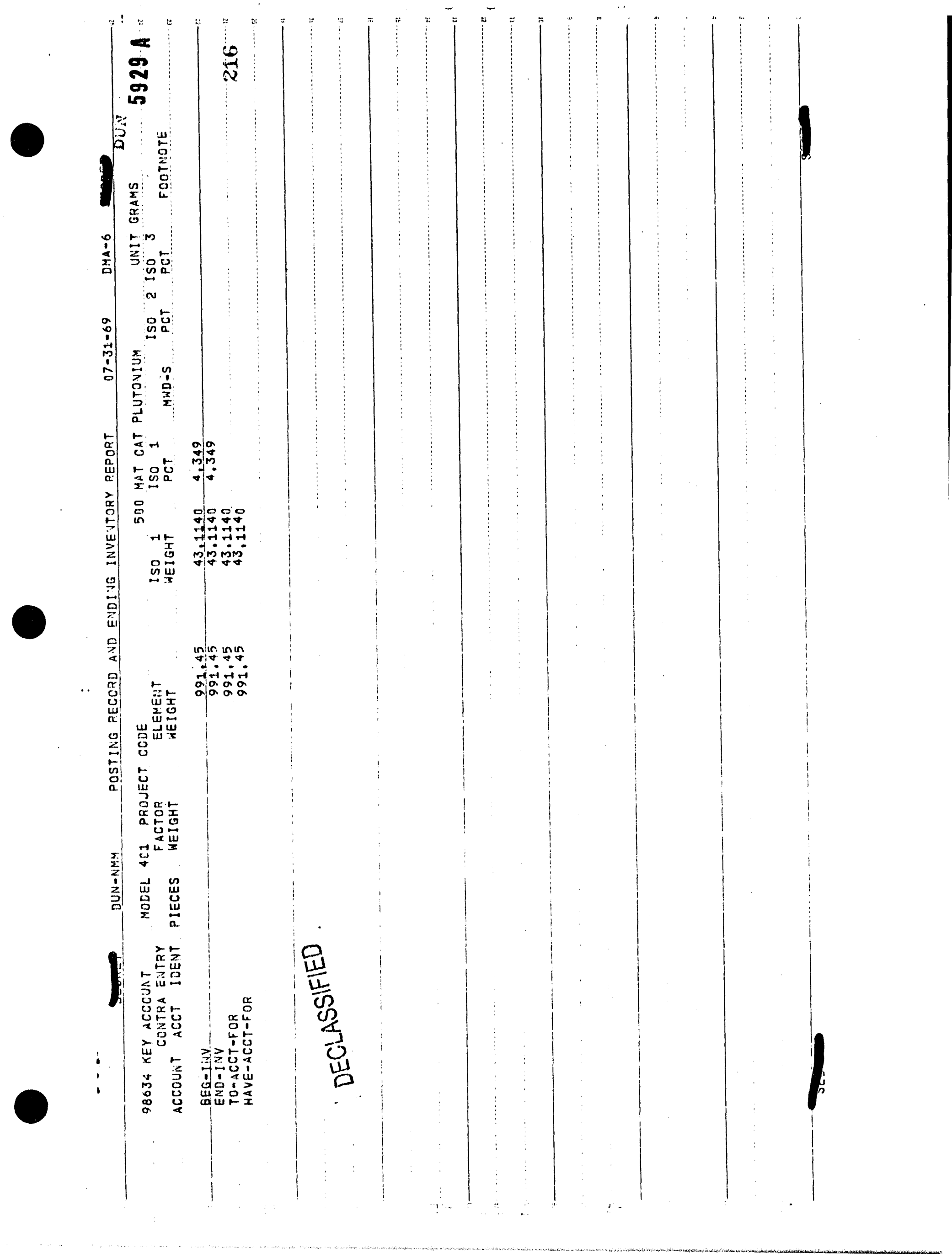




\section{$\therefore \quad \forall 626 \mathrm{G}$ NAa \\ 0킨ㄷㄴ70}

$2 \pi$

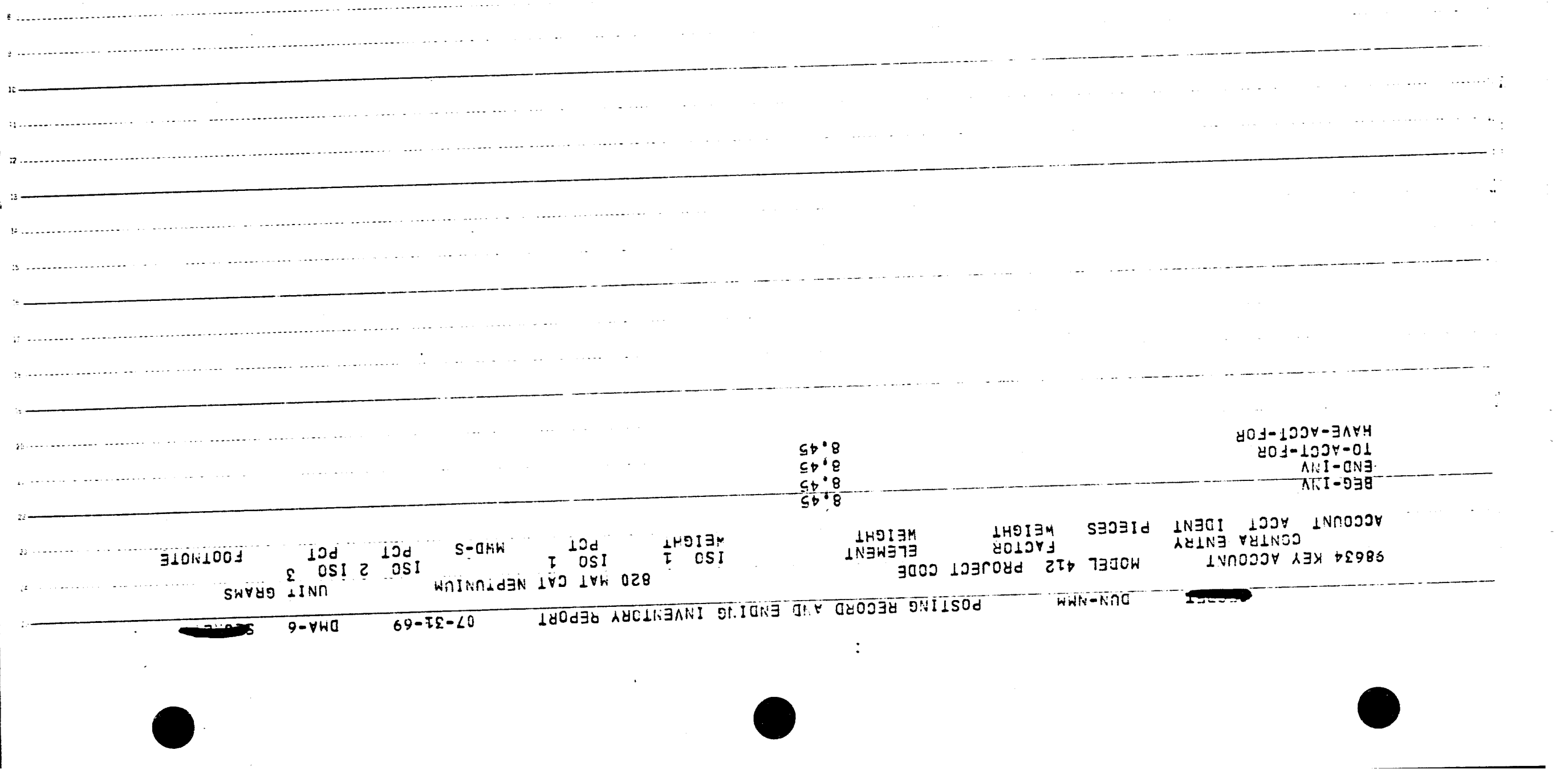


DUir-iMM

POSTIIO FECORD AYD ENDIVG INVEUTORY REPORT

$07-31-69$

DMA-6

DUN $5=927+4$

98666 KEY ACCOUNT

MODEL $\$ 14$ PROJECT CCDE

ELEMENT

200 HAT CAT ENRICHED URANIUM UNIT POUNDS

CONTRA ENTRY FACTOR

NEIGHT

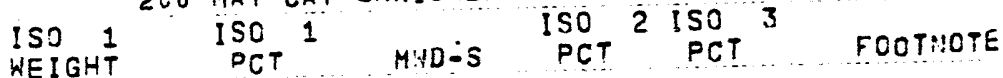
ACCOUNT ACCT IDENT PIECES WEIGHT

BEG-I:VY

TOMCACT-FOR

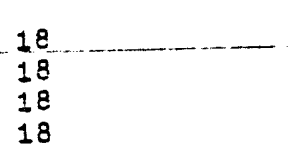

$\begin{array}{rrrr}731.18 & 5.3011 & .725 & 815.51 \\ 731.13 & 5.3011 & .725 & 815.51 \\ 731.13 & 5.3011 & & 815.51\end{array}$

$\begin{array}{rrrr}731.18 & 5.3011 & .725 & 845.51 \\ -731.13 & 5.3011 & .725 & 815.51 \\ 731.13 & 5.3011 & & 815 ! 51\end{array}$

$\begin{array}{lll}731.13 & 5.3011 & 815.51\end{array}$

HAVE-ACCT-FOR

200 MAT CAT ENRICHED URANIUM UNIT FOUNDS

98666 KEY ACCOUNT MODEL 915 PROJECT CODE

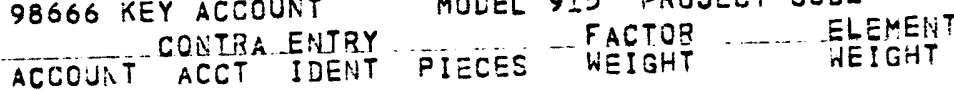

$\begin{array}{lr}\text { BEG-I:UV } & 28 \\ \text { END-IINV } & 28 \\ \text { TO-ACCT-FCR } & 28 \\ \text { HAYE-ACCT-FCR } & 28 \\ 98066 \text { KEY ACCOUNT } & \text { MOEEL } \\ \text { CONTRA ENTRY } & \\ \text { ACCOUNT ACCT IDENT PIECES } \\ \text { BEG-IIV } & 11 \\ \text { END-INV } & 11 \\ \text { TO-ACCT-FOR } & 11 \\ \text { HAVE-ACCT-FOR } & 11\end{array}$

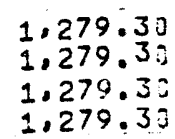

98 PROJECT CCDE

FACTOR ELEMENT

FEIGHT

:NEIGHT

WEIGH

GHT $\frac{\text { ISO } 1}{\text { PCT }}$ MWD-5

10.7397
10.7397
10.7397
10.7397

10.7397

4 MAT CAT ENRICHED URANIUM UNIT PCUNDS

WEIGHT 1 ISO 1 MCT MUDES ISO 2 ISO 3 PCT ${ }^{2}$ FOOTNOTE

$6.3852 \quad 1,110 \quad 345,36$

$6,3852 \quad 1,110 \quad 345,36$

$6,3852 \ldots \ldots \ldots \ldots 34,36$

345.36

\subsection{4}

575.24

$575 \cdot 24$

6.385

DECLASSIFIED 


\section{○}

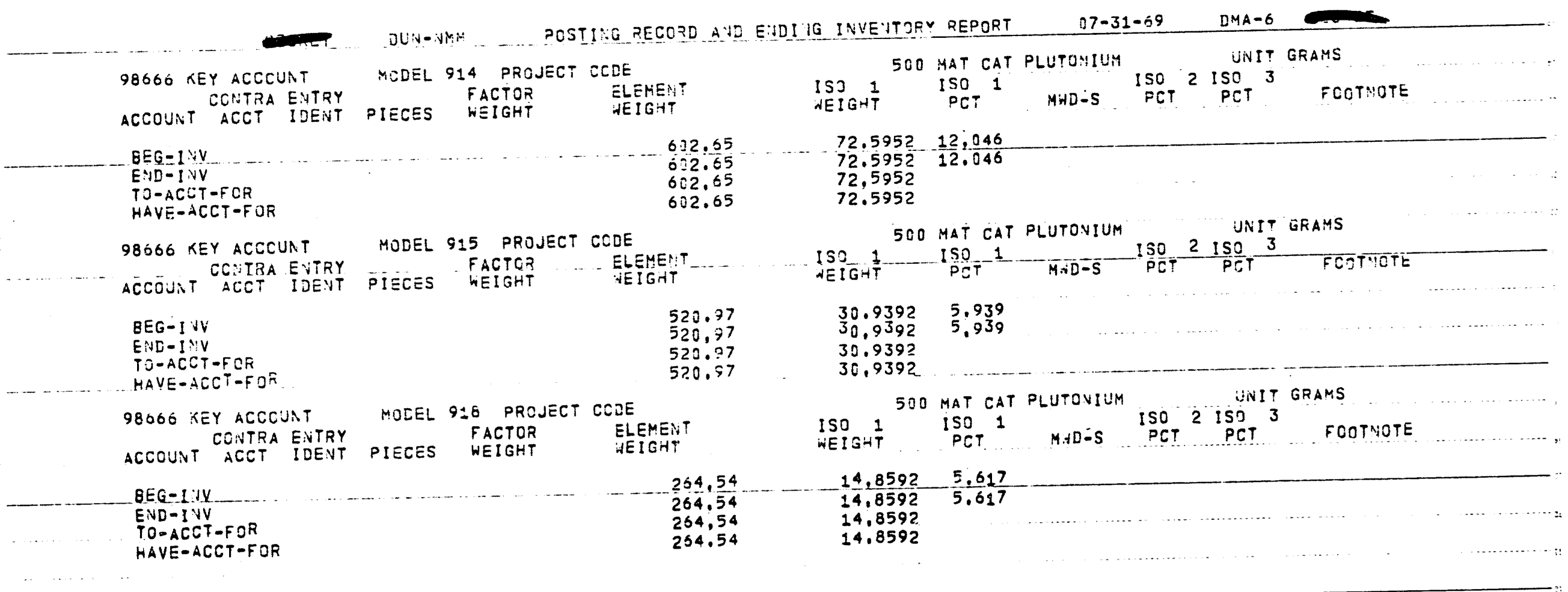




\section{$\bullet$}

DUN-SMM

POSTING RECORD AVD EVDIVG INVEVTOPYY REPORT

07-31-69

DMA-6

MOEEL 9:4 PROJECT COUE

98665 KEY ACCOUNT
COATRA ENTRY

FACTOR ELEMENT

820 MAT CAT NEPTUNIUM

IST 1 IS? 1

PCT

UNIT GRAMS

$5929 A$

ACCOUNT ACCT IDENT PIECES WEIGHT WEIGHT

5.53

5.53

END-INV

TO-ACCT-FOR

HAVE-ACCT-FOR

98066 KEY ACCCUNT

ACCOUN T CONIRA EIITRY

MODEL 915 PROJECT CODE

5.53

ACCOUAT ACCT IDENT

PIECES WACTOR . WEIGHT ELEMEN

ELEMENT

BEG-IUV

TOD-INV
TOACT-FOR

TO-ACCT -FOR
HAVE-ACCT -FOA

5.03

5.63

5.23

98666 KEY ACCOUNT

CONTRA ENTRY
ACCOUNT ACCT IDENT PIECSS WACTOR PIGT ELEMENT

MODEL $9 \pm 8$ PROJECT CODE

820 MAT CAT NEPTUNIUM

EEG $=$ IIIV

END-INV.

TO-ACCT-FOR

KAVE-ACCT-FOR

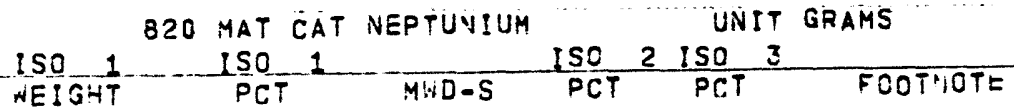

DECLASSIFIED

ISO 1 ISO 1 MNDES ISO 2 ISO 3 PCT FOT

$2.3 \frac{1}{31}$

2.31

2.31 
:

- DLT-NMM _. POSTING RECORE AVID EVDIIG INVEVTORY REPDRT O7-31-69 DHA-6

98670 KEY ACCOUNT MOLEL 713 PROJECT CODE
CONTRA ENTRY FACTOR M

MOLEL 713 PROJECT EODE
PIECES WETOR
PIGHT

200 MAT CAT EVRICHED URANIUH UNIT POUHES ACCOUNT ACCT IDENT

ISO 1 ISO 1 PCT 1 MND-S POT 2 ISO 3 FOT

END-IHV
EEG-IHV

END-IIN
TO-ACCT-FCR

HAVE-ACCT-FOR

98678 KEY ACCOUNT COATTRA ENTRY ACCOUNT ACCT IDENT PIECES WEIGHT HEIGHT
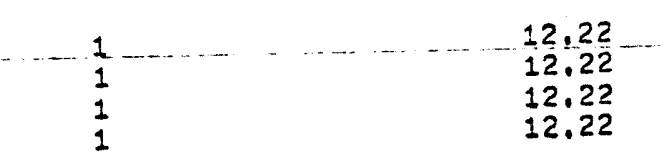

MOEEL 813 PROJECT CCDE

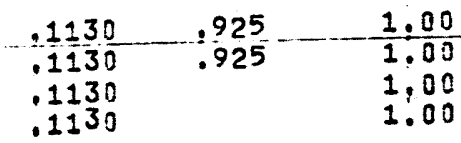

FACTOR -.. ELEMENT

200 MAT CAT ENRICHED URANIUM UNIT POUNES

$B E G-I N V$
END-INV
TO-ACCT-FCR
HAVE-ACCT-FOR

1
1
1

$$
\begin{aligned}
& 21.50 \\
& 21.50 \\
& 21.50 \\
& 21.50
\end{aligned}
$$

98678 KEY ACCOUNT

MOCEL 913 PROJECT CODE

ACCOUNT CONTRA ENTRY

MOCEL 913 PACTOR
PIECES WEIGHT ACCOUNT ACCT IDENT PIECES WEIGHT

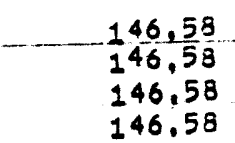

$\begin{array}{rrr}.1989 & .925 & 2,00 \\ .1989 & .925 & 2,00 \\ .1989 & & 2,00 \\ .1989 & 2,00\end{array}$

200 MAT CAT ENRICHED URANIUM UNIT POUNIS

\section{$B E G=I: Y$}

TO-ACCT-FOR

HAVE-ACCT - FOR

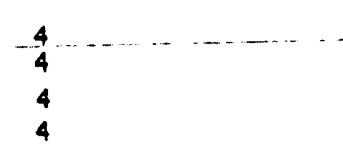

146,58

146,58

146.58

ISO 1 ISO

ISO 1 MOT 1 ISO 2 ISO

FOOTNOTE

$\begin{array}{rrr}1.3559 & .925 & 13,21 \\ 1.3559 & .925 & 13,21 \\ 1.3559 & & 13,21 \\ 1.3559 & & 13.21\end{array}$

20

${ }^{\text {DUN }} 5929$ A

$\neg \cdots \cdots$ 


\section{-}

\section{-}

$-\quad \because$ DUiv-iNM

POSTING RECORC AND EYDIYG INVEYTORY REPORT $07-31-69$ DMA-6

UNIT GRAMS

98673 KEY ACCCUNT MOLEL 713 PROJECT CCDE

CONTRA ENTRY
ACCOUNT ACCT IDENT PIECES WEIGHT

500 MAT CAT PLUTOVIUM

ISO 2 ISO 3

WEIGHT PCT MUD-S PCT PCT FOOTNOTE

222

EEG-1IV

END-INV

TOMACCT - FOR

1,03
1,00

1.00

HAVE-ACCT-FOR

1.30

98673 KEY ACCOUNT MODEL 813 PROJECT CCDE CONTRA ENTRY
ACCOUNT ACCT IDENT PIECES WEIGHT

BEG-INV

END-INV

TO-ACCT-FOR

IAAVE-ACCT-EOR

98678 KEY ACCCUNT MOLEL 913 PROJECT CODE

CONTRA ENTRY
ACCOUNT ACTOR PLT ELEMENT

$\frac{0117-1.170}{.0117}-1.170$

, 0117

.0117

500 MAT CAT PLUTONIUM

BEG-INV

END-INV

TO-ACCT-FOR

HAVE-ACCT-FOR

TT

is?

ISO 1

$\frac{150}{P C T}=\frac{2150 \quad 3}{P C T}$

UNIT GRAMS

$\begin{array}{lll}2.00 & .0235 & 1.175 \\ 2.00 & .0235 & 1.175\end{array}$

$2.00 \quad .0235$

.0235

2,09

\section{DECLASSIFIED}


DMA-6

98075 KEY ACECUNT MODEL 713 PROJECT CCOE

98075 KEY ACEOUNT ACCOUNT ACCT IDENT

GEG-IIV

END-IIIV

TO-ACCT-FCR

HAVE-ACCT-FOR

98673 KEY ACCOUNT

ACOOUA T COIITRA ENTRY

ACCT IDENT

$B E G-1$ IiV

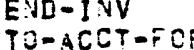

TO-ACCTEFCR
HAVE-ACCT-FOA

98678 KEY ACCCURT

CONTRA ENTRY
ACCOLNT ACCT IDENT

MOCEL 913 FROJECT SCDE

MODEL \&13 PROJECT CCDE

ELEMENT

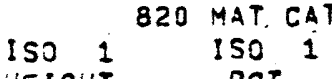

ISO 1

PCT.

PIECES WEIGHT HEIOHT NE!GHT

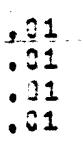

PIECES WACTOR ELEMEIT

ISO

TUVIUM

UIIT GRAMS $M+D-5$ ISO 2 IST 3

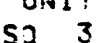

620 MAT CAT NEPTUNIUM

UNIT GRAMS

820 MAT CAT NEPTUVIUM

UNIT GRAIS

20 3

FOCTHOTE

-BEg-IiK

END-INV

TO-ACCT-FOR

HAVE-ACCT-FOR

ISO 1

150
PCT ISOT

FCOTHOTE

$2 \times 3$

DECLASSIF:EO

DUN : $2 \unlhd A$ 


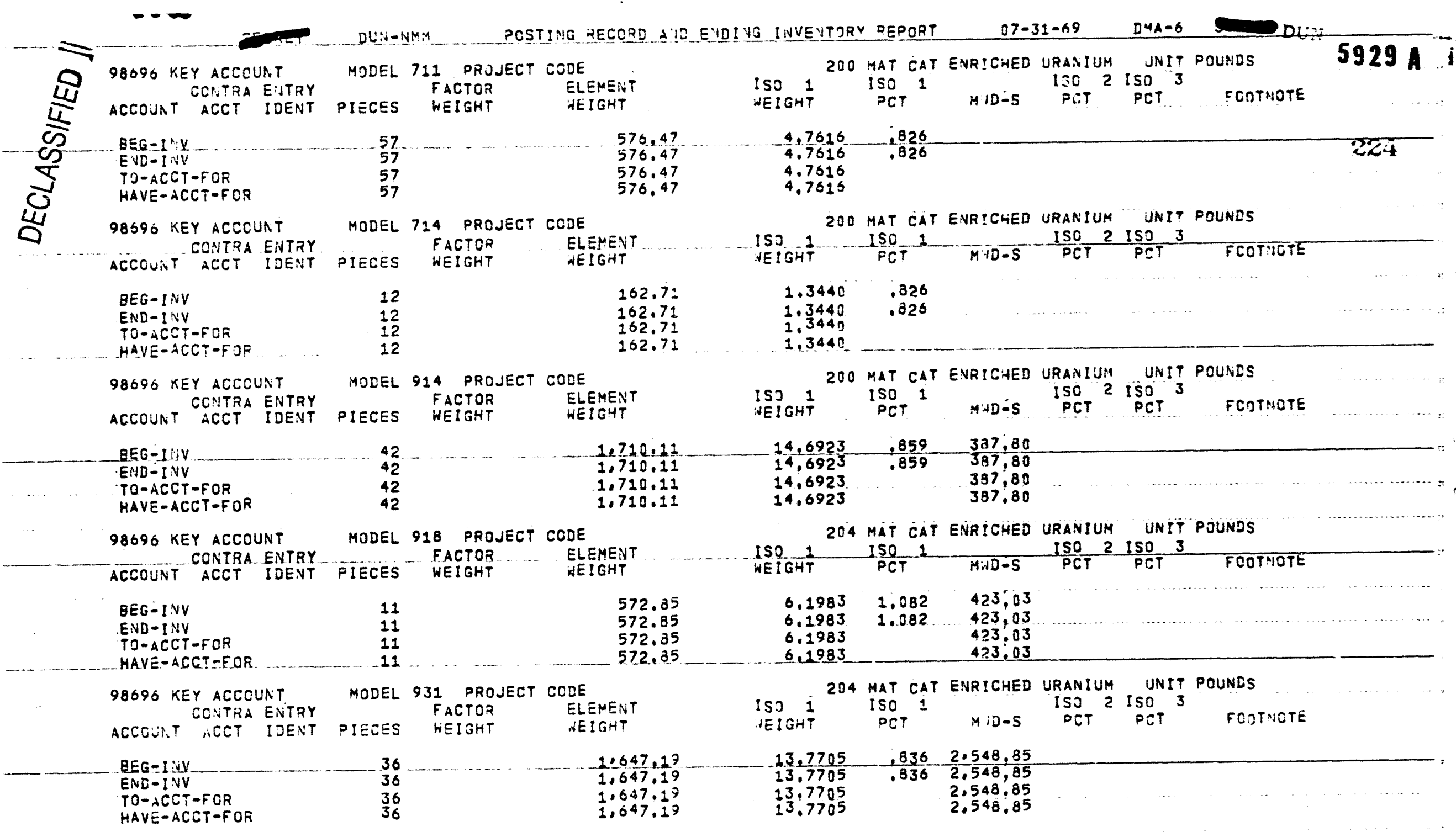




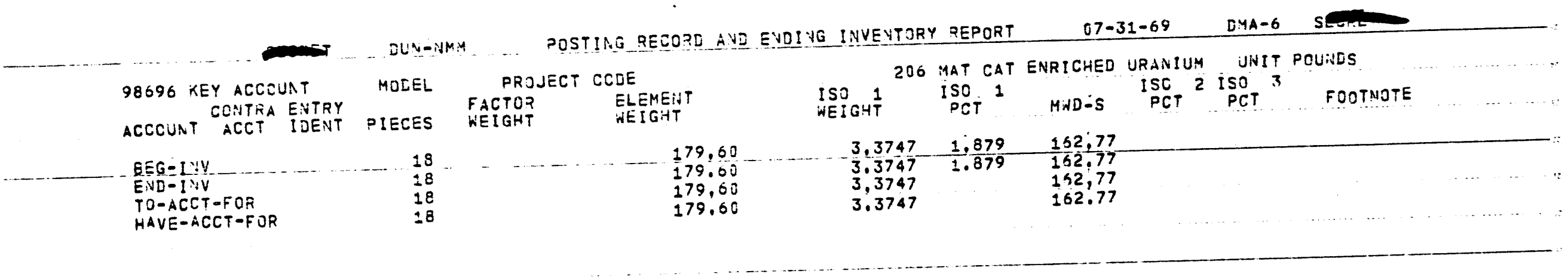




\section{DUN} ACOUNT ACCT IDENT PIECES WEIGHT

$$
\begin{array}{r}
91,36 \\
91.36 \\
91,36 \\
91,36
\end{array}
$$

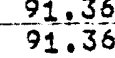

TO-ACCT-FOR
HAVE-ACCT-FOR

98696 KEY ACCOUNT

MODEL 711 PROJECT CCDE.

$\frac{4.7854}{4.7354}-\frac{5.238}{5.238}$

4,7854

PCT

FODTNOTE $5323 \mathrm{~A}$

ACCOUNT COLTRA ENTRY

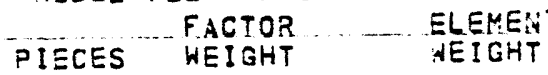

5 Jo MAT CAT PLUTONIUM

EEG-INV

TO-ACCT-FOR

TO-ACCT-FOR
HAVE-ACCT -FOR

98696 KEY ACCCUNT

ACCOUNT CONTRA ENTRY

MODEL 714 PROJECT ECDE
FACTOR

\subsection{6}

224.46

224.46

224,46

BEG $=I N Y Y$

TOMDOCCT-FOR

HAVE-ACCF-FOR

98696 KEY ACCOUAT

\begin{tabular}{|c|c|c|c|c|c|c|}
\hline IT & $\begin{array}{l}\text { ISO } \\
\text { WEIGHT }\end{array}$ & 500 & $\begin{array}{l}\text { MAT CAT } \\
\text { ISO } 1 \\
\text { PCT }\end{array}$ & $\begin{array}{l}\text { PLUTOVIUM } \\
\text { MHD }=5\end{array}$ & $\begin{array}{l}\text { ISO } \\
\text { PCT }\end{array}$ & 2 ISO 3 PET 3 FOOTNOTE \\
\hline $\begin{array}{l}65.17 \\
65.17 \\
65.17 \\
65.17\end{array}$ & $\begin{array}{r}3,2 \\
-3,2 \\
3,2 \\
3,2\end{array}$ & $\begin{array}{l}585 \\
585 \\
585 \\
885\end{array}$ & $\frac{5 ; 000}{5,000}$ & & & \\
\hline & ISO 1 & 500 & $\begin{array}{l}\text { MAT CAT } \\
\text { ISO } 1 \\
\text { PCT }\end{array}$ & $\frac{\text { PLUTONIUM }}{\text { MWD-S }}$ & $\frac{150}{P C T}$ & $\begin{array}{l}2 \text { ISO } 3 \\
\text { PCT } \\
\text { FOOTNOTE }\end{array}$ \\
\hline
\end{tabular}
CONTBA ENTRY

PIECES WEIGHT
ACCOUNT ACCT IDENT

MODEL 862 PROJECT CODE FACTOR
PIECES WEIGHT WLEMENT WEIGHT ISO 1

\section{O MAT CAT PLUTONIUM}

UNIT GRAMS

\section{BEG-INV}

END - INV
TO-ACCT -FOR

HAVE-ACCI-EOR

98696 KEY ACCOUNT CONTRA ENTRY

ACCOUAT ACCT IDENT

MOEEL 914 PROJECT CODE

FACTOR ELEMENT

\section{$11.2230^{\circ}$}

11,2230

11.2230

PCT MHD-S PCT $\frac{\text { ISO } 2 \text { ISO } 3}{\text { PCT }}$ FOOT TCTE

CCOUNT ACCT IDEUT PIECES WEIGHT

NEIGHT

.0001

.0 .001

.0001

BEG-INK

END-INV

TO-ACCT-FOR

HAVE-ACCT-FOR

537.51

537.51

537.51
537.51

ISO 500 MAT CAT PLUTONIUM

500 MAT CAT PLUTONIUM UNIT GRAMS

WEIGHT ISO 1 MCT MO-S PCT 2 PCT 3 FOOTVOTE

$31.2122 \quad 5.807$

$31.2122 \quad 5.807$

31,2122

31,212 ? 


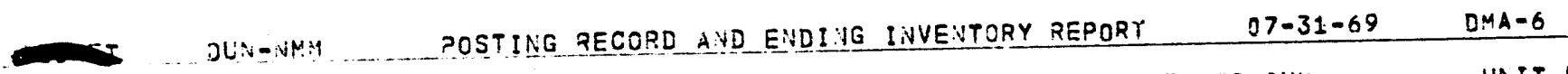

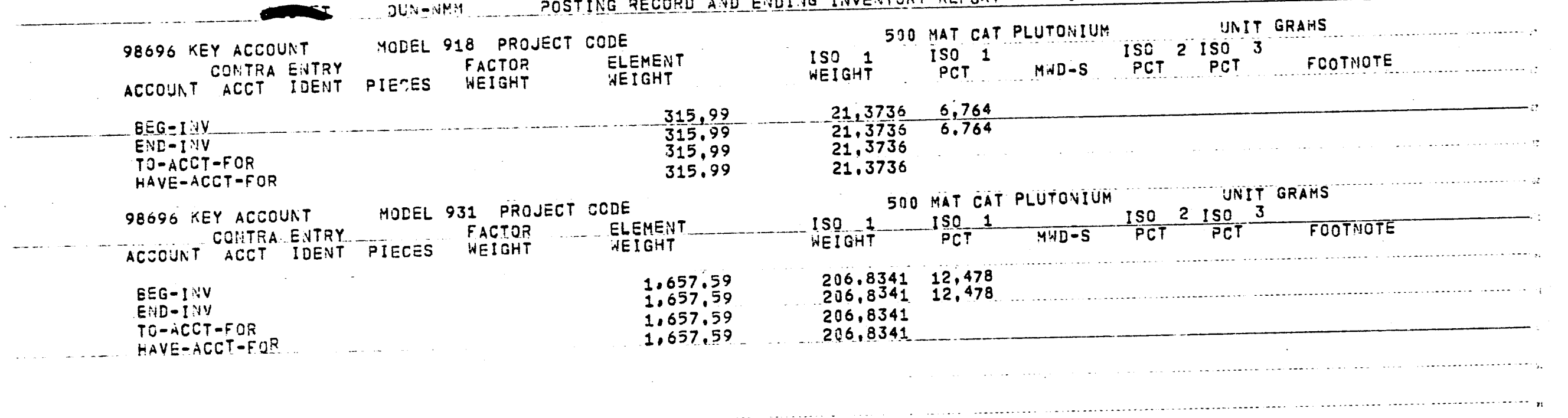

$2 \pi+4$ 
DLii-ivM4

PCSTING RECORD AND ENDIAG INVEYTORY REPORT

D.MA-6

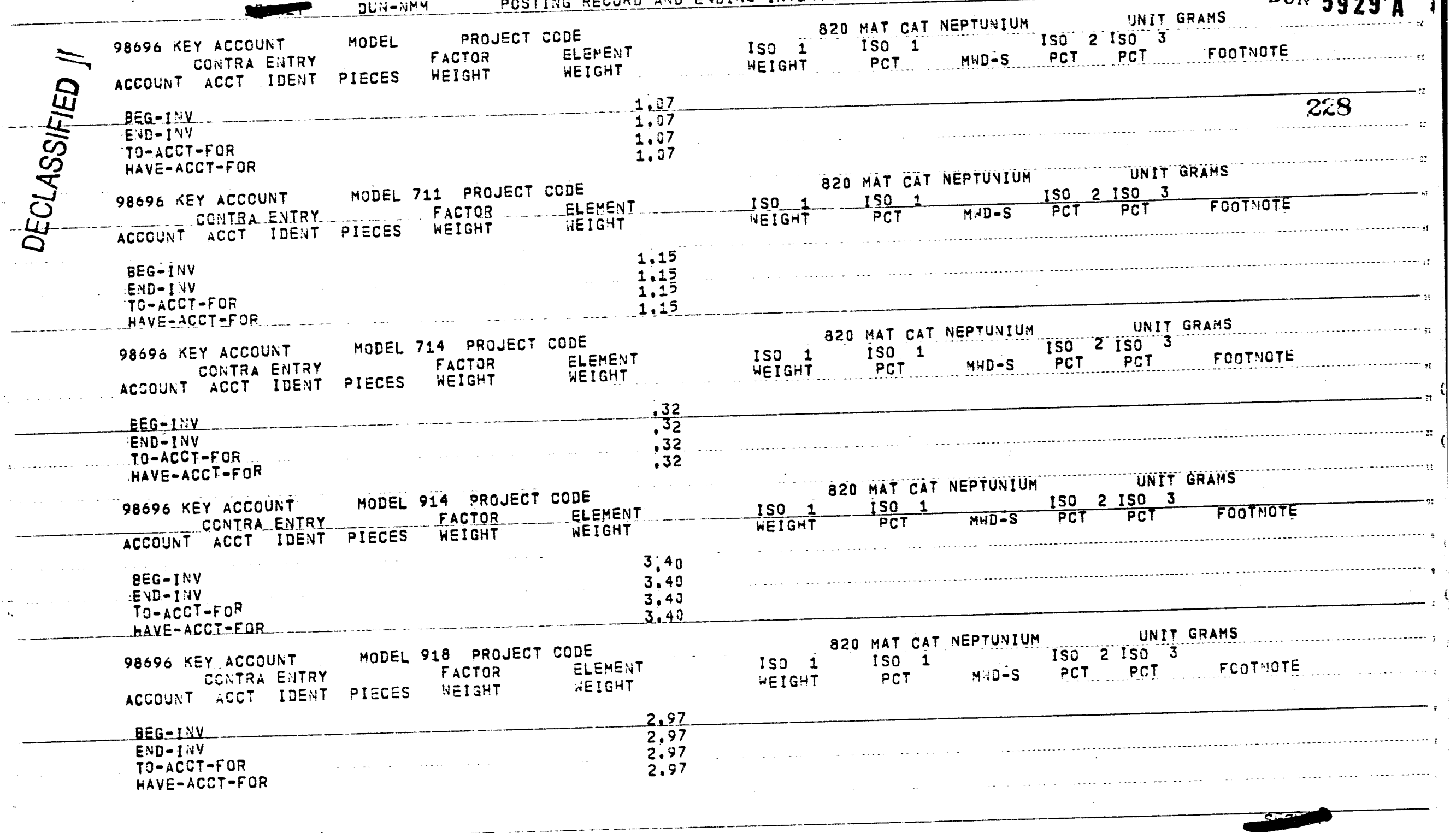


-
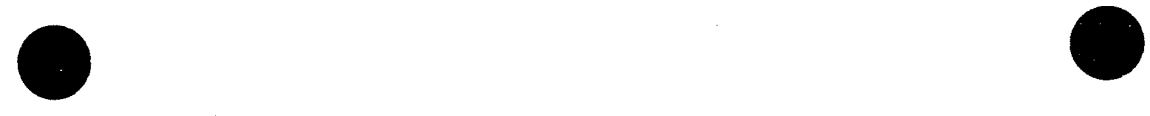

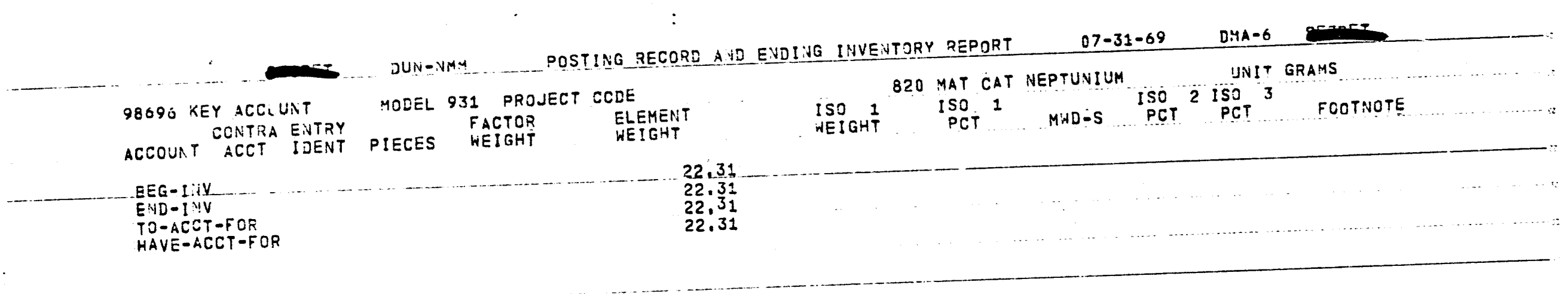

$2 * 9$

DECLASSIFIED 


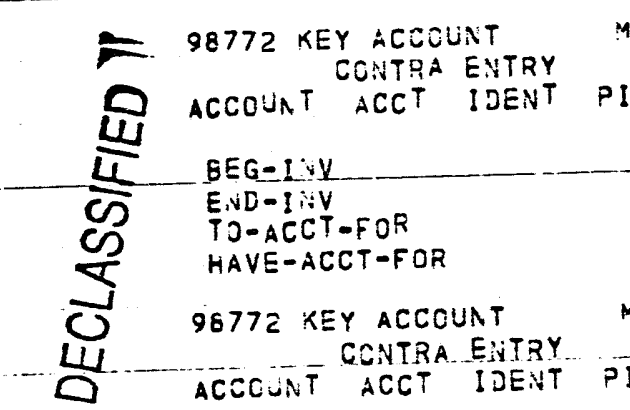

MOEEL 714 PROJECT CCDE

714 PROJECT CCOE
FACTOR
WEIGHT

ISO 1

ISOT 1

SO 2 ISO NEIGHT

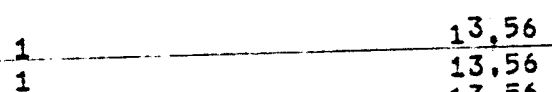

13.56

.1143

$$
.843
$$

$.1143 \quad .843$

.1143

.1143

MODEL 814 PROJECT CCDE

200 MAT CAT ENRICHED URANIUM UNIT POUNDS

DUM 5 : ? ?

I $\quad$ ISO

200 MAT CAT ENRICHED URANIUM ISO 3

\section{END-I:N
EEg-iNA}

END-I:NV

TO-ACCT-FOF

HAVE-ACCT - F 2 S

1
1
1
1

25.54
25.54
25.54
25.54 ISTT PCT

$\begin{array}{ccccc} & \text { ISO } 2 \text { ISO } 3 & \\ \text { PCT } & \text { MAD-S PCT } & \text { PCT } & \text { FOOTIIOTE }\end{array}$

$\begin{array}{lll}1 & & \\ 1 & \\ 1 & & \\ 1 & \ldots & \end{array}$

$\begin{array}{rrr}.2153 & .843 & 6,00 \\ .2153 & .843 & 6,00 \\ .2153 & & 6,00 \\ .2153 & & 6,00\end{array}$


$-$

DUM-THMM

POSTING RECORD AND EVDIVG INVEYTORY REPORT

$07-31-69$

DMA-6

98772 KEY ACCOUNT

CONTRA ENTRY
ACCOJNT ACCT IDENT MODEL 814 PROJECT CODE

MODEL 814 PROJECT
FACTOR
PIECES WEIGHT

500 MAT CAT PLUTONIUM

ISO I IST 1

UNIT GRAMS

10.05

EEG-INV

TO-ACCT-FOR

HAVE-ACCT-FOR

$.5711 \quad 5,583$

MUDOS

ISO 2 ISO

PCT 3 FOOTHOTE

10.05
10.05

10.05

.5711
.5711

.5711

.

$\therefore 1$

DECASEFED

-UN J323A 


\section{-}

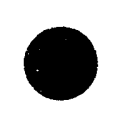

...

POSTING RECORD AYD EVDING INVEVTORY REPORT

$37-31-69$

DMA-6

98772 KEY ACCOUNT

CONTRA ENTRY

MODEL O14 PROJECT CODE MODEL O14 PROJECT CODE
FACTOR 820 MAT CAT NEPTUNIUM ACCOUNT ACCT IDENT ISO ISO 1

$-69$

UNIT GRAMSDUT 5929 A PIECES WEIGHT

$$
\begin{aligned}
& \text { EEG-IUV } \\
& \text { EN:D-I IV } \\
& \text { TO-ACCT-FOR } \\
& \text { HAVE-ACCT-FOR }
\end{aligned}
$$

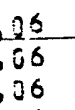

WEIGHT

PC

MWD-S

ISO 2 ISO 3 PCT ECOTNOTE
PCT

$29 \mathrm{~A}$ DECLASSIFIED; 


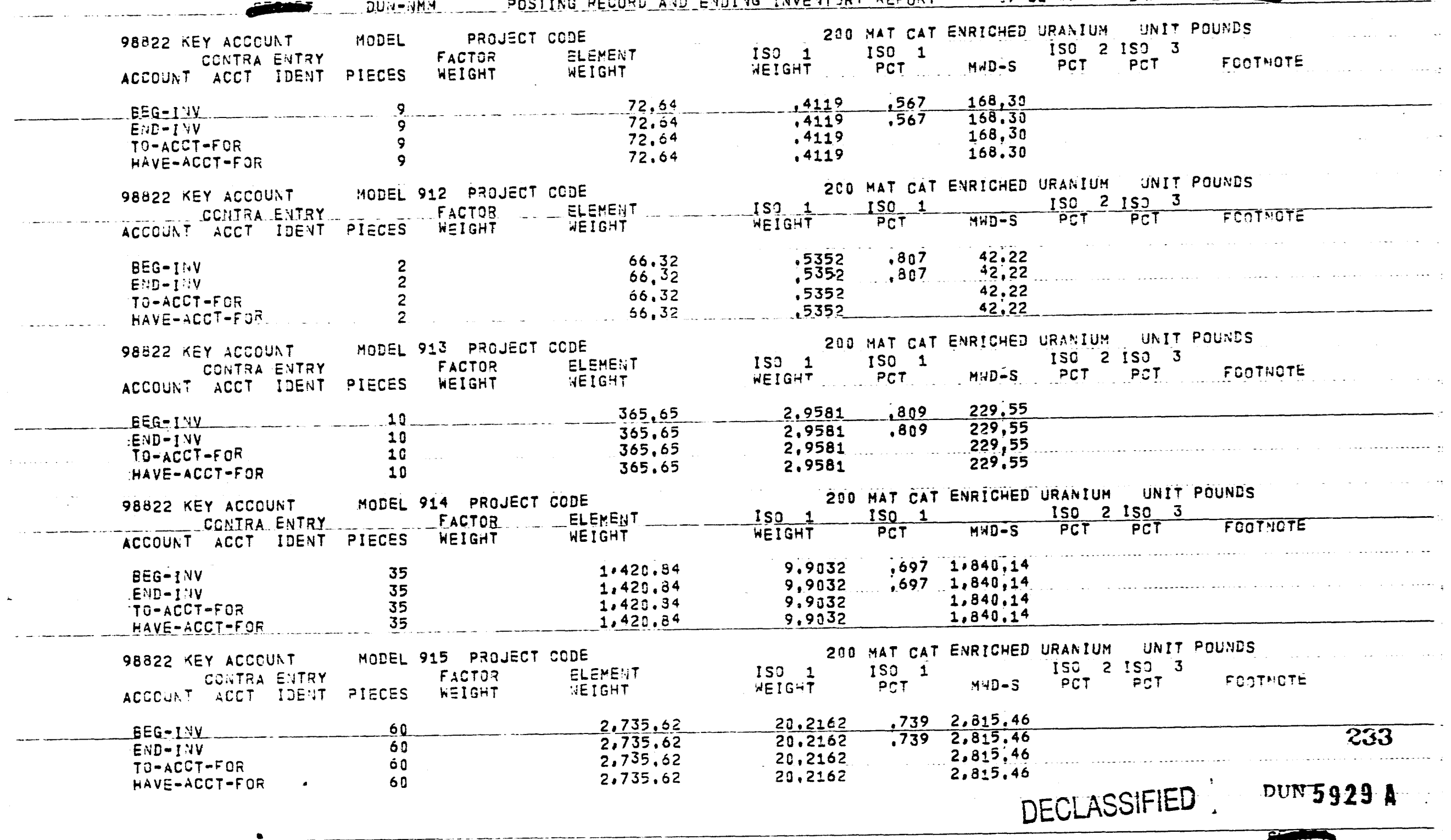


DUN-NMMH.

POSTING RECORD AND ENDIVG INVENTORY REPORT

07-31-69 DMA-6

MODEL 916 PROJECT CODE

98822. KEY ACCOUNT MACTOR
PIECES WEIGHT ACCOUNT ACNTRA ENTRY
IDENTT

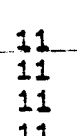
11 ISO 1 ISO

200 MAT CAT

UNIT POUNDS

OUfir

BEG-IUV

TO-ACST-FOR
END-INV

HAVE-ACCT -FOR

11

442.57

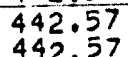

442,57
442.57

ISO 1 MADES ISO 2 ISO 3

FOOTNOTE

98822 KEY ACCCURT ACCOUNT CONIZA ENITRY

MOLEL 920 PROJECT CODE

FACTOB ___ ELEMENT

$3,2086 \quad .725 \quad 491 ; 65$

$3.2086 \quad .725 \quad 491.65$

$3,2086 \quad 491,65$

3.2086

491.65

200 MAT CAT ENRICHED URANIUM UNIT POUNDS ACCT IDEINT PIECES WEIGHT NEIGHT

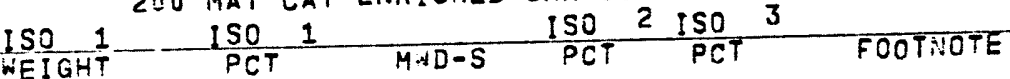

$\begin{array}{ll}\text { EEG-INV } & 27 \\ \text { END-INJ } & 27 \\ \text { TO-ACCT-FOR } & 27 \\ \text { HAVE-ACCT-FOP } & 27\end{array}$

$1,386.41$

$1,386.41$

$1,388.41$

98822 KEY ACCOUNT

CONTRA ENTRY

MODEL 918 PROJECT CCDE

FACTOR ELEMENT

GEG-IVY

END-INY

TOMD - INCT - FOR

HAVE-ACCT-FOR

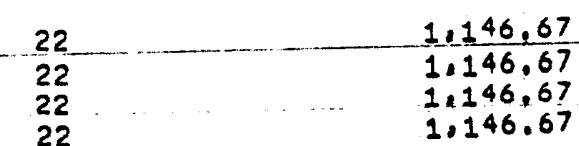

1.146 .67

1.146 .67

$1,146.67$

$\begin{array}{lll}10.8279 & .781 & 1,111,99 \\ 10.8279 & .781 & 1,111,99 \\ 10.8279 & & 1,111,99 \\ 10.8279 & & 1,111,99\end{array}$

204 MAT CAT ENRICHED URANIUM UNIT POUNES

ISO 1 ISO 1 MCT 1 ISO 2 ISO ${ }_{\text {PCT }}{ }^{2}$ FOOTYOTE

$\begin{array}{lll}11.2832 & .984 & 1.452,26 \\ 11.2832 & .984 & 1.452,26 \\ 11.2832 & & 1.452,26 \\ 11.2832 & & 1.452 .26\end{array}$

DECEASSIFIED 
:

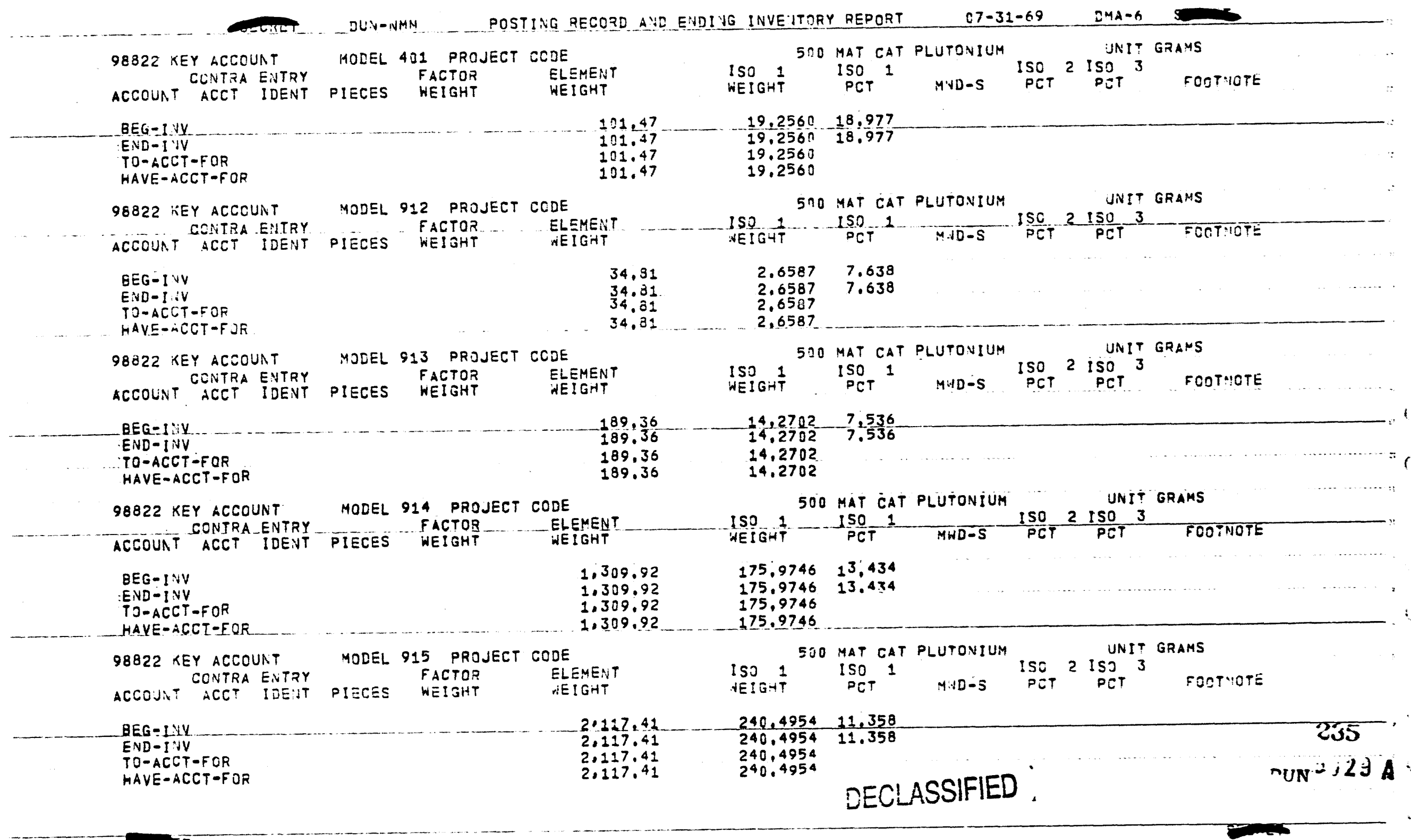




\section{0}

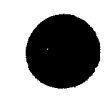

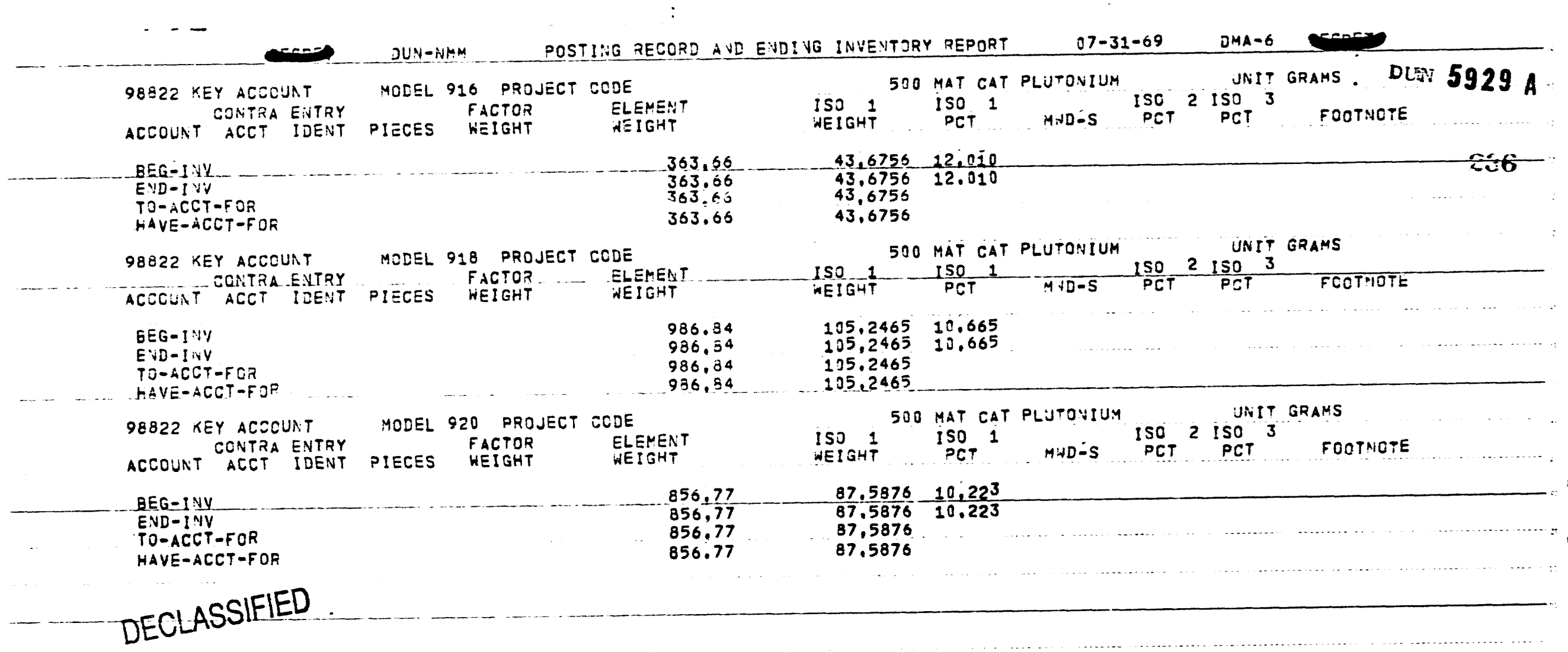


POSTING RECORD AND EUDING INVEUTORY REPORT

$07-31-69$

DYA-6

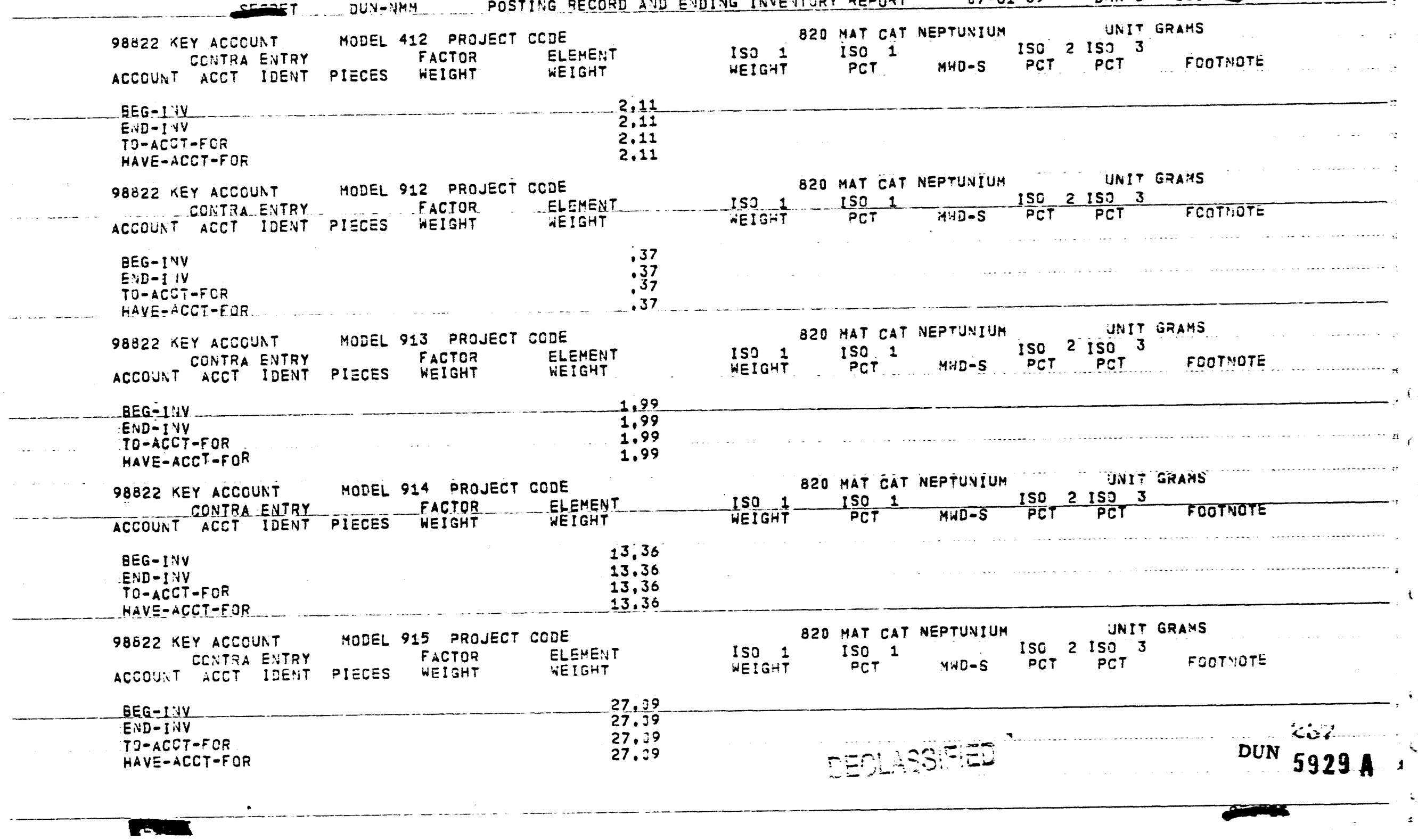


$27-31-69$

DYA-6

UNIT GRAMS

98822 KEY ACCOUNT MODEL 916 PROJECT CODE

CONTRA ENTRY FACTOR PLEMENT
EIECES WEIGHT WEIGHT

B20 MAT CAT NEPTUVIUM 820 MAT CA WE!GHT WEIGHT ISO

SO 3 ACCOUNT CONTRA ENTRY 4,82 4,82 6EG-1YY

END-INV

TO-ACCT-FOR

HAVE-ACCT-FOR

4.82
4.32

98822 KEY ACCOUNT MODEL 918 PROJECT CCDE 98822 KEY ACCOUNT MODEL
ACCOUNT CONTRA ENIRY ACCT IDENT PIECES WEIGHT

\section{MAT CAT NEPTUNIUM ….... UNIT GRAMS} ISO 1 ISO 1 MCT $\frac{\text { ISO } 2 \text { ISO } 3}{\text { PCT }}$

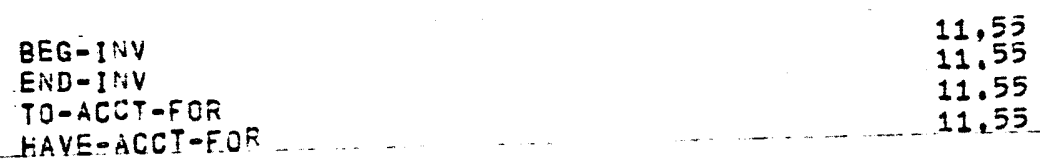

WEIGHT

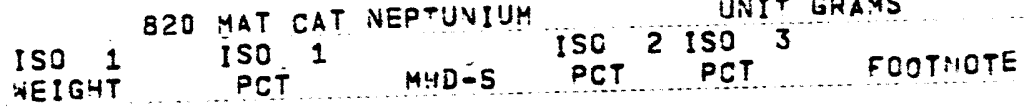

\section{DECLASSIFIED}


DUN-NMM POSTING RECORD A DD EIDIVG INVEVTORY REPORT

$07-31-69$

DMA-6

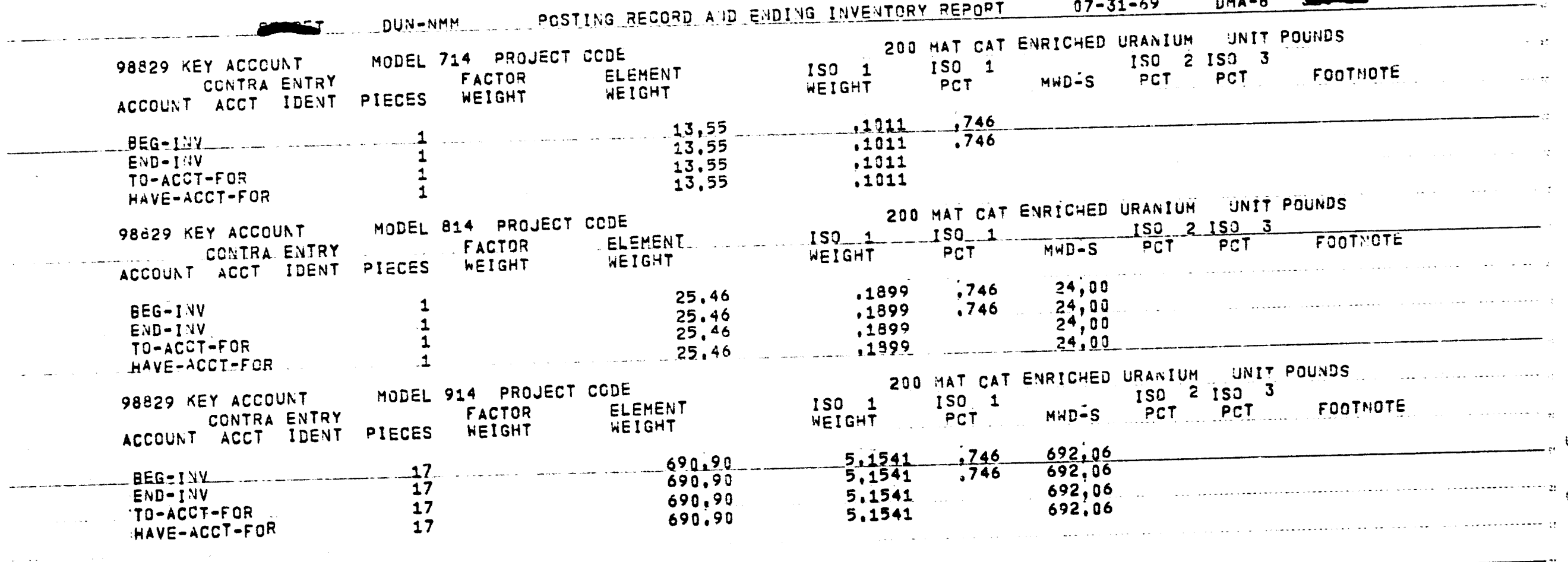




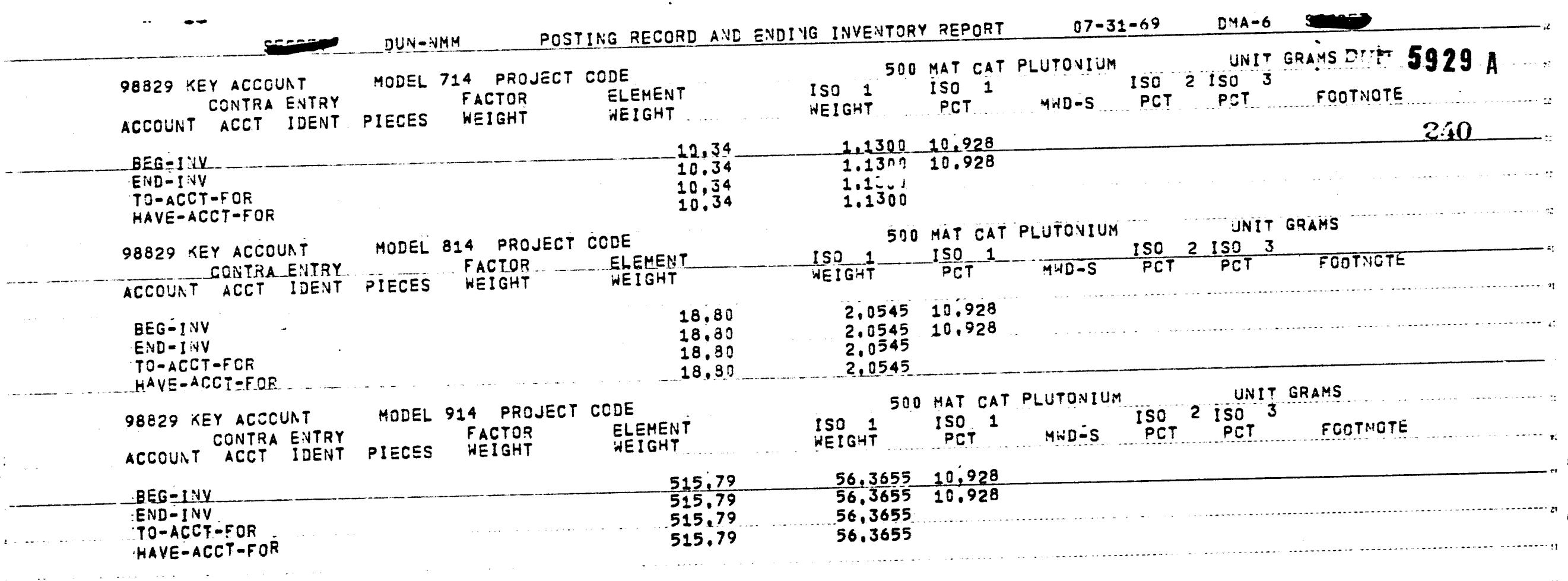

\section{ASSIFIED}


07-31-69 D:1A-6

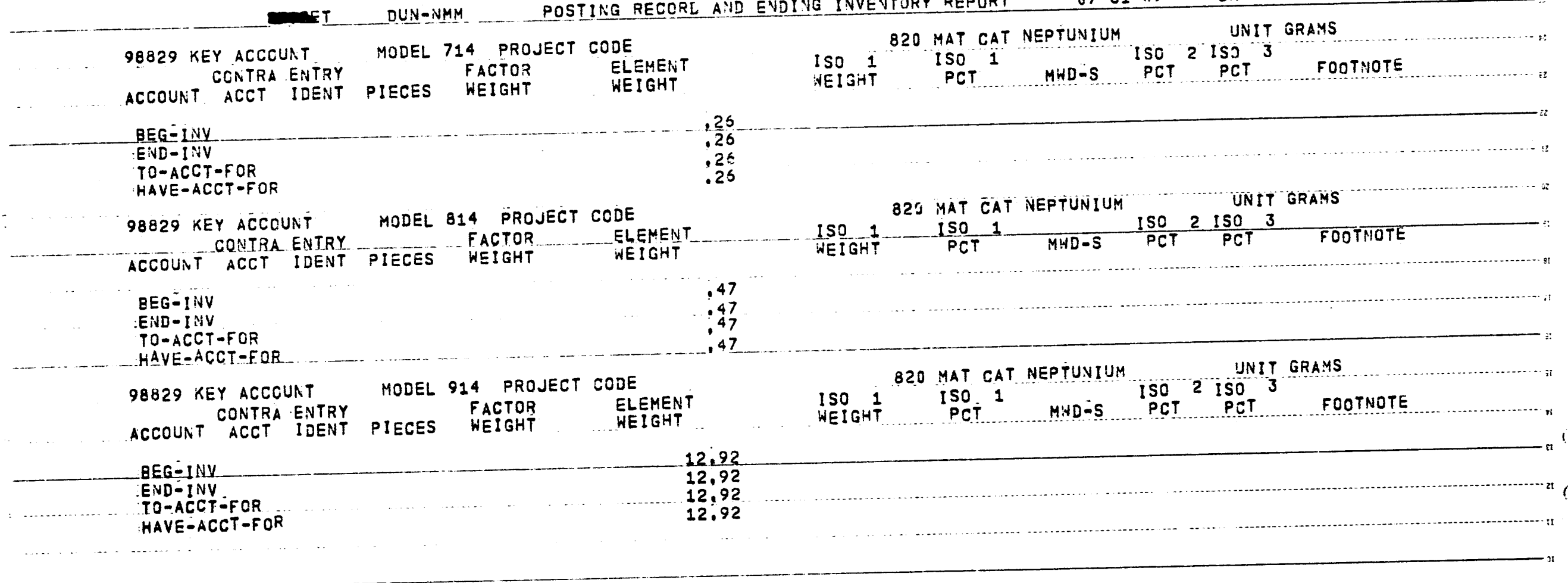


DUN-NMM

POSTING RECORD AVD ENDING INVEVTORY REPORT

$07-31-69$

DMA-6

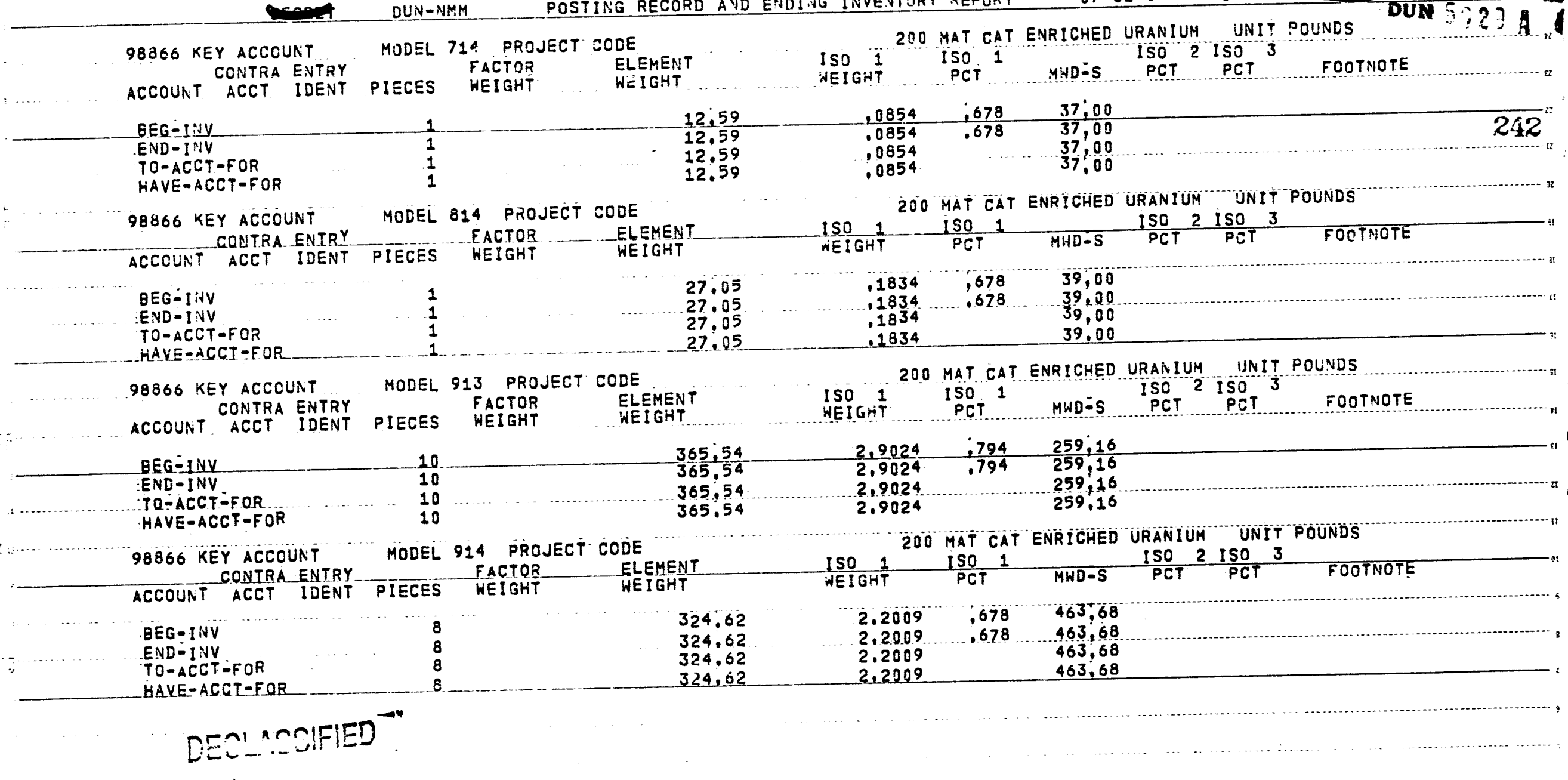




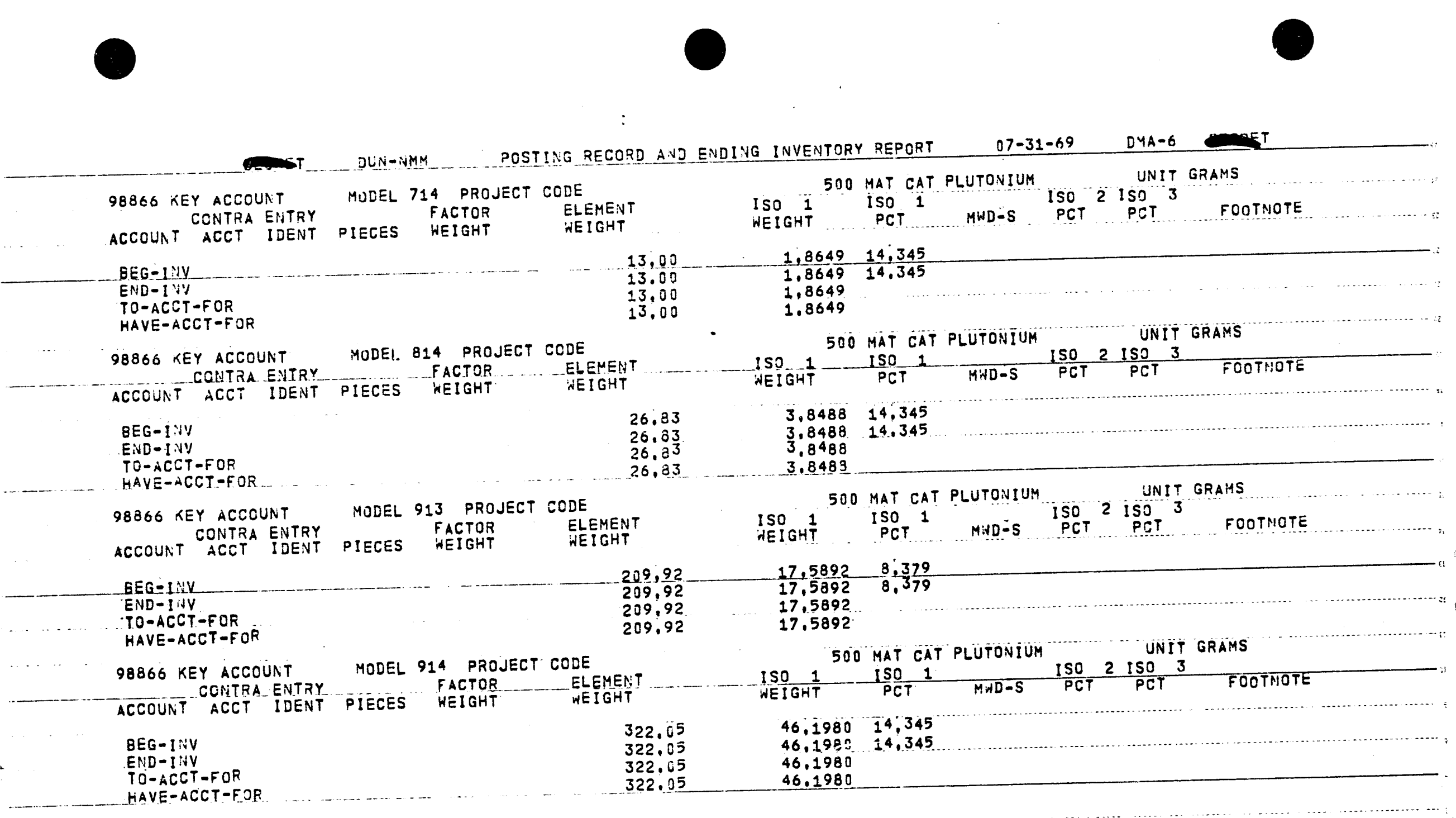




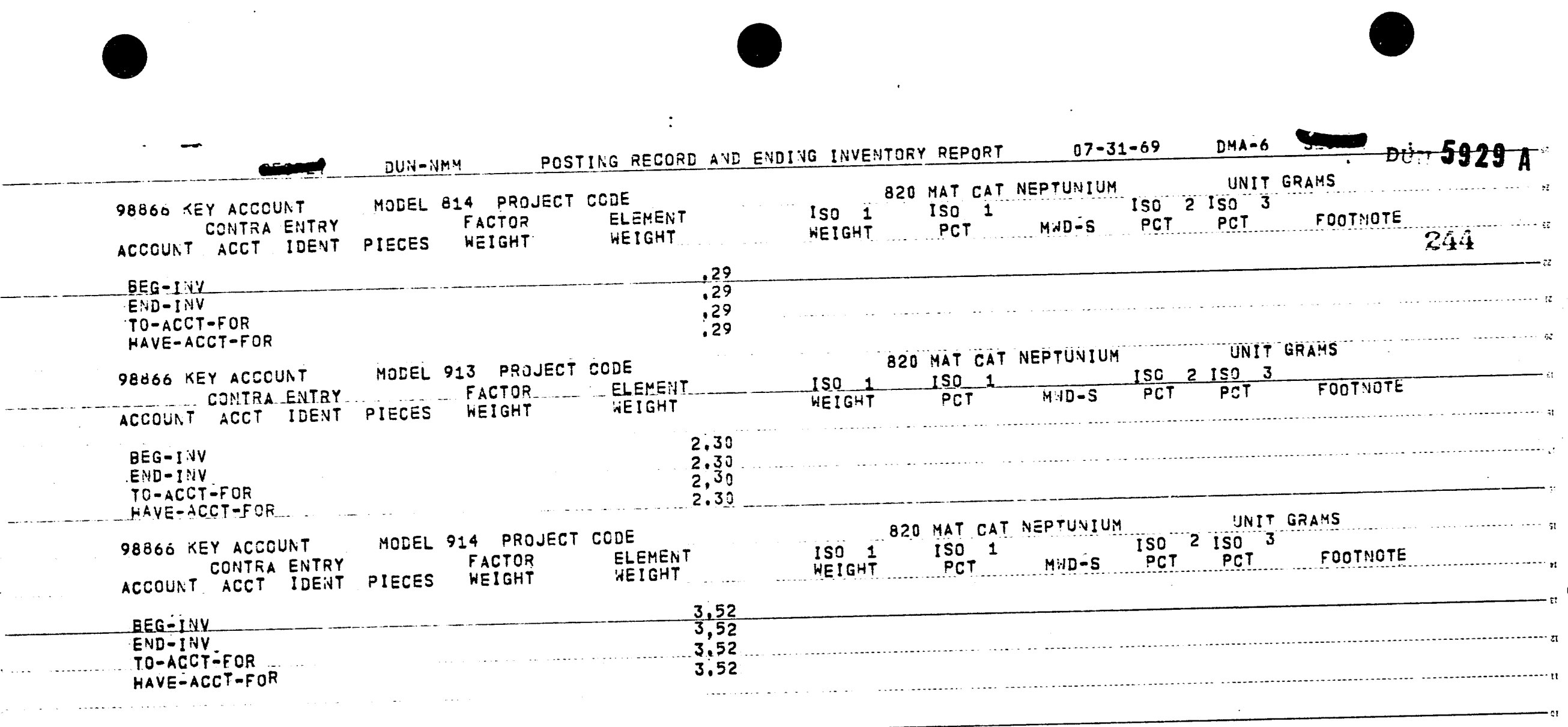

\section{DECLASSIFIED .}


DUV-NMM POSTING RECORD AYI EVDIVG INVENTORY REPORT

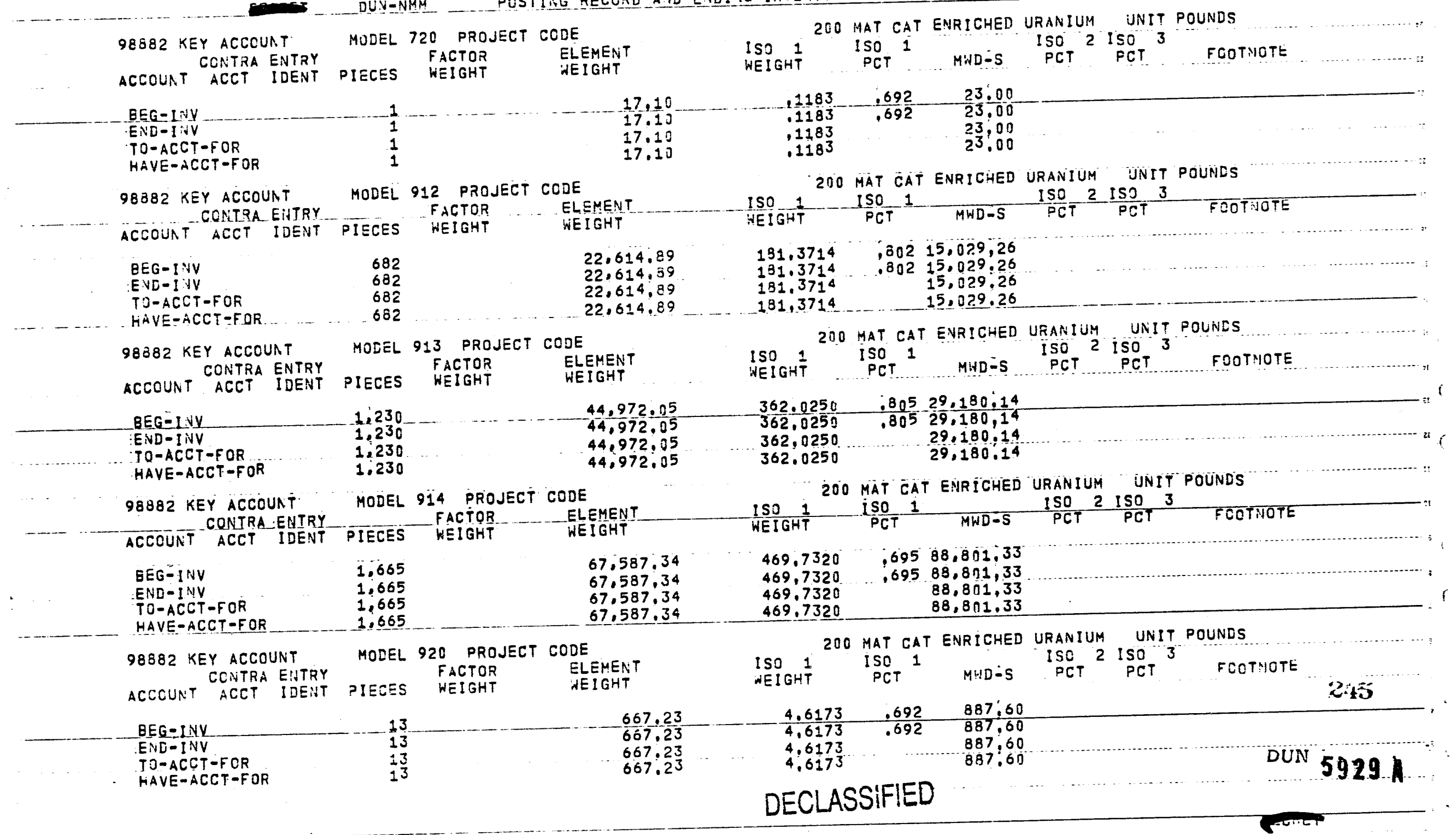


$m$

DUN-NMM

POSTING RECORD AND EVDING INVENTORY REPORT

$07-31-69$

DMA-6

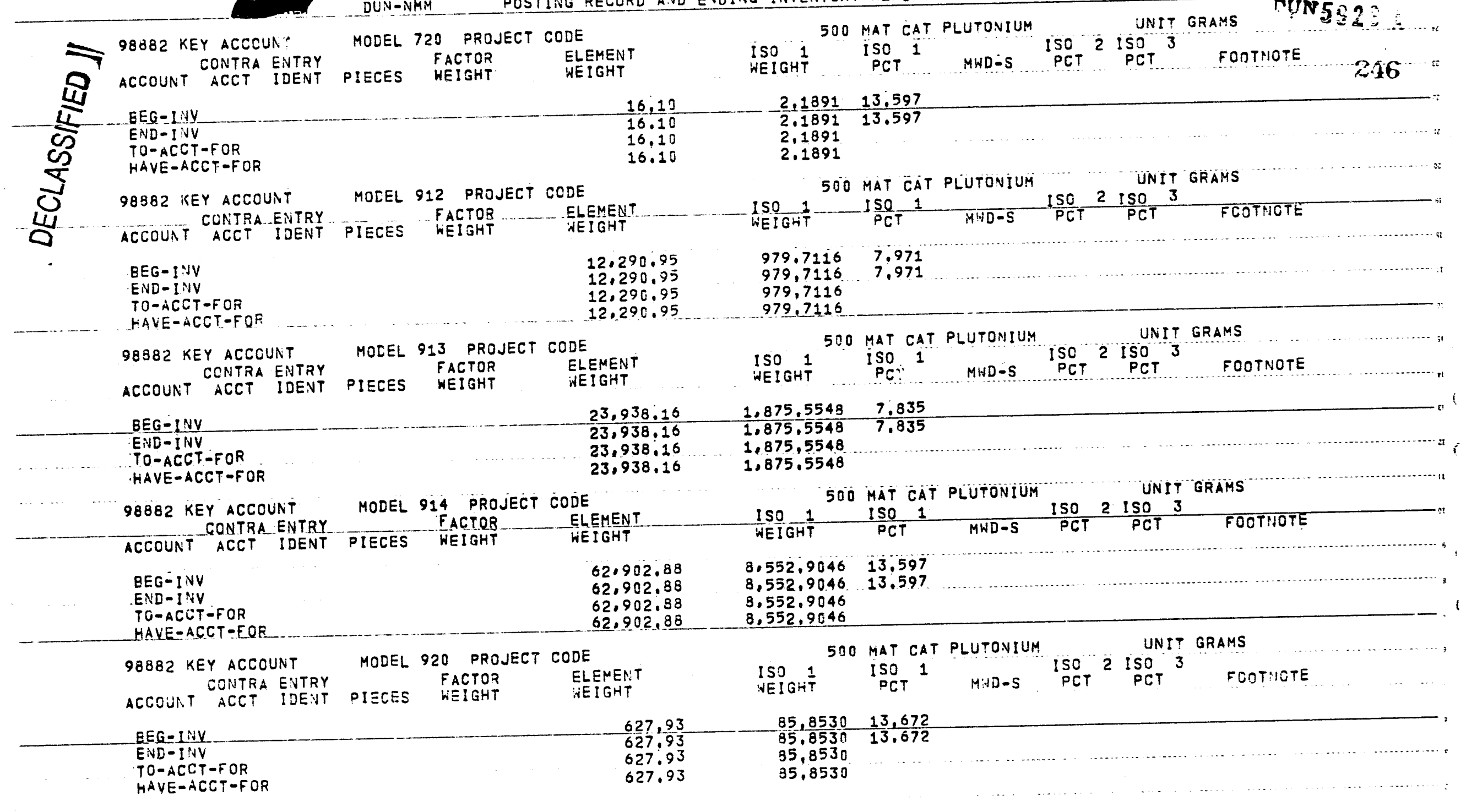


DUN-IUMM

POSTING RECCRD AYD EUDIVG INVENTORY REPORT

DMA-6

98882 KEY ACCCUP, T

MOLEL 720 PROJECT CODE

CONTRA ENTRY

CCNTRA ENTRY
ACCOUNT ACCT IDENT PIECES WEIGHT

E20 MAT CAT NEPTUNIUM

UNIT GRAMS

ISO 1 ISO 1 PCT MHDES ISO 2 ISO 3

FOCTNOTE

GEG $=I N Y$

TO-ACCT-FOR

HAVE-ACCT -FOR

WEIGHT PCT
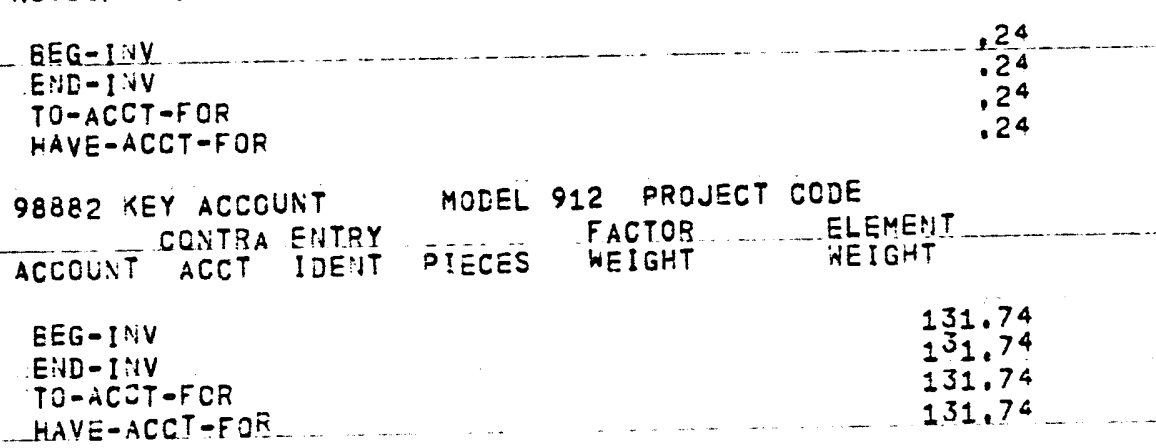

98882 KEY ACCOUNT

98882 KEY ACCOUNT ACCOUNT CONTRA ENTRY MOEEL 912 PROJECT CODE
PIECES WACTOR
WEIGHT WLEMENT

101.74
CER
131.74
$-\quad 131.74$

$$
131.74
$$

$\ldots .24$ .24

IT GRAMS

$98 \triangle 82$ KEY ACCOUNT MODEL 913 PROJECT CODE CCNTRA ENTRY
ACCOUNT ACCT IDENT PIECES WACTOR

EEG=INU

END-INV

TO-ACCT-FOR

HAVE-ACCT-FOR

98882 KEY ACCOUNT MOLEL 914 PROJECT CODE

CONTRA EVTRY ACCOUNT ACCT IDENT

PIECES WEIGHT - ELEMENT

254.91

$\frac{254.91}{254.91}$

254.91

254.91

ACCOUNT ACCT IDENT PIECES WEIGHT WEIGHT

651.18

651.13

651.13

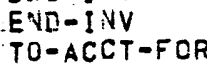

HAVE-ACCT-FOR

98882 KEY ACCOUAT MODEL 920 PROJECT CODE

98882 KEY ACCOUNT
CGNTRA ENTRY
ACCOUNT ACTT IDENT PIECES WEIGHT

820 MAT CAT NEPTUNIUM

820 MAT CAT NEPTUNIUM

UNIT GRAMS

ISO 11 ISO 1 MEIGHT ${ }_{\text {WCT }}$ ISO 2 ISO 3 PCT

BEG $=$ INV

9.14

$\begin{array}{llll}\text { ISO } 1 & \text { ISO } 1 & \text { ISO } 2 \text { ISO } 3 & \text { POT } \\ \text { WEIGHT } & \text { PCT } & \text { POTIIOTE }\end{array}$

END - IN

9.14

9.1

820 MAT CAT NEPTUNIUM

$\begin{array}{lllll}\text { ISO } 1 & \text { ISO } 1 & \text { ISO } 2 \text { ISO } 3 & \\ \text { WEIGHT } & \text { PCT } & \text { MWD-S PCT } & \text { PCT } & \text { FOOTHOTE }\end{array}$

HAVE-ACCT-FOR 


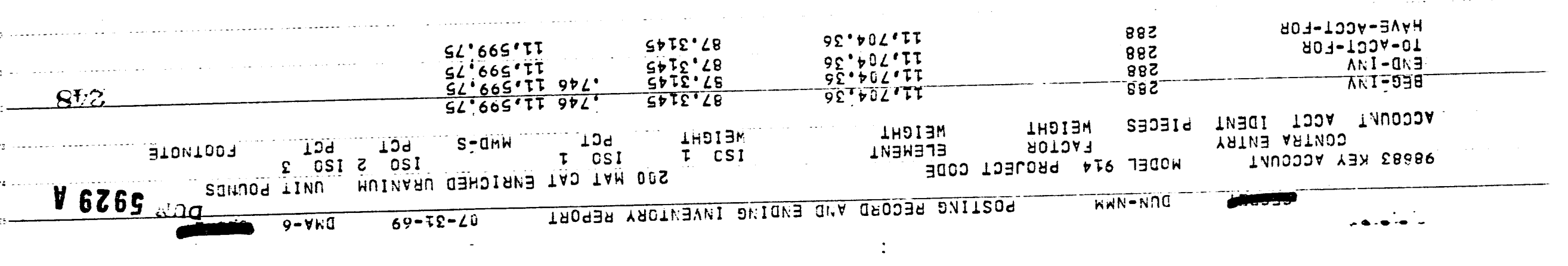


DLN-NMM

POSTING RECORD AVD ENDIVG IIVEENTORY REPORT ก7-31-69

DMA-6

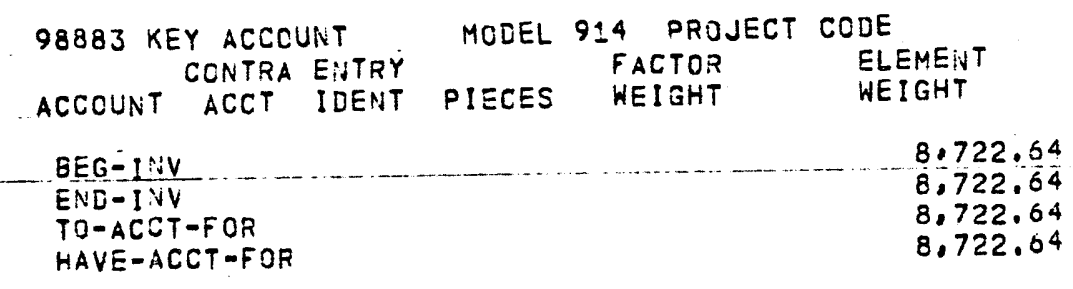

SOO MAT CAT PLUTONIUM $\begin{array}{ll}\text { ISO } 1 & \text { ISO } \\ \text { WEIGHT } & \text { PCT }\end{array}$

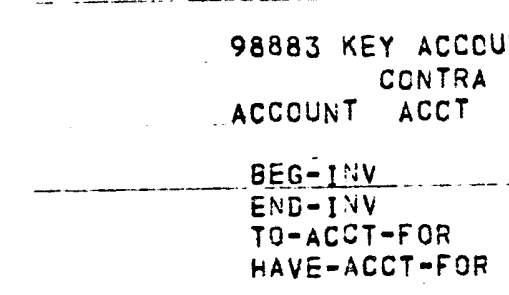

$934.6983 \quad 11.289$

$984.6988-11.289$

984.6988
984.6988

984.6988
934.6988
UNIT GRAMS

PCT 3 FOOTNOTE

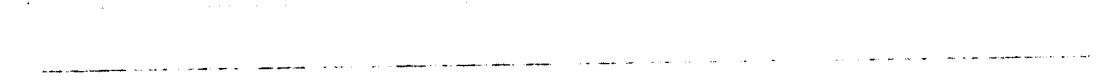

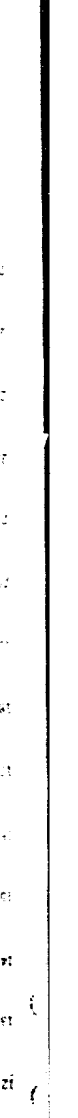


0

$07-31-69$

DMA-6

98883 KEY ACCOUNT MODEL 914 PROJECT CCDE

CCNTRA ENTRY
ACCOUNT ACCT IDENT PIECES WEIGHT PLTOR WEIGHT

820 MAT CAT NEPTUNIUM

ISO 1 ISO 1

HWD-S

UNIT GRAMS

222.11

222.11

ETID-INV

TO-ACCT-FOR

HAVE-ACCT-FOF

222.11

DECLASSIFIED : 


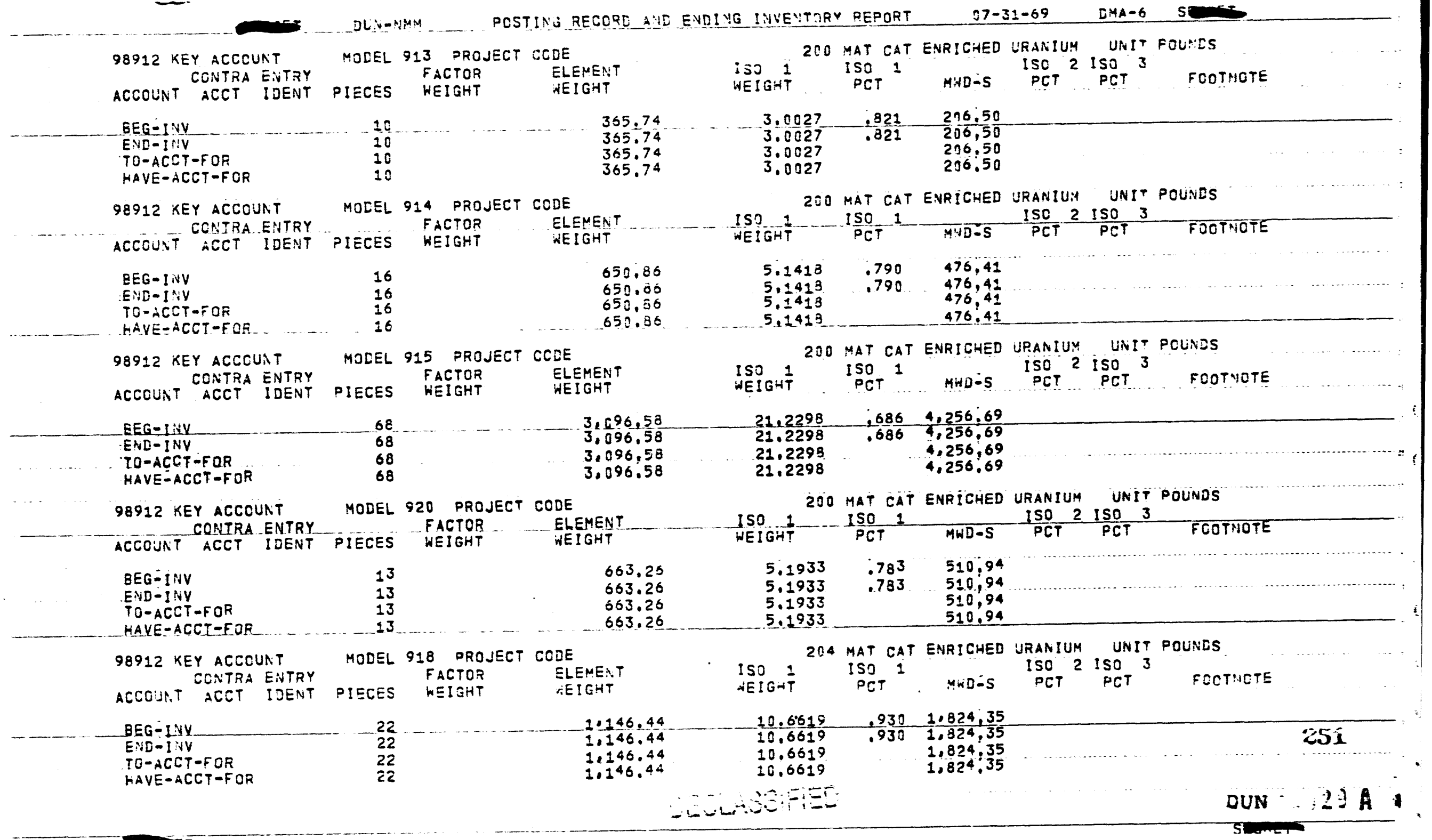




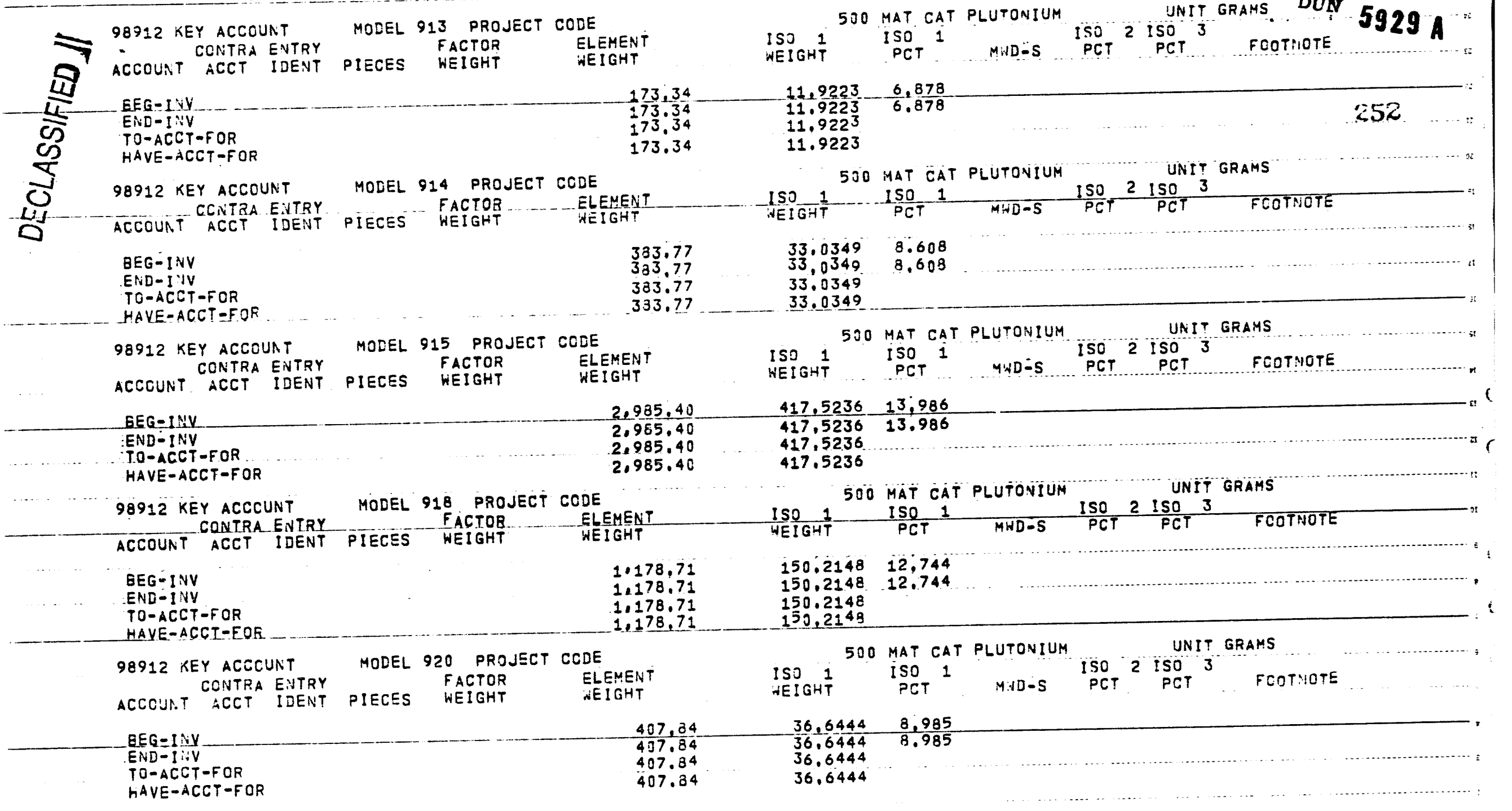




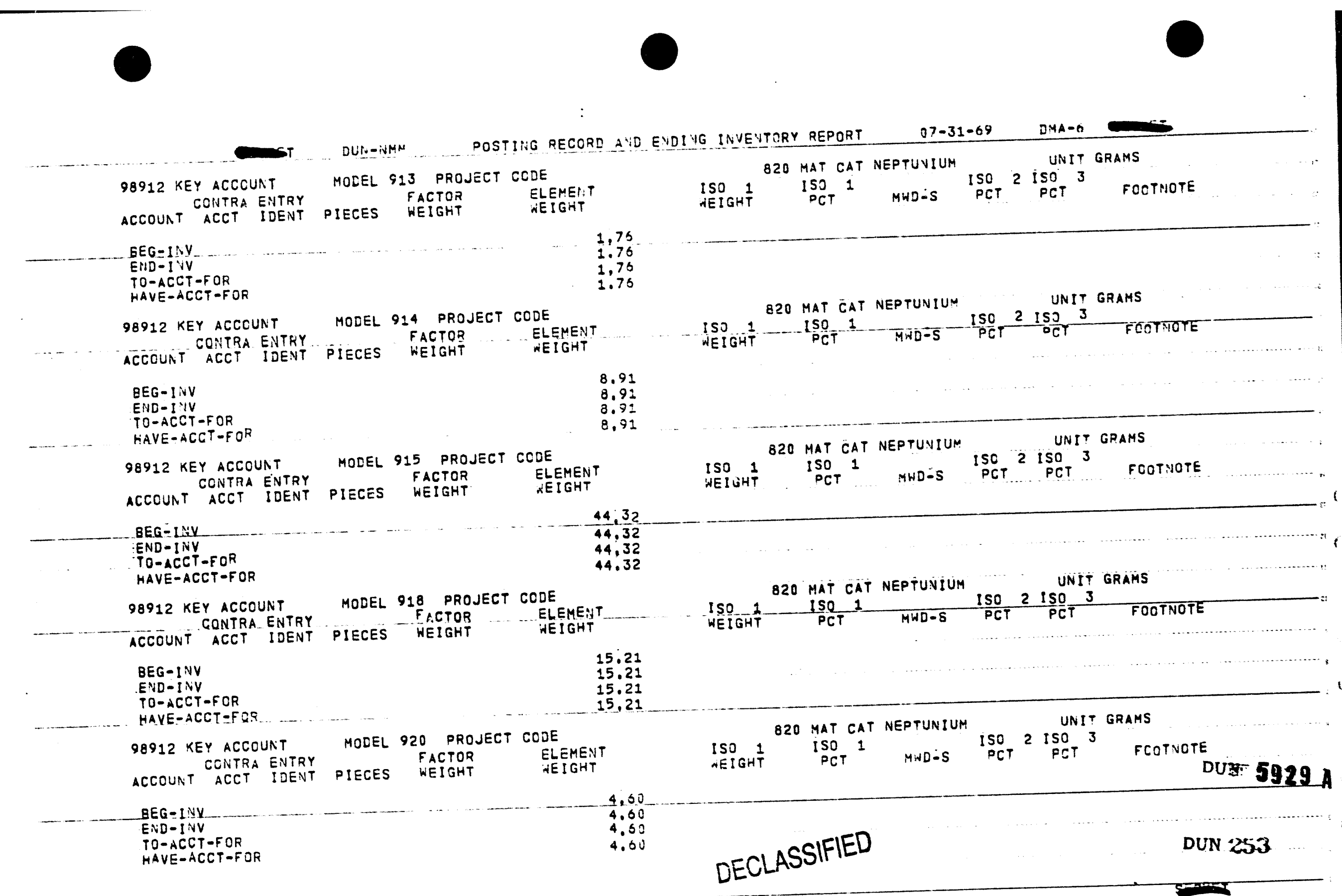


ISO 1 YWD-S ISO 2 ISOT

\section{DECLASSIFIED,}


DLN-INMI1 POSTING RECORD AID ENDING INVENTORY REPORT

$07-31-69$

DMA-6

98913 KEY ACCOUNT

CONTRA ENTRY MODEL 914 PROJECT CCDE ACCOUNT ACCT IDENT PIECES

$B E G-1 \operatorname{Ig} Y$

END-INV

TO-ACCT-FOR

HAVE-ACCT-FOR FACTOR ELEMENTT

WEIGHT

AEIGHT

ISO 1

WEIGHT

OO MAT CAT PLUTONIUM

ISO 1

NIND-S

ISO
UNIT GRAMS

ISO $^{\circ} 3$

FCOTNOTE

$305,73-37,3663 \quad 12,220$

$305.73 \quad 37.3663 \quad 12.220$

$305,79 \quad 37,3663$

$305.78 \quad 37.3663$

$\because$

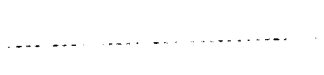

! 



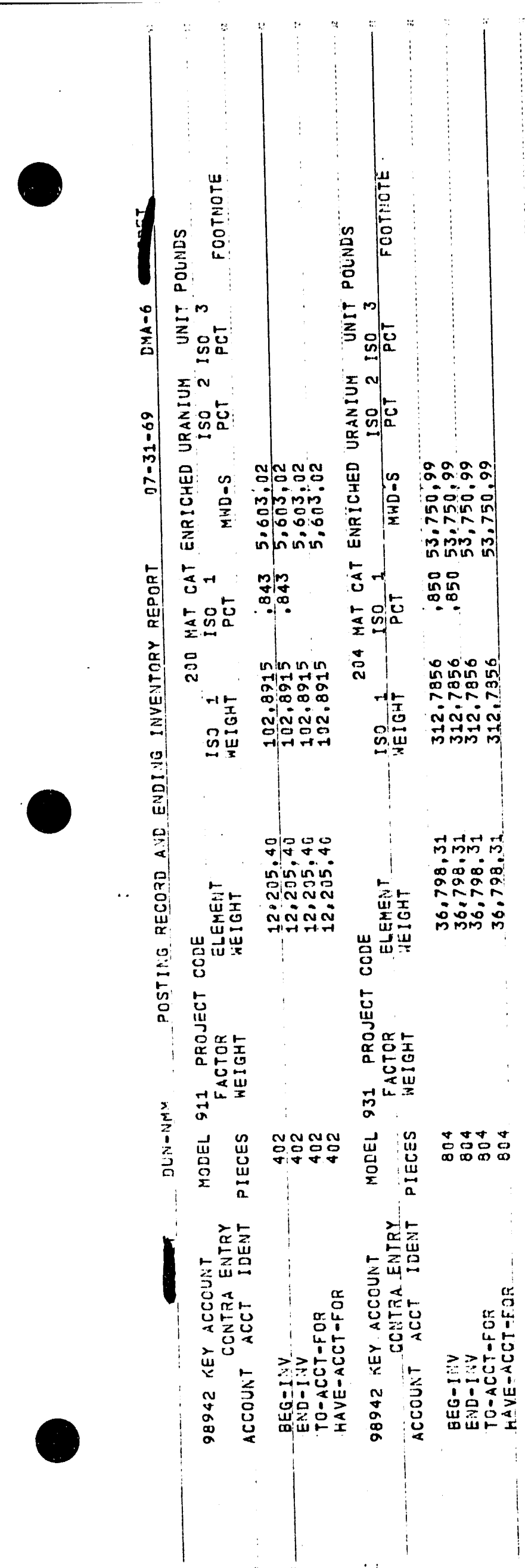




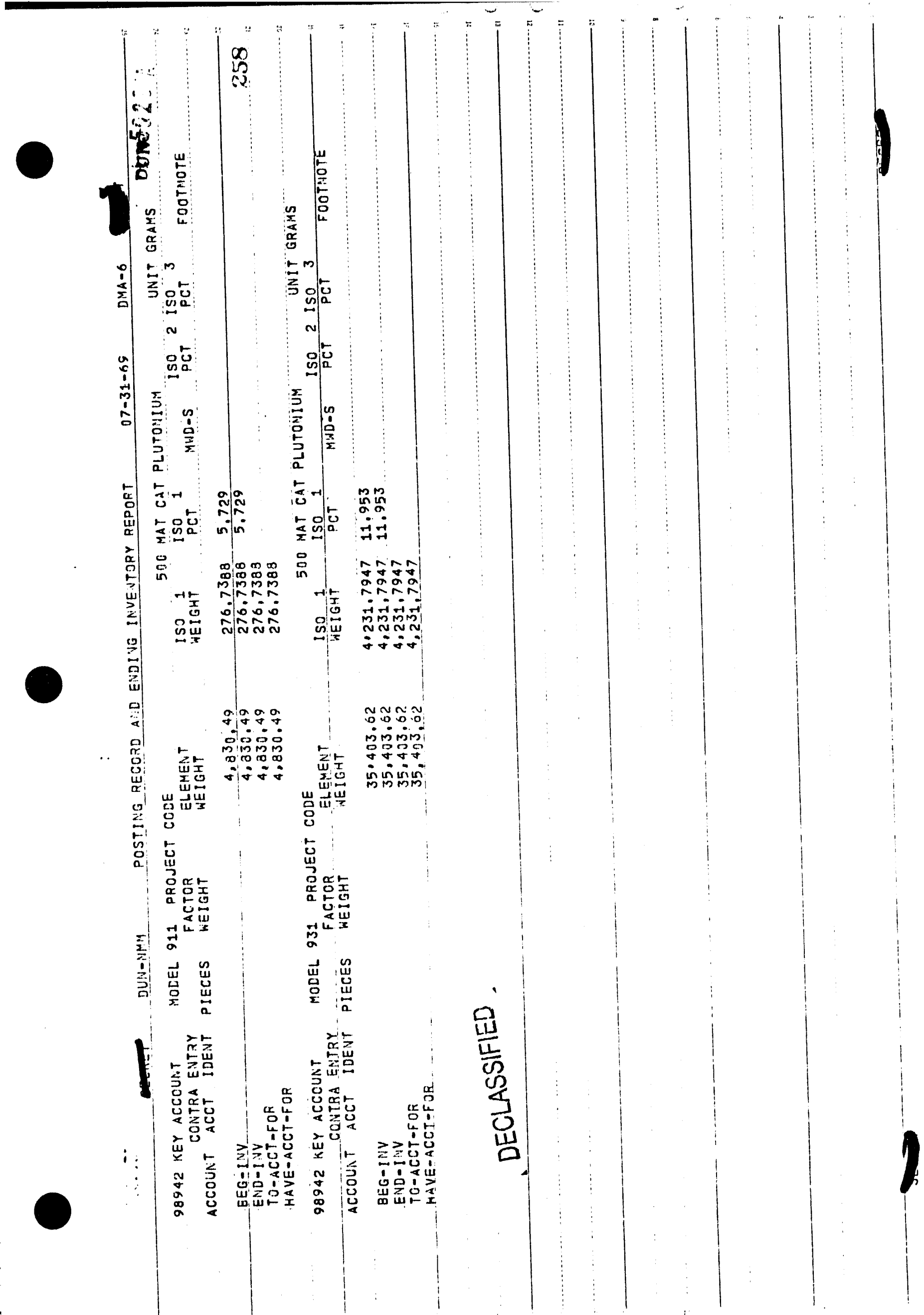




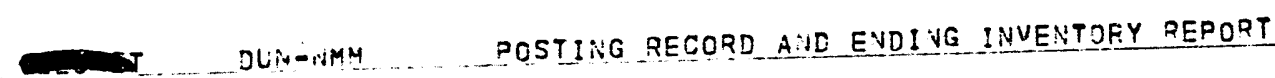

07-31-69

DMA-6

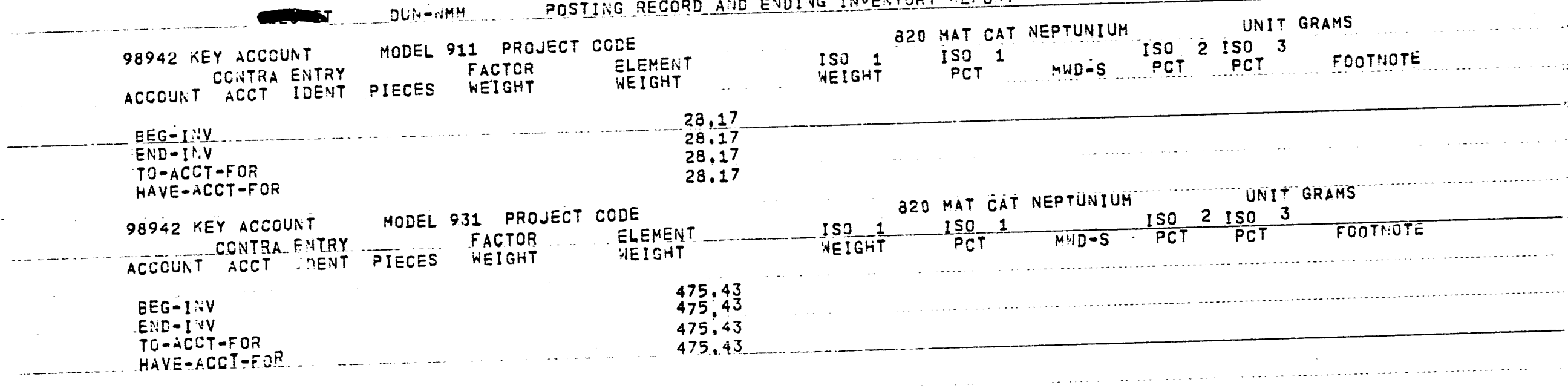




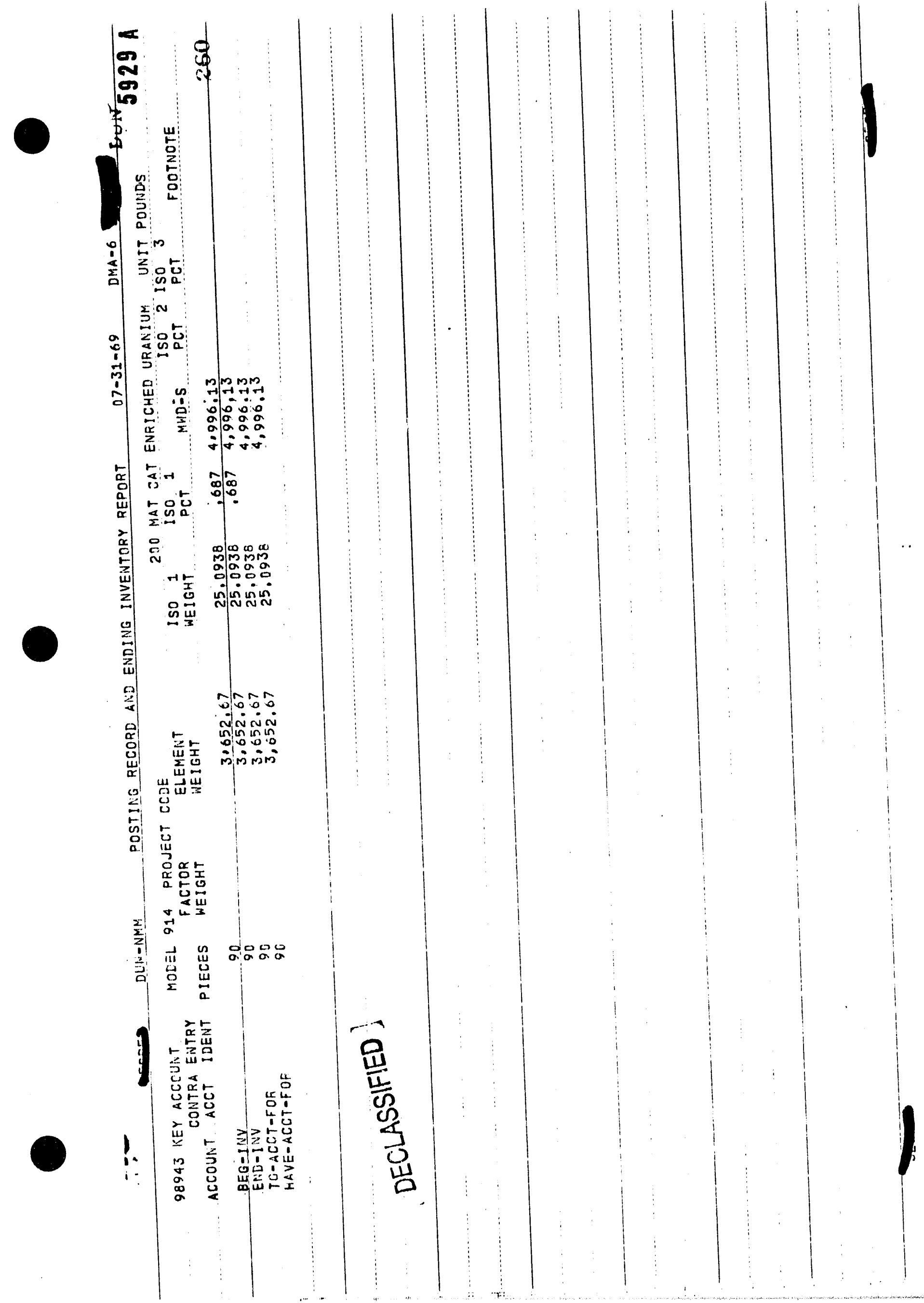




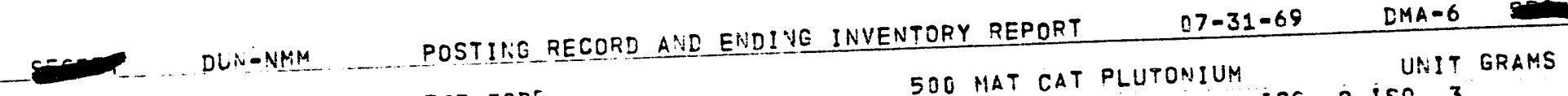
98943 KEY ACCCUNT MOEEL 914 PROJECT CODE
CCNTRA ENTRY FACTOR P BENT

ACCOUNT CCHTRA ENTRY

EEG-INY

END-INV

TO-ACCT-FOK

HAVE-ACCT-FOF

ISO 1 ISO 1

MUD-S

ISO 2 ISO 3

PCT 3 FOOTNOTE

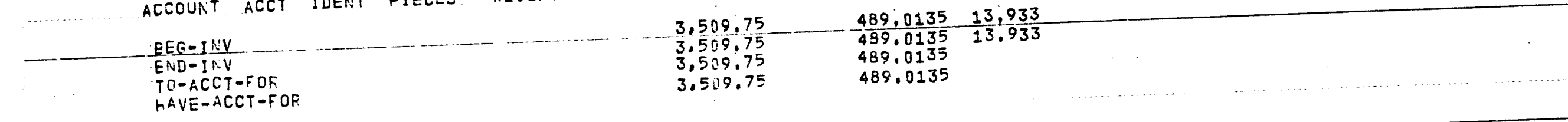




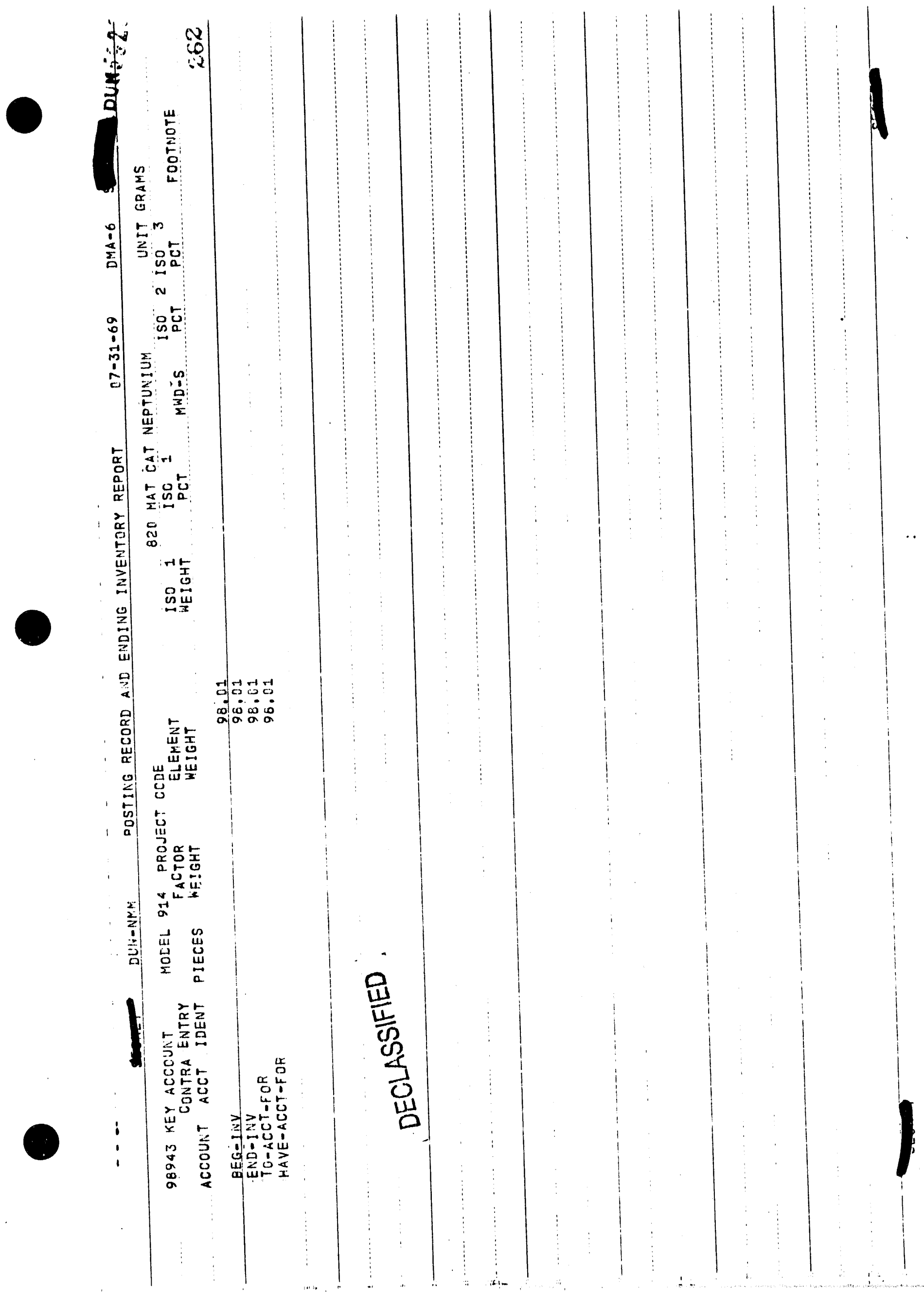


$07-31-69 \quad$ DMA-6

DUN-NYM POSTIRG RECORD AND ENDING INVENTORY REPOET UNIT POUNES

98944 KEY ACCCUNT CONTRA ENTRY MODEL 713 PROJECT CODE MODEL 713 PROTER
PIECES WEIGHT

$\begin{array}{llll}2 & \\ \text { ISO } 1 & \text { ISO } 1 & \text { ISO } 2 \text { ISO } \\ \text { WEIGHT } & \text { PCT } & \text { MHD-S } & \text { PCT PCT }\end{array}$

FOOTNOTE ACCOUNT ACET IDENT

EEG-INY

END-I IVV
TOACT

TU-ACCT
HAVE-ACCT $-F O R$

$1969 \quad 808 \quad 15 ; 40$

$\begin{array}{lll}1969 & .808 & 15,40\end{array}$

, 1969

200 MAT CAT ENRICHED URANIUM UNIT POLNDS

98944 KEY ACCOUNT MODEL 912 PROJECT CODE

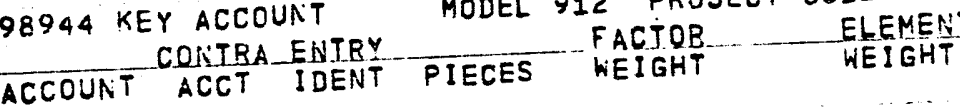
FACIOB -WEIGHT - WEIGH ISO 1 ISO 2 ISO

3 FOOTNOTE

$\begin{array}{lr}E E G-I N V & 231 \\ \text { END-INV } & 231 \\ \text { TO-ACCT-FOR } & 231 \\ \text { HAVE-ACCT-FOR } & 231\end{array}$

98944 KEY ACCOUNT

MODEL 913 PROJECT CODE CONTRA :ENTRY PIECES WEIGHT
ACCOUNT ACCT IDENT PIECS WATT

ACCOUNT ACCT IDENT PIECES

EEG:INY

908

908
908

PCT MHD-S

TE-ACCT-FOR

HAVE-ACCT-FOF

$33,201,02$

7.660 .67

7.660 .67

7.660 .67

$\begin{array}{lll}61.8216 & .807 & 4,883,71 \\ 61,8216 & .807 & 4,883,71 \\ 61,8216 & & 4,883,71\end{array}$

$\begin{array}{ll}61,8216 & 807 \\ 61,8216 & 4,883,71 \\ 61 & 4,883,71\end{array}$

61.8216

ACCOUNT MOLEL 914 PROJECT CODE

COLTRA ENTRY FACTO' ...... ELEMEUT ACCOUNT ACCT IDENT PIECES WEIGHT

WEIGHT

$\begin{array}{ll}\text { BEG-INV } & 1,170 \\ \text { END-IIUV } & 1,170 \\ \text { TU-ACCT-FOF } & 1,170 \\ \text { HAVE-ACCI-FOR } & 1,170\end{array}$

47.505 .57

47.505 .57

$47,5,5.57$

HAVE-ACCI-FOR

98944 KEY ACCOUNT MODEL 915 PROJECT CCD

CONTRA ENTRY

$\begin{array}{lll} & \text { FACTOR } & \text { ELEMENT } \\ \text { PIECES WEIGHT } & \text { WEIGHT }\end{array}$

EEG-IIVY

END-INIV

HAVE-ACCT-FOF

96
96
96
96

ISO 1 ISO 1 MCT ${ }^{2}$ ISO 2 ISO 3 PCT

268,2643 $808 \quad 20,975 ; 07$

$268.2643 \quad .808 \quad 20.975 .07$

$268,2643 \quad 20.975,07$

$\begin{array}{ll}268,2643 & 20.975 .07\end{array}$

200 MAT CAT ENRICHED URANIUM UNIT POUNDS

ISO 1 IEIGHT

$335.1661 \quad .70659 .024,23$

$335,1861 \quad .706,59,024,23$

$335.1861 \quad 59.024 .23$

335.1861
335.1861

$4,369,63$

$4,369,63$

$4,369,63$

4.369 .63

200 MAT CAT ENRICHED URANIUM UNIT POUNDS

PCT 1 MWDES PCT PCT FCOTNCTE

$29.1583 \quad .667 \quad 6.586,16$

$\begin{array}{lll}29,1583 & .667 & 6,586,16 \\ .667 & 6,586,16\end{array}$

$29,1563 \quad 6,586,16$

$29.1563 \ldots .586 .16$

$=$ MASTES 


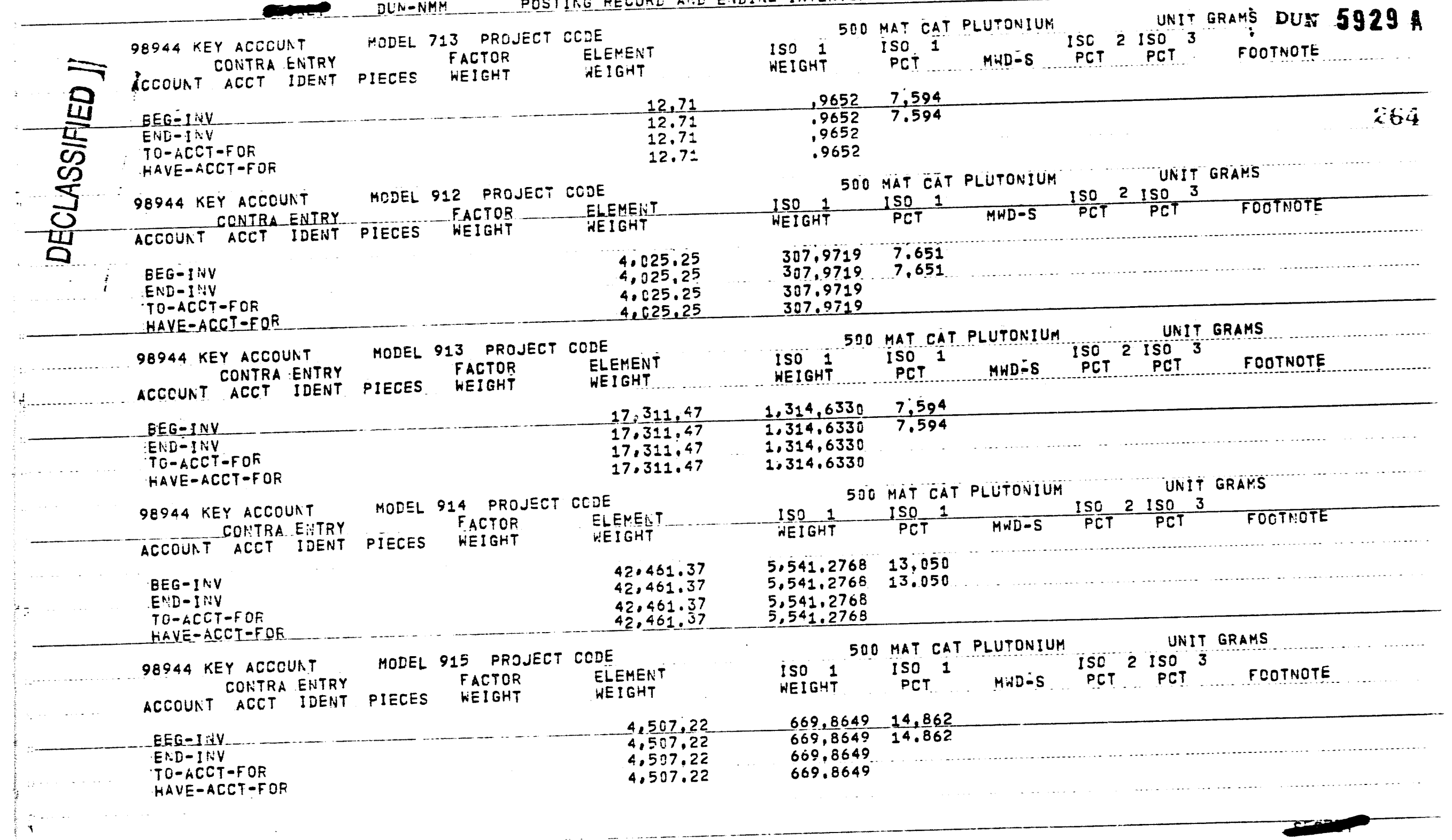


DULONMM

POSTINE RECORD ANE ENDING INVENTORY PEPORT

$07-31-69$

IMA-6 SIAOET

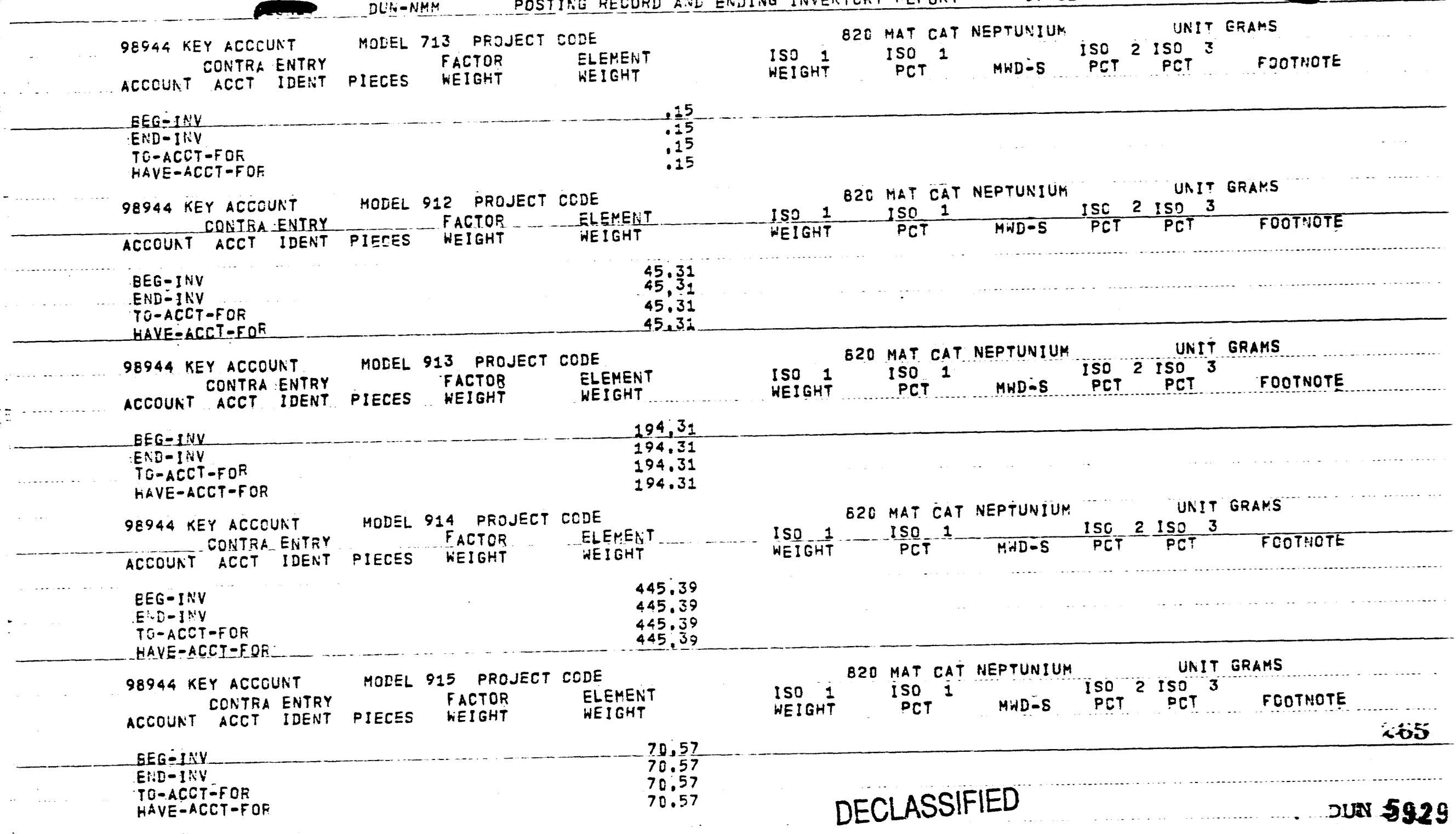


DUN-NMM POSTING RECORD AND ENDING INVENTORY REPORT

$07-31-69$

DMA-6

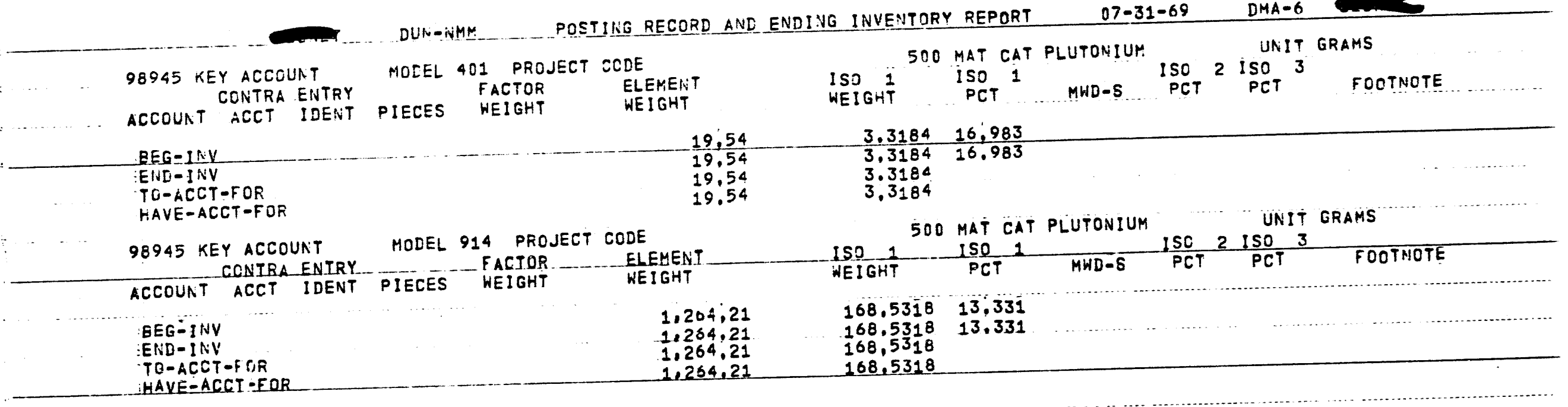


DUN-NMM POSTIL:G RECORD ANE ENDING INVENTORY REPORT O7-3

$07-31-69$

DMA-6 Steina

98945 KEY ACCOUNT, MOEEL 412 PROJECT CODE FACTOR ELEMENT ISO 1 ISO 1 UNIT GRAMS

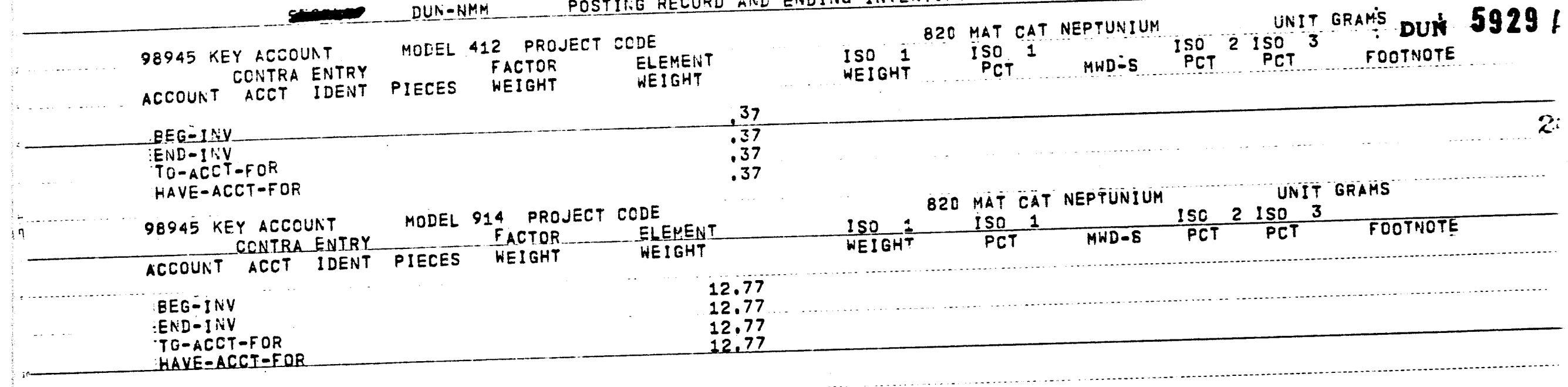

\section{DECLASSIFIED ,}




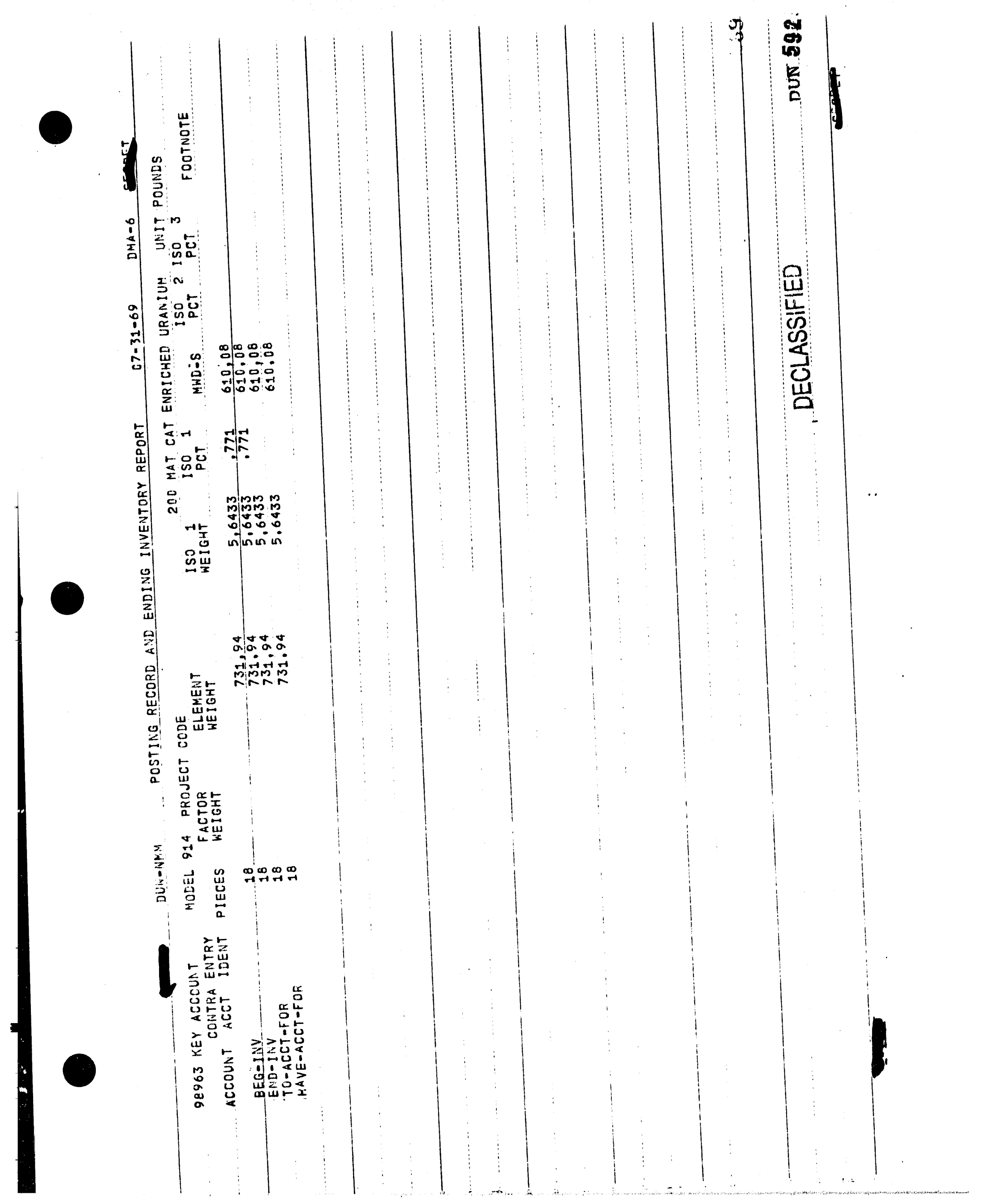




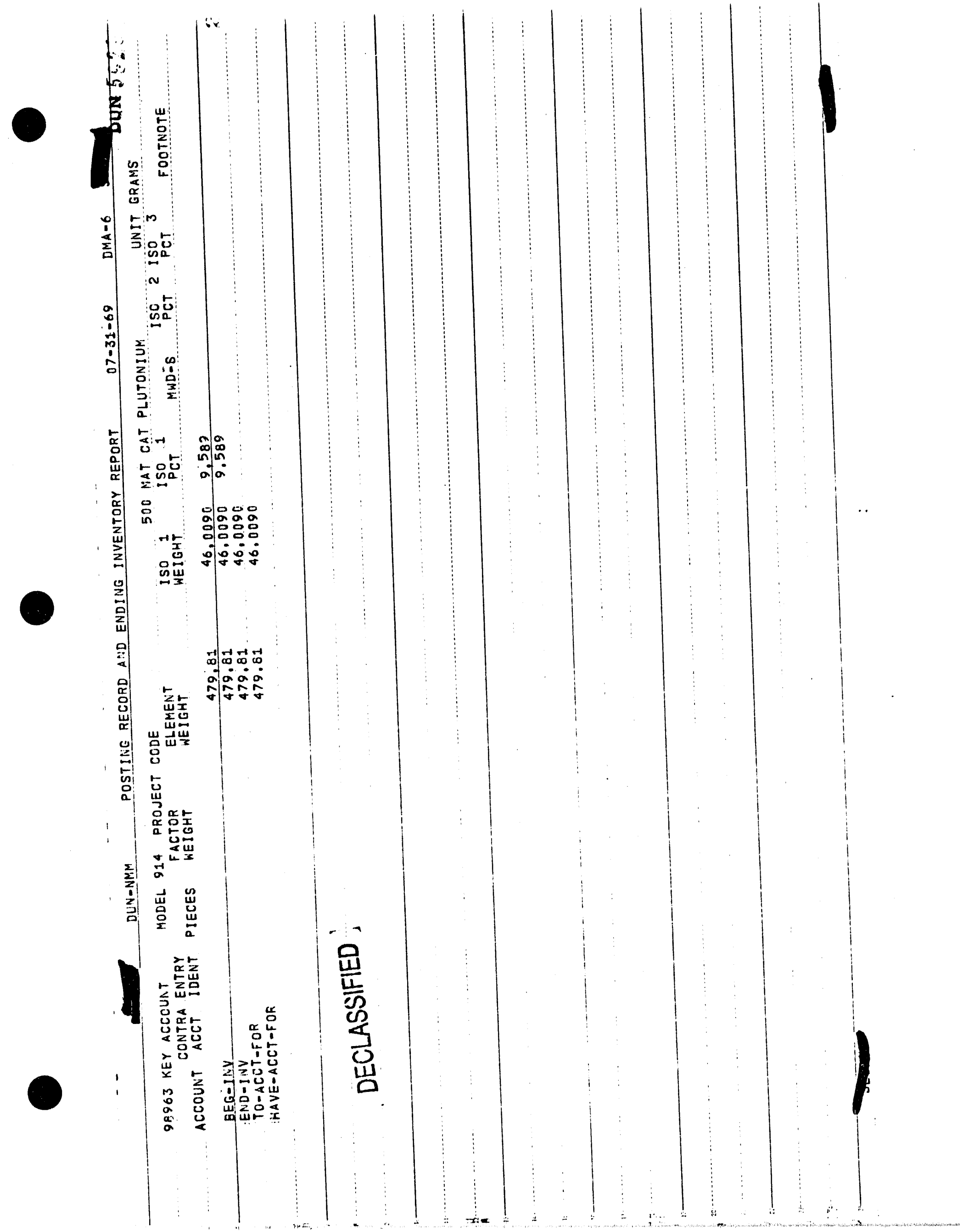


-

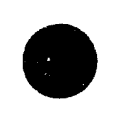

DUN-NMM ... POSTING RECORD AND ENDING INVENTORY REPORT

$07-31-69$

DMA-6

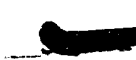

98963 KEY ACCOUNT CONTRA ENTRY MODEL 914 PROJECT CODE FACTOR ELEMENT ACCOURT ACCT IDENT PIECES WEIGHT

BEG-INY END-INV

TO-ACCT -FO

HAVE-ACCT-FOR

\section{MAT CAT NEPTUNIUM} ISO 1 ISO
UNIT GRAMS

iso 3

WEIGHT ISO

\section{FOOTNOTE}

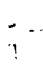

)

)

:a.

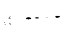

…….... 


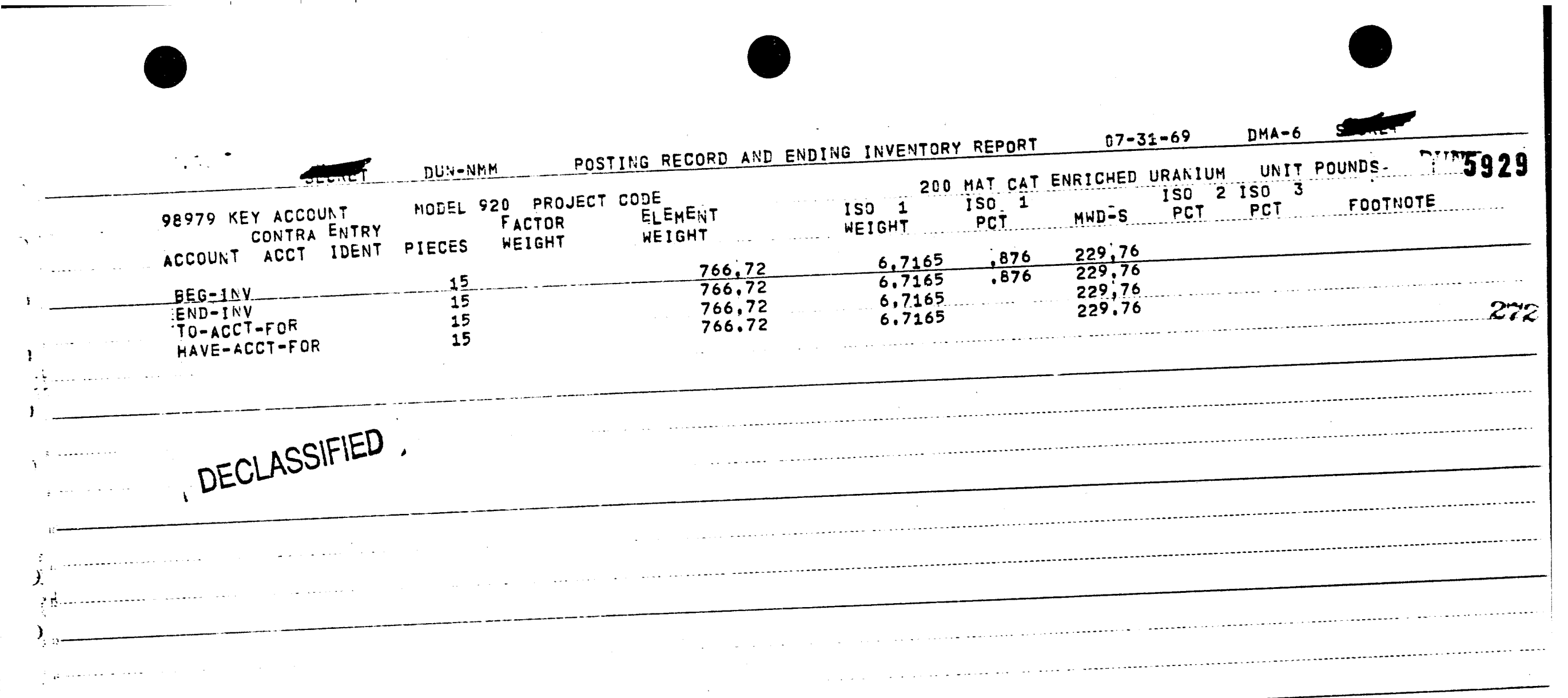




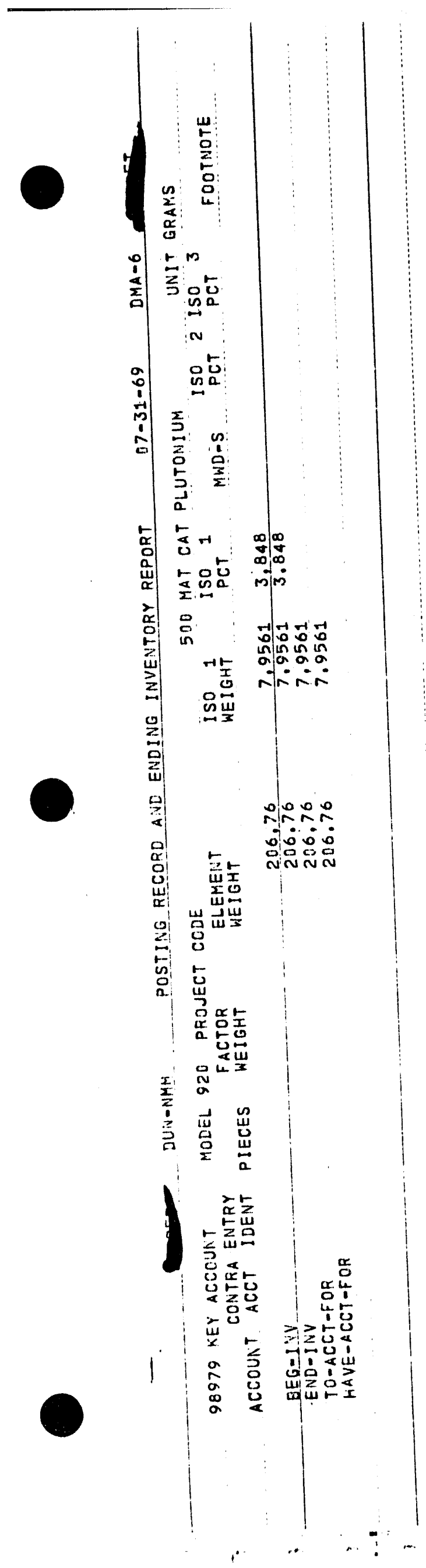

$\left|\begin{array}{cc}12 & 0 \\ 1 & \infty \\ \cdot 2 & 4 \\ & 3 \\ & 0\end{array}\right|$

$\frac{0}{5}$
$\frac{5}{5}$
$\frac{5}{4}$
岁 
DMA-6

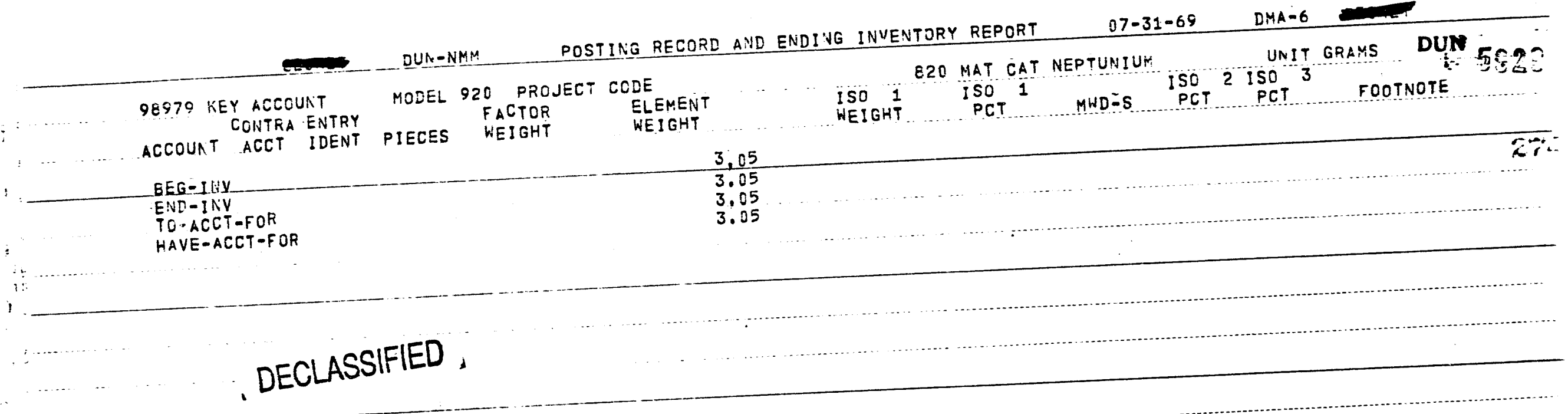

5 i i

) 


\section{' $0 \exists 1 \mid 1 S S \forall 70 \exists 0$}

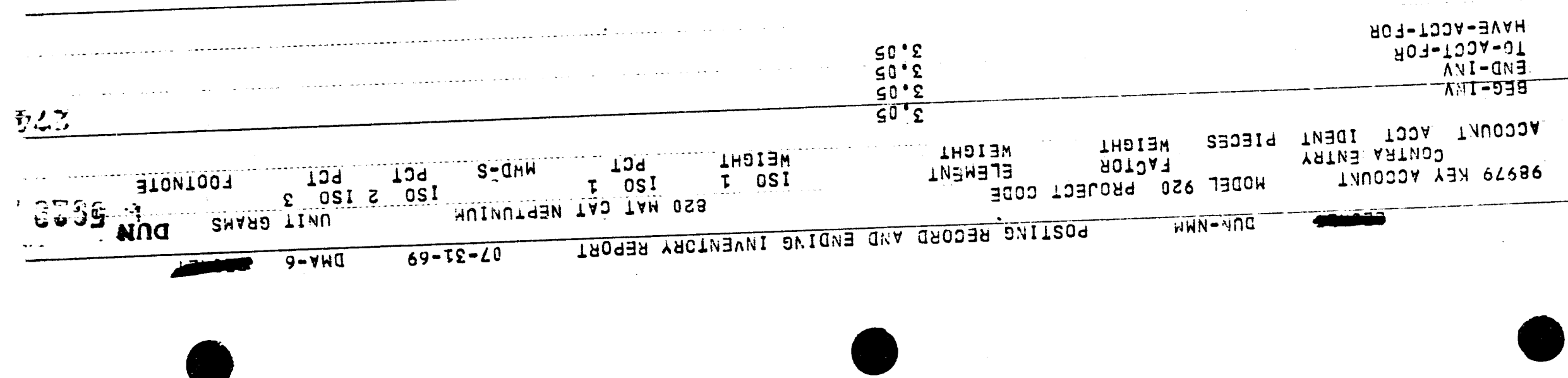




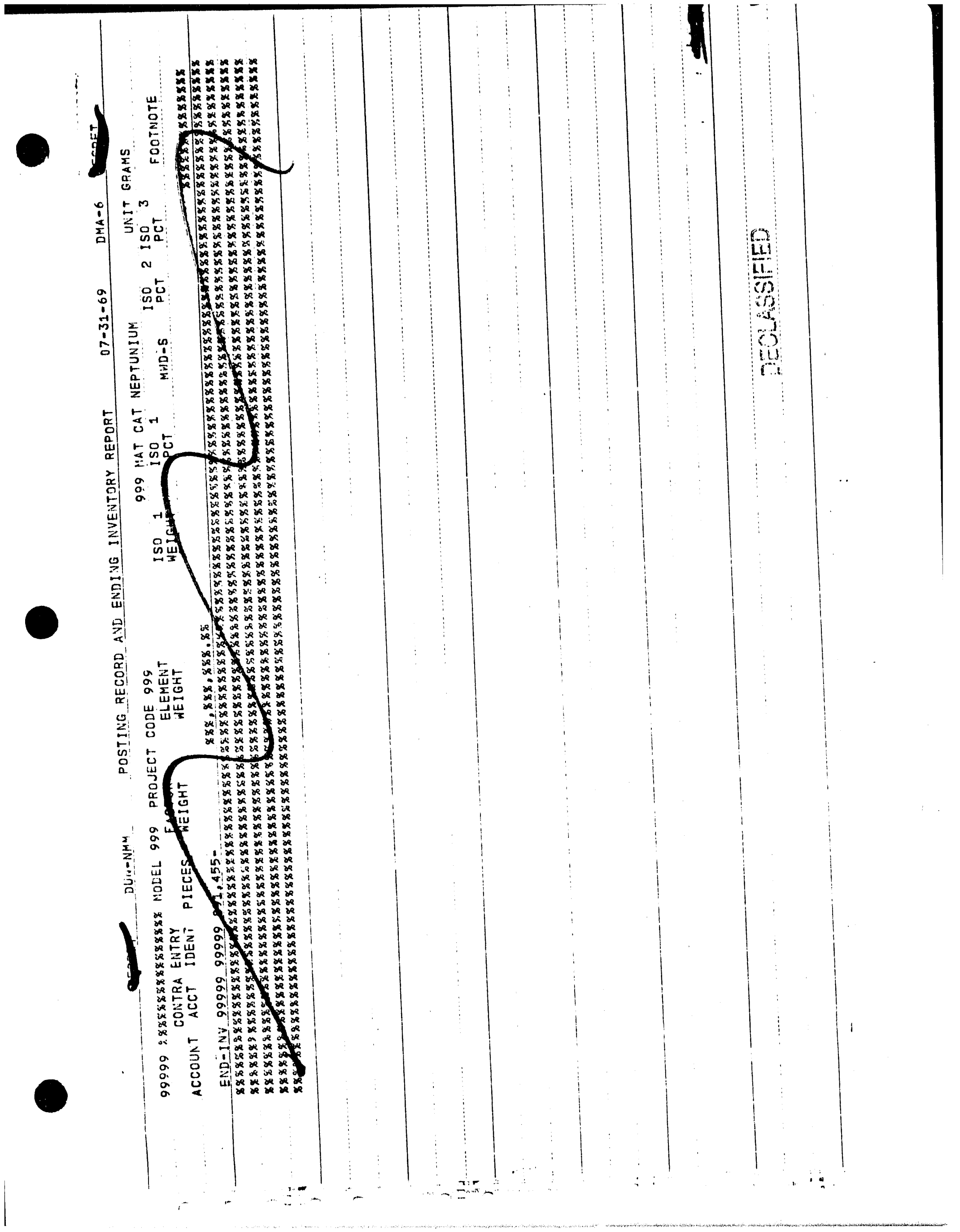


